
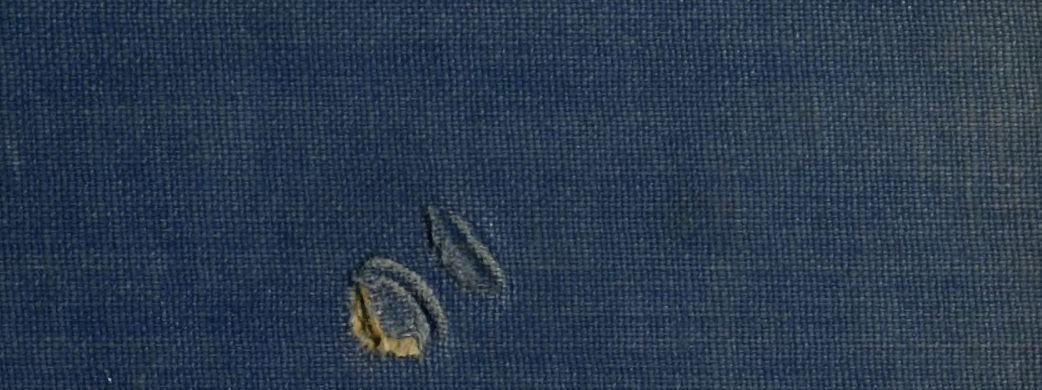

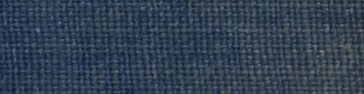
H.

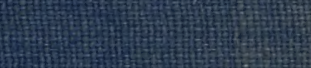

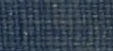

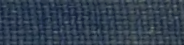
$+2$

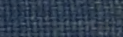
I"

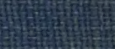

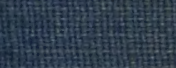

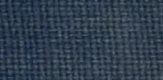

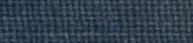

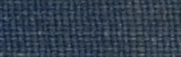




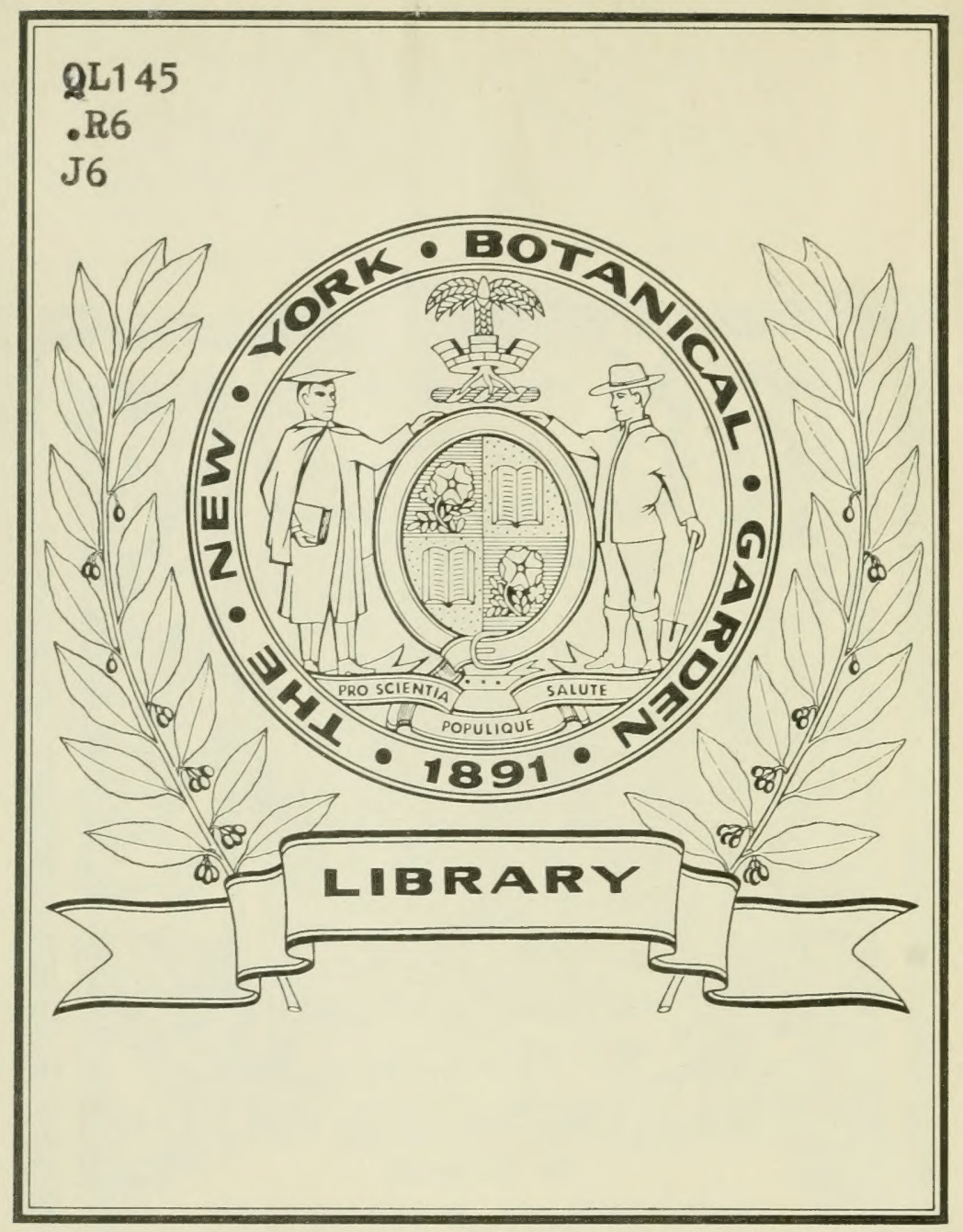

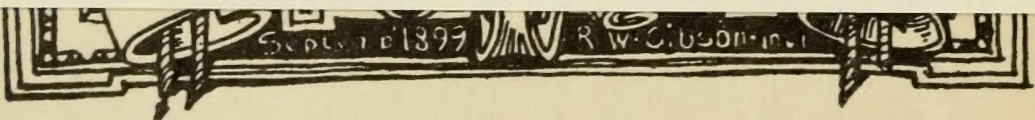


Sleaves GMarguaud

$$
1938
$$







\section{LA ROSE}

DANS L'ANTIQUITÉ ET AU MOYEN AGE 


\section{OUVRAGES DU MÊME AUTEUR}

Loi des finales en espagnol. Nogent-le-Rotrou, 1872 .

Du $c$ dans les langues romanes. Paris, 1874, in-8.

$n$

De rhotacismo in indoeuropaeis ac praesertim in germanicis linguis. Paris, 1875, in-8. . . . . . $3 \mathrm{fr}$.

Herder et la Renaissance littéraire en Allemagne au xvine siècle. Paris, 1875, in-8. . . . . . Épuisé

La littérature allemande au xvı1e siècle dans ses rapports avec la littérature française et la littérature anglaise. Aix, 1876, in-8. . . . . . . $1 \mathrm{fr} .50$

La légende de saint Alexis en Allemagne. Paris, 1881, in-8.

1 fr. 50

Essai sur le patois du Bessin, suivi d un Dictionnaire étymologique. Paris, 1881, in-8.

Des caractères et de lextension du patois normand. Étude de phonétique et d'ethnographie, suivie diune carte. Paris, 1883, in-8.

$6 \mathrm{fr}$.

Correspondance inédite du duc du Maine avec Lamoignon de Basville. Paris, 1883 , in-8. . . . . . Épuisé.

Mélanges de phonétique normande. Paris, 1884, in-8 . $3 \mathrm{fr}$.

Des rapports intellectuels et littéraires de la France avec l'Allemagne avant 1789. Paris, 1884, in-8 .... Épuisé.

La crise agricole en Normandie. Conférence faite au cercle SaintSimon. Paris, 1885 , in-8. . . . . $1 \mathrm{fr} .50$

J.-B. Tavernier, écuyer, baron d'Aubonne, chambellan du GrandÉlecteur Paris, 1886, in-8. . . . . . 7 fr. 50

Flore populaire de la Normandie. Caen, 1887, in-8. . . 6 fr.

Les dictionnaires du patois normand. Macon, 1887, in-8 . ”

Le livre des simples inédit de Modène et son auteur. Paris, 1888, in-8. . . . . 1 fr. 50

Les incantations botaniques du manuscrit F. 277 de la Bibliothèque de lÉcole de médecine de Montpellier. Macon, 1888, in-8. „

Le voyageur Tavernier (1670-1689). Paris, 1889, in-8. 1 fr. 50

Le P. Guevarre et les bureaux de charité au xvire siècle. Toulouse, 1889, in-8. . . . . . 2 fr. 50

Pierre et Nicolas Formont. Un banquier et un correspondant du Grand-Électeur. Paris, 1890, in-8. . . 2 fr. 50

La légende de la rose au moyen àge chez les nations romanes et germaniques. Macon, 1891, in-8.

CHARTRES. - IMPRIMEIE DURAXD, RUE FULBEIT. 


\title{
LA ROSE
}

\section{DANS L'ANTIQUITÉ ET AU MOYEN AGE}

\author{
HISTOIRE, IEGENDES ET SYMBOLISME
}

PAR

\section{CHARLES JORET}

Professeur à la Faculté des lettres d'Aix

Correspondant de I'Institut.

La rose

Fist plus que nule autre flors bele.

Chrestien de Troyes, Cligès, v. 208.

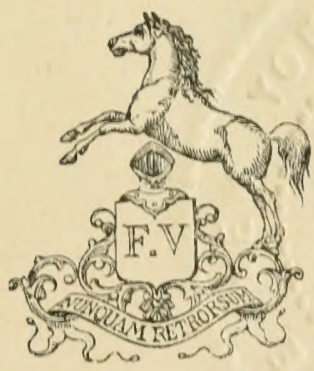

\section{PARIS}

EMILE BOUILLON, ÉDITEUR

67, RUE RICHELIEU, 67

1892

TOUS DROITS RÉSERVÉS

"IJPRARY

NWW YORK

BOTANICAL

OARDBN 
$580 \% 9$.

$-5762$ 


\title{
A MONSIEUR PAUL MEYER
}

\author{
MEMBRE DE L'INSTITUT
}

\section{Monsieur,}

C'est à votre bienveillante initiative que je dois d'avoir été nommé, en 1887, correspondant de l'Institut; permettez-moi, en témoignage de recomnaissance, de vous dédier cet essai sur la rose; s'il peut mériter votre suffrage, je ne croirai pas, en l'écrivant, avoir fait œuvre inutile.

Charles JORET. 



\section{PRÉFACE}

On a beaucoup écrit sur la rose et l'on peut se demander s'il était nécessaire d'ajouter un livre nouveau aux nombreux ouvrages dont elle a été l'objet; il m'a semblé néanmoins qu'il n'était pas impossible d'enrichir beaucoup l'histoire, moins connue qu'on ne le pense, de cette fleur aimée, et l'étude que je lui ai consacrée diffère peut-ètre assez de celles qui l'ont précédée pour que j’aie cru pouvoir l'entreprendre.

D'ailleurs le dernier et sans contredit le meilleur des ouvrages publiés en France sur la rose - je ne parle pas des essais plus anciens, incomplets ou introuvables, pas plus que du livre par trop élémentaire de M. J. Bel, paru il y a à peine quelques semaines, - celui du docteur Loiseleur-Deslongchamps, remonte à près de cinquante ans et est épuisé; on pouvait done songer à le remplacer. Je n'ai point eu cependant l'intention de le faire; c'est tout autre chose que j'ai essayé.

Loiseleur-Deslongchamps a voulu à la fois faire l'histoire de la rose et exposer les procédés employés pour la cultiver et l'embellir. Horticulteur, non moins que médecin distingué, la seconde partie de son livre est pleine de préceptes utiles, dont les amateurs de jardins peuvent profiter encore aujour- 
d'hui. Je n'ai ni l'expérience nécessaire pour le suivre dans cette roie, ni eu le désir de rivaliser avec lui. C'est l'histoire seule de la rose jusqu'à l'époque de la Renaissance, surtout son histoire poétique et légendaire, que je me suis proposé de raconter.

Loiseleur-Deslongchamps, qui l'a abordée dans la première partie de son livre, est loin de lui avoir fait la place à laquelle elle avait droit; comme ses précurseurs, il ne s'est presque occupé que de la rose dans l'antiquité et il a à peu près ignoré ce qu'elle devint au moyen âge. C'est à peine s'il a parlé des légendes dans lesquelles la rose figure chez les Orientaux; il n'a rien dit des traditions si curieuses dont l'ont entourée les peuples germaniques, et n'a guère mentionné davantage celles non moins curieuses où on la rencontre chez les nations romanes; enfin s'il a donné un recueil assez étendu des pièces de vers - faibles imitations bien souvent de leurs devanciers grees ou latins - dans lesquelles les poètes modernes l'ont chantée, il n'a point su ou a passé sous silence ce qu'ont dit de la rose les poètes du moyen âge, aussi bien français qu'étrangers.

Le dernier écrivain non francais, qui, avant l'ouvrage estimable, quoique non toujours original, de

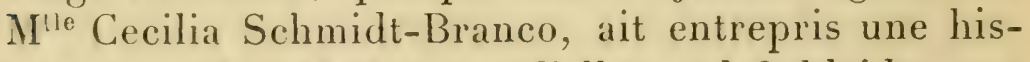
toire complete de la rose, l'allemand Schleiden, ne s'est point exposé aux mèmes critiques et il a accordé avec raison, dans le livre qu'il a publié en 1873, une place considérable aux légendes auxquelles elle a donné naissance chez les peuples anciens et modernes. J'ai suivi son exemple, sans 'l'imiter en tout et pour tout. 
Schleiden a retracé les destinées de la rose jusqu'à nos jours; je m'arrête à la fin du moyen âge; il s'est jeté parfois dans des digressions qui l'ont emporté hors de son sujet; je m'y suis scrupuleusement renfermé, comme je me suis gardé des assertions hasardées et des théories aventureuses, auxquelles il se complait trop souvent. Enfin, s'il a fait une large place aux traditions des peuples germaniques qui concernent la rose, il a passé trop rapidement sur ce qu'elle a été pour les nations romanes. J'ai tenu doublement à réparer cet oubli.

J'ai cru aussi que je ne devais pas, comme il l'a fait, me borner à quelques vagues indications sur la culture de la rose avant la Renaissance, ainsi que sur l'emploi si général qu'en a fait l'ancienne pharmacopée; j'ai de mème énuméré longuement les nombreux usages auxquels la rose servait dans la vie publique ou privée; mais ce sont les légendes aussi variées que gracieuses, dont elle a été entourée dans l'antiquité, comme au moyen àge, que je me suis attaché à recueillir et à coordonner avec tout le soin possible. C'est un chapitre du plantlore, je n'ose dire - le mot serait sans doute ici bien ambitieux — de la mythologie des plantes, que j'ai voulu écrire; j'ajouterai un chapitre détaché de l'histoire du monde végétal, à laquelle je travaille depuis plusieurs années. C'est en mème temps un complément à l'article sur la Légende de la rose au moyen âge chez les nations romanes et germaniques, publié dans le recueil que les élèves français de M. Gaston Paris ont, à l'occasion du vingt-cinquième anniversaire de sa réception au doctorat, offert au maitre des études romanes en France, article que les bornes qui 
m'étaient imposées ne m'ont pas permis alors de développer.

Le désir peut-être légitime de mettre en œuvre les nombreux matériaux que j'avais réunis pour ce travail et que je n'avais pu utiliser, celui de répondre au goût grandissant chez nous - encore qu'il soit loin d'approcher de ce qu'il est à l'étranger ${ }^{1}$ pour les traditions botaniques, m'ont engagé à écrire ce livre. Il m'a coûté de longues recherches; je serai assez récompensé de mes efforts, si l'on trouve que je ne suis pas resté trop au-dessous de ma tâche, et si cet essai peut avoir retenu quelque chose de l'intérèt et du charme qui s'attachent si naturellement à la fleur

$$
\text { plus que nule autre bele, }
$$

comme le dit un de nos vieux poètes.

Aix, le 25 décembre 1891.

1. Tandis que chaque année presque voit paraitre, en Allemagne et surtout en Angleterre, quelque nouvelle publication sur les légendes du monde végétal. nous n'avons à leur opposer que la Mythologie des plantes, de 11 . de Gubernatis, qui, si elle a été publiée en France, n'est pas l'œuvre d'un Français. 


\section{PREMIERE PARTIE.}

LA ROSE DANS L'ANTIQUITÉ.

\section{CHAPITRE PREMIER.}

DES ESPÉCES DE ROSES CONNUES DES ANCIENS.

Depuis plus de deux mille ans la rose a été célébrée à l'envi par les poètes; elle a été entourée des légendes les plus gracieuses; elle a pris place dans les traditions comme dans les usages de tous les peuples; cependant, son histoire présente les plus grandes obscurités et les origines de cette fleur charmante sont encore enveloppées d'un voile épais. La plus grande incertitude règne au sujet des espèces de roses dont les écrivains de l'antiquité ont fait mention, et on est loin d'ètre d'accord sur celles qu'ils ont pu connaitre ou qui ont été cultivées à cette époque, ainsi qu'au moyen âge. 
I.

Des nombreuses espèces du genre Rosa - Lindley en compte $73^{1}$ et $A$. de Candolle $146^{2}$, toutes non bien définies, il est vrai ${ }^{3}$, - et de ses variétés encore plus nombreuses ${ }^{4}$, bien peu furent connues des anciens. Ils ignorèrent, cela va sans dire, les roses qui sont propres ì la Chine ${ }^{5}$ et à l'Amérique, ainsi que celles de l'Asie et de l'Europe septentrionales; ils ne purent observer et par suite songer à cultiver que les espèces du sud de l'Europe, en particulier de la péninsule hellénique, ainsi que de l'Asie Antérieure. Nais les 46 espéces que Boissier ${ }^{6}$ a signalées dans

1. Monographie du genre rosier, traduite de l'anglais de M. E. Lindley, etc., par M. de Pronville. Paris, 1824, in-8. Aux 73 espèces primitives en ont été ajoutées 25 autres plus ou moins certaines.

2. Prodromus systematis naturalis regni vegetabilis. Parisiis, 1825, in-8, vol. II, p. 597-626.

3. Dans son Nomenclator botanicus, 1841, in-8, Steudel compte jusqu'à 217 espèces de roses. Toutes ces espèces appartiennent à lhémisphère boréal et leur habitat est compris d'une manière générale entre le $20^{\circ}$ et le $70^{\circ}$ degré de latitude ; il n'y a d'exception véritable que pour la Rosa abyssinica Brown, qui croît au sud du $12^{e}$ degré, mais dans une région montagneuse. La Rosa pimpinellifolir L., qui se rencontre en Islande, croit aussi bien au delà du 70 degré.

4. Lindley en énumère 329 et $\mathrm{A}$. de Candolle plus de 180 .

5. C'est de la Chine que vient en particulier la rose thé, type d'un si grand nombre de variétés, ainsi que la rose de Bengale.

6. Flora orientalis sive enumeratio plantarum in Oriente 
cette région sont loin d'avoir toutes fixé l'attention des naturalistes de l'antiquité ${ }^{1}$.

Sprengel ${ }^{2}$ a compté 8 roses connues ou cultivées par les anciens; Fraas n'en mentionne que 5 dans sa flore classique ${ }^{3}$; Lenz, 4 seulement ${ }^{4}$, Karl Koch a élevé ce nombre à 12 pour la Grèce seule ${ }^{5}$; mais de ces espèces, plusieurs sont douteuses, de l'aveu même de l'auteur, ou n'ont été découvertes que de

a Graecia et Aegypto ad Indiae fines hucusque observatarum. Gen.-Basil.-Lugd., 1872, in-8, vol. II, p. 672-682.

1. Il en est de mème des roses italiennes. Giovanni Arcangeli (Compendio della flora italiana, Torino, 1882, in-8), en compte 28 espèces, dont le quart sont des espèces de montagnes récemment découvertes; les autres sont presque toutes communes à la Grèce.

2. Les $R$. sempervirens, damascena, canina, provincialis, alba, gallica, villosa et spinosissima L. (Geschichte der Botanik. Leipzig, 1817, vol. I, p. 75, 123, 134, 150 et 168). Dans l'édition latine du mème ouvrage (Historia rei herbariae. Amst., 1807 , in-8), l'auteur mentionnait la $R$. centifolia et non la gallica.

3. Les $R$. sempervirens, canina, pimpinellifolia, gallica et centifolia L. (Synopsis plantarum florae classicae, oder übersichtliche Darstellung der in den classischen Schriften der Griechen und Rümer vorkommenden Pflanzen. München, 1845, in-8, p. 74-76).

4. Les $R$. gallica et centifolia, canina et sempervirens L. (Botanik der alten Griechen und Römer. Gotha, 1859, in-8, p. 691-700).

5. Les $R$. damascena et centifolia, avec les $R$. spinosissima, alpina, Heckeliana, Orphanidis, Heldreichii, canina, rubiginosa, repens Scop. (arvensis L.) et sempervirens: (Die Bäume und Sträucher der alten Griechenlands. Berlin, in-8, $2^{e}$ éd. 1884, p. 157-166). 
nos jours; en réalité, des 12 roses indiquées par le botaniste allemand, il n'y en a que 3 ou 4 qui aient pu ètre connues ou remarquées des anciens.

Sur ce nombre si petit, quelles furent les espèces qu'ils cultivèrent, celles que leurs poìtes ont, sinon exclusivement, du moins plus particulierement chantées? Dierbach ${ }^{1}$ - pour ne pas parler de Sprengel, sur lequel je reviendrai, - a regardé la rose à cent feuilles comme la seule qui figure dans les légendes gréco-romaines. Pour Fraas, cette espèce fut aussi et surtont cultivée dans l'antiquité, mais, suivant lui, la rose de Provins (R. gallica L.) l'aurait été en mème temps. Telle a été aussi la manière de voir de Lenz. Hehn², au contraire, considère, ainsi que Dierbach, la cent-feuilles comme la seule rose dont parlent les poètes anciens. Il n’en est pas de mème pour Koch. Si la rose à cent feuilles fut, il l'admet, célébrée par eux, ils auraient en mème temps chanté une autre espéce, non toutefois la rose de Provins ${ }^{3}$, mais la rose de Damas ( $R$. Damascena L.)

Cette maniere de voir n'est point nouvelle, c'était celle de Carl Bøetticher dans son étude sur le C'ulte

1. Dr. Joh. Heinrich Dierbach, Flora mythologica oder Pftansentiunde in Besug auf Wythologie und symbolik der Griechen und Rümer. Frankfurt-a.-M., 1833, in-8, p. 155-160.

2. Kulturpflanzen und Hausthiere in ihrem Uebergang aus Asien nach Griechenland und Italien sowie in das übrige Europa. Berlin, 1887 ( $5^{\circ}$ éd.), in-8, p. 200-210.

3. Il mentionne aussi, parmi les espèces de celte contrée, la rose de Provins, mais en passant et sans dire si elle y était oui ou non cultivée. 
des arbres chez les Hellènes ${ }^{1}$. Pour cet écrivain, la rose dont on faisait, nous le verrons, des offrandes sur les tombeaux, était, non la cent-feuilles de couleur claire, mais la rose simple de couleur foncée? malheureusement il ne dit pas quelle était cette rose. Quant à la cent-feuilles, s'il paraît en admettre l'existence, Bœtticher n'a rien dit cependant qui permette de savoir où elle était cultivée, ni même si elle l'était réellement. Koch a été plus affirmatif.

Il y eut en Grèce, remarque-t-il ${ }^{3}$, deux espèces de roses cultivées (Edelrosen), la rose de Damas dans la région du sud et la rose à cent feuilles au nord. ... La rose de Damas, ajoute-t-il plus loin, fut sans doute connue plus tôt en Grèce que la rose à cent feuilles; elle fut, à ce qu'il semble, importée directement, avec le culte d'Aphrodite $^{4}$, de la Syrie dans le Péloponèse d’abord, puis dans les îles de l'Archipel. Les Phéniciens la portèrent ensuite, mais vraisemblablement peu de temps après, à Pestum. De cette ville elle se répandit dans le nord de l'Italie, et, de lá, pénétra dans la plupart des pays soumis à la domination romaine... La cent-feuilles, dit-il encore plus loin,

1. Der Baumkultus der Hellenen nach den gottesdienstliahen Gebräuchen und den überlieferlen Bildwerken darosestellt. Berlin, 1856, in-8, p. 456.

2. "Die Rose, vornehmlich die einfache dunkel purpurrothe (nicht die helle Centifolie) wurde ein Liebesangebinde der Gräber », p. 457.

3. Op.laud., p. 158 et 159

4. Il aurait fallu prouver d'abord que le culte de l'Astarté phénicienne, d'où est sorti celui d'Aphrodite, était lié à la culture de la rose. 
remplace la rose de Damas dans la Grèce septentrionale; son berceau est le mont Rhodope, en Macédoine; peutêtre fut-elle aussi importée de l'Asie Mineure dans ce pays. Elle était consacrée à Cérès et à Bacchus - la rose de Damas à Aphrodite - et pénétra avec leur culte d'Asie Mineure en Grèce.

On ne saurait édifier un roman avec moins de souci de la vérité historique, ce qui n'a point empêché M. Josef Murr ${ }^{1}$ d'adopter tout récemment la manière de voir de Karl Koch. Les choses, en réalité, sont moins simples et se présentent sous un autre aspect. Le premier renseignement certain que nous ayons sur la rose de Damas nous a été fourni par Nicolas Monardes ${ }^{2}$, médecin espagnol du milieu du $\mathrm{xvI}^{\circ}$ siècle; d'après lui on en faisait un fréquent usage dans plusieurs pays d'Europe, mais on ne la connaissait en Espagne que depuis environ une trentaine d'années. L'était-elle dans le reste de l'Europe occidentale depuis beaucoup plus longtemps? On a, sans toutefois en donner de preuves, prétendu qu'elle aurait été apportée en Occident pendant les Croisades $^{3}$, mais cette date, si on l'admet, est la plus

1. Die Pflansenwelt in der griechischen Mythologie. Innsbruck, 1890, in-8, p. 78.

2. Ilarum rosarum apud Italos, Gallos, Germanos, diversasque gentes nunc est frequens usus, quas Damascenas rocant, quoniam ex Damasco nobilissima Syriae urbe credunt devenisse. Apud nos vero triginta fere sunt anni, de qua notitiam altingimus ». Nicola Monardi medict hispalexsis De rosis persicis seu Alexandrinis. Antverpiae, 1564, in-12, p. 30 b.

3. James Smith, cité par Lindley-Pronville, op. laud., p. 67. 
reculée qu'on puisse assigner à l'introduction de cette espèce de rose en Europe ${ }^{1}$ : comment donc aurait-elle pu être connue des Grecs et des Romains? Il n'en fut pas de même de la cent-feuilles, dont la Damascena n'est peut-être d'ailleurs qu'une simple variété $^{2}$; ici, point de désaccord ; tout le monde est unanime à y voir, sinon le type unique, du moins un type incontestable de roses cultivées par les anciens, l'espèce à laquelle « a été particulièrement donné le nom de reine des fleurs ${ }^{3}$ ), la première et probablement la seule fleur double qu'ils aient connue.

La rose à cent feuilles a été souvent considérée comme une simple variété de la rose de Provins; mais la forme et les dimensions des fruits et des fleurs, de mème que la contexture des feuilles, empèchent entre autres de la confondre avec cette dernière ${ }^{4}$. De plus, tandis que la rose de Provins est

1. Sans s'arrêter à cette difficulté, Sprengel a identifié cette espèce avec les roses de Pestum, chantées par Virgile au rve livre des Géorgiques, et $\mathrm{A}$. Fée arec la rose de Cyrène dont parle Pline après Théophraste, ce que Kioch s'est empressé de faire à son tour et sans plus de raison.

2. Poireau, dans l'Encyclopédie, vol. VI, p. 276, lui donne le nom de Centifolia bifera.

3. J. L. Loiseleur-Deslongchamps, La rose, son histoire, sa culture, sa poésie, p. 125.

4. “Ses pédoncules courts et rigides, l'absence de grands aiguillons, ses pétales plus petits et ses sépales, dit Lindley, op. laud., p. 7't, en parlant de la Rosa centifolia, la firent toujours distinguer" de la gallica. "Affinis centifoliae, dit à son lour de Candolle, Prodromus, vol. II, p. 603, de la R. gallica, sed 
indigène en Europe, la cent-feuilles y est exotique; Fraas dit ne l'avoir rencontrée en Grèce qu'à l'état cultivé ou échappée des jardins ${ }^{1}$. De quelle région a-t-elle été importée dans ce pays et dans les autres contrées de l'Occident? A quelle époque y remonte sa culture et celle des variétés qui en sont sorties?

II.

La rose à cent feuilles n'étant pas indigène en Grèce, pas plus que dans le reste de l'Europe, c'est en Asie ou en Afrique qu'il faut en chercher le berceau: quels sont les peuples de ces régions chez qui on l'a rencontrée d'abord? Auquel d'entre eux revient l'honneur d'aroir le premier cultivé cette fleur appelée à une si brillante destinée?

Aucune espéce de rose ne croit spontanément en Égypte; inconnue des anciens habitants de ce pars ${ }^{2}$, on ne rencontre cette fleur sur aucun des monuments, ni dans aucun tombeau de l'époque pharaonique ${ }^{3}$, et elle n'apparait dans la vallée du Yil cue sous les Ptolémées; elle y était done d'origine étrangère.

fructibus rotundis foliaceisque valde coriaceis, nervis frequentioribus promiscuis valde anastomosantibus ».

1. Flora classica, p. 76.

2. Franz Wœnig, Die Pflanzen im alten Aegypten. Leipzig, 1886, in-8, p. 18.

3. C'est par erreur que Schleiden, Die Rose, p. 18, dit qu'on la trouve mentionnée dans d'anciens hiéroglyphes. 
Les anciens Hébreux ne la connurent pas davantage; c'est par une erreur de traduction qu'on a cru la trouver dans le Cantique des Cantiques et dans Osée ${ }^{1}$; la Sagesse ${ }^{2}$ et le " livre de Jésus, fils de Sidrach ${ }^{3}$, - l'Ecclésiastique, — de beaucoup postérieurs à la captivité de Babylone, sont les premiers écrits des Juifs qui en parlent; c'est là qu'elle est mentionnée tout d'abord, ainsi qu'elle le sera plus tard dans la littérature talmudique ${ }^{4}$.

On ne voit pas de rose sur les monuments assyriens, où sont représentés tant d'arbres et d'arbustes indigènes, pas plus que sur les monuments pharaoniques; il est des lors plus que rraisemblable que cette fleur n'était point cultivée dans l'ancienne Mésopotamie, et que les espéces saurages qu'on rencontre dans le haut bassin du Tigre ou de l'Euphrate ne furent jamais revètues d'aucun caractère sacré.

La rose n'apparait pas non plus dans les Védas ${ }^{5}$,

1. Chap. II, v. 12 et chap. XIV, v. 5. Le mot Shoshanah, que Luther, entre autres a traduit par " rose », signifie " lis »- - le zpivor des Septante - el désigne probablement le Lilium chalcedonicum L. ou bulbiferum L. Dioscoride indique le mot sousan comme le nom syrien du lis. V. Hehn, Kulturpflanzen, p. 203. Deutsche Rundschau. Juli, 1890, p. 42.

2. Chap. II, r. 8. Cf. plus loin, chap. IV.

3. Chap. XXIV, v. 18 ; chap. XXXIX, v. 17 ; chap. L, v. 8.

4. Dr. M. Duschak, Zur Botanile des Talmud, p. 130. Ciet ouvrage ne mérite pas plus de confiance, il est vrai, qu'il ne témoigne de connaissances botaniques véritables.

5. Zimmer (Altindisches Leben, Leipzig, 1883, in-8), qui a fait la flore des Védas, ne parle point de la rose. 
qui mentionnent pourtant un si grand nombre de plantes, et le sanscrit n'a même pas de nom pour elle $^{1}$. L'auteur du Ritou-Sanhara, espèce de poème des saisons ${ }^{2}$, qui renferme l'énumération de tant d'espèces régétales indigènes dans l'Inde, ne la cite pas; Kalidâsa n'en parle pas davantage dans ses drames, où le monde des fleurs est si largement représenté, et, plusieurs siècles après notre ère, Suçruta lui-même, qui, dans sa thérapeutique ${ }^{3}$, a cité un nombre si considérable de remèdes végétaux, ne dit rien de cet arbuste, dont la fleur fournit des produits pharmaceutiques estimés, en même temps qu'elle est le plus bel ornement de nos jardins.

L'Hindoustan n'est donc point la patrie de la rose cultivée des Anciens. De fait, cette contrée ne possède, d'après Drury ${ }^{4}$, que deux espèces de roses indigènes, appartenant toutes deux à la région montagneuse ou septentrionale: la rose toujours verte (R. sempervirens L. $)^{\mathbf{5}}$ et la rose à fleurs involucrées

1. L'hindoustani gulab, mentionné par Roxburgh (Flora indica, Sérampore, 1832, in-8, vol. II, p. 513), comme nom de la $R$. centifolia, est dérivé du persan.

2. OEurres complètes de Kalidìsa, trad. par Fauche. Paris, 1860 , vol. II, p. 1-50.

3. Ajurvedas, Id est medicinae systema a venerabili Dнanrantare demonstratum, a Sugruta discipulo compositum, nunc primum ex Sanscrita in Latinum sermonem vertit... Fr. Hessler. Erlangae, 184 $4-50$, in-8.

4. Handbook of the Indian flora. Madras, 1864, in-8, vol. I, p. 377.

5. De Candolle (Prodromus, vol. II, p. 598) indique une variété de cette rose dans les monts Nilghiri. 
(R. involucrata Roxb. ${ }^{1}$; il n'est donc pas surprenant qu'elles aient échappé aux anciens habitants de l'Inde : la reine des fleurs leur fut sans doute inconnue, comme elle l'a été des Égyptiens, des Hébreux et des anciens Assyriens.

En fut-il de mème des Médo-Perses? Le ZendAvesta ne parle pas plus que les Védas de la rose; mais comme il ne cite point de noms de plante en particulier, on ne peut en conclure que les habitants de l'Iran n'aient point anciennement connu ou cultivé la rose; ce n'est pas toutefois chez eux, mais chez les Grees, qu'on rencontre la première mention de cette fleur charmante.

Le nom de la rose se trouve dans les plus anciens monuments de la poésie hellénique. Aphrodite, dans Ilomère, parfume le corps d'Ifector avec de l'huile de rose $^{2}$; mais le poète ne dit rien de la fleur ellemême dont était tiré ce parfum, et l'on s'est demandé s'il la connaissait ${ }^{3}$. Il donne, il est vrai ${ }^{4}$, ainsi que

1. Roxburgh (Flora indica, vol. II, p. 513) ne parle pas de la $R$. sempervirens, mais il mentionne comme indigènes les $R$. pubescens Roxb. et recurva Roxb. De Candolle indique aussi d'après Lindley les $R$. Brunonii et Lyellii.

2.

$$
\begin{aligned}
& \text { 'Apooditn }
\end{aligned}
$$

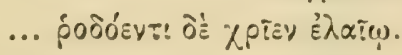

Ilias, XXIII, 186.

3. "Quapropter, lit-on dans Aulu-Gelle, rosam non norit, oleum ex rosa norit? " Noctes Atticae, lib. XIX, cap. 6.

4.

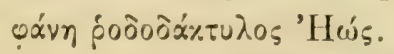

Odyssea, XVII, v. 1. 
le fera plus tard llésiode ${ }^{1}$, le nom d'Aurore " aux doigts de rose " à la déesse du matin ; mais c'est là une comparaison poétique, empruntée peut-ètre à des sonvenirs lointains, et qui, en tout cas, ne prouve pas que la rose était cultivée en Grèce du temps de ces poètes. Lorsque dans l'hymme à Déméter, ce monument vénérable du culte de cette déesse, vers le milieu du vir ${ }^{e}$ siècle $^{2}$, le poète nous montre Perséphone cueillant avec les filles de l'Océan, dans une " belle prairie » d' " aimables fleurs ${ }^{3}$ » : "le doux safran, des iris, l'hyacinthe, des roses et des lis, admirables à voir, avec le narcisse, que la vaste terre venait de produire ", pour séduire la jeune vierge, il nous présente un tableau de fantaisie, bien plus qu'il ne nous offre la peinture fidèle d'un jardin de son temps ${ }^{4}$.

On en peut dire autant de la description que Moschus, à son imitation, a, trois siécles plus tard, faite des "prés fleuris ", où eut lieu l'enlevement d'Europe 5 .

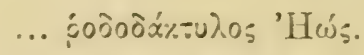

Opera et Dies, r. 610 , éd. Lehrs.

2. F. G. Welcker, Griechische Gotterlehre, Gottingen, in-8, vol. II (1859), p. 546. "Man kann unmöglich den Homerischen Hymnus bis gegen die 50. Ol. oder um das sechste Jahrhundert herabsetzen, sondern muss ihm ein höheres Alter zugestehen, etwa die 30. Ol. oder die mitte des siehenten Jahrhunderts ».

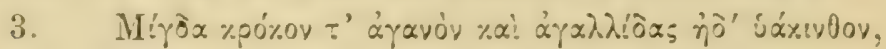

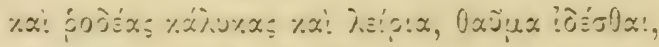

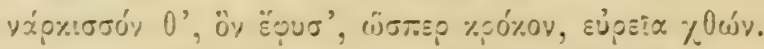

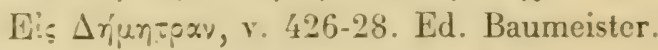

4. Il en a été le plus souvent de même chez les trouvères.

5. Idyllion, II, v. 63-70. 
Toutefois, si, comme son devancier, Moschus a réuni, dans ses vers, d'une manière arbitraire, les fleurs dont la vue frappe de joie Europe et ses compagnes, il les connaissait certainement; en était-il autrement pour l'auteur de l'hymne à Déméter? On n'est pas en droit de le penser, comme a paru le faire Hehn ${ }^{1}$; et sans doute, lorsque ce poète parle de la rose, c'est une fleur réellement connue de lui, tout comme les iris et l'hyacinthe, qu'il a en rue. Il en est à plus forte raison de mème quand Archiloque nous montre son amie " réjouie de la branche de myrte et des fleurs de rose " qu'elle reçoit ${ }^{2}$, ou bien quand Pindare nous parle de roses entrelacées dans les cheveux avec des violettes ${ }^{3}$. Il est impossible d'admettre qu'il ne s'agisse pas ici de fleur's comnues alors en Grèce; il n'est guère moins impossible de supposer que la rose dont ces poètes font ainsi mention ne soit pas déjà l'espèce cultivée, la fleur charmante qui oceupera désormais une si grande place dans les chants des lyriques grecs. Mais d'où venait-elle?

Le nom grec de la rose n'est point indigène; la forme éolienne plus complète $\beta$ só̀ov, celle même dont se servait Sappho, se rattache à l'arménien

1. Kulturpflanzen, p. 301.

2 .

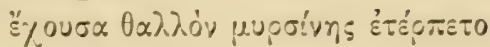

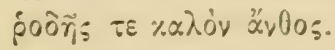

3.

Fragm. 29, éd. Bergk.

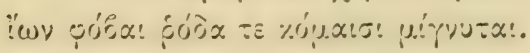

Dithyrambos II, v. 24-25 (Pindar's Werke, éd. J. A. Hartung. Leipzig, 1856, in-12; vol. IV, p. 218). 
vard ${ }^{1}$, lequel suppose, d'après Spiegel ${ }^{2}$, une forme zend rareda (fleur), d'où le vocable grec paraît dérivé ${ }^{3}$. Cette circonstance doit nous faire chercher la patrie de la rose vers le plateau de l'Iran. Or c'est précisément dans le Caucase ${ }^{4}$ oriental ainsi que dans le Khourdistan, qu'on a trouvé, à l'état spontané, la rose à cent feuilles, le type des plus belles espèces anciennement cultivées. C'est dans la région occidentale de l'Iran, en particulier dans le Mazendéran et le Farsistan, que la rose aussi atteint les proportions les plus grandes et qu'elle a le parfum le plus exquis; on ne peut guère douter dés lors que ce ne soit lì son berceau. De son pays d'origine, elle a d'un côté, à travers l'Asie Mineure, pénétré en Grèce, de l'autre, à travers la Mésopotamie, en Syrie et en Palestine.

La Vésopotamie l'avait déjà reçue au $\mathrm{v}^{\mathrm{e}}$ siècle avant notre ère. Ilérodote rapporte ${ }^{5}$ que les habitants de Babylone faisaient sculpter au haut du bâton

1. Kuhn und Schleicher, Beiträge zur sergleichenden Sprachforschung, vol. I (an. 1858), p. 318.

2. E. Røediger und Pott, Zeitschrift für die Kunde des Morgenlandes. Bonn, in-8, vol. VIII (ann. 1850), p. 119.

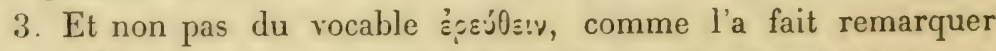

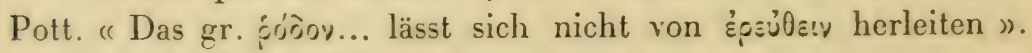
Z. F. K. d. M. vol. VII, 119.

4. Edm. Boissier, Flora orientalis, vol. II, p. 676. Marschall von Bieberstein (Beschreibung der Länder am Kaukasus, p. 78) l'a aussi indiquée à Kouban près de Schirvan dans la Perse septentrionale; mais y est-elle spontanée?

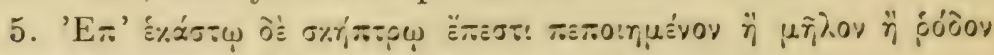

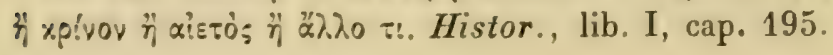


qu'ils portaient une pomme, une rose, une fleur de lis, un aigle ou quelque autre objet, preuve qu'ils connaissaient et qu'ils cultivaient sans doute aussi de son temps les roses et le lis. A la même époque la rose était déjà cultivée également au nord de la Péninsule hellénique. Les descendants de T'éménos, raconte Hérodote ${ }^{1}$, affligés de quitter Lébéa, "se retirèrent dans une autre partie de la Macédoine et s'établirent près des jardins qu'on dit ètre ceux de Midas, fils de Gordius; et où croissent spontanément des roses à soixante pétales, dont le parfum est plus suave que celui de toutes les autres espèces ». Audessus, ajoute-t-il, s'élève le mont Bermios. Un passage des Géorgiques de Nicandre complète celui des Histoires d'Hérodote et nous permet de suivre la marche de la rose depuis la région du Caucase jusque dans celle de l'Hémos.

Après avoir quitté la ville d'Asis en Phrygie, royaume de son père, Midas, nous apprend le poête alexandrin ${ }^{2}$, passa en Thrace et se fixa d'abord dans l'Édonide, puis dans l'Émathie; c'est dans cette dernière contrée, remarque-t-il, que se trouvaient

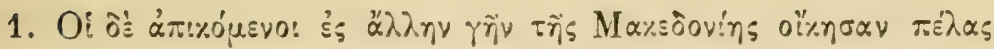

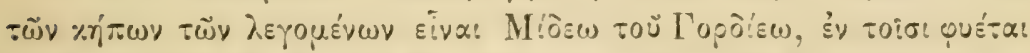

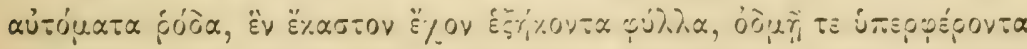

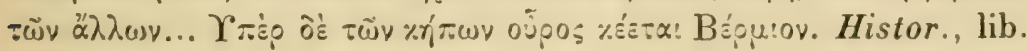
VIII, cap. 138.

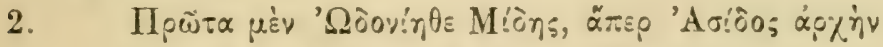

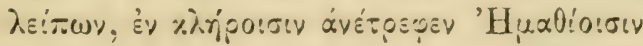

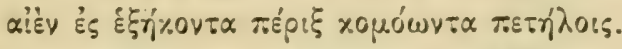

Georgica, ap. Athénée, lib. XV, cap. 31, 683 b. 
les jardins où ce prince a le premier élevé des roses ì soixante pétales. Ce récit symbolise l'importation de la rose, de l'Asie Mineure dans la presqu'île hellénique. Enfin le mont Bermios, au pied duquel Hérodote place les jardins de Midas, se trouvait dans la région mème où habitaient les Bryges, frères des Phrygiens d'Asie, suivant Strabon '. On roit comment de la Phrygie la rose fut transplantée dans le nord de l'Ilellade. Elle allait bientòt se répandre dans toute la Péninsule et de la dans le bassin de la Méditerranée tout entier.

Sappho parle des roses de Piérie ${ }^{2}$, province située au sud-est de la Macédoine, sur les frontières de la Thessalie. Théophraste a décrit les roses de Philippes en Macédoine, et vanté celles de Cyrène ${ }^{3}$. Nicandre a célébré à son tour les roses de Nisée et de Phasélis". Il y avait, d'après Pline ${ }^{5}$, des roses renommées a Trachine. Le naturaliste a aussi vanté celles de Préneste dans le Latium, et de la Campanie, ainsi que les roses de Carthagène en Espagne. Virgile ${ }^{6}$ et Columelle entre autres ont chanté celles de Pestum.

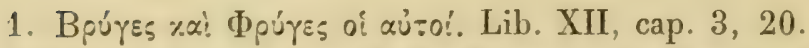

2. Lyrici Graeci, cur. J. Fr. Boissonnade. Parisiis, 1825, in-18, vol. XV, p. 59.

3. Hist. plantarum, lib. VI, 6. Voir pl. loin p. 17 et 18.

4. Georgica, ap. Athénée, lib. XV, cap. 31, 683, b.

5. Hist. natur., lib. XXI, cap. 10 (4), 2.

6. Georgic. lib. IV, v. 119.

7. De cultu hortorum, v. 37. (De re rustica, lib. X.) 
III.

Quelles étaient ces roses qui apparaissent ainsi dans les contrées les plus diverses de l'ancien monde? Appartenaient-elles à des espèces différentes ou n'étaient-elles que des variétés d'une seule et même espèce? On a répondu à ces questions de la manière la plus différente; le manque de précision et le vague des descriptions que nous ont laissées les anciens rendent trop explicable ce qu'il y a d'incertain et parfois même de contradictoire dans ce qu'ont hasardé les modernes sur cet obscur sujet.

Théophraste ${ }^{1}$, le premier et le plus exact des écrivains grees qui aient décrit la rose, s'est borné à distinguer entre elles les roses cultivées par le nombre plus ou moins grand de leurs pétales, leur parfum et quelques caractères extérieurs.

Les roses, dit-il², diffèrent beaucoup par le nombre de leurs pétales, leur plus ou moins de rudesse, la couleur et le parfum de leurs fleurs. La plupart ont cinq pétales, quelques-unes douze à vingt, d'autres encore un bien plus grand nombre, car il y en a auxquelles on donne le nom de cent-feuilles, du nombre de leurs pétales.

1. Hist. plant., lib. VI, cap. 6.

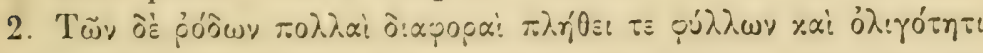

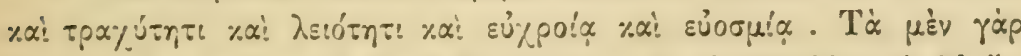

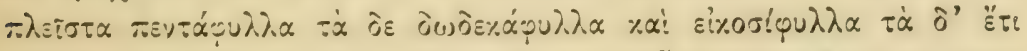

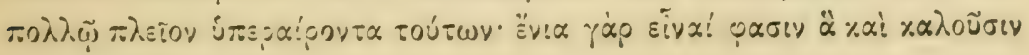

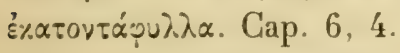

JoRET. La Rose. 
Et il ajoute que la plupart de ces dernières croissaient auprès de Philippes. Puis, après avoir dit qu'elles ne se faisaient remarquer ni par leur parfum, ni par la grandeur de leurs fleur's ${ }^{1}$, le disciple d'Aristote termine ainsi sa description ${ }^{2}$.

Les plus suaves d'odeur sont celles dont la partie inférieure (du calice) est hérissée. La couleur et le parfum des roses, dailleurs, dépend de la nature du sol où elles croissent, car on en trouve dans la même régrion de parfumées et d'autres qui n'ont pas d'odeur. Les plus parfumées sont celles de la Cyrénaïque.

Il n'y a dans ce passage rien qui puisse nous apprendre si Théophraste a eu en rue différentes espèces de roses, ou bien s'il ne parle que de variétés d'un mème type obtenues par la culture; mais une difficulté toute particulière se présente au sujet des roses de Philippes. Le naturaliste grec dit ${ }^{3}$ que les habitants allaient les chercher sur le mont Pangée, où elles croissaient en quantité, et qu'ils les plantaient dans leurs jardins. Si le renseignement est exact, il est presque impossible que les roses de Philippes aient été de rraies cent-feuilles,

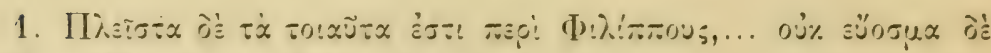

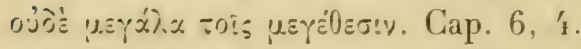

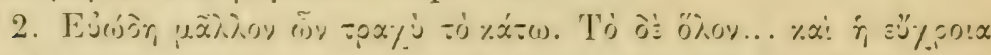

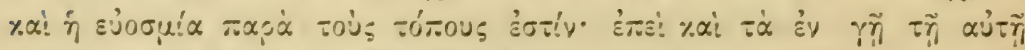

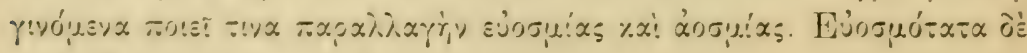

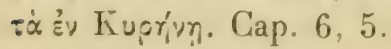

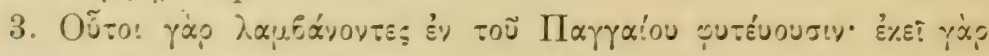

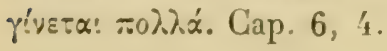


qui ne sont point indigenes en Grèce. Sprengel, après les avoir, dans la première édition de son Histoire de la Botanique ${ }^{1}$, identifiées avec cette espéce, a voulu y voir plus tard ${ }^{2}$ une forme anoblie de la rose toujours verte ( $R$. sempervirens L.) ; mais cette espèce fréquente sur le littoral de la G récene parait pas croître dans la partie montagneuse. Fraas regardait les roses de Philippes comme appartenant a l'espèce gallica, qu'il paraît confondre avec la centifolia, et que les Anciens, dit-il ${ }^{3}$, n'en distinguaient pas. Mais en mìme temps il voudrait voir dans les roses dont le calice est hérissé, suivant Théophraste, une forme de la rose à feuilles de pimprenelle (R. pimpinellifolia L., v. myriacantha Lindl.), hypothèse que rien ne justifie et qui parait reposer sur une interprétation erronce du texte de l'écrivain grec ${ }^{5}$.

1. Historia rei herbariae, 1807, vol. I, p. 93. "Rosa centifolia ad Philippos sponte crescere dicitur $»$.

2. Geschichte der Botanik, 1827, vol. I, p. 75. "Dies wird Rosa sempersirens sein, welche Sibthorp in Griechenland häufig wild fand $»$. On conviendra que la raison n'est guère suffisante.

3. "Die Alten unterschieden wohl R. centifolia et gallica als Arten nicht ». Op. laud., p. 76.

4. Ich ziehe diese Pflanze hieher, weil sie auf den nördlichsten Gebirgen die häufigste ist ». Op. Laud., p. 75.

5. Fraas a supposé que ces roses à calice hérissé étaient celles mêmes de Philippe, ce qui est inexact ; Athénée, l. XV, 682, en les appelant $\pi \varepsilon y \tau \alpha^{\prime}: 0 \lambda \lambda \alpha$ montre très bien qu il ne peut être question ici des roses doubles de Philippes; mais quelles étaient ces roses odorantes à cinq pétales? Serait-ce la Rosa sempervirens, dont le parfum est faible, mais très suave? 
Lenz ne s'est pas arrêté à la difficulté que j'ai signalée et il n'a pas hésité à regarder les roses de Philippes comme des cent-feuilles, il en a été de même de Hehn et aussi de Koch. Leur manière de voir est-elle conforme à la réalité des faits? Le texte de Théophraste est peut-être trop peu authentique ou exact, pour quion puisse se prononcer. Tout ce que nous apprend de certain l'Histoirc des Plantes, c'est qu'au iv siècle arant notre ère les roses de jardin variaient beaucoup par le nombre de leur's pétales, et qu'on en cultivait même de simples, c'est-à-dire à cinq pétales. Ces dernières étaient-elles de la mème espéce que les roses à fleurs doubles ou n'étaient-elles que des roses saurages non encore anoblies? Yous l'ignorons et le naturaliste grec n'a rien dit qui puisse nous l'apprendre.

Dans le livre VI de son Histoire des Plantes, à la fin de larticle sur les roses, Théophraste s'est borné à remarquer que les roses saurages ${ }^{1}$, - ̈̌p poz̀vix:, - dénomination sous laquelle il les comprend toutes, araient les feuilles et les tiges plus rudes, les fleurs moins colorées et plus petites que les roses cultives. Ailleurs ${ }^{2}$ il parait les designer, toutes également, sous le nom de \%uv́́obatoy - ronce de chien - , arbuste qui a, dit-il, un fruit rouge et semblable à une grenade, ce qui convient à peu

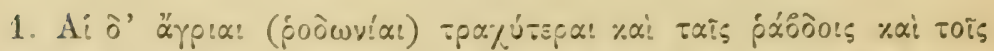

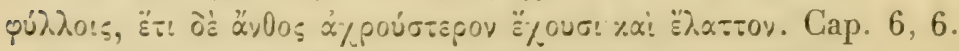

2. Hist. plant., lib. III, cap. 18, 4. Au livre IX, cap. 8, 5, il est fait également mention du fruit du cynosbaton. 
près au fruit de l'églantier, _ et dont "les feuilles, ajoute-t-il, ressemblent à celles du gattilier », ce qui s'applique bien à la ronce, mais non plus à l'églantier, encore que Théophraste ${ }^{1}$ ait sans doute voulu parler de cet arbrisseau. Mais quelle espèce

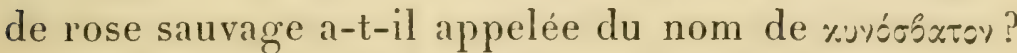

Théophraste n'est pas le seul écrivain qui se soit servi du mot; Dioscoride l'a également employé ${ }^{2}$, et la description qu'il a donnée du végétal auquel il l'attribue, si elle manque de précision, - il ne faut pas en demander aux naturalistes anciens, - convient néanmoins assez bien à un églantier, et en ce qui concerne le fruit et les graines ne saurait convenir qu’à lui.

Le cynosbaton, dit-il ${ }^{3}$, qu'on appelle parfois aussi oxyacantha, est beaucoup plus grand que la ronce; il devient en effet arborescent et a des feuilles beaucoup plus larges que celles du myrte. Ses rameaux sont garnis de robustes aiguillons, sa fleur est blanche ${ }^{*}$, son fruit

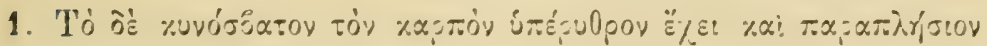

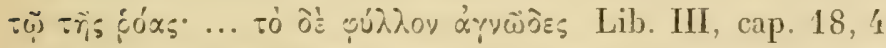

2. Galien (De alim. fucult. II, 1), a également employé ce nom pour désigner la rose sauvage, mais sans donner d'autre indication sur cet arbuste, sinon que le fruit en est astringent.

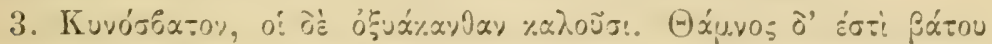
-

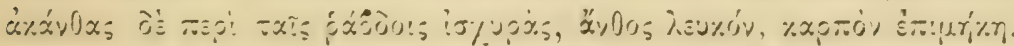

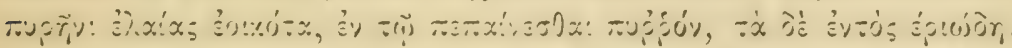
De medica mutrin, lib I, c-sp. 12:3. Lipsiac, 18:9, in-8, p. 117.

1. Cette couleur de la fleur a fait supposer à Sprengel que Dínscoride a eu ici en vue, ce qui est peu probable, la varićté 
oblong ressemble à une olive et devient rougre à la maturité; au milieu se trouvent des poils d'apparence laineuse.

Comme Théophraste, Dioscoride ne distingue pas plusieurs espéces d'églantier, et, comme pour son devancier, on ne sait pas laquelle des roses sauvages il avait en vue dans la description qu'il a donnée. Ce qu'il dit du fruit convient presque indifféremment à la rose de chien et à la rose toujours verte; il est plus que probable que l'auteur de la Matière médicale avait ru, comme Théophraste, ces deux espèces, qui se trourent également en Grèce: mais il est probable aussi qu'il les a confondues entre clles, ainsi peut-être qu'arec la rose rouillée (R. rubigginosa L.) qu'on rencontre, comme elles, dans la Péninsule hellénique, ainsi que daus les îles de l'Archipel et l'Asie Mineure ${ }^{1}$.

Dioscoride n'a pas plus essayé de distinguer les roses cultivées que les roses sauvages; il les comprend toutes sous le nom de rhoda, et comme il leur attribue a toutes les mémes proprictés médicinales, qu'il n'en a donné aucune description, on voit qu'on ne peut pas trouver chez lui le moindre renseignement sur ce qu'elles étaient ${ }^{2}$. Tout ce que nous

collina Jacq. de la $R$. canina. "Flores жuvor¿̨́x rou albi probant, Rosae caninae varietatem albifloram seu collinam Jacq., leucantham Lois. intelligi ». (Dioscoridis materia medica, vol. II, p. 399). Il aurait fallu commencer par montrer qu'il s'agit bien de la $R$. canina et non d'une autre espèce.

1. Edm. Boissier, Flora orientalis, vol. II, p. 67.

2. Sil fallait en croire Sprengel, qui a donné en 1829-10 une 
connaissons des roses cultivées par les Grees se réduit donc à ce que nous apprend Théophraste, et cela est trop peu pour que nous puissions en rien conclure. Le naturaliste grec a sans doute connu plusieurs races de roses cultivées, il a mème signalé entre elles des différences, mais rien n'indique qu'il ne les ait pas toutes rattachées au même type. Il en a été de même pour Dioscoride. C'est le point de vue où la science grecque en est restée. La science romaine est-elle allée plus loin?

Deux de ses représentants les plus autorisés, Columelle et Palladius, quand ils ont parlé de la culture de la rose, ne paraissent pas en avoir su plus que leurs devanciers de la Grèce, Théphraste et Dioscoride; ils n'ont rien dit du moins qui permette de penser qu'ils en distinguaient plusieurs espèces ${ }^{1}$;

nouvelle édition de la Matière médicale (Leipzig, in-8, 2 vol.), Dioscoride aurait connu deux autres espèces de rose ; on trouve en effet, dans cette édition (lib. I, cap. 130, vol. I, p. 124), le passage suivant. qui, manquant dans toutes les autres, semble ètre

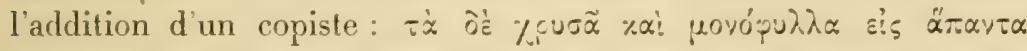

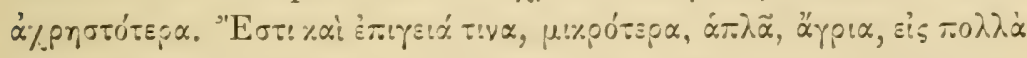

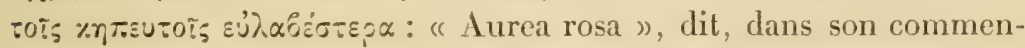
taire (vol. II, p. 40\%), Sprengel, qui ne paraît pas douter de l'authenticité de ce passage, encore qu'il n'en justifie pas l'origine, "est

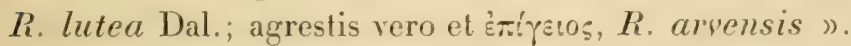

1. Il a fallu une singulière inadvertance de Sprengel /Geschichte der Botanik, vol. I, p. 130) pour parler de la rosa Sarrana; s'il avait lu plus attentivement ou moins perdu de vue le texte de Columelle, il aurait vu qu'il s'agit (lib. IX, 4) des "violettes pourpres » (Sarranae violae) on (lib. X, v. 287) de "'roses plus éclatantes que la pourpre de Sarra ») :

Jam rosa mitescit Sarrano clarior ostro. 
pour eux, il n'y a qu'une rose cultivée - la rosa comme il n'y a qu'une seule rose sauvage ou églantier, _ le cynosbatos ou sentis canis. - Reste Pline.

Le naturaliste latin distingue d'abord, mais d'une manière singulièrement vague, l'églanticr de la rose proprement dite ou cultivée. "Le végétal qui porte la rose est plutôt une épine qu'un arbuste », dit-il en parlant de la seconde ${ }^{1}$, mais cette fleur "vient aussi sur une ronce ", ajoute-t-il en faisant allusion à la première. Il connaissait aussi, comme Columelle, le nom cynoshatos, mais, tandis que celui-ci désigne évidemment l'églantier par ce nom² , la description qu'a faite Pline ${ }^{3}$ de l'arbuste auquel il l'attribue, ne saurait convenir qu'à une ronce ${ }^{4}$; il semble avoir réservé à l'églantier le nom de cynorrhodos ${ }^{5}$. Mais il confond toutes les roses sauvages sous cette dénomination, et on ne trouve rien dans son Histoire naturelle qui permette de croire qu'il en a connu, encore moins distingué, plusicurs espéces. Mais il

1. "Rosa nascitur spina verius quam frutice, in rubo quoque proveniens. Hist. natur., lib. XXI, cap. 10 (1).

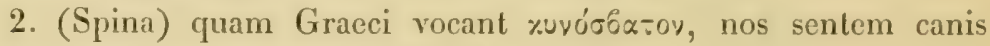
appellamus. De re rustica, lib. XI, cap. 3 .

3. Cynosbatos... folium habet restigio hominis simile. Fert et uvam nigram, in cujus acino nervum habet, unde nevrospatos dicitur. Hist. natur., lib. XXIV, cap. 7't.

4. Il faut dire toutefois qu'au livre XVI, chap. 71, il semble que le mot cynosbatos désigne bien l'églantier: "Rubi mora ferunt et alio genere similitudinem rosae qui vocatur cynosbatos ».

5. Radix silvestris rosae, quae cynorrhodos appellatur. IIist. natur., lib. VIII, cap. 43(41). - Radicem silvestris rosae, quam cynorrhodon vocant. Ibid. lib. XXV, cap. 6. 
a distingué plusieurs races ou formes, sinon plusieurs espèces, de roses cultivées.

Après avoir décrit, non sans originalité, l'inflorescence de la rose et parlé de quelques-uns des emplois de cette fleur, Pline ajoute ${ }^{1}$ :

Les espèces les plus célèbres parmi nous sont la rose de Préneste et celle de Campanie; d'autres ont ajouté la rose de Milet, qui est d'un rouge très vif et qui n'a pas plus de douze pétales: vient ensuite celle de Trachinie, qui est moins rouge, puis celle d'Alabanda, dont les pétales sont blauchâtres: la moins estimée est la rose épineuse (spineola), qui a beaucoup de pétales, mais très petits.

Après ces renseignements, qui paraissent lui appartenir en propre, Pline continue, en paraphrasant Théophraste, qu'il n'a pas toujours bien interprété $^{2}$ :

1. "Genera ejus nostri fecere celeberrima Prænestinam et Campanam. Addidere alii Milesiam, cui sit ardentissimus color, non excerlenti duodena folia. Proximam et Trachiniam minus rubentem. Mox Alabandicam viliorem, albicantibus foliis. Vilissimam vero plurimis, sed minutissimis spineloam » Ibid., lib. XXI, cap. 10 (4), 2 .

2. "Differunt multitudine foliorum, asperitate, laevore, colore, odore. Paucissima quina folia ac deinde numerosiora: quum sit genus ejus quam centifoliam vocant: quæ est in Campania Italiæ, Graeciae vero circa Philippos: sed ibi non suae terrae proventu. Pangaeus mons in vicino fert, numerosis foliis ac parvis: unde accolae transferentes conserunt, ipsayue plantatione proficiunt. Non autem talis odoratissima est ne cui latissimum maximumque folium ». Ibid., 3. 
Les roses different par le nombre des feuilles, par la rudesse, le poli, la couleur, l'odeur. Le nombre des feuilles, qui n'est jamais de moins de cinq, va toujours croissant, au point qu'il est une espèce à cent feuilles : elle vient en Italie dans la Campanie, et en Grèce aux environs de Philippes; mais dans ce lieu elle ne croît pas naturellement : elle vient du mont Pangée, qui est dans le voisinage, et qui produit des roses à pétales nombreux et petits; les habitants les transplantent et par là les améliorent. Cette espèce n'est pas très odorante, non plus que celle dont la feuille est trés large et trés grande.

Si Pline n'a pas eu un texte de l'Histoire des plantes différent de celui que nous possédons, il s'est permis avec Théophraste de singulières licences, et en transposant ou réunissant des phrases séparées, il a fait dire au naturaliste grec des choses auxcuelles celui-ci n'avait guère pu ni dû penser. Pline a emprunté au disciple d'Aristote la mention qu'il a faite de la rose si parfumée de Cyrène; mais je ne sais ou il a pris ce qu'il dit de la "rose greeque des Latins », - le lychnis des Grees, - "qui n'est pas plus grosse qu'une violette à cinq pétales, mais n'a pas d'odeur », de la grieculu, aux pétales réunis en peloton, de l'espèce appelée mosceuton, à la tige semblable à celle de la mauve et aux feuilles d'olivier, ainsi que de la coroneola "rose d'automme », qui tenait le milieu pour la grosseur entre les trois précédentes, et seule était odorante, tandis que les autres étaient sans parfum ". Il est impossible de recon-

1. "Est et quac Gracca appellatur a nostris, a Graecis lychnis, 
naître dans toutes ces fleurs des espèces ou variétés de roses ', et l'énumération qu'en a faite ici l'écrivain latin montre combien peu il tenait a l'exactitude.

On comprend d'après cela combien il est difficile d'identifier les variétés de roses mentionnées par Pline; Gaspard Bauhin ${ }^{2}$ l'a tenté, ainsi que l'Écluse, mais ils ne sont arrivés qu'à des hypothèses plus ou moins ingénieuses, et il était difficile qu'il en fût autrement, puisque les descriptions de l'Histoire naturelle sont incomplètes. Les conclusions de Bauhin n'en ont pas moins été acceptées par A. Fée dans sa Botanique de Pline ${ }^{3}$, ainsi qu'elles l'avaient été en partic dans l'édition allemande de l'Histoire de la Bolanique, de Sprengel. D'après ces deux derniers auteurs, la rose épineuse (spineola) serait la Rosa spinosissima L. ou myriacantha D. C., formes de la rose à feuille de pimprenelle, et tous voient dans

... unquam excedens quinque folia, violaeque magnitudine, odore nullo. Est et alia Graecula appellata, convolutis foliorum paniculis... Alia funditur e caule malvaceo, folia oleac habente, mosceuton vocant. Atque inter has media magnitudine autumnalis quam coroneolam rocant. Omnes sine odore, praeter coroneolam. » Ibid, , 4 .

1. La rose grecque ou Lychnis a été considérée comme étant le Lychnis coronaria L., la mosceuton, qui “certes n'est pas la rose musquée ", dit justement Hardouin (Pline de Lemaire, vol. VII, p. 1'́1, n. 25), est regardée par Desfontaines (ibid.) probablement comme l'Althaea rosea.

2. Pinax theatri botanici, sive Index in Theophrasti, Dioscoridis, Plinii et botanicorum qui a saeculo seripserunt opera. Basileae, 1623, in-8, p. 480.

3. A la suite du livre XXVI de Pline dans l'édition Panckoucke. 
la rose de Campanie la rose blanche (Rosa alba C. Bauh.); pour Sprengel, la rose de Nilet aurait été la rose de Provins (R. gallica L.); il en est de mème pour Fée, qui identifie aussi avec cette espèce les roses de Préneste et de Trachyne. Sprengel voyait, au contraire, avec l'Écluse, dans la première, une rose de Provence ( $R$. provincialis), et dans la seconde la rose de Damas ( $R$. damascena); c'est à cette dernière espèce que, d'après Fée, appartenaient les roses de la Cyrénäque. C'est à elle aussi qu'on a rattaché parfois les roses célibres de Pestum ${ }^{1}$. Enfin Sprengel regardait la rose d'Alabanda comme la rose velue ( $R$. villosa L.), espéce non cultivée pourtant ${ }^{2}$, et Fée voit dans la gracula, qui n'était probablement pas une rose, l'hypothétique Rosa silvestris de Bauhin. On ne pouvait donner plus libre carrière à la fantaisie ${ }^{3}$.

1. En particulier Sprengel (Geschichte der Botanik, v. I, p. 123), ainsi que Koch, on la vu. Pour Loiseleur-Deslongchamps (p. 137) c'est la " rose bifère ». Fée dans la "Flore de Virgile » (Virgile de Lemaire, vol. VIII, p. cLIv), s'est borné à dire que ce n'est pas la R. alba D.C.

2. Avec plus de raison Johan. Retzius, Flora Virgiliana, Lund, $1808,8^{\circ}$, l'a identifiée avec la var. pallida de la $R$. centifolia.

3. On trouve dans la Flore de Virgile par Fée un exemple curieux de ces créations arbitraircs d'espèces. Dans une de ses églogues, Virgile parle de roses pourpres.

Puniceis humilis quantum saliunca rosetis (V, v, 17).

Au lieu de voir dans l'adjectif puniceus un simple synonyme de purpureus, Fće, supposant d'ailleurs, on ne sait trop pourquoi, qu'un berger n'avait pu connaître de roses cultivées, a identifić la rose virgilienne avec la var. punicea de la rose jaune ( $\boldsymbol{R}$. Lutea, L.), encore qu'aucun auteur de l'antiquité ne paraisse avoir connu 
De toutes ces suppositions, la seule qui ait quelque fondement réel, c'est qu'à côté de la centfeuilles, regardée par Fée comme la forme type des espèces mentionnées par Pline, les Romains cultivaient sans doutc aussi la rose de Provins (R.gallica), de même peut-être que la rose à feuilles de pimprenelle. On doit admettre aussi qu'ils avaient probablement, quoique exceptionnellement, dans leurs jardins des roses rouges et des roses blanches! Quant aux dénominations diverses de roses qu'on rencontre chez les auteurs, elles désignent le plus souvent bien plus des centres de cultures que des variétés, encore moins des espèces différentes de cette fleur. Mais qu'importe ces distinctions, inconnues aux poètes de l'antiquité? Pour eux, sous ses diverses formes, la rose fut la reine des fleurs; c'est comme telle qu'ils l'ont chantée, sans se demandex à quelle variété appartenaient celles qu'ils connaissaient et qu'ils confondaient toutes, quelles qu'elles fussent, dans un même sentiment d'admiration.

cette espèce, mentionnée d'abord seulement par les écrivains arabes. Pietro Rubani, Flore Virgiliana, Firenze, 1868, 8, p. 99, n'a pas été plus heureux quand, faisant un contresens, il a voulu trouver des roses blanches dans le XII livre de l'Énéide, - il s'agit de lis blancs, - et qu'il n'a pas hésité à les identifier avec la $R$. moschata, L., espéce asiatique importée d'abord en Europe par les Arabes.

1. Il faut dire toutefois que Pline parle seulement de roses aux pétales blanchàtres (albicuntes), mais Ovide paraìt bien en connaître de blanches " Albentesve rosas ». Art. amat. lib. III, v. 182 . 


\section{CHAPITRE II.}

GULTURE DE LA ROSE DANS L'ANTIQUité.

Ilérodote est le premier écrivain de l'antiquité qui ait, mais en passant seulement, parlé de la culture de la rose; d'après lui, nous l'avons vu, cette fleur aurait été importée dans la péninsule hellénique par Midas, ce qui la fait remonter aux temps antéhistoriques. Mais, pour savoir en quoi consistait sa culture, à quels procédés elle avait recours, il faut descendre jusqu'à Théophraste, c'esta-dire $\mathrm{au} \mathrm{IV}^{\mathrm{e}}$ siècle avant notre ère. A cette époque la rose était répandue depuis longtemps déjà dans le monde grec tout entier, et l'influence hellénique avec le luxe croissant allait la faire pénétrer dans tout le monde connu des Anciens.

La mention, au vir ${ }^{\circ}$ siccle, de cette fleur charmante par Archiloque ${ }^{1}$ de Paros, l'éloge qu'en a fait Anacréon de Téos au siècle suivant, montrent que dés ces temps reculés elle était connue dans les îles de la mer Égée; celles de Samos fleurissaient deux fois l'an ${ }^{2}$; Rhodes parait lui devoir

1. Voir plus haut, p. 10 .

2. Athénée, Deipnos., lib. XIV, cap. 68 (654). 
son nom. Le témoignage de Pindare ${ }^{1}$ nous apprend qu'on la cultivait aussi dans la Gréce centrale. I.es roses de Trachine étaient renommées; celles de Lydie étaient encore plus célèbres. Nicandre place au second rang, après les roses d'Ĺmathie, celles de Nisée de Mégare; il vante aussi les roses de Phasélis, ainsi que celles qui "fleurissent sur les bords du Léthé de Magnésie ». Les roses de Wilet, tardives, mais d'un vif incarnat, étaient estimées, quoiqu'elles n'eussent que douze pétales". La pâle couleur de leurs fleurs faisait, au contraire, négliger les roses de la ville voisine d'Alabanda ${ }^{4}$.

Mais la culture de la rose ne resta pas longtemps renfermée dans les limites de l'Asie Mineure ou de l'Ilellade; les colons grees la transportèrent avec eux dans les établissements qu'ils formèrent loin de la mère patrie. C'est ainsi que la rose pénétra tour à tour en Sicile, où le poète Bion en racontera la naissance fabuleuse ${ }^{5}$, et où Ovide nous montre Perséphone la cueillant, quand elle fut enlevée par Pluton ${ }^{6}$; dans l'Italie méridionale, enfin dans la Cyrénaïque, où elle s'acclimata rapidement et pro-

1. Dithyrambi fragm. LIII, v. 17-18.

2. Athénée, Deipnos., lib. XV, cap. 31, 683, b. Le Léthé, affluent du Méandre, se jette dans ce fleuve près de Magnésie.

3. "Addidere alii Milesiam, cui sit ardentissimus color, non excedenti duodena folia. ") Pline, Hist. natur., lib. XXI, cap. 10(4), 2.

4. "Alabandicam viliorem, albicantibus foliis. " Pline, ibid.

5. Idyllion, II, v. 65-66.

6. Plurima lecta rosa est. Fast., lib. IV, v. 4 4t1. 
duisit une variété recherchée pour son exquis parfum ${ }^{1}$.

C'est peut-être de cette dernière contrée, que la rose, si elle n'y fut pas directement importée d'Orient, fut transplantée en Égypte; inconnue dans ce pars, nous l'avons vu, à l'époque des Pharaons, on l'y trouve cultivée sous les Ptolémées; la région d'Arsinoé - le Fayoum actuel - devint célèbre par les roses qu'elle produisait. On la rencontre aussi maintenant en Syrie, où elle était inconnue avant l'invasion babylonienne, et elle va prendre place dans la littérature juive, qui l'avait ignorée jusque-là.

C'est au moment où la rose apparaît ainsi dans toutes les provinces de la Grèce ou de l'Orient hellénisé que Théophraste a, le premier, fait connaître la culture de cette fleur charmante.

La rose, dit-il ${ }^{2}$, vient de graine. Puis, après avoir décrit le fruit de ce précieux arbuste, il ajoute: Mais, comme ce moyen de propagation est trop lent, on la reproduit plutôt par bouture. Taillée et passée au feu, elle donne des fleurs plus belles. Quand on la laisse intacte et qu'on l'abandonne à elle-même, elle se développe outre mesure et retourne à l'état sauvage. Il importe aussi de transplanter souvent les pieds de rosier; c'est le moyen, dit-on, de leur faire porter de plus belles fleurs.

C'est peu; ces préceptes cependant n'ont guère

1. "Cyrenis odoratissima est », dit Pline (Hist. natur., lib. XXI, cap. 10(4), 5), en traduisant Théophraste.

2. Hist. plantarum, lib. VI, cap. 6 . 
été dépassés dans l'antiquité, et Pline ne fera guère que les reproduire en les développant, cncore que de son temps la culture des roses eût une importance bien plus grande qu'ì l'époque de Théophraste.

Portée par les colons grecs dans leurs établissements de l'Italie méridionale, la culture de la rose y devint florissante; Lycophron parle des roses de Locres ${ }^{1}$; celles de Pestum ont été célèbres dans l'antiquité; elles fleurissaient, dit-on, deux fois l'an ${ }^{2}$. De la Grande Grèce, sous le nom de rosa, dérivé de posíx ou poscśs. le rosier pénétra dans la Campanie et le Latium: sa culture prit surtout une grande extension dans la première de ces provinces, qui rivalisa pour ses roses avec l'Égypte ${ }^{4}$. Dans le Latium, Préneste eut de bonne heure des roses renommées et tardives ${ }^{5}$; dans une pièce de vers connue, Martial parle aussi de celles de Tibur et de

1. Lycophron, Alexandra, v. 1429.

2. biferique rosaria Paesti,

Virgile, Georgic. lib. IV, v. 119. nec bifero cessuro rosaria Paesto.

Martial, Epigr. lib. XII, 31, v. 3.

Ovide, qui en parle aussi, se borne à les mentionner sans leur attribuer ce privilège.

tepidique rosaria Paesti. Metam. lib. XV, v. 708.

3. Pott, "Ueber altpersische Eigennamen. » (Zeitschrift der deutschen morgenl. Gesellschaft. Leipzig, in-8, vol. XIII (1859), p. 390). - II. Estienne, Thesaurus, s. v.

4. " Ab ea (Aegypto) Campania est, copia rosae. " Pline, Irist. natur. lib. XIII, cap. 6.

5. Pline, Hist. natur. lib. XXI, cap. 10(4), 5. Joret. La Rose. 
Tusculum ${ }^{1}$. Plus tard les roses de Milan aussi furent célèbres ${ }^{2}$, comme l'étaient dès longtemps celles de Malte ${ }^{3}$. Poursuivant sa marche vers l'Occident, la rose pénétra à son tour en Espagne, où Carthagène fut renommée pour l'espèce qu'on y cultivait", en Gaule, où Ausone la chantera, dans la Grande Bretagne même ${ }^{5}$.

Les écrivains latins ont laissé les témoignages les plus formels des progrès faits par la culture de la rose depuis la fin de la République, ainsi que les renseignements les plus curieux sur les soins dont elle était l'objet. Varron déjà recommandait de s'y livrer dans la campagne romaine ${ }^{6}$. Virgile n'a point oublić de faire cultiver les roses par son vieillard de Tarente ${ }^{7}$. Columelle prescrit à l'homme des champs de planter dans son jardin cette fleur dont " la couleur est l'image de la pudeur rougissante $^{8}$ »; il veut qu'au printemps elle " élargisse le jonc tordu de ses corbeilles », afin qu'il revienne de la ville, où il l'aura portée, ses poches chargées

1. Epigr. lib. IX, 61.

2. Flavius Vopiscus, Carinus, cap. 17.

3. Cicéron, In Verrem, V, cap. 11.

4. Pline, Hist. natur. lib. XXI, cap. 10(4), 5.

5. Voir pl. loin, IIc partic, chap. I et III.

6. "Itaque sul) urbe colere hortos late expedit, sic violaria ac rosaria. "De re rustica, lib. I, cap. 16, 3.

7. Primus vere rosam atque autumno carpere poma.

Georgic. lib. IV, v. 134 .

8. Ponatur ... nimium rosa plena pudoris.

De re rustica, lib. $\mathrm{X}, \mathrm{v}, 102$. 
d'argent ${ }^{1}$. Désormais la rose a sa place dans tous les jardins de Rome et de l'Italie. Dans la description connue qu'il a laissée de sa maison de campagne, Pline le jeune rappelle arec complaisance les bosquets de roses qui remplissaient l'intérieur du manège de sa villa ${ }^{2}$.

L'importance qu'on attachait à la rose fait comprendre le soin apporté à sa culture et explique les détails avec lesquels les agronomes latins en ont parlé. Comme on le voit d'après ce qu'ils en rapportent, on la pratiquait en grand, mais elle différait singulièrement de ce qu'elle est aujourd'hui.

Pour faire un plant de rosiers, dit Varron ${ }^{3}$, on choisit des pieds qui aient des racines; on les coupe à partir de la racine en brins d'une palme environ qu'on met en terre, et qu'on transplante plus tard, lorsqu'ils ont repris.

Columelle est plus bref: il se borne à dire que " le rosier se met en graine et par boutures dans des fosses d'un pied ${ }^{*}$ m. Mais il ajoute qu'il faut le labourer

1. Jam rosa distendat contorti stamina junci,

Acre sinus gerulus plenos gravis urbe reportet.

De re rustica, lib. X. v. 306 et 310 .

2. "Etiam rosas effert. » Epistol. lib. V, 6.

3. "Quod jam egit radicem, rosetum conciditur radicitus in virgulas palmares et obruitur. Ilaec eadem postea transfertur facta viviradix. " De agricultura, lib. I, cap. 35.

1. "Rosam fructibus ac surculis disponi per sulcos pedales convenit... Sed omnibus annis fodiri ante calend. Martias et interputari oportet. Hoc modo culta multis annis perennat. „) De arboribus, cap. 30 . 
et le tailler chaque année avant les calendes de mars; ainsi cultivé, remarque-t-il, il dure plusieurs années. Mais c'est grâce à Pline surtout qne nous savons ce qu'était la culture de la rose chez les Romains et les préceptes qu'il a donnés devaient faire loi encore au moyen âge.

Pour le rosier, dit-il ${ }^{1}$, on travaille la terre plus profondément que pour le blé, plus superficiellement que pour la vigne. Il vient, remarque-t-il d’après Théophraste, très lentement de graine... aussi préfère-t-on le planter de bouture. Lne seule espèce se plante comme le roseau par des yeux de racines; c'est le rosier à fleurs pâles et à cinq pétales, à branches épineuses, très longues ${ }^{2} .$. Tous les rosiers, ajoute-t-il encore daprès Théophraste, gagnent à être taillés et passés au feu. La transplantation les fait, comme la vigne, pousser très bien et très vite; on a des boutures de quatre doigts de long ou plus, Pline suit ici Varron, - on les plante après le coucher des Pléiades ${ }^{3}$, puis lorsque le Favonius souffle, on les

1. «Fodiuntur altius quam fruges, levius quam vites. Tardissime proveniunt semine ... ob id potius caule conciso inseruntur; et ocellis radicis, ut harundo, unum grenus inseritur, pallidae, spinosac, longissimis virgis, quinquefoliae, quae e Graccis altera est. ” Lib. XXI, cap. 10(4), 6.

2. Pline ajoute que " cette rose est la seconde des roses grecques »: que pouvait bien être cette espèce? Hardouin a supposé que c'était la même que la graecula; mais qu'était alors celle-ci?

3. "Omnis recisione atque uslione proficit : translatione quoque, ut vitis, optime ocissimeque provenit, surculis quaternum digitorum longitudine, aut ampliore, post Vereqiliarum occasum sata : 
replante à des intervalles d'un pied et l'on remue fréquemment la terre alentour.

Palladius a peu ajouté à ce qu'avaient dit Varron et Pline. Comme ce dernier, il fait venir les roses de boutures ou de graines, qu’il prescrit de déposer en férrier dans de petites fosses ou tranchées ${ }^{1}$. Il recommande également, pendant ce mois, de fouiller par le pied, avec des sarcloirs ou des dolabres, les vieux plants de rosier et d'en couper arec soin tout le bois sec ${ }^{2}$. Enfin il donne le conseil de renouveler les anciennes plantations devenues trop claires, en couchant en terre de jeunes branches en guise de scions $^{3}$. Dans les Géoponiques il n'est plus question de semer les rosiers ${ }^{4}$. On conseille de transplanter les pieds tels quels avec leurs racines, ou, après les avoir arrachés, de les couper à la hauteur d'une palme, - nous retrouvons ici le procédé de Varron,

dein per Favonium translata. pedalibus interallis crebroque circumfossa $)$.

1. "Hoc mense (feliruario) rosaria conseremus, quae sulco brevissimo aut scrobibus ponenda sunt, vel virgultis, vel etiam semine. " De re rustica, lib. III, cap. 21. Ailleurs (lib. XII, cap. 11), Palladius dit qu'on peut aussi planter les rosiers au mois de novembre, mais dans les terrains chauds.

2. "Si qua etiam sunt antiqua rosaria, hoc tempore circumfodiuntur sarculis vel dolabris, et ariditas universa reciditur. „) De re rustica, lib. III, cap 21.

3. "Nunc et quae rara sunt possunt ducta virgarum propagine reparari ").

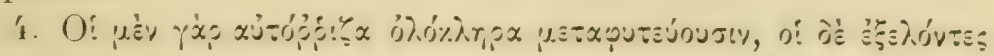

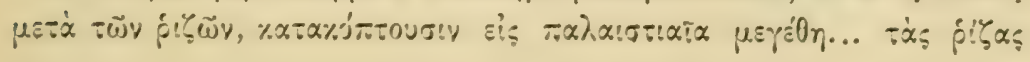


- et de planter pieds et racines à un interralle d'une coudée.

Palladius ne s'est pas borné à donner des préceptes pour la culture de la rose, il a indiqué encore le moyen de conserver fraìches les roses en boutons.

On fend, dit-il, ${ }^{1}$ un roseau vert sur son pied et on renferme les boutons dans sa cavité de façon que la fente puisse se rejoindre; on coupe ensuite le roseau, quand on veut avoir des roses fraîches. Il y a aussi des personnes, ajoute-t-il, qui renferment des roses dans un pot non verni et bien bouché; elles les conservent ainsi en les enterrant à l'air libre.

Ces précautions montrent le prix que les Romains attachaient à ces fleurs aimées et quel soin ils prenaient pour en avoir de fraîches.

Une préoccupation bien naturelle des jardiniers de Rome fut d'avoir des roses précoces; Pline, quand il parle de cette fleur, a bien soin de mentionner quelles variétés fleurissent de bonne heure et les lieux qui les produisent. Telles étaient les roses de Campanie, celles de Carthagène surtout, "précoces, dit le naturaliste ${ }^{2}$, pendant tout l'hiver ». Pour en

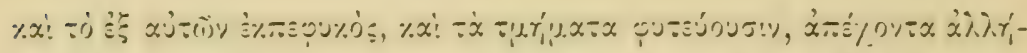
$\lambda \omega \nu$ is $\pi \pi_{\gamma}$.u. Geoponicorum sive de re rustica libri XX. Lipsiae, 1781, in-8. Lib. XI, cap. 18.

1. "Rosas nondum patefactas servabis, si in canna viridi stante fissa recludas, ita ut fissuram coire patiaris : et eo tempore cannam recidas, quo rosas virides habere volueris. Aliqui olla rudi condiLas ac bene munitas sub) dio obruunt ac reservant. „) De agricultura, lib. VI, cap. 17.

2. "Hieme tota praecox. " Hist. natur., lib. XXI, cap. 10(4), 5. 
obtenir en tout pays de semblables, on cut recours à un moyen ingénieux, mais qui peut nous paraître un peu primitif; Pline ${ }^{1}$ et Palladius ${ }^{2}$ nous le font également connaître. On creusait une fosse d'un pied autour du rosier et on y versait deux fois par jour de l'eau chaude, au moment où les boutons commençaient à pousser. Dans les Géoponiques ${ }^{3}$, on conscille, ce qui est plus simple, de mettre les plants de rosiers dans des vases en terre ou des caisses ${ }^{4}$, et de les placer à l'abri du froid dans des endroits exposés au soleil. C'est ce qu'on faisait, d'après Columelle ${ }^{5}$, pour avoir des concombres hâtifs.

Il y a loin de ces procédés à nos serres chaudes, dont on a roulu ${ }^{6}$ retrouver l'idée dans un passage corrompu de Sénérque. Le philosophe ne fait allusion évidemment qu'à l'emploi des arrosages d'eau chaude, conseillés par Pline ${ }^{7}$. Il n'est pas davantag'e question

1. "Qui praecocem faciunt, pedali circa radicem scrobe aquam calidam infundunt, germinare incipiente calyce. ") Hist. natur., lib. XXI, cap. 10(4), 6.

2. "Si rosam temperius habere volueris, duobus palmis ab ea gyrum fodies, ct aqua calida bis rigabis in die. ") Ibid.

3. Les Géoponiques préconisent aussi le procédé recommandé par Pline et Palladius, mais pour hàter la maturité des fruits.

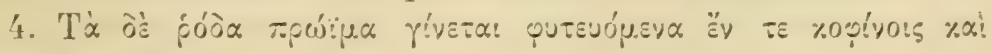
жspquío:ร. Ibid.

5. De re rustica, lib. XI, cap. 3 .

6. En particulier Loiseleur-Deslongchamps, La Rose, p. 52.

7. "Non vivunt contra naturam, qui hieme concupiscunt rosam fomentoque aquarum calentium et locorum mutatione brumalium florem vẹnum exprimunt? „ Epist. lib. XX, 5(122), éd. Fr. Haase. Les anciennes éditions donnent: " et calorum apta imita- 
de serres, quoi qu'en ait pensé IIehn ${ }^{1}$, dans un passage connu de Martial ${ }^{2}$, où le poète parle simplement de plaques de verre qui servaient à conserver la fraîcheur des fleurs en les préservant du contact de l'air, tout en permettant de jouir de leur vue.

Mais on n'en peut dire autant du procédé indiqué dans une autre pièce de vers du mème poète. Columelle recommandait de couvrir de plaques transparentes les plants de jeunes concombres, afin de pouvoir les mettre sans danger au soleil par les jours sereins, mais froids ${ }^{3}$. Ce procédé, importé en Italie par les Ciliciens, que Pompée arait transplantés en Calabre ${ }^{4}$, s'était bientôt répandu dans toute la Péninsule. Martial, dans une de ses épigrammes ${ }^{5}$, parle des plantations d'un de ses amis que " des plaques transparentes défendaient contre les vents d'hiver, tout en laissant pénétrer jusqu'à elles un jour pur et

tione bruma lilium », ce qui a fait croire qu'on obtenait des lis en plein hiver.

1. Kulturpflanzen, p. 206,

2. Condita sic pura numerantur lilia vitro,

Sic prohibet tenuis gemma latere rosas.

$$
\text { Epigr. lib. IV, 22, v. 5-6. }
$$

3. «Specularibus integi debebunt, ut etiam frigoribus serenis diebus tuto producantur ad solem. „ De re rustica, lib. XI, cap. 3.

4. “Voss zu Virgils Landbau, » IV, p. 773, ap. Sprengel, Geschichte der Botanik, vol. I, p. 116.

5. Pallida ne Gilicum timeant pomaria brumam

Mordeat et tenerum fortior aura nemus :

Hibernis objecta notis specularia puros

Admittunt soles et sine faece diem.

$$
\text { Epigr. lib. VIII, 14, v. 1-4. }
$$


les rayons du soleil ». S'il ne s'agit point encore la de serres chaudes, il faut y voir quelque chose d'analogue à nos chassis, ou mème à nos serres froides.

Ces procédés étaient bien imparfaits; ils permirent néanmoins aux Anciens d'avoir des roses bien avant ou après la saison qui les produit d'ordinaire, et ils les dispensèrent d'en faire venir des climats plus favorisés. Ce sont ces roses d'hiver, d'autant plus estimées qu'elles étaient plus rares ${ }^{1}$, dont parle Lucien $^{2}$, contre la recherche desquelles Sénèque s'est élevé dans son penchant habituel à la déclamation, et que Martial a célébrées comme le triomphe de l'horticulture de son temps.

L'hiver, dit-il en s'adressant à Domitien dans une de ses épigrammes ${ }^{3}$, vous offre, César, ses couronnes précoces; la rose était autrefois la fleur du printemps, elle est maintenant la vôtre.

Dans une autre piece de vers souvent citée, faisant allusion aux roses que les habitants de l'Égypte avaient envoyées à l'empereur à l'occasion de sa fète :

1. Rara juvant; primis sic maior gratia pomis ;

Hybernae pretium sic meruere rosae.

$$
\text { Martial, Epigr. lib. IV, 29, v. 3-4. }
$$

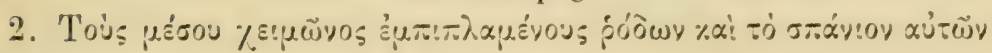

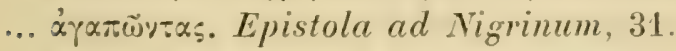

3. Dat festinatas, Ciesar, tibi bruma coronas;

Quondam veris erat, nune lua facta rosa est.

$$
\text { Epigr. lib. XIII, 127, v. 1-2. }
$$


L'habitant des bords du Nil, s'écrie le flatteur de Domitien', jaloux de vous faire sa cour, vous avait envoyé, César, des roses d'hiver, présent d'un genre tout nouveau. Mais on vit le nautonnier de Memphis rire des jardins de l'Épte, quand il eut passé le seuil de votre ville : telle était la douceur des parfums du printemps et la beauté de Flore, tant on pouvait s'y croire dans la splendeur des bosquets de Pestum! De quelque côté qu ill portât ses pas et ses regards, toutes les rues étaient éclatantes de roses tressées en couronnes. O Nil! puisque tes hivers sont forcés de céder aux hivers de Rome, envoie-nous tes moissons et accepte nos roses.

L'exagération est ici manifeste; mais ces ver's de Martial n'en sont pas moins un témoignage curieux et irrécusal)le des progriés qu'arait faits de son tomps la culture des roses. Elle devait en faire encore de plus grands. Si l'on en croit les Céoponiques, les anciens horticulteurs seraient parvenus à aroir des roses toute l'année, en arant soin de les transplan-

1. Ut nova dona tibi, Caesar. Nilotica tellus

Jiserat hibernas ambitiosa rosas:

Navita derisit Pharios Mempliticus hortos,

Urbis nt intravit limina prima tuae.

Tantus veris honos, et odore gratia Florae,

Tantaque Paestani gloria ruris erat.

Sic quacumque ragus, gressum oculosque ferchat,

Textilibus sertis omne rubehant iter.

At tu Romanae jussus jam cedere brumae,

Mitte tuas messes, accipe, Nile, rosas.

Epigr. lib. VI, 80. 
ter et de les fumer tous les mois ${ }^{1}$. Ce précepte devait du moins produire de bons résultats et plus certains assurément que celui du mème compilateur d'entourer d'aulx ${ }^{2}$ les pieds de rosiers, afin d'en rendre les fleurs plus odorantes.

Les Anciens ne cultivèent pas seulement la rose proprement dite ou de jardin, ils cultivaient aussi l'églantier; on s'en servait surtout en Italie, en y joignant parfois des ronces et des paliures", pour clore les jardins. On faisait tout à l'entour de l'endroit qu'on voulait protéger une double tranchée d'un pied et demi de profondeur, on y déposait à la fin de l'hiver des semences d'églantier enduites de farine d'ers, et, quand elles commençaient à pousser, on enfonçait entre les deux tranchées des appuis ou on y plantait une haie d'osier destince à supporter les jeunes plants ${ }^{4}$. On avait ainsi une clôture que

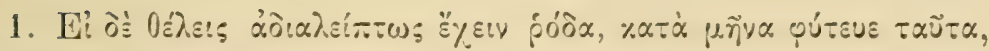

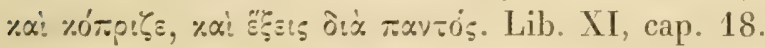

2. "La souefve odeur des roses est affinée par le voisinage des aulx, qui sont plantez près des rosiers "), dit François de Sales, tirant de cette prescription singulière une gracieuse comparaison. (Flore mystique de S. François de Sales. Paris, 1874, in-12, p. 220).

3. Ea sint vastissimarum spinarum, maximeque rubi et paliuri et ejus quam Graeci rocant \%uvó§ $\beta \alpha$ :ov, nos sentem canis appellamus. Columelle, De re rustica, lib. XI, cap. 3.

4. Melius erit rubi semina et spinae, quae rubus caninus vocatur, matura colligere, et cum farina ervi ex aqua macerata miscere... Ubi sepes futura est, duos sulcos tribus a se pedibus separatos, sesquipedis altitudine faciemus, et... semina obruemus levi terra. Palladius, De re rustica, lib. I, cap. 3't. 
rien, dit Columelle ${ }^{1}$, ne pousait détruire, si on ne l'arrachait, et que le feu mème ne pourait que fortifier. Nos haies d'aubépine cependant sont incontestablement préférables.

1. Hunc veprem manifestum est interimi non posse, nisi radicitus effodere velis. Caeterum etiam post ignis injuriam melius renasci nulli dubium est. Columelle, De re rustica, lib. XI, cap. 3. 


\section{CHAPITRE III.}

LA ROSE DANS LES LÉGENDES ET DANS LA POÉSIE DES GRECS ET DES ROMAINS.

La nature de la rose, dit l'auteur des Géoponiques ${ }^{1}$, a quelque chose de divin; ce n'était là que l'expression du sentiment général qu'inspirait aux anciens la reine des fleurs; les Grecs attribuèrent à la rose une origine surnaturelle; il en fut de même des Romains; objet pour ces deux peuples d'une espèce de culte, elle joua chez eux un rôle presque égal à celui du lotus dans les croyances religieuses des Egyptiens et des Ilindous, et l'imagination inventive de leur's poètes entoura sa naissance des plus gracieuses légendes.

\section{I.}

Une tradition, qui paraît bien avoir un caractère primitif, rapporte que le premier rosier aurait surgi

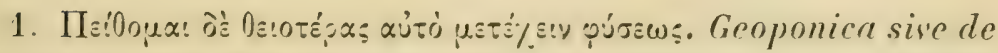
re rustica lib. XX, Cassiano Basso collectore. Lipsiae, 1781, in-8, p. 821, lib. XI, cap. 18, 15. 
de terre le jour même où Vénus sortit de l'écume des flots, et une goutte de nectar, versée par les dieux sur le jeune arbrisseau, aurait alors donné naissance a la rose ${ }^{1}$. D’après une légende probablement d'origine cyprienne, mais adoptée par Bion ${ }^{2}$ chez les Grecs, par Ovide ${ }^{3}$ et Servius ${ }^{4}$ chez les Romains, c'est, au contraire, du sang d'Adonis, blessé mortellement par un sanglier, que naquit la rose, tandis que des larmes versées par Vénus sur la mort de son favori serait née l'anémone. Suivant une autre tradition, Adonis lui-même aurait été changé en rosier ${ }^{5}$.

Mais la naissance de la rose avait été l'objet de bien d'autres légendes ${ }^{6}$; un poète latin de la déca-

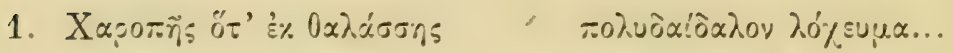

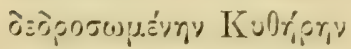

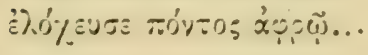

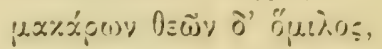

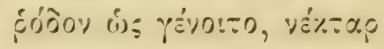

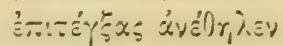

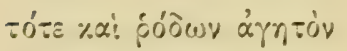

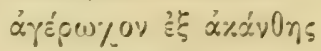

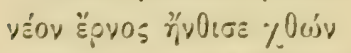

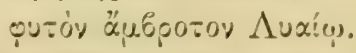
Anacreontea, LIII (53), v. 11-25. (Poetae lyrici graeci, rec. Th. Bergk, III, 1071.)

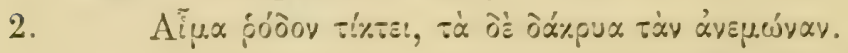
Idy.ll. I, v. 64 .

3. At cruor in florem mutabitur, fail dire Ovide (Metrem. X, 7.29)à T'énus s'adressant à Adonis expirant.

4. Sanguinem ejus vertit (Venus) in florem qui nunquam vento decuti dicitur. Ad Aen. cant. V, v. 72.

5. Servius, $A d$ eclog. X, v. 18. "Multi miseratione Veneris in rosam conversum (Adonidem) dicunt. »

6. Suivant Dierbach (Flora mythologica, Frankfurti, 1833, in-8, p. 156) qui, il est vrai, ne cite pas ses autorités, on aurait cru aussi que la rose était tombée de l'étoile du soir. 
LA ROSE DANS LES LÉGENDRS ET DANS LA POÉSIE. 47 dence en a réuni quelques-unes dans les vers suivants :

La rose, dit-il ${ }^{1}$, est ou née d'un sourire de l'Amour, ou l'Aurore la fit tomber de sa chevelure empourprée qu'elle peignait, ou bien elle naquit quand Cypris, arrêtée par des ronces cruelles, teignit de son sang leurs piquants aiguillons.

Toutes nombreuses qu'elles sont, ces fictions n'ont point encore paru suffisantes; les modernes n'ont pas hésité, chose bien superflue, à en inventer de nouvelles ${ }^{2}$, et les Anciens eux-mèmes en ont imaginé d'autres pour expliquer la couleur vermeille de la rose.

D'après une légende qui de bonne heure eut cours chez eux, les roses étaient blanches à l'origine;

1. Aut hoc risit Amor, aut hoc de pectine traxit Purpureis Aurora comis, aut sentibus haesit Cypris et hic spinis insedit sanguis acutis.

Poetae latini minores, vol. VII, p. 125, éd. Lemaire. Anthologia latina, ed. Al. Riese. Lipsiae, 1878, in-8, vol. I, p. 100.

2. Le marquis de Chesnel, entre autres (La Rose, p. 18), rapporte une prétendue " historiette grecque », d'après laquelle Roselia, s'élant mariée au " beau » Cymédore, quoique consacrée à Diane, aurait été frappée, en punition de ce parjure, d'un trait de la déesse et changée en rosier. Malheureusement Roselia n'est pas un nom grec et cette " historiette " paraît bien avoir été inventée par un moderne, comme celle de la métamorphose de Rhodanthe, reine de Corinthe, imaginée par le P. Rapin et popularisée par l'imagerie d'Épinal. 
mais, comme Vénus courait au secours d'Adonis, menacé par la jalousie de Mars, une épine lui pénétra dans le pied ${ }^{1}$, et le sang de sa blessure répandu sur les pétales de la rose en teignit en pourpre la blancheur primitive ${ }^{2}$. Une statue grecque du musée de Florence, qui représente Vénus arrachant de son pied l'épine qui l'avait blessée, semble avoir consacré le souvenir de cette légende ${ }^{3}$. On trouve du mème fait une explication toute différente dans les Géoponiques. Un jour, raconte l'auteur de cette compilation $^{4}$, que les dieux assistaient à un banquet dans l'Olympe, l'Amour, qui conduisait un chœur de danse, heurta de son aile et renversa un vase de nectar; la liqueur divine, en tombant sur le sol, donna à la fleur de la rose, blanche jusque là, sa couleur vermeille.

Ausone - on comprend qu'un pareil sujet l'ait tenté - a repris cette fiction et l'a transformée.

Un jour, dit-il", les héroïnes, victimes de l'Amour, errant tristes et aflligées sous les ombrages des Champs-

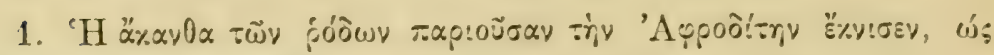

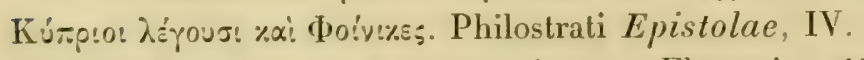

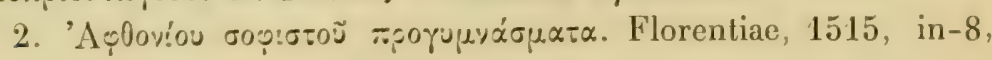
p. 26. - Geoponica, lib. XI, cap. 17.

3. Marquis d'Orbessan, Essai sur les roses. (Mélanges historiques et critiques, etc. Paris, 1768, in-8, vol. II, p. 307). Mus. florent. Statuae antiquae cum observ. Ant. Fr. Gorü. Flor. 1734, fol., tab. 33.

4. Lib. XI, cap. 17.

5. Idylle VI, éd. Nisard, 1887, in-8, p. 107-109 passim. 
LA ROSE DANS LeS LÉGENDES ET DANS LA POÉSIE. 49

Élysées, aux bords de leurs lacs immobiles et de leurs ruisseaux sans murmure, rencontrèrent l'Amour, venu étourdiment s'égarer au milieu d'elles; à sa vue elles sentent se réveiller dans leurs cœurs leurs anciennes douleurs; elles l'entourent, l'entrainent, l'attachent au tronc d'un myrte et, l'accablant de reproches, le soumettent à de longs tourments. Vénus, accourue au milieu du tumulte, au lieu de porter secours à son fils, se rappelant ses nombreuses trahisons, se joint aux infortunées, l'accuse à son tour et inexorable frappe d'un bouquet de roses l'enfant, qui pleure et tremble. Une sanglante rosée ${ }^{1}$, ajoute le poète, jaillit alors de ses membres meurtris sous les coups répétés de la rose flexible, qui, déjà teinte de sa pourpre, rougit de feux plus vifs son vermeil incarnat.

Cette scène nous transporte bien loin du sombre séjour des Ombres, tel qu'il nous apparaît dans les poèmes homériques; ce n'est pas l'auteur de l'Odyssée qui aurait, même s'il l'avait connue, placé la rose dans l'Érèbe. Mais à mesure que les mœur's deviennent moins rudes, que le luxe des jardins naît et se développe, la peinture des Champs-Élysées, qui en est l'image transformée, s'humanise; leurs paysages se diversifient et s'embellissent; la description qu'en font les poètes devient plus gracieuse; Virgile, tout fidèle qu’il est ì la tradition antique, met déjà dans le séjour des bienheureux, avec de frais bocages, un

1. Olli purpureum mulcato corpore rorem

Sutilis expressit crebro rosa verbere, quae, jam

Tincta prius, traxit rutilum magis ignea fucum.

v. $90-92$.

JoREt. La Rose. 
bois odoriférant de lauriers ${ }^{1}$. Avec Tibulle, la flore des Enfers s'enrichit encore; le gracieux poète ne craint pas d'y faire croître des régétaux inconnus de l'Europe, comme la case, et il en émaille le sol fécond de roses parfumées ${ }^{2}$. Properce nous montre aussi les doux zéphyrs caressant les roses des champs Élyséens ${ }^{3}$. Ces fleurs ne pouvaient pas plus manquer dans le séjour des IIéros que dans le palais des Dieux.

Mais c'était aux Dieux que la rose appartenait avant tout; présent fait par eux à la terre, son origine surnaturelle devait la leur rendre doublement chère. Elle devint en particulier, avec le mrrte, l'apanage et l'attribut habituel d'Aphrodite*. Tée arec elle ou créce par elle, teinte de son sang, elle fut sa fleur favorite. Le rhéteur Libanius a, dans une fiction ingénieuse, symbolisé la prédilection de Vénus pour la rose. Quand les trois déesses, raconte-t-il ${ }^{\circ}$, se disputèrent sur l'Ida le prix de la beauté, Ninerve et Junon ne voulurent pas se soumettre a la sentence de leur juge, qu'Aphrodite n'eût déposé sa ceinture,

Aeneidos lib. VI, v. 652

2. Fert casiam non culta seges, totosque per agros

Floret odoratis terra benigna rosis.

Elegiae, lib. I, 3, v. 61-62.

3. Mulcet ubi Elysias aura beata rosas.

Elegiae, lib. IV, 7, v. 60.

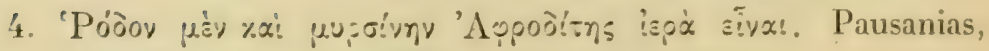
Descriptio Graeciae, lib. VI, cap. 24, 7.

5. Boissonade, Anecdota nova. Paris, 1813, in-8, p. 313 
dont le charme magique lui eût assuré la victoire. La déesse répondit que ses rivales avaient des attraits non moins puissants : Junon son diadème d'or, Minerve, son casque; elle consentait néanmoins à se dépouiller de sa ceinture, s'il lui était permis d'aller chercher une autre parure. On le lui accorda. S'étant alor's rendue au bord du Scamandre, Vénus, après s'ètre baignée dans le fleure, se mit à cueillir sur ses bords des lis, des violettes et d'autres fleurs; mais soudain, par son doux parfum, la rose attira son attention; alors, jetant loin d'elle les premières fleur's, elle se tressa une guirlande de roses et la posa sur son front, puis elle revint sur l'Ida. Mais les deux déesses n'attendirent pas le jugement de Pâris; elles enlevèrent à Aphrodite sa couronne, et après en avoir baisé les fleur's, elles la remirent sur la tête de leur rivale, comme seule digne de la porter, avouant qu'elle embellissait autant les roses que les roses l'embellissaient.

On comprend, d'après cette fiction, pourquoi on représentait parfois Iphrodite aree une couromne de roses'. Une tradition attribuait également à cette déesse un jardin merveilleux que l'on ne pouvait voir sans être épris d'amour ${ }^{2}$. C'était là qu'Éros

1. Pauly, Real-Encyclopädie der classischen Alterthumswissenschaft, vol. VI, p. 2463.

2. Hortus erat Veneris, roseis circumdatus herbis,

Gratus ager dominae, quem, qui vidisset, amaret.

Dum Puer hic passim properat decerpere llores...

Poetae latini minores, éd. Lemaire, vol. VII, p. 120. Alex.

Riese, Anthologia latina, $\mathrm{n}^{0} 82$, vol. I, p. 100. 
allait cueillir les fleur's dont il se parait ${ }^{1}$ et qu'étaient prises celles que Ténus envoyait a ses favoris². Les Grices et les Heures, dit Hégésias dans ses Cypriennes ${ }^{3}$, avaient trempé la tunique d'Aphrodite dans le nectar parfumé des roses.

Comme à Aphrodite, sa mère, la rose fut consacrée au dieu de l'amour ; " elle en était vraiment la fleur, dit Philostrate ${ }^{4}$, puisque, comme lui, elle était jeune et délicate comme lui ». Elle en était aussi l'emblème et l'ornement accoutumé. Le pseudo-Anacréon représente Éros “ son beau front ceint d'une couromne de roses, quand il se mèle aux chœurs des Grâces ${ }^{5}$. ))

La rose n'appartenait pas moins à Dionysos qu'à Aphrodite et à Éros ${ }^{6}$; son histoire n'est pas moins mèlée à celle de ce Dieu. Lui également avait un jardin, où les roses croissaient d'elles-mèmes, le jardin de Midas. Quand il se rendit de Thrace en Phrygie, son maître Silìne s'y enirra et y fut retenu

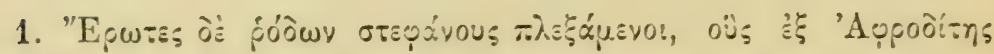

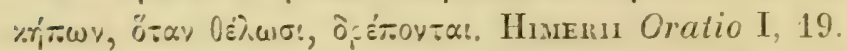

2. Glaudien, Opera, XL, v. 9-10, et XLVIII, v. 28-30.

3. Athénée, Deipnosoph., lib. XV, cap. 30(682).

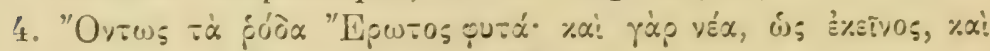

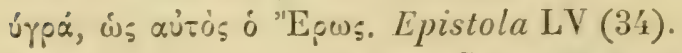

5 .

'Pójoy, đị rais ò KuOricn:

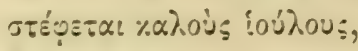

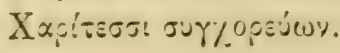

Ode, XLII (V), v. 9-11.

6. Fr. Lenormant, art. Bacchus, dans le Dictionnaire des antiquités grecques et romaines, vol. I, p. 623, 2. 
LA ROSE DANS LES LÉgeNdes ET DANS LA POÉSIE. 53

enchaîné ${ }^{1}$. Et une tradition nous le montre se présentant à Ariadne vètu d'une robe de pourpre et le front ceint d'une couronne de roses ${ }^{2}$. La rose aussi était chère à Dionysos; une mosaïque du Vatican le représente arrosant de ses mains un rosier ${ }^{3}$. Dans la statue colossale de la Villa Albani, qu'on a supposée être l'image de Bacchus, le dieu apparaît le front ceint d'une couronne de roses ${ }^{4}$.

La reine des fleur's était également l'attribut des Grâces. Le pseudo-Anacréon, dans une de ses odes, montre ces déesses s'en parant dans la saison fleurie des Amours". On les figurait parfois unies entre elles par des gruirlandes de roses. Dans un temple d'Élis, nous apprend Pausanias ${ }^{6}$, l'une d'elles sans doute Charis - était représentée une rose à la main. Cette fleur n'était pas moins chère aux Muses ${ }^{7}$.

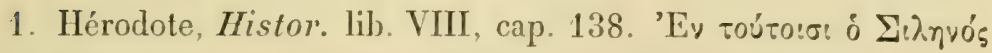

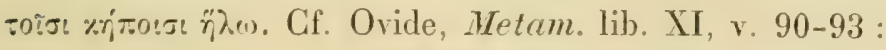

Titubantem annisque meroque

Ruricolae cepere Pliryges, vinctumque coronis

Ad regem duxere Midan.

2. 'A

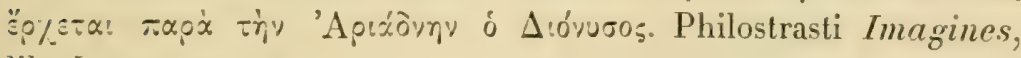
lib. I, cap. xiv, 2.

3. Emil Braun, Griechische Götterlehre. Gotha, 1854, in-8, p. 552 .

1. Roscher's Lexicon, p. 1102.

5 .

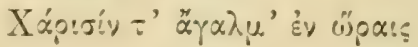

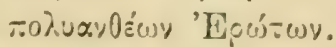

Ode LIII, v. 6-7

6. Eliaca, lib. V, cap. 24, 6

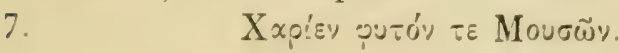

Anacreontea, Ode LIII, v. 9. 
Une couronne de roses, dit Plutarque ${ }^{1}$, leur etait attribuée. Dans la vision où il se croit transporté sous les ombrages de l'Ilélicon et prés des sources de l'Ilippocrène, Properce apercoit une des Muses tressant des guirlandes de roses ${ }^{2}$. Stace parle ${ }^{3}$ de la couronne de roses qui presse le beau front de Thalie. La Polymnie du musée du Tatican en porte une aussi ${ }^{4}$. On représentait également la déesse de la jeunesse, Ilébé, et Ganymède, l'échanson des dieux, couronnés de roses ${ }^{5}$. Sur le bas-relief d'un sarcophage romain ${ }^{6}$, on voit aussi des génies le front ceint d'une guirlande de cette fleur sacrée.

\section{II.}

La rose, il ne pouvait en ètre autrement, avait llace dans les fètes de Flore ${ }^{7}$; elle lui était consacrée comme à Vénus, qui, non moins qu'elle, prési-

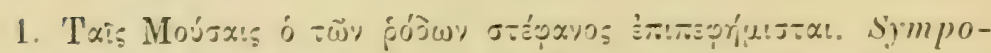
siaca, lib. III, questio I, 2, 10. Cf. Théoc., Epigr. I, v. 2.

2.

... illa manu texit utraque rosam.

3.

$$
\text { Elegiae, lib. III. 3 (II. 2). 1. :36. }
$$

crinemque decorum

Pressisset rosea lasciva Thalia corona.

Sylsae, lib. H, 1, v. 115-116.

4. Museo Pio-Clementino, Roma, in-fol, vol. 1, tav xxr.

5. Dierbach, Flora mythologica, p. 159.

6. Hus. capitol. vol. IV, tab. 57.

7. Ovide, Fast., lib. V, v. 336. 
LA ROSE DANS LES LËGENDES ET DANS LA POÉSIE. 55

dait aux jardins et était la déesse du printemps ${ }^{1}$. La rose était l'emblème de la saison des fleurs, comme clle en était la parure. Elle en annonçait le retour. C'est seulement quand elle s'épanouissait qu'on croyait le printemps revenu ${ }^{2}$. Aussi le moment de la floraison des roses était-il considéré comme un événement heureux. Dans plusicurs villes d'Italie, en particulier a Capone et a Rome, il y avait au mois de mai - le mois où elles fleurissent - une fète des roses. Il y avait aussi, les inscriptions en font foi, des fètes d'un caractère privé, données à cette occasion ${ }^{3}$.

Compagne du printemps, la rose figure dans toutes les descriptions que les poètes anciens ont fait de la saison des fleurs.

Vois comme à l'approche du printemps, s'écrie le pseudo-Anacréon ${ }^{4}$, les Grâces se couvrent de roses!

Il convient, dit-il encore ailleurs ${ }^{5}$, d'unir dans ses chants le printemps qui nous donne les couronnes et les douces roses.

La saison empourprée du printemps fleuri a souri,

1. L. Preller, Romische Mythologie. Berlin, in-18, 3e éd. 1881, vol. I, p. 433-411.

2. Cicéron, In Verrem, V. Cif. plus loin, p. 105.

3. L. Preller, Ramische \#/gthologie, vol. I, p. 433.

'

5.

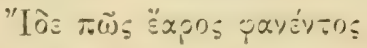

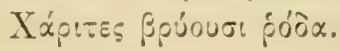

Ode XLIV, v. 1-2.

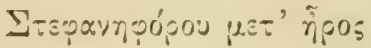

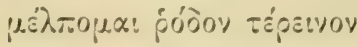

รงขอ์-

Ode LIII, y. 1-3. 
chante à son tour Méléagre 1; les prairies s’égaient au milieu des roses qui s'entr'ouvrent.

Et trois siècles plus tard, le poète Pancrate célébrera encore la rose comme la fleur qui s'épanouit au souffle des zéphyrs printaniers ${ }^{2}$.

Son charme, son doux parfum avaient aussi fait de la rose la reine des fleurs; son éclat, en effet, comme dit le poète Rhianos ${ }^{3}$, ne les efface-t-il pas toutes? Elle devint aussi le symbole et l'embleme de la beauté. C'est ainsi que Claudien compare Marie, l'épouse d'llonorius, et sa mère à deux roses épanouies en mème temps dans les jardins de Pestum ${ }^{4}$. Les poètes anciens sont revenus souvent sur cet attribut de la rose: mais ce qui les a peut-être encore frappés darantage, c’est la rapidité avec laquelle se fane et meurt cette fleur délicate, image trop fidele de l'instabilité des choses de ce monde.

La rose ne fleurit que pendant peu de temps, dit l'un

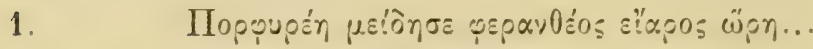

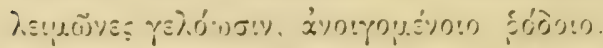
Anthol. palatina, éd. Dübner, cap. IX, n" 363, v. 3-6.

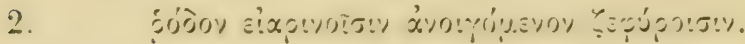

Athénée, Deipnos., lib. XV, cap. 21 (677).

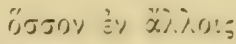

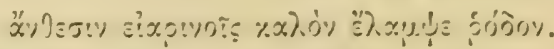

Anth. palatina, cap. XII, no 58, ․ 3-4.

'. Ceu genuinae Paestana rosae per jugera regnant.

De nuptiis Honorii et Hariae, r. 247. 
LA ROSE DANS LES LÉGENDES E'T DANS LA POÉSIE. 57

d'eux dans l'Anthologrie'; une fois passée, si tu la cherches, tu ne trouves qu'une épine.

$\mathrm{Ni}$ l'amour ni les roses ne vivent longtemps, remarque à son tour Philostrate ${ }^{2}$; le temps est l'ennemi de la beauté dans son été et de la durée des roses.

J'ai vu, chante Properce $^{3}$, les roses parfumées de Pestum, qui paraissaient devoir toujours durer, tomber brûlées dès le matin au souffle du Notus.

Un autre poète - on a cru que c'était Florus ${ }^{4}$ après avoir montré le bouton de rose qui, sous l'influence féconde du printemps, apparaît un jour, s'allonge et se gonfle le lendemain, entr'ouvre son calice le troisième jour et s'épanouit au quatrième, termine sa brève description par cette réflexion attristée, " si on ne la cueille ce matin mème, ce soir elle ne sera plus ».

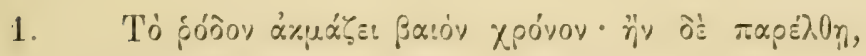

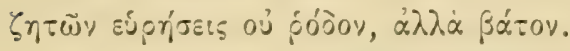

Anth. palatina, cap. XI, no 53.

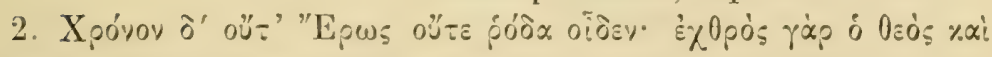

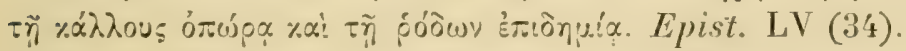

3. Vidi ergo odorati victura rosaria Paesti Sub matutino cocla jacere Noto.

$$
\text { Eleg., lib. V, 5, v. 61-62. }
$$

4. Venerunt aliquando rosae pro veris amœni Ingenium! una dies ostendit spicula florum,

Altera pyramidas nodo maiore tumentes,

Tertia jam calathos; totum lux quarta peregit

Floris opus, pereunt hodie, nisi mane legantur.

Anth. Latina, rec. Alex. Riese, vol. 1, p. 101. 
Heureuse, si je pouvais vivre pour un long destin, fait dire un autre poète à la rose elle-même ${ }^{1}$.

Rose, elle a fleuri et s'est fance aussitôt, lit-on également sur une inscription ${ }^{2}$ qui fait songer aux vers si connus de Malherbe ${ }^{3}$.

Cette vie si courte des roses a inspiré à Ausone, ce poite gracieux at affecté, l'une de ses plus belles idylles: au milieu de l'éloge de ces fleurs aimées, se rappelant la rapidité avec laquelle elles se fanent et meurent,

Jadmirais, dit-il', les rapides ravages du temps dans sa fuite et ces roses que je voyais éclore tout ensemble

1. O felix, longo si possim vivere fato!

Anth. latina, vol. I, p. 196.

2. Rosa simul floruit et statim periit.

Corpus Inscr. Rhen. 1053.

3. Et rose, elle a vécu ce que virent les roses, L'espace d'un matin.

Ode à Dupervier, XI, v. 15-16.

4. Mirabar celerem fugitiva aetate rapinam

Et, dum nascuntur, consenuisse rosas.

Ecce et defluxit rutili coma punica floris,

Dum loquor, et tellus tecta rubore micat.

Tot species, tantosque ortus, variosque novatus

Una dies aperit, conficit una dies

Quam longa una dies, actas tam longa rosarum,

Quas puljescentes juncta senecta premit.

Quam modo nascentem rutilus conspexit Eous,

Hane rediens sero vespere vidit anum. Idyllion, XII, Y. 35-40 et 13-46. Éd. Nisard. 
LA ROSE. DANS LES LÉGENDES ET DANS LA PÓESIE. 59

et vieillir. Et voici que la chevelure empourprée de la fleur radieuse se détache au moment où je parle et la terre brille jonchée de sa rouge dépouille. Et toutes ces naissances, toutes ces transformations variées, un seul jour les produit, un seul jour les enlève... La durée d’un jour est la durée de la vie des roses; pour elles la puberté touche à la vieillesse qui les tue. Celle que l'étoile du matin a vu naître, le soir, à son retour, elle la voit flétrie.

La courte durée de la rose qui a fait donner à cette fleur une de ses épithètes les plus ordinaires chez les poètes latins - celle de brevis ${ }^{1}$ — en a fait aussi le symbole de la fragilité des choses de ce monde. Un mème jour ne la voit-il pas s'épanouir, se faner et mouril, comme dit Ausone, nous rappelant que notre âge est passager comme elle, et nous engageant à cueillir sa fleur " pendant qu'elle est nouvelle et que nouvelle est notre jeunesse ${ }^{2}$ ).

1.

Nimium breves

Flores amœnae ferre jube rosac.

Horace, Carm. lib. II, 3, v. 13-14.

Aut imitata breves punica mala rosas.

Martial, Epigr. lib. II, 41, v. 6.

2. Collige, virgo, rosas, dum flos novus et nova pubes,

Et memor esto aevum sic properare tuum.

Ausone, Idyllion, II, v. 40-\{1.

Il faut rapprocher de ces vers ceux du Tasse, dans le xv1 livre de la Jérusalem délivrée:

Cogliam d'amor la rosa ; amiamo or quando

Esser si puote riamati amando.

․ 15-16. 
III.

Ainsi que de la fragilité et de la beauté, la rose fut aussi l'emblème de l'innocence virginale et de la pudeur rougissante; la grâce qui la pare, sa fraicheur si délicate. son doux incarnat, la prédestinaient à ce nouveau rôle. Mais là on la rencontre avec une autre fleur, originaire du plateau de l'Iran ${ }^{1}$, comme elle importée en Occident, et qui, comme elle aussi, a eu le rare privilège de fournir aux poètes les comparaisons les plus diverses: le lis blanc. Il est, coincidence qui n'a rien de fortuit, fait mention pour la première fois de cette plante nouvelle avec la rose, dans l'hymne ì Déméter ${ }^{2}$; c'est une des fleurs "belles à voir" que Perséphone cueille avec ses "douces compagnes " dans la prairie idéale décrite par le poìte. Depuis lors on trouve la rose et le lis constamment réunis dans les chants des poètes grees et latins ${ }^{3}$, comme dans les

1. V. Hehn, Kulturpflansen, p. 202.

2. Voir plus haut, p. 12. On lit dans les Géoponiques, lib. XI, cap. 19, que le lis doit sa naissance au lait tombé sur terre, quand Junon allaita Hercule; cette légende, toute d'origine grecque qu'elle est, semble bien indiquer l'origine lointaine du lis.

3. Claudien par exemple fait en mème temps naitre des lis et des roses, sous les pas de la reine Serena.

quamcumque per herbam

Reptares, fluxere rosac, candentia nasci Lilia.

Laus Serenae reginae, v. 89-91. 
LA ROSE DANS LES LÉgENDES E'T DANS LA poÉsie. 61 jardins, dont ces fleurs étaient l'une et l'autre l'ornement ${ }^{1}$.

Le contraste si doux offert par la couleur différente des deux fleurs frappa les Anciens; ils virent dans la blancheur du lis limage du teint immaculé de la vierge, dans la rose celle de l'incarnat de ses joues ou de la rougeur provoquée sur son front par la pudeur émue ou offensée. Virgile, voulant peindre l'ardente rougeur répandue sur le visage enflammé de Lavinie :

Comme rougissent les blancs lis, mêlés aux roses, dit-il ${ }^{2}$, ainsi éclataient les feux sur le visage de la jeune fille.

Et Ovide, parlant de la honte qui couvrait les joues coupables de sa maîtresse :

Tels, remarque-t-i ${ }^{3}$, brillent les roses au milieu des lis qui les entourent ${ }^{4}$.

1. C'est ainsi que dans le jardin du bouvier de Daphnis et

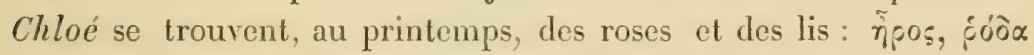
(※i) «piva. Longus, Pastoralium lib. II, 3.

2.

mixta rubent ubi lilia multa

Alba rosis, tales virgo dabat ore colores.

Aeneidos lib. XII, v. 68-69.

3. Conscia purpureus venit in ore pudor.

Quale rosae fulgent inter sua lilia mixtae.

Amorum lib. II, eleg. V, v. 34-37.

4. Le lis est parfois remplacé par le lait:

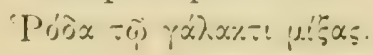

Inacr. Ode XV (28), v. 23. 
Les poites du moyen âge reprendront cette comparaison, qui s'est fidelement conservée depuis dix siceles: pour eux aussi, nous le verrons, la rose sera l'embleme de la grâce et de la beauté. Les Anciens les avaient devancés; chez eux la rose fut à la fois le symbole de l'innocence pudique comme de la beauté.

Cachée au fond des réduits secrets d'un jardin, dit Catulle dans des vers restés célèbres', ignorée des troupeaux, respectée par le soc de la charrue, tour à tour rafraîchie par la rosée, caressée par les zéphyrs et fortifiée par les rayons du soleil, la rose est la joie et le désir de tous; mais lorsque l'ongle tranchant, la séparant de sa tige, l’a flétrie, elle cesse de plaire et de séduire.

Utque rosae puro lacte natant folia.

Properce, Elegiae, lib. II, 3, v. 12.

ou par la neige:

Candida candorem roseo suffusa rubore

Ante stetit: niveo lucet in ore rubor.

Ovide, Amorum lib. III, Eleg. 3, v. 5-6.

1. Carmina, LXII, v. 39-47. Catulle se sert du mot générique " flos », mais il est évident, et ses imitateurs ont été unanimes à l'entendre ainsi, qu'il avait en vue la rose.

Ut flos in septis secretus nascitur hortis, Ignotus pecori, nullo contusus aratro,

Quem mulcent aurae, firmat sol, educat imber.

Multi illum pueri, multae optavere puellae;

Idem cum tenui carplus defloruit ungui,

Nulli illum pueri, nullae optavere puellae:

Sic virgo dum intacta manet, dum cara suis est;

Cum castum amisit polluto corpore florem,

Nec pueris jucunda manet, nec cara puellis. 
LA ROSE DANS LES LÉGENDES ET DANS LA POÉSIE. 63

Ainsi, ajoute-t-il, la jeune vierge, tant qu'elle reste chaste, est chère à tous les siens; mais a-t-elle, souillant ses charmes, perdusa fleur de pureté, elle cesse de plaire et de séduire.

L’idée de grâce et de fragilité attachée à la rose en avait fait l'emblème de l'innocence virginale, celle de beauté conduisit à lui comparer la personne aimée. Anacréon n'a pas cru pouvoir mieux louer son amie Myrille, qu'en disant qu'elle était "une rose entre les jeunesfilles ${ }^{1}$ ». L’amie de Méléagre, "l'aimable Zénophile, fleur parmi les fleurs, s'épanouit, semblable à la douce rose de la persuasion ${ }^{2}$ »). Les poètes de l'antiquité toutefois n'ont pas poussé plus loin la comparaison, et ils ne sont pas allés, comme leurs émules du moyen îgre, jusqu'ì personnifier leur bien-aimée dans la rose; mais la langue populaire l'a essayé: "ma rose » fut un terme de tendresse usité de bonne heure chez les Romains - on le rencontre déjà dans Plaute ${ }^{3}$ - pour désigner une persomne qui était chère.

Si les Anciens n'ont point chanté la rose comme l'image et la personnification même de la personne

1.

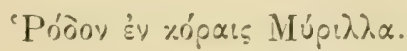

Fragmenta LV, éd. J.-B. Gail.

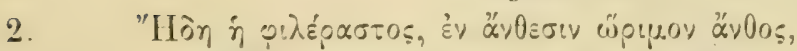

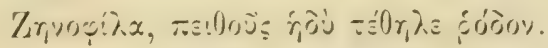

Anthologia palatina, cap. V, no 144.

3. Ubi tu lepide voles esse tibi, mea rosa, mihi dicito.

Bacchis, acte I, scène I, v. 148.

Cf. Asinaria, acte III, scène 3, v. 101. 
aimée, ils n'en ont pas moins fait de la reine des fleurs l'embleme de l'amour et l'attribut des divinités qui y président. C'est ainsi que l'Ifyménée fut représenté sous les traits d'un jeune homme couronné de roses $^{1}$ et un flambeau à la main. Ovide, dans les Amours ${ }^{2}$, dépeint Vénus répandant une pluie de roses sur son fils triomphant; et, dans ses Métamorphoses ${ }^{3}$, il nous fait le ravissant portrait de Cyllare et d'Ilylonomé, "la plus belle des filles des Centaures ", et nous les montre, symbole de leur mutuelle tendresse, "entremèlant leurs blonds cheveux de roses, de violettes, de romarin et quelquefois de blanes lis ).

Reposanius a embelli de lis et de roses le bocage, témoin des amour's de Vénus et de Mars ${ }^{4}$; Éros, sur l'ordre de la déesse, couve sa couche de roses; des

1. Sertis tempora vinctus
Hymen.
Ovide, Epist., VI, v. 4 4.

2. Laeta triumphanti de summo mater Olympo Plaudet, et appositas sparget in ora rosas.

Lib. I, 2, v. 40 .

3. Ut modo rore maris, mode se violave rosave Implicet; interdum candentia lilia gestet.

$$
\text { Lib. XII, v. 410-411. }
$$

4. Pingunt purpureos candentia lilia tlores...

Namque hic per frondes redolentia lilia splendent, Hic rosa cum violis, hic omnis gratia florum...

Tu lectum consterne rosis, tu serta parato,

Et roseis crinem nodis subnecte decenter..,

Lilia cum roseis supponit candida sertis.

De concubitu Martis et Veneris, v. 38, 41-42, 53-54 et 111. (Anth. latina, vol. I, p. 171-172.) 
LA ROSE DANS LES LEGENDES ET DANS LA POÉSIB. 65

couronnes de roses retiennent sa chevelure et c'est au milieu des lis et des roses qu'elle reçoit son farouche vainqueur. Dans la description qu'il a faite des amours des mèmes divinités ${ }^{1}$, Claudien pare le seuil de Vénus de branches de myrte et couvre de fleurs de roses sa couche que voile la pourpre nuptiale. Et aux noces d'Orphée ${ }^{2}$, il fait apporter par les colombes de Vénus des guirlandes faites de roses dérobées dans le jardin mème de la déesse.

L'auteur du Pervigilium Veneris, cette description affectée de la fète du printemps et de l'amour, va jusqu'à dire ${ }^{3}$ que " chaque matin les jeunes vierges, par l'ordre de Vénus, se marient aux roses ». Cueillir des roses, en tresser des couronnes, en effet, comme on le voit par un passage d'Aristophane ${ }^{4}$, était un signe qu'on était épris d'amour.

IV.

Consacrée à Bacchus, comme à Vénus, la rose fut

1.

Festa frondentia myrto

Limina cinguntur, roseisque cubilia surgunt

Floribus et thalamum dotalis purpura velat.

$$
\text { Magnes, v. 28-30. (Opera, XLVII.) }
$$

2. Furatae Veneris prato per inane columbac

Florea connexis serta tulere rosis.

Ad Serenam, Epist. II, v. 9-10. (Opera, XL.)

3. Ipsa jussit mane totae virgines nubant rosae.

v. 44, éd. Bücheler. Lipsiae, 1859, in-12, p. 53.

4.

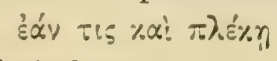

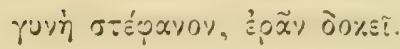

Thesmoph., v. 400-401.

JORET. La RoSe. 
le symbole et la compagne de la joie qui règne dans les banquets, comme elle était l'emblème de l'amour. C'est pour cette raison qu'on donnait à Comus, personnification de la gaité des festins, une couronne de roses ${ }^{1}$. La rose était considérée comme l'emblème de l'allégresse; sa présence seule en était la marque et le symbole, comme son absence était un signe de tristesse. "Que soudain les sommets des Alpes se couvrent de roses ", s'écrie Claudien", conviant l'univers entier à célébrer les noces d'Ilonorius. Pour nous peindre, au contraire, le deuil qui s'étendit sur la nature au moment de l'enlèvement de Proserpine, le même poète nous dira que "les roses meurent et les lis dépérissent ${ }^{3}$. Et présage du malheur qui va frapper le fils de Pélops, Sénèque fait dire à Thyeste que " les couronnes de roses sont d'elles-mêmes tombées de sa tête ${ }^{4}$.

Emblème de la joie et compagne du plaisir, la rose - la transition était naturelle - devint le signe et la marque de la mollesse. Pour donner une idée de la fermeté stoïque de Régulus, Cicéron dit que « la vertu le proclame plus heureux que Thorius»,

1. Philostrate, Imaginum lib. I, cap. II, 3.

2. Subitisque se rosetis

Vestiat Alpinus apex.

Fescennina, v. 8-9 (Opera, XII.)

3. Exspirare rosas, decrescere lilia vidi.

De raptu Proserpinae, lib. III, v. 241. (Opera, XXXVI.)

4. Vernae capiti fluxere rosae.

Thyestes, acte V, scène 2, v. 948 . 
-- un célèbre voluptueux de Rome - " "vidant sa coupe sur un lit de roses ${ }^{1}$ ). Et, dans Claudien, Ilonorius ne croit pas pouvoir mieux montrer à ses soldats à quel point le tyran Gildon était efféminé qu'en le leur représentant " marchant couronné de roses et oint de parfums ${ }^{2}$ ). Malgré cette attribution, si éloignće du caractère auguste et sacré qu'elle avait à l'origine, la rose conserva toujours la signification symbolique la plus haute et la plus grave. Dès longtemps elle était devenue la marque et le symbole du mérite ou d'une supériorité reconnue. Sappho dit à une femme ignorante qu'elle mourra oubliée parce que les roses de Piérie ne lui ont pas ceint le front ${ }^{3}$. Dans les Chevaliers, Cléon promet au peuple qu'il régnera couronné de roses sur toute la contrée ${ }^{4}$. La reine des fleurs servit de récompense aux vainqueurs dans les luttes poétiques, aussi bien que dans les combats sanglants de la guerre.

1. Clamat virtus beatiorem fuisse quam potantem in rosa Thorium. De finibus, lib. II, cap. 20.

2. Umbratus dux ipse rosis et marcidus ibit Unguentis.

De bello Geldonico, v. 44 . (Opera, XV.)

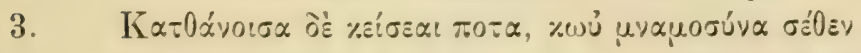

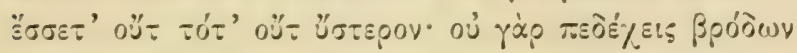
รสั)

Fragm. 68 (19). (Anthologia lyrica. Curavit Th. Bergk. Lipsiae, 1883, p. 204).

4.

$$
\ddot{x}_{p} \xi \alpha ! \quad \sigma \varepsilon \text { ò } \varepsilon
$$

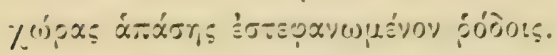

Aristophane, Equites, v. 965-66. 
Contraste qui pourrait surprendre, si les divinités auxquelles elle était consacrée n'avaient pas présidé a la mort ainsi qu'aux plaisirs de la vie: de symbole de joie et d'amour la reine des fleurs, avec le lis et les violettes, devint aussi un emblème funéraire. De bonne heure, hommage pieux qu'on rendait à des ètres chers, on orna de fleurs les tombeaux. Créon défend sous peine de mort de couronner de fleurs et d'ensevelir le corps de son ennemi Polynice ${ }^{1}$, qu'il poursuit de sa haine jusque dans la mort. Dans Electre ${ }^{2}$, au contraire, Chrysothémis reconnaît l'approche d'Oreste aux guirlandes de fleurs dont clle voit ornée la tombe paternelle.

Lorsque, dans la vision qui montre à Énée toute sa postérité, le guerrier apprend que Marcellus est voué par les destins à une mort prématurée, il demande des lis et des roses pour les répandre, vain et dernier hommage, sur les mânes de son petit-fils ${ }^{3}$.

1.

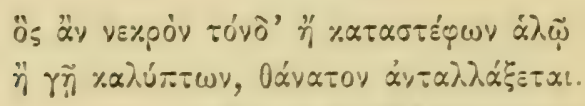

Euripide, Phoenissae, v. 1632-33.

2.

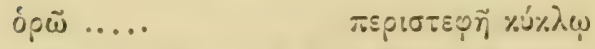

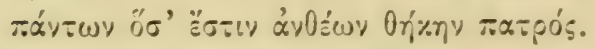

Sophocle, Electra, v. 894-96.

3.

Manibus date lilia plenis,

Purpureos spargam flores, animamque nepotis His saltem adcumulem donis el fungar inani Munere.

Aeneidos lib. VI, v. 883-86.

J'explique, ainsi que Servius, purpureos flores par roses, au lieu d'y voir arec M. Benoist une espèce de lis. 
Tibulle, dans une de ses élégies, souhaite que chaque annce une main amie orne de couronnes son monument $^{1}$, et Properce espère dormir plus doucement dans sa tombe, si l'on dépose ses restes sur de tendres roses ${ }^{2}$. Dans Alciphron ${ }^{3}$, Pétale se plaint de ne pas avoir d'ami qui pleure sa mort prématurée et orne son tombeau de couronnes de roses.

Ce ne sont point là de simples fictions poétiques, mais l'expression sincère du culte d'affection que les Anciens avaient voué aux défunts. Lucien, dans l'énumération qu'il a faite des devoirs qu'on rendait aux morts ${ }^{4}$, place au premier rang les couronnes de fleurs dont on parait leurs dépouilles, et saint Jérôme opposera aux roses, aux violettes et aux lis que les autres maris, dit-il ${ }^{5}$, répandaient sur les urnes qui

1. Atque aliquis senior, veteres veneratus amores,

Annua constructo serta dabit tumulo.

$$
\text { Elegiae, lib. II, 4, v. 47-48. }
$$

2. $\quad$ Holliter et tenera poneret ossa rosa.

$$
\text { Elegiae, lib. II, 17, v. } 22 .
$$

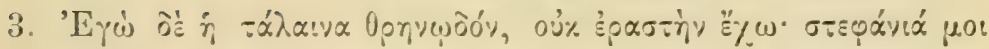

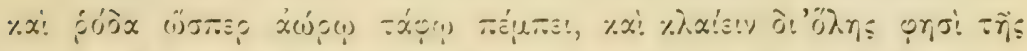
W\%rós. Alciphronis rhetoris Epistolae, éd. Rudolphus Hercher. Parisiis, 1873 , in-8, lib. I, 36, p. 57.

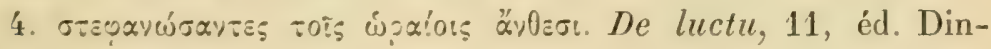
dorf. Opera, I, p. 567.

5. Caeteri mariti super tumulos conjugum spargunt violas, rosas, lilia, floresque purpureos, et dolorem pectoris his officiis consolantur. Pammachius noster sanctam favillam ossaque veneranda eleemosynae balsamis rigat. Epistola ad Pammachium. (Opera omnia, éd. Martianay. Paris, 1706, in-fol. vol. IV, p. 584$)$. 
renfermaient les cendres de leurs épouses, les ceures de charité par lesquelles Pammachius honore le souvenir de Paulina.

$$
\text { V. }
$$

Douce d'attributs si nombreux et si divers, consacrée aux Grâces et aux Muses, la rose ne pouvait manquer d'ètre chère aux poètes de l'antiquité; aussi l'ont-ils chantée à l'envi.

La rose, dit le pseudo-Anacréon ${ }^{1}$, est la plus belle des fleurs, le souci du printemps, la joie et les délices des dieux, la parure d'Aphrodite, et son fils en couronne ses beaux cheveux quand il se mêle aux chueurs des Grâces.

Et ailleurs ${ }^{2}$

La rose est est le parfum des Dieux, le charme des mortels, le plus bel ornement des Grîces dans la saison fleurie des amours, la parure d'Aphrodite; elle est

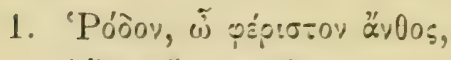

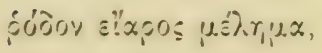

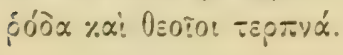

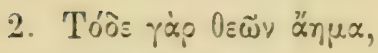

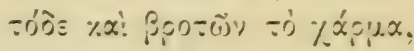

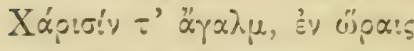

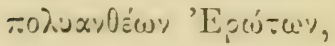

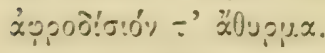

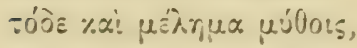

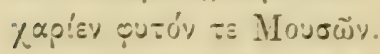

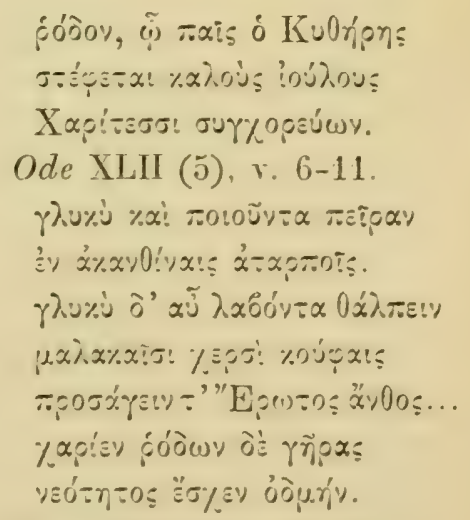


LA ROSE DANS LES LÉGENDES ET DANS LA POÉSIE. 71

l'objet du chant des poètes, l'arbuste chéri des Muses. Elle blesse de ses rudes épines et cependant on la cueille avec plaisir. On aime à tenir dans ses mains cette fleur consacrée à Éros et à respirer sa douce odeur... Agréable jusque dans sa vieillesse, elle y conserve le parfum de ses premiers jours.

Si Jupiter, dira à son tour Achille Tatius ${ }^{1}$, avait voulu donner une reine aux fleurs, la rose eut été leur reine ; elle est la parure de la terre, la splendeur des plantes, l'wil des fleurs, la pourpre de la prairie, l'éclair de la beauté. Elle exhale l'amour; elle attire et fixe Vénus: elle se couronne de feuilles odorantes; elle étale avec orgueil ses flexibles pétales, qui sourient légers aux zéphyrs.

Pour Philostrate encore la rose est le " monument d'Adonis, le sang de Vénus, l'œil de la terre „².

Les poètes de Rome ont rivalisé avec leurs émules de la Grèce dans ce concert de louanges en l'honneur de la rose; l'un d'eux ${ }^{3}$ ira jusqu'a l'appeler "l'astre des fleurs ». Mais nul ne l'a célébrée dans des vers plus gracieux, quoique non exempts d'afféterie, qu'Ausone. Dans une idylle ${ }^{4}$ qu'on a parfois attribuée a Virgile, il feint qu'il erre au milieu d'un jardin au moment où « la douce haleine du matin et sa piquante

1. Leucippe et Clitophon, liv. II, chap. 1. J'ai suivi en partie la traduction de M. Zévort

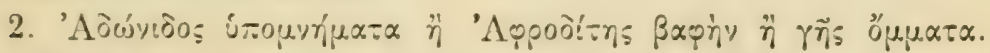
Epistola I.

3. " Haec florum sidus. " Anthologia latina, éd. Al. Ricse, v. I, p. 240. (De laude rosae centumfoliae.)

4. "Rosae. " Idjlle XII, éd. Nisard. Paris, 1887, in-8. 
fraîcheur annoncent le retour doré du jour. " Il y voit ${ }^{1}$ les rosiers que cultive Pestum briller humides au nouveau lever de Lucifer; et çà et là, sur les arbrisseaux encore chargés de brouillards, luit une blanche perle qui doit mourir aux premiers rayons du jour.

On doute, ajoute le poète ${ }^{2}$, si l'Aurore emprunte aux roses son éclat vermeil, ou si le jour naissant donne à ces fleurs la nuance qui les colore. Même rosée, même teinte, même grâce matinale à toutes deux; car l'étoile et la fleur ont pour même reine Vénus; même parfum peut-être; mais le parfum de l'une se dissipe dans les hautes régions de l'air ; plus rapproché, on n'en sent que mieux le parfum de l'autre. Déesse de l'étoile et déesse de la fleur, la divinité de Paphos a voulu leur donner à toutes deux la couleur de la pourpre.

On comprend d'après ces éloges enthousiastes quelle place la rose devait occuper dans les curres

1. Vidi Paestano gaudere rosaria cultu,

Exoriente noro roscida Lucifero.

Rara pruinosis canebat gemma frutetis,

Ad primi radios interitura die.

v. 11-14.

2. Ambigeres, raperetne rosis Aurora ruborem,

An daret; et flores tingeret orta dies.

Ros unus, color unus, et unum mane duorum

Sideris et floris, nam domina una Venus.

Forsan et unus odor : sed celsior ille per auras

Difflatur : spirat proximus iste magis.

Communis Paphic Dea sideris, et Dea floris,

Praecipit unius muricis esse habitum.

ง. $15-22$. 
LA ROSE DANS LES LÉGENDES ET DANS LA POÉSIE. 73

des poc̀tes anciens; ils lui ont aussi emprunté les plus charmantes comparaisons. Quand les dieux ou leurs faroris parlent ou sourient, un parfum de roses s'exhale de leur bouche ${ }^{1}$; s'ils agitent leur chevelure, il en tombe des roses ${ }^{2}$; des roses aussi naissent partout où ils portent leurs pas ${ }^{3}$.

Dans la langue des poètes, remarque le pseudoAnacréon ', l'Aurore a des doigts de rose, les Nymphes des bras de rose, Vénus un teint de rose.

Ce n'était là que la constatation de ce qu'on trouvait dans la réalité. Ilomère et Ilésiode nous montrent l'Aurore ourrant de ses doigts de rose les portes du matin. Théocrite a recours à la même figure pour décrire le lever du jour :

Les coursiers de l'Aurore aux bras de rose, dit-il" s'élancent dans le ciel, la ramenant de l'Océan.

1. Dum locquitur vernas efflat ab ore rosas.

$$
\text { Ovide, Fast. lib. V, v. 194. }
$$

2. motis flores cecidere capillis,

Accidere in mensas ut rosa missa solet.

Ovide, Fast. lib. V, v. 359.

3. Quidquid calcaverit hic rosa fiat.

Perse, Satira II, v. 38.

Quacumque per herbam

Reptares, fluxere rosae.

Claudien, Laus Serenae, r. 90. (Opera, XXIX).

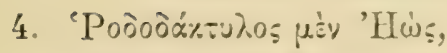

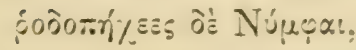

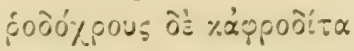

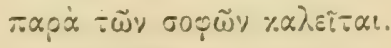

Ode LIV, v. 1-4 (LIII, v. 20-24).

5.

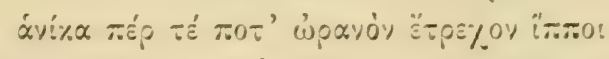

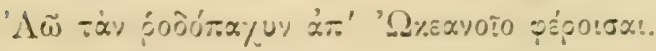

Idyllion II, v. 147-148. 
A l'exemple de leurs précurseurs de la Gréce, les poètes latins emploient, à chaque instant, la mème figure.

Le soleil, chante Lucrèce ${ }^{1}$, de ses feux couleur de rose ramenait la lumière dans le ciel.

Et Virgile ${ }^{2}$ :

L'Aurore, conduite par quatre coursiers coulcur de rose, avait déjà parcouru la moitié de la céleste carrière.

Ou encore ${ }^{3}$ :

Du haut des airs brillait la blonde Aurore, trainée par deux coursiers aux crins de rose.

Dans Tibulle aussi " l'Aurore ramène le jour brillant sur son char couleur de rose ${ }^{4}$ ).

L'Aurore à la chevelure de rose avait dissipé les ténèbres,

1. Dum rosea face sol inferret lumina caelo.

De natura rerum, lib. V, v. 97'́.

2. roseis Aurora quadrigis

Jam medium aetherio cursu, trajecerat axem.

Acneidos lib. VI, v. 355-56.

3. aethere ab alto

Aurora in roseis fulgebat lutea bigis.

Aeneidos lib. VII, v. 26.

4.

Aurora nitentem

Luciferum roseis candida portet equis.

Lib. I, 3, v. 93-9's. 
LA ROSE DANS LES LÉGENDES ET DANS LA POÉSIE. 75 dit également l'auteur du Moucheron ${ }^{1}$. Et dans Ovide :

La vigilante Aurore, ouvrant les portes resplendissantes de l'Orient, sort de son palais rempli de roses ${ }^{2}$.

Ailleurs, il la montre " blonde mère de Memnon, revenant visiter le monde trainée par des coursiers couleur de rose 3 ", ou bien " emportée par ses coursiers, ramenant dans le ciel la lumière rosée du matin ${ }^{4}$ ». L'Aurore, chez Valérius Flaccus, apparaît aussi " trainée par deux coursiers couleur de rose ${ }^{3}$ ".

Secouant de son bras de rose les rênes empourprées, dit Apulée ${ }^{6}$, l'Aurore lance ses coursiers dans les cieux ".

Stace parle aussi des "rênes de rose " qu'agite

1. Crinibus et roseis tenebras Aurora fugarat.

$$
\text { Culex, v. } 13 .
$$

2.

Ecce vigil rutilo patefecit ab ortu Purpureas Aurora fores et plena rosarum Atria. Metamorph. lib. II, v. 112-114.

3. veniet, terras visura patentes, Memnonis in roseis lutea mater equis.

Fastorum lib. IV, v. 713-71'.

4. roseam pulsis Hyperionis astris In matutinis lampada tollit equis.

Fastorum lib. V, v. 159-160.

5 . ut roseis Auroram surgere bigis Vidit. Argon. lib. II, v. 261.

6. Commodum punicantibus phaleris Aurora roseum quatiens lacertum, coclum inequitabat. Metamorph. lib. III. 
l'Aurore ${ }^{1}$ et Valérius Flaccus va jusqu'ì dire de Bacchus qu'il conduit arec des "rênes de rose " les nations vaincues ${ }^{2}$.

Comme à l'Aurore, on donna à la Lune un char couleur de rose; c'est ainsi que Stace la montre s'élevant au plus haut des cieux ${ }^{3}$. Valérius Flaccus la dépeint, guide propice à travers l'obscurité, remplissant les bois de sa lumière rosée ${ }^{4}$. Comme l'Aurore encore", la Lune ${ }^{6}$ recut, ainsi que le Soleil - Titan ${ }^{7}$ ou Phébus ${ }^{8}$ - l'épithète de " rosée » ou " couleur de rose ». L'un et l'autre, dit Claudien des deux astres " " nourrissons couleur de rose d'Ilypérion ", éclairent de leurs feux la plaine azurée.

1. roseasque moveluat habenas.

Punic. lib. I, v. 578.

2. roseis haec per loca Bacchus habenis Cum domitas acies... (duceret).

3. Scandebat roseo medii fastigia caeli

$$
\text { Argon. lib. III, v. } 538 .
$$

Luna jugo. Stace, Achill. lib. I, v. 818-19.

4. roseo talis per nubila ductor

Implet honore nemus. Argon. lib. VIII, v, 30-31.

5. Conscia nox sceleris roseo cedebat Eoo.

Silius Italicus, Punic. lib. IX, v. 180.

6. Cephalus roseae praeda pudenda Deae.

Ovide, Artis amat. lib. III, v. 84.

7 te roscum Titana rocari.

Stace, Thebaidis lib. I, v. 717

8. Ni roseus fessos jam gurgite Phobus Hibero

Tingual equos. Aeneidos lib, XI, v. 913.

9. Coeruleusque sinus roseis radiatur alumnis.

De raptu Proserpinae, II, v. 48. (Opera, XVII.) 
Le même poète nous montre ${ }^{1}$ encore les coursiers du soleil, les freins écumants, "lançant par les narines des feux aux teintes de rose ", ainsi que le Bélier céleste "à la corne de rose» qui ramène avec lui le fertile printemps ${ }^{2}$. Une couleur de rose est également attribuée par Stace à Lucifer, quand, au soir, il illumine les nuées de ses feux tardifs ${ }^{3}$. Valérius Flaccus donne aussi à ce dernier des " ailes rosées ${ }^{4}$ ». Les nuages prennent de même cette couleur.", lorsque la rapide Iris les traverse. L'expression "lumière rosée ${ }^{6}$ ", pour Stace, est synonyme d'Aurore, et Silius Italicus donne à l'Orient le nom de "levant couleur de rose " ${ }^{7}$. L'auteur des Guerres puniques parle encore des "feux rosés » qui s'élèvent des régions de l'Aurore au milicu de l'azur du ciel ${ }^{8}$.

1. Efflantes roseum frenis spumantibus ignem.

In consul. Probini et Olybrii, v. 5. (Opera, I.)

2. Phrixeus roseo producat fertile cornu Ver Aries.

De laud. Stilichonis, II, v. 463. (Opera, XXII.) roseus per nubila seras

Advertit flammas.

Thebaidis lib. II, v. 13:

4.

qualis roseis it Lucifer alis.

$$
\text { Argon. lib. VI, v. } 527 .
$$

5. Velocem roseis demittit nubibus Irin.

Valerius Flaccus, Argon. lib. IV, v. 77.

6. rosea sub luce reversi.

Silvar. lib. III, 1, v. 134.

7. Terminus huic roseos amnis Lageus ad ortus.

Punic. lib. I, v. 196.

8. ab aequore Eoo

Surgebant roseae media inter caerula flammae.

Punic. lib. IV, v. 481. 
Mais ce ne fut pas seulement à la lumière de l'Aurore ou des astres qu'on donnal'épithète de "rosée", on l'attribua aussi aux diverses parties du corps humain et surtout du visage. La bouche, les lèvres, les joues, le front, le cou, la face tout entière furent " couleur de rose »; on le dit également des seins et des bras, de la main comme du pied. Sappho donne aux Grâces des bras ${ }^{1}$, Himère des pieds de ruse $^{2}$. Catulle parle du " sein de rose 》 de sa mâ̂tresse $^{3}$; il montre les bandelettes de neige qui ceignent le front de rose des Parques ${ }^{4}$, et il nous décrit les accents plaintifs sortant des lèvres rosées d'Attis". Virgile ${ }^{6}$ comme son imitateur Silius Italicus ${ }^{7}$, fait parler Vénus d'une "bouche de rose ». Il en est de même d'Iris. Nous voyons aussi dans l'Énéide Vénus s'éloigneren détournant son cou ou sa face de roses, et Lavinie déchirer, dans sa douleur, ses joues de

1.

2.

3.

4.

5.

6.

T.
8. avertens rosea cervice refulsit.

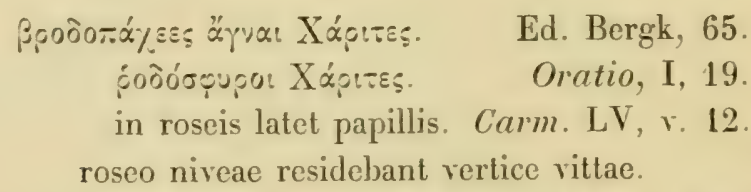

Carm. LXIV, v. 309.

Roseis ut huic labellis sonus editus abiit.

Carm. LXIII, v. 74.

roseoque haec insuper addidit ore.

Aeneid. lib. II, v. 593.

suspirans roseo Venus ore decoros

Adloquitur natos.

Punic. lib. VII, v. 448 .

roseo Thaumantias ore locuta est.

Acneid. lib. IX, v. 5.

Aeneid. lib. I, v. 402. 
LA ROSE DANS LES LÉGENDES ET DANS LA pOÉSIE. 79

rose $^{1}$. Horace parle aussi de la face de rose de Télèphe ${ }^{2}$.

Ovide, personnifiant l'Aurore, vante la bouche de rose de la déesse du matin ${ }^{3}$. Dans le premier livre de son Achilléide, Stace, voulant dépeindre l'éclatante beauté de Déidamie, fille de Lycomède, dit que les roses de son visage relèvent la pourpre de sa tunique ${ }^{*}$; ailleurs, il parle du léger duvet qui viendra ombrager les joues de rose du fils d'Atalante, Parthénopée", ou il rappelle quel vêtement Vénus inventa pour relever le teint de rose du visage ${ }^{6}$. Nartial met au rang des principaux traits de beauté des " lèvres rivalisant avec l'incarnat des roses de Pestum $^{\top}$ ». Dans une autre de ses épigrammes, il nous montre un jeune adolescent " effleurant une coupe

1.

2.

3.

4.

5.

roseas laniata genas.

Aeneid. lib. XII, v. 606. cervicem roseam (laudas).

Carm. lib. I, 13, v. 1.

roseo spectabilis ore.

Metam. lib. VII, v. 705. roseo flammatur purpura vultu.

v. 297 .

Dum roseis renit umbra genis.

Thebaid. lib. IV, v. 336.

Au lib. IX, v. 703, il est question des joues dont un duvet de rose a changé l'aspect :

mutatae rosea lanugine malae.

6. quae vestis roseos accendere vultus Apta. Silv. lib. III, 4, y. 51-52.

7. Paestanis rubeant aemula labra rosis.

Epigr. lib. IV, 42, v. 10 . 
de ses lères de rose ${ }^{1}$ ). On ne doit pas être surpris non plus qu'il soit question dans ses vers de " bouches de roses ${ }^{2}$ ). Plus tard on verra encore Claurlien réprésenter Achille peignant de sa " main de rose » sa blonde chevelure ${ }^{3}$.

Les poètes latins des derniers siècles ont prodigué l'épithète de "rosée » ou " de rose »; chez eux elle est derenue synonyme de l'adjectif " pourpre », ou mème simplement de "brillant ", " doré ", "beau ). Déjà Catulle parle d'un coussin d'ivoire que la pourpre de Tyr a recouvert d'un rernis couleur de rose ${ }^{\ddagger}$. Dans Valérius Flaccus, il est question d'une "jeunesse rosée" ». Claudien a chanté les "vallées de rose d'Henna ${ }^{6}$ "; il a vanté aussi le "Douro aux rives rosées ${ }^{7}$ ), et donné même des "crètes de rose " à

1. Et libata dabat roseis carchesía labris.

Lib. VIII, 56, v. 15.

2. roseo torserat ore puer.

$$
\text { Epigr. lib. XI, 56, v. } 12 .
$$

3. Thessalicos roseo pectebat pollice crines.

De nuptiis Honorii et Mariae, v. 19. (Opera, X.)

4. (pulvinar) Indo quod dente politum

Tincta tegit roseo conchyli purpura fuco.

Carm. LXIV, v. 48-49.

5. Mole nova et roseae perfudit luce jurentae. Argon. lib. V, v. 366 .

6. qualem roseis nuper convallibus Hennae Suspexere Deae.

De raptu Proserpinae, III, v. 85. (Opera, XXXVI.)

7. roseis formosus Duria ripis.

Laus Serenae, v. 72. (Opera, XXIX). 
des serpents ${ }^{1}$. Il y a eu sans doute quelque chose de conventionnel et de factice dans l'emploi ainsi généralisé de cette expression, et elle a cessé dès lors d'ètre un témoignage toujours manifeste de l'importance prise par la reine des fleurs dans la vie des Grecs et des Romains. Il n'en est pas de même de la place qu'occupe la rose dans quelques-unes des plus belles descriptions des poètes anciens, ni des images gracienses qu'elle leur a fournies. Une fleur aussi admirée et recherchée a pu seule les inspirer.

Pindare, par exemple, dit des ancêtres de Mélissos qu'ils " brillèrent comme la terre émaillée des fleurs pourpres de la rose ${ }^{2}$.»

Celui à qui Cypris n'a point donné un baiser ne sait point quelles fleurs sont les roses, dit la locrienne Nossis".

De douleur mon visage rougit, comme la rose sous la rosée,

s'écrie Théocrite ${ }^{4}$. Pour peindre Adonis au moment d'expirer et perdant ses forces et sa couleur, Bion

1. Erecti roseas tendunt ad carmina cristas. De raptu Proserpinae, I, v. 14 (Opera, XXXIII).

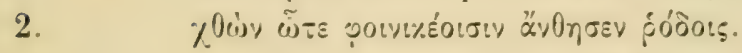

Isthmiques, IV, v. 330.

3.

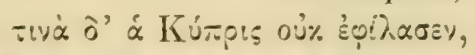

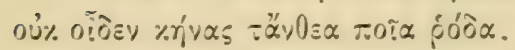

Anth palatina, lib. V, no 170, v. 3-4.

4

Idyllion XX, v. 16.

Joret. La Rose. 
dit que "les roses fuient de ses lèvres ${ }^{1}$." Et nous avons vu Rhianos comparer le jeune Empédocle, dont la beauté surpasse celle de ses compagnons, à la "rose qui brille entre les fleur's du printemps ${ }^{2}$. »

Ne me fuis pas, dit le pseudo-Anacréon à son amie ${ }^{3}$, parce que mes cheveux sont blancs; ne dédaigne pas mes présents, parce que tu es dans la fleur de l'âge. Vois comme, dans les couronnes, les blancs lis se marient agréablement aux roses.

Que ne suis-je une rose à la fleur empourprée, s'écrie, dans un sentiment tout moderne, un poète grec anonyme ${ }^{4}$, pour que tu me déposes sur ton sein blanc comme la neige.

Et Musée voulant décrire la beauté pleine de grâce de Héro :

1.

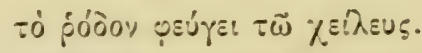
Epitaphium Adonidis, v. 11. Ed. Didot.

2.

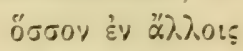

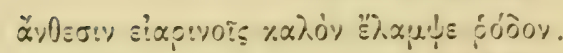

Anth. palatina, lib. XII, $\mathrm{n}^{\circ} 58$, v. 3-4.

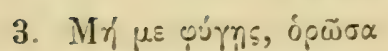

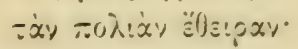

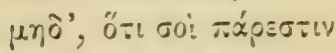

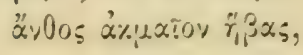

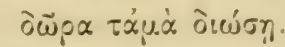

\%ँ \%

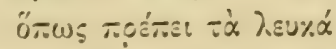

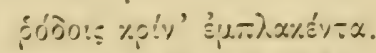

Ode XLIX (XXXIV).

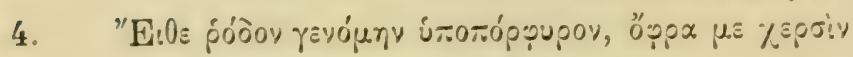

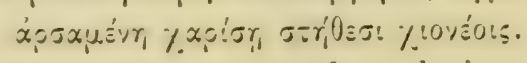

Anth. palatina, cap. V, no 84 . 
LA ROSE DANS LES LÉGENDES ET DANS LA POÉSIE. 83

Ses joues de neige, dit-il ${ }^{1}$, se couvrirent de rougeur, semblables à la rose à la double couleur, quand elle sort de son calice. On dirait que ses membres délicats étaient des champs de rose; une lumière rosée l'enveloppait; quand elle marchait, les roses brillaient sous les pas de la vierge vêtue de sa blanche tunique.

Sa bouche, dit Clitophon de Leucippe, dans Achille Tatius $^{2}$, était semblable à une rose qui commence à entr'ouvrir les lèvres de ses pétales.

La rapidité avec laquelle passe la rose fournit en particulier aux poètes les plus charmantes comparaisons :

La rose est belle et le temps la flétrit,

dit dans 'Théocrite ${ }^{3}$, un amant à l'amie qui le dédaigne et qu'il voudrait fléchir.

Et Straton ${ }^{4}$ :

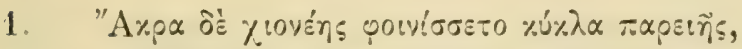

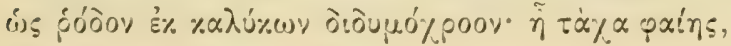

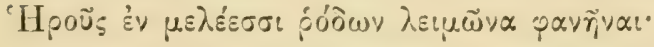

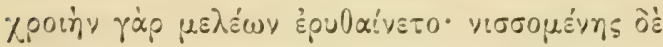

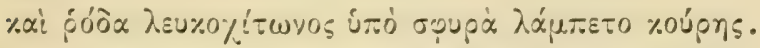

De IIerone et Leandro, éd. F. S. Lehrs, p. 4, v. 58-62.

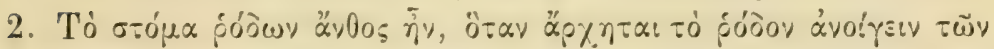

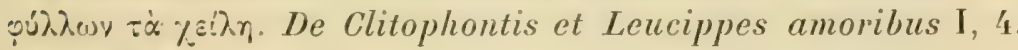

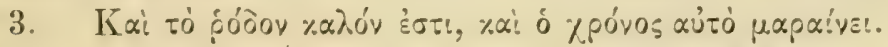
Idyllion XXIII, v. 28.

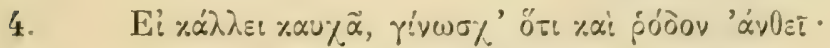

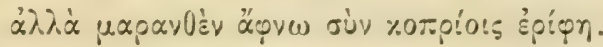

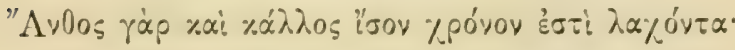

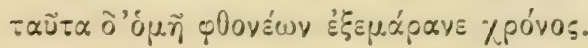

Anth. palatina, lib. XII, no 234 . 
Si tu t'enorgueillis de ta beauté, rappelle-toi que la rose aussi brille dans sa fleur; mais bientôt flétrie, on la jette au loin; les fleurs et la beauté ont reçu du sort la même durée; le temps envieux les flétrit en même temps.

Même pensée dans cette épigramme latine ${ }^{1}$ :

Après l'heure de la rosée, les violettes se fanent, la rose perd son parfum; après le printemps, les lis s'étiolent et perdent leur blancheur; redoute, je t'en supplie, ces exemples et paie de retour ton ami; il aime toujours celui qui toujours est aimé.

Telle encore cette autre épigramme toute empreinte de mélancolie ${ }^{2}$ :

Comme la rose apparaît en son temps dans sa beauté et en son temps se flétrit, ainsi tu commençais en ton printemps à être belle, mais soudain tu cesses d'être à moi.

De même, pour décrire la mort prématurée d'un

1. Marcent post rorem violae, rosa perdit odorem,

Lilia post vernum posito candore liquescunt.

Haec metuas exempla precor et semper amanti

Redde vicem, quia semper amat qui semper amatur.

Anthologia latina, rec. Al. Riese, vol. I, p. 82, no 24.

2. Ac veluti formosa rosa, cum tempore prodit,

Arescit certe tempore deinde suo.

Sic tu coepisti primo formosa videri

Tempore, sed subito desinis esse mea.

Anthologia seterum lat. epigrammatum cura P. Burmaxis. Amst., 1773, in-4, lib. IV, $n^{\circ} 152$. 
LA Rose dANS Les LÉgendes e'T dANS LA pó̉sie. 85 fils enlevé à ses parents dans la fleur de la jeunesse, Stace s'écrie ${ }^{1}$ :

Tels les lis inclinent leurs tiges pâlissantes ou les roses fraîches écloses meurent aux premiers souffles de l'Auster.

L'habitude que les Anciens avaient de vivre entourés de roses donna naissance aux locutions " vivre ", " dormir ", " être couché sur des roses" ", synonymes d'ètre heureux, vivre dans la tranquillité ou même dans la mollesse.

Cypris et la douce éloquence, dit Ibycus ${ }^{3}$, pour peindre l'heureuse jeunesse d'un ami, t'ont élevé au milieu des roses.

Et Martial conseille à Liber, "digne de vivre sans cesse environné de roses ", de se ceindre toujours le front de couronnes de fleurs4.

1. Qualia pallentes declinant lilia culmos,

Pubentesve rosae primos moriuntur ad Austros.

Silvarum lib. III, 3, v. 128-129.

2. An tu me putabas in rosa dicere? Cicéron, Tuscul. quaest. lib. V, cap. 26 .

3.

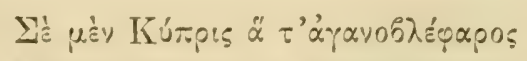

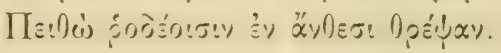

Athénée, Deipnosoph. lib. XIII, cap. 17 (564).

4. Liber, in aeterna vivere digne rosa,

.... cingant florea serta caput.

$$
\text { Epigram. lib. VIII, 77, v. 2-4. }
$$


Personne, remarque Sénèque ${ }^{1}$, ne s'exerce à ce que, en cas de nécessité, il couche tranquillement sur des roses,

donnant à entendre qu'il n'est point besoin d'apprendre à vivre dans la mollesse et l'oisiveté.

Ce que tu me dis est pour moi des roses ${ }^{2}$,

réplique l'Injuste au Juste quil'injurie dans les N'uées d'Aristophane, ce qui est l'équivalent de " tu me dis des choses agréables »)

Ici le mot rose est pris dans un sens métaphorique; on le retroure avec sa signification ordinaire dans plusieurs locutions proverbiales tirées de la nature et des qualités de cette fleur ou de l'arbuste qui la porte. Ainsi dans Théocrite :

Il ne faut pas comparer aux roses la fleur de l’églantier ou les anémones ${ }^{3}$,

pour indiquer qu'on ne doit pas mettre en parallèle des choses de valeur inégale.

L'oignon ne produit ni roses, ni hyacinthes ${ }^{6}$,

1. Nemo discit ut, si necesse fuerit, aequo animo in rosa jaceat. Epistola XXXVI, 9.

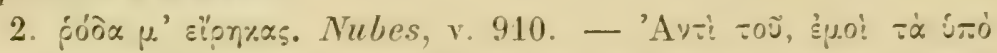

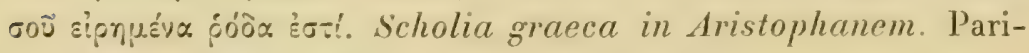
siis, 1843, in-8, p. 120.

3.

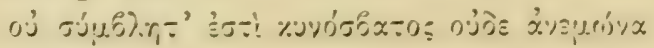

$\pi \dot{\alpha} \rho \dot{\rho} 0 \dot{\partial} \alpha$.

Idjellion V, v. 91-92.

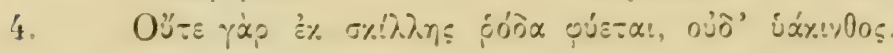

Gnomae. v. 537. 
LA ROSE DANS LES LÉGENDES ET DANS LA POÉSIE. 87 sentence de Théognis, qui a pour contre-partie cette pensée d'Ovide :

Souvent la rude épine produit de douces roses ${ }^{1}$.

Quand la rose est flétrie, on méprise l'épine ${ }^{2}$,

remarque encore le même poète.

Souvent, dit-il encore ${ }^{3}$, l'ortie croît près de la rose, sentence qui, nous le verrons, a été imitée dans la plupart des littératures modernes.

Tel ne trouve que des roses, a dit de son côté Pétrone ", tel autre des épines,

proverbe qu’il explique lui-mème, en remarquant qu'“ il n'est pas donné à tout le monde d'avoir ce qui plaît. )

1. Saepe creat molles aspera spina rosas.

Ex Ponto, lib. II, epist. 2, v. 34. Édition Lemaire; l'édition de Rod. Merkel ne donne pas ce vers.

2. Contemni spinam, cum cecidere rosae.

Fast. lib. V, v. 35'́.

3. urticae proxima saepe rosa est.

Remedia amoris, v. 46.

'́. non omnibus unum est quod placet, hic spinas colligit, ille rosas.

Petronii Arbitri Satirarum reliquixe, Berolini, 1862, in-8. Fragmenta, XXXV, p. 99. 


\section{CHAPITRE IV.}

USAGES DE LA ROSE CHEZ LES GRECS ET LES ROMAINS.

Si la rose occupe une place considérable dans la poésic des Grees et des Romains, elle en occupait une non moins grande dans leurs usages: "qui pourrait se faire sans la rose? ") dit le pseudo-Anacréon ${ }^{1}$; rien de plus vrai ; il n'est pas un acte de la vie des Anciens auruel cette fleur n'ait été associée; clle les accompagnait pendant leur existence tout entière et jusque au delà du tombeau. Sa beauté, son parfum, les propriétés qu'on lui attribuait, expliquent sans doute ce rôle immense de la rose dạns les usages, comme dans les légendes et la poésie des Grees et des Romains, il tient aussi à la place que, dès les temps les plus reculés, les fleurs prirent dans la vie de ces peuples; c'est sous forme de courounes qu'ils s'en servaient le plus souvent ${ }^{2}$.

1.

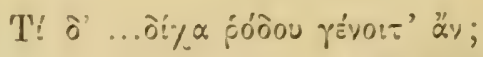

\section{Ode LIII (51), r. 19}

2. Voir sur l'emploi des couronnes chez les anciens le livre curieux de Carlo Pasquali (Paschalius) : Coronae, opus ... distinctum $X$ libris, quibus res omnis coronaria e priscorum eruta et collecta monumentis continetur. Parisiis, 1610, in-4 de 730 pages. 
On attribuait à Janus, preuve de leur haute antiquité, l’invention des couronnes '. Les plus anciens poètes lyriques de la Grèce du moins, Alcée, Sappho, Simonide, Anacréon, etc., en mentionnent l'emploi ${ }^{2}$. Aristophane parle, dans une de ses comédies ${ }^{3}$, de jeunes filles qui gagnaient leur vie en tressant des couromnes. Au siècle suivant, le poète Euboulos donna ia une de ses pieces le titre de "la Marchande de couronnes " ". La bouquetière Glycère, au rapport de Pline", inventa, vers la centieme Olympiade (380 av. J. C.), l'art de les varier par une heureuse combinaison de fleurs, qui en relevait la couleur et le parfum.

Les renseignements détaillés que tant d'écrivains, depuis Théophraste ${ }^{6}$ jusqu à Athénée ${ }^{7}$, ont donnés sur la composition des comronnes sont une preuve du prix qu'y attachaient les Grees; elles n'avaient pas moins d'importance aux yeux des Romains; mais l'emploi en était chez cux soumis à une règlementation sévere ${ }^{8}$ : ils en firent usage des les pre-

1. Athénée, Deipnosoph. lib. XV cap. 46 (692).

2. Athénée, Deipnosoph. lib. XV, cap. 11, 1't, 16.

3.

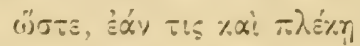

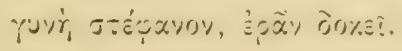

Thesmophoriazusae, v. 400-401.

4. Athénée, Deipnosoph. lib. XIII, cap. 6 (557).

5. Hist. natur. lib. XXI, cap. 3.

6. Hist. plantarum, lib. VIII, cap. 6.

7. Deipnosoph. lib. XV, cap. 8-17 (669-693). Il faut mentionner aussi Pollux, Onomasticon. lib. I, 10, et Lucien. Nigrinus, 32 et Ancecharsis, 9.

8. "Ingensque et hic severitas. " Hisl, natur. lib. XXI, cap. 6. 
miers temps de leur histoire. Caton l'ancien recommandait déjà de semer dans les jardins les plantes qui servaient à en tresser ${ }^{1}$. Les Romains, comme les Grecs, s'en servaient également d'ailleurs dans les cérémonies religieuses et dans les fètes profanes, an milieu de la joie des banquets ou du deuil des funérailles.

I.

Les couronnes figurèrent dès l'époque la plus ancienne dans le culte des Dieux ${ }^{2}$; ce fut à eux seuls, dit Pline", qu'on en offrit d'abord. Eux-mèmes avaient montré le prix qu'ils y attachaient; le poète Phérécyde disait que Saturne ${ }^{4}$ le premier avait porté une couronne. D'après Diodore, les Dieux auraient couronné de fleurs Jupiter, rainqueur des Titans". L'auteur des Cypriaques dépeint Vénus avec les Nymphes et les Grìces se tressant des couronnes de fleurs en chantant sur les sommets sourcilleux de

1. In hortis seri et coronamenta jussit Cato. Pline, lib. XXI, cap. 1 .

2. Coronac deorum honos erant. Plinc, lib. XXI, cap. 8.

3. Antiquitus nulla (corona), nisi Deo, dabatur. Hist. natur. lib. XVI, cap. 41.

1. Saturnum Pherecydes ante omnes refert coronatum, Iovem Diodorus post devictos Titanas (hoc munere a cacteris honoratum).

Tertulliani Liber de corona, cap. 7.

5. Athénée, Deipnosoph. lib. XV, cap. 31 (683). 
l'Ida ${ }^{1}$. Suivant Pline, c'est Bacchus qui, le premier de tous, se serait ccint le front d'une couronne de lierre ${ }^{2}$. Les couronnes aussi étaient agréables aux Dieux ; " ils se détournent, dit Sappho, de ceux qui n'en portent pas. » Et le poète Chérémion les appelait les " messagères des vœux " des mortels ${ }^{3}$; " les Prières, fait-il dire à l'un de ses personnages ", les placent devant les Dicux, comme expression de nos hommages $)$.

Les couronnes prenaient aussi place dans toutes les cérémonies religieuses. Les statues et les temples des Dieux en étaient ornés. Pausanias rapporte qu'il ne lui fut pas possible de distinguer la statue d'Ino dans le temple de cette déesse près de Thalamé, en Messénie ${ }^{5}$, tant elle était chargée de guirlandes. Les sacrificateurs ne se présentaient à l'autel que couronnés de fleur's ou de feuillages; les victimes clles-mèmes y étaient conduites parées de guirlandes ${ }^{6}$. Dans les fètes données en l'honneur de la fondation

1. "Ferunt primum omnium Liberum patrem imposuisse capiti ex hedera. "Hist. natur. lib. XVI, cap. 4.

2.

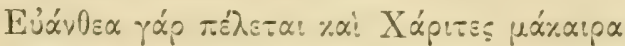

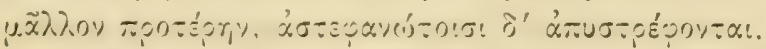

Athénée, op. laud., lib. XV, cap. 16 (674).

3.

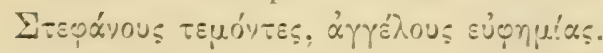

Dionysus, ap. Athénée, op. laud. lib. XV, cap. 19 (677).

4.

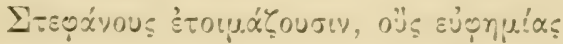

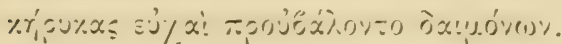

Centaurus, ap. Athénée, ibid.

5. Descriptio Craeciae, lib. III, cap. 26, 1.

6. "Deorum honori sacrificantes sumsere, victimis simul coronatis. " Pline, IIist. natur. lib. XVI, cap. '́. 
de Rome. le peuple tout entier portait des couronnes', comme on le voit par une note d'un calendrier du temps de Tibère. Parfois même on parait de fleurs, ainsi que nous l'apprend une inscription ${ }^{2}$, jusqu'aux places publiques.

Dans ce dernier exemple c'est de roses qu'il est question: ce sont elles aussi qui bientôt furent le plus employées dans les cérémonies du culte ${ }^{3}$. Elles le furent avant tout, on le comprend, dans celui qu'on rendait aux divinités dont cette fleur était l'attribut et l'apanage, comme Aphrodite ou Vénus et Bacchus. A Rome, d'après Ovide *, on offrait à Vénus les premieres roses du printemps. Le premier jour de la fète des Vinalia, célébrée aux calendes de mai, en l'homneur de cette déesse, les courtisanes lui offraient, avec des rameaux de myrte, des couronnes où “ le jonc s'entrelaçait à la rose ${ }^{5}$ ». Aux fites de Bacchus on portait aussi des couronnes de roses avec des guirlandes de pampre ou de lierre; et l'une des principales fètes des thiases diony-

1. Roma condita feriae coronatis omnibus. Bull. della Commis. Arch. mun an. IV, 1876, p. 16.

2. Nacellus rosa sumat. C. I. L. vol. I, p. 39'.

3. "Transiere deinde ad rosaria. " Pline, Hist. natur. lib. XXI, cap. 8.

4. Junc (Teneri) nova danda rosa est.

Frest. lib. IV, r. 138.

5. Cumque sua dominae date grala sisymbria myrto, Textaque composita juncea vincla rosa.

Ovide. Fast. lib. IV, r. 869-70.

Cf. L. Preller, Remische Wythologie 1881, 3e éd. v. I, p. '1'11. 
siaques de la région du Pangée, sous la domination romaine, portait le nom de Rosalia ${ }^{1}$.

Mais la rose ne figurait pas seulement dans le culte de Ténus et de Bacchus; elle prenait place aussi, Columelle le dit expressément ${ }^{2}$, dans celui de presque tous les autres Dieux. Aux fètes de Héra, a Argos, on comronnait de roses et de lis la statue de la déesse.

Ces roses couvertes de rosée et cette touffe de serpolet, chante Théocrite ", sont pour les Vierges de l'IIélicon ; à toi, Apollon Pythien, ces lauriers au sombre feuillage.

Lucrèce dépeint les adorateurs de Cybèle faisant tomber une pluie de roses sur la déesse et sur son cortege, lorsque, gage assure de salut, on promène, a travers les grandes citris, sa muette image'. L'auteur

1. Heuzey, Mission archéologique en Macédoine, etc. Paris, 1864-65, fol. p. 150, 152, 153.

2. Virgineas adoperta genas, rosa praebet honores

Coelitibus, templisque Sabaeum miscet odorem. De cullu hortorum, v. 261-62.

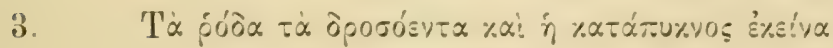

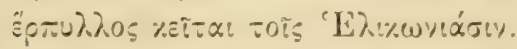

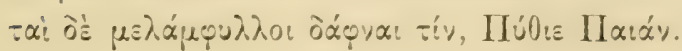

$$
\text { Epigram. I, r. 1-3. }
$$

4. Ergo cum primum, magnas invecta per urbes, Munificat tacita mortalis muta salute...

Largifica slipe ditantes, ninguntque rosarum Floribus. De natura rerum, lib. II, v. 624-28. 
de l'Aigretle rappelle dans ses ver's ${ }^{1}$ les roses dont il jonche le seuil des Nuses.

Dans les Mélumorphoses d'Apulée, Lucius parle des guirlandes de roses toutes fraîches qui ornaient la statue de la déesse Épone et qu'il s'efforça en vain d'atteindre 2. Aux fètes de la Déa Dia, déesse de la terre et des champs, identique avec Tellus et Cérès, dans la seconde moitié de mai, les frères Arvales faisaient sur son autel une offrande de pains couronnés de lauriers et de roses, et quand, après le festin, ils se séparaient en prononçant le mot de bon augure feliciler, les serviteurs enlevaient les restes du repas et les partageaient avec les roses entre les assistants ${ }^{3}$.

Les roses prenaient place en particulier dans les fêtes de Flore, la déesse qui les fait naître; parmi les réjouissances dont elles se composaient figurait une course où l'on portait des roses et dont la rapidité était comme l'emblème de la fragilité de ces fleurs charmantes ${ }^{4}$. Les roses servaient aussi sans doute à orner les lares domestiques et publies, qu'on parait, dans toutes les circonstances solen-

1. Sparsaque liminibus floret rosa. Ciris, v. 98.

2. Respicio pilae mediae... in ipso fere meditullio Eponae deae simulacrum residens aediculae, quod accurate corollis roseis et quidem recentibus fuerat ornatum. Hetamorph. lib. III.

3. Forcellini, Gloss. linguae latinae, s. v. arvalia. L. Preller, Remische Hythologie, vol. II, p. 33.

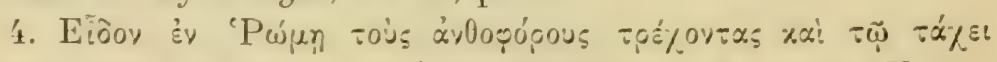

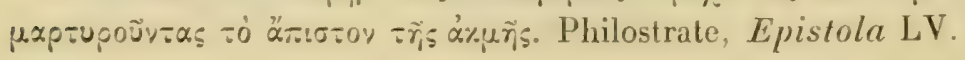


nelles, d'immenses couromnes de fleurs et de feuillage' ${ }^{1}$. Dans une de ses élégies, Tibulle nous montre l'habitant des champs ornant pieusement les siens de fleurs au retour du printemps ${ }^{2}$.

II.

Associées au culte des Dieux, les fleurs et surtout la rose le furent aussi aux fètes de l'Ilymen. Le jour des noces, les époux ${ }^{3}$, ainsi que tous ceux qui prenaient part à la cérémonie, portaient des couronnes ${ }^{4}$. Dans un tableau célèbre d'Aétion, décrit par Lucien, et qui représentait le mariage d'Alexandre et de Roxane, on voyait le prince macédonien offrant la couronne nuptiale à la fille de Darius". Plutarque décrit aussi le conquérant, le front ceint d'une couronne, présidant lui-mème au mariage de cent Macédoniens ou Grecs avec cent femmes Perses ${ }^{6}$. Sur le bas-relief d'un sarcophage étrusque ${ }^{7}$, on

1. Pline, Hist natur. lib. XXI, cap. 8. - Preller, Romische Mythologie, vol. II, p. 107

2. Rure puer verno primum de flore coronam Fecit et antiquis imposuil Laribus.

3.

$$
\text { Eleg. lib. II, 1, v. 59-60. }
$$

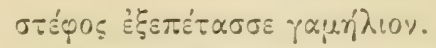

Bion, Epitaphium Adonidis, v. 88.

1. Em. Egger, art. Corona, dans le Dict. des antiquités grecques et latines, p. 1528, 1.

5. Iterodotus sive Aetion, 5. (Opera, XXI, éd. Didot, p. 243.)

6. Amatorius, cap. 26.

7. Monuments de l'Institut archéologique, 1863, pl. XIX. 
voit deux nouveaux époux, portés sur un char, et la tête couronnée de fleurs. Himère décrit les Amours ${ }^{1}$ tressant arec des roses cueillies dans les jardins d'A phrodite des couronnes dont ils parent la chambre nuptiale de Sérénos. Et Claudien², dans l'épithalame de Palladius et de Célérina, fait également répandre à pleines mains dans la chambre nuptiale une immense pluie de roses et des violettes cueillies elles aussi dans les jardins de Vénus.

C'est surtout comme embleme de l'amour que la rose prit ainsi place dans les fètes de l'llymen; elle était considérée à la fois comme un témoignage et un gage de tendresse et d'affection. Aussi, les amis en envoyaient à leurs amis, les amants en offraient à leur's maîtresses.

Portées sur leurs feuilles comme sur des ailes, écrit Philostrate à un ami ", ces roses se hâtent d'aller vers toi. Reçois-les avec faveur comme un souvenir d'Adonis, le coloris de V'énus ou les yeux de la terre. Une couronne d'olivier sied à un athlète, la tiare droite au grand roi, à un soldat le casque, la rose à un bel adolescent.

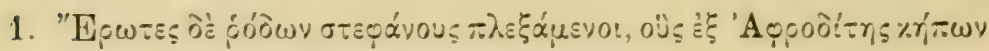

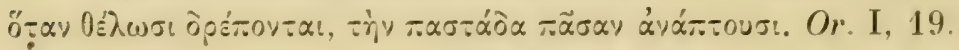

2.

Tum vere rubentes.

Desuper invertunt calathos, largosque rosarum

Imbres, et violas plenis sparsere pharetris

Collectas Veneris prato. v. 116-119. (Opera, XXXI).

3. Epistola I (29). Cf. Epist. II. "Je t'ai envoyé une couronne de roses, non pour te faire honneur, encore qu'il en soit ainsi, mais pour être agréable aux roses, en les empêchant de se faner. » 
Martial adresse de même à son ami Sabinus, comme le plus beau cadeau qu'il puisse lui offrir, une couronne de roses ${ }^{1}$, souhaitant seulement qu'il la croie faite des fleurs de son jardin. Ce sont des roses encore qu'il envoie une autre fois ceindre de leurs festons délicats le front de son cher Apollinaris $^{2}$. Et Properce, dans une de ses plus gracieuses élégies ${ }^{3}$, se représente enlevant de son front sa couronne de roses pour en ceindre pendant son sommeil les tempes de Cynthie.

C'est qu'il n'y avait pas d'offrande plus propre que les roses pour gagner l'affection; aussi les amants en déposaient-ils au seuil de leurs amantes, soit, dit Athénée ${ }^{4}$, pour leur faire honneur, en ornant de couronnes l'entrée de leur demeure, comme si c'eût été celle d'un dieu; soit qu'ils en fissent hommage, moins à leur's maîtresses qu'à l'Amour lui-même, dont elles représentaient la divinité et dont leur habitation était comme le temple. Et le grammairien grec cite un passage de Lycophronide où ce poète montre un chevrier "ípris d'une bergère

1. Epigr. lib. IX, 61. Gf. pl. haut p. 33.

2. I felix rosa, mollibusque sertis Nostri cinge comas Appollinaris.

$$
\text { Epigr. lib. VII, 89, v. 1-2. }
$$

3. $\quad$ solvebam nostra de fronte corollas,

Ponebamque tuis, Cynthia, temporibus.

$$
\text { Eleg. lib. I, 3, v. 21-22. }
$$

4. Deipnosoph. lib. XV, cap. $9(670)$.

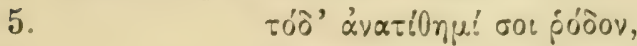

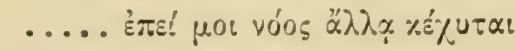

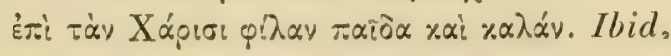

JORET. La Rose. 
"belle et chìre aux Grâces ), consacrant des roses devant sa demeure.

De Griee eet usage symbolique se répandit dans l'empire romain. Luerece peint ${ }^{1}$ l'adorateur rebuté inondant le seuil de son amante inflexible de larmes et de fleurs, de guirlandes et de parfums, et imprimant de lanentables baisers à sa porte orgueilleuse. Tibulle rappelle à sa maîtresse ${ }^{2}$ ses longues supplications et les couronnes de fleur's qu'il a déposées au seuil de sa demeure. Dans l'Arl d'aimer, Ovide donne à l'amant, qui a soupiré en vain à la porte de sa maitresse, le conseil de déposer sur le seuil, moyen assuré de la fléchir, les roses qui lui ornent le front.

Les amants se paraient aussi de roses, comme d'un embleme de plaisir dans leur's rencontres:

"Quel tendre adolescent, s’écrie Horace ${ }^{4}$, te presse,

1. At lacrumans exclusus amator limina saepe Floribus et sertis operit, posteisque superbos Unguit amaricino et foribus miser oscula figit.

De natura rerum, lib. IV, v. 1173-75.

2. Te meminisse decet quae plurima roce peregi

Supplice, cum posti florea serta darem.

$$
\text { Eleg. lib. I, 2, v. 13-14. }
$$

Tibulle, comme Lucrèce, ne parle que de fleurs, mais la citation d'Ovide montre que l'un et l'autre avaient évidemment en vue la rose.

3. Capiti demptas in fore pone rosas.

Art. amat. lib. II, v. 528.

4. Quis multa gracilis te puer in rosa 
ô Pyrrha, couvert de parfums et de roses, sous cette grotte voluptueuse?"

Palestre, dans l'Ane de Lucien ${ }^{1}$, s'empresse, en attendant Loukios, de courrir sa couche de fleurs et de feuilles, ainsi que de guirlandes de roses. Et dans Apulée ${ }^{2}$, quand Fotis va retrouver son cher Lucius, elle porte des guirlandes de roses dans les mains, une rose détachée sur le sein, elle l'enlace de ses couronnes et le couvie de fleurs.

\section{III.}

Non moins que dans les fètes de l'IIymen et de l'Amour, la rose était associée à la joie des banquets, comme elle figurait dans le culie du dieu qui y présidait. C'était sous forme de couronnes qu'elle était alors le plus souvent employée. De nombreuses espèces de fleurs entraient dans la composition des couronnes qu'on portait dans les festins; Théophraste $^{3}$ et Athénée $^{4}$ citent entre autres le lis, le

\section{Perfusus liquidis urget odoribus}

Grato, Pyrrha, sub antro?

$$
\text { Carm. lib. I, 5, v. 1-4. }
$$

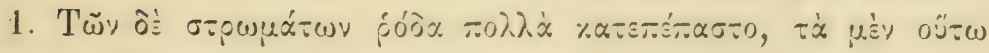

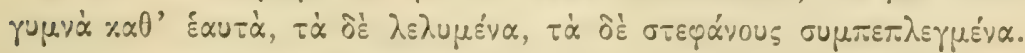
Lucius sive Asinus, cap. VII.

2. Fotis mea... proximat rosa serta el rosa soluta in sinu tuberante... Et corollis revinctis ac flore persperso. Hetamorph. lib. II.

3. Hist. plantarum, lib. VI, cap. 6 .

4. Deipnosoph. lib. XV, cap. 27 (680). 
faux-narcisse, la violette, l'anémone, l'asphodèle, l'hyacinthe, l'ache, ainsi que la menthe sauvage, le serpolet et la marjolaine. Pollux ${ }^{1}$ mentionne aussi le crocus et le lotus; mais, ainsi que Pline ${ }^{2}$, il met la rose au premier rang des fleurs ì couronnes.

La coutume de porter des couronnes dans les festins remonte à une époque reculée; en Grèce on la connaissait bien arant la guerre du Péloponèse. A Rome, on se servait déjà de couronnes au temps de la seconde guerre punique; mais on ne pouvait avec elles se montrer en public. Pline raconte ${ }^{3}$ que le banquier Lucius Fulvius, qui vivait à cette époque, accusé d'avoir, pendant le jour, regardé de son balcon dans le forum, en ayant une couronne de roses sur la tête, fut jeté en prison par ordre du sénat. Cette sévérité ne devait pas durer, et le relîchement des mœurs contribua rapidement à répandre l'usage des couronnes dans la vie ordinaire. A Athènes, on vit de jeunes voluptueux en porter en plein jour et se rendre avec elles dans les écoles des philosophes ${ }^{4}$. On trouve à chaque instant, chez les poètes anacréontiques de la Grèce et le Rome, la preuve de ce luxe croissant des couronnes dans les banquets et de la place nécessaire qu'y occupait la reine des fleurs.

Associons à Bacchus la rose dédiée aux Amours, dit

1. Onomasticon, lib. I, cap. 10.

2. Hist. natur. lib. XXI, cap. 10 (4) et 11 (5).

3. Hist. natur. lib. XXI, cap. 6.

4. Pline, Hist. natur. lib. XXI, cap. 6. 
LA ROSE CHEZ LES GRECS ET LES ROMAINS. 101

le pseudo-Anacréon ${ }^{1}$; buvons gaiement le front ceint des belles fleurs de la rose. »

\section{Et ailleurs ${ }^{2}$ :}

"Les tempes ceintes de couronnes de roses, enivronsnous gaiement. »

"Qu'il me serait doux, s'écrie Properce ${ }^{3}$, à son tour, d'enchainer mes esprits dans les flots de la liqueur de Bacchus et d'avoir tout le jour mon front demi-caché sous les roses du printemps. ")

Horace associe sans cesse dans ses vers la rose à ses joies et à ses plaisirs. Malgré la simplicité qu'il affecte parfois ", il ne veut pas que cette fleur manque à ses festins ${ }^{5}$. Que son ami Dellius fasse apporter " à l'ombre hospitalière d'un pin élancé et d'un

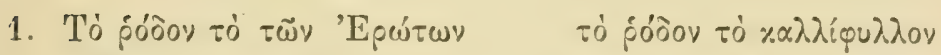

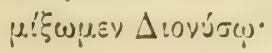

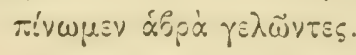

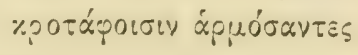

Ode XLII (5), v. 1-8.

2

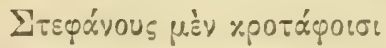

Sostivous surapurícavtes

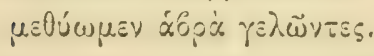

Ode XLI (6), v. 1-3.

3. Me juvat et multo mentem vincire Lyaeo

Et caput in verna semper habere rosa.

Elegiae, lib. III, 5, v. 21-22.

‘. Persicas odi, puer, apparatus.

5. Neu desint epulis rosae.

Carm. lib. I, 32, v. 1.

Carm. lib. I, 30, v. 15. 
pâle peuplier, près du lit sinueux d'une onde murmurante, du vin, des parfums et des roses sitôt flétries $^{1}$. »

"Pourquoi, lit-on dans une autre ode", les cheveux parfumés par la rose ou le nard d'Assyrie, ne buvonsnous pas nonchalamment couchés à l'ombre d'un haut platane ou d'un pin?

Dans la Cabaretière, cette piquante pièce de vers, qu'on a attribuée à Virgile, mais qui, si elle en rappelle le ton, n'en a point la sévère inspiration, le poc̀te énumérant tont ce qui, dans sa taverne, peut attirer les bureurs, place des roses à côté des coupes et des flutes ${ }^{3}$. Et quand, sous le nom de l'avenante hòtesse, il invite le passant à reposer à l'ombre des pampres ses nembres lassés, il l'engage avant tout ${ }^{*}$ a

1. Qua pinus ingens, albaque populus

Umbram hospitalem consociare amant

Ramis, et obliquo laborat

Lympha fugax trepidare rivo ;

Huc vina, et unguenta, et nimium breves

Flores amoenos ferre jube rosae.

Carm. lib. II, 3, v. 9-1'x.

Cur non sub alta vel platano vel hac

Pinu jacentes sic temere, et rosa

Canos odorati capillos,

Dum licet, Assyriaque nardo

Potamus uncti? Carm. lib. II, 11, v. 13-17

3. Sunt cupae, calices, cyathi, rosa, tibia, chordae.

$$
\text { Copa, v. } 7 .
$$

1. Eia age pampineo fessus requiesce sub umbra ;

Et gravidum roseo necte caput strophio. v. 31-32. 
nouer sur sa tète appesantic une couronne de roses.

Comme Horace, Martial représente son ami Flaccus ${ }^{1}$ couché sur un gazon émaillé de fleurs, près d'un ruisseau limpide, buvant, loin des fìcheux et le front couronné des fleurs empourprées de la rose, son vin frappé de glace. Une seule couronne, ni une couronne ordinaire ne suffit même plus à l'adulateur de Domitien, il lui en faut plusieurs et qui soient faites de pétales de roses cousus ensemble (sutiles).

Que dix fois, dit-il à son favori Calocissus ${ }^{2}$, les pétales assemblés de la rose serrent nos tempes, autant de fois que le nom de celui qui a élevé un temple à son illustre famille compte de lettres!

Si, par adulation, Martial cxagère ici, il n'en est pas moins rrai que l'usage de porter plusieurs couronnes, et des couronnes faites de pétales cousus ensemble, était connu depuis longtemps. Ovide fait allusion à ces dernières, Pline en parle aussi ${ }^{3}$. Anacréon recommande déjà aux convives d'avoir

1. Sic in gramine floreo reclinis,

Qua gemmantibus hinc et inde rivis

Curva calculus excitatur unda,

Exclusis procul omnibus molestis,

Perfundas glaciem triente nigro,

Frontem sutilibus ruber coronis.

$$
\text { Epigr. lib. IX, 91, v. 1-6. }
$$

2. Sutilis aptetur decies rosa crinibus, ut sit

Qui posuit sacrae nobile gentis opus.

$$
\text { Epigr. lib. IX, 9', v. 5-6. }
$$

3. Hist. natur. lib. XXI, cap. 8. 
trois couronnes, deux de roses, la troisieme naturatite, e'est-a-dire de myrte ${ }^{1}$. Le plus sourent on en portait deux, ordinairement de composition différente, l'une autour du front, la seconde, l'hypothémis, autour du cou. Les couronnes ne se portaient pas d'ailleurs pendant toute la durée du festin; c'était vers la fin du repas, quand on servait les vins fins, qu'on les présentait aux convives avec les parfums ${ }^{2}$; elles étaient ainsi les compagnes de l'ivresse; “ la rose régnait, suivant l'expression de Martial", lorsque Bacchus était dans toute son effervescence »).

Les convives, d'ailleurs, n'avaient pas seuls des couronnes, les esclares aussi en portaient; l'on en ornait jusqu'aux mets et aux coupes, en même temps que les murs de la salle du banquet étaient garnis de guirlandes de feuillage ou de roses. On ne s'en tint pas là; on alla jusqu'à répandre des roses effeuillées (rosae solutac; sur la table et même sur le pavé de la salle du banquet. Dans la description brillante et gracieuse qu'il a faite des festins qui accompagnaient les fètes de Flore*, Ovide n'a oublié

1.

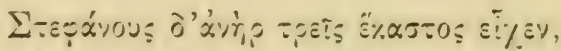

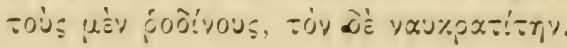

$$
\begin{aligned}
& \text { Fragm. XV. Ed. Gail. }
\end{aligned}
$$

2. Athénée, Deipnosoph., lib. XV, cap. 8 (669).

$3 . \quad$ cum furit Lyaeus,

Cum regnat rosa, cum madent capilli.

$$
\text { Epigr. lib. X, 19, v. 20-21. }
$$

4. Tempora sutilibus cinguntur tota coronis :

Et latel injecta splendida mensa rosa.

Fast. lib. V, v. 335-36. 
ni les couronnes faites de pétales cousus, ni la " pluie de roses sous laquelle les tables disparaissent".

Ce luxe des roses, auquel le contemporain d'Auguste fait ici allusion, comme de date ancienne, existait en effet bien avant lui et devait encore grandir après. Depuis longtemps les voluptueux de Rome avaient, comme ceux de la Grèce, pris l'habitude de vivre au milieu des roses. On raconte que Denys le jeune, alors retiré à Locres, fit joncher son palais de thym et de roses, et on le vit mème se vautrer au milieu de ces fleurs ${ }^{1}$. Dans un passage célèbre d'un de ses plus éloquents discours, Cicéron a flétri le raffinement que Verrès porta dans l'usage des roses:

Au printemps, dit-il ${ }^{2}$, et son printemps à lui ne datait pas du retour des zéphyrs ou de l'entrée du soleil dans tel ou tel signe, il ne croyait l'hiver fini que lorsqu'il avait vu des roses; alors il se mettait en marche. A l'exemple des anciens rois de Bithynie, mollement étendu dans une litière à huit porteurs, il s'appuyait sur un coussin d'étoffe transparente et tout rempli de roses de Malte. Une couronne de roses ceignait sa tête, une guirlande serpentait autour de son cou; il tẹnait à la main un réseau du tissu le plus fin, à mailles serrées et plein de roses, dont il ne cessait de respirer le parfum.

Le goût et l'emploi des roses ne pouvait qu'augmenter et se dépraver au milieu de la corruption dont les empereurs romains donnèrent si souvent

1. Athénée, Deipnosoph. lib. VII, cap. 58 (541).

2. InVerrem, V, cap. 10 et 11 . Trad. Nisard. 
l'exemple. Spartien rapporte ${ }^{1}$ qu'Elius Vérus avait fait faire un lit formé de quatre gros coussins, entouré de toutes parts d'un léger réseau et rempli de roses dont on avait enlevé l'onglet; il s'y couchait couvert d'un voile tissu de fleurs de lis et parfumé des plus suaves odeurs de la Perse. Héliogabale faisait parsemer ses salles à manger, ses lits et les portiques où il se promenait de roses, de lis et de violettes, d'hyacinthes, de narcisses et de toutes sortes de fleurs ${ }^{2}$. On a reproché au premier Gallien de " construire au printemps des chambres à coucher avec des roses ${ }^{3}$ ", et Carin, dit-on ${ }^{4}$, ne prenait ses repas que sur des lits garnis de roses de Vilan. L'invasion des Barbares et les progrès du christianisme devaient seuls mettre un terme à cette dépravation dans le luxe des roses, ainsi qu'à ce luxe lui-mème.

\section{IV.}

Mais toute profanée que fut ainsi parfois la rose, cette fleur divine n'en conserva pas moins toujours quelques-unes de ses plus hautes attributions. C'est ainsi qu'elle continua à servir de récompense aux vivants et à honorer les morts. On donnait une couronne de roses au vainqueur du dithyrambe dans

1. Aelius Verus, cap. 5. (Historia Augusta.)

2. Lampride, Antoninus Heliogabalus, cap. 19. (Ibid.)

3. Trebellius Pollion, Gallieni duo, cap. 16. (It)id.).

4. Flavius Vopiscus, Carinus, cap. 17. (Ibid.) 
les fètes de Bacchus au printemps ${ }^{1}$. Les soldats victorieux s'en paraient en revenant du combat; on en attachait des guirlandes à la proue du vaisseau qui rentrait heureusement dans le port ${ }^{2}$. On jetait aussi des roses sur le char ${ }^{3}$ et sous les pas des grands. Lorsque, quarante jours après sa victoire, Vitellius alla visiter le champ de bataille de Bédriac encore couvert des cadavres mutilés et en putréfaction des soldats d'Othon ${ }^{4}$, les habitants de Crémone, par une honteuse et horrible flatterie, jonchèrent de branches de laurier et de roses le chemin qu'il devait parcourir.

Mais la rose servait surtout à honorer la mémoire des morts. Elle prenait place au premier rang parmi les fleurs dont on entourait les monuments funèbres:

Grimpe doucement, ô lierre, sur le tombeau de Sophocle, s'écrie Simmias ${ }^{\circ}$, et que tout autour s'y épanouisse la fleur de la rose.

1. Lenormant, art. Bacchus, dans le Dictionnnaire des Antiquités.

2. Jam mea votiva puppis redimita corona.

Ovide, Amorum lib. III, 11, v. 29.

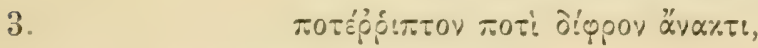

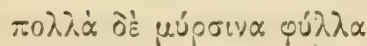

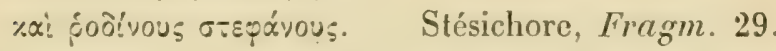

4. Intra quadragesimum pugnae diem lacera corpora, trunci artus, putres virorum formae, ...nec minus inhumana pars viae (fuam Cremonenses lauro rosisque constraverant. Tacite, Histor. lib. II, cap. 70 .

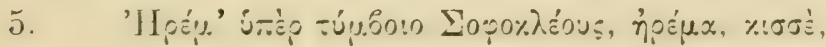

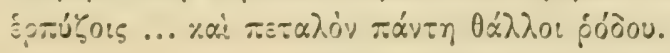
Anthol. palatina, cap. VII, no 22. 
Que les fleurs abondent sur ton tombeau nouvellement construit, dit aussi un autre poète ${ }^{1}$, non la ronce hirsute ni le stérile égilops, mais les violettes, la marjolaine, le tendre narcisse, et que les roses t'environnent en foule, ô Vibius!

Et autour du monument que la reconnaissance du pâtre, dans la pièce attribuće à Virgile, élève au moucheron qui l'a sauvé, entre les diverses plantes ou arbustes qu'y a mis le poète ${ }^{2}$, se voient au premier rang "les rosiers aux fleurs purpurines ».

La rose figurait aussi aux funérailles dans les " couronnes de fleurs de la saison ), dont on parait le corps ${ }^{3}$ et la tombe ${ }^{4}$ du défunt ou qu'on répandait sur ses restes. Mais c'était à la fète des Parentalia, que chaque année on célébrait, au mois de mai ou de juin, en l'honneur des morts, qu'elle jouait un rôle tout particulier." Dans le repas, dont cette fète se composait - les escae rosales ${ }^{6}$, - on distribuait

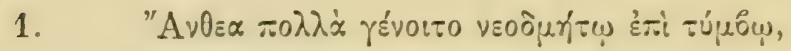

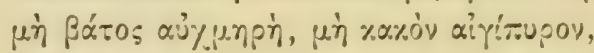

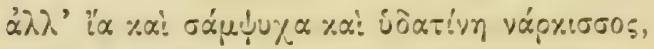

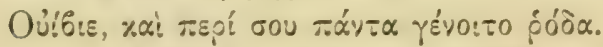

2. Anthol. palatina, vol. III, cap. II, no 238.

hic et acanthus

Et rosa purpureo crescit rubicunda colore.

Culex, v. 397-98.

3. Pline, Hist natur. lib. XXI, cap. 8. - Alciphron, Epist. lib. I, 36. Gf. pl. haut, p. 69.

4. Lucien, De luctu, 11. Cf. pl. haut, p. 69.

5. Virgile, Aeneidos lib. VI, v. 883 . Cf. plus haut, p. 68.

6. Marini, Gli atti e monumenti de' fratelli Arvali. Roma, 1795, in-4. Vol. II, p. 581. 
des roses entre les convives, puis on déposait des guirlandes sur le tombeau de celui dont on renait

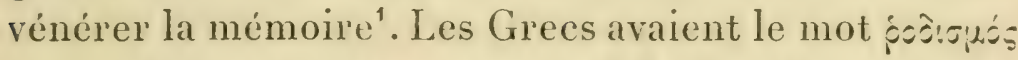
pour désigner cette cérémonie ${ }^{2}$; les Romains lui donnaient le nom de rosatio ${ }^{3}$, et la fète elle-même, ainsi que le jour où on la célébrait, portait celui de Rosalia ${ }^{4}$ ou même de Rosaria ${ }^{5}$. Les vers où Tibulle espère qu'une main amie, en souvenir d'une ancienne affection, ornera chaque année de couromnes son monument ${ }^{6}$, nous montrent cette coutume établie à Rome au premier siècle de notre ère, et les nombreuses inscriptions qui la constatent témoignent du prix qu'on attachait à cette cérémonie funèbre et du soin qu'on prenait pour en assurer l'exécution.

C'est ainsi qu'on voit ${ }^{7} \mathrm{Q}$. Titius Severus d'Adria,

1. Joach. Marquardt, Remische Staatsverwaltung, vol. III, p. 299. (Handbuch der romischen Alterthümer, Leipzig, 1878 , vol. VI.)

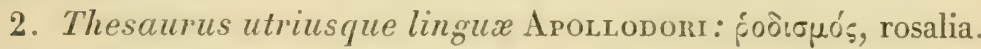

3. "Rosatio est rosarum sparsio. 》 Forcellini, Glossarium totius latinatis, s. v.

4. " Rosalia sunt solemnia rosarum seu dies, quo rosae in sepulcra ferebantur. » Forcellini, s. v.

5. Forcellini, Glossarium, s. v.

6. Atque aliquis senior, veteres veneratus amores, Annua constructo serta dabit tumulo.

7. D. M.

Q. TITIO. SERTORI ANO Q. TITIVS. SEVE RVS. FILIVS. QVI. ET

$$
\text { Eleg. lib. II, 4, v. 47-48. }
$$

COL. NAVT. M A DEDIT

$\overline{\mathrm{SN}}$ CCCC AD ROSAS ET

ESCAS DVCENDAS EI

OMNIBVS ANNIS.

C. I. L., t. V, 1, 2315 . 
fils de Q. Titius Sertorianus, léguer 400 sesterces au college des bateliers de cette ville pour offrir chaque année sur le tombeau de son père des roses et un repas funèbre. De même Claudia Severa, en souvenir de son mari " très cher », L. Magius Magianus, de son fils " picux " Cornelius Valerius, de son beau-père Magius Pricianus et d'elle-même, lègue au collège des bateliers de Brixia 60,000 sesterces pour répandre chaque année des roses sur leur tombeau ${ }^{1}$.

Dans une inscription de Ravenne souvent citée ${ }^{2}$, on voit également Titus Germanicus, fils de Drusus, "César, Auguste, » léguer, en souvenir de son père,

1. I. MAG. MAGIANO

CL. SEVERA. MARITO

KARISSIMO. ET, CORN.

VALERIO. FIL. PIENTIS

SIMO. ET. MAG. PRISCI

NIANO. SOCERO, B. M.
ET. SIBI. ET. IN. MEMO

RIAM. EOR. ET. SVI. COLI.

N. B. AD. ROSAS. ET. PRO

FVSIONES. Q. A. FAC

HS. N. $\overline{\mathrm{LX}}$. MIL. DEDIT.

C. I. L., t. V, 1, 4990 .

L'inscription $n^{\prime}\{017$ d'Aretica renferme un legs semblable.

2. TI. Clavdivs. DRVSI. F. CAES. AVG.

GERM. PONT. MAX. TRIB. POT. II.

COS. DESIG. III. IMP. III, P. P. DEDIT.

OB. MEMORIAM. PATRIS. SVI, DEC, VII.

COLLEGI. FABRVM. M. R. H. -S. CD. N.

DONAVIT. SVB. HAC. CONDITIONE. VT.

QVOTANNIS. ROSAS. AD. MONVMENTUM.

EIVS DEFERANT. ET. EPULENTUR.

DVMTAXAT. IN. V. ID. JVLIAS.

QVOD SI NEGLEXERINT. TVNC. AD VIII.

EIVSDEM. COLLEGII. PERTINERE DEBEBIT.

CONDITIONE, SVPRA. DICTA.

Orelli, Inscr. latin. selectarum amplissima collectio. Turici, 1828, in-8, vol. I, p. 175. 
LA ROSE CHEZ LES GRECS ET LES ROMAINS. 111

au septième collège des orfèvres, une somme de 1,000 sesterces en présent, à la condition d'offrir charque année, le 5 des ides de juillet, des roses sur son monument, et d'y fare un repas funebre, ajoutant que s’ils négligeaient de s'y conformer, cette somme devait, aux mêmes conditions, appartenir au huitième collège.

Le président d'Orbessan parle' d'une inscription de Torcello, dans laquelle ${ }^{2}$ un affranchi, Longius Patroclus, par un sentiment de pieuse affection, avait, de son vivant, légué au collège des Centum, une maison et les jardins qui y étaient attenants, a fin que leur revenu servît à offrir sur le tombeau de son patron et un jour aussi sur le sien, des roses ct un repas funèbres. Une inscription du Montferrat ${ }^{3}$

1. Essai sur les roses, p. 331 .

2.

$\begin{array}{cc}\text { L. OGIVS } & \text { SEPVLT A IVNCTOS } \\ \text { PATROCLVS } & \text { VIVOS DONAVIT VT } \\ \text { SECVTVS } & \text { EX REDITV EOR LAR } \\ \text { PIETATEM } & \text { GIVS ROSE. ET. ESCE } \\ \text { COL. CENT. } & \text { PATRONO SVO ET } \\ \text { HORTOS CVM } & \text { QVANDOQVE SIBI } \\ \text { AEDIFICIO HVIC } & \text { PONERENTVR. }\end{array}$

3. V. F.

$\mathrm{T}$. VETTIVS

C. I. L., t. V, 1, no 2176 .

T . L. HERMES

SEPLASIARIVS.

MATER GENVIT

MATERQ. RECEPIT

HI. HORTI. ITA. VTI. O. M.

QVE. SVNT. CINERIBVS

RATORES, SVBSTITVAM VTI. VESCANTVR. EX. HO RVM. HORTORVM. REDI TV NATALE. MEO ET. PER ROSAM. IN. PERPETVO HOS HORTOS NEQVE DITI DI VOLO NEQ ABALIENARI. SERVITE MEIS NAM. CV C. I. L., t. V, 2, no 7454. 
nous montre un parfumeur, nommé L. Vettius Hermès, laissant des jardins " excellents et vastes " - il défendait de les diviser et de les aliéner pour l'entretien de ses cendres. Des curateurs, auxquels il léguait pour vivre le revenu de ces jardins, deraient, le jour de sa naissance, faire à perpétuité une offrande de roses sur son tombeau. On connaît aussi une inscription grecque ${ }^{1}$, où un certain Longus laisse au sénat une somme d'argent pour offrir des roses (joz!"scoxı) sur son monument.

On roit, par ces exemples, qu'il eût été facile de multiplier, quelle place occupaient dans les honneurs funèbres les offrandes de roses, et combien on tenait à ce qu'elles fussent faites avec soin et au jour marqué. On y attachait une telle importance que ceux qui n'étaient pas assez riches pour laisser des legs analogues à ceux dont il vient d'ètre question faisaient, dit-on ${ }^{2}$, graver sur la pierre qui recouvrait leurs cendres une inscription par laquelle ils priaient les passants de répandre des roses sur leur tombeau, tant l'hommage de ces fleurs symboliques était regardé comme un deroir indispensable à la mémoire des morts.

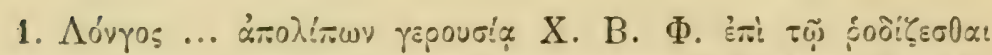
a'joóy. Corpus inscriptionum graccarum, éd. Aug. Bœckh. Berlin, 1848, in-fol. vol. II, p. 960.

2. D'Orbessan (Essai sur les roses, p. 312) cite une inscription de ce genre commençant par les mots :

Sparge, precor, rosas, supra mea busta, viator; mais il ne dit pas où il l'a remarquée. 


\section{V.}

Comme dans les usages de la vie et dans la poésie, la rose avait pris place dans les œurres d'art. On la rencontre surtout, cela s'explique, dans les représentations des divinités, à qui elle servait d'attribut, ainsi que dans celles des cérémonies religieuses ou des événements de la vie auxquels elle était associée. C'est ainsi que sur un sarcophage de la villa Albani ${ }^{1}$, qui représente les noces de Pélée et de Thétis, parmi les divinités qui viennent offrir des présents aux nouveaux époux, on voit l'Été apportant une immense guirlande de roses. Dans une fresque de Pompéi ${ }^{2}$, dont une des figures principales paraît être l'Arcadie, celle-ci porte une couronne où se trouvent deux roses parfaitement doubles. Un des monuments publiés par Édonard Gerhard ${ }^{3}$ nous montre aussi une déesse que l'on reconnaît pour Flore à la couronne de fleurs et peut-être de roses qui lui ceint le front. Sur un autre monument ${ }^{4}$ de la même collection, on voit une tête de femme également couronnée de roses.

1. Winckelmann, Monumenti antichi inediti spiegati ed illustrati. Roma, 1767, in-fol., vol. II, p. 151 et vol. I, pl. 111.

2. W. Zahn, Die schönsten Ornamente und Gemälde aus IIerculanum, Pompeii in Italien. Berlin, 1828-'15, in-fol. Vol. III, pl. 1.

3. Antike Bildwerke, München, 1827, in-fol. Tafel LXXXVII, 7.

4. Tafel CCGIII, 11. Text, p. 392.

JoRet. La Rose. 
C'est en particulier dans les scènes funéraires et nuptiales, souvent réunies dans une mème représentation, que l'on trouve des personnages avec des couronnes ou des guirlandes de roses. Ainsi le basrelief d'un sarcophage, encastré aujourd'hui dans le mur du Belvédère, représente à la fois une scène de mariage et une chambre funéraire, et l'un des deux génies qui figurent dans la cérémonie nuptiale semble porter une couronne de roses, tandis que le front de l'autre est ceint d'une branche de laurier ${ }^{1}$. De même parmi les génies bachiques du bas-relief d'un autre sarcophage, qui est aussi à Rome, on en voit un près de défaillir, poriant au cou une couronne funéraire formée de roses ${ }^{2}$. Sur le fragment d'une urne sépulcrale reproduite par Winckelmann, se trouve également un génie portant une guirlande de roses doubles ${ }^{3}$.

Mais ce n'est pas seulement sur les monuments et sous forme de couronne qu'on rencontre les roses; ces fleurs gracieuses araient pris place aussi, on le comprend sans peine, dans la décoration des palais, et même des maisons particulières. C'est ainsi qu'on les trouve sur diverses fresques et dans une mosaïque de Pompéi; elles y sont presque toujours repré-

1. Antike Bildwerke, Tafel LXXV, 2. Text, p. 314.

2. Antike Bildwerke, Tafel XCII, 2.

3. Honumenti antichi, vol. II, p. 265 et vol. I, pl. 203. Il est difficile de ne pas reconnaitre aussi une rose double au milieu des fleurs de lotus et, je crois, d'olivier, dont est composée la couronne que tient à la main l'Antinoüs colossal de la pl. 180 . 
sentées, suivant le Dr. Orazio Comes ${ }^{1}$, en bouton ou les pétales non encore développés, mais de couleur vermeille. Il en est de mème dans certaines fresques d'ITerculanum. Les fleurs rouges en bouton ou à moitié ouvertes, qu'on voit aux angles des losanges décoratifs des planches LIII, LV, et LVI du quatrième volume des Peintures antiques de cette ville $^{2}$, me paraissent, bien que les deux feuilles qui les accompagnent soient simples, être des roses.

Les roses figurent aussi, avec les autres fleurs cultivées à l'époque des Césars, dans les peintures représentant des jardins d'agrément, peintures qui décoraient souvent, ainsi que celles de paysages héroïques ou de convention, les murs des anciens palais de Rome ou des villas de la Campanie. C'est ce qu'on voit dans les belles fresques qui remplissent les quatre murs d'une vaste salle de la villa de Livie ad Gallinas, à la Prima Porta prés de Rome, et représentent, avec autant de réalisme que de ficlélité, un magnifique jardin de plaisance. Derrière le treillis qui borne le gazon situé au premier plan, s'étend une espèce de massif de plantes en fleurs, parmi lesquelles on reconnaît entre autres des rosiers et des grenadiers ${ }^{3}$.

On rencontre encore la rose en particulier sur les

1. Dr Orazio Comes, Illustrazione delle piante rappresentate nei dipinti Pompeiani. Napoli, 1879, in-4, p. 65.

2. Pitture antiche d'Ercolano. Napoli, 1757-95, in-fol.

3. Karl Woermann, Die Landschaft in der Kunst der alten Völker. München, 1876, in-8 , p. 331. 
monnaies des villes, dans l'histoire desquelles cette fleur occupe une place ou joue un rôle quelconque. On a sourent répété (qu'une rose figurait d'ordinaire sur les monnaies de l'île de Rhodes, ce que pourtant Spanheim avait mis en doute ${ }^{1}$; mais Mionnet n'en mentionne qu'une ${ }^{2}$ où il soit possible de reconnaître à peu près une rose; sur toutes les autres on distingue clairement le balaustion ou fleur du grenadier, la seule d'ailleurs que Spanheim y avait reconnue, la seule aussi que Victor Guérin, dans son Histoire de Rhodes ${ }^{3}$, dit expressément avoir vue, au lieu de la rose, sur les monnaies de cette île. Mais Mionnet indique cette fleur sur les monnaies de Rhoda, colonie de Rhodes en Catalogne, ainsi que sur celles de Tragilus ${ }^{*}$. Rasche la signale également sur les monnaies de Cyr'ène, d'Antissa dans l'île de Lesbos, de Béotie, de Naples, ainsi qu'un rosier sur celles de Pestum". Sur les statères d'argent de Nagidos, on voyait aussi, devant une Vénus assise, un rosier couvert de fleurs et de boutons ${ }^{6}$. Au con-

1. De praest. et usu numismatum antiquorum, vol. I, p. 315.

2. Description des médailles antiques grecques et romaines. Vol. VI, p. 589, no 180. "Rose ou fleur de balaustium, 》 dit-il.

3. Paris, in-12, $2^{e}$ éd. 1880, p. 47 et 61.

4. Op.laud, vol. I, 48 et 507.

5. Lexicon universale rei nummariae veterum praecipue Graecorum ac Romanorum. Lipsiae, 1789, in-8. Vol. v. IV, pars p. 1279.

6. Imhoof-Blumer und Otto Keller, Tier-und Pfanzenbilder auf Munzen und Ciemmen des Klassischen Altertums. Leipzig, 1889, in-8, 4, p. 62. Tafel X, 24. 
traire, sur une monnaie d'Antiochus VIII, roi de Syrie, où M. Ernest Babelon a cru reconnaître " une rose avec deux boutons sur sa tige ${ }^{1}$ ), il m'a été impossible de voir autre chose qu'une fleur de grenadier.

\section{VI.}

Si la rose n'occupe qu'une place secondaire, vu son importance, dans les auvres de l'art hellénique, elle en avait une considérable dans l'onomastique. Elle entre dans la formation d'un nombre considérable de noms de lieu. I'île de Rhodes paraît avoir tiré son nom des roses ${ }^{2}$ qui croissaient, dit-on, en abondance dans cette île ${ }^{3}$, nom qui se transmit à ses colonies de la Tarragonaise et des bords du Rhòne, - Rhoda. On retrouve probablement le radical qui sert à désigner la reine des fleur's dans Rhodia, nom d'une ville de Lycie, d'un territoire des Rhodiens en Carie, et d'une souree de la Troade, dans Rhodiac,

1. Les rois de Syrie, d'Arménie et de Commagène (Catalogue des monnaies grecques de la Bibliothique nationale). Paris, 1890 , in $-8^{\circ}$, p. $188, n^{\circ} 1418, \mathrm{pl}$. XXV, fig. 15. Quant à la rose qui se trouverait aussi sur une monnaie d'Antiochus IX (pl. XXVI, fig. 5), je n'en puis rien dire, car il m'a été impossible d'y distinguer aucune espèce de fleur.

2. V. Guérin, op. laud., met en doute cette étymologie, mais sans raison valable.

i. Eximias ea insula rosas habet, unde et Rhodum vocari quidem aiunt. Rasche, op. Inud., vol. IV, 1, p. 1027. 
ville des Peucétiens en Apulie, Rhodion, bourg des Ambianiens, tribu de la Narbonnaise, dans Rhodios, nom d'un petit fleuve voisin de Troie ${ }^{1}$, Rhodipolis, ville de la Tarragonaise, Rhodopolis, forteresse de la Colchide, Rhodos, nom de la ville située au nordest de l'île de ce nom, ainsi que d'une ville de Gérénia, Rhodountia, lieu voisin des Thermopyles, Rhodoussa, nom d'une ville d'Argolide et d'une île située sur la côte de Carie, enfin dans Rhodoussae, nom porté par deux îles de la Propontide, ainsi que dans Rhodope, chaine de montagne entre la Macédoine et la Thrace?

Le mème radical se rencontre bien plus fréquemment dans les noms de personnages historiques ou mythologiques, surtout dans les noms de femme, par exemple dans ceux de Rhodeia et Rhodopê, filles de l'Océan et de Téthys et compagnes de Perséphoné Phode, nom d'une fille de Poscidon et d'Amphitrite, qui, dit-on ${ }^{*}$, épousa le Soleil, ainsi que d'une fille de Danaos; c'était aussi le nom d'une Bassaris ou compagne de Bacchus". Une autre fille de Danaos et l'épouse de Lycon s'appelaient Rhodia ${ }^{6}$. Rhodoessa

1. W. Pape, Worterbuch der griechischen Eigennamen, $3^{\text {te }}$ Auflage neu bearbeitet von Gustay Eduard Benseler. Braunschweig, 1863-70, in-8, s. v.

2. William Smith, Diclionary of Greek and Roman Geography. London, in-8, vol. II, p. 712-713.

3. Hymni Homerici, V, in Cererem, v. 422.

4. Apollodore, Bibliolheca, lib. I, 4, 6 et II, 1, 5 .

5. Nonnus, Dionysiaca, cant. XIV, v. 223.

6. W. Pape, op. laud., s. v. H. Estienne, Thesaurus, s. v. 
et Rhodos étaient des noms de nymphes ${ }^{1}$. On rencontre encore les noms de Rhodanthê, Rhodinế, Rhodion, Rhodippe $\hat{e}^{2}$. Il est fait mention d'une athénienne, appeléc Rhodè, ainsi que la femme de Mégaclès ${ }^{3}$. On connait aussi plusicurs esclaves de ce nom. Il y a eu encore une danseuse appelée Rhodokleia ${ }^{4}$; une pythagoricienne connue se nommait Rhodopè ${ }^{5}$; on cite également une femme du nom de Rhodô et de Rhodon ${ }^{6}$.

Hérodote a rendu célèbre ${ }^{7}$ le nom de Rhodôpis, hétaïre de Thibes en Égypte, dont Élien a également raconté l'histoire ${ }^{8}$. Un jour qu'elle se baignait dans le Nil, elle donna ses vêtements à garder à ses suivantes, mais un aigle qui survint enleva une de ses pantoufles et l'emporta jusciu'à Memphis; là il la laissa tomber sur les genoux de Psammétique, qui rendait à ce moment même la justice. Rempli d'admiration pour la forme et l'álegance de cette chaussure, non moins que surpris par la maniere étrange dont il l'avait recue, le roi fit chercher dans tout son royaume celle à qui la pantoufle appartenait; on finit par découvrir Rhodopis ; Psammétique s'en

1. Etym. magnum, 507, 40. - Pindare, Olymp. VII, v. 130.

2. W. Pape, op.laud., s. v.

3. Corpus inscr., vol. I, no 730. - Longus, Pastoralia, IV, 36.

4. Anth. graeca, lib. V, no 73 .

5. W. Pape, op. laud., s. v.

6. Corpus inscr. graec, vol. IV, $\mathrm{n}^{\circ} 7468$ et 8038.

7. Histor. lib. II, cap. 135.

8. Var. histor. lib. XIII, cap. 33. 
éprit aussitòt qu'il l'eut vue et il l'épousa. Je terminerai cette longue énumération en mentionnant Rhodogoune et Rhodogune, noms grees de princesses perses ${ }^{1}$, qui traduisent sans doute ceux qu'ils portaient dans leur langue maternelle.

Tandis que les dérivés de rhodon, employés comme noms de femme, sont si nombreux, on en trouve assez peu, et cela se comprend, qui servent a désigner des hommes; j’ai relevé les noms de Rhodippos, un athénien et un pythagoricien de Crotone; Rhodiôn et plus souvent Rhodón notre Rosier, - Phodoliles, qu'on rencontre dans plusieurs inscriptions ${ }^{2}$. On peut citer encore Rhodopaeos et même Rhodopianos ${ }^{3}$, ainsi peut-ètre que Rhodophôn ${ }^{4}$.

Le nom de la rose, si fréquent dans l'onomastique des Grees, n'apparaît qu'exceptionnellement, au contraire, dans celle des Romains et seulement aux derniers temps de leur histoire. Ce n'est qu'à l'époque de la transformation du latin que les villes d'origine grecque comme la Rhodè ou Rhoda de la Tarragonnaise, changèrent leur nom en Rosa. Quant aux noms de personnes, tels que Rosa, Rosalia, tirés du radical qui sert à désigner la fleur du rosier,

1. W. Pape, op laud., s. v. Une Rhodogoune fut l'épouse de Darius, fils d'Hystape, et une autre, sœur de Phraate, épousa Démétrius Nicanor. Rhodogune était fille de Zopyre.

2. W. Pape, op. laud., s. v.

3. Corpus inser. graec, vol. II, no 280 ' et 2997.

4. Athénée, Deinosoph. lib. X, cap. 63 ('44). 
on les rencontre seulement dans les monuments chrétiens. Mais on a de bonne heure employé ce mème vocable pour dénommer des fleurs ou des choses qui offrent quelque ressemblance avec la rose. Ainsi Pline ${ }^{1}$ donne le nom de "rose grecque » (rosa graeca) à une plante qui paraît ètre un lychnis (L. coronaria L.); on appelait le lis " rose de Junon ${ }^{2}$ », et l'oléandre, - notre laurier-rose, - portait parfois le nom de rosa laurea ${ }^{3}$, traduction du mot grec équivalent rhododaphné. Enfin on donnait encore le nom de " rose » à une espèce d'érysipèle d'aspect rougeâtre, dénomination qui s'est conservée dans plusieurs idiomes modernes.

1. Hist, natur, lib. XXI, cap. 10(4).

2. Forcellini, Lexicon, s. v. rosa.

3. Apulée, Metamorph., lib. IV. 


\section{CHAPITRE V.}

LA ROSE DANS L'ANCIEN ORIENT.

Tandis que la culture de la rose prenait en Occident une extension que la décadence et la destruction de l'Empire devaient seules arrêter, elle se développait aussi en Orient; ce n'est plus seulement en Asie Mineure et en Mésopotamie qu'on en retrouve les traces incertaines, on la rencontre maintenant à la fois en Perse, en Syrie et en Égrpte. Des légendes se forment autour de la rose dans ces divers pays; elle y entre de plus en plus dans les usages de la vie et elle y devient un objet de luxe, comme dans la Grèce et l'Italie.

\section{I.}

Il nous est impossible de suivre les progrès que put faire la culture de la rose dans l'ancien Iran ; mais qu'elle y remonte à une époque reculée, c'est là ce dont on ne saurait douter. Comment cette fleur n'aurait-elle pas pris place dans les Parcedis célebres que les souverains de la Perse, comme nous le royons 
par l'exemple de Cyrus le jeune ${ }^{1}$, ne dédaignaient pas de cultiver de leurs propres mains. Le Bundehesh, dont le texte le plus ancien est seulement du vin ${ }^{e}$ siècle de notre ire ${ }^{2}$, mais dont certaines traditions ont un caractere primitif et nous reportent aux premiers temps du Zoroastrisme, connaît la rose à cent feuilles et la pose de chien ou rose sauvage; d'après lui deux amshapands différents veillent sur elles; la première, la rose à cent feuilles, est confiée a la garde de Dìn, la seconde, la rose de chien nestran - à la garde de Rashtu ${ }^{3}$; suivant une légende racontée dans le même livre sacré, la rose, comme tous les arbustes, aurait été crééc sans épines; ce n'est que depuis l'apparition d'Ahriman ou du génie du mal en ce monde qu'elle est armée d'aiguillons ${ }^{4}$, conception singulière dont nous retrouverons l'analogue chez plusieurs Pères de l'Église.

Un renseignement plus ancien nous permet d'affirmer que la rose fut non seulement cultivée avant notre èré, chez les Médo-Perses, mais qu'elle y devint un objet de luxe et de parure et y servait, comme chez les Grees, a faire des couronnes. Dans ses Questions de table;, Plutarque rapporte que le roi de Perse - c'était Artaxercès II Mnémon, -

1. Xénophon, Oeconomici cap. IV, 21.

2. The Pehlavi lexts, Part. I, Introduction, p. 27. (Vol. V des Sacred books of the East. Oxford, 1886, in-8.)

3. Bundehesh, chap. XXVII, 21.

1. Bundehesh, chap. XXVII, 1.

5. Symposiaca, lib. VII, probl. VIIJ, 4, 1' . 
envoya à Antalcidas une couronne faite de fleurs de rose et de safran, sur lesquelles on avait répandu des parfums, ce qui, remarque l'écrivain grec, avait outrageusement détruit la beauté naturelle de ces fleurs.

II.

Des faits non moins probants, mais contemporains de la domination hellénique, nous apprennent que la rose était cultivée et employée comme ornement en Syrie et en Judée, ainsi qu'en Grèce. Elle y était alors si connue que l'auteur de l'Ecclésiastique, Jésus fils de Sirach, qui vivait vraisemblablement au second siècle avant notre ère ${ }^{1}$, lui emprunte, comme les écrivains de la Grèce et de Rome, de nombreuses comparaisons :

Je m’élevai ${ }^{1}$, dit-il, comme le palmier sur le rivage, comme les rosiers de Jéricho.

Écoutez-moi ${ }^{2}$, vous qui êtes pieux, et croissez comme les rosiers plantés au bord des eaux.

Il a paru ${ }^{3}$ comme l'arc-en-ciel qui brille dans les nuées lumineuses et comme les roses du printemps, comme les lis qui croissent près d'une source jaillissante.

1. Éd. Reuss, La Bible. Ancien Testament, 6 ${ }^{\mathrm{c}}$ partic. Paris, 1878, in-8, p. 337.

2. Chap. XXIV, verset 18.

3. Chap. XXXIX, verset 17.

4. Chap. L, verset 8. 
Pour l'auteur du livre de la Sagesse, contemporain du fils de Sirach ou mème postérieur ${ }^{1}$, cet usage poétique de la rose ne suffit plus; comme un véritable anacréontique, il veut se couronner de roses :

Enivrons-nous de vin, s'écrie-t-il ${ }^{2}$, parfumons-nous d'huile de senteur; ne laissons pas passer la fleur du printemps! Couronnons-nous de roses avant qu'elles se fanent.

Ce n'est pas là une simple amplification de rhétorique, c'était la constatation du goût si général alors pour une vie voluptueuse et pour les roses, qui en étaient comme l'accompagnement obligé. Florus raconte $^{3}$ qu'Antiochus, roi de Syrie, ayant envahi la Grèce, établit son camp en Eubée, aux bords murmurants de l'Euripe; là, mollement installé sous des tentes d'or et de soie, entouré de joueurs d'instruments et d'une troupe de jeunes gens et de jeunes filles, afin que tout lui rappelât le luxe de sa patrie, il donna l'or'dre de rassembler, quoiqu'on fùt en hiver, des roses de tous côtés.

Le Talmud fournit également la preuve que la rose était, au commencement de notre ère, l'objet d'une grande culture en Judée. Le traité de Maaseroth fait mention d'un jardin près de Jérusalem, dans lequel croissaient de magnifiques figuiers, sur lesquels on

1. Édouard Reuss, op. laud., p. 513.

2. Chap. II, versets 7 et 8.

3. Epitome, lib. I, cap. 8. (Cap. 24, éd. K. Halm.) 
ne prélevait pas la dìme, parce que, pour préserver les roses, l'entrée n’en était permise à personne". D’aprés le Talmud, avant la destruction de Jérusalem par les Romains, l'époux portait une couronne de roses, de myrte ou d'olivier. ${ }^{2}$

III.

La culture de la rose se répandit en Égypte, où cet arluuste est exotique, peut-être après la conquête perse, et elle y prit sous les Ptolémées une grande extension; les roses de ce pays devinrent célèbres; c'est de là, au rapport de Pline ${ }^{3}$, qu'on en faisait venir à Rome pendant l'hiver, avant qu'on eût trouvé en Italie le mojen d'obtenir des roses dans cette saison. Cette découverte ne fut faite qu'au premier siècle de notre ère; aussi, comme le raconte Martial dans une épigramme que j’ai citée plus haut, quand les habitants de l'Égypte, qui l'ignoraient, crurent offrir à Domitien un présent digne de lui, en lui cnroyant, à l'occasion de sa fète, des roses à l'automne ${ }^{4}$ - Martial, par une licence poétique, dit au

1. Joh. Henr. Otho, Lexicon rabbin. philol., p. 302, ap. Schleiden, Die Rose, p. 31.

2. Sponsi corona erat sive rosea, sive myrtea, sive oleaginea. Joh. Selden, Uxor hebraica seu De nuptiis ct divortiis libri tres. Londini, 1646, in-4, lib. II, cap. 15.

3. Hist. natur., lib. XXI, cap. 3.

4. Domitien était né le 9 des 'calendes de novembre ou le 24 octobre. 
milieu de l'hiver, - leurs messagers furent surpris de trouver dans Rome ces roses qu'ils croyaient propres ì leur pays, et le poìte termine son récit en invitant ironiquement les habitants des bords du Nil à envoyer leurs moissons aux Romains, et à accepter leurs roses en retour.

L'Égypte, si elle continua d'envoyer ses blés à Rome, n'eut pas à lui demander de roses précoces ou tardives; son climat privilégié lui permettait d'en avoir avec moins d'efforts quel'Italie et elle suffit toujours à en fournir au luxe croissant de ses habitants et de ses princes. Une anecdote rapportée par Athénée ${ }^{1}$ montre à quel point ces derniers avaient poussé le goût et la passion des roses. Cléopatre, étant allée à la rencontre d'Antoine en Cilieie, lui donna pendant plusieurs jours des fètes d'une magnificence royale; le quatrième elle poussa même la somptuosité jusquà faire rassembler pour un talent ${ }^{2}$ de roses, et elle fit couvrir de ces fleurs, que retenaient des réseaux très fins, le pavé des salles du palais jusqu'à la hauteur d'une coudée.

La fleur pour laquelle un sourerain de l'Égypte faisait des dépenses aussi énormes avait dû prendre dans la culture de ce pays une importance considérable: elle y figurait maintenant au premier rang, comme en Italie et en Grèce, parmi les fleurs employées pour faire les couronnes; elle passait avant le lotus, si recherché autrefois, et quand ce

1. Deipnosoph. lib. IV, cap. $29(148, b)$.

2. Environ 5,600 francs. 
dernier, offert à Adrien qu'il surprit et ravit par sa beauté, recut par une honteuse flatteric le nom de " fleur d'Antinoüs "), le poète Pancrate put feindre, tant la rose était déjà connue depuis longtemps en Egypte, qu'elle y était indigène, tandis que le lotus aurait été de création ou d'importation récente.

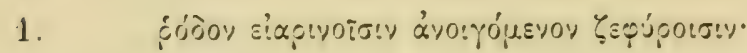

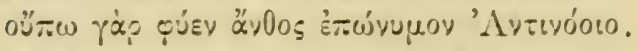

Athénée, Deipnosoph. lib. XV, cap. $21(677$, f). 


\section{CHAPITRE VI.}

LA ROSE DANS LA PHARMACOPÉE.

La rose n'était pas seulement recherchée des Anciens pour l'usage qu'on en faisait dans les diverses cérémonies du culte et dans les banquets, elle l'était encore à cause des propriétés médicinales ou magiques qu'on lui attribuait, ainsi que pour les produits qu'on en retirait ou qu'elle servait à préparer. L'emploi qu'on en faisait dans les banquets tenait d'ailleurs en partie à certaines vertus réfrigérantes qu'on lui supposait; comme telle, elle passait pour combattre l'ivresse ${ }^{1}$ et pour calmer les maux de tète qu'elle cause $^{2}$. Nais on lui reconnaissait des propriétés bien autrement puissantes; pouvait-il en être autrement avec l'origine surnaturelle qu'on lui attribuait?

Telles sont les vertus dont jouit cette fleur dans l'Ane de Lucien et dans les Métamorphoses d'Apulée. Lucius, héros de ce roman étrange, changé en âne par des pratiques magiques, ne peut recouvrer sa

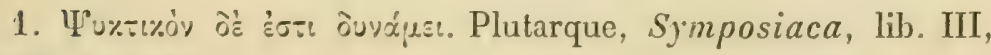
probl. I, 3, 16.

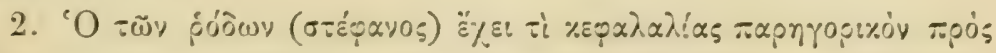

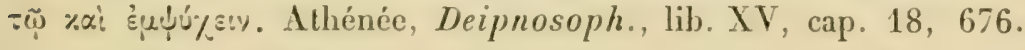
JoREt. La Rose. 
forme première qu'en mangeant des fleurs de rose. Longtemps le remède promis semble s'éloigner de lui, mais enfin le temps de ses épreures est terminé; la mère des Dieux et des hommes, la Nature ellemême, vient lui annoncer sa prochaine délirrance. Le lendemain, au milieu de la foule réunie pour célébrer la fète de la déesse, il aperçoit le grand-prètre qui tenait à la main une couronne de roses ${ }^{1}$; il s'approche, et, à peine a-t-il avidement saisi arec les dents et dévoré encore plus avidement cette couronne, qu'aussitôt il se voit débarrassé de son enveloppe difforme et reprend sa figure humaine : le charme qui l'avait métamorphosé est rompu par la vertu merveilleuse de la fleur divine.

Cette vertu attribuée ainsi à la rose, et à laquelle M. de Gubernatis ${ }^{2}$ roudrait reconnaître un caractère mythique, a sans doute du moins une origine ancienne; dès l'époque la plus reculée on reconnut d'ailleurs à la fleur du rosier, de mème qu'au rosier lui-mème, cultivé ou sauvage, de nombreuses propriétés médicinales. "Elle vient en aide aux malades, dit le pseudo-Anacréon ${ }^{3}$, et protège mème les morts. » Ilippocrate la prescrit dans un grand nombre de cas, et, cincl siècles après lui, Celse n'en recommandait pas moins expressément l'emploi. Suivant Pline, elle

1. Mctamorph. lib. XI, cap. 13. Coronam, quae rosis amœnis intexta fulgurabat, arido ore susceptam, cupidus cupidissime deroravi... Protinus mihi delabitur deformis et ferina facies.

2. Mythologie des plantes, vol. II, s. v. Rose.

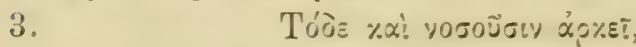

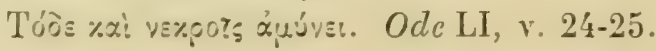


était astringente et réfrigérente ${ }^{1}$. Elle servait, soit seule, soit mêlée à d'autres ingrédients. Pline et Dioscoride, en particulier, ont décrit avec détail les diverses préparations dans lesquelles elle entrait.

La plus ancienne dont il soit fait mention est l'huile de roses, qu'Homère connaissait déjà ${ }^{2}$. Pour l'obtenir on faisait bouillir dans une certaine quantité d'huile d'olive ${ }^{3}$ du jonc aromatique, en agitant le tout arec soin, puis on coulait; après quoi on mettait dans le liquide ainsi préparé des pétales de rose bien desséchés; on remuait encore et agitait pendant un jour, puis on laissait reposer la nuit suivante et on filtrait de nouveau le mélange, qu'on versait alors dans un vase enduit préalablement de miel ${ }^{4}$.

Dissous tout simplement dans du vinaigre, les pétales de rose donnaient un autre ingrédient recherché, le vinaigre rosat. A propos d'une fumigation, Hippocrate parle aussi de l'eau de roses ${ }^{5}$; mais ni Dioscoride, ni Pline n'en donnent la composition; on la préparait sans doute d'une manière analogue it l'huile ou au vinaigre de roses. IIippocrate mentionne encore le miel rosat ${ }^{6}$; on l'obtenait, suivant Palladius ${ }^{7}$, en mèlant une livre de miel avec

1. Hist. natur., lib. XXI, cap. 73.

2. Ilias, cant. XX, v. 86 .

3. Palladius, De re rustica, lib. VI, cap. 14, dit une livre d'huile et une once de pétales.

4. Dioscoride, De materia medica, lib. I, cap. 44.

5. OEuvres, éd. Littré, vol. VII, p. 321.

6. OEuvres, éd. Littré, vol. VII, p. 177.

7. De re rustica, lib. VI, cap. 16. 
un sextarius de suc de roses, et on exposait cette composition pendant quarante jours au soleil.

Le vin rosat, breuvage très recherché, se fabriquait en pilant des pétales séchés de roses qu'on mettait ensuite, enveloppés d'un linge, dans du moût avec un poids pour les faire aller au fond; au bout de trois mois on coulait et on transvasait le liquide ${ }^{1}$. Pour le rendre plus parfumé, Iléliogabale au rapport de Lampride y ajouta des pignons broyés ${ }^{2}$. Dioscoride mentionne encore les pastilles de rose ${ }^{3}$

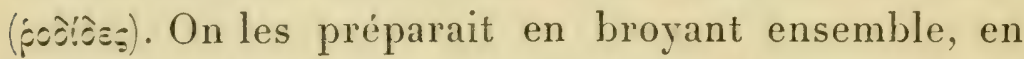
quantité déterminée, des pétales de roses frais cueillis, du nard indien et de la myrrhe. Quelquefois on y ajoutait du costus, de l'iris d'Illyrie et du vin de Chio avec du miel. On faisait de la pâte ainsi obtenue des pastilles qu'on mettait sécher à l'ombre dans un vase de terre bien bouché.

Plusieurs préparations se faisaient aussi avec les seuls pétales de la rose. Après les avoir pilés entiers ou séparés de leur onglet dans un mortier, on exprimait le suc à travers un linge et on le faisait réduire

1. Pline, op. laud, lib. XIV, cap. 19. - Dioscoride, op. laud., lib. IV, cap. 35. Palladius, VI, 13, donne une recette un peu différente : jeter cinq livres de roses, épluchées de la veille, dans dix sextarii de vin vieux et ajouter au bout de trente jours dix livres de miel écumé. Apicius Coelius, De arte coquinaria, lib. I, cap. 4 en donne encore une autre.

2. Rosatum..... pinearum attritione odoratius reddidit. Vila IIeliogabali, cap. xix.

3. De materia medica, lib. I, cap. 131. 
dans un vase d'airain jusqu'à consistance sirupeuse ${ }^{1}$, ou bien on le laissait simplement s'épaissir à l'ombre ${ }^{2}$. On employait aussi les pétales soit écrasés, soit brûlés ou desséchés, puis réduits en poudre, etc. Pausanias parle encore ${ }^{3}$ d'un onguent qu'on fabriquait à Chéronée avec des fleurs de rose, de lis, de narcisse et d'iris, comme d'un spécifique excellent contre les douleurs.

L'huile de roses était à la fois un remède et un parfum ; comme remède, on s'en servait soit seule, soit en composition. Hippocrate parle d'un pessaire composé avec de la farine et de l'huile de roses, et employé dans une affection strangurique ${ }^{4}$. Cette huile servait dans les injections, elle entrait aussi dans la composition des cataplasmes. Seule, on la prenait dans les irritations d'estomac ou d'entrailles; elle servait également en friction, pour calmer les douleurs d'oreilles" et les maux de dents. Contre ces derniers, Celse conseillait aussi le miel rosat avec du beurre ${ }^{b}$. L'huile de rose était aussi un émollient utile contre les ulcères ${ }^{7}$.

Le vin de roses, suivant Dioscoride ${ }^{8}$, était considéré

1. Pline, op.laud., lib. XXI, cap. 73.

2. Dioscoride, op. laud., lib. I, cap. 130.

3. Descriptio Graeciae, lib. IX, cap. 41, 6.

4. OEuvres, éd. Littré, vol. V, p. 429.

5. Théophraste, De odoribus, cap. VIII, 35.

6. Medicinae lib. VI, cap. 18, 2.

7. Dioscoride, op. laud, lib. I, cap. 53.

8. De materia medica, lib. I, cap. 130. 
comme un adjuvant de la digestion et comme souverain contre la dysenterie. D'après Théophraste ${ }^{1}$, il dissipait les pesanteur's de tête causées par les parfums. Pline conseillait contre les douleurs d'estomac, au lieu de la préparation ordinaire, du vin, dans lequel on s'était borné à mettre des fleurs de roses " en quantité suffisante pour le parfumer ». Il parle encore d'une infusion de roses dans de l'hydromel pour arrêter les crachements de sang, etc ${ }^{2}$. Quant au suc de roses, il servait à faire un collyre excellent pour les yeux, d'après Celse ${ }^{3}$ et Dioscoride mais il arait bien d'autres emplois; Pline le dit bon dans les maladies d'oreilles, en gargarismes pour les ulcérations de la bouche, les gencives, les amygdales; on s'en servait aussi contre les douleurs de tête, les maux de gorge, etc. On l'employait également dans la fièvre, seul ou dissous dans du vinaigre, pour combattre l'insomnie et les nausées.

Les pétales écrasés étaient employés en cataplasmes dans les inflammations, contre les phlegmons, les érysipèles, etc. Préalablement desséchés, puis réduits en poudre, ils servaient contre les excoriations de la peau. C'est ainsi qu'Aspasie, au rapport d'Élien", fut guérie d'une tumeur qui la défigurait dans sa jeunesse. Un médecin consulté par son père Hermo-

1. De odoribus, cap. X, 48.

2. Hist. natur., lib. XXI, cap. 73.

3. Hedicinae lib. VI, cap. 6,5 .

4. De materia medica, lib. I, cap. 130 .

5. Variae historiae, lib. XII, cap. 1. 
time avait demandé trois statères pour la traiter; Hermotime, qui ne possédait point cette somme, se retira avec sa fille. Aspasie revint à la maison paternelle dans un désespoir profond et refusa toute nourriture; cependant un sommeil bienfaisant s'étant emparé de ses sens, elle vit en songe une colombe, qui, prenant la figure d'une femme, lui dit d'avoir courage, de réduire en poudre quelques roses sèches d'une couronne consacrée à Vénus et de les appliquer sur le mal. Aspasie suivit le conseil et la tumeur disparut. Ainsi, grâce au secours de la plus belle des déesses, dit l'historien grec, Aspasie redevint la plus belle des femmes.

Suivant Pline ${ }^{1}$, les têtes de roses, c'est-à-dire sans doute les fleurs entières, prises en boisson, arrètent le flux de ventre et les hémorragies. Les fruits du rosier aussi étaient utilisés, d'après les naturalistes romains, qui les considèrent comme diurétiques; séchés à l'ombre après la maturité, on les appliquait sur l'estomac et sur les érysipèles récents; mis sous les narines, dit encore Pline, ils purgent le cerveau; on en frictionnait aussi les dents malades. Il n'y avait pas jusqu'aux étamines qui ne fussent employées; desséchées, on en saupoudrait les gencives dans les inflammations.

La rose n’était pas seulement employéc en médecine, on s'en servait également comme cosmétique et dans la cuisine. L'huile de roses en particulier, à cause de son parfum, était recherchée pour la toi-

1. Hist. natur. lib. XXI, cap. 73 . 
lette. On l'employait également, on le sait par le témoignage d'Ilomère, pour embaumer les morts. D'après Pausanias ${ }^{1}$, on en oignait aussi les statues de bois pour les préserver de la corruption.

J'ai parlé déjà des pétales desséchés et réduits en poudre; on s'en servait pour réprimer la sueur; on leur donnait le nom de diapasma; on en jetait sur la peau au sortir du bain; puis, au bout de quelque temps, quand celle-ci s'était imprégnée du parfum, on la lavait avec de l'eau froide ${ }^{2}$. On faisait encore avec les pétales desséchés et brûlés une espèce de cosmétique pour les paupières ${ }^{3}$. Mèlés à poids égal, avec du sel ammoniac, à un ingrédient composé d'encens, de nitre, de gomme, broyés et délayés dans du miel avec du fenouil et de la myrrhe, les pétales de rose formaient un cosmétique précieux pour conserver le teint ${ }^{4}$. Enfin, ils entraient dans la composition d'un parfum auquel cette fleur donnait son nom, et qui était un des plus recherchés et des plus répandus. On le fabriquait, nous apprend Pline $^{3}$, avec de l'omplacium ou jus d'olive, des fleurs de rose et de safran, du cinabre, du roseau et du jonc odorant, de la fleur de sel ou de l'anchuse et

1. Descriptio Graeciae, lib. IX, cap. 41,6 .

2. Pline, Hist. natur., lib. XXI, cap. 73.

3. Dioscoride, De materia medica, lib. I, cap. 130.

4. Ovide, De medicumine faciei, v. 91-98.

5. Hist. natur., lib. XIII, cap. 2, 5. Théophraste ne parle (De odoribus, V, 25) que de jonc odorant, daspalathe et de calanus. Ailleurs /llid., 33) il dit qu'on le colorait parfois avec de l'orcanette. 
du vin. Phasélis fut longtemps réputée pour celui qu'on y faisait, prééminence qui, rapporte Pline ${ }^{1}$, lui fut enlevée dans la suite par Naples, Capoue et Préneste. Le parfum des roses de Cyrène était également très renommé ${ }^{2}$; du temps de " la grande Bérénice », il passait pour le meilleur que l'on connût ${ }^{3}$.

La rose occupait une place non moins considérable dans la cuisine que dans la toilette chez les Anciens; on en confisait les pétales pour les manger ${ }^{4}$. On s'en servait également pour relever le goût des mets ou des boissons. Suétone raconte qu'un familier de Néron dépensa, dans un festin, plus de quatre millions de sesterces pour une boisson à la rose". Bouillis, les pétales entraient aussi dans la préparation d'une compote faite avec des coings cuits dans du miel et écrasés ensuite ${ }^{6}$. C'était un stomachique agréable et estimé. Le suc qu'on obtenait en pilant dans un mortier des pétales de rose, avec une quan-

1. Hist. natur., lib. XIII, cap. 2, 2. - Athénée, Deipnosoph., lib. XV, cap. $38,688$.

2. Théophraste, Hist. plant., lib. VI, cap. 6, 6. - Pline, Hist. natur., lib. XXI; cap. 10 (4), 5. " Thi unguentum pulcherrimum.»

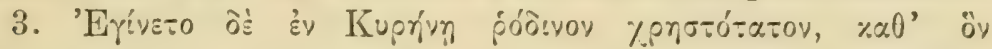

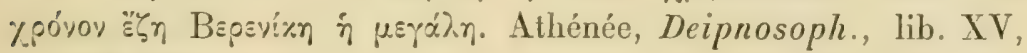
cap. 38,689 , a.

4. Pline, Hist. natur., lib. XXI, cap. 73. " Gibo quoque lapathi modo condiuntur. »

5. Indicebat familiaribus coenas, quorum uni mellita quadragies sestertio constitil, alteri pluris aliquanto absorptio rosaria. (En viron 500,000 francs.) Vita Neronis, cap. VII.

6. Pline, Hist. natur., lib. XXIII, cap. 54. 
tité d'eau déterminée, et en passant le résidu à travers un tamis, servait encore de principal condiment à un plat fait avec des cervelles et des œufs écrasés dans du vin et de l'huile, mets recherché dont Apicius a donné la recette, mais qu'il n'a pas inventé ${ }^{1}$. C'est le " plat aux roses ) du Banquet des Sophistes qu'Athénée fait décrire par le cuisinier qui l'a composé et qu'il leur apporte tout fumant dans la salle du festin ${ }^{2}$.

" Voici, dit-il, ce que j’appelle pâté aux roses; je l'ai ainsi préparé afin que rous eussiez tant sur la tète qu'intérieurement le parfum suave des couronnes, et que tout votre corps se sentît de ce régal. Après aroir pilé dans un mortier les roses les plus odorantes, jy ai ajouté des cervelles de volailles et de porc bien bouillies, dont j’ai ôté jusqu'au moindre fibre, des jaunes d'œuf, puis de l'huile, du garum, du poivre et du vin. Après avoir bien broyé le tout, je l'ai jeté dans une marmite toute neuve et l'ai placé sur un feu doux mais soutenu. En disant ces mots, il découvrit sa marmite; il s'en exhala une odeur suave qui parfuma toute la salle, et l'un des convires, tant l'odeur des roses était pénétrante, ne put s'empêcher de s'écrier avec le poète : “ Ce parfum agité remplit de sa vapeur le palais de Jupiter et se répandit dans le ciel et sur la terre s.

1. Apren Calr de obsoniis el condimentis sive arte coquinaria libri decem cum annotalionibus Mantrxi Lister. Amstelodami, 1709, in-12, lib. IV, cap. 2, p. 110.

2. Deipnosoph., lib. IX, cap. 70, 406, a. 
L'églantier, cynosbatos ou cynorrhodon, - les R. canina L., sempersirens L., ou rubiginosa L., si négligé par la poésie des Grecs et des Romains, - il en a été autrement, nous le verrons, chez les nations romanes et germaniques - avait, au contraire, sa place dans leur pharmacopée. Une décoction de sa racine était, assure Pline ${ }^{1}$, un remède infaillible contre l'hydrophobie, propriété, dit le naturaliste romain, révélée en songe à la mère d'un prétorien, qui avait été mordu par un chien enragé. Les fruits de cet arbuste, cuits dans du vin, arrètaient, suivant Dioscoride ${ }^{2}$, comme ceux de la rose cultivée, le flux de ventre. Enfin l'excroissance produite sur cet arbuste par la piqûre d'une espèce de cynips le bédégar - était, d'après Pline ${ }^{3}$, un remède excellent pour les calculeux. Le naturaliste latin dit aussi ${ }^{4}$ qu'on en employait la cendre mélée avec du miel, pour guérir l'alopécie. Ailleurs ${ }^{5}$ il conseille contre la mème affection, comme un spécifique merveilleux, ce mème produit avec de la graisse d'ours.

1. Hist. natur., lib. XXV, cap. 6.

2. De materia medica, lib. I, cap. 123.

3. Hist. natur., lib. XXIV, cap. 74.

4. "Spongiolae, quae in mediis spinis ejus nascitur, cinere cum melle, alopecias capitis expleri. » Hist. natur., lib. XXV, cap. 6. Littré traduit par "la cendre du fruit », mais le mot spongiola ne peut désigner ici que le bédégar et le bédégar n'est pas un fruit.

5. « Silvestris (rosae) pilulae cum adipe ursino alopecias mirifice emendant ». Littré traduit pilulae par " têtes », mais il est évident que ce mot est ici synonyme de spongiolae; Forcellini, s. v. Spongia, 11, ne laisse pas de doute à cet égard. Il s'agit donc encore du bédégar dans ce passage. 
La rose qui possédait, d'après les Anciens, tant de propriétés salutaires, passait aussi à leurs yeux pour en avoir à l'égard de certains ètres, ou dans certaines circonstances, de contraires ou de malfaisantes. Pline prétend ${ }^{1}$ que les scarabées fuient l'odeur de la rose. Élien ${ }^{2}$ va jusqu'à dire qu'on tue ces insectes en jetant dessus des pétales de roses. De mème, suivant Artémidore ${ }^{3}$, c'était un signe de mauvais augure pour les malades que de rêver de couronnes de roses; la fragilité de ces fleurs présageait leur mort prochaine.

1. “Fugantur... scabaei rosa. » Hist. natur., lib. XI, cap. 115.

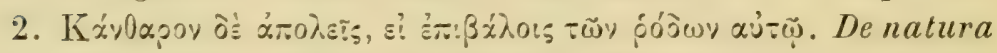
animalium, ex recogn. Rud. Hercheri. Parisiis, 1858, in-8, lib. IV, 18.

3. Artémidore, Oncirocritica, lib. I, cap. 79. 


\title{
SECONDE PARTIE
}

\author{
LA ROSE AU MOYEN AGE.
}

\section{CHAPITRE PREMIER.}

CUlture de LA rose dANS L'ORIENT ET DANS L'OCCIDENT.

Les deux grands faits, qui ont changé la face du monde au commencement des temps modernes, l'avènement du christianisme et l'invasion des Barbares, font aussi époque dans l'histoire de la rose. Sa culture poussée si loin dans tout l'empire romain dut être, sinon abandonnée complètement, du moins négligée, au milieu des guerres presque continuelles qui le désolèrent. Une autre cause devait lui être fatale; ce fut le discrédit dans lequel cette fleur charmante, mais profane, tomba auprès des partisans chaque jour plus nombreux du christianisme. Une religion, fondée sur la mortification de la chair, ne pouvait manquer de condamner l'usage que les paiiens faisaient de la rose; n'était-elle pas d'ail- 
leurs associée anx pratiques d'un culte proscrit, comme aux plaisirs coupables d'une vie condamnée par les nouveaux croyants?

C'est là ce qui explique le dédain dont la reine des fleurs fut tout d'abord l'objet auprès des chrétiens ${ }^{1}$. Hais cette opposition disparut bientôt avec l'abus qui l'avait fait naître et provoquée, et elle fera place à un sentiment où le symbolisme se mêlera avec je ne sais quoi de mystique et de respectueux chez les nations chrétiennes de l'Occident. Les destinées les plus brillantes étaient en mème temps réservées à la rose chez les peuples musulmans.

\section{I.}

Tandis que la culture de la rose avait été arrètée pour longtemps, sinon détruite, en Europe, par les calamités et les troubles de l'invasion, elle prenait une extension chaque jour plus grande dans l'Asie antérieure. Nous l'avons vue se répandre avant notre ère dans tous les pays des bords de la Méditerranée; elle s'étend maintenant des plaines de la Mésopotamie jusqu'au delà de l'Ilindou-Kouch et au Sindh.

La conquête arabe, qui détruisit l'ancienne civilisation hellénique dans l'Asie-Mineure et la Syrie, ainsi que la religion de Zoroastre et la civilisation persane dans l'Iran, ne porta pas atteinte, ou

1. Voir plus Ioin, chap. II. 
ne porta atteinte que pour un temps, à la culture de la rose dans ces contrées; cette fleur gracieuse ne tarda pas à être en aussi grand honneur auprès des vainqueurs qu'elle l'avait été près des vaincus, et les contrées soumises à la domination de l'Islam furent celles où la rose, au moins dans les premiers siècles du moyen âge, fut cultivée avec le plus de soin et prit la plus grande extension. "Elle vient en abondance en pays arabe ", — c'està-dire musulman —, affirme Ibn-el-Beithar ${ }^{1}$, qui écrivait en Espagne au xıI siècle, mais avait parcouru presque tous les pays soumis à l'Islam; on ne doit pas être surpris aussi que les auteurs musulmans de ces diverses contrées, qui se sont occupés de botanique ou d'agronomie, aient parlé de la rose.

Ishak Ibn-Amrâm, l'un des plus anciens, - il florissait au $\mathrm{xx}^{\mathrm{e}}$ siècle - parle de deux espèces de roses cultivées, la blanche et la rouge, désignées l'une et l'autre sous le nom de djoul - le persan gul -. Abou Hanifah, originaire de l'Irak, son contemporain, outre la rose cultivée, mentionne une espèce qui vient dans les campagnes et la rose de montagne ${ }^{1}$. Ibn Tamîm, cité par Ibn el-Beithar, connaît une rose jaune et il ajoute qu'il y en avait aussi, lui avait-on affirmé, une noire dans l'Irak ${ }^{3}$.

1. Traité des simples, art. 2274. Ouard, Rose, (Notices et extraits des manuscrits, vol. XXVI, p. 406).

2. Ap. Ibn el-Beithar, op. laud., p. 405. Malheureusement Abou Lanifah ne dit pas quelle différence il y a entre la rose des campagnes et celle de montagne.

3. Ibn el-Beithar, op. laud., p. 406. 
Quoique antérieur à Ibn el-Beithar, - il appartient au $\mathrm{XII}^{\mathrm{e}}$ siècle -, Ibn el-Awam est bien mieux renseigné :

" Il y a, dit-il, d'après l'andalou Abou-el-Khaïr, une de ses principales autorités ${ }^{1}$, le rosier de montagne ${ }^{2}$, le rosier rouge, le rosier blanc, tous deux à fleurs doubles, et le rosier de Chine ${ }^{3}$. La fleur du rosier de montagne est formée de cinq pétales. La rose double est la plus estimée de toutes les espéces; elle se fend sans s'épanouir complétement; elle est blanche, panachée d'un rouge plus foncé que celui de la rose de montagne; elle se compose de cinquante pétales ou de quarante au moins."

Jusqu'ici tout paraît aussi exact que vraisemblable; il n'en est pas de même de ce qui suit:

" Il y a en Orient, c'est toujours Abou el-Khaïr qui parle, la rose jaune et la rose bleue et une autre espèce dont les pétales sont rouges à l'extérieur et bleus à

1. Le livre de l'agriculture (Kitab-al-felah), traduit par J.-J. Clément-Mullet. Paris, 186'́, in-8, vol. I, chap. VI, art. 26, p. 281 .

2. Ciest, d’après Clément-Mullet. l'églantier, mais quelle espèce d'églantier?

3. Clément-Mullet ne dit pas quelle était cette espèce de rose. Ibn el-Beithar, op. laud., nos 2222 et 2282 , parait dire que c'est la rose de chien, nisrin ou nasrin; il est bien plus vraisemblable que la rose de Chine (Ouard Siny) est une tout autre plante, probablement une malvacée; Ibn el-Beithar donne lui-mème le nom de rose (Ouard) à la pivoine, la guimauve, l'anémone et à la renoncule. 
l'intérieur ; enfin une quatrième espèce dont les pétales, rouges à l'extérieur, sont d'un jaune blanc à l'intérieur. Cette espèce est cultivée dans les environs de Tripoli de Syrie. La rose jaune se trouve dans les parages d'Alexandrie. "

De ces deux dernières espèces, la première est incontestablement la rose capucine, la seconde est la rose jaune ordinaire ou soufrée. Mais que faut-il penser des roses bleues ou rouge bleu, dont parle Abou el-Khaïr ${ }^{1}$ ? Avant de répondre à cette question, voyons ce que Ibn el-Façel, autre botanisie du $\mathrm{xi}^{\mathrm{e}}$ siècle, cité aussi par el-Awam, dit de la rose et de sa culture:

“ On connaît, suivant cet écrivain ${ }^{2}$, quatre espèces de roses: la rose blanche camphrée ${ }^{3}$; elle est généralement connue sous le nom de rose double; dans une seule fleur on compte plus de cent pétales; la rose jaune, de la couleur du narcisse; la rose violette foncée, la rose rouge, la blanche nuancée de rouge ${ }^{*}$, dont le parfum est plus agréable et plus pénétrant que celui de la rose jaune et de la rose couleur foncée. »

1. Elles ont beaucoup embarrassé Loiseleur-Deslongchamps, qui ne connaissait que de seconde main et par extraits Ibn el-Awam. Voir son chap. sur la «Culture des roses chez les Maures d'Espagne ». Op. laud., p. 141.

2. Le livre de l'agriculture, vol. I, p. 282.

3. Clément-Mullet y voit la rose musquée ( $R$. moschata L.).

4. Sans doute couleur chair, comme le remarque avec raison Clément-Mullet. 
Cela fait cinq et non quatre variétés, analogues d'ailleurs, comme nuance, à celles qui sont cultivées de nos jours. Mais quelles étaient les roses bleues ou bleues et rouges, mentionnées par el-Awam d'après el-Khaïr et dont el-Façel ne dit rien?

Dans le chapitre XV de son livre, chapitre ou el-Awam examine les "recettes et procédés» à l'aide desquels on peut communiquer aux arbres ou aux plantes des qualités qu'ils n'ont pas de nature, l'agronome musulman indique, d'après Hadj de Grenade, le "moyen d'obtenir des roses jaunes ou bleues ${ }^{1}$ »; ce moyen consiste à soulever l'écorce des racines principales et à introduire entre l'aubier et l'écorce soulevée, soit du safran, soit de l'indigo trituré dans un mortier, suivant qu'on veut avoir des roses jaunes ou bleues; on lie ensuite les racines sur lesquelles on a opéré et on les recouvre de terre. Sans rechercher si par ce procédé on obtient, comme parait le dire el-Awam, à volonté, des roses jaunes ou bleues, on voit que pour lui les secondes au moins, car les premières existaient réellement, auraient été une production tout artificielle, non un produit de la nature.

El-Awam n'a point décrit seulement la rose double ou rose de jardin, il mentionne aussi, d'après elKhair, la rose saurage, que celui-ci appelle, comme Abou Hanifah, rose de montagne ${ }^{2}$; d'après lui, il y en a deux espèces, l'espèce à íleur blanche, sans

1. Le livre de l'agriculture, vol. I, p. 602.

2. Le livre de l'agriculture, vol. I, chap. VI, art. 26, p. 282. 
aucun mélange de rouge, - on peut croire qu'il s'agit de la rose toujours verte ( $R$. sempervirens L.), indigène dans le midi de l'Europe et dans l'Afrique septentrionale, - et l'espèce à fleur rouge, connue sous le nom de "rose des Mages ), laquelle, ajoutet-il, est la "rose d'Orient, du pays de Ghaur ou de Syrie "; j’inclinerais à y voir une des formes non cultivées de la rose de Provins ou de Damas. La fleur de ce rosier, dit encore el-Awam, est formée de cinq pétales. Ailleur's ${ }^{1}$, tant ses renseignements concordent peu, il ne parle que d'une seule espèce de rose sauvage, l'églantier ou rose de chien, le nasrin des médecins arabes. "Son fruit - dalik en arabe - est rouge, dit-il, et ressemble à la datte qui commence à mûrir; dans l'intérieur, il y a une espèce de laine; la fleur est celle du rosier, et d'un blanc nuancé de rose ${ }^{2}$. »)

Après cette description de l'églantier, el-Awam donne quelques renseignements sur la culture de cet arbuste et sur les moyens de le propager ${ }^{3}$; il en a donné de bien autrement longs et détaillés, preuve de l'importance qu'elle avait prise, - sur la culture du rosier proprement dit. Ces deux

1. Le livre de l'agriculture, vol. I, chap. VI, art. 54, p. 377.

2. El-Awam, qui cite ici Abou Hanifah et Avicenne, oublie ce quil il avait dit précédemment des roses sauvages rouges et blanches " sans aucun mélange de rouge ».

3. En particulier par semis. Il semble qu'il ait suivi Pline et Palladius. 
cultures offraient d'ailleurs la plus grande ressemblance.

Le rosier, dit-il, d'après Ibn el-Façel ${ }^{1}$, "se propage de graines, de branches éclatées, entières ou coupées, de rejetons enracinés; on l'obtient encore de rejetons marcottés pour leur faire prendre racine. »

Puis, après aroir décrit longuement ces divers procédés, en particulier la reproduction par semis, elAwam donne quelques conseils sur le traitement des pieds vieillis; il affirme qu'en les brûlant en octobre au moment de la sécheresse, on pouvait leur rendre leur ancienne vigueur; au printemps, ils poussaient, Dieu aidant, des rejetons, qui ne tardaient pas à se couvrir de nombreuses fleurs. Ce qui est plus curieux, c'est le procédé à l'aide duquel on peut, d'après elAwam, donner au rosier l'aspect arborescent.

"Pour l'ornement des jardins, dit-il", on plante au mois d'octobre des pieds de rosiers de diverses espèces. Quand la reprise est bien assurée et la végétation bien établie, on les enferme dans des tubes réunis par groupes de six ou huit environ disposés verticalement, de deux coudées de hauteur environ, et qu'on peint de diverses couleurs. Le sommet des rosiers s'élève au-dessus des tubes quil dépasse. On a soin de remplir ceux-ci de terre meuble ou de sable entretenu dans un degré convenable d'humidité. On laisse se déployer au sommet de

1. Le livre de l'agriculture, vol. I, ch. VI, art. 26, p. 283287.

2. Le livre de l'agriculture, vol. I, p. 286. 
ces tuyaux la tête des rosiers et lorsque leurs boutons s'épanouissent, ils ressemblent à des arbres portant des fleurs de diverses couleurs.

Cet exemple suffit pour montrer que les Maures d'Espagne, car ce sont eux qu'el-Awam avait en vue surtout quand il donnait ces conseils, cultivaient les roses, non seulement à cause de leurs propriétés médicinales, mais encore comme plantes d'agrément. Il en était de même dans tous les pays musulmans de l'Asie antérieure. Mais c'est surtout dans la partic occidentale du plateau de l'Iran que la rose a dès longtemps été cultivéc avec succès et donné ses produits les plus merveilleux. Celles de Chiras et du Farsistan, dont cette ville était regardée comme le jardin, ainsi qu'elle en est la capitale, sont restées célèbres ${ }^{1}$.

La terre de Chiraz, chante le poète $\mathrm{Hafiz}^{2}$, qui composa ses vers à l'ombre de ses bocages fleuris, ne cessera jamais de porter des roses et jamais le rossignol ne s'en éloignera.

La ville sainte de Koum est comme ensevelie sous des buissons de roses. Rien de comparable, au rap-

1. "Rosam uti Persia ex omnibus mundi partibus maxime copiosam ac suave olentem gignit, ita Sijrasum ejusque pagus praclaudatus, prae cacteris Persiae provinciis, fert copiosissimam ac fragrantissimam. "Engelbert Kaempfer, Amoenitates exoticue politico-physico-medicae. Lemgoviae, 1712, in-4, p. 373.

2. Schleiden, Die Rose, p. 264. 
port des voyageurs, pour leur grandeur et le parfum de leurs fleurs, aux rosicrs musqués de Téhéran, qui atteignent 15 et parfois mème jusqu'à 30 pieds de haut ${ }^{1}$. Les roses du Kourdistan et de l'Aderbeidjan ne sont ni moins belles, ni moins parfumées. On en peut dire autant de celles de la région de la Caspienne,

"Que le Mazenderan, mon pays, soit célébré, " fait dire Firdousi à un div devant Kiei Kaous ${ }^{2}$, un des héros de l'Iran, que ce chant décidera à entreprendre la conquête de cette province fortunée, "que le Mazendéran soit célébré, que ses plaines et ses campagnes soient toujours cultivées. La rose ne cesse de fleurir dans ses jardins et la tulipe et l'hyacinthe croissent sur ses montagnes. L'air y est doux et la terre y est peinte de fleurs. Il y règne un printemps éternel et sans cesse le rossignol chante dans ses jardins. Tu dirais que dans ses rivières coule l'eau de rose qui réjouit l'âme de son odeur. "

Au nord-ouest de l'Iran, la rose, cela était naturel, fut aussi cultivée en Arménie, la patrie véritable, peut-être, de l'espèce à cent feuilles, et l'on institua, dit-on, dans cette contrée en l'honneur de la reine des fleurs une fète particulière, appelée vartesar de son nom indigène, et qui, si elle ne date pas de

1. Ker Porter, cité par Schleiden, Die Rose, p. 264.

2. Livre des rois, publié, traduit et annoté par Jules Mohl. Paris, 1838, in-fol., vol. I, p. 489.

3. Félix Lajard, Recherches sur le culte du cyprès pyramidal. (Mémoires de l'Académie des Inscriptions, vol. XV, 2, p. 74.) 
l'époque fabuleuse que lui assigne Moöse de Khoren, n'en remonte pas moins à une haute antiquité.

Mème spectacle au nord-est de l'Tran, dans le Cachemir; les roses de ce pays sont célèbres et leur éclat et leur beauté, dit Georges Forster ${ }^{1}$, sont depuis longtemps passés en proverbe dans l'Orient. Chaque année les habitants célèbrent par de grandes réjouissances l'époque où les boutons de rose commencent à s'épanouir. Ce jour là, raconte-t-on, des jeunes gens et des jeunes filles parcourent les rues des corbeilles de roses à la main, et jettent leurs fleurs aux passants; celui qu'ils atteignent leur doit un présent et il le donne d'autant plus volontiers, qu'ètre touché par une rose est réputé porter bonheur. Les roses de la vallée de Péchawer, dans le Caboul, n'étaient pas moins renommées que celles du Cachemir; en y entrant, Babour, dit-on ${ }^{2}$, fut rempli d'admiration à la vue de celles qu'il y apercut.

La rose à cent feuilles devait aussi pénétrer dans la presqu'ìle hindoustanique; mais le nom qu'elle y porte, gulab ${ }^{3}$, trahit son origine étrangère et occidentale; c'est de l'Iran qu'elle a été importée au delà de l'Indus et qu'elle y est venue disputer au lotus son empire si longtemps incontesté. Mais elle n'a dû y pénétrer que depuis l'établissement, au

1. Voyages du Bengale à Pétersbourg..., traduit de l'anglais par L. Langlès. Paris, 1802, in-8, vol. I, p. 29 '.

2. Ritler, Erdliunde von Asien, vol. VI, p. 558.

3. William Roxburgh, Flora indica. Serampore, 1832, in-8, vol. II, p. 513 . 
$\mathrm{xI}^{\mathrm{e}}$ siècle, des Ghaznévides dans le nord-ouest de la Péninsule; ce n'est même qu'à une époque relativement récente, au $\mathrm{xvir}^{\circ}$ siècle, qu'on en trouve mentionnée la culture. Tahcîn-Uddin, poète hindoustani de cette époque, dans le récit des Aventures de Kâmrûp, a eu soin de mettre des roses et des lis avec des hyacinthes et des violettes dans le jardin de la princesse Kala, que son héros voit en songe ${ }^{1}$, et Mir Haçan de Dehli, écrivain du mème siècle, dans la description du Gulzar-i-Iram (Jardin d'Iram) ${ }^{2}$, parle aussi de la vue attrayante des roses qu'il y apercut avec des milliers de tulipes.

\section{II.}

Tandis que la culture de la rose était poussée si loin, dans les pays musulmans, que devint-elle, au moyen âge, chez les nations chrétiennes? Fut-elle, comme on l'a supposé ${ }^{3}$, inconnue, au moins de

1. Les aventures de Kâmrûp, trad. par M. Garcin de Tassy. Paris, 1834, in-8, chap. III, p. 16. Au chap. XXII,p. 130, il est aussi question des " jardins embellis par les fleurs demi-ćpanouies de la rose rouge et blanche. "Il y là, sans doute, une licence poétique, car les fleurs que Tahcin-Lddin place ainsi dans les jardins de Ceylan n'appartiennent pas à la flore de cette contrée, mais sil n'en avait pas vu dans l'Hindoustan, il n'aurait point eu lidée d'en parler dans ses fictions.

2. Garcin de Tassy, Histoire de la littérature hindoui et hindoustani. Paris, in-8, vol. II, 1847, p. 493.

3. Schleiden, Die Rose, p. 195. 
plusieurs d'entre elles, jusque vers la fin de cette époque? Le fait est déjà invraisemblable à priori; il est de plus en contradiction absolue avec le témoignage formel des contemporains. Il est difficile de mettre en doute que les roses dont ont parlé, au $\mathrm{v}^{0}$ siècle, l'Africain Dracontius ${ }^{1}$ et le Gaulois Avitus ${ }^{2}$, au siècle suivant Ennodius ${ }^{3}$ en Italie, Grégoire de Tours $^{4}$ et Fortunat ${ }^{\circ}$ en Gaule, ainsi que, au vir ${ }^{\circ}$ siècle, Isidore de Séville ${ }^{6}$ et même l'Anglo-saxon Aldhelm ${ }^{7}$, - je reviendrai plus loin sur ce qu'ils en ont dit, - aient bien été la fleur qu'avaient connue et chantée les anciens, et qui avait continué d'être cultivée d'une manière ininterrompue, quoique non générale peut-être, depuis les derniers temps de l'Empire romain.

Si ce n'est là qu'une induction, on ne peut en contester la légitimité, et l'on peut dire que, si la culture de la rose fut, conséquence de la destruction de l'Empire, négligée dans l'Europe romane, durant les premiers siècles du moyen âge, elle ne disparut pas plus dans l'Occident que dans l'Orient; elle devait d'ailleurs bientôt y ètre remise en honneur, et avec la culture des jardins, elle allait même, sous

1. Carmen de Deo, lib. I, v. 437.

2. De mundi initio, r. 13 f.

3. Carm. XLIII, v. 146 ; CLXIV, v. 11. (Monumenta germaniae historica, vol. VII, p. 14.)

'. Hist. Francorum, lib. VI, cap. 4́, B.

5. Carm., lib. VI, 8 ; lib. XI, 11.

6. Etymol. lib. XVII, cap. 9. De rebus aromaticis, no 17.

7. De laudibus virginum, éd. Migne, p. 241 D, 242 в. 
son nom latin, pénétrer bientôt chez toutes les nations germaniques.

Les ordres religieux contribuerent pour une large part à cette diffusion de la rose. Au milieu des ruines dont linvasion des Barbares courrit le sol de l'Empire, le clergé recueillit les débris de la civilisation antique, et les monastères, qui s'élevèrent peu à peu dans toute l'Europe chrétienne, lui servirent d'asile et de refuge. Construits le plus souvent dans des sites, qui témoignaient, chez leurs fondateurs, d'un véritable sentiment des beautés de la nature $^{1}$, on y trouvait toujours, à côté de spacieux bâtiments, un jardin destiné aux besoins du couvent ${ }^{2}$; s'il renfermait avant tout des légumes, qui servaient a la nourriture des cénobites, des arbres qui leur donnaient des fruits et de l'ombre, avec des herbes aromatiques ou médicinales cultivées pour les remèdes qu'elles fournissaient, on y trouvait aussi quelques fleurs destinées - l'expression est d'Albert le Grand ${ }^{3}$ - au plaisir des yeux et de l'odorat, ainsi qu’a parer les autels aux jours de fètes; comment parmi celles-ci aurait-on oublié le lis et la rose, que leur signification symbolique associait si naturellement au culte? C'est ainsi que ces fleurs, d'origine orientale, pénétrèrent, au moyen âge, dans tout

1. Ferdinand Cohn, Die Geschichte der Gärten. Berlin, 1856, in- 18, p. 36.

2. Charles Blanc, Grammaire des arts du dessin. Paris, 1867, in-8, liv. I, chap. xxri, p. 328.

3. "Ob delectationem.... visus et odoratus ". Alberti Magni, De segetabilibus, lib. VII, tract. I, cap. 14, 119. 
l'ouest et le nord, comme à l'époque romaine, elles avaient pénétré dans le sud de l'Europe; déjà au temps d'Aldhelm elles paraissent avoir été connues en Angleterre. Elles le seront bientôt aussi en Allemagne.

Du jardin des monastères la rose et le lis ne tardèrent pas à pénétrer dans celui des burges et des chatteaux des grands. Dans leur admiration pour la civilisation romaine, les rois mérovingiens ne pouvaient manquer d'en imiter le luxe; celui des jardins ne leur fut pas étranger. Fortunat a chanté le jardin de la reine Ultrogothe, veuve de Childebert, "où l'air, dit-il ${ }^{1}$, est embaumé du parfum des roses du Paradis ». Les soucis de la guerre et le soin de leur grandeur laissèrent peu de loisirs aux premiers Carolingiens pour s'intéresser à la culture des jardins; mais bientôt les choses changèrent.

Charlemagne, qui, dans ses expéditions en Italie, arait puisé le goùt des constructions somptueuses, y puisa aussi, il semble, celui des jardins; ils embellirent, dit-on ${ }^{2}$, sal résidenee d'.ix-la-Chapelle; toutes

1. Paradisiacas spargit odore rosas.

Carm. lib. VI, 6. De horto Ultrogothonis reginae, v. 2.

2. Arthur Mangin, Histoire des jardins. Tours, 1888, in-8, p. 70. Malheureusement II. Mangin, qui ajoute Ingelheim, ne dit pas où il a puisé ce renseignement. Ermoldus Nigellus, qui vante le palais d'Ingelheim, ne parle pas de ses jardins et il se borne mème à dire de la résidence impériale d'Aix-la-Chapelle qu'elle était entourće d'arbres el d'un frais gazon.

Consitus arboribus, quo viret herba recens.

Carmen in honorem Hludosici, lib. III, v. 586. Ed. Ern. Duemmler. 
les métairies impériales en possédèrent. Dans un de ses capitulaires ${ }^{1}$, le grand empereur n'a pas dédaigné d'énumérer les espèces régétales qu'on y devait cultiver; à côté des plantes potagères et médicinales, ainsi que des arbres fruitiers dont la place $y$ était marquée d'avance, il eut le soin de recommander d'y mettre des lis et des roses ${ }^{2}$. Il s'agit évidemment ici, quoi qu'en ait dit Schleiden ${ }^{3}$, de la rose à cent feuilles; tous les historiens de la botanique, depuis Sprengel jusqu'à Ernst Meyer, sont unanimes sur ce point: comment supposer que Charlemagne aurait pu, ainsi que le prétend l'auteur de La Rose, recommander de planter dans les jardins de ses villas l'églantier, qui croît au bord de tous les chemins, et dans toutes les haies de l'Allemagne et de la France? Yon, c'est bien la rose cultivée, - la cent feuilles - qu'il avait en vue dans ses prescriptions, la mème qu'à cette époque également, Alcuin célébrait comme l'ornement, avec le lis, de l'humble

1. "Capitulare de villis imperialibus ", cap. 70. (Migne, Patrol., vol. XCVII, p. 358.)

2. "Volumus quod in horto omnes herbas habeant, id est lilium, rosas, etc. »

3. Die Rose, p. 196. D'après lui il serait question dans ce capitulaire, non de la $R$. centifolia. mais de la $R$. canina, parce que "Charlemagne n'y recommande pas la culture d'une seule plante qu on ne doive considérer que comme plante d'ornement. ") Je ne rois pas dans quel autre but cependant on aurait planté le lis, dont la culture est recommandée par le monarque franc, comme celle de la rose. 
jardin de sa cellule ${ }^{1}$, mais qui était loin, ainsi que le montre l'inventaire dressé, en 812, par l'ordre du monarque franc ${ }^{2}$, de se trouver dans tous ceux des résidences impériales.

C'est encore évidemment la rose cultivée ou à cent feuilles que Walafrid Strabus a chantée, au $\mathrm{rx}^{\mathrm{e}}$ siècle, dans son Hortulus ${ }^{3}$ comme la "fleur des fleurs ». Cette qualification ne peut guère convenir qu'à cette espèce, et le sens mystique qu'il lui attribue empêche de voir dans ses vers, comme on l'a prétendu ${ }^{4}$, un simple pastiche ou une amplification des louanges données à la rose par les poètes de l'antiquité.

On ne peut douter non plus que ce ne soit bien la rose double que l'auteur anonyme d'une traduction

1. Lilia cum rosulis candida mixta rubris.

Carm., 23. (Poetae lat. medii aevi, éd. Ern. Dümmler. Berlin, 1881, in-8, vol. I, p. 243.)

2. Beneficiorum fiscorumque regalium describendorum formulae, ap. Pertz, Monumenta Germaniae historica, legum tomus I, p. 180.

3. "Hortulus ad Grimaldum." Migne, Patrol., vol. GXIV, no 24, p. 1123.

4. Schleiden, Die Rose, p. 199. "Walafridus Strabus hat nur (!) das Wort "Rose » und macht dazu aus den Versen der Alten einige neue Verse. " Est-ce dans "les vers des Anciens» que Walafrid a trouvé l'éloge de la Vierge et du Christ? On serait tenté de croire que Schleiden n'a pas lu l'Hortulus qu'il traite avec tant de dédain. Biese (Die Entwickelung des Naturgefühls im Mitlelalter, Altona, 1888, in-8, p. 81), qui l'a lu, n'a pas hésité à reconnaitre ce quill y a de personnel et d'original dans les descriptions de ce petit poème. 
de la Genèse en rieil allemand, faite au $\mathrm{x}^{\mathrm{e}}$ siècle ${ }^{1}$, place, avant tout autres plantes, avec le lis, dans le Paradis terrestre, dont il a fait un jardin semblable à ceux qu'on voyait dans tous les coủvents de l'époque.

L'éloge que Macer Floridus fait de la rose, dans son traité des "Vertus des simples " ${ }^{2}$; ce qu'il dit de ses propriétés curatives ne pent s'appliquer aussi qu'à l'espèce cultivée. Il en est de mème de la rose, dont l'abbesse de Saint-Rupert près Bingen, Hildegarde, a décrit au $\mathrm{xiI}^{\mathrm{e}}$ siècle, dans son livre Des Plantes $^{3}$, les propriétés médicinales. Hildegarde ne parle pas par oui-dire, mais en connaissance de cause; c'est la religieuse habituée à préparer des remèdes qu'on entend ici; on ne peut mettre en doute dès lor's qu'elle n'ait récllement connu, sinon mème cultivé, les roses dont elle indique l'emploi et les vertus ${ }^{4}$; et cette circonstance qu'elle traite aussitot après des propriétés du lis montre encore que dans la première de ces deux plantes, il ne peut

1. E.-G. Graff, Diutiska. Denkmäler deutscher Sprache und Literatur. Stuttgart, 1829, in-8, vol. III, p. 48.

2. De herbarum viribus, cap. xxı. Parisiis, 1506, in-18, c. 3. Bibl. Méjanes, G. 1953.

3. Liber subtilitatum diversarum naturarum creaturarum et sic de aliis quammultis bonis. Physica. Lib. I. De plantis, cap. 22. (Migne, Patrol., vol. CXCVII, p. 1139.)

4. On devine que ce n'est pas là la manière de voir de Schleiden; pour lui Hildegarde n'a pas vu les plantes dont elle parle, et elle n'a fait que copier dans d'autres ourrages des mots qu'elle ne comprend pas. Il est impossible de pousser plus loin le parti pris. Cf. Die Rose, p. 220, note 246. 
être question pour la pieuse et docte abbesse que de la rose à cent feuilles, inséparable depuis si longtemps du lis dans la culture des jardins, comme dans la poésie.

Tous les auteurs du moyen âge, qui se sont occupés d'horticulture, ont parlé de ces deux fleurs. Dans l'énumération que l'un des plus anciens, Alexandre Neckam, a faite ${ }^{1}$ des plantes que devait, suivant lui, renfermer un jardin plus idéal, il est vrai, que réel, et que Thomas Wright ${ }^{2}$ a eu le tort de regarder comme le modèle d'un verger anglais au xn ${ }^{e}$ siècle, - on y voit des arbres tels que le citronnier et le dattier, qui n'ont jamais pu ètre cultivés dans un parterre de la Grande-Bretagne, - le religieux anglais a, lui aussi, mis au premier rang des rares fleurs qu'il connaissait, les roses et les lis, auxquels il a joint la violette ${ }^{3}$ et, chose singulière, la fabuleuse mandragore, ainsi que la pivoine qu'il paraît ranger parmi les herbes aromatiques.

1. Hortus ornari debet hinc rosis et liliis, solsequio, violis et mandragoris, inde petroselino, et costo, et maratro, et abrotano, et coriandro, salvia, et satureia, hysopo, menta, ruta, ditacmo, ..... pionia. " De naturis rerum, etc., edited by 'Thomas Wright. London, 1863, in-8, lib. II, cap. 166, p. 274. (Rerum Britannicarum medii aevi scriptores, vol. XXXIV.)

2. A history of domestic manner's and sentiments in England during the middle ages. London, 1862, in-8, p. 297.

3. Au lieu de violis, Th. Wright avait lu d'abord molis, mot qui n'exíste pas plus que ortulano, qui n'est que l'adjectif qualificatif de nasturtio (du cresson de jardin), mais dont il a fait, par une singulière inadvertance, un nom de plante. 
Jean de Garlande ${ }^{1}$, au siècle suivant, avait aussi, dans son jardin, qui semble avoir été celui d'un bourgeois de Paris, à cette époque, des roses et des lis, ainsi que des violettes. C'étaient là les principales et presque les seules fleurs d'agrément cultivées de son temps. Albert le Grand n'en mentionne guère davantage. On ne s'occupait à peu près alors que de la culture des plantes aromatiques et potagères avec celle de quelques arbres fruitiers. Alexandre Neckam en a donné une liste assez longue, Albert le Grand a suivi son exemple; mais il a fait plus; il nous a laissé une description précieuseje pourrais dire scientifique - du jardin, tel qu'on le comprenait et qu'il existait alors ${ }^{2}$.

L'horticulture avait fait bien des progrès depuis les premiers siècles du moyen âge; les expéditions en Orient avaient révélé l'existence d'espèces végétales, jusque-là inconnues dans l'Occident, et dont quelques-unes y furent importées; Thibault IV, comte de Champagne, en particulier, rapporta, diton $^{3}$, de Syrie la rose de Provins cultivée jusqu'en ces

1. Dictionarium, no LXXIII, ap. Géraud, Paris sous Philippe le Bel. Paris, 1837, in-8, p. 609.

2. De vegetabilibus libri VII, historiae naturalis pars XVIII. Editionem criticam ab Ernesto Meyero coeptam absolvit Carolus Jessen. Berolini, 1867, in-8, p. 636-38, lib. VII, tract. I, cap. 1'́.

3. "Il alla au secours des chrétiens... et rapporta de la TerreSainte la fameuse rose rouge dont nous voyons l'espèce se perfectionner à Provins. "Félix Bourquelot, Histoire de Prosins. Provins. 1839-'10, in-8, p. 179. "Le plant en a été apporté de la 
dernières années dans cette ville et aux environs. Ce ne fut pas là sans doute un fait isolé. Le sentiment croissant de la nature dont témoignent les ouvres des poètes contemporains, comme le bien-être grandissant, ne pouvaient que développer le goût des jardins. Chaque demeure seigneuriale ${ }^{1}$ et, bientôt, chaque habitation bourgeoise en possédèrent un, comme chaque monastère. C'est dans le "verger " féodal que se déroulent le plus souvent les scènes héroïques ou gracicuses décrites par les poètes. Les assemblées les plus graves s'y tenaient, comme les réunions les plus gaies et les plus joyeuses. Le jardin occupait dans la vie tout entière une place trop grande pour que les écrivains du moyen âge n'aient point songé à 'le faire connaître. Albert le Grand n'y a pas manqué.

Le savant allemand a consacré un chapitre de son traité des végétaux à la "plantation du verger ${ }^{2}$. "

Syrie par Thibaut VI et ce n'est qu'à Provins que ces fleurs ont conservé la belle couleur pourpre et le parfum qui leur sont propres, ainsi que toutes les propriétés médicinales. ) Christ. Opoix, IIstoire et description de Provins, 2 e édit., 1846, in-8, p. 388. Malheureusement Opoix ne cite aucun document, ce qui eủt bien mieux valu que de chercher à identifier la rose de Provins avec les " roses milésiennes » de Pline, ou ce qui est encore plus étrange, avec les prétendues "roses de Saron » du Cantique des Cantiques.

1. "Der Garten fehlte an keiner Burg." Alvin Schultz, Das höfische Leben zur Zeit der Minnesinger. Leipzig, 2c éd., 1888, vol. I, p. 43.

2. Lib. VII. tract. I, cap. 14, par. 119-125, " De plantatione viridariorum. »" 
Il comprendra d'abord, dit-il, un gazon d'une herbe fine, soigneusement sarclé et foulé aux pieds, vrai tapis de verdure, dont rien ne doit dépasser l'uniforme surface ${ }^{1}$. A l'une de ses extrémités, du côté du midi, se dresseront des arbres: poiriers, pommiers, grenadiers, lauriers, cyprès et autres de ce genre, où s'enlaceront des vignes, dont le feuillage protégera en quelque sorte le gazon et fournira une ombre agréable et fraîche ${ }^{2}$. Derrière le gazon, on plantera en quantité des herbes aromatiques et médicinales, par exemple la rue, la sauge, le basilic, dont le parfum viendra réjouir l'odorat, puis des fleurs, telles que la violette, l'ancolie, le lis, la rose, l'iris et d'autres semblables ${ }^{3}$, qui par leur diversité charment la vue et excitent l'admiration ${ }^{4}$. Enfin, à l'extrémité du gazon, dans l'espace réservé aux fleurs,

1. "Caespite macro subtilis graminis totus locus impleatur... et conculcentur gramina a pedibus in terram, donec... vix aliquid de ipsis possit considerari ; tunc enim paulatim erumpent capillariter et superficiem ad modum panni viridis operient. » 120.

2. "In caespite etiam contra viam solis plantandae sunt arbores aut vites ducendae, ex quarum frondibus quasi protectus caespis umbram habeat delectabilem et refrigerentem... piri et mali et mala punica et lauri et cypressi et hujusmodi. „ 122.

3. «Per quadratum aromaticae herbae, sicut ruta et salvia et basilicon plantentur, et similiter omnis generis flores, sicut viola, aquilea, lilium, rosa, gladiolus et his similia. ”

4: “Post caespitem sit magna herbarum medicinalium et aromaticarum diversitas, ut non tantummodo delectet ex odore secundum olfactum, sed et flores diversitate reficiant visum, et ipsa multimoda sui diversitate in admirationem trahant se aspicientes. » 123. 
Albert le Grand recommandait de relever le terrain de manière à y former un siège verdoyant et "fleuri », où l'on pourrait venir s'asseoir et se reposer doucement l'esprit ${ }^{1}$.

Tel était, d'après le célèbre dominicain, le verger ou jardin d'agrément du moyen âge. Qu'on le restreigne a l'espace planté d'herbes aromatiques et de fleurs, en y joignant quelques légumes, oignons, poireaux, ail, bettes, melons, concombres et autres ${ }^{2}$, et l'on aura le jardin d'un bourgeois du xim ${ }^{\mathrm{e}}$ siècle, comme celui de Jean de Garlande, comme l'était encore à peu près, à la fin du siècle suivant, celui de l'auteur du Ménagier de Paris ${ }^{3}$ - lequel, outre les quelques fleurs déja mentionnées, cultivait aussi la lavande et la giroflée, plantes inconnues avant lui, mais qu'on trouve désormais dans tous les jardins. Qu'on agrandisse, au contraire, ce verger, qu'une fausse poterne y conduise de la demeure seigneuriale ${ }^{4}$, qu'on l'entoure d'une enceinte de murs, qu'un parc peuplé de fauves et où retentit le chant des oiseaux, s'y joigne

1. "Inter quas herbas et caespitem in extremitate caespitis per quadratum elevatior sit caespis florens et amoenus et quasi per medium sedilium aptatus, cum quo reficiendi sunt sensus et homines insideant ad delectabiliter quiescendum. „ 121.

2. De naturis rerum, lib. II, cap. 166, p. 274.

3. Le ménagier de Paris, traité de morale et d'économie domestique composé vers 1393 par un bourgeois parisien.. Paris, 18'6, in-8, vol. II, p. 43.

4. Et desfreme un guicet d'une fauce posterne Par ou ele sieut issir et les soies pucheles, Quant vient el mois de mai, por colir la florete. Elie de Saint-Giles, v. 1405-1407. 
à l'occasion, enfin qu'une source limpide l'arrose, et l'on aura "l'enclos ${ }^{1}$ " attenant à tout château féodal, arec ses arbres fruitiers, ses bosquets et ses quelques fleurs, en particulier les roses et les lis, tel que nous le décrivent les poèmes chevaleresques ${ }^{2}$.

Albert le Grand n'a pas seulement le mérite de nous avoir fait connaitre le rerger du moyen âge, avec ses herbes et ses fleurs; le premier écrivain de cette époque, il nous a laissé, dans son traité des Plantes ${ }^{3}$, une description complete et exacte dans ses traits généraux des roses qu'on y cultivait; le premier il les a nettement distinguées des roses saurages. Après quelques renseignements sur la nature du rosier, son port et ses dimensions, Albert ajoute $^{4}$ :

1. Léon Gautier, La Chevalerie. Paris, 1889, in-8, p. 526.

2. Par exemple le Karl Meynet, p. 184, v. 1.

An eynen bungart fier

Dar standen lilien und rosiere

Zederboum und Olyvere

Und ander gode bome vele.

On peut rapprocher de cetie description la définition qu'llugues de Saint-Victor fait du jardin : "Ortus circumfoditur... arborum distinctione ornatus, floribus jucundus, gramine viridante suavis... umbrarum amoenitatem praestans, murmure fontis delectabilis, fructibus variis refectus, volucrum cantu laudabilis. „). De bestiis et aliis rebus. lib. IV, cap. 13. (Migne, Patr., rol. CLXXVII, p. 154.)

3. Lib. VI, tract. I, cap. xxxil, par. 212-216. Ed. E. MeyerJessen, p. 445-47.

4. "Flos ejus vocatur rosa, et est flos primum habens siliquam viridem quinque foliorum, quae cum aperitur, egreditur rosa 
“ On donne le nom de rose à sa fleur; cette fleur est renfermée dans un calice à cinq sépales verdâtres; quand il s'ouvre, apparaît une corolle composée d'un grand nombre de pétales, si c'est une rose des jardins, en particulier la rose blanche, qui a souvent jusquà cinquante ou soixante pétales, tandis que la rose sauvage n'en compte que cinq. "

Quelle est cette rose sauvage ou “champêtre» que le dominicain allemand oppose d'une manière si expresse à la "rose des jardins"? A-t-il connu de l'une ou de l'autre plusicurs espèces ou variétés? Albert a été frappé, et cela était naturel, par le grand nombre de pétales de la rose cultivée à fleurs blanches; il revient à deux reprises sur ce caractère. Il a été également frappé par les dimensions qu'acquiert le rosier qui la porte: “C'est un arbre, dit-il ", dont le trone atteint parfois la grosseur du bras; il est très rameux et les branches en sont touffues, mais longues et minces. ") Quant à la rose à fleurs purpurines, Albert n'en parle qu'indirectement, en paraissant dire qu'elle a moins de pétales que la rose blanche et il se borne à remarquer, comme en passant, que

multorum foliorum, quando est hortensis, et maxime rosa alba, quae frequenter excedit numerum quinquaginta foliorum vel sexaginta. Sed tamen in campestri rosa non inveniuntur nisi quinque folia. "Op. laud, lib. VI, cap. xxxi, 213.

1. "Illa quae fert rosas albas multorum valde foliorum, pro certo arbor est, cujus stipes efficitur sicut brachium hominis... Et est arbor valde ramosa; et sunt rami eius spissi, sed parvi el ongi sicut surculi rubi. „ Cap. xxxrr, 212. 
sa fleur d'abord verte devient rouge à la fin ${ }^{1}$. Il n'y a là rien, on le voit, qui permette d'en déterminer l'espèce. Il peut se faire qu'il s'agisse de la $R$. gallica, comme de la centifolia. Pour la rose à fleurs blanches on serait tenté de l'identifier arec la R. alba de Linné ${ }^{2}$; mais il est impossible de rien affirmer. Ceci n'a d'ailleurs qu'un intérêt secondaire; ce qui importe, c'est que la rose double, Albert le Grand nous l'apprend, était cultivée en Allemagne au xin ${ }^{\circ}$ siècle, et qu'elle y produisait les fleurs les plus belles. Le fait sans doute n'était pas nouveau; Albert ne le donne pas non plus comme récent; mais il est intéressant de le trouver aussi incontestablement, quoique si tardivement, constaté.

Ce qui n'offre pas moins d'intérèt, c'est que le savant encyclopédiste distingue nettement, ce que n'avaient point fait les anciens et ce qu'avaient fait si mal les écrivains arabes, plusieurs espèces de roses sauvages. Dans la " rose champêtre » opposée par lui, à cause du nombre de ses pétales, à la rose des jardins ou cultivée, Carl Jessen a reconnu, avec toute raison, je le crois, la " rose des champs »Rosa arvensis de Linné ${ }^{3}$. - Cette tige unique -

1. «Flos rosae incipit primo a virore et terminatur in ruborem. "Cap. xxxir, 213.

2. C'est ce que fait Jessen, tandis qu'il n'assigne aucun nom particulier à la rose rouge d'Albert le Grand.

3. Ernst Meyer, dans son histoire de la botanique, avait cru pouvoir lidentifier arec la $R$. villosa. (Geschichte der Botanik. Königsberg, 1857, in-8, vol. IV, p. 73). 
culmus unus -, qui se dresse au milieu de la fleur ${ }^{1}$, paraît bien désigner les styles soudés en colonne de cette espèce, que le pollen vient, au moment de la fécondation, recouvrir d'une poussière jaunâtre respersio crocea. - Le fruit arrondi de cet églantier est bien aussi celui de la Rosa arvensis.

Mais Albert le Grand connaissait encore deux espèces de roses saurages auxquelles il ne donne pas, il est vrai, le nom de rose, - il les appelle l'une bédégar, l'autre tribulus, - et qu'il a, on ne sait trop pourquoi, dans un chapitre a part, décrites comme des épines et séparées de la rose des jardins, à laquelle il a, au contraire, réuni la rose des champs, quoiqu'elle ne lui ressemble pas darantage. Il ne lui est pas échappé néanmoins tout ce qui les rapprochait, au moins la première, de la "rose champêtre " et de la rose des jardins.

" La rose, disait-il , en commençant la description de cette dernière, est un arbre ou un arbrisseau, pourvu de nombreuses épines, tout comme le bédégar, auquel elle ressemble d'ailleurs par la forme de ses feuilles. Mais les épines de la rose sont plus faibles et ses feuilles plus larges que celles du bédégar.

Et en parlant du fruit de la rose champètre, Albert

1. "In medio ejus (rosae campestris) est respersio crocea, stans in culmo uno simul. » Cap. xxxir, 213.

2. "Rosa est arbor aut frutex cum spinis multis sicut et bedegar, cui etiam per omnia habet folia similia. Sed spinae rosae debiliores sunt et folia eius latiora, quam folia bedegar. ") Cap. xxxir, 212. 
remarquait ${ }^{1}$ qu'il était " fait comme celui du bédégar, seulement qu'il était plus arrondi ».

Le savant encyclopédiste avait insisté déjà, dans sa description du bédégar, sur la ressemblance de cet arbrisseau et du rosier.

"Ses feuilles, remarque-t-il ", ainsi que la fleur et le fruit ressemblent à ceux du rosier, mais la fleur est plus petite. "

\section{Et il ajoute ${ }^{3}$ :}

Les feuilles exhalent, surtout au printemps et quand elles sont fraîches, une odeur vineuse.

Ce dernier caractère ne permet pas de se méprendre sur la nature du bédégar; par ce nom Albert le Grand désigne évidemment le rosier odorant ou rouillé - la $R$. rubiginosa de Linné, la Weinrose des Allemands, la sweet briar des Anglais, - arbuste auquel, par une confusion étrange, il a attribué le mot arabe, qui désigne, nous l'avons vu, l'espèce de galle produite sur l'églantier par la piqùre d'un ichneumon, le Cynips rosae.

Il n'est pas plus difficile d'identifier l'espèce de rosier sauvage auquel Albert a assez singulièrement

1. "Cum perficitur pomum eius, est sicut pomum bedegar, nisi quod est rotundius illo. » Cap. xxxir, 213.

2. "In foliis similis est rosario et similiter in flore et fructu, nisi quod flos ejus parvae est latitudinis. » Lib. VI, cap. xI, 42 , p. 358.

3. "In foliis praetendit quasi odorem vini et maxime in vere, quando recentia sunt folia. » 
donné le nom de tribulus, mot qui sert d'ordinaire à désigner la macre ou châtaigne d'eau.

C'est, dit-il ${ }^{1}$, une autre espèce d'épine, plus robuste que le bédégar, mais qui lui ressemble par la forme de ses feuilles et de ses épines; sa fleur aussi est plus grande. Quant à son fruit il est plus allongé que celui du bédégar ou de la rose. Mais sous le rapport de la couleur et des graines, les fruits de la rose, du bédégar et du tribulus - pourquoi n'a-t-il pas ajouté de la rose champêtre? - sont entièrement semblables.

Albert, tout en disant, erreur assez peu explicable, que le tribulus n'est pas vraiment de la nature de la rose, ajoute néanmoins qu'on lui donne parfois le nom de rose sauvage ${ }^{2}$. On se serait attendu à ce qu'il eût dit "rose de chien », - Rosa canina, car c'est évidemment de cette espèce si commune qu'il s'agit ici.

Nalgré ce qu'il y a d'incomplet et d'inexact dans les descriptions d'Albert le Grand, elles témoignent, le fait est incontestable, d'une observation personnelle de la nature. C'est un spectacle curieux de voir le dominicain du $\mathrm{xm}^{\mathrm{e}}$ siècle, devançant les

1. "Est aliud genus spinae, quod quidam vocant tribulum, quod est maioris ligni quam bedegar, sed in foliis et spinis est isti simile; et flos eius latior est quam flos istius... Pomum autem illius est longius quam pomum bedegar vel rosae. Sed in colore et granis sunt similia pomum rosae et bedegar et tribuli. „) Cap. Ix, 43.

2. "Hoc quidam vocant rosam silvestrem, sed non est vere de natura rosae. » Cap. Ix, 43. 
botanistes modernes, s'appuyer, pour distinguer les différentes espèces de roses, sur la forme du fruit. Ce qu'il remarque de la situation de la fleur par rapport à celui-ci ${ }^{1}$, ainsi que des divisions du calice, des laciniures qu'elles présentent d'un côté, de leur alternance avec les pétales ${ }^{2}$, de leur persistance enfin, tant que le fruit n'est pas arrivé à maturité ${ }^{3}$, est nouveau et original; on n'avait rien dit de semblable depuis Théophraste et Théophraste était loin d'avoir si bien et tant observé. Toutefois il ne faut demander à Albert le Grand aucun renseignement particulier sur la culture de la rose 4 , mais il a exposé longuement, il est rai presque exclusivement d'après Aricenne, les propriétés médicinales de cette fleur ${ }^{5}$.

1. "Suus flos super pomum suum sicut in cucurbite et malo granato. " Cap. xxxir, 213.

2. "Quodlibet foliorum (siliquae) ex una parte est barbatum pluribus barbis... et in alia parte est planum sine barbis... Sub qualibet comparinatione duorum foliorum siliquae subjicitur recte medium dorsum unius folii rosae. " Cap. xxxir, 21'4.

3. "Siliqua rosae non cadit quidem cum foliis rosae, sed cadit, quando maturatur pomum ejus. „ Cap. xxxir, 215.

4. Je ne puis regarder comme tel, du moins, ce qu'Albert, sur l'autorité prétendue du fabuleux Hermès, dit de la manière d'obtenir des roses en hiver, laquelle consisterait à planter dans une terre mêlée de sang et arrosée avec du sang les rosiers dont on lierait les branches au printemps. Lib. VI, cap. xxxrr, 217, et lib. IV, cap. III, 160.

5. Cap. xxxur, 216. Dans ce chapilre Albert mentionne une " rose fétide », quiil ne parait connaître que par ouï-dire et dont la racine serait brủlante comme le pyrèthre. "Quaedam est quac 
Ce sont elles aussi qui avaient presque exclusivement fixé l'attention de l'auteur De la nature des choses $^{1}$, Thomas de Cantimpré. Dans ce traité, qui dut être écrit, comme l'a montré M. Léopold Delisle ${ }^{2}$, avant 124 et qu'il avait mis, dit-il, quatorze ans à composer, ce "compilateur $)^{3}$, - c'est le nom qu'il se donne lui-même -, n'a rien dit de la culture de la rose; il ne l'a pas décrite davantage: tout ce qu'on y trouve c'est que le "rosier est plutôt un arbuste qu'un arbre ${ }^{4} »$. On ne pouvait donner moins au point de vue botanique et l'on s'étonnerait de cette indigence de renseignements sur une fleur si bien étudiée par Albert le Grand, dont Thomas de Cantimpré avait été un instant le disciple, si le livre De la nature des choses, de l'élève, n'avait

nominatur rosa foetida et radix cius est sicut piretrum adustiva. ” G'est évidemment l'Ouard montin de Razès et d'Ibn el-Beithar, probablement l'Anison (anis), c'est-à-dire tout autre chose qu'une rose. Traité des simples, no 2276.

1. De natura rerum, tel est le titre que la plupart des manuscrits donnent à cet ouvrage; une note du manuscrit 14720 de la Bibliothèque nationale lappelle De naturis rerum; c'est ainsi également que le manuscrit de Gotha, étudié par Ernst Meyer, (Geschichte der Botanik, vol. IV, p. 92), désigne ce traité.

2. Léopold Delisle, Histoire littéraire, vol. XXX, p. 377. Dès lors le De natura rerum dut ètre écrit arant le De vegetabilibus d'Albert le Grand, composé seulement après 1249.

3. « Revolvi autem librum illum de natura rerum, quem ipse multo labore per annos XIIII de diversis auctoribus utilissime compilavi. "Liber apum aut de apibus mysticis, s. 1. n. d., in-fol. (Incunable de la Méjanes, 18131).

4. “Rosa est potius frutex quam arbor. " Man. 14720, fol. 134. 
probablement été composé avant le Traité des plantes, du maître ${ }^{1}$.

Les livres du Miroir de la nature ${ }^{2}$, de Vincent de Beauvais, qui traitent du règne végétal, sont-ils aussi antérieurs à l'ourrage d'Albert le Grand sur le mème sujet? Cela est rraisemblable; du moins Vincent n'y cite point son illustre contemporain auquel il a tant fait d'emprunts dans son histoire des animaux. Composé d'extraits des auteurs qui araient dans l'antiquité ou durant les siècles précédents écrit sur la rose, les chapitres consacrés par Vincent à cette fleur ne renferment aucun renseignement nouveau ${ }^{3}$. Vincent n'a point essayé de la décrire, et il en est encore à Pline, pour ce qui regarde sa culture; il n'y a à apprendre chez lui quau sujet des usages longuement énumérés de la rose dans la médecine et des préparations pharmaceutiques qu'on en retire. Heureusement il en est tout autrement, au point de rue botanique, de ce qu'en a dit Barthélemy l'Anglais.

Dans le chapitre 1:36 du dix-septiéme livre de son traité Des propriétés des choses ${ }^{4}$, livre consacré aux

1. Cf. au sujet de la chronologie des œurres d Albert, Ernst Meyer, Op. laud., vol. IV, p. 33.

2. Speculum naturale. Duaci, 1624, in-fol. Lib. X-XV.

3. Lib. X. cap. 131, De Rosa; cap. 132, De rosarum cultura; cap. 133, De operatione rosae in medicina; cap. 134, Iterum de codem; cap. 135 , De his quae procedunt vel conficiuntur ex rosa. P. - 761-63.

4. Bartholomat Axglici de genuis rerum caelestium, terrestrium el infernarum proprietatibus libri XVIII, opus 
plantes, Barthélemy, comme Albert le Grand, s'est d'abord attaché ì distinguer la rose des jardins ou "domestique " de la rose saurage ou champètre.

“ La rose des jardins, dit-il ${ }^{1}$, différe de la rose sauvage par son parfum, sa couleur, les vertus et le nombre de ses fleurs." "Les pétales des roses sauvages, ajoutet-il ${ }^{2}$, sont planes, larges, d une teinte blanchâtre mêlée d'un peu de rouge; ils sont aussi moins odorants et moins efficaces en médecine. Les pétales de la rose des jardins, au contraire, sont nombreux, serrés et appliqués les uns contre les autres, entièrement rouges ou entierement blancs, d'un parfum exquis, d'une saveur stiptique et quelque peu mordante et d'une grande efficacité en médecine. ")

On a ici une description de la reine des fleurs, dont les principaux traits appartiennent bien à Barthélemy, et l'auteur anonyme du Pö̈me moralisé sur les propriétés des choses, dont M. Gaston Raynaud a publié des extraits, dans le tome XIV de

incomparabile, ac theologis, jurisconsultis, medicis, omniumque disciplinarum et artium alumnis utilissimum. Francofurti, 1619, in-8.

1. « Est autem rosa duplex, quia quaedam est domestica sive hortensis et quaedam est sylvestris. Differt autem rosa hortensis a sylvestri in florum multitudine, in odore, in colore et in virlute. » Op. laud., p. 913.

2. "Folia sylvestrium sunt plana, lata, subalbida, pauco rubore admixta, minus odorifera et minus operantia in medicina. Folia autem hortensis rosae sunt multa mutuo cohaerentia et connexa, omnino rubra vel omnino albissima, mire redolentia, in sapore stiptica et quodam modo mordicantia el magnae efficaciae in medicina. » 
la Romania ${ }^{1}$, n’a pas hésité à les lui emprunter, sans en conserver la précision et l'exactitude.

En ce que Barthélemy nous apprend de l'inflorescence et du développement de la rose de jardin, ainsi que de sa culture, il y a encore plus d'un trait ou d'un renseignement que le savant du moyen âge peut revendiquer comme sien. S'il dit, par exemple, que la rose se reproduit par semis, par bouture ou par greffe ${ }^{2}$, ce qu'on trouve déjà dans Pline, il remarque, ce qui n'est pas dans l'auteur latin et paraît dès lors être de lui, que "la rose sauvage peut donner par la culture et de fréquentes transplantations des "roses véritables " $^{3}$, c'est-ì-dire évidemment des roses doubles: preuve, il semble bien, qu'on était, dès cette époque, parvenu à anoblir et ì doubler les roses sauvages. »

1. Année 1885, p. 442-484.

Il (le rosier) est d'une double substance,

Si com demonstre l'apparance:

L'un en jardin croist, l'autre as chans.

De jardin est mieux odourans

La rose, que ne fait icele

Qui est as chans et est plus belle:

L'une est blanche, l'autre vermeille.

$$
\text { Rosier, v. 5-12, p. 455, Art. XXXI. }
$$

2. "Nascitur arbor rosae quandoque per seminationem, quandoque per plantationem, quandoque per insertionem.» Iljid., p. 914 . "Le rosier naist aucunes fois par semer et aucunes fois par planter », se borne à dire Jean Corbichon dans la traduction qu'il a donnée de l'ouvrage de Barthélemy.

3. "Agrestis rosa per frequentem mutationem et culturam efficitur vera rosa. » 
Une autre remarque qui n'est pas davantage dans Pline, et où, circonstance curieuse, Barthélemy se rencontre avec 'Théophraste, qu'il ne pouvait connaître, c'est que " la rose des jardins restée sans culture et non débarrassée de ses rameaux superflus revient à l'état sauvage ${ }^{1}$ ). Ne semble-t-il pas aussi qu'on entende l'amateur des jardins et des fleurs, quand Barthélemy dit ${ }^{2}$ que la rose, - raison pour laquelle elle sert à couronner la partie la plus noble de l'homme, c'est-à-dire la tête, - occupe le premier rang parmi les fleurs, à cause de sa beauté, de son parfum, de sa douceur et de ses vertus. “Car, ajoute-t-il ${ }^{3}$, par sa beauté elle réjouit la vue, par son parfum elle affecte agréablement l'odorat, par sa douceur elle plaît au toucher; enfin, grâce à ses propriétés médicinales, elle prévient ou guérit nombre d'indispositions et de maladies. »

Barthélemy l'Anglais est l'écrivain du moyen âge qui, avec Albert le Grand, fournit les renseignements les plus complets, comme les plus originaux, sur la rose. On aurait pu s'attendre à ce que, en sa qualité d'horticulteur et d'agronome, Pierre de Cirescence en aurait donné d'aussi étendus, sinon de

1. "Si remanserit inculta et a superfluis non purgata, degenerat in sylvestrem. )

2. "Flos rosae inter flores obtinet principatum et ideo solet principalis pars hominis, scilicet caput, rosarum floribus coronari et hac ratione decoris, odoris, suavitatis et virtutis. »)

3. "Nam sua pulchritudine aspectum reficit, suo odore olfactum afficit, suavitatis mollicie tactum delinit, sua virtute multis languoribus et morbis obviat et succurrit. ») 
plus importants; il n'en est rien; le savant italien, peut-être paree qu'il la regardait comme suffisamment connue ${ }^{1}$, s'est borné, dans son traité d'agriculture $^{2}$, à donner quelques vagues indications sur cette fleur.

"Rosiers, dit son traducteur français ", sont arbres bien congneuz et en sont les ungs blancs et les autres rouges, et aussi en sont les uns francs (cultivés) et les autres saurages. Les blancs, soient francs ou sauvages, font très bonnes et fortes haies pour ce quils ont bonnes branches et fortes espines et si se reteurdent et entrelacent en telle manière que l'on ne peult passer parmy pour les espines qui arrestent a force."

Puis, après quelques mots sur cet arbuste, - j'y reviendrai plus loin —, Pierre de Crescence termine, comme ses devanciers, par l'exposé des propriétés médicinales de la rose et des dirers produits pharmaceutiques qu'on en retire.

C'est peu sans doute; on n'en trouve pas davantage ou encore moins dans le Livre de la nature", de Conrad de Mesgenbers, "traduit du latin ", il

1. "Rosaria nota sunt », dit-il lui-mème p. 273.

2. De agricultura omnibusque plantarum et animalium generibus libri XII, auctore optimo agricola et philosopho Petro Crescentrexsi. Basileae, 1538, in-4, lib. V, p. 273.

3. Le livre des prouffitz champestres et ruraulx. Paris, 1486 , in-fol. p. I, ch. 22.

4. Konrad von Megenberg, Das Buch der Natur, hgg. von Franz Pfeiffer. Stuttgart, 1861, in-8. 
l'avoue lui-même ${ }^{1}$, vers le milieu du xiv ${ }^{\mathrm{e}}$ siècle, non toutefois d'Albert le Crand, comme il le croyait", mais de Thomas de Cantimpré, puisque le Liber de natura rerum, qu'il a suivi, est de ce dernier et non d'Albert, ainsi que l'avait avancé déjà Ernst Meyer ${ }^{3}$, et que l'a prouvé d'une manière irréfutable M. Léopold Delisle ${ }^{4}$; mais il a traduit ce traité avec une grande liberté, car on trouve dans sa version des passages et même des chapitres entiers, qui ne sont point dans l'original, comme celui-ci en contient en retour, mais en bien moins grand nombre, qu'on cherche en vain dans la traduction. De mème que son modèle, Conrad n'a point donné de description de la rose, ni parlé de sa culture; il s'est attaché seulement à en faire connaitre les propriétés médicinales; mais il y a joint, ce qu'on ne trouve pas dans Thomas de Cantimpré, une longue énumération des comparaisons mystiques qu'on a établies entre la reine des fleurs et la reine des cieux.

1. Alsô trag ich ein puoch von latein in däutsehen wort. P. 2, v. 6-7.

2. Daz hat Albertus meisterlich gesammet von den alten.

Une note de la dernière page du manuscrit 14720 de la Bibliothèque nationale, comme le manuscrit de Gotha dailleurs, attribue expressémont le Traité de la nature des choses à Albert: " Explicit liber de naturis rerum, quem composuit frater Albertus, ordinis fratrum Praedicatorum doctor eximius ). Daunou (Histoire littéraire, vol. XIX, p. 184), mettait encore en doute que le De natura rerum fùt de Thomas de Cantimpré.

3. Geschichte der Botanik, vol. IV, p. 200.

4. Histoire littéraire, vol. XXX, p. 377. Jonet. La Rose. 
L'ourrage de Conrad de Megenberg présente une autre différence avec celui de Thomas de Cantimpré; tandis que ce dernicr ne parle point de la rose sauvage, son imitateur allemand a consacré un chapitre à l'églantier, le bédégar ou hagedorn ${ }^{1}$. Sous ce nom, il désigne évidemment, comme Albert le Grand, le rosier rouillé ou odorant; car le "goût vineux », que les feuilles de cet arbuste, d'après lui ${ }^{2}$, ont " surtout au printemps », ne saurait s'appliquer qu'à cette espèce de rose. Outre le bédégar, Conrad connaît aussi un autre églantier, qu'il appelle velıdorn et qu'il en distingue. "Les fruits du premier, dit-il ${ }^{3}$, sont plus petits que ceux du second; il en est de même des fleurs ». Faut-il d'après cela identifier le veltdorn de Conrad avec le tribulus d'Albert le Grand, c'est-ì-dire avec la Rosa canina, comme l'ont pensé Schleiden ${ }^{4}$ et Pritzel-Jessen"? Il est difficile de se prononcer, mais j'inclinerais à voir dans eet églantier la $R$. arvensis L., dont les fleurs, sinon les fruits, sont plus grandes que celles de la R.rubiginosa.

Si Conradde Megenberg ne nous apprend rien au

1. "Bedegar haizet ain hagedorn oder weithagen ». Cap. IV, Von den Paumen, p. 316, 8.

2. "Des hagdorns pleter habent ainen weinsmack und allermaist in dem lenzen, die weil si new sint $)$.

3. «Der paum... hat pleter gleich aim rosendorn oder aim veltdorn, iedoch sint sein frülıt kleiner wan des veltdorns früht, ez sint auch sein ròsen kleiner wan des veltdorns rôsen ").

4. Die Rose, p. 199.

5. Die deutschen Volksnamen der Pflanzen. Hannover, 1882, in-8, p. 199. 
sujet de la culture de la rose, on ne trouve pas plus de renseignements à cet égard dans les Livres des simples remedes latins ${ }^{1}$, Ies Arboristes ou IIerbiers francais ${ }^{2}$, ainsi que dans les Arbolayres ${ }^{3}$ ou Grants Herbiers ${ }^{4}$, qui ne sont que la reproduction imprimée de ces derniers, ou dans l'Herbarius de Mayence" et le Jardin de santé ${ }^{6}$, l'un en haut-allemand, imprimé en 1485 , l'autre en bas-allemand, publié en 1492 à Lubeck. Issus tous d'une source commune, le Circa instans primitif de Platéaire, auquel sont venus s'adjoindre des emprunts faits aux médecins arabes ou aux encyclopédistes du xır siècle, ce sont des traités de pharmacopée; comme tels, ils énumèrent soigneusement les propriétés médicinales de la rose, mais ils ne nous apprennent rien sur son histoire ou sur sa culture.

Les témoignages si nombreux et d'ordre si divers qui précèdent ne laissent aucun doute sur l'existence

1. Par exemple, le Liber de simplici medicina ou Circa instans de Platearius, publié à Ferrare en 1488, et le Tractatus de herbis, manuscrit de Nodène, étudié par M. Jules Camus. (L'opera Salernitana Groca instans ed il testo primitivo del Grant Herbier en francors secondo due codici del secolo xiv conservati nella regia bibliotheva estense. Modena, 1886, in-4x).

2. Ainsi l'Herbier, Arboriste ou Traité des plantes des manuscrits du commencement du xve siècle, 1307, 9136, 12319 et 12320 de la Bibliothèque nationale.

3. Arbolayre contenant la qualitey et vertus, proprietey des herbes, arbres, gommes, et semences, etc., s. 1.n. d. in-fol.

4. Le Grant Herbier en francois contenant, etc. Paris, in-fol.

5. Herbarius cum herbarum figuris. Moguntiae, 1484, in-4.

6. Der ghenocliche Gharde d' Suntheit. 1485, in-4. 
de la rose double au moyen âge; e'est par un parti pris inexcusable que Schleiden ${ }^{1}$ et par une inadvertance peu explicable qu'Alvin Schultz, d'ordinaire si bien informé, en ont paru contester la présence à cette époque. Lorsqu'Alvin Schultz, par exemple, pour nier l'existence des roses doubles au temps des Minnesænger, s'appuie sur le témoignage de l'un d'eux ${ }^{2}$, lequel dit que, quand les roses auraient les étamines, - qui sont jaunes comme l'on sait, - aussi rouges que les pétales, la bouche de sa bien-aimée serait encore plus vermeille", le savant allemand oublie que dans les roses mème les plus donbles, à plus forte raison dans celles qui ne le sont qu'ì moitié, comme les roses de Provins en particulier, toutes les étamines ne sont pas transformées en pétales ${ }^{4}$. De ce que les

1. Die Rose, p. 195 : "Gänzlich unbekannt waren unseren Vorfahren die im Süden schon früh geschätzten gefüllten Spielarten, die erst ganz allmälig am Ende des Mittelalters sich nach und nach, erst in Frankreich und Holland, dann in Deutschland einbürgerten ». Cf. Hehn, Kulturpflanzen, p. 207: «Im Mittelalter... blieben Rose und Lilie, beide verhältnissmässig leicht zu erziehen, in den Gärten gewöhnlich. »

2. Das höfische Leben zur Zeit der Minnesinger, vol.I, p. 50.

3. Le poète grec Philé, qui vivait au $\mathrm{xII}^{\mathrm{e}}$ siècle, parle aussi de l'union charmante des pétales vermeilles de la rose et de la couleur jaune des étamines :

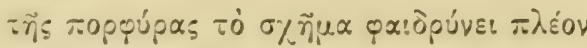

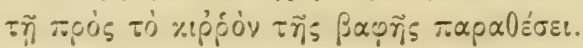

Peut-on dire que Philé ne connaissait pas les roses doubles?

4. Il est à remarquer que l'enlumineur des Heures de la reine Anne, dans les roses quilil y a peintes avec tant de vérité et auxquelies 
roses, dont parle le poìte de Der rôte Mund, avaient encore des étamines et étaient par suite incomplètement doubles, il ne s'ensuit pas qu'elles ne le fussent pas du tout, ni qu'il n'y eût point à cette époque, en Allemagne et en France, de roses de jardin entièrement doubles: le témoignage d'Albert le Grand et de Barthélemy l'Anglais prouve le contraire.

Ce qui est vrai; c'est que depuis le xm10 siècle le culture de la rose parait avoir pris une extension considérable dans toute l'Europe romane et germanique. C'était la conséquence naturelle de l'usage de plus en plus grand qu'on faisait alors de cette fleur et de ses produits. En Italie il en est déjà question, au commencement de ce siècle, dans une espèce de tournois donné à Trévise ${ }^{1}$, et cent ans plus tard on se servait mème dans la Péninsule, Pierre de Crescence en fait foi, des rosiers à fleurs blanches pour faire des clotures; Boceace parle aussi, à plusieurs reprises ${ }^{2}$, de rosiers blancs et vermeils, qui, avec les jasmins, bordent les allées des jardins, et à chaque instant il fait mention de roses dans ses ouvrages. En Espagne la culture de ces fleurs con-

il ne donne pas moins de cinquante ou soixante pétales, a eu grand soin de figurer au centre de chacune d'elles un petit buisson d'étamines jaunàtres.

1. Alvin Schultz, Op. laud., 2c éd., vol. I, p. 578. Cf. plus loin, au chap. v, le Siège du Chàteau d'amour.

2. "La latora delle quali vic tultedi rosai bianchi e vermigli et di gelsomini erano quasi chiuse ». Decamerone. Giornata terza, novella 1. Firenze, 1827, in-8, vol. II, p. 15. "Da spessissimi gelsominie da pugnenti rosai sono per tutto cinte. ) Ameto, vol. XV, p. 87. 
tinua d'ètre l'objet de soins assidus après l'expulsion des Arabes, qui l'araient poussée si loin. Nicolas Monardès parle de nombreuses variétés de roses qu'on rencontrait dans la Péninsule; il cite en particulier celles de Tolède comme surpassant toutes les autres en éclat et en parfum ${ }^{1}$.

En France l'impulsion donnée à l'agronomie et à l'horticulture par Charles $V$ ne fut pas arrètée par les troubles qui suivirent ce règne réparateur. Depuis lors la culture des roses prit la plus grande extension. Sauval rapporte ${ }^{2}$ que Charles VI fit, en 1398, planter dans le jardin du Champ-au-Plâtre, à l'hôtel de Saint-Pol, trois cents gerbes de rosiers blancs et rouges, avec trois cents oignons de lis, autant d'oignons de flambes iris) et huit lauriers. En 1432, dit-il encore, le due de Bedford fit de mème planter dans le jardin de l'hótel des Tournelles " une infinité de rosiers blancs. »

Le roi René de Provence, qui “ joignait à des gouts chevaleresques celui de la culture des fleurs ", rivalisa avec eux. Dans les jardins de ses chàteaux d'Aix, d'Angers, de Baugé, des Ponts-de-Cé, on voyait, dit son historien ${ }^{3}$, arec des arbres fruitiers

1. "Superant quae Toleti leguntur omnes Hispaniae rosas et fragrantia et rubore. ") De rosa libri tres in latinum donati a Carolo Clusio. Antverpiae, 1611, in-fol., p. 45.

2. Histoire et antiquités de la ville de Paris. Paris, 1725, in-fol. Liv. VII, vol. II, p. 283.

3. A. Lecoy de La Marche, Le roi René, sa vie, son administration, ses travaux artistiques el littéraires. Paris, 1875, in-8, vol. II, p. 8, 9, 3̈3 35 et 50 . 
des plantes variées, spécialement des rosiers, et Bourdigné ${ }^{1}$ a été jusqu'à attribuer à ce prince le mérite peu probable d'ailleurs d'avoir le premier importé dans l'Anjou les roses de Provins. Les relations de nos rois avec l'Italie à la fin du $\mathrm{xv}^{\mathrm{e}}$ et au commencement du xvi ${ }^{\mathrm{e}}$ siècle contribuèrent à développer, en mème temps que l'amour des constructions luxueuses, le culte des jardins; les enluminures des Heures de la reine Inne ne sont pas seulement un monument unique de l'art contemporain, mais encore un témoignage manifeste de la passion croissante qu'on avait alor's pour les fleurs, en particulier pour les roses.

Partout les plantations de rosiers vont se multipliant, partout se répand la culture de ces fleurs aimées. Les femmes surtout aimaient à s'y livrer, goût que leurs maris étaient d'ailleurs loin de contrarier :

Sachiez, dit à sa femme un bourgeois de Paris ${ }^{2}$, de la fin du xıve siècle, que je ne prends pas desplaisir, mais plaisir, en ce que vous aurez a labourer rosiers, a garder violettes et a faire chapeaulx.

Et au $\mathrm{xrr}^{\mathrm{e}}$ siecle Louis Vivès recommandait encore aux jeunes filles la culture de ces mèmes fleurs".

1. «Pour certain il futle premier quid'estrange pays feist apporter en France... fleurs de œilletz de Provence, roses de Provins et de muscadetz... ignorées en Anjou auparavant. „ Hystoire agregative des Annalles et C:oniques d'Anjou. Angers, 1529, in-fol., p. 168, 1.

2. Le Ména oier de Paris, vol. I, p. 2.

3. "Aussi sera honneste à la jeune fille de cultiver herbes et vio- 
Il serait intéressant de connaitre quels furent, au moyen àge, les principaux centres de culture de la rose; malheureusement on ne trouve que bien peu de renseignements à cet égard dans les écrivains du temps, et l'on a encore à peine songé à recueillir dans les Archives ceux qu'elles pourraient fournir". Les historiens de Provins, MM. F. Bourquelot et Opoix, ont omis de dire ce que fut autrefois la culture de cette fleur dans cette ville, où elle a dû avoir tant d'importance, et ils ne parlent des produits de la rose et du présent qu'on en faisait aux rois à leur passage par Provins qu’à partir du xvi ${ }^{\mathrm{e}}$ siècle ${ }^{2}$.

Que Provins ait été depuis une époque reculée un lieu de culture renommé par ses roses, le fait est incontestable; mais bientot Paris rivalisa avec lui. Un document, dont je dois la connaissance à une bienreillante communication de M. Siméon Luce,

lettes es courtils et jardins selon leur vocation et exercice, pour les induyre par après a meilleur et plus grand besongne "). Institution de la femme chrestienne, composée par Loys Vives et nouvellement traduite en langue francoise (par Pierre de Changy). Lyon, s. d., in-12, chap. iII, p. 19.

1. Je ne connais qu une exception, ce sont les recherches faites par M. Léopold Delisle dans les dépôts publics de Normandie. Malheureusement cet exemple si utile n'a pas été suivi et la Normandie est la seule province de France jusqu'ici dont nous connaissions bien l'état de la culture au moyen àge.

2. “En 1574, la Reine-Mère et les princes du sang... arrivèrent à Provins... On offrit des conserves et des roses sèches... Henri IV, le 16 avril 1607 , arriva au chàteau de Montglas, près Provins... Le corps de ville... lui offrit des conserves et des sachets de roses $)$. Opoix, p. 136. 
nous apprend qu'à la fin du $\mathbf{x v}^{0}$ siècle on achetait également les roses dont on avait besoin a Paris et à Provins. C'est ainsi que, sur l'ordre de Louis XI, qui se trouvait alors à La Mothe d'Esgry, dans l'Orléanais, deux messagers furent envoyés en même temps dans ces deux villes " querir des rozes et boutons »".

Mais il y avait alor's bien d'autres centres de culture de la rose en France. Au Homme près Rouen, elle avait pris déjà au $\mathrm{xun}^{\mathrm{e}}$ siècle assez de développement pour que le curé perçut la dìme sur les rosiers ${ }^{2}$. L'usage si répandu des chapeaux de rose, auxquels les poètes des $\mathbf{x u n}^{\circ}, \mathbf{x i v}^{\mathrm{e}}$ et $\mathrm{xv}^{\mathrm{e}}$ siècles font sans cesse allusion, les fréquentes mentions des redevances quion en faisait ${ }^{3}$, montrent que la culture de la rose était générale en France à la fin du moyen âge.

Cette fleur occupe une trop grande place dans la poésie allemande ou néerlandaise contemporaine

1. “A Nicolas Mesnagier, varlet de fourrière du dit scigneur (le roi Louis $\mathrm{XI}$ ), la somme de vingt-sept livres douze solz huit deniers tournois que le dit seigneur lui a ordonnée ou dit mois pour le rembourser de pareille somme qu'il a baillée du sien par l'ordonnance et commandement du dit seigneur, c'est à savoir vi livres xvin solz vi deniers tournois pour avoir envoyé deux hommes à cheval de la Mothe d Esgry à Paris et à Prouvins querir des rozes et boutons ». 1480, juin, Arch. nat., registre KK 64, folio 62 .

2. "Le prestre prend toutes autres diesmes comme de pois ramiers, de rosiers... » Livre des jurés de S. Ouen, ap. Léopold Delisle, Études sur la condition de la classe agricole et de l'agriculture en Normandie au mojen âge. Évreux, 1851, in-8, p. 491, note 36 .

3. Léopold Delisle, op. laud., p. 492. Voir pl. loin chap. v. 
pour qu'il n'en fût pas de même dans les pays de langue germanique. Depuis Frédéric II, qui en fut le protecteur, l'horticulture avait fait de grands progrès en Allemagne, la faveur dont au siècle suivant elle jouit auprès de Charles IV contribua encore à la développer ${ }^{1}$. Nais le goût des fleurs ne fut pas le privilège des princes et des grands; les riches marchands de la Souabe et de la Bavière, ainsi que de la vallée du Rhin, le partagèrent de bonne heure; les jardins des villes d'Augsbourg, Ulm, Nuremberg, Bâle, Cologne, furent célèbres à la fin du moyen age $^{2}$; on y voyait les plantes les plus diverses, mais surtout des rosiers; les roses d'Ulm furent renommées de bonne heure, et le nom de rose de Batavie ou de Hollande, donné à une variété de la centfeuilles ${ }^{3}$, témoigne de l'importance que vers l'époque de la Renaissance prit aux Pays-Bas la culture de cette fleur. Dans toute l'Europe centrale il n'y aura pas désormais de jardin, si humble soit-il, qui n'ait quelques rosiers.

Mème spectacle en Angleterre. Un roman du $\mathrm{xu}^{\mathrm{e}}$ siècle nous apprend que déjà alors les roses y étaient employées comme fleurs d'ornement ${ }^{4}$; un manuscrit du British Museum nous les montre au

1. Oscar Teichert, Geschichte der Ziergärten in Deutschland, Berlin, 1865, in-8, p. 12 et 21.

2. K. W. Volz, Beiträge zur Kullurgeschichte, ap. Schleiden, Die Riose, p. 197.

3. Car: Clusius, Rar plantarum historia, Antverpix, 161, p. 114.

4. Thomas Wright, A history of domestic manners and sentiments in England during the middle Ages, p. 243. 
siècle suivant cultivées, non seulement pour l'agrément, mais encore pour servir à la distillation ", ainsi que cela se faisait d'ailleurs en France. Ainsi qu'en France et en Allemagne aussi, depuis la fin du moyen âge les roses se multiplient en Angleterre: nul pays n'en connaissait un plus grand nombre d'espèces vers la fin du $\mathrm{xvi}^{\ominus}$ siècle.

Mais à quels procédés avait-on recours dans la culture des rosiers? Le Ménagier de L’aris se borne à recommander de les planter, ainsi que les oignons de lis et les groseilliers, après la septembresse ${ }^{2}$. Pierre de Crescence est plus explicite; il n'a guère fait cependant que résumer les enseignements de Pline et de Palladius.

On plante les rosiers, dit son traducteur ${ }^{3}$, par plantes et vergètes divisées en petites parties et mises en semoir ou semence jetée en semoir ${ }^{4}$. La semence est recueillie dedans les boutons rouges et congnoist on leur meureté quant apres vendanges les dictz boutons deviennent jaunes et molz. Se les rosiers sont vieilz on les doit fouyr autour et retailler le secq et peuvent estre retaillez (reproduits) les rosiers tendres et foibles par leurs gettons ${ }^{\circ}$.

Pierre de Crescence ayant été bientôt connu en

1. Thomas Wright, op.laud., p. 301.

2. Vol. Il, p. 49. La septembresse est Notre-Dame de septembre.

3. Liv. V, chap. $22 b$, fol. p. i.

4. "Plantantur autem plantulis et virgultis in parvas particulas divisis, et in seminario satis seminibus m. P. 273. On voit que par semoir le traducteur reut dire pépinières, et quil a fait un demi contresens.

5. "Si qua sunt antiqua rosaria circumfodiantur, et ariditas 
original dans tous les pays et traduit dans presque toutes les langues de l'Europe, ses préceptes peuvent ìtre considérés comme l'expression exacte de ce que dut être dans les derniers temps du moyen âge la culture de la rose chez les nations romanes et germaniques. On ne la poussa point d'abord jusqu'a créer des espèces nouvelles et pendant longtemps on se contenta des roses blanches et rouges que l'on possédait depuis des siècles. On sut encore moins obtenir des roses remontantes; mais comme on désirait néanmoins avoir ces fleurs en toute saison, on s'efforça de les conserver bien après l'époque de leur floraison. Le Ménagier de Paris donne une recette pour " garder les roses en yver ${ }^{1}$ ». Elle consistait a mettre des boutons non encore completement épanouis dans une bouteille ou un tonnelet, avec du sable ou sans sable; on bouchait hermétiquement le vase, puis on le plongeait dans un courant d'eau vire.

Ce procédé primitif ne pouvait longtemps satisfaire; les Géoponiques avaient d'ailleurs, ce qui valait mieux, indiqué le moyen d'avoir des roses précoces et en toute saison. Quand ce recueil précieux, mais resté ignoré de l'Occident jusqu'à l'époque de la Renaissance, y fut enfin connu, on mit en pratique les préceptes qu'il contenait; Mizauld les énumère longuement dans son live De la culture des jardins ${ }^{2}$;

reciditur universa. Item quae rara sunt, possunt virgarum propagine reparari. »

1. Vol. II, p. 52 et 252.

2. Hortorum secreta, culus et auxilia. Lutetiac, 1575, in-8, p. $48-49$. 
avec les Géoponiques, il recommande en particulier, pour obtenir des roses de bonne heure, de mettre les rosiers dans des caisses ou des vases, tenus à l'abri du froid et à une bonne exposition. Ce sont aussi les préceptes du même recueil que l'auteur du Théátre d'Agriculture, Olivier de Serre, suivra pour la culture des roses et surtout des roses précoces ${ }^{1}$ :

Pour avoir des rozes de hastiveau ou fort primeraines, dit-il, convient avancer les Roziers par le fumier. Ce moyen est, qu'estans les Roziers plantés joignant quelque muraille au regard de Midi et par la à l'abri de la bize, soient au mois d'Octobre ou de Novembre deschaussez profondément, et la fosse remplie de bon fumier de cheval meslé parmi un peu de bonne terre; apres durant l'Hyver, tous les huict jours une fois, seront arrousés avec de l'eau tiede... Par telle adresse dans l'Hyver aurez des Rozes, pourvu que les preserviez des gelees, les tenant couvertes.

Les soins apportés ainsi maintenant à la culture des roses devaient finir par en multiplier les espèces; on en recut d'ailleurs des contrées lointaines. La rose de Damas se répandit alor's dans toute l'Europe; il en fut de même de la rose musquée, venue comme elle d'Orient; d'après Mizauld ${ }^{2}$, ces deux espèces étaient communes de son temps dans les jardins des gens riches. Claude Mollet connaissait

1. Le théâtre d'agriculture et, mesurage des champs, Genève, 1639 , in-4, p. 490 .

2. "Divitum hortis hodie familiares ». Op. laud., p. $48 \mathrm{~b}$. 
aussi déjà, sinon la première, du moins la seconde ${ }^{1}$; le célèbre Fugger l'importa à Augosbourg ${ }^{2}$. Le même Claude Mollet, dans son Thécitre des plans et jardinages, distinguait huit espèces de roses cultivées, les mèmes à peu près que le bressan Agostino Gallo ${ }^{3}$; mais ce dernier ne parle pas de la rose jaune à fleurs doubles (R. sulphurea L.), que mentionne, au contraire, Claude Mollet. L'Écluse ne la recut qu'en $1607^{4}$; jusque-là il ne la connaissait que par ouïdire $^{5}$. Olivier de Serre, qui parle de la rose jaune à fleurs simples ( $R$. eglenteria L.), ne fait pas encore mention non plus de la rose jaune double, bien qu'elle ait dû être importée dans la prescqu'ìle des Balkans peu de temps après la conquête de Constantinople.

\section{III.}

Tandis que la culture des roses se répandait et se développaitainsi chaque jour davantage dans l'Europe

1. Théâtre des plans et jardinages. Paris, 1563, in-4, p. 172. Peut-être par " roses incarnates qu'on emploie pour l'eau de rose » désigne-t-il les roses de Damas.

2. Schleiden, Die Rose, p. 201.

3. Gité par Loiseleur-Deslongchamps, La Rose, p. 173.

4. Curae pesteriores seu plurimarum non ante cognitarum aut descriptarum stirpium... novae descriptiones. Raphelengii, 1611, p. 12.

5. "Accipio flavas rosas multiplici foliorum scrie praeditas ex- 
occidentale et centrale, elle pénétrait aussi chez les peuples scandinaves, comme le montre sa présence, avec le lis, dans leurs légendes et leurs chansons populaires. Elle y fut, ce semble, importée par les ordres religieux, les véritables créateurs, dans les pays du Nord, de l'horticulture, restée à peu prés inconnue des populations paiennes de ces contrées, mais l'une des occupations favorites des cénobites chrétiens ${ }^{1}$. A quelle époque la rose commença-t-elle à être cultivée dans les jardins des couvents scandinaves? Il est difficile de le dire; mais il est vraisemblable que les religieux étrangers établis en Scandinavie ne purent manquer longtemps d'y faire venir des rosiers, avec les autres plantes, arbres ou arbustes qu'ils prirent à cour d'importer dans leur patrie d'adoption ${ }^{2}$. C'est ce que fit entre autres, à la fin du xı $^{\mathrm{e}}$ siècle, Guillaume, abbé d'Eskilsö, religieux de l'abbaye de Sainte-Geneviève de Paris. Appelé, en 1165 , par l'évèque Absalon en Danemark ${ }^{3}$, on le voit, dans une lettre écrite par lui, entre 1192-1194, au moine Etienne, "s'en remettre aux bons soins » de

tare. "Rariorum plantarum historia. Antverpiae, 1601, in-fol., p. 114.

1. F. C. Schübeler, Die Culturpflanzen Norwegens, mit einem Anhange über die altnorwegische Landwirtschaft. Christiania, 1862, in-4, p. 164.

2. “Die Mönche brachten Ostbäume, Wurzeln, Kräuter und Blumen vom Auslande mit sich ». F. C. Schübeler, ibid.

3. Il mourut en 1202. Rasmus Nyerup, Historisk-statistike Skildringer af Tilstanden i Danmark og Norge $i$ aeldre og nyere Tider. Kjöbenhavn, 1803, in-12, vol. I, p. 166. 
ce frere pour lui envoyer, de France probablement, des graines de divers légumes et herbes, ainsi que des drageons et des greffes d'arbres ${ }^{1}$ I avait-il parmi ces arbres ou arbustes des rosier's? Je l'ignore; mais moins d'un demi-siècle plus tard, Ilenri Harpestreng, dans sa pharmacopée ${ }^{2}$, énumérait longuement les propriétés médicinales de la rose, et comme il parle d'une préparation faite arec des roses fraîches, il n'est guère douteux que ces fleurs ne fussent déjà cultivées en Danemark.

Elles ne durent pas tarder non plus à l'être en Norvège et en Suède. La culture fut poussée très loin dans les jardins des couvents que les religieux de l'ordre de Cîteaux fondèrent dans le premier de ces deux pays; et on rencontre aujourd'hui encore naturalisées dans la contrée où se trouvait en particulier celui de Ilovedö, près Christiania, des plantes non indigènes qui n'existent ailleurs que cultivées. Lorsque les poètes nationaux parleront de la rose et lui emprunteront, ainsi qu'au lis, quelques-unes de leurs plus gracieuses comparaisons, ils pourront bien le faire à l'imitation de leurs modeles de France ou d'Allemagne, mais ils seront compris de tous

1. "Caeterorum olerum semina et herbarum diversarum atque radicum et arborum surculos tuae nobis prudentiae providere relinquimus ». Jacobus Langenbeck, Scriptores rerum danicarum medii aevi. Hauniae, 1786, in-fol. vol. VI, p. 75, ep. Lxxvi.

2. Danske Laegebog fra det 13de Aarhundrede, fürste Gang udgivet efter et Pergamenthaandskifif $i$ der store hongelige Bibliothek, med Indledning, Anmürlininger og Glossarium, af Christian Molbech. Kjöhenharn, 1826, in-8, p. 88. 
leurs compatriotes, puisqu'ils chanteront des fleurs connues aussi d'eux tous.

La rose fut connue chez les peuples slaves, comme chez les nations scandinaves, après leur conversion au christianisme, et peut-être même plus tôt chez quelques-uns d'entre eux; il faut ici, en effet, distinguer entre les Slaves méridionaux et les Slaves septentrionaux et occidentaux. Les premiers trouvèrent la rose cultivée à leur arrivée dans le nord de la péninsule des Balkans, habitée à la fois par les Grecs de la Macédoine et par les populations romanes de la Mésie et de la Dacie; c'est d'eux qu'ils reçurent la rose; de là les deux noms que cette fleur porte chez ces peuples; l'un d'origine hellénique,

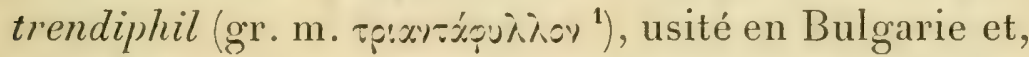
sous la forme trö̈anda, dans l'Ukraine et la Russie méridionale, l'autre d'origine latine ${ }^{2}$, slavon roja ${ }^{3}$, bulg. rou $\stackrel{\sim}{i}$, serbe rouja et rosa, rousa dans le dialecte de Raguse ${ }^{4}$.

1. Bern. Langkavel, Botanik der spaeteren Griechen. Berlin,

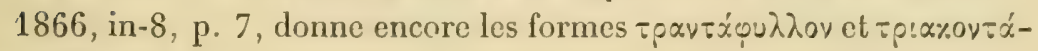

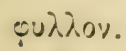

2. Le nom roumain de la rose est rosa et dans certaines localités rousa. A. T. Laurienu si J. G. Massimu, Dictionariulu limbei romane. Bucuresci, 1876, in-8, s. v.

3. Miklosisch, Lexicon palæo-slovenico-graeco-latinum. Vindebonae, 1862-65, in-8, s. v. A ces mots il faudrait ajouter le vocable gjul, emprunté plus tard aux Turcs par les Bulgares.

4. "Das Serbische hat fur die Rose eine zweifache Benennung ruza und rosa, rusa; in beiden Formen ist es entlehnt, nur ist es in der ersteren eine jungere, in der letzteren, in Dalmatien ïblichen Joret. La Rose. 
Quant aux Slaves septentrionaux et occidentaux, il est évident qu'ils ont connu la rose beaucoup plus fard que leurs fières du Sud, et ils l'ont peut-ètre reçue à la fois par l'intermédiaire de ces derniers et des Allemands; mais de quelque côté qu'elle leur soit venue, les noms qu'elle porte chez eux sont, comme chez les Slaves méridionaux, d'origine latine: russe roja, pol. ro:a, lith. ro $\cong e$, tchèque rouะe. II en est de même chez les Hongrois, qui appellent la rose cultivée rocza. Quant à la rose sauvage, son nom slave est chipla ou chipak ${ }^{1}$.

A quelle époque remonte la culture de la rose chez les Slaves? Il m'est impossible de répondre à cette question faute de documents, en ce qui concerne les Slaves septentrionaux et occidentaux; le nom de la rose apparaît, pour la première fois, avec certitude, au commencement du $\mathrm{xv}^{0}$ siècle, dans un écrit de Jean IIuss ${ }^{2}$, qui n'est encore qu'une traduction de la Bible. Il est probable néanmoins qu'elle était connue depuis longtemps déjà en Bohême, ainsi qu'en Pologne, et peut-ìtre mème en Russie. Les Slaves méridionaux, eux, cultivèrent ou du moins

Form rosa, rusa eine ältere Entlehnung aus dem Romanischen ». Miklosisch, Die Rusalien (Sitzungsberichte der lais. Akademie, vol. XLVI, an. 1864, p. 405).

1. Karadschitsch, Lexicon serbico-germanico-latinum. Vindebonae, 1852 , in-8, s. v.

2. “Je n'ai pu trouver dans notre littérature ancienne un seul mot surl' histoire de la culture de la rose en Pologne, dit M. Kaczynski, dans son livre Ro $\cong$, publié en $1880 »$; il m'est permis de n'être pas plus avancé. 
connurent la rose, sans doute, dès les premiers temps de leur histoire. Le mot slavon roja apparaît déja dans un document du $x^{\mathrm{e}}$ siècle; mais ils devaient, quelques-uns d'entre eux du moins, la connaître bien plus tôt.

C'est aussi sans doute d'assez bonne heure que prirent naissance les légendes dont les Slaves, comme les peuples germaniques et les nations romanes, ont entouré la rose; la plupart d'entre elles devaient exister avant la fin du moyen âge, encore qu'on ne les rencontre le plus souvent que dans des monuments postérieurs. C'est la raison qui m'en fera rapporter quelques-unes. 


\section{CHAPITRE DEUXIÈME.}

LA ROSE DANS LES LÉGENDES ET DANS LA POÉSIE DE L'ORIENT AU MOYEN AGE. LA ROSE ET LE ROSSIGNOL.

C'est depuis l'époque seulement où l'hellénisme s'établit en Orient que nous rencontrons la rose dans les traditions de l'Asie antérieure, et que nous pourons en suivre les destinées dans cette vaste contrée. Mais, à quelle époque a-t-elle pris place dans les traditions de cette région et en particulier de l'Iran? Il est impossible de le dire; toutefois j’ai cru pouvoir rattacher les légendes où elle apparait, dans le Bundehesch, aux anciens temps de l'histoire religieuse de la Perse; en tout cas, bien arant notre ère, la rose, nous l'avons vu, fut connue et aimée des peuples de ce pays. Ce n'est que vers le milieu du moyen àge cependant qu'elle figure dans leur littérature; mais à partir de là et durant quatre siècles les poètes de l'Iran n'ont point cessé de la chanter. Il cn fut de mème chez les Arabes et plus tard chez les Osmanlis; pour eux la rose est une fleur d'élection; elle règne sans rivale sur le monde des plantes, à la place du lotus, obligé de lui céder le premier rang.

Une légende a symbolisé, sous une forme ingé- 
nieuse, cet avènement de la rose à l'empire et l'admiration profonde qu'elle a inspirće aux peuples de l'Orient. Les fleurs, raconte-t-elle ${ }^{1}$, allèrent un jour se plaindre à Allah que le lotus dormît toute la nuit ${ }^{2}$, et elles lui demandèrent une autre reine. Allah écouta leur prière et il créa, pour régner sur elles, la rose à la blancheur virginale; mais afin de protéger cette fleur charmante et délicate il l'entoura d'aiguillons ; quand le rossignol aperçut la nouvelle reine, il s'éprit pour elle d'un si vif amour que, hors de lui, il s'envola au milieu des buissons de roses, sans prendre garde aux aiguillons qui les défendaient; blessé à mort, il exhala sa faible vie en accents plaintifs et doux, et son sang, en coulant sur la fleur, objet de son amour, en colora en rouge les blanes pétales ${ }^{3}$.

I.

On a là un exemple des fictions dont l'imagination orientale a entouré la rose au moyen âge. Arabes, Persans surtout, Osmanlis, ont rivalisé dans l'éloge

1. Warnke, Pflanzen in Sitte, Sage und Geschichte, p. 108.

2. Allusion à la propriété du lotus de fermer ses fleurs pendant la nuit.

3. "Les aiguillons acérés et les flèches aiguës de mes épines me blessent, et répandant mon sang sur mes pétales, les teignent d'me couleur vermeille "), fait dire Azz-Eddin el Mlocadessi à la rose ellemème. Les oiscaux et les fleurs, allégories morales, traduites par Garcin de Tassy. Paris, 2e éd. 1876, in-8, p. 11. 
qu'ils en ont fait ou dans les légendes qu'elle leur a inspirées.

Quand les Arabes pénétrèrent en Syrie et en Perse, ils furent frappés par la beauté et l'éclat des roses qu'on y cultivait; cette fleur nouvelle fut pour ces fils du désert, émerveillés de son parfum et de sa grâce, comme plus tard pour les Tures, plus qu'une fleur ordinaire; elle eut à leurs yeux quelque chose de divin. L'admiration presque superstiticuse des Osmanlis pour la rose est connue. On rapporte qu'ils ne souffrent pas qu'on jette à terre les pétales de cette fleur rénérée ${ }^{1}$. Quand par hasard ils en rencontrent, ils les ramassent soigneusement et, après les avoir approchés arec respect de leur bouche, ils les déposent dans la fente de quelque muraille, afin de les préserver de toute espéce de profanation.

Lorsque la rose était pour les Mahométans l'objet d'une si grande rénération, il ne pouvait manquer que des légendes se formassent autour de cette fleur si admirée. En voici une où elle est rattachée au souvenir de l'ancêtre de leur race. Abraham, ditelle, ayant refusé de sacrifier aux Divinités de la Chaldée, Nemrod le fit jeter dans une fournaise ardente. Mais Dieu ne permit pas que le feu lui fit aucun mal; à la place où se trouvait le patriarche, il fit jaillir une source d'eau fraîche et pousser des roses et d'autres fleurs, et il envoya l'archange Gabriel, qui resta près du patriarche jusqu’ì ce que

1. Rosenberg, Rhodologia, p. 15. 
le feu fùt éteint ${ }^{1}$. Nous rencontrerons dans l'histoire de la rose chez les nations chrétiennes une légende analogue, dont l'héroïne est la fille mème d'Abraham.

L'histoire de Hahomet est aussi mêlée à la légende des roses; suivant une tradition qui a cours chez les Tures ${ }^{2}$, mais qu'on rencontre également chez les Persans, la rose, conception plus singulière que gracieuse, serait née de la sueur mème du prophète. D'après une autre tradition, sur la prétendue tombe d'Nli le rival de Mahomet ne pouvait être oublié dans la légende des roses - près de l'antique Bactres, se voient de merveilleuses roses rermeilles, qui surpassent en beauté et en parfum toutes les autres, mais qui ne peurent aussi, dit-on ${ }^{3}$, c̀tre transplantées ni croître ailleur's.

Ces traditions ont éridemment pris naissance ehez les Persans; ce peuple, à l'imagination inventive et gracieuse, a entouré la rose, cette fleur qu'il a aimée et chantée entre toutes, de légendes qui rappellent parfois les légendes germaniques ou romanes; c'est ainsi que la rose est, comme dans ces dernières, devenue l'embleme et le signe de la chasteté conservée. Le Livre du perroquet, du poète Nachshebi, raconte qu'un guerrier, réduit à la

1. G. Weil, Biblische Legenden der Muselmänner. Frankfurta.-11., 18'15, in-12, p. 75. Cf. J. von Hammer, Rosenöl. Wien, 1813.

2. Rosenberg, Rhodologia, p. 15.

3. Ersilia Caetani Lovatelli, La festa delle rose. (Antologia nuoya, vol. XVIII, p. 7).Vambéry, Reise, in Mittelasien, p. 188. 
misère, hésitait néanmoins à se séparer de sa jeune femme, pour aller chercher fortune ${ }^{1}$. Celle-ci finit toutefois par le décider à s'éloigner en lui donnant un bouquet de roses, qui, lui dit-elle, ne se fanerait pas, tant qu'elle lui resterait fidèle. Le guerrier part et entre au service d'un seigneur d'une contrée voisine. Le maître remarque bientôt avec étonnement que son serviteur portait toujours sur lui un bouquet de roses, fraîches même au milieu de l'hiver; il l'interroge sur leur prorenance et leur nature. Le guerrier lui raconte son histoire; le gentilhomme ne peut s'empêcher de rire de sa crédulité et lui dit que sa femme est une sorcière.

Cependant il envoie en secret un de ses deux cuisiniers essayer de la séduire. Le cuisinier trouve moyen de pénétrer jusqu'à la femme du guerrier; celle-ci, pour se soustraire à ses sollicitations, feint de lui accorder un rendez-vous; mais elle le fait tomber dans un souterrain et elle le force d'avouer son dessein et le mobile qui le fait agir. Surpris de de ne pas voir revenir le premier cuisinier, le seigneur envoie le second dans la ville du guerrier; mais il n'est pas plus heureux que son camarade et a bientôt le même sort. Le gentilhomme, sous un prétexte, se rend alors lui-même, en compagnie de son serviteur, dans la ville de ce dernier. Le guerrier ayant, dans une entrevue arec sa femme, appris

1. Reinhold Köhler, Zu der Erz̈̈hlung Adams von Cobsam. (Jahrbuch für romanische und englische Literatur, vol. VIII (année 1867), p. '1'i-65). 
de sa bouche ce qui était arrivé, invite son maître a un repas, où il est servi par ses deux cuisiniers, déguisés en esclaves; il les reconnaît cependant à la fin et leur témoignage met hors de doute la fidélité et la vertu de la femme du guerrier.

Cette légende était trop curieuse pour ne pas se répandre chez les peuples voisins de la Perse; on la rencontre dans un recueil de contes tures appelé Al faradj bad ash shidda (La joie après la tristesse); toutefois ce n'est pas un bouquet de roses, mais une branche verte de cyprès, ce qui offre un caractère moins frappant et surtout moins poétique, qui doit rendre témoignage de la vertu de la femme absente, Le traducteur turc du Livre du Perroquet a, au contraire, conservé la fleur de l'original; mais il a remplacé le bouquet par une seule rose. C'est aussi une rose que nous retrouverons dans la forme francaise de cette légende merveilleuse. Dans un conte hindou de Samadeva Bhatta, l'histoire de Devasmita, c'est un lotus rouge, au contraire, donné par Civa aux deux époux, qui doit être par sa fraîcheur conservée le gage de leur mutuelle fidélité ${ }^{1}$.

La rose n'est pas seulement dans les traditions persanes le gage de la constance pendant la vie, sa naissance miraculeuse est devenue aussi l'emblème de l'affection conservée mème dans la mort. 'Tajin, le favori du chef des Khaled, Zein-eddi, et son ami

1. Jahrbuch für rom. u. englische Literatur, vol. VIII, p. 47 . Cf. Édélestand du Méril, Floire et Blanceflor, poèmes d" $\mathrm{x}_{\mathrm{n}} \mathrm{e}^{\mathrm{e}}$ siècle. Paris, 1856, in-18. Introduction, p. 167-169. 
Mem, ayant, raconte une légende kourde ${ }^{1}$, aperçu, dans une procession solennelle, les deux soeurs de ce prince, en devinrent éperduement épris ; Tajin, d'une famille illustre, obtint sans peine la main de son amie; il n'en fut pas ainsi de Mem, de naissance obscure, et il fut réduit à aimer en secret la sœur de Zein-eddi, la belle Zin. Trahi par un serviteur, il fut, après bien des dangers, jeté en prison. Cependant Zein-eddi, le voyant près de mourir et redoutant la vengeance de Tajin, lui rendit la liberté et consentit à l'unir à sa sœur. Mais Mem expira de joie à la vue de sa chère Zin; celle-ci mourut bientôt elle-mème emportée par le chagrin et, sur son désir, fut enterrée à côté de Mem. Sur chacun de leurs tombeaux on vit croître un rosier, dont les branches s'entrelacèrent, en témoignage de leur immuable affection.

Dans les légendes hindoues, la rose n'a pas pris un caractère moins merveilleux que dans celles de la Perse, mais on ne l'y rencontre qu'à une époque relativement récente, comme celle où cette fleur a été cultivée dans la Péninsule. Telle elle apparaît en particulier dans le conte de Gul i Bakâyalì La rose de Bakâvalî̀ ${ }^{2}$. Le roi Zaïn-ulmulûk, frappé de cécité, ne peut être guéri qu'au contact d'une rose, qui se

1. Transactions of the Ethnological Society, vol. II, p. 24t et suivantes; ap. Schleiden, p. 263.

2. La rose de Bakâwali, traduit de l'hindoustani par Garcin de Tassy. (Allégories, récits poétiques et chants populaires, p. $307-421$.) 
trouve dans le jardin de Bakâvalî, fille du roi des Péris. Les fils du monarque se mettent à la recherche de cette fleur miraculeuse. Les aînés ne peuvent atteindre au but de leur entreprise; mais plus heureux, le cadet, Tâj-ulmulukk, après de longues aventures et grâce à l'aide des devas, pénètre dans le jardin de la péri. Dans une des salles de son magnifique palais se trouve un bassin plein d'eau de roses; au centre se dresse une fleur épanouie d'une extrême beauté et d'une odeur délicieuse; Tâj-ulmulukk comprend que c'est là la rose de Bakâvalì; sans hésiter il ôte ses vètements, entre dans le bassin et va cueillir la fleur. Revenu sur le bord, il reprend ses vêtements, serre la rose dans sa ceinture, et, après avoir visité le palais enchanté de la péri, il se met en route pour regagner le palais paternel. De nouvelles aventures l'attendent; Tajulmulukk en triomphe comme des premieres. Et au contact de la rose qu'il a si heureusement conquise, les yeux du roi aveugle deviennent "lumineux comme des étoiles ).

\section{II.}

La rose n'a pas été seulement entouréc par les Orientaux de gracicuses légendes, elle a été le thème favori des chants de leurs poètes. Depuis son réveil au $\mathrm{xI}^{\mathrm{e}}$ sicecle jusqu'a l'époque de sa décadence au $\times v^{\mathrm{e}}$,

1. Joseph von IIammer, Geschichte der schönen Redekünste Persiens. Wien, 1818, in-4, 
la poésie persane n'a point cessé de célébrer cette fleur, parure des jardins de l'Iran: avec le eyprès, le narcisse, la tulipe et le jasmin, mais bien avant eux et au-dessus d'eux, elle l'a exaltée dans ses chants; s'il y a quelque chose de conventionnel et d'affecté dans l'espèce de culte qu'elle lui a voué, si l'éloge perpétuel qu'elle en fait tombe parfois dans la mièvrerie et frise la banalité, par l'importance et la place qu'il y a prise, le culte de la rose n'en est pas moins un des traits caractéristiques de cette poésie facile et voluptueuse. Tous ses représentants ont associé cette fleur à leurs fictions, il n'est point une de leurs œuvres où elle n'ait sa place; ils lui doivent quelques-unes de leurs peintures les plus charmantes.

Écoutons, dans une description du printemps, Firdousi ${ }^{1}$, l'un des plus grands et des plus anciens poètes de la Perse, - il vivait au $\mathrm{xi}^{\mathrm{e}}$ siècle - :

Les jardins de l'éclat des roses rougissent; les collines sont couvertes de tulipes et d'hyacinthes; dans les bosquets se plaint le rossignol ; la rose répond en soupirant à ses chants.

Et Nisami, traitant, un siècle après Firdousi, le même sujet :

Jardinier, s'écrie-t-il ${ }^{2}$, viens renouveler notre joie, en ouvrant ton verger à la rose... dont les lèvres

1. Cité par Schleiden, Die Rose, p. 238.

2. Joseph ron Hammer, Op. laud., p. 118, 
blanches comme le lait sont pénétrées du parfum de l'ambre... Colore des teintes du safran tes jasmins ${ }^{1}$... Conduis l'eau limpide au milieu des plates-bandes..., les roses y rougissent comme des lampes allumées.

Djelal-eddin-Roumi, au $\mathrm{x}^{\circ} \mathrm{I}^{\circ}$ siècle, ne montre pas moins d'enthousiasme ni d'affectation dans son éloge de la rose :

C'est aujourd'hui, dit-il ${ }^{2}$, le jour de la joie, la saison de la rose... Le bocage sourit, les narcisses sont enivrés de la splendeur de beauté et de l'éclat de la rose. La langue des lis raconte à l'oreille des cyprès les secrets du rossignol et de la rose. Le monde ne saurait comprendre tout ce qu'est la rose, l'imagination ne saurait concevoir la rose. La rose est un messager du jardin des âmes; la rose est un monument de beauté. La sueur du prophète s'étale en perles sur la rose ${ }^{3}$; la rose est une pleine lune faite de nouvelles lunes. Une vie nouvelle agitera l'esprit, aussi souvent qüil sentira le doux parfum de la rose. Comme Abraham anima de son souffle les oiseaux, au souffle du printemps naît la rose.

Hafiz aussi, l'Anacréon de la Perse, a chanté la rose comme la plus charmante des fleurs. Djelaleddin Roumi avait fait prendre le deuil aux violettes, "parce qu'clles sont séparées de la rose ${ }^{4}$ ), IIafiz nous les montre se prosternant devant elle pour

1. Il s'agit ici de jasmins jaunes.

2. Joseph von Hammer, Op. laud., p. 186.

3. Allusion à l'origine de la rose d'après la tradition musulmane.

4. Schleiden, Die Rose, p. 248. 
l'adorer. Mais la reine des fleurs a surtout été pour le poète voluptueux, malgré les épines qui l'entourent ${ }^{1}$, l'emblème et la compagne de la joie et du plaisir :

"Dieu nous garde, s'écrie-t-il dans un de ses ghazels", de renoncer au vin dans la saison des roses. ")

Amis, dit-il ailleurs ${ }^{3}$, les jours des roses sont venus, le mieux maintenant est de se réjouir.

Et dans un autre ghazel $^{4}$ :

Ciest la fête des roses; échanson, apporte du vin! Qui jamais dans la saison des roses s'assit près d'un verre vide... saisis joyeux l'occasion en ce jour de la rose! Cherche la gaieté près du vin: n'es-tu pas du reste amoureux?

Maintenant, dit-il encore ${ }^{5}$, que les roses déplient leur centuple corolle et que le calice des narcisses nous offre, enivré, sa coupe, heureux entre tous est celui qui, affranchi de tout souci, s'en va, semblable à une bulle de vin, bondissant dans la salle des festins.

Comme son émule de la Grèce, Hafiz, "la rose

1. “Hafiz, dit-il dans un de ses ghazels, où est le buisson de roses qui n'ait pas d'épines? ”)

2. Der Diwan von Mohammed Schemsed-din Hafis, aus dem Persischen übersetzt yon Joseph ron IIammer. Stultgart. 1812, in-12, vol. II, p. 181, XXV, v. 1-2.

3. Der Diwan von Hafis, vol. II, p. 197, XXVIII, v. 1-2.

4. Der Diwan von Hafis, vol. II, p. 348-49, XIII, v. 1-4 et 13-16.

5. Der Disan ron Hafis, rol. II, p. 51, V, v. 14. 
sur le front, la coupe à la main ${ }^{1}$ "), a varié ce thème sur tous les tons :

Échanson, s'écrie-t-il ², buvons gaement, gaement causons dans ce bosquet de roses; rompons nos vœux, car des roses voici le temps. Videz à l'ombre de ces arbres la coupe insoucieuse; que la joie ne se fasse pas attendre; les roses l'ordoment. Quand le printemps revient, souviens-toi du changement de l'année et demande du vin à notre échanson, sous ce berceau de roses.

Prends exemple sur les roses, chante-t-il encore ${ }^{3}$, sur le clair visage du soleil, sur la rosée matinale et les caresses des doux zéphyrs, qui ne cessent et ne cesseront jamais. Vois comme ils sourient, les pétulants, aussi longtemps qu'ils vivent, clairs et brillants, ils ne s'inquiètent, joyeux, ni de Moïse, ni des prophètes.

Homme pieux, dit-il ailleurs ${ }^{4}$, viens et cueille des roses, suspends ton capuchon aux épines; troque la vie amère de ton ordre contre de doux et agréables vins. Rejette loin de toi, au son du luth, les usages et les mœurs du cloître; donne des couronnes... et verse du bon vin aux buveurs ".

Si les dévots, dit-il une autre fois ${ }^{*}$, nous accablent de reproches et entourent d'épines le sentier de notre vie, gaiement nous voulons en échange d'une telle conduite gratifier de roses ces hommes sombres.

Et ailleurs, se rappelant à la fois la beauté et la fragilité de la reine des fleurs:

1. Der Diwan von Hafis, vol. I, p. 64, XV, v. 1.

2. Jolowicz, Der poetische Orient. Leipzig, 1853, in-8, p. 553.

3. Jolowicz, Der poetische Orient, p. 555.

4. Der Diwan von Hafis, vol. II, p. 77, XIV, v. 1-8.

5. Cité par Schleiden, Die Rose, p. 248. 
Certes, belles sont les roses! s'écrie-t-il ${ }^{1}$; il n'est rien de plus beau. Il est beau aussi que la coupe soit près de ta main! Allons! bois le vin au milieu de ces bocages fleuris! Et (souviens-toi) que fugitive est la durée de la rose!

Parfois aussi toutefois la vue de la rose, loin d'être pour Ilafiz un encouragement au plaisir, lui inspire des pensées graves et sérieuses; tel est le ghazel suivant, où rien ne rappelle le chantre du vin ${ }^{2}$ :

Les lis et les roses font de ce monde une vie éternelle; mais qu'en retirons-nous, nous qui ne pouvons vivre éternellement sur terre? Que la rose, comme Salomon, chevauche sur les ailes du zéphir et que, le matin, le rossignol, comme jadis David, chante des psaumes!

Unn des caractères distinctifs de la poésie de Hafiz, c'est le parallèle constant qu'il établit entre la rose et son amie; toutefois au lieu d'emprunter des traits a la reine des fleurs pour peindre sa bien-aimée, c'est à cette dernière qu'il en demande pour dépeindre ou plutôt pour rabaisser la rose :

Quand au milieu des bosquets de roses, soupire-t-il ${ }^{3}$, tu te balances, semblable au cyprès, les roses de jalousie en dévorent leurs épines.

Ailleurs, il dit que " les boutons de roses se referment couverts de honte a la rue de son amie ".

1. Der Diwan von Hafis, vol. I, p. 335, XG, v. 1-8.

2. Schleiden, Die Rose, p. 250.

3. Der Diwan yon Hafis, vol. II, p. 441, XVII, v. 1-4.

4. Der Diwan von IIafis, vol. II, p. 531, XXXIII, v. 1-2. 
ou encore que « confuse de l'éclat de ses joues, la rose de dépit se couvre de gouttes de sueur ${ }^{1}$ ». Et dans un autre ghazel, devançant Gongora et les cultistes du xvir $^{\mathrm{e}}$ siècle :

Les boutons de rose, chante-t-il ${ }^{2}$, referment leurs calices par toi couverts de honte; les hyacinthes rougissent enivrés de ta présence. Ah! comment la rose pourrait-elle se comparer à toi? L'éclat que le soleil lui donne, tu le donnes, toi, au soleil.

La rose était devenue un élément tellement indispensable à la poésie persane que Saadi, voulant composer un recueil de sentences morales et religieuses, n'a pas cru mieux faire que de lui donner le nom de "Jardin ou Parterre des roses » - Gulistan 3. - II se représente, au mois d'arril, dans un verger plein d'arbres magnifiques et de fleur's parfumées, au milieu desquelles chante le rossignol, mais il n'oublie pas que toutes ces choses si douces sont périssables et il offre à son ami, en échange des roses, des basilics, des hyacinthes et des amaranthes, dont il avait rempli le pan de sa robe, le livre du "parterre des roses ", sur les feuilles duquel le vent de l'automne n'étend pas sa violence et pour lequel les révolutions du temps ne changeront pas les plaisirs du printemps en tristesses de l'automne.

Un autre poète qui florissait au milieu du xı ${ }^{\circ}$ siecle,

1. Der Diwan yon Hafis, vol. II, p. 433, LI, v. 1-2.

2. Der Diwan von Hafis, vol. II, p. 531, XXXIII, v. 1-4.

3. Gulistan ou le parterre des Roses par Sadi, traduit par Ch. Defremery. Paris, 1858, in-12. 
Azz-Eddin-el Mocadessi, dans un recueil d'allégories morales, oì " les oiseaux et les fleurs ${ }^{1}$ » - de là le titre de son livre - donnent des conseils à l'homme, fait ainsi parler la rose :

En exhalant son parfum, la rose mannonça sa douce venue et s'exprima ainsi dans son langage muet: Je suis l'hôte qui vient entre l'hirer et l'été et ma visite est aussi courte que l'apparition du fantôme nocturne; hâtez-rous de jouir du court espace de ma floraison et souvenezvous que le temps est un glaive tranchant... J'embaume celui qui respire mon haleine; je cause à linnocente beauté qui me reçoit de la main de son ami une émotion inconnue. Le temps de ma durée est comme une courte visite que je fais aux hommes et celui qui espère me posséder longtemps est dans l'erreur.

III.

Figurant dans les légendes des Arabes et des Osmanlis, la rose derait prendre place dans leur poésie, comme dans celle des Persans, leurs modèles. On la trouve déjà mentionnée par le poète arabe Omar Ibn Elbi Rebia el-NIachsami, mort en 711 ou 719. "De charque jardin, lit-on dans une de ses pièces de rers ${ }^{2}$, s'élève ver's moi un parfum de rose ou de jasmin. " Asmai, qui florissait un siècle

1. Les oiseaux et les fleur's, allégories morales d'Azz-EDdrx EL Mocadessi, trad. par Garcin de Tassy. Paris, 1876, in-8, p. 7.

2. Joseph ron Hammer, Geschichte der arabischen Literatur. Wien, 1851, in-4, vol. II, p. 399. 
plus tard, dit de sa couche qu'elle lui est un " paradis de roses ${ }^{1}$ ». Un poète anonyme et peut-être postérieur a célébré la rose capucine, " parée d'une double beauté, coupe de rubis à l'extérieur, remplie à l'intérieur d'un or pâle ${ }^{2}$ ».

Un conte des Mille et une nuits renferme comme le résumé de ce que les poètes arabes ont, à l'imitation de leurs modèles de l'Iran, pensé et chanté de la rose. Dans l' "Histoire de Noureddin et de l'esclave Miriam ${ }^{3}$ ", le narrateur nous montre une compagnie d'amis se promenant dans un jardin rempli des fruits les plus savoureux et des fleurs les plus belles. "On y voyait, dit-il, des jasmins, des jacinthes, des myrtes et des roses de mille espèces. "Ces dernières surtout excitent leur admiration; ils en demandent au maître du jardin; mais celui-ci leur répond que la coutume était de ne donner des roses qu'à ceux qui les méritaient par quelques saillies piquantes ou quelques pensées heureuses. Alors chacun des amis, pour en obtenir, chante tour à tour les louanges de ces fleurs charmantes:

J'aime et j'admire la rose, dit le premier, comme la plus belle des fleurs. Elle en est la reine. Sa présence annonce le triomphe de la belle saison.

Elle répand le parfum du muse, répondit le second.

1. "Mein Lager wird zum Rosenparadies ». Jolowicz, Dc:" poetische Orient, p. 388.

2. Jolowicz, Op. laud., p. 429.

3. Contes inédits des mille et une nuits, trad. par Tréвutrex, Paris, 1828 , in-8, vol. II, p. 356 et suivantes. 
Semblable à une vierge timide, elle cache sa tête, en rougissant, dans une enveloppe de verdure.

Son aspect réjouit les cocurs, reprit le troisième; elle renferme la quintessence des plus suaves odeurs. Son bouton qui s'entr"ouvre ressemble aux lèvres d'une jeune beauté qui s'apprête à donner un baiser à son ami.

Voyez le rosier couvert de ses fleurs, poursuivit le quatrieme; n'est-ce pas comme un flot de rubis qui brillent au milieu de l'or el des émeraudes.

Par la couleur de ses pétales et de ses étamines, continua le cinquieme, la rose ressemble aux joues d'une amante ornée de sequins.

Dieu a donné à la rose l'éclat du métal le plus brillant et l'odeur des parfums les plus suaves, dit le suivant.

O merveille de la nature! s écria un autre, l'argent de la rosée dont se nourrit la rose se change en or sur sa face.

Je compare le feuillage vert et les fleurs rouges de la rose et ses vertes épines à un carquois d'or et à des flèches d'émeraude, reprend le suivant.

La rose offre les brillantes couleurs de l'aurore, ajouta le dernier, et jette les plus doctes dans I'embarras; car ils ne peurent dire si une coupe remplie d'un vin couleur de pourpre et couronné de roses leur donne son éclat ou le reçoit d'elles.

On roit quelle admiration la rose inspira aux poètes arabes; néanmoins elle est loin d'occuper dans leurs vers une place comparable à celle qu'elle a prise dans la poésie ottomane; formée sur le modele de celle de l'Iran, il était naturel que cette poésie d'imitation lui empruntàt ce motif d'inspiration avec son style de convention et ses grâces affectées. On 
retrouve déjà ces caractères distinctifs de la littérature persane dans les cuvres des plus anciens poètes ottomans. Ahmedi, qui appartient encore au $\mathrm{x}^{\mathrm{e}} \mathrm{v}^{\mathrm{S}}$ siecle - il est mort en 1412 - et qui a écrit un long poème en l'honneur d'Alexandre, dit luimème de cette œurre que c'est un "Gulistan, rempli de roses entr'ouvertes, de fraîches tulipes et d'hyacinthes ». Et quand il va chanter la rivalité sanglante d'Mlexandre et de Darius, il n'invoque pas la muse qui lui est inconnue, mais le chantre harmonieux des nuits, cet amant de la rose:

Allons, s'écrie-t-il, rossignol ", voici la saison des roses, que ta douce plainte retentisse; des odeurs du musc et de l'ambre l'air est embaumé, de chaque rameau résonne une douce voix. Les roses resplendissent des teintes du rubis et une bordure d'ambre sert de chevelure aux hyacinthes.

Sheichi, qui vient aussitôt après Mhmedi, rivalise avec les poètes les plus raffinés de l'Iran:

Des roses de ton visage, dit-il dans une de ses pieces de vers ${ }^{2}$, le jour se pare; il jette les siennes aux vents, admirant le miracle de ta beauté; le zéphyr est un joaillier, qui fond en émail la poussière de tes pieds et en forme des couronnes d'or pour la tête des roses et des lis. Quand Sheichi chante au milieu des bosquets de roses à

1. Joseph von Hammer, Geschichte der osmanischen Dichtliunst. Pesth, 1836, in-8, vol. I, p. 103.

2. Joseph von Hammer, Op. laud., vol. I, p. 108, traduit par: "Des feuilles de ton risage le jour se fait un memento" 
la bouche de rubis, aussitôt les doux accents des rossignols lui répondent.

Pour les poètes ottomans, comme pour les poètes anacréontiques de la Perse, ainsi que de la Grèce ou de Rome, la rose est la compagne de la joie et du vin, le signe du plaisir:

Le printemps est venu, jouissons de la vie, dit Nihami dans un de ses ghazels ... Oublions le passé, il est temps de jouir des roses et du vin... Buvez sous ces bosquets de roses, au milieu des narcisses... Ne laissez pas passer les roses sans en jouir.

Voici quelques strophes d'une pièce de vers célèbre, dans laquelle Messihi, contemporain de Nihami, chante, à son exemple et avec une grâce affectée, mais charmante, la rose du printemps ${ }^{1}$ :

Écoutez le chant des rossignols, voyez le printemps descendre vers nous. Sur les plaines d'alentour il se dresse des berceaux de roses. Les amandiers répandent leurs fleurs d'argent sur son chemin. Jouissez, jouissez de ce que l'amour nous offre; elle fuit, elle fuit la saison de la rose! - 3. Les roses resplendissent dans le bocage entourées de la gloire et de la magnificence du prophète. Les hyacinthes et les tulipes brillent sous la splendeur de leurs couronnes sacrées; la joie, la joie règne aujourd hui. Jouissez, jouissez de ce que l'amour nous offre; elle fuit, elle fuit la saison de la rose. - 5. Que sont donc les

1. Je traduis d'après l'adaptation qu'a faite Wieland de cette pièce de vers et que de Hammer lui-même (Op.laud., vol. I, p. 299), a cru devoir reproduire. 
roses? Ce sont des vierges (timides). Voyez, comme de leur oreille, par un fil léger, pend en perles d'argent la rosée! Les roses fleuriront-elles toujours? Non, comme les vierges, bientôt elles se faneront et feront place à de plus jeunes. Jouissez, jouissez de ce que l'amour nous offre; elle fuit, elle fuit la saison de la rose!

D’importation étrangère et récente dans l'Inde, la rose ne pénétra que plus tard et lentement dans la poésie, comme dans les légendes de la Péninsule, où avaient d'abord exclusivement figuré les plantes de la flore indigène; mais de même que peu à peu elle prit place à côté d'elles dans les jardins des princes et des grands, elle finit aussi par prendre place avec elles dans les cuvres des poètes hindoustanis. Mir Haçan, nous l'arons vu', la mentionne dans la description du jardin d'Iram; nous la rencontrons également dans l'Arä̈sch-i-Mahfil Topographic de l'Inde); l'auteur de cet ouvrage, pour peindre la venue du printemps", dit que "les manguiers fleurissent et que les roses s'épanouissent innombrables au milieu des jardins ».

L'éclosion de la rose sert aussi à Jawan pour décrire cette saison charmante, en son roman de Barah Maca ${ }^{3}$ :

Le printemps arrive pompeusement dans le monde. La rose à cent feuilles sépanouit en tous licux. La beauté

1. Voir plus haut, chap. I, seconde partic, p. 15.

2. Garcin de Tassy, Itistoire de la littérature hindoui et hindoustani, vol. II, p. 314.

3. Garcin de Tassy, Op. laud., vol. II, p. 455 et suivantes. 
de la rose en bouton fait l'admiration du monde et lui donne le contentement. Assis au milieu des roses, tous se revêtent d'un vêtement printanier.

Et après aroir énuméré les divers arbres ou plantes dont les fleurs parfument alor's les airs ou parent les jardins, tels que les maulsari (Himusops elengi), les manguiers, les jasmins jaunes, etc., le poète dit que “ la rose - il s'agit de l'espèce à cent feuilles les illumine, en y montrant réunies " les couleurs de l'amant et de sa maitresse ${ }^{1}$ ), et en y répandant un parfum qu'on sent jusqu'au Khoutan ${ }^{2}$.

Son cœur se dilate, ajoute-t-il, quand il se proméne au milieu des roses, ou quarec ses amis il boit un vin couleur de rose.

Ailleurs ${ }^{3}$ il nous montre une jeune fille plaçant une rose a son oreille, tandis qu une autre en attache un bouquet à son corsage. Il vante aussi la Séolila rose glanduleuse - " qui remplit les jardins de son éclat ).

Ainsi la rose à cent feuilles et ses variétés ont fini par pénétrer a la fois dans la culture et dans la poésie de l'Ilindoustan; toutefois ce n'est que longtemps après la fin du moyen âge que j'ai réussi à en trouver

1. Le vert du calice et le rose des pétales.

2. Partic de la Tartarie où l'on trouve le musc.

3. Op. laud., vol. II, p. 495.

1. Rosa glandulifera Roxb. Elle était à lleurs blanches. Roxburgh (Flora indica, vol. II, p. 514) dit qu'on lui donne le nom do sheouti gulab (rose dnuce). 
la trace. Aussi je ne crois pas devoir m'étendre plus longuement sur le róle qu'elle y a joué et je n'en ai parlé que pour montrer comment elle a pris place successivement dans la littérature de tous les peuples indo-européens.

IV.

La rose occupait une trop grande place dans les fictions des Orientaux pour que leurs poètes, comme ceux et encore plus que ceux de la Grèce et de Rome, ne lui aient pas emprunté les images les plus gracieuses et les plus variées. Ils comparent leur amie à une rose ou même à une feuille de rose; e'est pour eux une rose de beauté; ils parlent des roses de son front et même de ses oreilles, de la rose délicate de ses joues ${ }^{1}$, de ses lèvies et de son visage, de l'émail rose de ses joues ${ }^{2}$, mème de son éclat gloricux de roses ${ }^{3}$. Sa bouche et ses lèvres sont pour eux comme un bouton de rose, ses joues comme les roses de l'Éden; elles en ont l'éclat rougissant. Ils les comparent à un bouquet de roses entr'ouvertes ; pour eux enfin sa bouche et ses levvres ressemblent à une rose vermeille qui sourit, son sein à la nesrin

1. "Ses joues se composent de tendres feuilles des roses. " Firdousi, ap. Jolowicz, Op. laud., p. 462.

2. Der Diwan 'on Hafis, vol. II, p. 23, XIV, v. 3.

3. Anszari, Wamik und Asra, das ist der glühende und die kühlende, überselst son .Joseph von Hammer. Wien, 1833, in-8. p. 17 . 
ou rose blanche ${ }^{1}$. Son visage, remarque l'un d'eux, rougit comme la rose pourpre au lever de l'aurore.

Sa taille élancée, dit Anszari ${ }^{2}$, se balance comme les bosquets de roses et les moissons d'œillets au souffle du printemps.

Et Djami n'a pas hésité à dire d'une de ses hérönes, Suleika, que "la rose de son bonheur s'épanouit dans toute sa fraîcheur »; il parle également d'une "rose cueillie sur le rosier de l'espérance $^{3}$ ).

Mais les poètes orientaux ne se sont pas bornés à comparer leur amie it la rose, ils comparent aussi, ce qui leur est particulier, la rose à leur amie. Nous avons $\mathrm{vu}^{4}$ un poète arabe dire que le bouton de rose qui s'entr'ourre est semblable aux lèves d'une jeune beauté; on pourrait croire que c'est là une simple imitation de vers connus du persan Anwarî ${ }^{5}$ :

La brise embaumée du matin répandait sur ce parterre une odeur suave; le jasmin qui l'ornait avait les charmes des joues de ma maîtresse; le bouton de la rose entr'ouvert par le zéphyr matinal était semblable à la jeune beauté qui entr'ouvre à demi les lèvres pour sourire à son ami.

1. Joseph von Hammer, Geschichte der schönen Redekünste Persiens, p. 29-32.

2. Wamik und Asra, p. 12.

3. Schleiden, Die Rose, p. 253.

4. Cf. plus haut p. 212.

5. Traduction de Garcin de Tassy, cité par Trébution. Op.laud., vol. II, p. 357 , note. 
Le contraste offert par la beauté de la rose et les épines qui l'entourent devait frapper les poètes de l'Orient, comme ceux de l'antiquité. Hafiz entre autres en a tiré plus d'une comparaison ingénieuse:

On ne peut vivre, dit-il, par exemple, dans un de ses ghazels ${ }^{1}$, sans que les gens parlent de vous; on ne peut cueillir de roses sans que les épines vous piquent.

Les poètes hindous modernes devaient rivaliser dans l'emploi métaphorique de la rose avec leurs modèles de l'Iran.

Sa bouche, fait dire Tahcin-Uddin de Kamrup à la suivante de Kala ${ }^{2}$, ressemble à un bouton de rose.

Le bouton du cœur, dit un autre poète hindou ${ }^{3}$, s'épanouit comme une rose.

Et ailleurs" :

Dans le jardin du monde sont disséminées les roses de l'espérance, que les hommes cueillent à l'envi.

V.

Les poètes de la Perse ne se sont pas bornés à chanter dans la rose la reine des fleurs, à en faire,

1. Cité par Schleiden, Die Rose, p. 246.

2. Les aventures de Kamrup, traduites de l'hindoustani par Garcin de Tassy. Paris, 1834, in-8, p. 20.

3. Garcin de Tassy, Histoire de la littérature hindoui, vol. II, p. 473.

4. Garcin de Tassy, Op. laud, vol. II, p. 477. 
comme leurs devanciers de la Grèce et de Rome, le symbole de la beauté et de la grâce, ils lui ont donné une vie réclle et ont placé à côté d'elle le rossignol comme son admirateur et son ami.

Cette fiction des amours du rossignol et de la rose, dit Joseph von Hammer ${ }^{1}$, est un des mythes les plus anciens et les plus gracieux de la poésie persane, un mythe aussi gracieux et aussi ancien que les bocages de roses de l'Iran, où, déjà avant Firdousi, le rossignol s'exprimait en pehlvi ${ }^{2}$ ou en zend ${ }^{3}$. La rose aux cent feuilles (Gül sad berg) est la reine de beauté dans l'empire des fleurs, le rossignol aux mille voix (Hesar das istan), le roi des oiseaux chanteurs, et tous deux sont les compagnons du printemps, la saison de la jeunesse et de la joie. Alors la rose brille dans son orgueil et sourit dans sa joie, tandis que le rossignol gémissant et suppliant dit à la nuit les douleurs de son amour. Là où fleurissent les roses, gazouillent aussi les rossignols, sans cesser, sous les mille formes changeantes de leur chant harmonicux, de déclarer leur amour à la rose, tandis que celle-ci se réjouit, insoucieuse, de la vie, et ne prend pas garde à la plainte attristée du rossignol. Sans trêve ce dernier, quoique non payé de retour, chante de son amour, et, modèle d'amour et de fidélité, il invite à l'amour le voyageur. Aussi est-il à vrai dire la seule

1. Geschichte der schönen Redekünste Persiens, p. 25.

2. «Hier encore, dit le poète Hafiz, perché sur les branches d'un haut cyprès, le rossignol tenait en pelhvi les assises de l'amour. ” Joseph von Hammer, Der Diwan von M. Schemsed-din Ilafis, vol. II, p. 389.

3. C'est-à-dire, dans la langue des anciens Perses; de Hammer se sert du mot altpersisch. 
muse des orientaux, qu'ils ne manquent jamais d'invoquer au début de chacun de leurs chants.

On ne pouvait mieux caractériser cette fiction, laquelle, suivant le mot de Gœthe ${ }^{1}$, remplace pour les peuples de l'lran la mythologie qui leur fait défaut. On comprend d'aprés cela la place considérable qu'elle occupe dans les ouvres de leurs poìtes. On la rencontre chez les plus anciens, comme chez. les plus récents d'entre eux. Déjà Firdousi lui a donné place dans ses plus belles descriptions:

Le rossignol, dit-il ², dans une pièce de vers que j’ai citée en partie, se plaint dans le bocage; à ses chants la rose répond en soupirant.

Ce poète s'est même servi de la succession des amours du rossignol et de la rose pour compter le temps :

Soixante-dix fois, fait-il dire à un de ses personnages ${ }^{3}$, la rose avait fleuri, soixante-dix fois elle s'était fanée, et le rossignol l'avait chantée et sétait tu soixante-dix fois.

A la même époque, tant cette fiction était devenue d'un emploi ordinaire, on voit le poète Anszari comparer les doux entretiens de deux amants à ceux

1. Westijstlicher Diwan (Noten und Ablandlungen. Allgemeines): "Rose und Nachtigall nehmen den Platz ein von Apoll und Daphne. »

.2. Voir plus haut, p. 130.

3. Jolowicz, Der poetische Orient, p. 442, 2. Trad. de Heine. 
qu'au retour du printemps le rossignol a dans le bocage avec la rose ${ }^{1}$.

Mais c'est Ferid-eddin-Attar, qui le premier a donné a cette gracieuse fiction tout son développemen; dans la Diète des oiseaux, il nous montre "le rossignol ivre d'amour » et, ravi par la beauté de la rose, oubliant abîmé dans sa passion sa propre existence :

Je ne pense, dit-il ², quà l’amour de la rose, ne désire rien que la rose... Le rossignol suffit à la rose; pour lui s épanouit sa centuple corolle. A mon gré la rose fleurit et me sourit avec une douce joie. Quand elle me sourit dans sa fleur, la joie éclate sur mon front. Que serait une seule nuit passée loin de ma bien-aimée?

Ces derniers vers nous montrent clairement le sens de l'allégorie qui se cache sous cette fiction; gul - la rose - n'est autre que l'amie du poète, bulbul - le rossignol - le poète lui-même. Ce sont ses amours que celui-ci raconte ou chante sous ces noms empruntés; c'est là "le secret, comme s'exprime Djelal-eddin-Roumi", que la langue du lis raconte à l'oreille des cyprès ». C'est ainsi que Hafiz en particulier a entendu les amours du rossi-

1. So pflegten diese Liebenden zu kosen, Wie in dem Rosenhain, zur Rosenzeit, Die Nachtigallen sprechen zu den Rosen In ungetrübter Ruh' und Heiterkeit.

Wamik und Asra, p. 33.

2. Joseph von Hammer, Geschichte der schönen Redeliünste Persiens, p. 144.

3. Voir plus haut, p. 167. 
gnol et de la rose. Comment se méprendre sur le sens qu'il y attache, quand, à la fin d'un de ses ghazels, il s'écrie : "Viens et sois une rose pour le rossignol?"

Le poète anacréontique revient sans cesse sur cette fiction, à laquelle il doit peut-ètre ses plus beaux vers :

Plains-toi, plains-toi, Bulbul, s'écrie-t-il dans un de ses ghazels ${ }^{1}$, si tu es mon ami; tous deux nous sommes épris, la plainte nous sied."

Et dans un autre ghazel ${ }^{2}$ :

De bonne heure, je suis allé dans mon jardin pour cueillir des roses; la voix du rossignol est venue frapper mes oreilles. Ah! l'infortuné est comme moi épris des roses, aussi pleure-t-il en accents plaintifs dans le bocage.

Et ailleurs encore ${ }^{3}$ :

Séduit par le parfum des roses, de grand matin je suis allé dans leurs bosquets, pour calmer, semblable au rossignol, ma tête enivrée. D'un œil fixe j’ai regardé face à face et dans les yeux la rose qui à l'aube brillait comme une lampe. Elle était fière et de sa beauté et de sa jeunesse, parce que le rossignol est tout à elle.

Jamais Hafiz n'a été mieux inspiré que par cette fiction; qu'on en juge par le ghazel suivant, où se

1. Der Diwan von Hafis, vol. I, p. 101, XXXVII, v. 1-2.

2. Der Dis an von Hafis, vol. II, p. 405, XXXVIII, r. 1-5.

3. Der Diwan von Hafis, vol. II, p. 110, I, v. 1-2. 
fait entendre, chose rare chez lui, un accent sincire et profond ${ }^{1}$ :

J'ai salué au matin la plaine couverte de perles de rosée; la nature de son sourire faisait par myriades éclore les roses. Alors j'ai entendu les douces plaintes du rossignol. Elles révélaient les tourments que son cour éprouve... O rossignol, ton chagrin, que je le comprends bien; pour nous deux l'amour n'est que peine.

Quoi de plus gracieux encore que ces vers, qu'on prendrait pour une épigramme de l'anthologie grecque $^{2}$ :

Sache-le, ô rose, il ne te sied pas d'être si fière de ta beauté, que dans ton orgueil tu ne daignes même pas t'informer du triste rossignol.

Cette note attristée et résignée à la fois se retrouve avec le mème charme dans les deux ghazels suivants :

Écoutez, le rossignol chante de nouveau dans les branches des cyprès; qu'un oil malveillant n'ose point regarder la rose! Rose, dans l'ivresse du bonheur d'être une sultane de beauté, ne t'éloigne pas si fièrement des pauvres rossignols ${ }^{3}$.

1. Jolowicz, Der poetische Orient, p. 557.

2. Der Diwan ron Hafis, vol. Il, p. 19, XI, v. 1-4. Der poetische Orient, p. 553.

3. Jolowicz, Der poetische Orient. On reconnait là la source des vers célèbres de Byron dans le Giaour, v. 21-24.

The rose o'er crag or vale

Sultane of the nightingale,

The maid for whom his melody

His thousand songs are heard on high. 
Le rossionol songe comment il pourrait faire de la rose son amie; mais la rose ne pense qu’à faire souffrir le rossignol ${ }^{1}$.

Cette fiction offrait aux poètes persans un moyen trop commode d'exprimer leurs sentiments cachés, pour quils ne lui aient pas eu toujours recours; on la rencontre aussi à chaque instant dans leurs vers ; parmi ceux qui s'en sont servis après Hafiz je n'en citerai qu'un, Kiatibi, qui a rivalisé de grâce, mais encore plus d'afféterie avec son prédécesseur; il appartient à la période de décadence de la littérature persane ${ }^{2}$. Dans son Prème des roses, il nous montre Bulbul - c'est-à-dire lui-mème —, chantant, retenu par l'amour, sur un eyprès. ”

O toi, s'écrie-t-il ${ }^{3}$, dont la bouche est un vrai bouton de roses, tant que tu demeures ici, je n'ai point d'ailes (pour menvoler), blessé que je suis par l'enivrement de ton regard.

C'est là d'ailleurs le seul passage où l'on trouve quelque vérité de sentiment; le reste du poème est un simple jeu de l'imagination, et le monde des fleurs et des animaux n'y est qu'une machinerie dont le poète se sert pour éblouir le lecteur.

1. Der Diwan von Hafis, vol. II, p. 59, III, v. 1-4.

2. Kiatibi mourut de la peste en 1435 .

3. Joseph von Hammer, Geschichte der schönen Redekünste Persiens, p. 282. Voici le début du poème de Kiatibi ; íl peut donner uneidée de sa manière : " Les roses reviennent dans la plaine, les flacons du ciel versent sur terre de l'eau de rose; le printemps prend une livrée verte et rouge, etc. ") 
Tout autre avait été Saadi; l'auteur du Gulistan a, lui aussi, mis en cuvre l'allégorie des amours du rossignol et de la rose, mais pour en faire sortir une de ces vérités morales qui lui sont chères. Dans sa fable du rossignol et de la fourmi, sujet de la première des fables de La Fontaine, qui a remplacé le roi des oiseaux chanteurs par la cigale, Saadi nous montre le rossignol qui voltige jour et nuit autour des bosquets de rose, en répandant ses chants aux mélodies enchanteresses ${ }^{1}$. Tandis que la fourmi peine et fatigue, il s'entretient de ses secrets arec la rose et prend le zéphir pour confident. Cependant l'automne vient, les vents dépouillent les arbres de leur parure, les feuilles jaunissent et tombent. Les frimas ont succédé aux tièdes zéphyrs. Le rossignol revient visiter les parterres qui l'avaient charmé, mais il n'y retroure plus, ni l'éclat des roses, ni le parfum des jasmins. Le cœur lui défaille en présence du bocage dépouillé de ses leuilles, sa voix s'arrète dans son gosier au milieu du silence universel et cesse de se faire entendre. Nu et affamé, il n'a d'autre recours que d'aller demander à la fourmi l'aumône de quelques graines, mais l'économe ouvrière le refuse :

Tu passais les nuits, lui dit-elle, en entretiens amoureux, tu n'élais occupé que des charmes de la rose et des joies du printemps; ne savais-tu pas qu au printemps

1. Joseph von Hammer, Geschichte der schünen Redekünste Persiens, p. 207. 
succède l'automne et que tout chemin conduit au désert?

Ici les amours du rossignol et de la rose servent seulement de cadre au récit du poète; ils n'en sont pas le sujet mềme. Chez Saadi, ainsi que chez ses prédécesseurs ou ses émules, l'allégorie, quelque. complète qu'elle soit, n’en est pas moins renfermée dans des proportions modestes; il n'en fut pas de même chez leurs imitateurs ottomans, qui lui ont donné les développements les plus vastes.

L'Iran a été subjugué tour à tour, on le sait, par les Arabes et les Osmanlis, mais tour à tour aussi ces peuples ont subi l'influence littéraire de ceux qu'ils avaient vaincus. Les Arabes ont emprunté aux poètes persans leur langage de convention et quelques-unes de leurs fictions; c'est ce que, six siècles plus tard, a fait également et bien plus encore la poésie ottomane, ì qui la poésie iranienne a servi surtout de modèle ${ }^{1}$. Facile, aimable et voluptueuse conme elle, comme elle cherchant ses inspirations dans le monde des sens et de la nature, elle ne pouvait manquer de lui emprunter la fiction des amours du rossignol et de la rose; mais ce que n'avait point fait la poésie persane, elle devait, en l'adoptant, lui donner les proportions de l'épopée.

1. Parmi les livres que lit de préférence la princesse Rose, l'héroïne du poème de Fasli, Gul et Bulbul, figurent le Beharistan de Djami et le Gulistan de Saadi, avec beaucoup de Diwans. Hammer-Purgstall, Geschichte der osmanischen Dichtiunst, vol. II, p. 311. 
Un poète de cette nation, Fasli, s'en est emparé au $\mathrm{xvi}^{\mathrm{e}}$ siècle $^{1}$ et en a fait un long poème moral, Gul et Bulbul, dont la Rose est l'héroïne, le Rossignol le héros, et où figurent personnifiés la rosée du matin, le zéphyr, le cyprès, le narcisse, l'hyacinthe, la violette et l'épine avec les quatre saisons de l'année. Le Rossignol éloigné par la fierté de la Rose et repoussé par l'orgueil du roi Printemps, indisposé par l'Hyacinthe, philosophe grognon, fuit désespéré dans la solitude. Il est même jeté en prison, au moment où la Rose, gagnée par le Narcisse et touchée par une lettre de son admirateur, se déclare prète à l'écouter. Tandis qu'il gémit dans la captivité, n'ayant pour confident que la Violette, les armées du roi leté ravagent les états du Printemps; l'Automne s'interpose, mais bientòt l'Iliver vient achever de détruire les bosquets de roses dévastés par l'Été. Cependant l'année nouvelle ramène le printemps; la Rose revient tròner au milieu des fleurs qui composent sa cour. Avertie que le Rossignol languit toujours en captivité, elle prend la résolution de le visiter, et, conduite par le Zéphyr, elle se rend auprès de son amant, qu'elle trouve amaigri et si faible qu'il s'évanouit à sa vue. Elle enroie aussitòt demander sa délivrance à son père. Le Rossignol amené devant le roi est remis en liberté. Il se hate de se rendre auprès de son amante; tandis qu'il raconte ses arentures et ses chagrins au Cyprès,

1. Fasli est mort en 1563. Hammer-Purgstall, $O_{P}$. laud, vol. II, p. 309. 
gardien du palais, la Rose tient une diète, dans laquelle elle confesse devant toutes les fleurs assemblées son penchant pour le Rossignol; celui-ci est introduit par le Zéphrr et vivra désormais heureux dans la société de la Rose.

Tel est ce poème gracieux, sous les fictions duquel se cache, nous dit Fasli, un sens profond, car la rose y personnifie l'esprit engendré par la raison, et le rossignol, le cœur, dont l'accord avec la raison constitue le bonheur de la vie humaine. Cette allégorie, si elle était entièrement exacte, eût jeté de la froideur sur l'œuvre de Fasli; heureusement l'explication qu'il a donnée a été faite après coup; en réalité la rose, dans tout son poème, n'est que la reine des fleurs, sous l'emblème de laquelle se cache la personne aimée. Telle elle apparaît encore plus dans le poème du contemporain de Fasli, Muidi, Gul et Newrous ${ }^{1}$.

Newrous (le printemps), fils du chah Ferrouch, qui a vu Gul en songe, en est épris et le Rossignol, devenu messager d'amour, porte ses lettres à son amie. Rapprochés par ses soins, les deux amants voient leur bonheur troublé par un message du Zéphyr qui demande la main de Cul pour son maître, Désespérés ils prennent la fuite. Attaqués par des brigands au passage d'un fleuve, ils parviennent ì leur échapper, arrivent au bord de la mer et s'embarfuent; mais ils font naufrage; Gul tombe entre

1. Hammer-Purgstall, Op. la:ld, vol. II, p. 527-529. 
les mains d'un marchand, qui la conduit au roi Rebii, Newrous est vendu comme esclave au chah de l'Yémen, Bebii, par le pècheur qui l'a sauré. Cependant la guerre éclate entre le chah Rebii et Bedii; le général de ce dernicr est tué par Newrous; Gul, pour venger la mort du héros, attaque Newrous, à son tour; mais les deux amants se reconnaissent bientòt et désormais, réunis, ils passent le reste de leur vie dans la joie et le bonheur. 


\section{CHAPITRE $\cdot$ III}

LA ROSE DANS LES LÉGENDES CHRÉTIENNES

La rose avait été trop intimement mèlée aux pratiques du paganisme, elle avait joué un rôle trop considérable dans ce que la vie des anciens avait de plus profane, pour n'avoir pas été tout d'abord suspecte au christianisme; elle fut proscrite aussi par les premiers docteurs de l'Église. Tertullien, en blâmant l'emploi des couronnes: condamnait par là mème l'usage des roses; Clément d'Alexandrie proscrivait également l'emploi des couronnes de fleurs, en particulier de roses et de lis, ainsi que l'usage des parfums ${ }^{2}$. Prudence se vante de ne se servir, dans ses repas, ni de roses, ni d'aromates ${ }^{3}$, et il félicite sainte Eulalie d'aroir toujour's dédaigné et méprisé

1. "Neminem dico fidelium coronam capite nosse alias extra tempus tentationis. „) De corona, cap. II, éd. Migne, vol. II, p. 77.

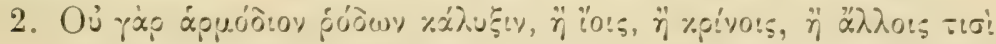

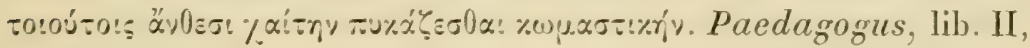
cap. 8 ( $/ 8)$.

3.

Hic mihi nulla rosae spolia,

Nullus aromate fragrat odor.

Cathemerinon, Hymnus ante cibum, v. 21-22. 
les couronnes de roses, aussi bien que les ornements d'ambre et les colliers d'or ${ }^{1}$.

Mais cette opposition prit fin avec le spectacle des excès auxquels la rose avait été associée. Merveille du règne végétal, cette fleur devait bientôt, avec toutes les autres, prendre place dans le culte qu'on rendait au Créateur et aux saints; les poètes de la religion nouvelle la chantèrent comme l'avaient fait autrefois ceux du paganisme; le mysticisme chrétien lui attribua une signification symbolique et elle en devint un des emblèmes les plus chers.

Parure de la terre, la rose ne pouvait manquer dans le Paradis terrestre; elle y figure au premier rang avec le lis dans toutes les descriptions que nous en ont données les écrivains du moyen ìge. Mais, par une conception qui rappelle une des traditions du Bundehesch ${ }^{2}$, saint Basile ${ }^{3}$ et saint Ambroise ${ }^{\ddagger}$ ont supposé qu'elle était alors sans épines”.

1. Spernere succina, flere rosas,

Fulva monilia respuere.

Peristephanon, Hymnus in honorem Eulaliae, v. 21-22.

2. Voir plus haut, II e partie, chap. I, p. 1'í0.

3. De peccato, cap. 10. Dans une épitre adressée à Libanius, Basile paraît dire, au contraire, que la nature a donné des aiguillons aux roses pour exciter le désir de ceux qui les cueillent.

4. Surrexerat ante floribus immixta terrenis sine spinis rosa et pulcherrimus flos sine ulla fraude vernabat. Ilexameron, cap. xi, 48.

5. Milton a soigneusement conservé cette légende, quand il parle 
Elles n'auraient apparu qu'après la désobéissance d'Adam et d'Éve, fiction qui devait ètre la source d'innombrables comparaisons.

L'un des poètes les plus anciens qui aient décrit le Paradis terrestre, Dracontius, nous montre Adam et Eve se promenant dans ce jardin délicieux " au milieu des fleurs et des vastes bosquets de roses ${ }^{1}{ }^{2}$.

L'hiver, dit-il ailleurs ${ }^{2}$, y produisait des roses; en plein été seépanouissaient encore les lis empourprés; toutes les fleurs y étaient parfumées et jamais l'éclat des violettes à la pudique pâleur ne s'y ternissait.

Les lis, chante également Avitus ${ }^{3}$, y brillent sans que le soleil les flétrisse, aucun souffle n'y ternit les violettes et les roses vermeilles y conservent toujours une grâce inaltérable.

Dans la description du Paradis que Madoïnus* adresse à son ami Théowulf, l'érèque du raI $^{\mathrm{e}}$ siècle décrit aussi ce séjour de délices et ses ruisseaux au

des fleurs de toute nuance et de roses sans épines qui remplissent les vallées de l'Éden :

Flowers of all hue and without thorn the rose.

$$
\text { Paradise lost, book IV, v. } 256 .
$$

1. Ibant per flores et lata rosaria bini.

Carmen de Deo, lib. I, v. 137 (Migne, Patrol., vol. IX, p. 754).

2. Et rosulas proferret hiems, servaret et aestas

Lilia purpurea, tlos omnis staret odore,

Nec marceret honos violae pallore decenti.

$$
\text { Ibid., lib. II, v. 442-44. }
$$

3. De initio mundi, Éd. Migne, p. 328.

4. Florigeras sedes, jucundo et murmure rivos

Undique stipatos floribus atque rosis.

De Paradiso, v. 5-6 (Poclae aevi Car. rec. Duemmler, I, 573). 
doux murmure tout bordés de fleurs et de roses. Deux siécles plus tard, l'auteur de la traduction allemande de la Genèse, dont j’ai parlé plus haut, place également dans le Paradis terrestre des roses et des lis, ainsi que toutes sortes d'herbes aromatiques ${ }^{1}$. Milton est resté fidèle à cette tradition et il nous montre le berceau fleuri, sous lequel sommeillent Adam et Éve, répandant sur eux " une pluie de roses que renouvelle chaque matin »:

on their naked limbs the flowery roof

Showered roses, which the morn repaired ${ }^{2}$.

1. Dans le Paradis, où le conduit son royage miraculeux, saint Brandan aperçoit de

Bels gardins et grant praerie

Qui tousjours est verde et florie,

Ses flors souef et moult bon flairent.

Der Brandan des Arsenal publ. par Th. Auracher, v. 1651-53 (Zeitschrift für rom. Philologie, vol. II, p. 456). De même dans la " terre nouvelle » que visite le saint, d'après la version en ancien anglais, " le soleil est plus brillant, la joie règne,... chaque herbe est couverte de fleurs et chaque arbre de fruits s:

Euerech herbe was ful of flowres: and ech treo was ful of fruyt. Vita sancti Brandani abbatis de Hibernia (The early southenglish Legendary or lives of saints... ed. by Carl Horstmann. London, 1887, in-8, p. 222). Nais le saint, chose remarquable, n'y voit ni lis, ni roses. Le chevalier Owen n'en aperçoit pas davantage dans le Paradis terrestre où il aborde après sa visite au Purgatoire :

Si cum uns prez fust cis païs Herbes i out de bone odur De flors e d'arbres planteis, E gentils fruiz de grant valur.

Le Purgatoire de saint Palrice, v. 1587-90. (Poésies de Marie de France... publićes par B. de Roquefort. Paris, 1831, in-8, rol. II, p. 4z2).

2. Paradise lost, book IV, v. 772-73. 
Par une analogie qui s'explique de soi, on mit aussi des roses dans le séjour des bienheureux, qu'écrivains et poètes, l'identifiant avec le Paradis terrestre, représentent à l'envi comme un lieu de délices, un parterre rempli d'arbres verdoyants et de fleurs parfumées.

Il est, dit une inscription funéraire ${ }^{1}$, entré, ô Paradis, dans tes demeures angéliques et tes royaumes brillants d'or, au milieu de tes richesses, et de tes parterres aux gazons embaumés de fleurs divines.

Sedulius dépeint également le Paradis et "ses champs remplis de fleurs éternelles ${ }^{2}$, le charme de ses bosquets entretenu par des eaux toujours vives et ses jardins ravissants où les fruits ne manquent jamais ». "C'est un verger immense, dit Saturus dans les Actes de sainte Perpétue", rempli de rosiers et de fleurs de toute espèce ». Ailleurs" il nous

1. Angelicasque domos intravit et aurea regna,

Divitias, Paradise, tuas, flagrantia semper

Gramina et habentes divinis floribus hortos.

2 .

$$
\text { C. I. L., vol. XII, no } 9 \text { ' } 9 \text {, p. } 122 \text {. }
$$

Gramineus blanditur ager, nemorunque voluptas

Irriguis nutritur aquis, interque benigne

Conspicuos pomis non deficientibus hortos.

Opus paschale, lib. V, v. 222-25. Cf. Éd. du Méril, Poésies populaires latines. Paris, 1843 , in-8, p. 113 , note 2.

3. "Spatium grande... quasi vividarium, arbores habens rosae, et omne genus floris. "Acta primorum martyrum sincera et selecta collecta opera et studio Th. Ruinart. Amsteld., 1713, in-fol., cap. xı, p. 98.

4. "In vividario, sub arbore rosae... universi odore inenarrabili 
peint les martyrs, réunis dans ce jardin céleste, " sous un rosier, et se nourrissant à satiété de parfums inénarrables. »

Dans la patrie des justes, chante Prudence ${ }^{1}$, célébrant l'aurore de la Pâque, la terre est tout embaumée de rosiers aux fleurs empourprées qui la couvrent, et, arrosée par des sources vives, elle y produit de brillants soucis, de molles violettes et le tendre safran.

Fortunat, parlant des vierges qui, au ciel, célèbrent dans de divins banquets leurs vœux exaucés, les montre cueillant “ l'une des violettes, l'autre des roses $)^{2}$; et des roses et des lis y sont aussi l'éternel aliment de leurs yeux ${ }^{3}$.

Dans les demeures éclatantes d'or et de pierres précieuses, lit-on dans un hymne de Pierre Damien, mais attribué parfois à saint Augustin ${ }^{\prime}$, jamais ne sévissent,

alebamur, qui nos satiabat ». Ibid., cap. XIII. p. 99. Cf. Martigny, Dictionnaire des antiquités chrétiennes, s. v. Paradis.

1.

Illic purpureis tecta rosariis

Omnis fragrat humus, calthaque pinguia

Et molles violas et tenues crocos

Fundit fonticulis uda fugacibus.

Cathemerinon. V. De novo lumine Paschalis sabbati, v. 113-11' .

2. Per paridisiacas epulas sua rota canentes, Ista legit violas, carpit et illa rosas.

De virginitale, v. 29-30, lib. VIII.

3. Floribus aeternis oculos rosa, lilia pascunt.

Ad virgines, v. 11, lib. VIII.

'́. Hiems horrens, aestas torrens illic nunquam saeviunt.

Flos purpureus rosarum ver agit perpetuum,

Candent lilia, rubescit crocus, sudat balsamum.

Rhythmus de gloria paradisi, v. 13-15, éd. Migne, vol. LI, 
ni les frimas de l'hiver, ni les chaleurs de l'été ; un printemps éternel y produit sans cesse la fleur vermeille de la rose; sans cesse y brillent la blancheur du lis et la pourpre du safran et sans cesse y coule le baume.

Adam de Perseigne dépeint également le " jardin du ciel » comme rempli de fleurs mystiques au milieu desquelles brille la "rose d'amour " ${ }^{1}$. Et un vieux poète italien, dans la description de la “ Jérusalem céleste $~ " ²^{2}$, la montre arrosée par un beau fleuve, dont les rives sont ombragées d'arbres verdoyants et embellies de lis, de roses, de violettes et d'autres fleurs qui exhalent les plus doux parfums.

II.

La rose ne fut pas seulement considérée par les écrivains mystiques du moyen àge comme la parure

p. 915-16. Migne en avait fait d'abord le chap. xxvi du Livre des méditations de saint Augustin.

1. Fragmenta Mariana (Migne, Patrol., vol. CGXI, p. 750).

$2 . \quad$... per meco un bello flumo ge cor

Lo qual e circundao di molto gran verdor

D'albore et de çigi e d'altre belle flor

De rose et de viole ke rendo grande odor.

De Jerusalem celesti et de pulcritudine ejus, etc., v. 89-92. Mussafia, Monumenti antichi di dialetti ilaliani. Sitzungsberichte der Alademie der Wissenschaften. Wien, vol. XLVI (1864), p. 137. (Il faut rapprocher de cette description celle du jardin céleste dont parle Jacopone de Todi, avec ses forêts chargées de fruits, ses ruisseaux qui fuient parmi les gazons et les fleurs. Ozanam, Des sources poétiques de la Divine Comédie (OEurres complètes, vol. V, p. 418). 
et l'ornement du paradis et du ciel, ils la regardèrent encore comme le prix de toute action noble et belle, l'emblème de ce qu'il y avait de plus auguste et de plus vénéré dans les croyances chrétiennes. C'est ainsi qu'elle devint le symbole et l'apanage de la Vierge et du Christ.

A l'exemple de saint Paul qui exhortait les fidèles à acquérir par une vie exemplaire " une couronne de gloire incorruptible ${ }^{1}$, "s saint Cyprien encourageait les confesseurs et les martyrs de son église à gagner de blanches couronnes de lis et des couronnes vermeilles de roses.

Dans les camps célestes, écrivait-il ${ }^{2}$, la paix et la lutte ont leurs fleurs propres, dont le soldat du Christ se couronne dans sa gloire.

Et rappelant dans un autre ourrage que jamais Dieu ne laisserait nos mérites sans récompenses.

A ceux qui vainquent dans la paix, disait-il ${ }^{3}$, il donnera, en retour de leurs bonnes œuvres, une couronne de lis blancs ; à ceux qui triomphent dans la persécution, en récompense de leur mort, il accordera une couronne de roses vermeilles.

1. Epist. ad Corinthios, lib. I, cap. $\mathrm{rx}, \mathrm{v}, 25$.

2. “Floribus ejus (ecclesiae) nec lilia nec rosae desunt... Accipiant coronas vel de opere candidas, vel de passione purpureas. In caelestibus castris et pax et acies habent flores suos, quibus miles Christi ob gloriam coronetur. "Epistola ad marty'res et confessores. (Epist., lib.. I, 8, Migne, p. 249-50).

3. "In pace vincentibus coronam candidam pro operibus dabit, in persecutione purpuream pro passione geminabit. " Liber de opere et eleemosynis, cap. 26 (Éd. Migne, p. 646). 


\section{Saint Jérôme tient le même langage :}

Le sacrifice d'un cœur pur, écrit-il à Eustochius ${ }^{1}$, est un martyr, aussi bien que l'effusion du sang pour confesser sa foi; par celle-ci on se tresse une couronne de roses et de violettes, par celle-là une couronne de lis.

Dans une autre épître adressée à Rusticus, il fait encore de la rose l'emblème de la pudeur, ainsi que du lis celui de la pureté ${ }^{2}$. Et ailleurs ${ }^{3}$ on le voit associer les "violettes » des veuves aux " lis des vierges » et aux "roses des martyrs ».

Saint Augustin, à son tour, rappelant combien l'Église honore, et la dignité de la vie, et la gloire du martyr, ainsi que la pureté inviolée de la foi, ajoute que ni les roses ni les lis ne manquent aux récompenses qu'elle donne". Ailleurs encore, il dit que

1. "Non solum effusio sanguinis in confessione reputatur, sed devotae quoque mentis servitus immaculata quotidianum martyrium est. Illa corona de rosis et violis plectitur, ista de liliis. ) E Eist., GVIII, 31. Ad Eustochium, Epitaphium. Paulae matris. Éd. Migne, vol. I, p. 905.

2. "Non mihi laborandum (est) uti ostendam tibi variorum pulchritudinem florum, quid in se lilia habeant puritatis, quid rosa verecundiae possideat; quid violae purpura promittat in regno. » S. Hieronymi, Epist., CXXV, 2. Ad Rusticum monachum. Éd. Migne, vol. I, p. 1073.

3. "Suscipe viduas, quas inter Virginum lilia et Martyrum rosas, quasi quasdam violas, misceas. "E Epist., LIV, 14. Ad Furiam, De viduitate servanda. Éd. Migne, vol. I, p. 557. "Est quocque, dit aussi S. Ilildefonse, candidum lilium flos Virginum, rosae purpurantis sanguinis martyrum, violae gratia continentium. » Du Cange, s. v. Rosa.

4. "Floribus ejus nec rosae, nec lilia desunt. " Sermo CCIX. In festo omnitim Sanctorum, 1. Éd. Migne, vol. V, p. 2135. 
Dieu qui, dans la persécution, donne des couronnes de fleurs vermeilles, récompense du martyre, donnera aussi à ceux qui vivent dans la paix de blanches couronnes, récompense de leurs mérites de justice ${ }^{1}$.

On voit par là comment la rose est devenue le symbole du martyre, comme elle était la récompense de ceux qui le souffrent; mais elle devait prendre bien d'autres significations:

Il y a trois roses mystiques, suivant Pierre de Mora ${ }^{2}$, qui a fait en quelque sorte la théorie du symbolisme de la reine des fleurs; la première est le chœur des martyrs; la seconde, la Vierge des Vierges; la troisième, le médiateur de Dieu et des hommes ${ }^{3}$. La premiere est rouge, ajoute-t-il,... la seconde, blanche... la troisième, rouge et blanche... La première est bonne, la seconde meilleure, la troisième excellente. Et encore: la première rose naît des épines de la persécution et des hérétiques. La seconde est sortie des épines de la perversion des Juifs, dou le proverbe : comme l'épine produit la rose, ainsi la Judée a engendré Marie. La troisième est née sur la tige sortie de la racine de Jessé, suivant la

1. “Qui coronam in persecutione purpuream pro passione donavit, ipse in pace viventibus pro justitiae meritis dabit et candidam. " Ibid., vol. V, p. 2137. "Flos rosae, écrit S. Grégoire, qui mira est fragrantia, rutilat et redolet ex cruore martyrum. » Homil. VI, lib. I.

2. E rosa alphabelica seu ex arte sermocinandi, ap. J. B. Pitra, spicilegium Solesmense complectens sanctorum patrum scriptorumque ecclesiasticorum' anecdota hactenus opera. Paris, 1855, in-8, vol. III, p. 490.

3. "Qualis est rosa? Prima rosa est chorus martyrum; secunda, Virgo Virginum; tertia, mediator Dei et hominum. „) 
LA ROSE DANS LES LÉgendes CHRÉtiennes. 241

prédiction d'Isaïe: "Une verge sortira de la racine de Jessé et une fleur surgira de cette racine ${ }^{1}$ "».

On a là comme le résumé de ce que les écrivains mystiques ont dit et chanté de la rose depuis les premiers siècles jusqu’à la fin du moyen âge. Prudence compare les saints Innocents voués à la mort, " au seuil même de la vie, ) par le " persécuteur du Christ », à des roses naissantes que l'ouragan détruit et disperse ${ }^{2}$.

Le jeune martyт qui vint au devant du captif chrétien, conduit par Agapius au banquet céleste, portait autour du cou une couronne de roses merveilles ${ }^{3}$. Peu de temps arant son martyre, la bienheureuse Digne vit en songe sainte Agathe portant des roses et des lis dans la main *. "Je suis Agathe, lui dit celleci, qui jadis ai souffert pour le Christ les plus affreux tourments; je viens t'apporter une partie de ces présents empourprés, ) ajouta-t-elle, en lui offrant des roses vermeilles, présage de son prochain martyre.

Saint Ambroise a été jusqu”a dire que la rose est

1. "Prima rosa nascitur ex spinis persecutionis et haereticorum. Secunda rosa orta est ex spinis Judaicae pravitatis, unde

Sicut spina rosam, genuit Judaea Mariam.

Tertia rosa nata est ex virga de radice Jesse. Isaias. Egredietur virga de radice Jesse et flos de radice ejus ascendet. )

2. Salvete, flores martyrum, Christi insecutor sustulit,

Quos lucis ipso in limine Ceu turbo nascentes rosas.

Cathemerinon, XII, Hymnus de Epiphania, v. 125-128.

3. E. Le Blant, Les songes et les visions, p. 9.

4. Acta sanctorum, rol. III, p. 646, $c$.

Joret. La Rose. 
l'image du sang ou plutôt le sang même du Seigneur". Si Walafrid Strabus se borne i remarquer que le Christ " a coloré les roses par sa mort ${ }^{2}$ ), un poète latin du $\mathrm{xw}^{\circ}$ siècle, reprenant la tradition de saint Ambroise, salue " la rose du sang sorti a torrents de la chair divine du Sauveur ${ }^{3}$ ). Les mystiques parlent à chaque instant du “sang couleur de rose du Christ" ). "L'effusion de son sang sacré, dit de Jésus l'auteur de la Vigne mystique ${ }^{5}$, a rougi les feuilles de la rose sanglante de sa souffrance ».

Et un poète du $x^{e}$ siècle compare à des roses vermeilles les cinq plaies sanglantes par lesquelles le Christ a racheté tous les hommes ${ }^{6}$. Le piétisme

1. "Carpis rosam, hoc est, domini corporis sanguinem ». Commentarium in psalmum 118.

2 .

$\ddot{3}$

Morte rosas tingens.

Hortulus, XVI, Rosa, v. 35.

Ave rosa sanguinis

Fusi more tluminis

De carne Salvatoris.

Philipp Wackernagel, Das Deutsche Kirchenlied von der ältesten Zeit lis zu Anfang des xvir. Jahrbunderts. Leipzig, in-8, vol. I, 1864, no 283.

4. "Nundamur roseo sanguine, Ghriste, tuo, " lit-on au-dessous d'une image du Christ à Nuremberg.

5. “ Rosa passionis effusionibus crebris sacratissimi sanguinis psius specialiter fuit rubricata. „ Bernardi opera, III, 712 (480).

6. Merkt, ihr Kristenleute!

Die Rosen ich euch deute;

Das sein fünf Wunden roth und zart.

Damit er uns erlöset hat,

Die Frauen und auch die Mann.

J. Görres, Altdeutsche Vollis-und Meisterlieder, p. 239. 
LA ROSE DANS LES LÉGENDES CHRÉTIENXES. 243

allemaìd a, mème après la Réforme, fidèlement conscrvé cette comparaison: au $\mathrm{xvII}^{\mathrm{e}}$ siècle Paul Gerhard s'en servira encore. Elle ne suffit pas à l'ardeur mystique du moyen âge. Saint Bernard est allé plus loin; il a fait de la rose l'image mème du Christ dans sa passion :

Contemplez, s'écrie-t-il dans une de ses homélies sur les Évangiles ${ }^{1}$, cette divine rose, où la passion et lamour se disputent pour lui donner son vif éclat et sa couleur pourprée. Celle-ci lui vient sans nul doute du sang qui coule des plaies du Sauveur... Comme durant une nuit froide la rose demeure fermée et s'entr'ouvre le matin aux premiers rayons du soleil, ainsi cette délicieuse fleur qui est Jésus-Chrit a paru se refermer comme par le froid de la nuit, depuis le péché du premier homme, et lorsqu'est venue la plénitude des temps, elle s'est épanouie soudain au soleil de l'amour. Autant de plaies sur le corps du Sauveur, autant de roses! Regardez ses pieds et ses mains, n y voyez-vous pas des roses? Mais contemplez surtout la plaie de son cœur entrouvert! Ici c'est plus encore la couleur de la rose, à cause de l'eau qui coule avec le sang, quand la lance a percé son côté!

Écoutons un autre mystique, qu'on a voulu parfois identifier avec saint Bernard ${ }^{2}$ :

1. Homeliae in Esangelia, lib. II, cap. 38, ap. Mgr. de la Bouillerie, Étude sur le symbolisme chrétien de la nature. Paris, 1866, in-8, 2e édit., p. 267. IV. "Jésus-Christ assimilé à la rose à cause de sa passion. "

2. "Floret in vite nostra, benigno Jesu, nihilominus rosa rubens et ardens: rubens sanguine passionis, ardens igne charitatis, 
Sur notre vigne - le divin Jésus - fleurit une rose vermeille et ardente: vermeille du sang de la passion, ardente du feu de la charité, humide de la rosée des larmes du doux Jésus.

Noble rose, dit également un cantique des frères Moraves ${ }^{1}$, grand est ton amour; Seigneur, tes plaies couleur de rose ont triomphé du péché, du démon et de la mort.

Un vieux lied, qu'on rencontre à la fois en Allemagne et en Hollande, en Suède et en Danemark, a mis en œuvre d'une manière dramatique ces comparaisons mystiques de la rose et des plaies du Sauveur $^{2}$. A la première heure du jour, la fille du sultan est allée cueillir des fleurs dans le jardin de son père; la vue des belles fleurs, toutes brillantes de rosée, élève son cœur vers celui qui les a créées; elle l'adore sans le connaitre et souhaite de le voir. Et voilà que le jour suivant à minuit, le Christ lui

roscida effusione lacrymarum dulcissimi Jesu ». Vitis mystica, cap. xxxur, 121 (Migne, Patrol., vol. 184, p. 708). "Il nous faut, dit-il encore, unir la rose de la souffrance à la rose de la charité, afin que la rose de la charité rougisse dans la souffrance et que la rose de la souffrance brùle du feu de la charité. » Necessarium habemus rosum passionis rosae charitatis conjungere : ut rosa charilatis in passione crubescat et rosa passionis igne charitatis ardescat. Cap. $\operatorname{xxxy}, 126$.

1.

Du edle Ros', Dein Lieb ist gross,

Herr, durch die rosinfarbnen Wunden

Hast du Sünd, Teufel und Tod überwunden.

Paul Cassel, Riose und Nachtigall, p. 22.

2. Hofmann von Fallersleben, Viederlaendische Volkslieder. Hannover, 1856, in-8, no 199, p. 345-353. 
apparait sous la figure d'un beau jeune homme; elle s'étonne et lui demande comment il a pénétré dans la demeure royale. Et Jésus lui répond qu'il a quitté pour elle le royaume du ciel et qu'il est le maître des fleurs. Seigneur, s'écrie-t-elle alors, y a-t-il loin d'ici au jardin de ton père? Je voudrais bien en soigner les fleurs pendant toute l'éternité. "Mon jardin, reprend Jésus, est situé à des milliers, milliers de lieues, " et il lui offre une couronne vermeille, présent de fiançailles, qu'elle accepte. A ce moment les blessures du Christ s'ouvrent. "O bien-aimé, s'écrie-t-elle, pourquoi ton cœur est-il si rouge? Tes mains se couvrent de roses? - " Mon cœur saigne pour toi, répond Jésus ${ }^{1}$, pour toi je porte ces roses; je les ai cueillies dans la mort, en versant mon sang pour toi. Mon père m'appelle, ô fiancée; viens, je t'ai conquise depuis longtemps. „ Elle a eu confiance en l'amour de Jésus, dit le poète en terminant; une couronne lui est tressée au ciel.

Nais quelque habituelle que soit la comparaison de Jésus et de sa passion avec une rose, la comparaison de la Vierge avec cette fleur se rencontre encore plus frérfuemment au moyen àge. Symbole de la grâce pudique, la reine des fleur's devint alors, il en sera de même et plus encore à l'époque de la Renaissance, l'apanage naturel de la reine des anges;

1. Mein Herz, das ist um dich so roth,

Für dich trag' ich die Rosen

Ich brach sie dir im Lichestod,

Als ich mein Blut vergossen.

Des Knaben Wunderhorn, Berlin, 1873, vol. I, 61. 
elle en fut la compagne inséparable, comme l'emblème habituel. Chaque fois qu'elle se manifeste aux hommes, des roses naissent sous ses pas. Des roses sont sa parure accoutumée. Suivant une tradition, peu ancienne il est vrai, quand Narie monta au ciel, elle laissa son tombeau rempli de roses ${ }^{1}$. C'est à une rose aussi que depuis le $\mathrm{x}_{1}{ }^{\circ}$ siècle la comparent le plus souvent les écrivains mystiques, poètes ou prosateurs.

Pour Fortunat, Marie n'est encore que la verge sortie de la racine fleurie de Jessé et qui porte des fruits ${ }^{2}$. Fulbert de Chartres ne voit aussi en elle que la verge dont Jésus est la fleur divine:

Jessé $^{3}$ a produit une verge, et cette verge une fleur; sur cette verge repose l'esprit saint; la verge est la Vierge, mère de Dieu, et la fleur son fils.

Mais les choses changent quand on arrive à saint

1. Jacob de Voragine l'ignore encore: " Sepulcrum aperientes, dit-il. corpus minime invenerunt, sed tantum vestimenta et sindonem reperunt ». Legenda aurea, rec. Th. Graesse. Dresdae, 18'16, in-8, cap. cxrx, De assumtione sanctae Mariae Virginis. Dans la première moitié du xvie siècle, Ribadeneyra ne parle pas davantage de cette légende; on la trouve déjà représentée cependant dans des enluminures du siècle précédent.

2.

Radix Jesse floruit

Et virga fructum edidit.

Miscellanea, lib. VIII, cap. 9.

3. Stirps Jesse virgam producit virgaque florem,

Et super hunc florem requiescit spiritus almus,

Virgo, Dei genitrix, virga est, flos, filius ejus.

De beata Virgine, v. 4-6 (Hymni et Carmina ecclesiastica, éd. Migne, XI, p. 356). Cette comparaison se rencontre à charque instant 
LA ROSE DANS LES LÉGENDES CHRÉTIENNES. 247

Bernard; avec lui la Vierge est devenue une rose et telle elle apparaitra désormais. Après avoir opposé à Ève, "l'épine qui a apporté la mort dans le monde, "Marie, "la rose source de salut pour tous les hommes, " le célèbre mystique poursuit ainsi cette comparaison de la mère du Sauveur avec la reine des fleurs ${ }^{1}$ :

Marie a été une rose, blanche par sa virginité, vermeille par sa charité; blanche par la chair, vermeille par l'esprit; blanche par la pratique de la vertu, vermeille par l'écrasement du vice; blanche en purifiant les passions, vermeille par l'esprit ell mortifiant les appétits charnels; blanche par l'amour de Dieu, vermeille par sa compassion pour le prochain.

Si les poètes religieux n'ont point imité toutes ces antithèses scholastiques, ils ont varié à l'infini

chez les écrivains mystiques, poètes ou prosateurs, du moyen àge. Voici comment l'a mise en œurre un trouvère du xrıI ${ }^{\mathrm{e}}$ siècle.
Se nos dist Isaïe
en une profesie
c'une verge d'Egipte
de Jesse espanie
istroit par signorie
de tres grant biaulteit.
celle verge d'Egipte
est la virge Marie,
la flor nos senefie
de ceu ne doutes mie.

Wilh. Wackernagel, Altfranzoesische, Lieder und Leiche,

Basel, 1846, in-8, p. 66, no XLII, str. 2 et 3.

1. Maria rosa fuit candida per virginitatem, rubicunda per charitatem ; candida, carne; rubicunda, mente ; candida, virtutem sectando; rubicunda, vitia calcando ; candida, aflectum purificando ; rubicunda, actum carnalem mortificando; candida. Deum diligendo; rubicunda, proximo compatiendo. Sermo de beata Haria, vol. III, p. 1020, éd. Vigne. 
les comparaisons de la Vierge ${ }^{1}$ arec la rose. La double couleur de cette fleur, le vermeil éclat de sa corolle, son parfum, les épines au milieu desquelles elle croìt, offraicnt autant d'images allégoriques qu'ils ont à l'envi appliquées à la mère du Sauveur. Adam de Saint-Victor la salue comme " un myrte de tempépérance, une rose de patience, le nard odorant ${ }^{2}$ »; pour saint Bonarenture c'est " une rose sans épines, remède des pécheurs ${ }^{3} n$; elle lui apparait comme " douée d'une beauté suprème ", et, comparaison souvent répétée ${ }^{4}$, "plus vermeille que la rose, plus blanche que le lis ${ }^{3}$ ».

Narie, dit Albert de Regensbourgo ${ }^{6}$, est une rose fleurie sortie de la souche cuopable dìve, notre mère commune..." "Elle est, dit-il encore, pourpre comme la violette, brillante de rosée comme la rose, blanche à l'égal des lis ${ }^{\top}$.

1. Léon Gautier, Prières à la Vierge. Paris, 1874, in-32, p. 339.

2. Salve, decus Virginum, myrtus temperantiae, rosa patientiae, nardus odorifera.

Philipp Wackernagel, Das deutsche Kirchenlied, no 194, IV, ง. $1-4$.

3. Eia, rosa sine spina, peccatorum medicina.

$$
\text { Ibid, , no 229, XI, v. 1-2. }
$$

4. On la trouve encore dans l'hymne anonyme $n^{\circ} 297$.

5. Tu es illa speciosa, qua nulla est pulcrior, rubicunda plus quam rosa, lilio candidior.

Ibid., no 228, I, v. 5-8. Cf. Man. lat. 1196, fol. $456 a$ de la Bibl. Nat.

6. A rea virga primae, processit Maria.

7. Purpura ut viola. candens ut lilia. matris Evae florens rosa Ibid., no 22 ', 1 , v. 1-3. roscida ut rosa, Ibid., nم 244, II, v. 4-6. 
LA ROSE DANS LES LÉgENDES CHRÉTIENNES. 249

"Salut, rose suave, s'écrie Conrad de Gaming", annoncée par le buisson de Moïse ». "Réjouis-toi, belle rose, dit un autre poète ${ }^{2}$, livre-toi à l'allégresse, ô rose unie maintenant aux lis ». C'est la " rose du ciel ", lit-on dans un hymne ${ }^{3}$ ). Pierre Damien l'appelle également une " rose du Paradis" ${ }^{4}$ ).

Les poètes provençaux et français ont rivalisé avec les mystiques latins. Pour Pierre de Corbiac, la Vierge est " une rose sans épines, la plus odorante des fleurs. »

\section{Roza ses espina \\ Sobre totes flors olens ${ }^{5}$.}

Elle est pour lui encore " l'églantier que Moïse trouva verdoyant au milieu des flammes ardentes ${ }^{6}$ ».

Le trouvère Gautier de Coincy, cela se comprend de cet écrivain mystique, s'est complu à ces comparaisons de la Vierge avec la rose. Pour lui, e'est une

1. Ave, rosa delicata..., rubo Moysi signata.

$$
\text { Ibid., no 271, III, v. 1-3. }
$$

2.

Gaude, rosa speciosa,

Gaude fruens deliciis,

Nunc rosa juncta lilio.

F. J. Mone, Lateinische Hymnen des Mittelalters. Freiburg-i-

B. 185 ' , in-8, no 458 .

3. “ Caeli rosa. » Ph. Wackernagel, no 300, IV, v. 1.

4. Index Marianus. (Migne, Patrol., vol. CGXIX, p. 509).

5. Karl Bartsch, Chrestomathie prosençale, p. 207, v. 10-11. 6. l'aiglentina

Que trobet vert Moysens Entre las flammas ardens.

Karl Bartsch, Chrestomathie provencale, p. 207, v. 24-27. 
Rose en toute douceur emmiellée et souciée, ainsi qu'il s'exprime dans une de ses Prières ${ }^{1}$. Il faut, dit-il en parlant d'elle, aimer

la fresche rose,

La fleur espanie,

En qui sainz Espirs repose ${ }^{2}$.

"Elle est », dit-il de mème dans son Proloğue ,

Elle est la fleur, elle est la rose

En cui habite, en cui repose

Et jour et nuit sainz Esperiz.

Et, dans un cantique ${ }^{4}$,

C'est la fleur, la violete,

La rose espanie.

Il l'appelle encore, dans un de ses miracles",

La flor de lis et d'englentier,

L'odorant fleur, l'odorant rose,

Qui souef i out seur tote chose.

1. L'abbé Poquet, Les miracles de la Sainte Vierge, traduits et mis en vers par Gautier DE Corrcy. Paris, 1857, in-4, p. 760, v. 81 .

2. Chanson pieuse, v. 61-63, ap. Paul Meyer, Recueil d'anciens textes bas-latins, prosencaux et français. Paris, 187477, in-8, p. $381 a$.

3. L'abbé Poquet, op. laud., p. 5. Prologue, v. 91-94. Et de même ailleurs, Man. fr. 1530, fol. 6 a 2, de la Bibl. nat.,
Rose fresche et chicre
De saint Esperit plene.

4. Chanson pieuse, v. 20-21, ap. Paul Meyer, op. laud., p. 380 .

5. Du clerc qui fame espousa et puis la laissa, v. 2.-4, ap. l'abbé Poquet, op. laud., p. 631. 
Ailleurs ${ }^{1}$ il la salue comme une

Flors d'églantier, flors de lis, fresche rose, Flors de toz biens, flors de totes flors.

Ou encore ${ }^{2}$ comme

Cele qui la rose est des roses.

L'auteur des Miracles de la Vierge revient à chaque instant, et sans craindre de fatiguer, sur ces images, qu'il a parfois développées outre mesure ${ }^{3}$; mais il n'est pas le seul à s'en servir; elles paraissent tellement naturelles qu'on les rencontre chez tous les poètes du temps. Tout sceptique et volage qu'il est parfois, Rustebeuf les a employées comme Gautier de Coincy :

Tu es rosiers qui porte rose

Blanche et vermeille,

dit-il, par exemple dans son Ave Maria ${ }^{4}$. Et dans les Neuf joies de Nostre Dame:

1. Man. fr. 1530, fol. 8, a 2 .

2. De presbitero quem beata virgo defendit ab injuria, ap. Karl Bartsch, La lanģue et la littérature françaises. Paris, 1888, in-8, p. 366 , v. 21.

3. Par exemple au début du Miracle de sainte Léochade, v. 6367 (L'abbé Poquet, p. 112):

La mere Dieu est la grant rose

En cui toute douceur repose,

Ceste rose est de tel douceur

Et si plaine de bone oudeur

Qu'ele refait le cors et l'ame.

1. V. 116-117. Gedichte nach den IIandschriften der Pariser Metional-Bibliothek hege. von Dr. Adolf Kressner. Wolfenbüttel, 1885, in-8, p. 196. 
Tu es li buissons Sinaï...

Liz et trones au roi de gloire...

Olive, eglantier, flors d'espine...

Et ysopes d'umilité

Et li cedres de providence,

Et li lis de virginité

Et la rose de pacience ${ }^{1}$.

Dans le miracle de Théophile enfin il l'appelle tout comme Gautier,

Flors d'eglantier et lis et rose

En qui le fils Dieu se repose ${ }^{2}$.

On lit de mème dans un vieux cantique anonýme en l'honneur de Marie :

Tu es la flour, tu es la rose,

Tu es celle en qui se repose

La doulceur qui tout aultre passe ${ }^{3}$.

Un autre cantique de la mème époque nous offre accumulées comme à plaisir toutes les comparaisons si chères aux mystiques de la Vierge avec la rose et les fleurs :

Tu ies roze colorée, Tous jours nete et pure...

Tous tens est vermoille... Tu ies roziers,

$\mathrm{Tu}$ ies lis et violete Tu ies vergiers,

Tu ies li tres dous paradis ${ }^{4}$.

1. V. 44, 74, 79 et 141-144. Ibid., p. 202-204.

2. Gedichte, p. 220, v. 555-56.

3. Bibl. nat, man. fr. 13167 , fol. 138 b.

4. Wilh. Wackernagel, Altfranzösische Lieder und Leiche, p. $69, n^{\circ}$ xis, str. 3 et 4 . Billl. nat., fr. 1688, fol. 86 . 
LA ROSE DANS LES LÉGENDES CHRÉTIENNES. 253

Une prière $d u x^{e}$ siècle salue également en la Vierge la

Rose tres odorissant

Et vray lis de securité,
Violette tres flourissant Marguerite d'humilité... ${ }^{1}$

Enfin, dans une autre prière plus ancienne, elle est aussi invoquée comme une

Rose de grace et de douceur ${ }^{2}$.

La rose ne joue pas un rôle moins grand dans les invocations adressées à la Vierge par les poètes des pays germaniques". Pour eux aussi c'est une "rose sans épines " " ou "sans les épines du péché », une " rose de Jéricho ${ }^{5}$ ", " du Paradis ${ }^{6}$ » ou " du ciel ${ }^{7}$ »; ou encore une " noble rose épanouie ${ }^{8}$ ", une "rose vermeille ${ }^{9}$ » ou " parfumée ${ }^{10}$ », une "fleur souriante de rose ${ }^{11}$ », ou bien une " rose baignée de la rosée

1. Catalogue Durel 1889, no 355 . J'ignore ce qu'est devenu le manuscrit d'où sont tirés ces vers.

2. Bibl. nat., fr. 13167, à la fin.

3. W. Grimm a réuni, sans les épuiser, les comparaisons mystiques dont la Vierge a été l'oljet, dans la préface de son édition de la Forge d'or de Konrad de Wurzbourg, p. 25-53.

4. Ph. Wackernagel, vol. II, nos 59, 179, 541, 711, etc.

5. Iloffmann von Fallersleben, Niederlaendische geistl. Lieder des XV Jahrhunderts, no 20. - Konrad v. Wurzbourg, Die goldene Schmiede, v. 422 , etc.

6. Niederl. geistl. Lieder, no 28 , str. 3, v. 8.

7. Wernher v. Tegernsee, Maria.

8. Loblied auf die Jungfrau, str. 1'́4, 7.

9. Ph. Wackernagel, vol. II, no 541, 2.

10. Joh. der Münch v. SalzJurg. ap. Ph. Wackernagel, II, 583, 10.

11. Gottfried v. Strasljurg, Lobgesang, st. 14, 2. 
de mai ${ }^{1}$ », ou " du ciel ${ }^{2}$ ». D'autres fois même ils l'appellent une "couronne de roses ${ }^{3}$ », une "vallée ${ }^{4}$ » ou un " champ $^{5}$ ), un " parterre $^{6}$ » ou bien encore un " jardin de roses gardé par Dieu même ${ }^{7}$ », etc. Mais les anciens poètes allemands ne se sont pas bornés à ces courtes comparaisons de la Vierge avec la rose; ils l'ont chantée sous ce gracieux symbole dans des lieds étendus et nombreux.

Un rosier, dit l'un d'eux ${ }^{8}$, est sorti d'une souche délicate; comme les anciens l'ont annoncé, rejeton de la race de Jessé, il a porté une fleur au milieu de l'hiver et de la nuit; la fleur dont je parle, c'est Marie la Vierge pure qui l'a portée.

Un autre ${ }^{9}$ a chanté la naissance de la mère du Sauveur comme celle d'une rose miraculeuse, sortie du sein de sainte Anne et plus belle que toutes les

1. Konrad v. Würzburg, Ave Maria, str. 36 .

2. $\mathrm{Ph}$. Wackernagel, op. laud., II, no 180 , etc.

3. Meister Sigeher, ap. Ph. Wackernagel, II, n० 185, str. 4, 10.

4. Gottfried v. Strasburg, Lobgesang, str. 17, 1.

5. Ph. Wackernagel, II, no 199. - Mariengrüsse, v. 97. Gœdeke, Deutsche Dichtung in Mittelalter, p. 151.

6. W. Grimm, Einleitung, p. xurr.

7. Hoffm.v. Fallersleben, no 60 , st. 4, v. 1 .

8. Es ist ein Ros entsprungen Mitten im kalten Winter. Aus einer Wurtzel zart Das Röslein das ich meine Aus Jesse kam die Art, Und hat ein blümlein bracht

Hat uns gebracht alleine

Marie die reine Magd.

Ludw. Uhland, Alte hoch-und niederdeutsche Volkslieder, Stuttgart, 1845, in-8, vol. II, p. 176, no 340.

9. Mittler, Deutsche Volkslieder, Frankf. a. M. 1856, in-8, $298, n^{\circ} 368$. 
roses que le printemps produisit jamais, plus salutaire au cour et à l'esprit, plus secourable à l'heure de la mort. Un troisième, feignant que Dieu a donné a la "Vierge pleine de grâces » sept roses, emblème des sept principaux actes de sa vie, lui en a tressé une mystique couronne ${ }^{1}$.

Dans un autre lied, qui unit dans un même symbolisme le Christ et sa mère, celle-ci est représentée comme ayant apporté au monde trois roses d'une beauté sans égale; la première parut lorsqu'elle mit au monde Jésus; la seconde fut cueillie le jeudi saint, quand le Christ institua le sacrement de vie; la troisième enfin s'épanouit, quand il rendit l'esprit sur la croix ${ }^{2}$.

Ce lied est $d u x^{e}$ siècle; il offre un exemple curieux des images que la rose a fournies aux mystiques allemands de cette époque. En voici un autre non moins frappant, tiré de la littérature néerlandaise. C'est Bertha d'Utrecht ${ }^{3}$ qui parle. :

Mon fiancé, dit-elle ${ }^{\ddagger}$, voit avec complaisance les lis,

Der Maid Genaden voll...

Gott, der gab ihr sieben rosen fein,

Damit mach' ich das Kränzlein.

Die sieben Rosen der heiligen Jungfrau, ap. J. Görres, op. laud., p. 313.

2. Mittler, op.laud., no 361 .

3. Née en 1457 , morte en 1514.

4. Die lelyen siet hi gaerne, die minre mijn,

Als si te rechte bloeyen ende suver sijn.

Als die rode rosen daer onder staen,

So laet hi sinen sueten dau dacrover gaen.

Moll, Joannes Brugman, vol. II, p. 187. 
qui s'épanouissent dans leur beauté et leur pureté, et quand les roses vermeilles s'y mêlent, il fait tomber sur ces fleurs sa douce rosée.

Il s'en faut qu'on trouve dans l'ancienne poésie religieuse de l'Espagne ou de l'Italie cette abondance de comparaisons entre la Vierge et la rose; Gonzalo de Berceo les ignore complètement. On en trouve, au contraire, comme un écho affaibli dans le refrain d'une des Cantigas d'Alphonse le Sage, où la Tierge est représentée comme la " rose des roses et la fleur des fleurs ${ }^{1}$ ).

Si Juan Ruiz, l'archiprêtre de Mita, l'appelle surtout une fleur - "fleur non ternie »" ou " la fleur desfleurs $)^{3}$-, il lui donne aussi le nom de (rose $)^{*}$. Dans la vie de Marie l'Égyptienne, imitée, il est vrai, d'un poème français, on rencontre également la comparaison de la Vierge avec la reine des fleurs :

1. Rosa das rosas e fror das frores.

Cantigas de Santa Maria de don Alfonso el Sabio. Madrid, 1889 , in-4, $n^{\circ} \times$. Il est probable que si nous possédions les Cantares dont parle le “Sage » monarque, nous y trouverions plus d'une autre comparaison de ce genre.

$2 . \quad$ Santa flor non tannida.

Del Ave Maria de Santa Maria. Libro de cantares del arciprestre de Fita, str. 1639, 2. (Biblioteca de autores españoles. Madrid, in-8, vol. LVII, 1864. Poetas castellanos anteriores al siglo $\mathrm{xv}$ ).

3. Quiero seguir á ti, flor de las flores.

Cantica de loores de Santa Maria. Libro de cantares, str. 1650,1 .

O bendicha fror e rosa.

Del ave Maria de Santa Maria, str. 1636, 8. 
Ce fut, dit la sainte, dans sa prière à Notre Dame ${ }^{1}$, chose merveilleuse que de l'épine sortit une rose et de cette rose le fruit par qui tout le monde a été sauvé.

La comparaison de la Vierge avec la rose et le lis se retrouve encore dans un cantique espagnol anonyme, mais évidemment fort ancien. La Vierge apparaît au vieux poète comme " plus belle que les fleurs ", " plus remplie de grâce que le lis et la rose fleurie $)^{2}$.

Cette même comparaison ne se rencontre qu'assez tard et assez rarement chez les poètes italiens du moyen âge; l'un d'eux, qui semble être de la fin du xu ${ }^{\mathrm{e}}$ siècle, se borne à saluer la Vierge comme la " rose brillante du Paradis, plus odorante qu'aucun autre parfum $)^{3}$. Disciple et émule des mystiques du moyen âge, Dante ne pouvait oublier de leur emprunter une de leur's images les plus ordinaires; un passage célèbre de la Divine Comédie représente la

1.

Fue marauillosa cosa,

Que de la espina ssallió la rosa

Et de la rosa ssallió friçió

Porque todo el mundo saluó.

Biblioteca de autores españoles. Vol. LVII, p. 311 a.

2 .

Mas hermosa que las flores...

Venis con más galanía

Que lirio y rosa florida.

J. Nic. Böhl de Faber, Floresta de rimas antiguas castellañas. Hamburgo, 1828, in-8, vol. 5, p. 27.

3.

Rosa encoloria del parais

Aolente piu ke n'è eonsa nesuna.

Lodi della Virgine, v. 9-10. (Won. antichi di dialetti italiani, p. 192).

Joret. La Rose. 
Vierge, dans " le beau jardin qui fleurit sous les rayons du Christ ", comme "la rose dans laquelle le Verbe divin s'est fait chair ",

La rosa, in che 'I Verbo Divino carne si fece, entourée des " lis dont l'odeur enseigne le bon chemin ${ }^{1}$.

\section{III.}

La rose ne fut pas seulement l'attribut de Jésus et de la Vierge, elle prit place aussi dans la vie des Saints; elle est le témoin de leur innocence méconnue ou persécutée; elle sert à manifester la vertu ou la gloire des élus et sa naissance ou son apparition miraculeuse proclame aux yeux de tous leurs mérites. De nombreuses légendes illustrent cette signification de la rose.

Après sa mort, rapporte l'une d'elles ${ }^{2}$, saint André Corsini apparut à un chanoine de ses amis, revètu d'habits magnifiques et d'une blancheur éclatante, un bouquet de roses et de lis à la main. Et comme le chanoine lui demandait pourquoi il portait un bouquet de fleurs, chose peu séante pour un évêque, André répondit qu'il portait ces roses et ces lis en témoignage de la pureté et de la chasteté de sa vie.

Une vierge d'une grande sainteté, dit une autre

1.

Quivi son li gigli,

$\mathrm{Al}$ cui odor s'aprese 'l buon cammino.

Il Paradiso. Canto XXIII, v. 73-75.

2. Acta sanctorum, vol. II, p. 1069, 25. 
légende ${ }^{1}$, étant venue vers Suson, ce " maître de sagesse » au $\mathrm{xIv}^{\mathrm{e}}$ siècle, craignait de ne pouvoir le trouver au milieu de ses frères, mais la voix qui l'avait envoyée lui dit qu'elle le reconnaitrait sans peine à la couronne de roses vermeilles et de roses blanches qu'il portait sur la tête, symboles, les premières de sa patience au milieu de ses nombreuses afflictions, les secondes de sa chasteté.

Suson est encore le héros de la légende suivante, qui, elle aussi, met bien en évidence la signification mystique des roses miraculeuses. Un jour une religieuse nommée Anne vint le trouver et lui raconta qu'elle avait vu " en esprit » un immense rosier tout couvert de fleurs magnifiques et vermeilles. Au milieu de l'arbuste se trouvait l'enfant Jésus, le front ceint d'une couronne de roses également vermeilles. Au pied était assis Suson lui-mème. Et l'enfant Jésus, cueillant des roses, les jetait sur son serviteur, qui en fut bientôt tout couvert. Anne ayant demandé ce que signifiaient ces roses, l'enfant répondit: "Ces roses nombreuses signifient les croix innombrables que Dieu enverra à son serviteur et qu'il acceptera avec douceur et supportera avec patience $)^{2}$.

Mais ce n'est pas seulement sous forme allégorique que la rose figure dans les légendes religieuses du

1. Acta sanctorum, vol. II, p. 667,62 .

2. Laurentius Surius, Vita Susonis ap. Acta sanctorum, vol. II, p. 677,99 . 
moyen âge, elle y apparaît aussi comme fleur véritable. Le ciel lui-même pour justifier, ou honorer les saints, en envoie parfois sur terre. "Sainte Dorothée, raconte Jacques de Voragine', ayant été conduite devant le proconsul Fabrice, celui-ci voulut la forcer à abjurer: "Choisis, lui dit-il, ou de sacrifier et de vivre, ou de périr dans les tourments. " Mais la sainte répondit: " Je suis prète à souffrir tout ce que tu voudras et je le ferai pour Jésus-Christ, mon époux avec lequel je jourai de la vie éternelle; j’ai cueilli dans son jardin des roses et des fruits délicieux. ) Le tyran la condamna alors à la mort. Comme on la menait au supplice, le greffier du tribunal, Théophile, lui demanda comme par dérision de lui envoyer des roses du jardin de son époux, ce qu'elle promit. Au moment ou elle allait tendre la tête au bourreau, un enfant se montra près d'elle tenant une corbeille ou il y avait trois roses et trois pommes. Alors Dorothée dit: "Seigneur, je rous supplie d'avoir pitié de Théophile. " Et elle reçut la mort. Théophile, étant entré dans le palais du proconsul, recut les roses; il crut en Jésus-Christ et il obtint la couronne du martyre. »

Vincent de Beauvais raconte de Valérian et de sa fiancée sainte Cécile une histoire analogue ${ }^{2}$, que

1. La légende dorée, traduite du latin, par M. G. B. Paris, 1843, in-12, vol. II, p. 284. L'histoire de Dorothée a été mise en vers allemands à l'éporque de la Réforme. Mittler, op. laud., no 509.

2. Vingentir Bellovacensis Speculum historiale, lib. X, cap. 22 . 
la poésie religieuse a popularisée ${ }^{1}$. Cécile était chrétienne, mais Valérian était païen; il se convertit et le jour de son baptême un ange apparut, portant deux couronnes, l'une de fleurs de lis, l'autre de roses; il donna la première à Cécile, l'autre à Valérian. "Gardez, leur dit-il, d'un cœur et d'un corps purs ces couronnes apportées du Paradis de Dieu; jamais elles ne se faneront ni ne perdront leur parfum. »)

Ce sont encore ces roses célestes « dont la beauté jamais ne passe ni disparaît ", que l'épouse du drame italien de saint Thomas apercoit sur un " arbre merveilleux » du Paradis "2, symbole de la récompense promise à quiconque croit et se dévoue au Christ.

Sous l'influence du redoublement de ferveur mystique qui marqua la lutte engagée contre la Réforme la légende des roses se transforma. Ce n'est plus du ciel qu'elles viennent; c'est au ciel qu'on envoie celles de la terre. On conte que Rose de Lima $^{3}$, ayant lancé vers le ciel des roses qu'elle avait cueillies de ses propres mains, comme pour en faire hommage au "Suprème jardinier », ces fleurs

1. Elle fait l'objet du $38^{\mathrm{e}}$ des Niederlaendische Geistliche Lieder, publiés par Hoffmann von Fallersleben.

2.

Vidi una pianta in ciel maravigliosa

Qual sopra ogni cosa felice assurge.

Questa a ciascun di noi dava una rosa,

La cui bellezza mai trapassa o fugge.

Alessandro d'Ancona, Sacre rappresentazioni dei secoli xiv, xv $e$ xvi. Firenze, 1872, in-12, vol. I, p. 436.

3. Acta sanctorum, vol. XXXVII, p. $970, d, c$. 
bénies restèrent suspendues dans les airs et s'y réunirent en forme d'une croix entourée d'un limbe lumineux, signe que Jésus acceptait son offrande.

Un jour que la fille du commandant de Grosswardein se promenait, attristée qu'on la voulût marier, dans le jardin de son père, Jésus se présenta à elle et lui mettant un anneau au doigt: " Je veux, lui dit-il, que tu sois ma fiancée ». La jeune fille devint toute rouge de joie et, cueillant une rose, elle l'offrit à son céleste fiancé. Mais Jésus, la prenant par la main, reprit: "Viens, que je te montre le jardin de mon père », et il l'emmena dans le Paradis ${ }^{1}$. La légende ajoute qu'elle ne revint sur terre qu'au bout d'un siècle et pour mourir aussitôt.

Le plus souvent l'apparition miraculeuse des roses est destinée à témoigner du mérite des saints, surtout de leur mérite méconnu ou ignoré. Suivant une tradition locale "les gouttes de sang du chef » de saint Lucien, martyr et premier évèque de Beauvais, se seraient changés en rosiers couverts de roses vermeilles ${ }^{2}$. Un auteur espagnol raconte la mème

1. Büsching, Volkssagen, p. 163, ap. W. Menzel, Christiche Symbolik, vol. II, p. 195.

2. "C'est chose véritable que les gouttes de sang du chef de nostre martyr, dont la terre fut empourprée, engendrèrent tello quantité de rosiers garnis de roses vermeilles, que le lieu du martyre s'appelle encore la Rosière, pour signifier, comme dit Tertullian, que le sang des martyrs est une graine et une semence des belles fleurs du Paradis. " Louvet, Histoire et antiquités du diocèse de Beauvais. Beauvais, 1631, in-8, vol. I, p. 387. Louvet ignorait que rosière signifie bien plutôt un lieu rempli de roseaux, que planté de rosiers. 
chose du martyr saint Magin; des gouttes de son sang seraient nés des rosiers dont les fleurs, dit son biographe ${ }^{1}$, portaient en témoignage de leur origine des taches couleur de sang.

Quand on ouvrit, quelque temps après sa mort, le tombeau de saint Lucius, on apercut trois ${ }^{2}$ roses sur sa poitrine, qui disparurent aussitòt qu'on voulut les enlever ${ }^{3}$. On conte la mème chose du bienheureux Gandolf, évêque de Milan. Les fidèles, ayant voulu transporter sa tombe dı milieu de l'église, où elle était foulée aux pieds, au chevet du chœur, un nuage épais et un parfum délicieux remplirent la basilique, et quand on vint à ouvrir le sépulcre du saint, on y trouva une rose d'une beauté merveilleuse et d'une odeur exquise, aussi fraîche, ajoute le chroniqueur, que si on venait de la cueillir ${ }^{4}$. Suivant une ancienne tradition ", on aurait trouvé aussi une rose dans la bouche de saint Louis, de 'Toulouse. D'après la légende, beaucoup plus récente, de saint Antoine de Stronconio, ce n'est plus une fleur naturelle, mais une rose de chair qu'on trouva dans sa main ${ }^{6}$.

Dans l'histoire de saint Julien de Vienne et de

1. Acta sanctorum, vol. XXXVII, p. 119, e. Suivant une légende anglaise des fleurs aussi narpuirent sur la colline où fut décapité saint Alban. Ém. Montégut, Sir John Maundeville. (Revue des Deux Mondes, 15 nov. 1889, p. 300.)

2. Acta sanctorum, vol. XXXVI, p. $28 \mathrm{f}$.

3. Acta sanctorum, vol. XXII, p. پ̈81 $a$.

4. Les petits Bollandistes, vol. X, p. 35.

5. Les petits Bollandistes, vol. X, p. 31.

6. Waddingus, Annales minorum, vol. XIII, p. $470,20$. 
sainte Agnès de Monte-Pulciano, la légende a pris une forme toute différente. Une nuit, rapporte Grégoire de Tours ${ }^{1}$, le diacre de la basilique où saint Julien était enterré entendit un grand bruit, comme si on eùt ouvert et fermé la porte de l'église avec violence; il n'y fit d'abord aucune attention; mais le bruit ayant recommencé, il se rendit tout inquiet auprès du tombeau du bienheureux; quelle ne fut pas sa surprise de voir le pavé de l'église tout couvert de roses vermeilles, "aussi fraiches, remarque l'historien, bien qu'on fût au mois de novembre, que si on venait de les cueillir sur la branche ».

Deux ermites, attirés par la réputation de sainteté d'Agnès de Monte-Pulciano, étaient allés lui rendre visite $^{2}$; aprés de longs et pieux entretiens, Agnès les reçut à sa table. Au moment où l'on servait le repas, ils aperçurent tout à coup, à leur grand étonnement, au milieu de l'écuelle placée devant la sainte, une rose d'une merreilleuse beauté et d'un parfum délicieux. On conte ${ }^{3}$ que des moines de Norwich ayant, vers la Saint-Michel, planté près du tombeau de saint Guillaume l'enfant une branche d'un rosier de leur courent, ce rameau prit bien vite racine et se courrit de fleurs, qui persistèrent jusqu'au jour de la Saint-Edmond (16 ou 20 novembre). Un ouragan survint alors et les emporta toutes, une

1. De miraculis S. Juliani, cap. 46. (Miraculorum lib. II, p. 826, éd. Migne.)

2. Raymond de Capoue, ap. Acta sanclortem, vol. X, p. $800 f$.

3. Acta sanctorum, vol. VIII, p. 590 e. 
seule exceptée qui resta épanouie jusqu’à la Noël. Cette forme nouvelle de la légende se rencontre encore dans l'anecdote suivante.

Un jour que saint Jacques de Venise, se trouvant en compagnie de quelques religieux, vint à passer devant un rosier privé de feuilles et de fleurs, on était au milieu de l'hiver, - une rose de toute beauté et d'un parfum délicieux apparut tout à coup sur le rosier. Il la cueillit et la présenta aux religieux du couvent, que remplit d'admiration la vue de cette fleur en une pareille saison ${ }^{1}$. Ja bienheureuse Rita étant restéc malade au couvent de Cascia, une de ses parentes vint lui rendre visite et lui demanda, en la quittant, si elle ne désirait rien. “ Je voudrais, reprit Rita, une rose de mon jardin de Rocca-Porena. " Or on se trouvait au mois de janvier; sa parente crut aussi que la sainte était dans le délire et elle s'éloigna en souriant. Nais combien grand fut son étonnement, quand, de retour à RocacPorena, elle apercut une fleur fraiche et vermeille sur un rosier du jardin. Se rappelant alors le désir exprimé par Rita, elle s'empressa de cueillir cette rose et la lui envoya à Cascia ${ }^{2}$.

On comprend que les roses miraculeuses durent prendre place de bonne heure dans les légendes de la Vierge. Elles y apparaissent, pour révéler le salut non soupconné que la mère de Dieu ne manque pas d'assurer à ceux qui lui ont toujours été fideles.

1. Acta sanctorum, vol. XVIII, p. $465 c$.

2. Acla sanctorum, vol. XVI, p. 226 b. 
Durant toute la durée de son séjour au couvent de Déols ${ }^{1}$, raconte Thomas de Cantimpré, le moine Josbert n'avait jamais omis de réciter chaque jour les cinq psaumes qui commencent par les lettres du nom de Marie ${ }^{2}$. Le jour de la fête Saint-André 1156 , le prieur ne l'ayant pas vu à la chapelle se rendit dans sa cellule; il l'y trouva mort, une rose dans la bouche, dans les yeux et dans les deux oreilles, chac une portant gravée une des cinq lettres du mot Marie.

Cette légende a été célèbre au moyen âge; Gautier de Coinci l'a rapportée à son tour ${ }^{3}$; mais il l'attribue

1. Bonum universale de apibus. Duaci, 1627, in-8, p. 289, lib. II, cap. 29.

$$
\begin{aligned}
& \text { De ces cinq saumes sont li non } \\
& \text { Magnificat. Ad Dominum. } \\
& \text { Retribue servo tuo. } \\
& \mathrm{Li} \text { carte est In convertendo. } \\
& \text { Ad te levavi lo cinquisme. }
\end{aligned}
$$

Gautier de Coinci, li Hiracle de Nostre Dame, Bibl. nat. fr. 22928, fol. 42, 2.

3. Je devrais dire traduite, puisque, sous sa forme primitive, cette légende, ainsi que les suivantes, se présente d'abord sous une forme latine. Il faut en rapprocher celle des manuscrits lat. 1'457 de la Bibliothèque nationale de Paris et 612 de la Bibliothèque de Metz, où il s'agit d'un frère convers qui ne sait réciter que l'Ave .Iaria et du cœur duquel. après sa mort, sort un arbuste (tumba parit quasi ficum de dulci corde fratris), sur les feuilles duquel se trouvaient inscrites les lettres A. M. A. Mussafia, Studien zu den mittelalterlichen Marienlegenden, III. 9. (Sitzungsberichte der Akademie der. Wissenschaften, vol. CXIX, an. 1889). Dans les rédactions allemandes de cette dernière léfende. c'est un lis qui sort de la bouche du mort et sur chacune de ses feuilles se tronve gravé en lettres d'or Ave Maria. Godeke, Deutsche Dichtungr im Mittelalter, 140. 
LA ROSE DANS LES LÉGENDES CHRÉTIENNES. 267

à un moine inconnu et ignorant, et, dans le récit qu'il en a fait, c'est dans la bouche du mort qu'on trouve “encloses») les cinq roses

Cleres, vermeilles et foillies

Con se luez droit fuissent coillies ${ }^{1}$.

Cette forme de la légende était trop conforme à l'esprit du mysticisme de l'époque pour n'avoir pas fait fortune. Les Miracles de Nostre Dame de Gautier de Coinci en présentent plusieurs variantes curieuses. Un clerc, raconte-t-il ${ }^{2}$,

Des lettres iert bien enbeuz

Mais tant iert soz et durfeuz ${ }^{3}$

Qu'il ne pensoit a nul bien faire;

son oncle, abbé d'un riche monastère, après s'être efforcé en vain de le ramener au bien, l'abandonne à son sort. Le clerc se livre à tous les déportements et se trouve bientôt réduit à la plus grande pauvreté; il se repent alors et revient vers son oncle; il lui promet mème de dire chaque jour une prière i la Vierge ${ }^{4}$; mais bientot il retombe dans ses anciens désordres et l'on est contraint de l'excommu-

1. Bibl: nat., man. fr. 22928, fol. 99, b 2.

2. Bibl. nat., man. fr. 1530, fol. 107, b 1. Cf. l'abbé Poquet, Les Miracles de la sainte Vierge traduits et mis en vers par Gautier de Coincy. Paris, 1857, in-4, p. 363.

3. Deditus illecebris vite factisque superbis.

Man, d'Erfurt, 4'́ . Cf. Man. de Heiligenkreuz (A. Mussafia, op.

laud., I, p. 936, et III. p. 1'1).

4. Daprès le man. de la Bibliothèque nationale lat. 12593, no 35 , c'est la prière $O$ intemerata. 
nier. Il mourut sans avoir pu, malgré son repentir, se réconcilier avec l'Église, et

Honteusement a grant misere

En un fossé jeter le firent.

Mais Notre-Dame, qu'il n'avait point cessé d'invoquer au milieu de ses plus grands dérèglements, touchée de l'affront fait à son serviteur, apparaît par trois fois au doyen; elle lui reproche le traitement indigne infligé au clerc, et, comme marque de son mérite méconnu, elle ajoute qu'on trouvera une " fraîche rose ) dans la bouche du mort. Le doyen et tout le peuple s'étonnent à cette nouvelle; on se rend au lieu où le clerc était enterré, et, ô surprise,

Une rose fresche, novele,

Maintenant qu'il le deffoirent,

Troverent en sa bouche et virent ${ }^{1}$.

Ce fut sans doute aussi une rose que la fleur

Si fremiant et si florie

Com se lues droit fust espanie ${ }^{2}$,

1. Comme l'avait fait Gil de Zamora dans son Liber Mariae, cap. V, no 2, Gonzalo Berceo, qui a raconté ce iniracle, parle seulement d'une fleur :

Yssieli por boca una fermosa flor

De muy grant fermosura, de muy fresca color.

Milagros de nuestra sennora, III, str. 112.

2. Bibl. nat., man. fr. 22928, fol. 78, a 2. Le man. 1530 , fol. $38, b 2$, donne :

Com ce fust rose espanie.

Dans la rédaction allemande il est aussi seulement question diune 
trouvée, trente jours après sa mort impénitente, dans la bouche de ce clerc de Chartres

Orgueilleux et despers

Et du siecle moult curieus,

mais dévot à la Vierge, dont Gautier de Coinci a également raconté l'histoire, et que la mère de Dieu, en récompense de sa fidélité, honore ainsi aux yeux de la foule émerveillée.

Il faut rapprocher des légendes qui précèdent celle de la pieuse Ada, rapportée par Thomas de Cantimprét. Pendant une absence que fit son mari, un lépreux las et épuisé vint lui demander l'hospitalité; non seulement elle s'empressa de le recevoir, mais elle ne crut pouvoir mieux farre que de lui donner, pour se reposer, le propre lit de son mari. Il y était a peine, que celui-ci revint de son voyage et se dirigea vers ses appartements. A cette vue Ada s'effraya; son mari surpris et inquiet forca la porte; mais quel fut son étonnement, en pénétrant dans sa chambre, de trouver, bien qu'on fût en hiver, sa couche toute couverte de roses parfumées.

fleur qu'on trouve sur le palais du clerc volage dont la langue est restée fraiche et rose comme s'il était encore en vie :

Man vant dó einen blumen

Vrisch ûf sime gumen

Ligen in sinem munde.

Franz Pfeiffer, Marienlegenden. Wien, 1863, in-8, no XI, v. 99101, p. 81 .

1. Liber apum aut de apibus myslicis... in-fol.s. 1. n. d., lib. II, cap. 17, fol. 39. J. W. Wolf (Niederländische Sagen. Leipzig, 1843, in-8, p. 281) lui donne le nom d Ada de Belomeir. 
On trouve dans la vie de saint Jean l'Ange une légende encore plus merveilleuse ${ }^{1}$. Pendant son séjour au couvent de Cavacurta, au diocèse de Lodi, on l'accusa de ne se rendre si souvent au jardin, voisin d'une des rues de la ville, que dans un but profane; le prieur le fit surveiller; mais les religieux qu'il avait enroyés pour l'observer le trouvèrent, à leur grand étonnement, en prières au milieu de rosiers en fleurs, encore qu'on fût en hiver et que la terre fût toute couverte de neige.

Ici l'apparition merveilleuse des roses rend éclatante la vertu soupconnée de saint Jean l'Ange. Le rôle de ces fleurs miraculeuses apparaît d'une manière non moins frappante dans l'histoire suivante racontée par le prétendu voyageur John Mandeville 2 . Une jeune fille de Bethléem, accusée d'avoir enfreint les lois de la chasteté, avait été condamnée au feu. Déjà le bûcher était dressé quand elle invoqua le Seigneur, le priant, si elle était sans faute, de lui venir en aide et de manifester son innocence aux veux de tous. Puis elle entra dans le feu; mais aussitôt les flammes s'éteignirent et les brandons qui brûlaient déjà se changèrent en rosiers couverts de fleurs vermeilles, ceux qui n'étaient pas encore allumés devinrent autant de rosiers à fleurs blan-

1. Acta sanctorum, vol. LVI, p. $887 b$.

2. The buke of John Maundeville being the travels of sir John Maundeville, knight, 1322-1356, from the m. copy h. s. Egerton 1982, together with the french text... by George Fr. Warner. London, 1889, in-fol. ch. Ix, p. 35. 
ches. "Et ce furent, ajoute naïvement le narrateur, les premiers rosiers et les premières roses qu'on eût encore vues. "

Bien que le pseudo-Mandeville ${ }^{1}$, pour donner plus de crédit à ce miracle, dit que le nom de "Champ flori » porté par le lieu où se serait passée la scène, en avait conservé le souvenir, il semble avoir tout simplement arrangé et intercalé dans le récit de son voyage une légende qui avait cours de son temps. On la rencontre sous une autre forme, dans un poème inédit sur le Nouveau Testament ${ }^{2}$. Ici il s'agit d'une fille d'Abraham, devenue grosse pour avoir respiré le parfum d'un arbre du jardin de son père, - l'arbre de la science du bien et du mal - ; elle est condamnée à être brûlée; mais quand elle monte sur le bûcher, les brandons s'éteignent aussitôt et se couvrent de fleurs et de roses :

Onques n'i ont .r. sol tison,

Tant bien espes, ne vif charbon,

Ne fussent roses de rosier

Et flor de lis et aiglentier;

Le feu estaint, c'est verites.

Cette naissance miraculeuse des roses, leur subs-

1. Le voyage mis sous le nom de John Maundeville est probablement l'œurre du médecin liégeois Jean de Bourgogne, dit à la Barbe, - "Johannes de Burgundia, aliter vocatus cum Barba »qui se sera caché sous ce nom. Edward B. Nicholson, The Academiy, no 623,12 april 1886, p. 261.

2. Renfermé dans le manuscrit de Grenoble 1137. Jean Bonnard, Les Traductions de la Bible en vers françois au mojen äge. Paris, 188' , in-8, p. 181. 
titution à d'autres objets, se produit surtout quand il s'agit de soustraire un saint aux reproches ou au châtiment qu'il aurait encourus pour une infraction faite dans un but de charité. Sainte Casilde, fille du roi de Tolède, Aldemore, dans l'ardeur de sa charité, portait aux esclaves chrétiens les mets qu'elle pouvait dérober à la table royale; un jour son père la surprit, mais lorsque, irrité, il souleva son manteau, à la place des vivres que celui-ci recouvrait, il n'apercut que des roses ${ }^{1}$. Sous cette forme dramatique la légende de la naissance de roses miraculeuses devait bien vite se répandre; on la retrouve dans tous les pays.

Sainte Rose de Viterbe ${ }^{2}$ portait un jour, suivant sa coutume, du pain aux pauves; son père la rencontra et lui demanda ce qu'elle avait dans les pans de sa robe ; elle l'entr'ouvrit aussitôt et la lui montra remplie de roses de diverses couleurs. Saint Nicolas, moine du couvent de Tolentino, fut apercu par le prieur, comme il portait aussi du pain aux paures; le prieur lui ayant demandé ce que renfermait sa corbeille, il la découvrit, et, encose qu'on fût en hiver, elle se trouva pleine de roses vermeilles et parfumées ${ }^{3}$.

La légende qui concerne sainte Élisabeth de IIongrie est surtout célèbre, depuis que la peinture l'a popularisée :

1. Les petits Bollandistes, vol. IV, p. 305 .

2. Acta sanctorum, vol. XL, p. $434 c$.

3. Acta sanctorum, vol. XLI, p. $642 f$. 
Elle aimait, dit son historien ${ }^{1}$, à porter elle-même aux pauvres non seulement l'argent, mais encore les vivres et les autres objets qu elle leur destinait... Un jour qu'elle descendait, accompagnée d'une de ses suivantes favorites, par un petit chemin très rude, portant dans les pans de son manteau du pain, de la viande, des œufs et d'autres mets, pour les distribuer aux pauvres, elle se trouva tout à coup en face de son mari qui revenait de la chasse. Étonné de la voir ainsi ployant sous le poids de son fardeau, il lui dit: "Voyons ce que vous portez," et en même temps ouvrit malgré elle le manteau qu'elle serrait tout effrayée contre sa poitrine; mais il n'y avait plus que des roses blanches et rouges, les plus belles qu'il eût vues de sa vie; cela le surprit d'autant plus que ce n'était pas la saison des fleurs.

On raconte une histoire analogue de sainte Élisabeth de Portugal; mais celle-ci pour s'excuser ne recule pas devant un pieux mensonge. Un jour qu'elle portait dans sa robe une grosse somme d'argent pour la distribuer aux pauves, elle rencontra son mari, qui lui demanda ce qu'elle cachait. Elle répondit: "Ce sont des roses, ) et en effet, dit son biographe ${ }^{2}$, dépliant sa robe, il se trouva que c'en était réellement, quoiqu'on fût dans un temps où il ne pouvait y en avoir.

Si l'on en croit son récent historien ${ }^{3}$, sainte Rose-

1. Le comte de Montalembert, Vie de sainte Élisabeth de Hongrie. Paris, 1836, in-8, p. 57.

2. Les petits Bollandistes, vol. VIII, p. 35.

3. Le comte $\mathrm{H}$. de Villeneuve-Flayosc, Histoire de sainte Joret. La Rose. 
line de Villeneuve aurait aussi, pour excuser sa charité, répondu à son père par un mensonge semblable, et les aliments qu'elle distribuait aux pauvres se seraient également changés en roses fleuries.

Saint Pierre Régalat ne craignit pas davantage de mentir dans une circonstance analogue et il est le héros d'un pareil miracle ${ }^{1}$. Il portait un jour du pain et des viandes à une pauvre malade, quand il se trouva en face du prieur de son couvent. "Pierre, lui dit celui-ci, tu parais bien occupé, que portes-tu là?" “ Ce sont des roses, répondit-il tout troublé, destinées à une paurre affligée. " Or on n'était pas à la saison des roses. "Montre-les », reprit aussitôt le prieur. Et comme Pierre, tout couvert de confusion, dit: "Les voici, » en entr'ouvrant sa robe, le pain qu'il portait se changea en roses blanches et les viandes en roses vermeilles.

Le miracle de la naissance des roses a pris place également, en se transformant, dans la vie du bienheureux Eelke Liaukama, abbé du couvent de Lidlum en Frise ${ }^{2}$. Poursuivi par des frères convers, auxquels il avait reproché leur's désordres et qui avaient juré sa mort, il essaya en vain de les fléchir. Mais quand

Roseline de Villeneuve. Paris, 1867, in-8, p. 296. Sainte Roseline naquit en 1263.

1. Acta sanctorum, vol. VIII, p. 862 . On raconte une histoire semblable, à part le mensonge, de sainte Germaine Cousin; mais ce sont de simples fleurs dont son historien nous montre rempli son tablier.

2. Acta sanctorum, vol. VIII, p. 397 e. - J. G. Wolf, Deutsche Sagen, 1861, in-8, p. 587. 
ils saisirent et retournèrent les manches de sa robe pour voir s'il n'y avait rien caché, ils les trouvèrent remplies de roses.

La rose figure en particulier dans les légendes destinées à glorifier l'intervention de la Vierge en faveur de ceux qui lui sont fidèles. En voici une dont le but d'édification est manifeste, telle qu'Alphonse le Sage l'a traduite du latin en langue portugaise ${ }^{1}$; il s'agit d'un gentilhomme qui avait fait vœu d'offrir chaque jour une couronne de roses à la Vierge, ou, si cela lui était impossible, de dire en son honneur un Ave Maria. Un jour qu'il traversait une vallée sauvage, il s'arrèta pour faire sa guirlande accoutumée; tandis qu'il était tout à cette pieuse occupation, des ennemis survinrent pour le tuer; mais au moment de mettre leur criminel dessein à exécution, ils aperçurent auprès du gentilhomme une dame d'une grande beauté, qui faisait elle aussi une couronne de roses. Cette vụe les frappa d'étonnement et de crainte et ils se dirent qu'ils n'araient qu'à s'éloigner au plus vite, car il ne plaisait pas à Dieu qu'ils tuassent ce gentilhomme ${ }^{2}$.

Dans une rédaction allemande de cette légende ${ }^{3}$ il s'agit d'un jeune écolier si indolent qu'il ne voulut

1. "Milagro do cavaleiro que fazia a guerlanda das rosas a santa Maria. "Cantigas de santa Maria de don Alphonso el Sabio. Madrid, 1886, in-4, $\mathrm{n}^{0}$.cxxr.

2.

Tornemos d'aqui logo, pois esto non praz a Deu que est' ome nos matemos.

3. Franz Pfeiffer, Marienlegenden, no xxi. 
et ne put rien apprendre; mais en dépit de sa paresse il avait la bonne habitude d'aller tous les jours aux champs cueillir des fleur's et d'en faire une couronne dont il ornait une image de la Vierge qui se trouvait dans sa ville natale. 'Touché par la grâce, il entra dans un couvent de l'ordre de Cîteaux et s'y fit remarquer par son intelligence et sa vertu. Aussi gagna-t-il la confiance de l'abbé et celui-ci le chargea un jour d'une affaire hors du couvent. Son voyage le conduisit au milieu d'un bocage délicieux, plein de fraîcheur et d'ombre. Il s'y arrèta et, descendant de cheval, il se mit à réciter les cinquante Ave qu'il avait oublié de dire ce jour-là. Pendant ce temps deux voleurs de grand chemin vinrent pour lui dérober son cheval. Nais en s'approchant ils apercurent la Vierge toute rayonnante de beauté et parée des plus beaux atours debout près du religieux, cueillant sur ses lères, à mesure qu'il les récitait, les Ave changés en roses, et elle en fit une couronne qu'elle placa sur la tète du pieux moine, lorsqu'il eut fini sa prière, puis elle disparut ${ }^{1}$. Les voleurs étonnés renoncèrent à leur dessein et, s'approchant du religieux, ils lui demanderent quelle était cette dame merveilleuse; il leuir raconta ce qu'il renait de faire; alors frappés du miracle dont il avait été

1. Als der munich hete entsaben ein àvê Marjà unde gesprach, seht, welch ein wunder dà ges[chah! wan ez wart zeiner rôsen. mit griffen harte lôsen die vrouwe dó begunde. im brechen von dem munde cine rôsen nach der andern.

v. 278-285. 
LA ROSE DANS LES LẺgENDES CHRÉTIENNES. 277

l'objet, au lieu de le dépouiller ils se jetèrent à ses pieds, lui confessèrent leurs péchés et se retirèrent dans son couvent pour y faire pénitence.

Il existe diverses variantes de cette légende en Allemagne; l'une d'elles rapporte qu'un jeune homme d'une grande dévotion envers la Vierge succomba dans une forêt sous les coups des brigands; un ange recueillit sur ses lèvres expirantes ses dernières prières, sous forme de douze roses blanches et trois roses rouges et les réunit en couronne ${ }^{1}$ : telle aurait été l'origine du rosaire.

En France, la même légende a été également populaire; au $\mathrm{xiv}^{\mathrm{e}}$ siècle elle revêtit mème la forme dramatique. Un marchand, qui traversait une forêt, allait ètre tué par un voleur qui l'épiait, quand il se rappela qu'il avait oublié de faire ses dévotions accoutumées à la Vierge; il s'arrêta aussitôt pour remplir ce pieux devoir. Pendant qu'il récitait ses prières, s'approche de lui, sans être vue,

\section{une femme,}

Plus belle et de plus noble arroy

Conques ne fut femme de roy...

Un chapel de roses faisoit

Et les prenoit la dame doulce

De ce marchant dedanz la bouche,

Puis li assist dessus son chief:

récompense de sa dévotion et des couronnes qu'il

1. Binterim, Denkwürdigkeiten, vol. VIII, Th. 1, 2, p. 98, ap. Schleiden, p. 105. 
avait si souvent offertes à la mère du Sauveur. Ce spectacle, quand il en a l'explication, fait rentrer le larron en lui-même; il abandonne son dessein criminel et renonce à sa vie de meurtres pour se faire ermite ${ }^{1}$.

L'apparition soudaine de roses se rencontre dans bien d'autres légendes; parfois elle révèle la présence ou l'approche d'un saint ou d'un être divin, d'autres fois elle est destinée à faire connaitre quelque chose de mystérieux ou de caché. Quand François d'Assises visita le couvent de San Subiaco, où pour se mortifier saint Benoît avait coutume de se rouler sur des épines, à son approche celles-ci se changèrent en rosiers qui se couvrirent de roses vermeilles, miracle dont une fresque de la chapelle du couvent a perpétué le souvenir ${ }^{2}$.

Marie, dit un ancien lied ${ }^{3}$, traversait une forêt, qui depuis sept ans n'avait point poussé de feuilles; que por-

1. Miracles de Nostre Dame par personnages, publ. par Gaston Paris et Llysse Robert. Paris, in-8. Hiracle de un marchant et un larron, vol. II, 1877, p. 90-119.

2. J.-B.-L.-G. Seroux d'Agincourt, Histoire de l'art par les monuments. Paris, 1823, in-fol. - Peinture, pl. C, no 7, vol. III, p. 121. Dans le Lileraturblatt für rom. et germ. Literatur du mois d'aoùt 1891, p. 272, M. Suchier parle aussi de roses qui seraient nées sous les pas de saint Patrice, quand il traversait la mer; mais je ne sais d'où vient cette légende, et n'ai pu en trouver l'origine ni la trace.

3. Maria durch den Dornenwald ging.

Der hatte sieben Jahre kein Laub getragen,

Was trug Maria unter ihrem Herzen? 
tait-elle sous son cœur! Un petit enfant sans douleur. Quand elle entra dans la forêt, les épines se couvrirent de roses.

Une nuit, raconte une autre légende ${ }^{1}$, on aperçut tout à coup au pied du Kirchberg près de Ludge trois roses couleur de feu; elles restèrent visibles pendant quelque temps, puis disparurent. Comme l'apparition se répéta plusieurs fois, on creusa en cet endroit et l'on y trouva une antique image en pierre de la Vierge, a laquelle on bâtit une chapelle. Ici tout est merveilleux; la légende suivante a un fondement réel; le rosier célèbre de la cathédrale d'Hildesheim.

Louis le Débonnaire ayant, dit-on ${ }^{2}$, perdu dans une chasse son reliquaire, envoya un de ses serviteurs à sa recherche; celui-ci le trouva accroché à un rosier saurage, mais ne put parvenir à l'emporter. L'empereur étonné s'y rendit alors en personne et apercut, au milieu de la forèt en feuilles, un champ de neige, qui avait la forme du vaisseau d'une église; à l'extrémité se dressait, tout couvert de fleurs, le rosier où était accroché le reliquaire. Louis fit bâtir à cette place même une église, en

Ein kleines Kindlein ohne Schmerzen.

Als das Kindlein durch den Wald getragen,

Da haben die Dornen Rosen getragen.

Paderboner Liederbuch, no 99, ap. Mittler, op. laud., nº 382.

1. Ritter von Perger, Pflanzenlegenden. Stuttgart, 1864, in-8, p. 231 .

2. Cette légende est racontée de la manière la plus diverse. Cf. Schleiden, p. 98 et A. Ritter von Perger, p. 233. 
recommandant de ne point toucher au rosier. Quand au $\mathrm{xi}^{\mathrm{e}}$ siècle l'évèque Hézilo fit reconstruire cette basilique détruite par un incendie, il prit soin qu'on préservât les racines du rosier et en fit appliquer les branches contre le mur de l'abside. On y roit encore aujourd'hui un églantier haut de 25 pieds et qui recourre tout un pan de la muraille du choeur ${ }^{1}$.

Les roses miraculeuses ont pris place aussi dans les légendes slaves. Sainte Hélène, raconte l'une d'elles, s'étant mise à la recherche du tombeau du Christ, arriva au sommet d'une montagne couverte d'arbres; du bois de ces arbres elle fit faire deux croix et une église, ainsi que trois cercueils. Dans l'un de ceuxci elle fut déposée elle-mème; mais au-dessus de sa tombe poussa un rosier qui bientôt se couvrit de fleurs. Et la légende ajoute que de l'une de ces fleurs, sortit un oisean - Jésus-Christ lui-mème qui s'élança vers le ciel $^{2}$.

Par une opposition d'idées que nous avons déjà rencontrée dans les légendes de l'antiquité, en même temps qu'elles sont un signe de salut et de joie, les roses ont été aussi parfois considérées comme un symbole de deuil et un présąe de mort. Quand un des chanoines de Lubeck était près de sa fin, il trouvait, trois jours auparavant, sous le coussin de sa stalle dans la salle du chapitre, une rose blanche ${ }^{3}$.

1. C'est une Rosa canina. Alex. v. Humboldt, Ansichten der Nalur. Stuttgart, 1860, in-18, vol. II, p. 82.

2. Zienkienicz, Recucil de chansons populaires, p. 31, ap. Potebnia, I, p. 762.

3. Gebr. Grimm, Deutsche Sagen, Bd. I, p. 352. 
La Vierge, d'après une croyance populaire suédoise ${ }^{1}$, apparaît aux enfants malades et leur donne des fraises, s'ils doivent guérir, une rose, s'ils sont près de mourir.

Les légendes religieuses dont la rose est entourée, le caractère sacré qu'elles lui donnent, suffisent pour faire comprendre l'horreur qu'elle est supposée inspirer au démon; son odeur, dit-on, suffit pour le mettre en fuite. Les possédés ne peuvent davantage supporter le parfum de cette fleur et on ne saurait les contraindre à passer près d'un bosquet de rosiers ${ }^{2}$. Les sorcières aussi en redoutent le voisinage et elles n'oseraient cueillir de roses blanches, de peur d'ètre aussitót reconnues. Pour la même raison un loupgarou ne peut conserver son déguisement et reprend la forme humaine, dès qu'il lui arrive seulement de toucher un églantier ${ }^{3}$.

Il est question ici, comme dans la légende d'Hildesheim, on le voit, d'un rosier sauvage; ainsi que le rosier cultivé, il a pris place, en effet, dans les légendes religieuses. L'une d'elles, répandue dans certaines provinces septentrionales de l'Allemagne ${ }^{4}$, rapporte que quand Lucifer fut précipité sur terre, il fit pousser un églantier, afin de se servir de ses

1. Afzelius, Sagen und Lieder aus Schweden, übersetzt von Ungewitter. Leipzig, 1842, Th. III, p. 240.

2. Rosenberg, Rhodologia, pars II, cap. xix, p. 232.

3. Ritter von Perger, Deulsche Pflanzensagen, p. 232.

4. K. Müllenholf, Sagen, Mürchen und Lieder der Herzogthümer Schleswig, Holstein und Lauenburg. Kiel, 18't5, in-8, p. 358, n $^{\circ} 479$. 
aiguillons pour remonter au ciel; mais Dieu ayant remarqué son dessein abaissa vers la terre les branches de l'églanticr et en recourba les aiguillons. Suivant une autre version, ce serait Lucifer lui-même qui dans son dépit aurait recourbé les épines de l'arbuste ${ }^{1}$.

D'après une légende, qui a cours surtout aussi dans les pays germaniques, ce serait à un églantier que Judas se pendit, et c'est depuis lors que les aiguillons en sont courbés ${ }^{2}$. Enfin suivant une tradition qu'on rencontre également dans les mêmes contrées, la couronne du Sauveur aurait été faite de branches entrelacées d'églantier ; c'est pour cette raison que cet arbuste jouirait du privilege de protéger contre la foudre celui qui se met à l'abri sous ses rameaux ${ }^{3}$.

Tout autre est le rôle de l'églantier dans la légende suivante. Après avoir tué sa femme, qu'il accusait à tort de lui ètre infidèle, le comte de Bergue fit exposer dans un désert les enfants qu'il en avait eus, afin qu'ils fussent dévorés par les ours et les loups. Mais la Vierge, prenant en pitié ces innocentes créatures, les entoura d'une épaisse haie d'églantiers, afin qu'aucune bête sauvage n'en pût appro-

1. A. Ritter von Perger, op. laud., p. 236.

2. A. Ritter von Perger, op. laud., p. 236.

3. T. F. Thiselton Dyer, The Folk-lore of plants. London, 1889, in-12, p. 256. Inutile de rappeler que les traditions qui concernent la composition de la couronne d'épines varient avec les divers pays. 
cher. Un jour qu'il était allé à la chasse, le comte retrouva ses enfants en pleine santé; il reconnut alors son erreur, ramena chez lui les pauvres petits, et en signe de son repentir, il remplaça le sceptre d'or qui était dans ses armes par un rosier sauvage $^{1}$.

Dans ces légendes, c'est de l'églantier ordinaire (R. Canina L.) surtout qu'il s'agit; la suivante se rapporte à l'églantier rouillé (R. rabiginosa L. $)^{2}$. Pendant la fuite en Égypte, la Vierge Marie étendit un jour les langes de l'enfint Jésus sur cet arbrisseau, et c'est à cette circonstance, dit-on, qu'il doit cette odeur vineuse ou de pomme de reinette qui le caractérise $^{3}$. De cet églantier ainsi sanctifié, des rejetons auraient été portés au loin ; l'un d'eux, d'après une tradition ${ }^{4}$, se verrait encore près de Marienstein en Alsace; il porte un bouton, qui ne s'ourre que dans la nuit de Noël, mais répand alors au loin un suave parfum et une vive lumière. En Souabe l'églantier rouillé porte le nom de “ couronne d'épines du Sauveur ", dénomination qui s'explique d'elle-même, et les points rougeâtres qu'on

1. Montanus, Deutsche Volksfeste, p. 148 b, ap. Ritter von Perger, p. 237.

2. A. Ritter von Perger, op. laud., p. 239, la rapporte, lui, à l'églantier à fleurs blanches, sans dire, il est vrai, quel est cet églantier.

3. Friedrich, Symbolik und Mythologie, vol. I, p. 402, ap. Schleiden, p. 97.

4. August Stöber, Sagen aus dem Elsass, no 6. (Wolffs Zeilschrift für deutsche Mythologie, vol. I, p. 402). 
remarque sur ses feuilles sont regardés comme les marques du sang de Jésus ${ }^{1}$.

Il est difficile de dire à quelle époque remontent ces dernières légendes; si quelques-unes paraissent anciennes, la plupart le sont peut-être assez peu; il en est de même des suivantes qui se rapportent à la naissance même de la rose ou de quelques-unes de ses variétés. D’après l'une d'elles ${ }^{2}$ les roses auraient été originairement rouges; mais les pleurs de Marie Magdeleine, pendant la passion, en tombant sur ces fleurs, en auraient décoloré les pétales, et c'est ainsi que les roses blanches auraient pris naissance. Suivant une autre légende ${ }^{3}$, au contraire, la couleur primitive des roses aurait été le blanc; c'est depuis la faute d'Adam et d'Eve qu'elles auraient pris la couleur vermeille.

Si l'on en croit une tradition répandue en Angleterre, les gouttes de sang tombées de la couronne du Saureur, faite de branches entrelacées d'églantier, se seraient changées en roses, en touchant le sol 4 . Suivant une autre version, mais celle-là évidemment récente, comme la belle variété dont elle prétend expliquer l'origine, la rose moussue derrait sa naissance aux gouttes de sang tombées de la couronne d'épines sur la mousse qui se trouvait au pied de la croix ${ }^{5}$.

1. A. Ritter von Perger, op. laud,, p. 239.

2. Wolfgang Menzel, Christliche Symbolik. Regensburg, 185't in-8, vol. II, p. 283.

3. Wolfgang Menzel, op. laud., II, p. 281.

4. Fraser's Magazine, an. 1870, p. 714 .

5. Wolfgang Menzel, op. laud., vol. II, p. 283. 


\section{CHAPITRE IV.}

LA ROSE DANS LES LÉGENDES PROFANES ET DANS LA POÉSIE.

Les traditions si nombreuses dont la rose avait été entourée dans l'antiquité ne périrent pas toutes avec la civilisation hellénique et latine; beaucoup persistèrent chez les Grecs du moyen âge comme chez les nations romanes, et elles ne devaient pas tarder, avec la fleur aimée qui en était l'objet, à pénétrer chez les peuples slaves et germaniques; elles prirent place dans le folk-lore de ces peuples a còté des légendes indigènes qui s'étaient déjà formées ou allaient se former autour de la rose sauvage, seule espèce qui ait un nom indigène chez elles $^{1}$, la seule qu'ils connurent jusqu'au jour où la rose à cent feuilles fut importée dans leur pays.

Les Germains, en particulier, lui attribuaient un caractère sacré; ils l'avaient consacrée à Frigga; aujourd'hui encore elle porte dans certaines contrées de l'Allemagne, entre autres dans la vallée inférieure

1. All. hagedorn ou hagebutte, angl. briar ou bramble, norv.-dan. klongtorn ou nypetorn, suéd. törnbuske ou nynponbuske, slav. tchipaka. La rose saurage a seule aussi un nom indigène chez les nations romanes; fr. églantier, esp. agavanzo, pg. escaramujo. 
du Rhin, le nom de Frigdorn - épine de Freya ${ }^{1}$, et elle passe pour croître de préférence sur l'emplacement d'anciens bois sacrés ${ }^{2}$. Pendant tout le moyen âge, même quand la rose à cent feuilles eut été importée et cultivée en Allemagne, la rose sauvage ne cessa pas d'ètre chantée en même temps qu'elle par les minnesaenger. Il en fut de même en France, et il est souvent difficile de dire de laquelle des deux roses, la sauvage ou la cultivée, il est question dans les légendes, comme dans les vers des poètes. Il faut d'ailleurs distinguer entre les époques.

La rose cultivée est inconnue à notre ancienne poésie épique; cela se comprend; la culture de cette fleur était trop peu répandue dans les contrées ou notre épopée nationale a pris naissance pour qu'elle y pût figurer; elle ne pousait prendre place dans la description des luttes gucrières, matière principale - de nos vieux poèmes, et clle ne se trouvait guère aux lieux où se tenaient les délibérations qui préparaient ou suivaient les combats des héros de notre ancienne poésie. C'est en ple.n air qu'elles avaient lieu, dans un "vergier ", au pied ou à l'ombre d'un

1. A. Ritter von Perger, Deutsche Pflanzensagen, p. 235.

2. Oscar Teichert, Gesehichte der Zisrgärten und der Ziergärtnerei, p. 4, dit même, mais sans citer ses autorités, que le sanctuaire d'Irmensul en était entouré. 
LA ROSE DANS LES LÉGBNDES PROFANES. 287

arbre, soit le pin du nord ${ }^{1}$ ou l'olivier du midi ${ }^{2}$, remplacés parfois, le premier par un if ${ }^{3}$, le second par un laurier ${ }^{k}$; tout au plus le rosier sauvage peutil exceptionnellement s'y rencontrer' :

Desuz un pin, delez un eglentier

Un faldestoed i out fait tout d'or mier ${ }^{5}$.

ainsi plus tard que l'aubépine :

il trouverent

Devant aus souz une aube espine Seant la bele Clarmondine ${ }^{6}$.

Il en est de mème dans les poésies lyriques des premiers temps du moyen âge; la seule flore qu'elles connaissent est la flore des champs; c'est elle qui pare et embellit le "pré verdoyant » ou "fleuri » où les poètes d'alors ou leurs personnages viennent prendre leurs ébats. Mais bientôt tout change. L'importance prise par la vie de château, le luxe qui bientôt l'accompaz̧na, la recherche du bien-être et de ce qui fait le charme de l'existence, ont transformé les paysages poétiques de l'àge nouveau.

1. Li cuens Rollanz se jut desuz un pin.

La Chanson de Roland, v. 2375.

2. Guenes chevalchet suz une olive halte.

La Chanson de Roland, v. 366.

3. En Sarraguce descendent suz un if.

La Chanson de Roland, v. 406.

4. Suz un lorier ki est en mi un camp.

La Chanson de Roland, v. 2651.

5. La Chanson de Roland, v. 114.

6. Li roumans de Cléomadès publ. par A. von Hasselt. Bruxelles 1865, in-8, v. 6668-70, vol. I, p. 208. 
Le verger des anciennes épopées ne connaissait guère que quelques arbres forestiers ou cultivés ${ }^{1}$, les fleurs des champs émaillaient seules le pré verdoyant des premières pastourelles; le jardin ${ }^{2}$ des poèmes chevaleresques et allégoriques, ainsi plus tard que des chansons populaires, a, comme le jardin qui avoisine d'ordinaire le château seigneurial ou la maison bourgeoise, une flore autrement variée; il est rempli d'arbres d'agrément ou à fruits ${ }^{3}$, parfois mème de végétaux exotiques ${ }^{4}$, et il renferme un certain nombre de flcurs cultivées ${ }^{5}$; au premier rang

1. Aux arbres dont il a été fait mention il faut ajouter l'orme ou ormel et l'ente :

Dedens l'aigue de Sore, droit deles un ormel.

Bueves de Commarchis, éd. A. Scheler, v. 2623.

Et vient a Charlemaigne desoz l'ombre d'une ente.

Karls Reise, éd. Ed. Koschwitz, v. 795.

2. Il porte encore souvent le nom de "vergier », ou se confond avec celui-ci. Cf. Max Kuttner, Das Naturgefühl der Altfranzosen und sein Einfluss auf ihre Dichtung. Berlin, 1889, p. 62.

3.

Ou vergier ot arbres domesches,

Qui chargeoient et coins et pesches,

Chastaignes, nois, pommes et poires,

Nefles, prunes blanches et noires,

Cerises fresches vermeilletes,

Cormes, alies et noisetes.

Roman de la Rose, éd. Fr. Michel, v. 1355-60.

4. C'est cil cui est cil biax jardins

Qui de la terre as Sarrasins

Fist ça ces arbres aporter.

Roman de la Rose, v. 595-97.

5. Le lis, la pivoine, l'ancolie, auxquels se joignent, au xive 
LA ROSE DANS LES LÉGENDES PHOFANES. 289

figure la rose. Point de paysage poétique où celle-ci n'apparaisse désormais.

Au milieu des plantes rares, dont l'auteur de $L a$ Mort Aymeri de Narbonne a paré la plaine qui environne la ville d'Esclabarie, il n'a eu garde d'oublier la reine des fleurs :

Soz la cité est une praerie...

La croist la mente et la rose florie ${ }^{1}$.

Des rosiers " de roses bien cargiés " parent, avec les arbres les plus " chiers », le verger du Dieu d'amour, dans le fableau de ce nom ". Guillaume de Lorris a assigné aussi à la rose une place d'élection dans le jardin qui occupe une si grande place dans son poème. Planté des arbres les plus divers et rempli des fleurs les plus gracieuses, il renferme encore $^{3}$,

$$
\begin{gathered}
\text { en un long détor } \\
\text { D'une haie clos tot entor, }
\end{gathered}
$$

des " rosiers chargiés » de roses "savorées, "

siècle, la giroflée et la lavande, puis au $\mathrm{xv}^{\mathrm{e}}$, l'œillet. Voir plus haut deuxième partie, chapitre premier, p. 130.

1. V. 2423-25. Éd. J. Couraye du Parc, Paris, 188', in-8, p. 105.

2. N'i ot arbres, ne fust pins u loriés,

Cyprès, aubours, entes et oliviers:

Ce sont li arbres que nous tenons plus ciers.

Fuelles et flors ont tos tans li ramier

Et sont de roses bien carchié li rosier.

Li Fablel dou dieu d'Amours public par Achille Jubinal. Paris, 1834, in-8, str. 10, v. 2-6.

3. Le Roman de la Rose, v. 1623-25 et 1646.

Joret. La Rose. 
Si beles ne vit homs sous ciaus.

Froissart aussi n'a point omis, dans le Paradis d'amour, de mettre des lis et des roses sur le bord du ruisseau où il va s'abandonner à sa rèverie ${ }^{1}$. Et des

Roses blanches comme lys et vermeilles

brillent entre toutes les fleurs allégoriques du Jardrin salutaire de Jean Joret ${ }^{2}$. C'est la rose aussi qu'on trouve dans les parterres fleuris des légendes germaniques.

Les burgs seigneuriaux de l'Allemagne possédaient un jardin comme les châteaux féodaux de la France $^{3}$; comme dans ceux-ci également on y cultiva de bonne heure des arbres à fruits et des fleurs; la rose n'y manquait pas plus que le lis; mais, d'importation étrangère, elle dut être d'abord considérée comme un objet de luxe, quelque chose de rare et de précieux; il n'est pas surprenant aussi que la légende s'en soit emparée et qu'elle lui ait donné place dans l'épopée germanique rajeunie. C'est ainsi qu'a pris naissance, sans doute vers la fin du xıı ${ }^{0}$ siècle $^{4}$, la

1. Tant alai et haut et bas Que je vins dessus un ruissiel Oủ il avoit maint arbrissiel.
Moult par estoit le lieu jolis ; Anquelies, roses et lys A l'environ d'illuec croissoient.

Poésies publiées par Auguste Scheler. Bruxelles, 1870 , in-8, vol. I, p. 2, v. 48-53.

2. Éd. J.-G.-A. Luthereau. Paris, 1844, in-8, p. 111.

3. Alvin Schultz, Das höfische Leben zur Zeit der Minnesünger, vol. I, p. 50. Cf. pl haut, deuxième partie, chap. I, p. 140. 4. Ce n'est pas l'opinion de Schleiden qui attribue aux Rosen- 
tradition du parterre ou jardin des roses - Rosengarten - de Worms. Planté dans une île du Rhin par la fille du roi Kibich, la belle Kriemhild, il avait une lieue de long sur une demi-lieue de large; un tilleul s'élevait au milieu, sous lequel cinq cents nobles dames pouvaient trouver un abri; des roses éclatantes le remplissaient ${ }^{1}$. Il n'avait pour le protéger qu'un fil symbolique de soie ${ }^{2}$; mais douze héros en défendaient l'entrée; quiconque triomphait de l'un d'eux recevait en récompense de sa vaillance un baiser de la bouche même de Kriemhild et une couronne de roses.

La légende du jardin des roses de Worms se répandit vite en Allemagne; à son imitation on en attribua un au roi des nains Laurin $^{3}$; il se trouvait près de Méran, dans le Tyrol; il avait quatre portes, avec un tilleul au milieu et une enceinte formée d'un simple fil de soie, comme le Rosengarten de Worms.

garten germaniques une origine orientale, analogue à celle des gulistans persans, mais cette manière de voir ne soutient pas l'examen. " Der Rosengarten kann nicht wohl eine ältere Sage enthalten ", dit avec raison Gervinus, Geschichte der deutschen Dichtung. Leipzig, in-8, vol. II, p. 81. Cf. K. Godeke, Grundriss, $2^{e}$ éd., p. 245.

1. Sie heget einen anger mit rôsen wol bekleit, der ist einer mîle lang und einer halben breit, dar umme gêt ein mûre, daz ist ein borte fìn : trutz sì allen fürsten, daz ir einer kume drîn.

Der Rosengarte, von W. Grimm. Göttingen, 1836, v. 165-68.

2. "Symbolisch zu binden reichte ein... seidenfaden hin. " J. Grimm, Deutsche Rechtsalterthümer. Göttingen, 1828, p. 182.

3. Zingerle, Künig Laurin, Innsbruck, 1850, in-8, p. 21. 
Des rossignols en charmaient les bocages, et les roses en étaient si merveilleuses que leur vue suffisait pour remplir de joie ceux qui étaient affligés et leur faire oublier leur tristesse ${ }^{1}$.

Mais il existait bien d'autres jardins de roses en Allemagne et même en dehors de l'Allemagne; on en trouvait à Rohrschach, à Constance, à Nunich, près de Combourg dans le Kiocherthal, dans la forêt de Thuringe, auprès d'Osnabrück et de Rostock ${ }^{2}$, ainsi qu'en Suède ${ }^{3}$. Heinrich Frauenlob visita celui que le margrave Woldemar de Brandebourg avait planté à Rostock; " sept tilleuls au milieu d'un parterre de roses", que l'on voit encore sur le sceau de cette ville ${ }^{4}$, en ont perpétué le souvenir.

Se rattachant à la fois à ce que l'épopée germanique avait de plus héroïque et à la fète du printemps, la légende du jardin des roses resta populaire en Allemagne pendant de longues années; elle se trouve intimement mèlée à celle de l'établissement du Meistergesang au $\mathrm{xiv}^{0}$ siècle. Les douze maîtres qui passent pour l'avoir fondé avaient, dit-

1. Der plân hete fröude vil, swer in solde sehen an

der muoste all sîn trûren làn. V. 920-22.

2. Uhland, Zur deutschen Heldensage. (Germania, vol. VI (a. 1861), p. 321, note 23.)

3. Le héros d'une ballade suédoise porte le nom de "Sven i Rosengård ». Sivenskia Folk-Wisor utgifne af E. G. GerJer och A. A. Afzelius. Stockholm, 1814-16, vol. III, p. 2.

4. "Seren Linden up den Rosengahrden ». Bechstein, Deutsches Sagenbuch, n ${ }^{\circ}$ 65. ap. Schleiden, p. 138. 
on, recu en garde un jardin des roses. Les fleurs qui y brillaient étaient l'emblème de leurs ingénieuses poésies ${ }^{1}$; une couronne de roses était la récompense et l'insigne destiné à celui que ses vers rendaient digne de prendre rang dans la poétique corporation ${ }^{2}$. C'était aussi le prix que recevait le vainqueur dans les défis que se donnaient alors les poètes.

Pour inviter au chant, dit Regenbogen, l'un d'eux ${ }^{3}$, je suspends ici une couronne de roses, quiconque fera entendre une sage parole et des sons mesurés, gagnera cette couronne et je le proclamerai maître.

Par un air joyeux je veux commencer, dit un autre ${ }^{4}$; à la main je tiens un étendard où se trouve dessinée une

1. Diestöck diestunden rosen voll. Ir komen vil hernach.

Das was ir kluegs geitchte Sie lasen pluemen auf der vart,

Die zwölff hätten es gerichte. Das was ein Meisterschaft.

J. Görres, Altdeutsche Volks- und Meisterlieder aus den Handschriften der Heidelberg Bibliothek. Frankfurta.-Main, 1817, in-8, p. 225.

2. Wilt du im Garten wetten,

Und treiben Meisterschaft,

Man setzt dir uff der Ehren ein Kranz,

Bist du mit Künste behafft.

$$
\text { Görres, Ibid., p. } 226 .
$$

3. Umb singens willen heng ich usz ein rosenkranz,

Wer singet wise wort und auch der töne schanz,

Und mir den cranz gewinnet an, den meister wil ich kennen.

$$
\text { Uhland, Schriften, etc., vol. III, p. } 310 \text {. }
$$

4. Frölich wil ichs heben an, Einkranz von rosen wol getan, In meiner hant fur ichs ein van, Wer mir den abgewinnen kan Daran findt man gezieret stan Mit schallen und mit singen.

Görres, Ibid., p. 226, Der Kranz, v. 1-7. 
élégante couronne de roses, qui veut la gagner par ses chants et ses mélodies?

Cependant la légende du Rosengarten finit par s'obscurcir; à la fin du moyen âge, le mot n'éveille plus que l'idée d'un parterre où l'on aime à se promener et à se réunir, et le nom resta dans la langue comme l'équivalent de "lieu de plaisance » ou "de joie "; "être dans un jardin de roses " fut en allemand une locution synonyme d'ètre heureux et content ${ }^{1}$, analogue à celle de "vivre au milieu des roses " chez les Latins.

Tu me réjouis le cœur au fond de la poitrine, dit une chanson $\mathrm{du} \mathrm{xv}^{\mathrm{e}}$ siècle $^{2}$, agréable passe-temps pour un oisif que d'être dans un jardin de roses!

On employa même, dans la poésie allemande, l'expression de "Rosengarten », comme celle de "pré fleuri ${ }^{3} »$ dans la poésie française, pour désigner le ciel :

Là-haut, dans ce jardin de roses, dit une chanson

1.

Gy Heren weset alle fro

Gy sint in dem rosengarden,

lit-on dans la " Dispute de Lunebourg». Uhland, Schriften, III, 539.

2.

Du erfreust mirs Herz im Leib,

wohl in dem Rosengarte

dem Schlemmer sein Zeitvertreib!

Lhland, Schriften zur Geschichte der Dichtung, vol. III, p. 439.

3. M'ame la m'amie sivra En camp flori la trovera,

$\mathrm{Ou}$ el queut encontre moi fiors.

Floire et Blancheflor. Éd. Edél. du Méril. Paris, 1854, in-18, v. 777-79. 
populaire $^{1}$, j’attendrai mon fiancé; là, dans ce séjour éternel, est déjà prête ma couche.

Ce qui a assuré à la rose une si grande place dans les légendes et la poésie du moyen âge, comme de l'antiquité, c'est à la fois la grâce qui en a fait le symbole de la beauté, et cette circonstance que, apparaissant au moment où le printemps est dans toute sa splendeur, elle en a été considérée comme la compagne et l'emblème: comment, dès lors, n'aurait-clle pas été chantée par les poètes? Tous aussi en ont célébré le doux charme et l'ont saluée comme la parure de la saison des amours et des chansons. Un ménestrel anglo-saxon ${ }^{2}$ a dit d'elle qu' " elle croît sur terre unique dans sa grâce ». Elle

Est sor totes flors la plus belle,

1.

Dort in jenem Rosengarten

Will ich mein Bräutigam erwarten;

Dort in jener Ewigkeit

Steht mein Brautbett schon bereit.

W. von Plönnies, Volksgesang aus dem Odenwald, ap. Schleiden, Die Rose, p. 142.

2. Aenlic on eordhan tyrf wynlic veaxedh.

Rätsel, XII, v. 25-26 (G. W. M. Grein, Bibliothek der engelsächsischen Poesie. Göttingen, 1858, in-8, vol. II, p. 388). 
s'écrie un trouvère ${ }^{1}$. C'est encore, comme l'appelle une chanson des Dithmarses "la noble fleur " ${ }^{2}$.

Cet éloge de la reine des fleurs a retenti pendant tout le moyen âge; c'est seulement sous l'influence de préoccupations étrangères au sentiment esthétique qu'on a paru déprécier la rose. C'est parce qu'il lui oppose la fleur mystique de l'obéissance, que l'auteur de la V'ie de sainte Martine ${ }^{3}$, Hugo von Langenstein, a cru devoir la rabaisser. Et si, dans la Plaidoirie de la rose et de la violete, Froissart place audessus de la première et de "toutes fleurs " le lis", c'est que ce dernier est l'emblème de la royauté et non parce que le poète le considérait réellement comme plus beau que la rose, qu'il met ailleurs il ne faisait en cela que se conformer à la tradition" sus toutes fleurs »".

Comme les poètes romans, les poètes germa-

1. La Patenostre d'Amors, v. 39. (Barbazan et Méon, Fabliaux et contes des poëtes français $d u \mathrm{xin}^{\mathrm{e}}$ au $\mathrm{xm}^{\mathrm{e}}$ siècle. Paris, 1808, in-8, vol. IV, p. 44. Beaudoin de Condé (Li contes de la rose, v. 343, ap. A. Scheler, Les dits et contes de Beaudoin et de Jean de Condé, vol. I, p. 145), dit de mème

Rose est sor toutes flors la fine.

2.

De adlige rôsenblòme.

\section{Uhland, Volkslieder, no 128.}

3. Martina hgg. v. Ad. Keller. Stuttgart, 1858, in-8, p. 66.

4. ... Beaus advocas jolis. Qu on doit bien tenir en chierté, La noble et haulte flor de lys, N'a elle souveraineté

Sus la rose et sus toutes flours?

Poésies pub. par 1. Scheler. Bruxelles, 1870, in-8, vol. II, p. 233.

Paradis d'amour, v. 1627 (Poésies, vol. I, p. 49). 
niques ont été unanimes à assigner à la rose, et aussi bien à la rose sauvage qu'à la rose cultivée, le premier rang parmi les fleurs du printemps.

La bruyère, dit le minnesaenger Gotfrid de Nîfen ${ }^{1}$, s'est revêtue de sa ravissante parure, les roses en sont le plus bel ornement.

Dans la ballade danoise de " la fière Mettetil »", les roses forment avec les lis la parure du jardin symbolique que "le noble Pierre " avait planté et qu'en son absence un cerf est venu dévaster, foulant aux pieds les fleurs et détruisant l'unique plante qui pouvait donner de la joie à son cœur. Les roses, il est à peine besoin de le dire, figurent encore au premier rang des fleurs qui servent à construire les demeures fantastiques que les poètes romans et ger-

1.

$$
\begin{aligned}
& \text { Nust diu heide wol bekleidet } \\
& \text { mit vil wunneclichen kleiden : } \\
& \text { rôsen sint ir besten kleit. }
\end{aligned}
$$

Karl Bartsch, Deutsche Liederdichter, p. 156, v. 51-53.

2. Ich hab gepflanzt ein Würzgärtlein mit Blumen und adlichen

[Rosen :

Nun ist noch andres dazwischen gewachsen, dieweil ich nach

[Rom gezogen.

In meinem Garten ist gewesen ein Hirsch der die Blumen hat niedergetreten,

Er hat mir verwüstet das einzige Kraut, das Freude meinem

[Herzen konnt geben.

Altdänische Heldenlieder, Balladen und Mürchen übersetzt

v. Wilh. Carl Grimm. Heidelberg, 1811, p. 283, n ${ }^{\circ}$ 69, v. 18-21. 
maniques donnent parfois aux héros de leurs chansons amoureuses ${ }^{1}$.

En faisant de la rose l'emblème de la beauté, les poètes du moyen âge se sont montrés les continuateurs et les disciples fidèles des poètes de l'antiquité. Mais, plus que de la beauté, ceux-ci avaient été frappés de la fragilité de la rose; la courte durée de cette fleur, au contraire, a été à peine remarquée par les trouvères et les minnesaenger, et les rares allusions qu'ils y font leur ont été inspirées par leurs précurseurs de la Gréce ou de Rome. Telle est cette comparaison d'un trouvère belge :

Pucele est com flors de rose

Qui tost vient et tost trespasse ${ }^{2}$.

De mème quand le ménestrel anglo-saxon Aelfred déplore que la violence de la tempête détruise la beauté de la rose ${ }^{3}$, il ne fait que traduire Boèce, et ce dernier s'était lui-même inspiré des poètes anciens pour chanter "le bocage qui, au souffle des

\section{Ainsi dans le Tristan:}

De flors et de roses sans giel

Iluec ferai une maison. Éd. Fr. Michel, vol. I, p. 222. Et dans un vieux lied allemand :

Got gebe uch ein gute nacht, von rosen ein dach von liligen ein pet. Uhland, Schriften, vol. III, p. 360 .

2. Aug. Scheler, Trouvères belges, $2^{\text {e }}$ série. Bruxelles, 1879, in-8, p. 29.

$3 . \quad$ Se stearca storm, thonne hê strong cyndh, nordhan and eàstan, thaere ròsan vlite hê genimedh hradhe Metra, VI, v. 11-13.

Grein. Bibliothek der angelsächsischen Poesie, vol. II, p. 301. 
tièdes zéphyrs, se pare des roses du printemps, dont l'impétueux auster vient dépouiller bientôt leur tige fleurie $"{ }^{1}$.

Mais si ce motil poétique n'a été qu'exceptionnellement mis en cuvre au moyen âge, il en est un autre que les poètes romans et germaniques de cette époque ont abordé aussi souvent et plus souvent mème que ceux de l'antiquité : c'est le rapport de la rose et du printemps. Emblème préféré de la saison qui réveillait en eux l'inspiration, la rose leur servit, avant toutes les autres fleurs, à en marquer le retour et à en embellir la fête.

ja estoit passez yvers

Et l'aubespine florissoit

Et (que) la rose espanissoit,

dit l'auteur du Roman du Renard'.

$\mathrm{Ce} f u$ en mai que la rose est fleurie,

L'oriol chante et le rossignol crie, remarque le poète du Siège de Narbonne.

Bertrand de Bar s'exprime de même ${ }^{4}$ :

1. Cum nemus flatu zephyri tepentis vernis inrubuit rosis,

Spiret insanum nebulosus auster : iam spinis abeat decus.

De consolatione Philosophiae, lib. II, cap. 3, v. 114-17.

2. Éd. Méon. Paris, 1826, in-8, vol. II, v. 9660-63.

3. Bibl. nat. man. fr. 24369, v. 54-55.

4. Girart de Vienne, ap. K. Bartsch, La langue et la littérature francaises, p. 333 , v. 19. Adenet le Roi, au contraire, fait fleurir les roses à la fin de juin :

Entour la saint Jean que la rose est florie Berte, v. 36. Ed. Aug. Scheler. 
Ce fu en mai qu'il fait chaut et seri, Que l'erbe est verz et rosier sont flori.

Adenet le Roi, dans Bueve de Commarchis, a également eu recours à l'épanouissement des roses, ainsi qu'à la longueur des jours, pour marquer le retour de l'été :

En esté quant li jour sont bel et lonc et clerc,

Que la rose est florie et bele a esgarder ${ }^{1}$.

Après avoir rappelé que le froid hiver est fini, que les nuits sont courtes maintenant et longs les jours, qu'un temps ravissant vient remplir de joie le monde entier, Nithard ajoute ${ }^{2}$ :

Un brillant spectacle s'offre à nos yeux, les roses, vraie merveille, ont paru sur la bruyère.

Mai vient avec sa multiple parure, s’écrie de son côté Gotfrit de Nîfen ${ }^{3}$, la douce bruyère se revêt et de fleurs et de roses vermeilles.

En tous pays, chante également un autre minnesaenger, Ulrich de Winterstetten ", monts et vallées sont

1. Ed. Aug. Scheler. Bruxelles, 1874, in-8, v. 53-54.

2. Komen ist uns ein liehtiu ougenweide : man siht der rôsen wunder ûf der heide.

Karl Bartsch, Deutsche Liederdichter, p. 109, v. 215-16.

3.

Meie kumt mit maniger bluot, nu hàt aber diu liebe heide beide bluomen unde rôsen rôt.

Karl Bartsch, Deutsche Liederdichter, p. 155, v. 5-7.

4. Berc und tal in allen landen sint erlòst ùz winters banden, heide rôte rôsen treit.

Karl Bartsch, Deutsche Liederdichter, p. 163, v. 74-76. 
LA ROSE DANS LES LÉGENDES PROFANES. 301

affranchis des liens de l'hiver, la bruyère se couvre de roses vermeilles.

Et dans un lied anonyme recueilli par Gœrres ${ }^{1}$ :

Maintenant verdoient dans les forêts les douces fleurs, dans les champs (brillent) les roses aux mille couleurs.

Sois le bienvenu, ô mois de mai, dit un autre lied ${ }^{2}$, aussi loin que s'étend le monde, poussez, roses fleuries.

Même spectacle dans la poésie néerlandaise et scandinave, mais ici la rose et le lis annoncent, non le retour du printemps, mais celui de l'été :

Avec lui, dit un lied néerlandais ${ }^{3}$, l'été a ramené mainte fleur couverte de rosée, elles rendaient un si plaisant éclat que le monde en était tout éclairé; le trésor des parfums est ouvert; jy ai vu de belles roses

1. Es grünen jetzt in den Wäldern Die Rosen auf den Feldern Die Blümlein fein, Von Farbe mancherlein.

Frühlingscur, Altdeustche Volkslieder, p. 36, str. 3.

2. Maie, sei wilkommen! All so weit die Welt ist, Spriesset, ihr Rosenblumen! Schleiden, p. 141.

3. Der zomer bracht in den hove zin

Bedauwet menich bluemelin

Die gaven so wonnenlichen schin,

Das ze verlichten die werolt al.

Outlossen wart der zalden scrin,

Darin so sach ich rosen fin

vurich blenchen zam ein robin;

van vruden zanc der nachtegal,

da hoert man menigen rychen scal.

G. Kalff, Het Lied in de middeleeuwen. Leiden, 1884, in-8, p. 299. 
briller comme des rubis. De joie chante le rossignol, et l'on entend maint accent joyeux.

Voici la saison si douce de l'été, lit-on dans une vieille chanson danoise ${ }^{1}$; le froid hiver est passé; les roses et les lis s'épanouissent et les bois se couvrent d'une gaie verdure.

A Thann en Alsace, c'était la coutume qu'au premier jour de mai une petite fille, la "Rose de mai ", toute couverte de fleurs et de rubans, parcourût les rues avec une amie chargée de recueillir dans une corbeille des dons, pour la fète du printemps, tandis que leurs compagnes chantaient ${ }^{2}$ :

Rose de mai, tourne-toi trois fois, fais-toi voir et revoir! Rose de mai, viens dans la verte forêt! Réjouissons-nous, mai nous ramène au milieu des roses.

En Provence, suivant une coutume très ancienne, dit César de Nostredame ${ }^{3}$, on choisit le premier mai

1. Her stunded saa blid en sommer i Aar,

forgangen er Vinter hin kold:

der springer ud Roser og Lillier,

og Skoven hon stander saa bold.

Vore Folkeviser fra middelalderen. Studier over Visernes. Aesthetik, rette Form og Alder af Joh. C. H. R. Steenstrup. Kjöbenhavn, 1891, in-8, p. 149.

2.

Maienröslein, ker dich dreimal'rum,

Lass dich beschauen 'rum und num!

Maienröslein, komm in grünen Wald hinein,

Wir wollen alle lustig sein.

So fahren wir vom Maien in die Rosen.

Aug. Stöber, Elsässisches Volksbuchlein. Strassburg, 1842, in-8, p. 42. - Böhme, Altdeztsches Liederb., no 497 a.

3. Mistral, Lou tresor dou Felilrige, s. v. maio. 
LA ROSE DANS LES LÉGENDES PROFANES. 303

une petite fille, la mayo, qu'on habille de blane et qu'on pare d'une couronne et de guirlandes de roses; on la place ensuite sur une sorte d'estrade ou de trône élevé et chaque passant lui donne quelque menue pièce de monnaie ${ }^{1}$.

Symbole et parure du printemps - et, dans le Nord, même de l'été - le retour de la rose provoque le poète à chanter dans cette saison des amours et lui rappelle l'amie dont il est séparé :

Quant voi le douz tens venir,

La flor en la prée,

La rose espanir,

Adonc chant, pleur et sospir,

s'écrie Philippe de Nanteuil ${ }^{2}$.

Quant voi la glaie meure La rousée resplendir,

Et le rosier espanir

Lors souspir

Et sur la bele verdure

Pour celi qui tant desir

Et aim, las, outre mesure,

chante également Raoul de Soissons ${ }^{3}$.

Mème inspiration chez 'Thibaud de Blazon":

Quant je vois esté venir Au point du jor,

Et sa verdor

Et la rose espanir Adonques souspir Et plaing et desir.

1. Cf. Alfred de Nore, Coutumes, mythes et traditions des provinces de France, Paris, 1846, p. 17.

2. Chansonniers de Champagne, Reims, 1850, p. 102, Lxxvi, v. $1-4$.

3. Eduard Mätzner, Altfranz̈̈sische Lieder. Berlin, 1853, in- 8, p. $18, n^{\circ} 10$, v. 1-7.

4. Chansonniers de Champagne, p. 127, v. 1 à 6. 
Et Thibaud de Champagne dit à son tour ${ }^{1}$ :

Au renouvel de la douçor d'esté, Que resclarcit li dois en la fontaine, Et que sont vers bois et vergiers et prés, Et le rosier en mai florist et graine, Lors chanterai que trop maura grevé Ire et esmai qui m'est au cueur prochaine.

Mais la vue de la rose et le retour du "temps nouveau " ne suggéraient pas toujours au poète des pensées de tristesse ou de regret, ils étaient aussi pour lui une invitation à s'abandonner à la gaieté et à la joie :

Le tens qui raverdoie Et la rose nouvele
Si que tot en sautele; Talent m'est pris de chanter, Fet mon cueur estre en joie Car bone amor m'i semont, s'écrie un ancien poète ${ }^{2}$.

"Par deu ", dit également l'auteur anonyme d'une vieille pastourelle ${ }^{3}$ :

Par deu, belle compaignete, Et espanir la rosete Voi le tens renoveler Ke nos semont de juer,

Pour Colin Muset, la vue de "la rose espanie » est un simple encouragement à boire "vin sus lie, " ainsi qu'à mener "bone vie " ${ }^{4}$. L'inspiration ne

1. Chansons de Thibaut IV, comte de Champagne et de Brie. Reims, 1851, in-8, p. 7, no Iv, v. 1-6.

2. Les Chansonniers de Champagne, p. 121.

3. Karl Bartsch, Romanzen und Pastorellen. Leipzig, 1870, in-8, p. 139. II. no 24, v. 12-15.

4. Les Chansonniers de Champagne, p. $89, \mathrm{n}^{\circ} 66$, str. 4. 
pouvait ètre plus réaliste ni plus vulgaire, et la signification de la reine des fleurs plus rabaissée. C'est, au contraire, une joie toute pure que la vue des roses vermeilles, toutes couvertes de rosée au milieu de la verte prairie, inspire à un des héros de l'épopée allemande ${ }^{1}$, spectacle qui attire et charme ses regards.

De même, dans le roman de Guillaume de $\mathrm{Pa}$ lerne $^{2}$, la vision d'une rose offerte suffit pour éloigner la douleur du cœur du héros et y ramener la joie :

$$
\text { Si li ert vis }
$$

Que de la chambre issoient fors

Alixandrine et Meliors,

Dessi en droit a lui venoient,

Une rose li aportoient.

Tantot com recevoit la flor,

Ne sentoit paine ne dolor,

Travail, grevance ne dehait.

\section{III.}

Ce qui réjonit ainsi le cœur de Guillaume, c'est que la rose apportée par Alexandrine et Mélior lui apparaît, ce qu'elle était réellement, comme une marque de sympathie et d'affection. La rose était, en

1. Mìn ougen vuoren mir schiezen rôte rôsen in dem touwe Als sie sachen entspriezen in einer grüenen ouwe

Ges. Abenteuer hgg. v. der Hagen, vol. III, p. 123, v. 448-51, 2. Id. Michelant. Paris, 1876, in-8, v. 1452-57.

JoRet. La Rose. 
effet, avant tout et surtout l'emblème de l'amour; en cueillir et encore plus en offrir était le signe d'un cœur épris ou capable de s'éprendre. C'est ainsi qu'une vieille chanson nous montre un amant, séparé de celle qu'il aime ${ }^{1}$,

Quant se vient en mai, ke rose est panie, Allant coillir par grant druerie,

pour apaiser sa douleur, cette fleur symbole de son amour.

"Où est la jeune fille qui m'aime tant? Elle est dehors dans son jardin et cueille des roses "), dit un lied allemand ${ }^{2}$, montrant ainsi l'étroit rapport des roses et de l'amour. De même dans une chanson néerlandaise ${ }^{3}$, il est question d'une jeune fille dont le cour est épris et qui va cueillir des roses vermeilles sur la bruyère. Même motif dans une romance espagnole ${ }^{4}$ :

1. Wilhelm Wackernagel, Altfranzösische Lieder und Leiche. Basel, 1846, in-8, p. 84, no 51, v. 1-2.

2. Wo ist dann das Mädchen, das mich so lieb hat?

Es is draussen im Garten, pflückt Röselein ab.

Fr. L. Mittler, Deutsche Volkslieder, no 938, v. 1-2.

$3 . \quad$ Rode rooskens woude si plucken die aen der heiden staen.

Antwerpener Liederboek, no 22, 1.

4. Cual es la niña la rosa florida, que coge las flores si no tiene amores? Cogia la niña el hortelanico prendas le pide si no tiene amores.

Böhl, Floresta de rimas antigurs castellanas. Hamburgo, 1821, in-8, vol. I, p. $303, \mathrm{n}^{\circ} 278$. 
Quelle est la jeune fille qui cueille des roses, si elle n'a pas d'ami? Voyant la jeune fille cueillir la rose fleurie, le petit jardinier lui demande un gage, si elle n'a pas d'ami.

Et dans une autre romance également espagnole ${ }^{1}$, l'ami va aux bords du ruisseau cueillir en soupirant sur le rosier fleuri des roses, qui lui rappellent ses amours.

De même dans un chant grec $^{2}$, nous voyons Melpomène, "la belle au corps d'ange ", presser le nautonnier qui l'aime de la conduire sur la rive, afin qu'elle puisse, signe qu'il lui est permis d'espérer, y cueillir des roses, avant que le soleil se lève. Et dans une autre chanson également greeque ${ }^{3}$, l'ami rêve que son amie cueille avec ses compagnes, dans une belle prairie, des roses et des fleurs de myrte couvertes de rosée, emblème du sentiment qu'elle éprouve et que le baiser qu'elle lui donne vient confirmer.

C'est à cause mème de cette signification symbolique que la rose occupe une place d'élection dans le jardin du Dieu d'amour; voilà pourquoi aussi Guillaume de Lorris en fait porter une couronne aux principaux personnages de sa cour ${ }^{4}$, depuis le dieu lui-même,

1. A riberas d'aquel rio viera estar rosal florido...

Cogi rosas con sospiro.

Böhl, Floresta, vol. I, p. 302, no 273, v. 6-10.

2. Chants du peuple en Grèce, par M. de Marcellus. Paris, 1851 in-s, vol. I, p. 373, no 10, Melpomène.

3. Op. laud., vol. II, p. 309, $\mathrm{n}^{\circ} 20$, Chansons des roses.

4. Le Roman de la Rose, éd. Fr. Michel. Paris, 1864, in-12, v. 899-900, 833-34 et 557-58. 
Qui ot ou chief un chapelet De roses, jusqu'à Déduit, à qui “Léesce » en a aussi donné :

Li ot s'amie fet chapel

De roses qui moult li sist bel, et à Oyseuse, " la noble pucelle, » laquelle

Ung chapelet de roses tout frois At dessus le chapel orfrois.

"Celui dont le coeur brûle d'amour », dit le Tannhäuser ${ }^{1}$ faisant en quelque sorte la théorie de cette coutume, " doit porter une couronne de roses". Chez tous les poìtes des derniers siècles du moyen âgie, un " chapel ) de roses apparaît comme la parure ordinaire des amants :

Si voit de la forest issir,

Tot bellement et a loisir

Dusc' a irj.xx. damoiselles.....

Capeaux de roses avoient

En lor chiés mis et d'aiglentier

Por le plus doucement flairier.....

Et sur .j. destrier de lès lui

Avoit cascune son ami,

lit-on dans un ancien lai ${ }^{2}$, dont il est superflu d'expliquer le sens allégorique.

1. $\mathrm{Ob}$ im sin herz von minne enbran, der soll von ròsen einen kranz

tragen. Minnesinger, vol. II, no 83, v. 19-21.

2. Lai du trot, v. 76-85 et 112-113, publié par Francisque Michel. Paris, 1845, p. 74́ et 76. 
Au jardin du voisin je suis entré, dit le héros d'une chanson morave ${ }^{1}$, je m'y suis couché et endormi, et j'ai rêvé de ma belle amie. Quand je me suis réveillé, personne près de moi, rien que deux roses vermeilles épanouies au-dessus de ma tête. J'ai cueilli ces roses, j’en ai tressé une couronne, les ai mises à mon chapeau et suis allé à la danse nuptiale.

Cette couronne mise ainsi par le jeune Morave à son chapeau, qu'est-elle, sinon le signe manifeste des sentiments d'amour qui l'animent?

Une chanson serbe ${ }^{2}$ nous montre également Mitza, " la belle», et " qui a la maison la plus blanche », cueillant des roses et en faisant des bouquets; elle en offre un au faucon gris, c'est-à dire à son fiancé, comme gage de son amour. Dans un chant tchèque ${ }^{3}$ nous voyons de même une jeune fille cueillir une rose, qu'elle offre successivement à son père, à sa mère, à son frère; mais cette fleur n'est point faite pour eux; enfin elle la présente à son fiancé, à qui seul elle doit appartenir. Les chansons de l'Ukraine parlent aussi d'une jeune fille qui ne veut donner la rose qu'elle a cucillie à aucun des siens, mais la garde pour son fiancé $e^{4}$, tant la rose était devenue le symbole et l'emblème de l'amour.

1. Uhland, Schriften, vol. III, p. $2: 2$.

2. Kurelac, Jačke, no 50, ap. Potebnia, Obiasnenia, etc.(Explication des chansons populaires de l'Ukraine). Kharkov, 1887, in-8, vol. I, p 488.

3. Plohl-Hordvig, Hrvatske narodpiesne, no 53, ap. Potebnia, op. laud., vol. II, p. 489.

4. Goloratzki, Recueil de chansons populaires, ap. Potebnia, op. laud., vol. I, p. 488. 
Ne peut-elle s'épanouir, c'est la marque d'un amour dédaigné ${ }^{1}$; vient-elle à s'effeuiller, e'est un signe d'abandon ${ }^{2}$. Les amants se jettent des roses ${ }^{3}$ en signe d'affection. C'est sous un bosquet de rosiers que l'amie attend son ami-éloignét. Si des amants jettent sur un cours d'eau des pétales de rose et que deux d'entre ceux-ci surnagent sans se séparer, c'est signe que leur mariage est prochain".

Dans une ballade danoise, le noble Tidemand, ne pouvant gagner l'amour de la befle Blidelille, grave des runes puissantes sur des roses et les jette à la mer près du rivage où les porte le flot; la belle Blidelille trouve ces roses, elle les porte chez elle et les place sur sa couche ${ }^{6}$; mais voilà qu'à minuit elle s'éveille, violemment agitée par un songe; c'est l'amour du noble Tidemand qui pénètre à son insu dans son cœur. Ici les roses exercent une action magique qu'elles doivent peut-ètre à la présence des runes, et qui est inconnue aux autres traditions

1. Chanson tchèque, ap. Sobotka, Rostlinstvo a jeho uyznam vnarodnich pisnich... slovansliych. V Praze, 1879, in-8.

2. Chanson tchèque. Ibid.

3. Chanson de la Lusace. Ibid.

4. Chanson tchèque. Ibid.

5. A. Ritter von Perger, Deutsche Pflanzensagen, p. 232.

6. Det var Jomfrud Blidelille, Tog hun op de Roser to, hun ganger ud med den Strand: Stak dem i Aermelin, fandt hun dèr de Roser to, saa bar hun dem i Bure hjem, de flöde ind for det Land: lagde dem paa Sengen sin. Svend Gruntrig, Danmarks Folkeviser $i$ Udvalg, II, no 23. Hr. Tidemands Runer, p. 290. 
romanes ou germaniques, dans lesquelles elles figurent.

Mais il n'était point besoin de runes pour que les roses servissent à gagner l'affection; le présent de ces fleurs était le plus agréable et le plus propre à l'obtenir. "Fleur, recois cette fleur », dit dans un chant anonyme du xur ${ }^{\mathrm{e}}$ siècle un ami à son amie, à qui il présente des roses ${ }^{1}$. C'est à cause de cette signification symbolique reconnue de tous qu'on offrait des roses à l'ami ou à l'amie dont on recherchait l'affection :

En no jardin je suis entrée

Trouvay la rouse espanouye :

Si doucement je l'ay cueillie

Et l'ay donnée à mon amy,

lit-on dans une chanson du $\mathrm{xv}^{\ominus}$ siecle ${ }^{2}$. Pour gagner l'amour de sa dame, Froissart aussi se rend dans un jardin rempli de fleurs,

Et la une vermeille rose

Coillit sus un moult vert rosier $^{3}$,

et "sans point noisier") il va l'offrir à celle à qui il veut plaire.

Et la jeune reine du Chritiment des Dames de Fran-

1.

Suscipe Flos florem

quia flos designat amorem.

Carmina Burana, p. 217, $\mathrm{n}^{\circ}$ 147. La vignette qui accompagne ces vers représente une double branche, l'une qui porte des lis, l'autre des roses.

2. Chansons du $\mathrm{xv}^{\mathrm{e}}$ siècle, p. 74, $\mathrm{n}^{0} 76$, str. 2.

3. L'espinette amoureuse, v. 986-87.(Poésies, vol. I, p. 115.) 
cesco da Barberino envoie une guirlande de roses au roi, signe de l'amour qu'elle lui donne et qu'elle lui avait d'abord refusé ${ }^{1}$.

Faisons chose plaisante, fait dire Nithard à un gai compagnon qui brigue la main d'une villageoise 2 ; allons au milieu des fleurs cueillir des roses et tressons-en une couronne qu'en ce mois de mai nous porterons à la danse.

Une chanson allemande, plus récente ${ }^{3}$, il est vrai, parle aussi de roses cueillies et envoyées pour la danse des fiançailles par un ami à son amie. Un autre lied nous représente l'ami endormi dans un jardin, rèvant de celle qu'il aime et vers laquelle se reportent toutes ses pensées; mais à son réreil il n'apercoit que des roses rermeilles; il en cueille ce qu'il peut et les donne à son amie, qui en fait une couronne et la lui place sur le front; longtemps cette couronne fait sa joie; enfin elle se fane; mais que lui importe, puisque celle qu'il aime est maintenant à lui ${ }^{4}$.

1. Del regimento e de' costumi delle donne. Roma, 1815 , in-8, p. 121 .

2.

Nu tuo wir gemelichiu dinc

unt gê wir in die bluomen

brechen ròsen z' einem kranz,

die wir in dem meien tragen zuo dem tanz.

Minnesinger, vol. III, p. $236 a$.

3. Ich brach mir die röslein abe $z u$ einem kranze.

Ich schickt sie meinem feinen lieb zum lobetanze.

Franz M. Böhme, Altdeutsches Liederbuch, no 140.

4. Franz M. Böhme, op.laud., no 176. 
Dans l'ancienne poésie allemande et néerlandaise, l'expression "cueillir des roses »- il en est de mème de celle de "boire du vin frais ${ }^{1}$ " - est synonyme de briguer l'amour de quelqu'un ou de se marier. Dans un lied allemand ${ }^{2}$, deux amants s'entretiennent de leur séparation prochaine : "Quand reviendras-tu, mon bien-aimé, cueillir des roses vermeilles et boire du vin frais?" - "Quand il neigera de vermeilles roses, ma bien-aimée, et pleuvra du vin frais. "Restée seule, l'amie se rend un jour dans le jardin de son père; elle s'y couche, s'y endort et rève qu'il pleut du vin frais. Mais quand elle se réveille, elle voit seulement des roses en fleur audessus de sa tête. Alors elle se fait construire une maison - sa dernière demeure; - quand elle est terminée, elle s'y endort, une couronne de roses à la main et après avoir bu le vin du Seigneur. Cependant son ami revient enfin; il se rend au jardin avec une couronne de roses et une coupe de vin. Mais il heurte du pied une tombe; il chancelle. "Alors, dit le poète, il neigea des roses et il plut du vin."

Partout la rose apparaît ainsi comme l'emblème de l'amour qu'on éprouve ou qu'on réclame: c'est aujourd'hui encore la coutume, dans le midi de la France, - on la rencontre aussi dans d'autres provinces, - d'attacher, le premier jour de mai, une

1. Par un souvenir, comme le remarque Hoffmann von Fallersleben (Niederländische Volkslieder, préface, p. xiv), de l'ancienne coutume d'offrir du vin aux noureaux mariés le lendemain des noces.

2. Des Knaben Wunderhorn, vol. II, p. 52. 
rose à la porte de la jeune fille dont on recherche l'amour ${ }^{1}$. En Allemagne, on jetait une rose dans la chambre de celle qu'on aimait ${ }^{2}$, ou on l'invitait à venir voir les fleurs de son jardin et à en cueillir, ou on en cueillait pour elle.

L'été est venu, mes belles roses sont en fleur; ne veux-tu pas venir les voir dans mon jardin? dit un vieux lied allemand ${ }^{3}$. Il lui cueillit les plus belles roses; un baiser, un baiser elle lui donna en retour.

\section{Et dans un autre lied ${ }^{4}$ :}

Mon trésor, va mattendre dans le jardin, nous y dirons de douces choses et cueillerons des roses en ce beau lieu.

1. Mistral, Lou tresor dou Felibrige, s. v, roso.

2.

Er thät ein Röslein brechen,

Zum Fenster stiess er's hinein.

Uhland, Schriften, vol. III, p. 422.

3. Der Sommer ist da, meine Rosen blühn schön :

Willst du nicht meine Rosen im Garten ansehen?

Die schönsten Rosen pflückte er ihr,

Einen Kuss, ein Kuss gab sie ihm dafür.

Fr. L. Mittler, Deutsche Volkslieder, n ${ }^{\circ} 310$, str. 16 et 17.

'. Schatz, geh in Garten, Ein angenehmes Wort,

Da sollst du warten. Und Rosen brechen

Da wollen wir sprechen An jenem Ort. Mittler, Ibid.

Dans un autre lied. le poète invite sa dame à venir avec lui dans la prairie, où fleurissent de belles roses :

Ach Jungfrau! wollt ihr mit ihm gan?

Da wo die schönen Röslein stan,

Draussen auf jener Wiesen.

Görres, op.laud, p. 190. 
Le même motif se rencontre également dans les vieilles chansons néerlandaises:

Ah! jeune fille, voulez-vous venir avec moi? dit l'une d'elles ${ }^{1}$; je vous conduirai là où fleurissent les roses.

Trois roses qui se trouvent sur la mème tige, ce qu'on appelle en Allemagne un "roi des roses" (Rosenkönig), - étaient considérées comme le présage d'un mariage ${ }^{2}$; le présent de l'une d'elles avait dès lors une valeur toute particulière :

Si j’avais à faire trois souhaits, trois nobles souhaits, dit une chanson néerlandaise ${ }^{3}$, je me souhaiterais trois roses sur la même tige; $j$ 'en cueillerais une, je laisserais la seconde; la troisième je la donnerais à l'amie que j'ai.

Ces trois roses sont parfois aussi représentées comme quelque chose de rare ou même d'impossible à trouver, surtout en hiver. C'est ainsi qu'au chevalier qui lui demande son amour une jeune fille répond qu'elle ne sera à lui que s'il lui apporte " trois roses, qui aient fleuri dans l'année, entre les

Och maechdelijn, woudi met mi gaen, ic soude u leien daer rooskens staen.

Antwerpener Liederbuek, $\mathrm{n}^{\circ}$ 61, 4.

2. A. Ritter von Perger, Pflanzensagen, p. 231.

3. Had ic nu drie wenschen, Die ene soude ic plucken, drie wenschen also eel, so soude ic nu gaen wenschen drie rosen op enen steel. die ander laten staen, die derde soude ic schenken der liefster die ic haen. Hoffmann von Fallersleben, Niederlëndische Volkslieder, $\mathrm{n}^{\circ} 103$, str. 6 et 7. 
jours gras et Pâques $)^{1}$. Le chevalier, n'ayant pu découvrir ces roses, en fait peindre trois; il les porte à la jeune fille, qui se met à pleurer". "J'ai dit, reprend-elle, ces paroles en plaisantant; je ne croyais pas que tu pusses trouver ces roses." “ Si tu as dit ces paroles en plaisantant, sérieusement tu dois les tenir; je suis à toi comme tu es à moi ; remets-t'en du reste à Dieu ${ }^{3}$. »

Dans un conte allemand les trois roses ont une signification merveilleuse toute différente ${ }^{4}$. Un homme avait deux filles, l'une méchante, l'autre bonne. Un jour qu'il allait à la foire, la première lui demanda de lui apporter une robe de soie, la seconde ne lui demanda que trois roses sur une tige ${ }^{5}$. Le

1. Dein eigen das en werd ich nicht,

Du bringst mir denn drei rosen,

Die in dem jar gewachsen sein

Zwischen fastnachten und ostern.

Franz M. Böhme, op. laud., no 62, str. 2.

2. Dans une autre version, $n^{\circ} 61$, c'est en hiver que les roses doivent avoir fleuri; le chevalier finit néanmoins par en découvrir. et quand il les apporte à la jeune fille, elle se met à rire.

3 .

Und da das mägdlein die röslein ansach

hub an heimlichen zu weinen :

"Ich habe ein wort im schimpf geredt,

ich meint', du fündest ir keine. »

". Hastu ein wort im schimpf geredt, im ernst solstu es mir halten!

so bin ich dein und du bist mein,

drum lass dir den lieben gott walten. »

'́. Meier, Volkssagen, p. 202, ap. Ritter von Perger, p. 233.

5. Suivant une autre version, il ne s'agit que d'une seule rose, 
père acheta la robe, mais il ne put sur tout le marché trouver les trois roses. Il s'en revenait tout attristé de n'avoir pu satisfaire au désir de sa douce et bonne fille, quand il passa devant un jardin rempli de rosiers et aperçut trois roses épanouies sur la tige de l'un d'eux; il s'empressa de les couper. Mais soudain un monstre apparut devant lui, le saisit et ne le laissa aller qu'il n'eût promis de lui donner sa fille comme épouse. Le père, effrayé, le promit et, laissé libre, revint chez lui avec les roses. Sa fille, qui ignorait à quelle condition il les avait obtenues, se réjouit beaucoup de les avoir. Tout paraissait oublié lorsque, à quelque temps de là, un char s'arrêta devant la maison; c'était la jeune fille qu'il venait chercher et elle partit au milieu des larmes des siens. Elle finit cependant par s'accoutumer à sa nouvelle existence et à la société du monstre, et un jour que celui-ci ne revint pas comme à l'ordinaire, elle se mit tout inquiète à sa recherche; elle le découvrit étendu sans vie près d'un étang; une violente douleur la saisit à cette vue; elle se baissa en versant d'abondantes larmes sur le mort; mais à peine l'eurent-elles touché qu'il revint à la vie sous la forme d'un beau jeune homme. L'amour avait levé le charme qui le tenait transformé.

Dans nos vicilles chansons il n'est point question de trois roses croissant sur la même tige, mais seu-

mais demandée pendant l'hiver. Paulus Cassel, Rose und Nachtigall, p. 1. 
lement de "trois fleurs d'amour", lesquelles néanmoins étaient sans doute des roses :

Hier au matin mi levai

En notre jardin entrai,

Trois fleurs d'amour j'y trouvai,

Une en prins, deux en laissai,

A mon ami l'envoirai,

Qui sera joieux et gay ${ }^{1}$.

Dans une variante de cette chanson, c'est l'ami qui trouve les roses, et de celles qu'il cueille il tresse une couronne pour son amie ${ }^{2}$ :

Ung chapelet fait en ay;

De trois rens le començay

Et a quatre l'achevay

A m'amye le donray

En ceste nouvelle saison.

Les roses n'étaient pas seulement un gage d'affection; elles étaient aussi regardées comme s'associant à la joie des amants dans leurs rencontres; un minnesaenger ${ }^{3}$ est allé jusqu'à dire qu'elles poussent de terre à la vue de l'ami qui presse son amie dans ses bras; elles leur sourient du moins et s'associent à leur allégresse.

Comme la rose était le gage et l'emblème de

1. Uhland, Schriften, vol. III, p. 519, note 199.

2. Chansons du xve siècle, p. 9, no 8 .

3. Die boum begonden krachen und der ritter nåch neic... die rosen sêre lachen... Vil rôsen ûz dein grase gienc, Dò diu vrouve nider seic dó liep mit armen liep enphienc.

Ges. Abenteuer, vol. I, p. 46女́, v. 345-46, 349-50, 353-54. 
l'amour, un poète a pu supposer qu'il était interdit aux moines de s'en parer:

Vert ne vermeil porterés, Bouqués, roses ne marjolaines.

Il leur défend même de se reposer à l'ombre d'un rosier ou d'un églantier, ainsi que de prêter l'oreille aux chants du rossignol:

En printemps nouvellet, Quant par boys ou champs passerés, Des que orrez le rossignolet, Vos vigilles des mors dirés, Et (que) jamais ne dormirés Soubs aubespins ou esglantiers, Autre part tant que vous vouldrez, Pourveu qu'il n'y ait des rosiers ${ }^{1}$.

En Allemagne, c'est aux vilains qu'il était interdit de se parer de roses, et un poète leur assigne en échange des couronnes d'orties, marque de dédain et de mépris :

Vilain, dit-il à un paysan arrogant ${ }^{2}$, laisse ces roses; elles ne sont pas pour toi; c'est une couronne d'orties quill te convient de porter.

Kriemhild, dans une des rédactions du grand

1. L'amant rendu cordelier à l'observance d'amour, publ. par $\Lambda$. de Montaiglon. Paris, 1881, in-8, v. 1399-1400 et 1369-;6.

2. O Bauernknecht! lass die röslein stan!

Sie sein nit dein;

Du tregst noch wol von nesselkrant

Ein krenzelein. Böhme, op. laud, no 222. 
Rosengarten ${ }^{1}$, fait dire aussi aux guerriers de Berne qu'ils pourraient bien emporter de Bourgogne une couronne d'orties, au lieu de roses vermeilles.

Dans un de ses poèmes les plus gracieux, Christine de Pisan suppose que la déesse de Loyauté a été chargée par le dieu d'Amour d'établir un "ordre », destiné à prendre la défense des dames contre leurs détracteurs. Elle apparait tout à coup au milieu d'une noble assemblée, réunie, le 14 férrier 1401 , chez le duc d'Orléans, et offre aux assistants, de la part du dieu, des " roses odorables, blanches et vermeilles ". Ce sont les insignes de l'ordre nouveau, et chaque membre, avant de "prendre la jolie rose" qui lui revient, fait le vœu suivant:

A tousjours mais la bonne renommée Je garderay de dame en toute chose,

Ne par moy ja femme n'y ert diffamée:

Et pour ce prens je l'Ordre de la Rose ${ }^{2}$.

On comprend que la rose ait été choisie comme insigne d'un ordre supposé établi par le dieu d'Amour lui-même; ce qui est plus surprenant, c'est qu'elle ait aussi servi d'emblème de paix et d'amitié. Dans nos plus anciens poèmes, un rameau d'olivier

1. Kriemhilt hat iuch entbotten und heisset iuch mere sagen, Ir möhtent lieber heimen ein kranz uz neslen getragen, Den da zuo Burgentriche die liechten rosen rot.

Éd. v. d. Hagen, v. 207-209.

2. Le dit de la Rose, v. 201-204. OEuvres poéliques de Christine de Pisan publiées par Maurice Roy, vol. II, Paris, 1891 , p. 35. 
en était, ainsi que chez les Grecs et les Romains, regardé comme le symbole :

Branches d'olive en vos mains porterez,

Ce sinefiet pais et humilitet,

dit Marsile, dans la Chanson de Roland ${ }^{1}$, aux députés qu'il envoie auprès de Charlemagne. Dans le roman de Renaud de Montauban, au contraire, ce sont des roses que les fils Aymon, se voyant dans l'impuissance de résister à Charlemagne, portent dans leurs mains en allant implorer la clémence du redoutable monarque ${ }^{2}$ :

Es plains de Vaucolors nos covient chevaucier...

Mais nos n'i aurons ja palefroi ne destrier,

Sor nos muls aragons irons esbanoier:

Aurons rosses es mains et flors par amistié.

Ils partent avec leur offrande pacifique et se rendent rassurés et contents vers le grand empereur :

Or chevalchent li conte a joie et a baldor, Chascuns porte en sa main une molt bele flor ${ }^{3}$.

Non seulement la rose formait les liens de l'amour, elle pouvait servir encore à réunir les amants que le sort avait séparés. Telle elle apparait dans le ro-

1. Laisse V, v. 72, éd. Léon Gautier.

2. Renaud de Montauban oder die Haimonskinder, altfranzösisches Gedicht nach den Handschr. hgg. 'on Dr. H. Michelant. Stuttgart, 1862, in-8, p 170, v. 29, 32-34.

3. P. 175, v. 1-2: Dans un autre passage, c'est un rameau de pin qui sert d'emblème :

Chascuns porte en sa main .r. rain de pin petit

Ce fu senefiance de joie et de delit. $\quad$ P. 59, v. 8 .

JoRet. La Rose. 
man si célèbre au moyen âge de Floire et Blancheflor ${ }^{1}$, noms qui rappellent la rose et le lis ${ }^{2}$, ainsi que la saison dans laquelle les deux amants vinrent au monde ${ }^{3}$. Élevés ensemble, malgré la différence de condition et de croyance, — Floire est le fils d'un roi sarrasin, Blancheflor a pour mère une esclave chrétienne, - les deux enfants éprouvent l'un pour l'autre la plus tendre affection. Le père de Floire, qui redoute pour son fils les effets de ce penchant, l'envoie étudier au loin et, en son absence, il vend Blancheflor à des marchands étrangers. Mais Floire ne peut supporter les tourments de l'absence; il revient à la cour, et quand il apprend le départ de Blancheflor, il se met à sa recherche. Après de longues aventures, il arrive à Babylone, dont l'émir

1. Floire et Blancheflor, poèmes du $\mathrm{xin}^{\mathrm{e}}$ siècle publiés d'après les manuscrits..., par M. Édélestand du Méril. Paris, 1856, in-18. Outre une double rédaction française, on connait encore une rédaction en haut et bas allemand, une version tchèque et flamande. plusieurs rédactions scandinares, une traduction anglaise, l'imitation qu'en a donnée Boccace dans le fillocopo et une version italienne en vers, une rédaction espagnole en prose et un poème en grec moderne. Introduction. p. 28-89. Cf. Emil Hausknecht, Floris and Blanchefleur, mittelenglisches Gedicht aus dem 13. Jahrhundert. Einleitung, p. 1-89.

3. Flos bedudet eine blome schone, ghelikent einer gulden kronen, Blanckflos bedudet eine witte blome wool.

Van Flosse en Blancflosse, v. 102-103.

2.

Le jour de la Pasque florie...

Li doi enfant, quand furent né,

De la feste furent nomé.

Floire et Blancheflor, v. 161 et 169-70. 
a acheté son amie et la tient renfermée dans une tour. Floire gagne le gardien de la tour, et après avoir revêtu des vêtements couleur rose, il se place dans une corbeille pleine de roses ${ }^{1}$ et on le porte ainsi dans la chambre de Blancheflor. Il est découvert, mais le sultan lui pardonne, et désormais les deux amants réunis vivent heureux. Sur leur tombeau commun on les représenta sous la figure de deux jeunes gens, Floire offrant à son amie une rose vermeille, tandis que son amie lui donne un lis blanc ${ }^{2}$.

Mais la rose ne rapproche pas seulement les amants éloignés, elle peut servir même à désigner l'époux marqué par le sort. C'est ainsi que dans un conte sicilien ${ }^{3}$, un roi en mourant recommande à son fils, quand une de ses sœurs se voudrait marier, de jeter dans la rue une fleur cueillie sur le beau rosier de la terrasse; celui qui la ramassera sera l'époux qui lui est destiné. La rose peut encore

1. Le trouvère français parle seulement de fleurs et non de roses, v. 2033 :

De fleurs assez a fait cueillir;

mais son éditeur croit qüil faut lire " de roses» et son imitateur allemand, Konrad Flecke, parle de roses encore couvertes de rosée:

Wir wurde nie sô müede

Von sô vil rôsen noch sô laz. Ich wæne sie wurden naz

Gelesen in dem touwe. $\quad$ V. 5556-62.

2.

Flore höveschlîche

Sỉner friundìn eine rôse bôt gemachet ûzer golde rôt,

Dà wider bôt im sîn friundìn ein gilje. V. 2002-2006.

3. Laura Gonzenbach, Sicilianische Märchen, 1870, vol. II, p. 111. Die Geschichte von Peze e fogghi. 
révéler la présence non soupconnée d'un ami caché ou déguisé ${ }^{1}$; sa vue rappelle à l'ami le souvenir de son amie absente ${ }^{2}$, à l'amie le chagrin que la séparation a causé à son $\mathrm{ami}^{3}$; enfin elle encourage la première à supporter l'absence du second et à lui rester fidèle ${ }^{4}$. Aussi le délaissé prend-il cette fleur à témoin de sa douleur ${ }^{5}$; l'amie lui demande des nouvelles de son ami éloigné ${ }^{6}$, son éclat terni ou conservé révèle le sort d'un être cher $^{7}$; elle peut même devenir le gage de la constance et de la fidé-

1. Schleiden, Die Rose, p. 158.

2.

Ich sach dà Rosenblumen stân

Die manent mich der Gedanke vil,

Die ich hin zu einer vrowen hàn.

Dietmar von Aist, Minnesinger, vol. I, p. 98, $b, 4$.

3. Ich sach boten des sumeres, daz wâren bluomen also rôt : weistu, schœne vrouwe, waz dir ein riter enbôt?... im trûret sîn herze, sît er nu jungest von dir schiet.

Minnesinger, vol. I, p. 220 b, 12.

'́.

Es sten dri rosen in jenem dal die rufent jungfraw an :

Got gesegeneuch, schöne jungfraw, und nement kein andern man!

Franz M. Böhme, op. laud., no 156, str. 12.

5. Klag' Alles, das Der Himmel beschlos!

Klag Röslein fein !

Gœrres, Volkslieder, p. 73.

6. Nun sag', nun sag', gut Röslein roth, lebet mein Buhl' oder ist er todt?

Er lebet noch, er ist nit todt,

er liegt vor Münster in grosser Noth.

Uhland, Schriften, vol. III, p. 428.

7. J. G. von Hahn, Griechische und albanesische Märchen, Leipzig, 1864, in-8, vol. I, p. 231. 
lité conjugale; c'est ce que montre l'histoire du chevalier Margon dans le Roman de Perceforest.

D'une condition humble, mais vaillant, Margon avait épousé la belle Lisane, fille d'un des seigneurs les plus puissants de la Grande-Bretagne. Mais se voyant, à cause de sa pauvreté, dans l'impossibilité de tenir un rang convenable, il résolut, sur le conseil de sa femme, de chercher fortune à la cour du roi. Une chose le retenait toutefois, l'amour qu'il avait pour Lisane et la crainte des dangers que sa jeunesse pouvait courir dans l'isolement. Mais Lisane le rassura, en lui donnant, renfermée dans une boîte d'ivoire, une " rose de telle nature que s'il advenait qu'elle méfît de manière à mériter reproche, cette rose deviendrait toute sèche. (Mais) tant qu'elle demeurerait loyale envers lui la rose resterait fraîche, comme si elle fût nouvellement cueillie du rosier et tant tiendrait sa couleur $)^{1}$. Rassuré par la possession de ce talisman, Margon se rendit auprès du roi Perceforest, dont il gagna bientôt les bonnes grâces. Mais la faveur dont il jouissait ne lui fit pas oublier sa chère Lisane, et plusieurs fois le jour on lc voyait se retirer à l'écart, et là il contemplait la rose qu'elle lui avait donnée et s'abandonnait à la joie de la "trouver toujours vermeille et odorante». Son bonheur devait bientôt être troublé.

Deux chevaliers de la cour d'Angleterre, Méléan

1. Anciennes croniques Dangleterre faicts et gestes du tres pieux et redoubté en chevalerie le noble roy Perceforest A. Paris, 1582, lib. IV, chap. 16, fol. $45, b 2$ et $50, a 1$. 
et Nabon, jaloux du crédit de Margon, épièrent ses démarches et accusèrent sa conduite auprès du roi; mais quand Perceforest eut appris de la bouche de Margon pour quelle raison celui-ci se retirait chaque jour loin de la foule, il n'en eut que plus d'estime pour ce chevalier et conçut en même temps la plus grande admiration pour Lisane. L'envie que Méléan et Nabon ressentaient pour Margon n'en fit que grandir, et, dans leur haine, ils formèrent le dessein de séduire sa femme et de ruiner par là la faveur dont il était entouré. Miéléan partit le premier et se présenta devant Lisane, comme envoyé par son mari. Après plusieurs jours passés au château de Margon, il crut le moment venu d'accomplir son projet; Lisane feignit de l'écouter, mais l'enferma un soir dans une tour dont elle emporta la clef. Le lendemain, à son réveil, Méléan se trouva prisonnier, et sur la muraille il lut une inscription qui le condamnait par "pénitence » à rester dans la tour et à y filer pour "gagner son pain ${ }^{1}$ ). En même temps il aperçut sur une table une quenouille et du lin, avec des fuseaux. Cette vue le remplit d'abord de fureur; mais bientôt, la faim venant, il se calma et finit même par se résigner à son sort.

Cependant Nabon, n'ayant point vu revenir son ami NIéléan, se rendit à son tour au château de Margon. Comme Méléan, il fut bien accueilli par Lisane; comme lui, il chercha à la séduire; mais, comme

1. Anciennes croniques Dangleterre, etc., liv. IV, chap. 16 , fol. $47, b 1$. 
lui encore, il fut enfermé dans la tour et obligé de filer pour obtenir à manger. Un an presque s'écoula ainsi. Margon commença à s'inquiéter et demanda au roi Perceforest la permission de retourner dans son pays. Arrivé chez lui, sa femme n'eut rien de plus pressé que de le conduire à la tour où étaient enfermés Méléan et Nabon; là il apprit de leur propre bouche le récit de leur criminelle tentative et de leur punition. Il revint ensuite à la cour de Bretagne, mais cette fois accompagné de sa femme, qui se vit fètée et honorée comme la " dame qui avait appris aux chevaliers à filer ${ }^{1}$ ».

Il faut rapprocher de cette légende celles de la tunique toujours blanche et inusable du soixanteneuvième récit des Gesta Romanorum², donnée par une mère à son gendre en gage de la fidélité de sa femme, ou du portrait de l'héroïne de la vingt et unième nouvelle de Bandello ${ }^{3}$, qui doit rester frais et brillant, tant que sa vertu sera immaculée, mais se ternira si elle trahit sa foi, ainsi que du fableau

1. Anciennes croniques Dangleterre, etc., liv. IV, chap. 18 , fol. $51, a, 2$.

2. Camisia ista talem virtutem habet, quod nunquam toto tempore vitae tuae lotione indiget, nec frangi potest, nec consumi, nec colore mutari, quamdiu inter te et filiam meam sit amor fidelis; si vero (quod alssit!) aliquis ex volsis matrimonium violaverit, statim camisia omnes ejus virtutes amiserit. P. 108, éd. Keller.

3. Si la moglie tua non ti romperà la fede maritale, vedrai sempre la imagine si bella e si colorita... ma se per sorte ella pensasse sottoporre a chi si sia il corpo suo, la imagine diverrà pallida. Fol. 141 vo, éd. de Lucques, 1550. 
du Mantel mautaillé ${ }^{1}$, qui s'adapte de lui-même à la taille des épouses qui ont gardé la foi conjugale et s'allonge ou se raccourcit pour ne pas aller aux femmes infidèles"; mais elle rappelle surtout les talismans qui figurent, nous l'avons $\mathrm{vu}^{3}$, dans le Livre du perroquet du persan Nachshebi et dans le recueil turc intitulé La Joie après la tristesse, et elle est sans doute, comme ces contes eux-mêmes et le récit des Gesta Romanorum, d'origine orientale ${ }^{4}$; mais elle dut passer de bonne heure en Occident et elle y devint populaire, en particulier en Angleterre. L'auteur anonyme du roman de Perceforest dit qu'elle fut chantée par les Bretons dans un lai " qu'on appela le lai de la rose ${ }^{\sharp}$ ». Ce lai est perdu; mais on possède encore l'imitation qu'Adam de Cobsam a faite de l'histoire merveilleuse de la rose de Lisane dans un de ses plus jolis contes: La chaste

1. La fée fist el drap une oevre Qui les fausses dames des[cuevre :
Se ele a de rien messerré Vers son seigneur, se ele l'a, Ja puis a droit ne li seira.

Ja fame qui l'ait afublé,

Ferd. Wolf, Ueber die Lais, p. 346.

2. Il y a bien d'autres légendes de ce genre. Cf. Édélestand du Méril, Floire et Blanceflor, Introd. p clxvir.

3. Partie II, chap. III, p. 120.

4. Après l'avoir dit dans sa belle étude sur le conte d'Adam de Cobsam, II. Reinhold Köhler semble admettre, dans un post-scriptum, que cette légende, au contraire. a passé de Grèce en Orient. Jahrbuch f. rom. u. engl. Literatur, vol. VIII, p. 167.

5. Anciennes croniques Dangleterre, etc., liv. IV, ch. 18, fol. $51, a, 2$. 
femme du charpentier ${ }^{1}$. Ici seulement, ce n'est pas une simple fleur, mais une couronne de roses, qu'une pauvre veuve donne à son gendre, joyau précieux dont la fraîcheur conservée doit l'assurer de la fidélité de sa femme.

\section{VI.}

Comme dans la poésie orientale, la rose est devenue aussi, dans la poésie occidentale du moyen âge, l'emblème et la personnification de la personne aimée. L'auteur anonyme du poème latin de Phyllis et Flore appelle son amie " la fleur des jeunes filles et la rose des roses ${ }^{2}$ "; pour un autre poète ${ }^{3}$, également latin et anonyme, son amie est une " rose" ", la "rose

1. The wrightes chaste wife or a fable of a wright that was mayde to a pore wydows dowtre, the which wydow having noo good to geve with her gave as for a precious Johelle to hym a Rose garland, the which she affirmed wold never fade while she kept truly her wedlock. A merry tale by Adan of Cobsam, ed. by Fred. Furnival. London, 1865.

2.

Flos est puellarum

Et rosa rosarum.

Carmina Burana. Lateinische und deutsche Lieder und Gedichte einer Handschrift des XIII. Jahrhunderts aus Benedictbeuren. Stuttgart, 1847, in-8, $n^{\circ} 65$, p. 208, str. 140-3.

3. L'auteur du poème appelé Carmen de rosa par M. Ernest Langlois, Origines et sources du Roman de la rose. Paris, 1891. p. 37.

4. per quandam vetulam rosa prohibetur,

L't non amet aliquem atque non ametur.

Carmina Burana, p. 151, no 50, str. 4, v. 3-6. 
du monde ${ }^{1}$ ), ou encore une "rose de mai, la fleur des fleurs, plus belle que toutes les autres ${ }^{2}$ ». Pour un trouvère, aussi anonyme et leur contemporain,

C'est la rosete, c'est la flor,

La violete de douçor ${ }^{3}$.

Un autre l'appelle

Flor de lis, rose espanie,

Taillie por esgarder ${ }^{4}$.

L'auteur du Dit de la Rose a expliqué lui-même cette allégorie charmante :

Par la rose puet l'en entendre,

La belle qui assez plus tendre

Est et fresche come rose en may,

Et je suis cil qui esté ai

En si grant desir longuement

D'avoir s'amor entirement ${ }^{5}$.

La bien-aimée, pour laquelle le véritable amant se “ dueil », n'est-elle pas aussi “ sans pareille » à ses yeux, comme la rose,

1.

2.

Ave mundi rosa. Ibid., str. 8, v. 6 . vidi florum florem,

Vidi rosam madii, cunctis pulchriorem.

$$
\text { lbid., str. 6, v. 2-4. }
$$

3. Recucils de motets français des $\mathrm{xr}^{\mathrm{e}}$ et $\mathrm{xm} \mathrm{II}^{\mathrm{e}}$ siècles, publiés par Gaston Raynaud. Paris, 1881, in-12, vol. I, p. 150.

4. Recueil de motets, vol. I, p. 146. Adenet dit aussi de Clarmondine qu'elle est une

Flor de lis et rose espanie. Cléomadès, v. 6034 .

5. Bartsch, La langue et la littérature françaises. Paris, 1887 , in-8, p. 606, v. 27-33. 
Qui plus bele est sus toutes choses...

Et par coleur et par odeur

Vaut [ele] miex que nule fleur ${ }^{1}$.

Dans le Roman de la Rose, où Guillaume de Lorris a repris la donnée du Dit de la Rose, développée une première fois dans le poème de Vénus la déesse d'amour ${ }^{2}$, nous voyons l'amant poursuivre le dessein longtemps traversé de cueillir le bouton de rose, dont la beauté et l'éclat l'ont charmé dans le jardin du Dieu d'amour,

Car une color l'enlumine,

Qui est si merveille et si fine,

Com nature la pot plus faire,

et

les maux d'amer,

Qui lui soloient estre amer,

ne s'apaisent que quand, secouru par Vénus,

Ung baisier dous et savoré

A[i] pris de la Rose erraument ${ }^{3}$.

Guillaume de Lorris ne pouvait désigner sous une forme plus gracieuse et plus claire son amour et celle,

tant digne d'estre amée

Qu'el doit estre rose clamée ${ }^{4}$,

qui en était l'objet.

1. Dit de la rose, Bartsch, p. 609, v. 6 et p. 610, r. 2-6.

2. De Venus la deesse d'Amor, altfranzösisches Minnegedicht aus dem XIII. Jahrhundert nach der Handschrift B. L. F. 283 der Arsenalbibliotheck in Paris zum ersten Male hgg. v. Wendelin Foenster. Bonn, 1880, in-12.

3. Roman de la Rose, v. 1667-69, 4093-94 et 4088-89.

4. Vers 43-44, vol. I, p. 2. 
Nos anciens poètes sont restés fidèles à cette allégorie $^{1}$; c'est, dit l'un d'eux de son amie,

La doulce fleur que mon cueur aime tant.

Je suis, chante un autre,

Je suis amoureulx d'une rouse ${ }^{2}$.

L'imitation italienne du Roman de la Rose dans Il fiore ${ }^{3}$ et les traductions qui furent faites de ce poème en anglais ${ }^{4}$ et en néerlandais ${ }^{5}$ portèrent bien au delà de nos frontières cette ingénieuse fiction; on la rencontre dans toutes les littératures du moven âge. La personne aimée est la "rose sur la bruyère » des minnesaenger allemands ${ }^{6}$; pour eux encore " elle ressemble à un rosier " et "fleurit comme une $\operatorname{rose}^{7}$ ». " $O$ toi, mon étoile du matin à son lever, ma fleur, ma rose ", s'écrie Schionatulander, en parlant à son amie ${ }^{8}$.

1. Chansons $d u \mathrm{xv}^{\mathrm{e}}$ siècle, p. $78, \mathrm{n}^{\circ} 80, \mathrm{v} .3 ;$ p. $74, \mathrm{n}^{\circ} 76, \mathrm{v} .1$.

2. Villon de mème appelle son amie " $m$ 'amour ma rose $», n^{\circ} \mathbf{L x x x}$, v. 1 .

3. D'Ancona, Varietà storiche et letterarie. Milano, 1885, vol. II, p. 1-31.

4. W. W. Skeat, Essays on Chaucer, Chaucer's Society, 1884, vol. V, p. 437-53.

5. Petit, Bibliographie der meddelnederlandsche Taalen Letterliunde. Leiden, 1888, no 468, p. :2-73. Le traducteur néerlandais le plus célèbre du roman de la Rose fut Heinrike van Aken.

6. Rôsen aûf der heide. Nithard, éd. Ben., v. 441.

7. Sie gleicht wol einem rosenstock...

Sie blüet wie ein röselein.

Franz Böhme; Altdeutsches Liederbuch, no 147, v. 1 et 5.

8. Dû mîn ûfgender morgensterne,

Dû bluom', dû rôs'. Títurel, éd. F. Pfeiffer, str. 220. 
Pour les poètes néerlandais, imitateur's des trouvères et des minnesaenger, leur amie est un "rosier » ou un " églantier odorant ${ }^{1}$ ». Dans les lieds danois la bien-aimée du poète est aussi une "fleur de rose», une "rose vermeille ${ }^{2}$ ». Ulva, la fille du petit nain, dans la ballade suédoise du noble Thinne, est une " rose au milieu des lis ${ }^{3}$ ". La bien-aimée des poètes italiens ${ }^{4}$ et espagnols ${ }^{5}$, est également une " rose fraîche " et "parfumée ", "belle et chère".

Même symbolisme chez les poètes slaves ${ }^{6}$. Leur amie est une "fleur de rose ${ }^{7}$ », une "rose fleurie ${ }^{8}$ ", ou encore une "rose vermeille, ni plantée, ni greffée ${ }^{9}$ ", elle est " belle comme une fleur de rose ${ }^{10}$ " ou "comme une rose double" $"$. Dans la poésie

1.

wel rieckende eglentier

Antw. Liederboek, no 7, ap. Kalff, Het lied, p. 335.

2. Rosens blomme, rose röd, etc.

Uhland, Schriften, vol. III, p. 496, note 143.

3. Eine Rose unter Lilien.

O. L. B. Wolff, Proben altholländischer Volkslieder, Greiz, 1832, in-18, p. 115, str. 9.

4.

Rosa fresca ed aulentissima.

Ciullo d'Alcamo, Il contrasto, v. 1.

5. Rosa fresca, rosa fresca,

Tan garrida y con amor.

Böhl, Floresta, vol. I, p. 256, no 149.

6. Sobotka, liostlinstio a jeho yzlum v narodnich pisnich -.slovanskych. V Praze, 1879, in-8.

7. Chanson de la Petite Russie.

8. Chanson bulgare.

9. Chanson serbe.

10. Chanson tchèque.

11. Chanson de la Petite Russie. 
hongroise aussi la personne aimée est une rose. " Ma chère rose, que fais-tu? ) dit une vieille ballade $^{1}$. Et dans une chanson populaire ${ }^{2}$ :

Scarabée de mai, petit scarabée d'or, je ne te demande pas quand viendra l'été; dis-moi seulement, ma rose sera-t-elle à moi?

"O ma chère rose vermeille », dit aussi un poète

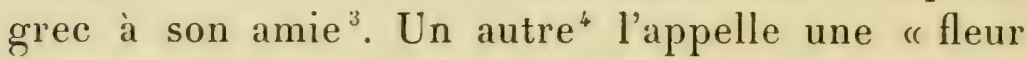
charmante et une rose parfumée ». Pour un troisième c'est " une blanche petite rose ", une "rose effeuillée, fleurette parmi les fleurs ${ }^{5}$ ).

\section{VII.}

Quand la rose servait ainsi, dans la poésie du moyen âge, à personnifier la bien-aimée du poète, on pourrait s'attendre à ce que celui-ci y apparût, ainsi que dans la poésie persane, sous les traits du rossignol et à ce que les troubadours et les trou-

1. Ballades et chansons populaires de la Hongrie, traduites par Jean de Néthy. Paris, 1891, in-18, Ballade $n^{\circ}$ XXVII.

2. Ibid. Chanson no I, 1.

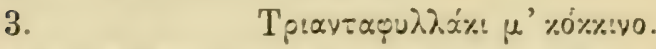

M. de Marcellus. Chants du peuple en Grèce, vol. I, p. 342. 4.

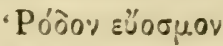

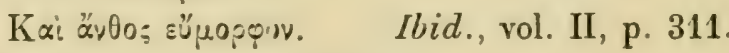

5.

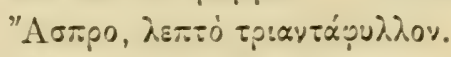

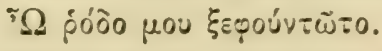

Émile Legrand, Recueil de charsons populaires grecques. Paris, 1873, in-8, p. 38,9 et p. $366,88$. 
LA ROSE DANS LES LÉGENDES PROFANES. 335

vères ou les minnesaenger aient raconté, comme les poètes persans et turcs, leurs amours sous l'allégorie transparente des amours du rossignol et de la rose. Il n'en est rien. Les poètes de l'Occident ont bien fait une place au rossignol dans leurs chants, mais ils ne se sont pas, comme leurs émules de la Perse, identifiés avec lui et ils n'en ont pas fait l'interprète de leur amour.

Héraut du printemps, la saison des amours et des fleurs nouvelles, son " doux chanter " provoque ${ }^{1}$ et " semont ${ }^{2}$ ) le poète à se faire entendre; il lui remet en souvenir ses propres amours ${ }^{3}$, et le fait penser

1. Quan lo rius de la fontana s'esclarzis, si cum far sol, e par la flors aiglentina el rossignoletz el ram volf e refraing et aplana son dous chantar et affina

Dreitz es qu'eu lo meu refraigna.

Jaufre Rudel, ap. Bartsch, Chrest. provençale, 61, v. 7-13.

Rossignor cui joi chanteir...

Me fait mon chant renovelleir.

Wilh. Wackernagel, Altfr. Lieder, $\mathrm{n}^{\circ} 30$.

2.

Li noviauz tens et mais et violete

Et rossignols me semont de chanter.

Le chàtelain de Coucy, chanson VI (IX), v. 1-2. (Éd. Fr. Michel, Paris, 1830, in-8, p. 33. - Éd. Fritz Fath. Heidelberg, 1883, p. 54).

Li rosignox m $\mathrm{i}$ semont

que j'aime loiaument.

K. Bartsch, Altfranzösische Romanzen und Pastourellen. Leipzig, 1870, in-8, I, no 52, v. 7-8.

3.

Bel m'es quan lo vens m'alena

En abril ans qu'intre mais,

E tota la noit serena

chantal rossinhols el jais... 
A la plus belle, a la millor

Ke soit dont jai ne pertira(i $)^{1}$.

Le rossignol est sans doute représenté plus d'une fois comme chantant au moment même où

la belle rose est en bruit ${ }^{2}$,

dans le jardin où elle naît et fleurit ${ }^{3}$, ou même au milieu de ses rameaux :

Par la flors aiglentina,

El rossignoletz el ram

volf e refraing e aplana ${ }^{4}$;

mais il ne chante pas pour elle, comme chez les poètes de la Perse. Il n'en joue pas moins un rôle considérable dans notre vieille poésie; elle le fait

non posc mudar nom sovena

d'un' amour per qu'eu sui jais.

Arnaut de Maroill, ap. Bartsch, Chrest. provençale, p. 87 et 88 , v. 37-28, et p. 89, v. 6-7.

1. Chanson anonyme, ap. Bartsch, La langue et la littérature françaises, p. 517, v. 21-22.

2. Phil. de Beaumanoir, La Manekine, éd. H. Suchier. Paris, 1884 , in-8, vol. I, p. 69 , v. 2158.

$3 . \quad$ En la huerta nace la rosa

Quiérome ir alla

Por ver al ruiseñor

Como cantaba. Böhl, Floresta, vol. I, p. 272.

4. Jaufre Rudel. Voir p. 335, note 1. De même dans un vieux lied allemand :

Auf dem Kirchhof steht ein Rosenbaum...

Darauf setzt sich Frau Nachtigall.

Karl Simrock, Die deutschen Volkslieder, no 86, v. 1-3. 
servir de messager à l'amant auprès de son amie ou à l'amante auprès de son ami :

Rossignol, en son repaire

Miras ma domna vezer,

$\mathrm{E}$ diguas lil men afaire,

s'écrie le troubadour Peire d'Alvernhe ${ }^{1}$.

Rossignol, va, si li di

Les maus que je sent por li;

Di li qu'il avra m'amor,

fait dire également à son héroïne un vieux trouvère anonyme ${ }^{2}$. Et dans une chanson du $\mathbf{x v}^{\mathrm{e}}$ siecle un autre poète s'écrie ${ }^{3}$ :

Roussignolet sauvaige Je t'en prie par ta foy:

Qui chante de cueur gay; Va dire à mon amy

Va moi faire un messaige, Qu'il m'a mys en oubly.

Parfois aussi l'ami ou. l'amie demande au doux chanteur aide ou conseil :

Roussignolet du bois ramaige, Conseille moy et je t'en $p^{\prime} y^{4}$.

Et encore :

1. Bartsch, Chrest. provencale, p. 73, v. 1-3. Dans une «nou velle s) d'Arnaut de Carcasses, c'est un perroquet qui porte le message d'Antiphanor à son amie, enfermée par un mari jaloux. Dans les lieds allomands le mème ròle est assigné aussi au coucou, à la colombe ou à n importe quel oiseau.

2. Alfred Jeanroy, Les origines de la poésie lyrique en France au moyen âge. Paris, 1889, in-8, p. 467-68.

3. Chansons $d u \mathrm{xv}^{\mathrm{e}}$ siècle, p. 70, no 72, v. 19-24

4. Chansons du xve siècle, p. 116, no 117, v. 33-34.

Joret. La Rose. 
Roussignolet mon amy,

Par amour je te prie,

Ne prens repoux ne demy, Tant que puisse parvenir

Mais tousjours chante et crye...

A ce que je désire ${ }^{1}$.

Même spectacle chez les poètes germaniques. Quand, au retour du printemps, les tièdes zéphyrs font épanouir la reine des fleurs, le rossignol -Frau Nachtigall - fait entendre ses accents joyeux ${ }^{2}$; parfois mème, perché sur un tilleul, il chante de l'amour qu'il ressent"; alors le poète va dans le vert bocage l'interroger sur l'éloignement de son amie ${ }^{4}$; il l'engage à chanter pour la dame qui « a son cœur et le laisse sans joie et sans courage ${ }^{\circ}$ ", ou bien il

1. Chansons du xve siècle, p. 135, no 132, v. 39-48.

2. Van vruden zanc der nachtegal

da hœrt man menigen rijchen scal.

Lied van den zomer en van den winter, ap. Uhland, Schriften, rol. III, p. 41, note 7. Cf. p. 301.

... in meines Vaters Hof da steht eine grüne Linde, darauf so singt die Nachtigall, sie singt so wol von Mlinne.

Uhland, Volkslieder, vol. II, no 17.

'. Nun will ich zichn in den grünen Wald,

die stolze Nachtigall fragen :

ob sie alle müssen geschieden sein,

die einst zwei liebchen waren.

Antwerper Liederboek de 154'4, no 193, ap. Uhland, III, 92. 5.

Nahtegal, guot vogellin,

miner frowen solt du singen in ir òre dar,

sìt si hàt daz herze min

und ich àne fröide und àne hôhgemüete var.

Hêr Ileinrich v. Stretelingen (K. Bartsch, Deutsche Liederdichter, $\mathrm{n}^{\circ}$ LXI, v. 1-4). 
l'envoie porter ses souhaits et ses vœux à sa toute aimée ${ }^{1}$.

Tandis que la poésie populaire de l'Europe occidentale ne sait rien des amours de la rose et du rossignol, cette fiction a pénétré dans celle de l'Europe orientale, qui l'a empruntée sans doute aux poètes persans ou turcs, chez lesquels elle occupe, nous l'arons vu, une si grande place. On la rencontre dans plusieurs chants serbes et grecs. L'un de ces derniers ${ }^{2}$ nous montre le rossignol, silencieux pendant dix longs mois, retrouvant sa voix harmonieuse au retour du printemps; il appelle la fleur, cause de sa souffrance; enfin il aperçoit la rose qui l'attend; il vole aussitôt vers elle; mais à la vue des épines qui l'entourent, comme autant de glaives, il s'arrète l'àme désolée et sa voix n'a plus d'accents que pour la douleur et la plainte.

Mais si l'ancienne poésie des nations romanes et

1. Frau Nachtigall, du kleins waldröglein...

ich wolt', du soltst mein bote sein...

und faren zu der herzallerliebsten mein.

Böhme, Altdeutsches Liederbuch, no 166, sir. 4-5.

Dans un lied néerlandais, c'est une amie qui l'envoie à son amie :

O nachtegael, clein roghel, woudt ghijder mijn bode wel sijn en vlieghen tot den ruiter, den alderliefsten van mijn en segghen dat hi comt alhier?

Niederländische Volkslieder, gesam. yon Hoffmann ron Fallersleben, no 56 , str. 5.

2. Chan's du peuple en Grèce, par M. de Marcellus, vol. II, p. 271, $\mathbf{n}^{\circ}$ xin : "Le rossignol et la rose. » 
germaniques - leurs poètes modernes aussi ont emprunté cette fiction à l'Orient ${ }^{1}$ - ignore les amours de la rose et du rossignol, clle a donné à la reine des fleurs une amie dans une plante, qui, venue comme elle de l'Orient ${ }^{2}$, a été aussi presque constamment et partout cultivée arec elle, le lis, dont l'union étroite arec la rose est comme l'emblème de leur origine et de leur culture communes, et est devenue, dans la tradition portugaise, un véritable amour ${ }^{3}$.

O cravo (lis) por simpatia Foram laços tâo estreitos A' linda rosa se uniu, Que amor perfeita sahiu.

\section{VIII.}

Comme dans l'antiquité, la rose a été au moyen àge, ainsi que de la grầce et de la beauté, le symbole de l'innocence pudique et de la chasteté. "Comme le vainqueur dans la lutte, dit Aldhelm", recoit au milieu du cirque des couronnes de lis et de roses à la couleur sanglante, ainsi la chasteté, victorieuse de la chair rebelle, portera, dans le rovaume du Christ, des couronnes de ces fleurs brillantes. "Saint Médard, évèque de Xoyon au $r^{\mathrm{e}}$ siècle, voulut, d'après

1. Voir plus haut, partie II, chap. r, p. 3 .

2. Cf. plus haut, partie I, chap. III, p. 60.

3. Leite Vasconcellos, Etnographia popular portugueza, p. 116, ap. A rosa na vida dos porss por Gecilia SchmidtBraxco, p. 25.

4. De laudibus virginum, Patrologie, vol. LXXX, p. $242 \mathrm{~B}$. 
une tradition ${ }^{1}$, accorder à l'innocence vertucuse cette récompense future dès cette vie. Il résolut de donner chaque année une couronne de roses et une dot a la jeune fille de ses terres de Salency reconnue pour la plus rertueuse. Cet honneur échut d'abord à sa sœur, qui fut ainsi la première des Rosières.

I.es anciens poètes romans et germaniques font souvent allusion à ce symbolisme nouveau de la rose, qui devait inspirer à l'Arioste des vers célèbres $^{2}$, imités des vers non moins célèbres de Catulle. Isote, dans le Tristan allemand, dit que son cour est toujours resté insensible à tous les hommes, excepté à celui qui a eu la fleur de rose de sa virginité ${ }^{3}$. Mème figure dans une chanson populaire francaise, dont l'héroïne pleure sur sa chasteté perdue 4 .

1. J.-L.-A. Loiseleur-Deslongchamps, La rose, p. 70.

2 .

La virginella è simile a la rosa,

Ch' in bel giardin su la nativa spina

Mentre sola e sicura si reposa

Nè grege nè pastor se le avvicina.

L'aura soave et l'alba rugiadosa,

L'acqua, la terra al suo favor s'inclina.

Orlando furioso, canto I, v. 329-34.

3.

hiule und jemer alle man vor minem herzen sint rerspart niwan der eine, dem dà wart der èrste rosenbluome von minem magetuome. V. 14766-70.

4. E. Rolland, Recueil de chansons populaires. Paris, 1883, in-8, vol. I, p. 202, n ${ }^{\circ}$ CVI, 5. Il faut dire que d'autres variantes de cette chanson présentent un sens tout différent. 
En revenant des noces J'étais bien fatiguée; Au bord d'une fontaine Je m'y suis reposée. Sur la plus haute branche Le rossignol chantait; Chante, rośsignol, chante, Toi qui as le cœur gai.
Car moi je ne l'ai guère, Mon amant m'a quittée; Pour un bouton de rose Que trop tôt j’ai donné. Je voudrais que la rose Fût encore au rosier Et que mon ami Pierre Fût encore à m'aimer.

Au lieu d'un bouton ou d'une fleur, l'emblème de la chasteté était le plus souvent une couronne de roses: aussi, l'infortunée qui s'était laissée séduire n'avait plus le droit d'en porter une et elle était la première à s'en dépouiller.

Elle arracha sa couronne, dit un vieux lied allemand ${ }^{1}$, et la jeta dans l'herbe verte. "Je t'ai portée volontiers tant que jétais vierge. " - Elle enleva sa couronne et la jeta au milieu du tréfle vert. "Dieu te bénisse, ma couronne, jamais je ne te reverrai. »

Les poètes germaniques ne se lassent pas de recommander aux jeunes filles, et souvent de la façon la plus piquante, de bien conserver leur couronne et d'éviter avec soin tout ce qui pourrait la leur faire

1.

Da zog sie ab ihr Kränzelein, warf's in das grüne Gras :

" ich han' dich gerne tragen, dieweil ich Jungfrau was. "

Auf hub sie wohl ihr Kränzelein, warf's in den grünen Klee : " gesegen' dich Gott, mein Kränzelcin, ich seh' dich nimmermeh.

Mittler, op.laud., Gœrres, Volkslieder, p. 182. 
perdre. Tantôt c'est un oiseau, d'autres fois un arbuste qui les conseille. Une jeune fille voudrait forcer au silence un rossignol, mais il répond qu'il est un oiseau de la forêt et que personne ne peut le contraindre ${ }^{1}$. "Si personne ne peut te contraindre, reprend-elle, le givre et la neige glacée, ainsi que le tilleul dépouillé de ses feuilles, te contraindront bien. — — "Si le tilleul perd son feuillage, répond le rossignol, il garde du moins ses rameaux; penses-y, jeune fille, et tiens ferme ta couronne. ) Et il termine en lui recommandant de ne pas se laisser tromper en écoutant les éloges menteurs qu'on pourrait lui donner.

Dans un autre lied ${ }^{2}$, nous voyons une jeune fille qui, voulant aller à la danse, va chercher des roses sur la bruyère; au bord du chemin elle apercoit un coudrier tout courert de feuilles; elle s'itonne et lui demande pourquoi il est si verdorant; le coudrier à son tour lui demande pourquoi elle est si belle; c'est, dit-elle, qu'elle mange du pain blanc et boit du vin frais. "Si tu es si belle paree que tu manges du pain blane et bois du vin frais, sur moi tombe la fraîche rosée, voilà ce qui me r’end si verdoyant. ) — "Prends garde, prends garde, cher coudrier, que je ne te fasse abattre; j'ai deux frères qui pourraient bien te couper.» - "S'ils me coupent en hiver, en

1. Uhland, Volkslieder, vol, I, no 17.

2. Böhme, Altdeutches Liederbuch, n 65. Nittler, Deutsche Volkslieder, nos 621 et 623 . 
été je reverdirai; quand une jeune fille perd sa couronne, elle ne la retrouve jamais plus. »

$\mathrm{Si}$, sous sa forme connue, ce beau lied est moderne, le motif en est d'origine ancienne, comme le montre une rédaction anglaise $\mathrm{du} \mathbf{x r r}^{\mathrm{e}}$ siècle, où le coudrier est remplacé par l'aubépine ${ }^{1}$. Il en existe aussi une rédaction suédoise, qui met en scène un tilleul $^{2}$. Dans une chanson vende ${ }^{3}$, au contraire, c'est une simple branche d'arbuste, qui, menacée par une jeune fille qu'elle a frappée au visage, d'être coupée par ses frères, lui donne le conseil de bien garder sa couronne.

Ce conseil n'est pas le seul que renferment les chansons populaires à l'adresse des jeunes filles; elles leur recommandent de ne point quitter seules la maison paternelle pour aller chercher des roses, et, comme dans la ballade écossaise des trois sœurs" leur montrent d'une manière tragique le danger auquel elles s'exposent alors. Un lied allemand du Kuhlaendchen en particulier les met en garde contre le péril de cueillir des roses et d'en faire des couronnes pour la danse le jour consacré au Seigneur".

1. Ritson, Ancient songs and ballads. London, 1829, in-8, vol. II, p. 44.

2. Svenska Folkwisor, vol. III, p. 115.

3. Volkslieder der Wenden, vol. I, p. 88, ap. Uhland, Schriften, vol. III, p. 427.

4. Minstrelsy, vol. IiI, p. 56, ap. Uhland, III, 520.

5. Uhland, Schriften, vol. III, p. $\$ 26$. 


\section{IX.}

Par une opposition d'idées dont nous avons déja eu un exemple, la rose a été aussi regardée parfois comme un présage funeste, en mème temps qu'elle est devenue un emblème funéraire. Quand elle fleurissait à l'automne ou en hiver, - on ne connaissait pas alors les roses remontantes —, c'était un signe de malheur. Grégoire de Tours place à côté des "prodiges " et des "dévastations " de l'année 584 les roses que l'on vit au mois de janvier ${ }^{1}$. Suivant une croyance populaire, les roses qui paraissaient ainsi hors saison annonçaient un décès dans la maison du possesseur du jardin où elles avaient crû.

L'apparition soudaine de roses, surtout de roses rouges, était aussi considérée comme un signe de mauvais augure on de mort. Un ami, dans un lied allemand $^{2}$, attend son amie, couché sur le vert gazon; tout à coup deux roses rouges de sang lui tombent sur les genoux; à cette vue, il s'inquiète et se demande si son " trésor » est encore en vie ou s'il est mort. Il vit, mais il est près d'un autre et à jamais perdu pour lui.

Une légende allemande parle de deux sœurs et d'un frère qui avaient choisi chacun un arbre dans

1. «Hoc amno multa prodigia adparuerunt in Galliis vastationesque multae. Nam mense Januario rosae visae sunt. „) Mistoria Francorum, lib. VI, cap. 4́́. Éd. Bouquet, p. 289.

2. Mittler, Deutsche Vollislieder, no 789, 790 et 1091. 
le jardin de leur père; quand le printemps fut venu, les arbres des deux sœurs se couvrirent de fleurs blanches, signe qu'elles seraient religieuses; l'arbre du frère porta une rose rouge, emblème de mort; dans la suite aussi il fut martyrisé à Prague par les Hussites ${ }^{1}$.

Il est souvent question, dans les chansons populaires germaniques, de roses répandues ou de rosiers plantés sur les tombeaux; les amants souhaitent de reposer au milieu de ces fleurs si chères, faible symbole de leur inaltérable fidélité.

Je sais que je dois mourir, dit l'un d'eux dans un lied allemand ${ }^{2}$, mais mon trésor plantera sur ma tombe un rosier rouge après ma mort.

Dans un autre lied ${ }^{3}$ il s'agit de rosiers qu'une fille en larmes a plantés sur la tombe de sa mère.

Souviens-toi des heures passées, dit ailleurs une fiancée à son fiancé qui lui survit ${ }^{4}$; répands sur mon tombeau des roses et des violettes. Ne m'oublie pas.

1. Mannhardt, Baumkultus der Germanen. Berlin, 1875, p. 49.

2. Weiss wohl dass ich sterben muss,

Und ein Röslein rosenroth

Pllanzt mein Schatz nach meinem Tod.

$$
\text { Nittler, Vollislieder, } n^{\circ} \text { \%62. }
$$

3. Die Rosen die pllanzt ich ihr weinend auls grab.

Mittler, Volkslieder, no 310 , str. 4, 2.

'. Gedenk an jene Stunden!

Bestreu das Grab mit Blumen,

Mit Rosen und Veilchen. Vergiss mein nicht!

Mittler, Volkslieder, n 833 , str. 5. 
Dans un lied néerlandais, un amant demande, quand il sera mort, à ètre enterré sous des rosiers à fleurs rouges ${ }^{1}$.

Les deux amants sont morts, dit un autre lied également néerlandais ${ }^{2}$, où les enterra-t-on ? Sous un églantier, ainsi leur tombeau portera des roses.

\section{Et dans une chanson serbe ${ }^{3}$ :}

Creusez, dit un amant désespéré, creusez-moi une tombe dans la plaine! Qu'au-dessus de ma tête on plante un rosier! Qu'à mes pieds on fasse couler une source limpide! Si par lá passe un jeune homme, qu'il se pare de roses! Si un vieillard y passe, qu il puisse étancher sa soif" !

On répandait aussi des roses dans le cercueil des défunts; mais suivant une croyance populaire, le rosier sur lequel elles avaient été cueillies se fanait

1. Och sterve ic nu, so ben ic doot, So graeft mi onder die rooskens root.

Antwerper Liederboek, $\mathrm{n}^{\circ} 63$, str. 3.

2 . Nu sijn daer twee gheliefkens doot, och waer sal men se graven? al onder enen eglentier, dat graf sal rooskens draghen.

Hoffmann v. Fallersleben, Niederl. Volkslieder, no 17 , str. 9.

3. Grabt, grabt ein Grab mir Leitet eine Quelle?

Auf dem weiten Felde!

Ueber meinem Haupte

Pllanzet eine Rose!

Unter meinen Füssen
Geht vorbei ein Jüngling, Schmücke er sich mit Rosen! Wenn ein Greis vorbei geht, Lösch' er scinen Durst dort!

Vollislieder der Serben, Metrisch übersetzl (... Von T'Talvj.) Leipzig, 1853, 2 ter. Theil, p. 82. 
alors et mourait. Quant aux roses qui poussaient sur les tombeaux, elles étaient parfois regardées comme une incarnation de l'âme même des défunts. "Mais ce n'est pas une fleur ", dit dans une chanson lithuanienne ${ }^{1}$, une mère à sa fille, qui lui apporte une rose cueillie sur la tombe de son bien-aimé, ) c'est l'âme de ton fiancé. »

La poésie regarda aussi comme doués de sentiment ou animés des passions des êtres aimés les arbres et les fleurs plantés sur leur tombeau. Suivant le continuateur de Gotfrid de Strasbourg, Heinrich de Freiberg, après que Tristan et Isolt eurent été enterrés non loin l'un de l'autre, le roi Mark fit planter un rosier sur la tombe de Tristan et une vigne sur celle d'Iseult ${ }^{2}$ :

Le rosier et la vigne, ajoute le poète, s'enracinèrent

1.

Das ist ja die Rose nicht,

Ist des Jünglings Seele.

Koberstein, Fortleben. (Weimarer Jahrbuch, vol. I, p. 96, ap. Perger, p. 13.)

2. Uf Tristan den werden liez der künic ùz erkorn pelzen einen rùsendorn... und einen grüenen winreben liez er ùf Isôten pelzen; den zwein tòten gelieben edel und hùchgewurzelten schòne an der stunt ieglìchem in sins herzengrunt, da noch der glüende minne「tranc in den tòten herzen ranc und sîn art erzeigete. ieglich ris da neigete

[born, dem andern ob den grebern der winrebe und der ròsen[sich,

dorn und in ein ander minnenclich vlehten... Herxuch's von Freiberg Tristan, hgg. von Bechstein. Leipzig. 1877, in-12, v. 6822-6839. 
bientôt jusqu'au fond du cœur des deux chers et nobles morts; l'ardent breuvage amoureux, qui couvait encore dans ces cours éteints, montra sa force; les arbustes s inclinèrent l'un vers l'autre et s'entrelacèrent amoureusement au-dessus des deux tombes.

Cette gracieuse légende avait déjà été acceptée par Uhich de Turheim ${ }^{1}$, le premier continuateur de Gotfried, et le Livre populaire ${ }^{2}$, consacré à l'histoire des deux amants infortunés, l'a soigneusement conservée.

Cette fiction du sentiment communiqué par les morts aux fleurs et aux plantes destinées à honorer leurs restes ne suffit pas à l'imagination populaire; elle en fit sortir de leurs cendres elles-mêmes. Dans une vieille ballade anglaise, la ballade de la « belle Marguerite» et du "doux Guillaume », morts, la première d'amour, le second de chagrin, et enterrés, elle en bas, lui en haut du sanctuaire, un rosier naît des restes de Marguerite, un églantier de ceux de Guillaume, et, en croissant, leurs branches s'élèvent jusqu'au faìte de l'église, se rejoignent et s'enlacent ${ }^{3}$, manifestant ainsi aux regards étonnés le lien indestructible qui unissait les deux amants.

Dans une ballade écossaise, celle de «lord Tho-

1. Karl Gœdeke, Deutsche Dichtung im Miltelalter. Dresden, 1871, in-8, p. 816, col. 1 .

2. Karl Simrock, Deutsche Volksbücher, p. 179.

3. Fair Margaret dyed for pure true love, Sweet William dyed for sorrow.

Margaret was buryed in the lower chancel, And William in the higher; 
mas » et de la "belle Annette », un bouleau croît sur la tombe du lord, un églantier sur celle d'Annette, et, en se rapprochant, leurs branches montrent aussi que là reposent deux amants ${ }^{1}$.

On les enterra tous deux à côté l'un de l'autre, dit une chanson serbe en parlant de deux amants malheureux, leurs mains unies sous terre, et dans leurs mains on plaça des pommes encore vertes. Et voilà quau bout de quelques mois sélève au-dessus de la tombe du jeune homme un vert sapin, au-dessus de celle de la jeune fille un rosier à fleurs rouges, et le rosier s'enlaça autour du

Out of her breast there sprang a rose,

And out of his a briar.

They grew till they grew unto the church top,

And then they could grow no higher;

And there they tyed in a true lovers knot,

Which made all the people admire.

Thomas Percy, Reliques of ancient english poetry. Leipzig, in-12, vol. III, p. 130-131, v. 65-76.

1. And ay the grew, and ay they threw

As they wad faine be neare;

And by this ye may ken right weil,

They were twa lovers deare.

Francis James Child, The english and scoltish ballads. Boston, s. d., in-4, vol. III, p. 183, v. 117-120. La poésie populaire de la Grèce connait des légendes semblables, comme celle de l'ami qui se tue de désespoir en apprenant la mort de son amie; mais sur sa tombe croit un cyprès, sur celle de son amie un roseau et non un rosier.

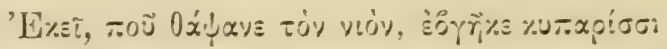

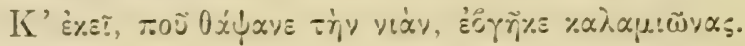

Chants du peuple en Grèce, par M. de Marcellus, vol. II, $\mathrm{n}^{\circ} \mathrm{xII}$ 
sapin, comme un fil de soie autour d'un bouquet d'immortelles ${ }^{1}$.

Une romance portugaise, plus récente, il est vrai, nous montre encore un rosier poussant sur la tombe d'un amant mort de désespoir, mais c'est un jasmin qui croît sur celle de son amic ${ }^{2}$. Perger ${ }^{3}$ parle, mais sans en donner la source, de trois roses sorties de la tombe d'une jeune fille, et que son bienaimé seul peut cueillir. Et dans la ballade suédoise de "Petite Rose ${ }^{4}$ ), des lis croissent sur son tombeau et sur celui de son ami, et de leur bouche sort une rose.

C'est encore la naissance miraculeuse des roses, fait si fréquent dans les traditions chrétiennes, que

1. Sie begruben beide zu einander,

Einten ihre Hände durch die Erde,

Legten in die Hände grüne Aepfel.

Und nach wenig Monden ob dem Jüngling

Hob empor sich eine grüne Fichte,

Ob der Jungfrau eine rothe Rose;

Und die Rose schlang sich um die Fichte,

Wie ein seiden Band um Immortellen.

P. von Götze, Serbische Volkslieder. Leipzig, 1827, p. 93.

2. Alvaro Rodriguez de Azvedo, Romanceiro de Archipelajo de Madeira. Funchal, 1888, p. 122.

3. Pflanzensagen, p. 13. Malheureusement Perger est si peu soucieux de l'exactitude qu'on ne peut accepter qu'arec défiance ce qu'il dit.

4. Perger, Pflanzensagen, p. 12. Je ne sais d'où Perger a tiré cette légende; dans le recueil des anciens lieds suédois de Erik G. Geijer et Arvid Aug. Afzelius, il est seulement question d'un liileul qui croilt sur la tombe des deux amants réunis. 
nous montre la légende allemande du Tannhäuser'. Poussé par la curiosité, il n'avait pas craint de pénétrer dans la montagne de Ténus et avait succombé aux séductions de l'enchanteresse. Aprés de longs efforts, il parvient enfin à s'éloigner de ce séjour coupable, et, plein de repentir, va à Rome demander pardon au pape. "Je suis, lui dit-il, resté un an auprès de dame V'énus, j’en viens faire pénitence et demander pardon. ") Le pape tenait à la main un bàton blanc, fait d'un rameau desséché : "Quand ce bâton portera des roses, répliqua-t-il, tu obtiendras ton pardon. ) A ces mots, le chevalier se retira triste et désespéré. Cependant, le troisième jour, des roses poussèrent sur le bâton du pape; surpris et repentant, il fit chercher partout le 'Tannhäuser. On le retrouva enfin, mais mort au milieu de la montagne de Vénus, ou il restera jusqu’au jour du jugement.

La légende de sainte Rose de Lima et celle de l'Autel de Seefeld dans le Tyrol offrent le plus grand rapport avec la naissance miraculeuse des roses de l'histoire du Tammäuser. Lorsqu'on proposa, dit-on, au pape de canoniser la sainte du Pérou, il répondit

1. Wolfrang Menzel. Zur deutschen .Yythologip. Berlin, 1855, in-8, vol. I, p. 312, ap. Schleiden, p. 166. Le rolkslied publié par Uhland, p. 761, dans le Des Knaben Wunderhorn, I, 53, ainsi que par Simrock (Die deutschen Volkslieder, $n^{0} 4$ ), parle de feuilles et non de roses, qui poussent sur le bàton du pape; il est, au contraire, question de roses dans le lied néerlandais «Herr Daniel s (Viederlündische Volkslieder, no 4), imitation du lied allemand du Tannhäuser. 
qu'il ne croirait pas à la sainteté d'une Indienne, même s'il pleurait des roses. Il avait à peine prononcé ces paroles qu'une pluie de roses couvrit le Vatican, et ne cessa que quand, se rendant à l'évidence, il eut prononcé la formule de canonisation ${ }^{1}$. Le seigneur de Seefeld, Arnold Müller, s'étant, le jour du jeudi saint 1384, rendu coupable d'un sacrilège, allait être englouti sous terre, lorsqu'il se repentit; mais l'hostie qui avait touché ses lèvres fut aussitôt couverte de sang. Sa femme, à qui on raconta ce qui s'était passé, refusa d'y croire, disant que c'était aussi peu vrai que si des roses pouvaient pousser et fleurir sur un bâton desséché et pourri. Mais aussitôt le bâton qu'elle portait, tout sec qu'il était, se couvrit de feuilles et de belles roses blanches ${ }^{2}$.

L'histoire transformée du Tannhäuser se retrouve aussi dans une légende suédoise ${ }^{3}$. Un soir un prêtre, en traversant un pont, entendit retentir les sons harmonieux d'une harpe; il regarde autour de lui et apercoit au milieu du torrent un jeune homme blond, nu juscu'ì la ceinture, un bonnet rouge sur la tête et une harpe d'or à la main. Il reconnut un esprit des eaux et dans son zèle il lui cria : "Comment en es-tu venu i jouer des airs si joycux sur ta harpe? Le bâton desséché que je porte à la main reverdira et se couvrira de fleurs, avant que tu obtiennes ton

1. Cecilia Schmidt Branco, A rosa na vida dos povos, p. 76.

2. Gebr. Grimm, Deutsche Sagen, no 356.

3. Afzelius, Volkssagen aus Schweden, deutsch von Ungewitter (1842). Thl. II, p. 327-29.

Joret. La Rose. 
pardon. " A ces mots, l'infortuné musicien jeta tout troublé sa harpe au milieu de l'eau et s'assit au bord du fleuve en pleurant amerement. Le prètre cependant poursuivit son chemin; mais a peine avait-il fait quelques pas qu'il vit son bàton se couvrir de fenilles et au milieu d'elles apparaitre les plus belles roses. Il reconnut là un avertissement du ciel et revenant en hate vers l'esprit en pleurs, il lui montra son bâton tout verdorant: "Vois, lui dit-il, mon bâton verdit et fleurit comme un rejeton du jardin des roses, ainsi doit fleurir l'espérance au cœur de tous les êtres créés. "L'ondin, consolé, saisit de nouveau sa harpe et fit entendre toute la nuit sur la rive de joyeux accents.

Les traditions scandinares parlent aussi de roses qui naissaient en signe de joie. Ainsi dans une chanson populaire suédoise ${ }^{1}$, un francé décédé apparait à sa fiancée éplorée, et, après avoir blàmé l'excès de sa douleur, il lui dit pour la consoler : "Chaque bonheur, qui t'émeut le caur, remplit ma tombe de roses parfumées. " La ballade danoise de Aage et d'Elfe nous offre la mème pensée? : "Chaque fois

1. Mohnike, Volkslieder der Schweden, ap. Schleiden, Die Rose, p. 163.

2.

Men hver en Gang du kvaeder, din Her er glad:

da er min Grav far inden omhaengt med Rosensblad.

IV. Grimm, Altdänische Volkslieder. Heidelberg, 1811, in-8, p. 73. - Svend Gruntvig, Danmarks Folkeviser $i$ Udvalg. Kjöbenhavn, $1 \varepsilon 82$, in-12, $n^{\circ} 30,19$, p. 334 . 
que tu te réjouis, dit Aage à son amie, et que ton cour s'égaye, ma tombe se remplit de fleurs de roses. "Mais les roses dont il est ici question n'ont point d'existence réelle et ne sont guère que des expressions métaphoriques.

Un attribut inconnu de l'antiquité et qu'on a, surtout en Allemagne et en Angleterre, donné à la rose vers la fin du moyen âge, est celui d'être l'emblème du secret qu'on doit à ses amis. Un poète anonyme, dont on ignore l'époque, mais qui n'était point un ancien ${ }^{1}$, quoiqu'on ait parfois joint ses vers à ceux d'Ausone, a feint que l'amour donna une rose à Harpocrate, le dieu du silence, afin de l'engager à taire les larcins de sa mère 2 . Ce serait, paraît-il, pour cette raison, encore qu'on ne la comprenne guère, que la rose serait devenue l'emblème de la discrétion. Quoi qu'il en soit, on la suspendait au-dessus de la table des festins pour signifier que tout ce qu'on y entendait devait ètre gardé secret par les

1. "Neque ipsius proverbii memoriam in antiquitate exstare, neque morem rosae e lacunari suspendendae satis antiquum esse arbitror "), dit avec raison Saumaise. Poelae latini minores, éd. Lemaire, vol. VII, p. 125, note 1.

2. Est rosa flos Veneris, cujus quo furta laterent.

Harpocrati matris dona dicavit amor.

Inde rosam mensis hospes suspendit amicis,

Convivae ut sub ea dicta tacenda sciant. 
convives. (Dit) " sous la rose ), - sub rosa, under der Rosen, - est une expression familiere aux écrivains allemands $d u \mathrm{xv}^{\mathrm{e}}$ et $d \mathrm{u} \mathrm{xv}^{\circ}$ siècle, équivalente de ( dit sous le sceau du secret ${ }^{1}$ ). Murner, entre autres, l'a employée, en l'expliquant, comme si elle était encore peu connue de son temps : "Dis-le, remarque-t-il ${ }^{2}$, sous la rose ou sous le sceau de la confession. ")

On ne se borna pas à répéter cette sentence, on l'inscrivit au plafond de la salle des festins ${ }^{3}$; on $y$ peignit également ou sculpta une rosef. On trouve aussi sur des verres à boire antiques cette autre sentence qui n'est que le développement de la premiere: "Que tout ce que nous disons ici reste sous la rose ${ }^{5}$. " Par une raison analogue, on a représenté une rose au plafond de la salle des délibérations de certains hôtels de ville; on la voit encore sur la porte

1. "Die rose bezeichnet nicht sowohl das urtheil, als die heimlichkeit und stille des gerichts. "Jacob Grimm, Deutsche Rechtsalterthümer. Göttingen, 1854, in-8, p. 941.

2. "Sprich das unter der Rosen oder bichtwys. "

3. J. G. Rosenberg, Rhodologia seu philosophico-medica generosae Rosae descriptio. Francofurti, 1631, in-12, p. 14.

4. Verisimile est morem illum profectum, ut multis in locis Germaniae in cœnaculis rosa lacunaribus supra mensae verticem affixa conspiciatur, quo quisque fit secreti tenax, ne quid temere effutiat, sed omnia reticenda meminerit : hinc proverbium quoque illud pervulgatum apud Germanos : haec sint sub rosa acta sive dicta. " Joh. Guil. Stuckins, Antiq. convival. Tiguri, 1597, lib. III, cap. 16, p. 371 .

ร.

Was wir all hier thun kosen,

Das bleibe unter der Rosen. 
de quelques vieux confessionnaux, dans les églises allemandes ${ }^{1}$.

\section{XI.}

Aux légendes de la rose se rattache le mythe slave des Rusalky. Le mot Rosalia avait pénétré chez les populations à moitié romanisées de l'ancienne Macédoine et de la Thrace. On le rencontre dans une inscription découverte par M. Heuzey à Drama, près Philippes ${ }^{2}$. Cette ville avait, de temps immémorial, été un des sanctuaires du culte de Bacchus. Deux habitants de Drama lèguent aux thiases de ce dieu - Liber Pater Tasibastenus, comme il est appelé d'un surnom local - , une somme d'argent dont le revenu devait être employé à faire chaque année sur leur tombeau une offrande de roses - rosalia et un repas funèbre. Ce monument précieux nous montre à la fois le vocable rosalia porté dans la péninsule hellénique et les offrandes de roses qu'il désigne mêlées au culte de Bacchus ${ }^{3}$.

Iais le sens que l'on trouve encore ici attribué au mot rosalia se perdit peu à peu et ce mot ne désigna bientôt plus que celui de fètes où figuraient les

1. Stieglitz, Altdeutsche Bauliunst, p. 18', ap. Schleiden, p. 191.

2. Revue archéologique, juin 1865, vol. XI, p. 451.

3. Cf. Tomaschek, Ueber Brumalia und Rosalia.(Sitzungsberichte der philos.-historischen Klasse der liais. Akademie der Wissenschaften. Vol. IX, an. 1868, p. 370.) 
roses; il devint ainsi l'équivalent de l'expression

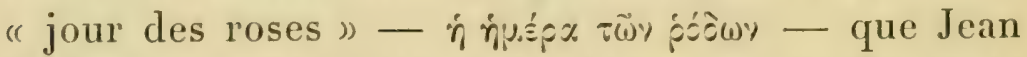
de Gaza ${ }^{1}$, au $v^{*}{ }^{e}$ siècle, a chanté comme la fìte de la joie et du printemps. C'est avec cette signification que furent adoptées les rosalia, transformées en rousalia par les tribus slaves, qui s'installerent au nord de la péninsule hellénique, et peu à peu par tous les peuples de la même race. Miklosich ${ }^{2}$ en a constaté la présence successive au nord de l'Mlbanie, chez les Roumènes, les Slovènes et les Serbes, puis chez les Russes blanes et les petits Russiens, les Ruthenes, les Sloraques et même chez les Lithuaniens.

A la fin du $x_{1}^{0}$ siècle, époque où, pour la première fois, Théodore Balsamon en a signalé l'existence, les Rousalia, par une coutume païenne, se célébraient dans les campagnes aprés la Pâque ${ }^{3}$; au commencement du xim siecle elles avaient lieu le dimanche rqui suit la Pentecôte et, d'après le témoignage de Démétrios Chomatianos, archerêque d'Achrida ${ }^{f}$, elles étaient accompagnées de réjouissances, de danses et de représentations scéniques. On voit que, si l'époque ou l'on célébrait les Rousalia semble les rattacher, comme l'a voulu Miklosich, à la fète

1. Poetae lyrici graeci, éd. Th. Bergk, vol. III. p. 1082, nos 4 et 5 .

2. "Die Rusalien. » (Sitzungsberichte der kaiser. Akademie der Wissenschaften, vol. XLVI, an. 186i, p. 389 et suir.)

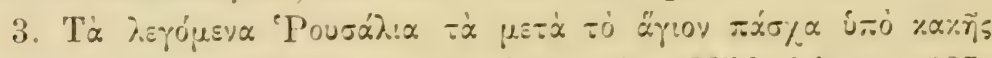

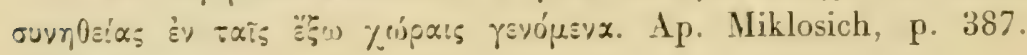
Cf. Du Cange, s. v. rosalia.

4. Miklosich, op. laud., p. 388. 
de la Pentecôte, la pascha rosarum des Italiens et des Espagnols ${ }^{1}$, elles rappelaient bien plutôt le culte de Bacchus, comme le "jour des roses » chanté par Jean de Gaza.

Nais le mot Rousalia derait subir une transformation bien plus grande encore; à une époque qu'on ne peut préciser, il a cessé de désigner des réjouissances, et, sous la forme altérće rusalky, il est devenu, chez les Russes blancs, les petits Russiens et les Tchèques, la dénomination habituelle d'êtres surnaturels, esperces d'esprits des eaux ${ }^{2}$, qu'on rencontre dans les légendes de ces peuples.

\section{XII.}

Entourée de légendes si nombreuses, regardée comme la compagne et le simbole du printemps et de l'amour, l'emblème de la personne aimée, la rose a occupé une place considérable dans la poésie du moxen âge; comme leurs précurseurs de l'antiquité, les poètes de cette éporque lui ont emprunté les comparaisons et les images les plus variées et lui sont rederables de quelques-unes de leurs plus belles descriptions.

1. «Pentecostes die, qui Hispanis aeque ut Italis rosarum pascha dicitur. " Acta sanctorum, vol. IXXVIII, p. 902, col. 2.

2. "Die Rusalky waren nach dem Glauben der alten Slaven die Götlinnen der Gewässer, namentlich der Flüsse und Bäche. s) Jos. Virgil Grohmann, Sagen-Buch son Bühmen und .Hëhren. Prag, 1863 , in-8, p. 136. 
Revêtue d'une pourpre brillante, dit un poète latin du $\mathrm{xm}^{\mathrm{e}}$ siècle $^{1}$, la rose est la gloire, la joie et l'honneur des jardins au printemps. Fleur des vierges, elle n'est pas moins chère aux jeunes gens. Elle est l'emblème de la pudeur virginale. Ornement sitôt fané des beaux jours, la rose est limage de la jeune beauté, et, chose passagère, elle nous apprend que tout ici-bas est passager. Agréable par son aspect, non moins agréable par son parfum, cette fleur l'emporte encore par le nombre de ses merveilleuses propriétés.

Cette description de la rose est presque isolée dans la littérature du moyen âge; celle de Mathieu de Tendòme, où le poète scolastique se borne à dire que "dans sa riche parure la douce rose fleurit, compagne délicieuse de l'odorat ${ }^{2}$, ne peut guère compter, et c'est à peine si on peut rapprocher des distiques de Neckam les vers du trouvèrę Beaudoin de Condé, tant ils sont rudes et dénués de poésie ${ }^{3}$.

1. Et rosa, purpureo restita rubore decenter,

Vernans est horti gloria, laetus honos.

Flos est virginibus aptus, gratusque juventae,

Grataque virginei signa pudoris habet.

Virginis est speculum, rosa vernans sed cito marcens,

Et quod sit species res fugitiva docet.

Flos gratus specie, flos gratus odore, sed eius

Virtutum celebris gloria pluris erit.

Alexandri Neckam De laudibus divinne sapientiae, v. 295302. Oper. p. 295-302).

2. Natali tumulo dulcis rosa dives amictu.

Vernat odoratus deliciosa comes.

Desiriptio loci, Bibl. nat. lat. 15155, fol. 60.

3. Li contes de la rose, r. 3ł3-51. (Les dits et contes de 
Rose est sor toutes flors la fine

Et la rousée qui l'afine

Quant nature de tans l'esclot,

Les fuelles rousée les clot

Et joint ensamble, et si les garde

Si em biauté quant on l'esgarde

Que trop li fait grace aquellir

Et trop plaist la rose a quellir

Por la biauté de la coulour.

Le plus souvent les poètes français ou allemands se sont bornés à célébrer, sans la décrire, la beauté de la rose, beauté qui leur paraît surtout admirable, quand cette fleur est couverte de rosée :

En mai la rosée que nest la flor

Que la rose est belle au point du jour!

s'écrie Guillaume de Champagne'. Et Reinbot de Durnen ${ }^{2}$ :

La rose humide de rosée resplendit comme une lumière, quand le doux éclat du soleil vient l'éclairer.

Mème sentiment d'admiration, mais plus motivé, chez Wolfram d'Eschenbach.

Beaudoin et de Jean de Condé, éd. A. Scheler. Bruxelles, 1866, in-8, vol. I, p. 145).

1. Les chansonniers de Champagne, p. 23, XVII, v, 1-2.

2. Die Rose in dem touwe ein licht auzuschauen. wann sie anget süsser sonnenschein.

Der heilige Georg, v. 402.

"Qui égale la joie que cause la vue d'une rose couverte de rosée?" dit aussi le minnesaenger Der von Wildonje (Bartsch, Deutsche Liederdichter, p. 211):

Was gelichet der wonne

dà ein rôse in touwe stàt? V. 10-11. 
Au milieu de la rosée, la rose, du fond de son calice, brille d'un nouvel éclat, où la pourpre se mêle à la blancheur de la neige ${ }^{1}$.

Il est, dit un autre minnesaenger ${ }^{2}$, une fleur nommée rose qu'on aime à porter à la main à cause de son doux parfum; elle est si belle en tout temps qu'on la regarde volontiers; mais quand elle est couverte de rosée elle est encore beaucoup plus belle.

C'est surtout dans les comparaisons que la rose a pris place dans les œuvres des poètes du moyen âge ${ }^{3}$ : "Comme une rose embaumée, il me charme le cœur, ") dit de son mari, qui lui a sauvé la vie ${ }^{4}$, la femme de Digénis Acritas, dans le poème grec du $\mathbf{x i}^{\mathrm{e}}$ siècle, composé en l'honneur de ce héros.

Plus bela que bels jors de mai... roza de mai, ploja d'abriu,

1. von dem süezen touwe diu rôse uz ir bälgelín blecket niuwen werden schîn, der beïdiu wiz ist unde rôt.

\section{Parzival, IV Buch, v. 268-71.}

2. Ein bluome ist ròse genant, si ist so schoene allen tac, Die treit maneger in der hant daz man si gerne schouivet : durch ir vil süezen smac. dò si aber wirt betouwet, sô ist si schoener vil dan ê.

Haupt's Zeitschrift, vol. VII, p. 327.

3. Cf. Max Kuttner, Das Naturgefühl der Altfranzosen, 1889, p. 13-17 et 23-26. - Heinrich Drees, Dic poetische Naturbetrachtung in den Liedern der deutschen Minnesänger, 1888, p. 31-3' . - Otto Lüning, Die Natur... in der altgermanischen und mittelhochdeutschen Epik, 1889, p. 156-159.

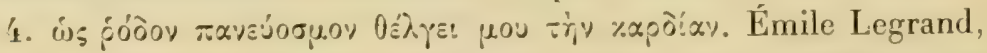
Les exploits de Basile Digénis Acritas. Paris, 1892, in-8, ch. vi, v. 108. 
chante de son amie Arnaut de Maroil ${ }^{1}$.

Et la sœur de Charlemagne, Erminjart, a

La color fresca com roza de rozier ${ }^{2}$.

Elle est plus gracieuse ne soit la rose en mai, remarque de son héroïne l'auteur de Berte ${ }^{3}$.

Il ot miex coulouré le vis

Que n'est la rose el tens demai,

dit également de Girard Gerbert de Nontreuil".

Le poète de Renaut de Montauban, voulant faire le portrait de

Clarisse la cortoise au gent cors envoisié,

la montre

Plus vermeille que rose de rosier ${ }^{5}$.

1. Bartsch, Chrestomalhie provençale, p. 94, v. 8-10.

2. Daurel et Beton, éd. Paul Meyer. Paris, 1880, in-8, v. 144.

3. Éd. Aug. Scheler. v. 1407. Le poète d'Aliscans dit de même de son héroïne :

La rose samble, en mai, la matinée, v. 2852.

4. Roman de la Violette, éd. Fr. Michel. Paris, 1834, in-8, p. 11, v. 167-68.

5. Ed. Michelant, p. 170, v. 9-10. De même le poète de la Chanson du Chesalier au cygne chante de la fille de la duchesse de Bouillon,

La pucele est plus blance que n'est flor d'aiglentier

Et assez plus vermeille que rose de rosier.

Ed. Hippeau, Paris, 187' dit plus simplement de la femme du maréchal dans Equitan:

Sa face avait couleur de rose, v. 39.

Die Lais der Marie de France, p. 42. 
Suivant l'auteur de Fierabras, Floripas, " la fille l'amiré ",

La car ot tenre et blance comme flours en esté, La face vermellete comme rose de pré ${ }^{1}$.

Et dans une estampie, publiée par M. Paul Meyer, il est question

De lèvres vermoilletes

Plus ke la rose n'est en mai 2 .

L'amie du poète du Dit de la Rose aussi

coleur a fresche et novele

Plus que n'est pas la rose en may ${ }^{3}$.

De mème la " clère façon " de l'hérö̈ne du Comte de Poitiers

plus est bele enluminée

Que ne soit rose encolorée ${ }^{4}$;

image que l'on retrouve, tant ces comparaisons se répètent comme autant de lieux communs, dans la peinture que le Roman de la V'iolette fait de la frâ̂cheur d'Euriant :
La rose qui naist en esté,
Quant s'aeuvre la matinée,
N'est pas si bien enluminée ${ }^{5}$.

1. Ed. Krœber et Servois, v. 2008-2009.

2. Recueil d'anciens textes, p. $372, \mathrm{n}^{\circ} 44$.

3. Bartsch, Langue et littérature françaises, 605, v. 17-18.

4. Éd. Fr. Michel. Paris, 1831, in-8, p. 24, v. 516-17.

5. Éd. Fr. Michel, p. 49, v. 879-81. De même le biographe de sainte Élisabeth dit qu'elle 
Chrestien de 'Troyes s'est aussi servi de la même comparaison, mais en la développant, pour montrer combien Enide surpasse toutes les autres dames:

Mais ensinc come la clere jame

Reluit desor le bis chaillo

Et la rose sor le pavo,

Aussi est Enide plus bele

Que nule dame ne pucele ${ }^{1}$.

Beaudoin de Condé a fait, dans son Conte de la Rose, comme la théorie de cette comparaison de la beauté avec la rose :

Biens et biautés en dame ensamble

Bien affierent, et moi en samble

C'on doit comparer à la rose

La biauté 2 .

(Des grands biens », ajoute-t-il dans son rude style,

Tant a biauté k'en la rose a

Sour le rosier a la rosée.

fut tant bele et colorée

come rose est la matinée.

Sainte Ysabiel l'estoire. Bibl. nat. fr. 19531, fol. 1226, b, v. 73.

1. Édit. d'Imm. Bekker, v. 2400-405. (Haupt's Zeitschrift, vol. X.) Chrestien de 'Troyes a eu aussi recours à la rose pour montrer que la largesse est la plus grande des vertus :

Meis tot ausi come la rose

Est plus que nule autre flors bele,

Quant ele neist fresche et novele :

Einsi la ou largece vient,

Desor totes vertuz se tient.

Cligès, éd. W. Fœerster, Halle, 1888, in-8, v. 208-212.

2. Dits el contes, vol. I, p. 144, v. 331-34 et 336-37. 
Mais le plus souvent, dans la description qu'ils font de la beauté de leur amie ou des héroïnes qu'ils chantent, les poètes du moyen àge, à l'imitation de leurs précurseurs de l'antiquité, - les poètes modernes suivront à leur tour leur exemple ${ }^{1}$ - nous montrent la blancheur du lis s'unissant à l'éclat de la rose. "Elle est plus belle que le lis ou la rose", dit un poète latin ${ }^{2}$ du $\mathrm{xir}^{\mathrm{e}}$ siècle.

"Elle est vermeille comme la rose et surpasse en parfum le lis de la vallée ${ }^{4}$ ", lit-on dans un autre.

Plus fresca que rosa ne lis,

remarque ì son tour Cercalmont de la dame quiil aime $^{5}$.

Roza de pascor sembla de sa color et lis de sa blançor,

chante Peire Vidal de son amie ${ }^{5}$.

L'auteur de Berte nous la peint

Vermeille come rose, blanche com flours de lis ${ }^{6}$.

L'héroïne des Enfances Ogier aussi

Com flours de lis estoit blanche et polie

1. Voyez si de son teint les roses et les lis

Dans l'hiver de la mort sont bien ensevelis,

dit entre autres Mairet, dans la Sophonisbe, acte V, scène 8, en transformant la comparaison en métaphore.

2.

pulchrior lilio vel rosa.

Carmina burana, p. 145, no II, v. 22.

3. Carmina burana, p. 200.

4. Bartsch, Chrestomathie provençale, p. 46, v. 1.

5. Chant IV, v. 61-63. Éd. Karl Bartsch, Berlin, 1857, in-8.

6. Éd. Aug. Scheler, r. 789. 
Et plus vermeille que n'est rose espanie ${ }^{1}$.

Et, suivant Marie de France, l'amie de Lanval,

Flur de lis et rose nouvele,

Quant ele pert el tens d'esté,

Trespassot (ele) de bealté 2 .

Dame, mar vi le clair vis et la fache

$\mathrm{Ou}$ rose et lis fleurissent cascun jour,

s'écrie aussi le Châtelain de Coucy ${ }^{3}$, en s'adressant à son amie. Et, pour donner une idée de la beauté de Soredamors, Chrestien de Troves dit à son tour ${ }^{4}$ :

Qui poïst la façon descrivre

Del nes bien feit et del cler vis,

Ou la rose cuevre le lis,

Einsi qu'un po le lis efface,

Por miauz anluminer la face?

Adenet aussi, voulant peindre le teint de roses et de lis de Clarmondine, n'a pas craint d'avoir recours à cette métaphore prétentieuse ${ }^{5}$ :

La rose forment se penoit

De la flour de lis honnorer

En son tres douz viaire cler.

Dans ces comparaisons le lis est remplacé parfois par l'églantier, plus souvent par la neige ou par le

1. Éd. Aug. Scheler. Bruxelles, 1874, in-8, v. 1470-71.

2. Lanval, v. 94-96: Die Lais der Marie de France, p. 90.

3. Ghanson XI, p. 48, éd. Fr. Michel. - Chanson XV, p. 70, éd. Fritz Fath, str. 4, v. 1-2.

4. Cligès, v. 816-820.

ј. Cléomadès, v. 1'33:9-81. 
cristal, ou même à la fois par l'un et l'autre. Ainsi le traître Gombaut dit de la compagne d'Aiol ' :

La dame, ele est plus blanche que n'est flor d'aiglentier, S'a la color rovente plus que rose en rosier.

Dans le Mystere d'Adam, au contraire, le diable, voulant dépeindre la nature délicate et fragile d'Éve, la compare à la rose, ainsi qu'au cristal et à la neige :

$\mathrm{Tu}$ es fieblette et tendre chose

Et es plus fresche que n'est rose ;

Tu es plus blanche que cristal,

Que neif qui chiet sor glace en val .

Les comparaisons tirées de la rose et du lis se rencontrent dans la poésie anglaise, comme dans la nôtre; c'est ainsi que Chaucer dit de Cléopâtre qu'elle

1. Aiol, chanson de geste, publiée par Jacques Normand et Gustave Raynaud. Paris, 1877, in-8, v. 6696-97.

2. Adam, mystère du $\mathrm{xi}^{\mathrm{e}}$ siècle, texte critique accompagné d'une traduction par Léon Palustre. Paris, 1877, in-4, p. 40. Parfois nos poètes n'ont établi de comparaison qu'entre la blancheur du teint et le lis, auquel se joint souvent l'aubépine, ou qui est remplacé par la neige ou la glace, le cristal, l'argent et l'ivoire.

gola e peitrina

Blanca com neus e flors d'espina.

Arnaut de Mareuil (Mahn, Troubadours, I, 153.)

ele ot plus blanc col et poitrine

que flour de lis, ne flour d'espine.

Roman de la Violelte, v. 888-89.

Neis la gorge contreval

Sanbloit de glace ou de cristal.

Guillaume au Faucon, v. 105, etc., etc. 
était " belle comme est la rose en mai»", comparaison dont il faut rapprocher la locution anglaise "frais comme une rose en juin " ". Chaucer dit même du soleil à son lever qu'il est "vermeil comme une rose $^{3} »$. Le mème poète, pour peindre la beauté d'une des héroïnes des Canterbury tales, en montre le teint rivalisant avec le doux éclat de ces fleurs ${ }^{4}$ :

Émilie, qui était plus belle à voir que ne l'est le lis sur sa verte tige et plus fraîche que le mois de mai avec ses fleurs nouvelles (car son teint le disputait à la couleur de la rose et je ne sais lequel des deux était le plus admirable).

La rose, cette "fleur favorite " des minnesaen$g^{\prime} \mathrm{r}^{5}$, joue dans leur poésie un rôle encore plus grand que dans celle des minestrels, le même que chez les troubadours et les trouvères; mais les com-

1. And she was faire, as is the rose in May.

The legend of Goode Women, I, v. 34.

2. Hazlitt, English Proserbs and proverbial Phrases. London, $1882,2^{\text {e éd., p. } 68 .}$

$3 . \quad T h e$ sonne, that roos as rede as rose.

The legend of goode Women. Prol. v. 112. Cf. William Hacckel, Das Sprichwort bei Chaucer, p. 57. (Erlanger Beiträge zur englischen Philologie, fasc. VIII, 1890.)

4. Emily that fairer was to seen

Than is the lily, on her stalke green,

And fresher than the May with flowers new,

(For with the rosè colour strove her hue,

I n'ot which was the finer of them two).

The Knight's tales, éd. John Saunders. London, 1889, in-12, p. 177.

5. Otto Lüning, op. Laud.. p. 156. Cf. Karl Finsterwalder, Die JoRet. La Rose. 
paraisons qu'ils lui ont empruntées ont parfois un caractère plus sentimental ou plus tendre.

Ses joues de neige, dit le poète de Wolfdietrich ${ }^{1}$, brillaient comme la rose vermeille, quand elle va s'entr'ouvrir.

Amoureusement colorés de l'éclat vermeil de la rose, chante Konrad von Kilchberc ${ }^{2}$, fleurissent de cette belle les joues, la bouche et le menton.

D'amour, lit-on dans les Nibelungen ${ }^{3}$, le clair visage de Kriemhild devint vermeil comme une rose.

Rien ne me paraît aussi bon, ni aussi digne d'être loué, fait dire un minnesaenger à sa dame ${ }^{4}$, que la rose brillante et l'amour de mon mari.

La vue des dames réjouit plus le cœur, chante un autre ${ }^{5}$, que celle d'une rose encore humide de rosée.

Rose, eines der drei Wahrzeichen deutscher Dichtung, 1882, in-8.

1. Ir wiziu wangel lủhten an derselben stàt reht als diu liehte rôse swenn si erste ûf gât.

Wolfdietrich, B. v. 576-77.

2. Minneclich gevar in ròsen rœe

Blüet der schœnen wengel, munt, ir kinne.

Karl Bartsch, Deutsche Liederdichter des zwölften bis vierzehnten Jahrunderts. Stuttgart, 1870, in-8, p. 265, v. 17-18.

3. Wart ir lieht antlitze vor liebe rôsenrôt.

$$
\text { Nibelungenlied, v. } 340 .
$$

4. Mich dunket niht sô guotes noch sô lobesam,

Sô diu liehte rôse unt diu minne mìnes man.

Karl Bartsch, Deutsche Liederdichter, p. 289, v. 39-40.

5. Vrouwen vröuwent verre baz danne ein rose in touwe naz.

Minnesinger, Adesp. I, v. 2. 
La chasteté, remarque Reinmar von Zweter ${ }^{1}$, est la parure de la femme, comme la rose celle de l'épine.

Comme nos anciens poètes, les minnesaenger ont aussi désigné par la métaphore de la rose et du lis la blancheur et l'éclat du visage, ainsi que la rougeur qui le couvre parfois. Walther von der Vogelweide $^{2}$, parlant d'une jeune fille à laquelle il vient d'offrir une couronne de fleurs: "Ses joues, dit-il, devinrent rouges, semblables à la rose qui est près du lis. " Et voulant dépeindre la beauté du teint de son amie, il dit qu'on voit briller sur ses joues " ici l'éclat de la rose, là la blancheur du lis $n^{3}$. Il a mème inventé le mot liljeróserarwe "couleur de lis et de rose $~^{4}$, pour désigner l'éclat blane et vermeil d'un beau teint. Un autre poète, Der Durner, substituant la neige aux lis, parle des "roses vermeilles répandues sur la blanche neige des joues de son amie $)^{5}$.

Mais l'union des lis et des roses est pour les minnesaenger la marque et l'emblème de la beauté suprême du corps comme de l'âme.

1. Ap. H. Drees, Die poetische Naturbetrachtung, p. 31.

2.

Ir wangen wurden rôt

Same diu rôse, dà si bì der liljen stàt.

Éd. Pfeiffer, VI, v. 19-20.

3.

Hie roeselocht, dort liljenvar.

Éd. Pfeiffer, XVII, v。24.

4. Éd. Pfeiffer, LXXVI, v. 19.

5. rôsen ròt geströit ûf wîzen snê sint der lieben under ougen.

K. Bartsch, Deutsche Liederdichter, p. 279, XC, v. 6-7. 
Je voudrais qu'il me fût possible, s'écrie Walther von der Vogelweide dans les vers où il implore les secours de Frédéric II ${ }^{1}$, de me chauffer à mon propre foyer. Ah! comme je chanterais alors des oiseaux, de la bruyère et des fleurs, ainsi que j’en chantais jadis; à la belle dame qui m’en prierait je ferais briller sur ses joues les roses et les lis.

A notre avis, dit-il ailleurs ${ }^{2}$, la constance est la couronne de la femme; quand la joie de l'âme sunit (chez elle) à la pureté, on dirait que le lis se marie à la rose.

La rose entre aussi, quoique assez rarement, dans les comparaisons des poètes italiens et espagnols. "Péricon, raconte Boccace ${ }^{3}$, avait un frère beau et frais comme une rose. " Dans une romance espagnole, une " gentille dame ) dit que son teint a les nuances de la rose sur le rosier $)^{4}$. Mais le plus souvent, dans ces comparaisons, à la rose se joint le

1. Gerne wolte ich, möhte ez sîn, bì eigem fiure erwarmen. Zahî wie 'ch dann sunge von den vogellinen, von der heide und von den bluomen, als ich wilent sanc. Swelch schœne wîp mír danne gæbe ir habedanc, der lieze ich liljen unde rôsen ùz ir wängel schînen.

Éd. Franz Pfeiffer, CXLIX, v. 3-7.

2. Wir wellen, daz diu staetekeit iu guoten wiben gar ein kròne sì

kumt iu mit zühten sìn gemeit, sô stêt diu lilje wol der rôsen bî.

Éd. Franz Pfeiffer, XVI, v. 17-20.

3. Aveva Pericone un fratello bello et fresco, come una rosa. Decamerone, Giornata II, novella 7.

千́.

La color tengo mezclada como rosa en el rosel.

Primavera de romances, $n^{\circ} 145$, vol. II, p. 65 . 
lis. Bonagiunta Urbiciani chante de sa dame que " son visage esmeré et brillant porte des lis et des roses $)^{1}$. Dante et, à son exemple, Pétrarque ont remplacé les lis par la neige :

Ah! le doux sourire, s'écrie le premier ${ }^{2}$, à travers lequel perce la blanche neige au milieu des roses toujours vermeilles.

Je verrais, dit le second en parlant de Laure ${ }^{3}$, apparaitre sous la neige les roses vermeilles de son teint.

Et ailleurs il vante " les roses de son visage éparses sous les doux flocons d'une neige vivante »'

Boccace, au contraire, est resté fidèle à la comparaison empruntée aux lis :

Ses joues, dit-il, dans la Théséide, de la belle Émilie ${ }^{5}$,

1. Gigli e rose novelle Vostro viso ha portate

Si smerato e lucente,

Vincenzio Nannucci, Manuale della letteratura del primo secolo della lingua italiana. Firenze, 1874, in-12, vol. I, p. 149.

2.

Ome! lo dolce riso

Per lo qual si vedea la bianca neve

Fra le rose vermiglie d'ogni tempo.

Canzone 233. Il canzoniere di Dante Alighieri publ. da Pietro Fraticelli... Firenze, 1873, in-12.

3. Le rose vermigli infra la neve

Mover (vedrei). In vita di Laura, son. CI, v. 9-10.

4. rose sparse in dolce falda

Di viva neve. Ibid., CXIV, v. 5-6.

5. Le guance sue... eran delicate e graziose

Bianche e vermiglie, non d'altra misura

Che in tra gigli le vermiglie rose.

Canto XII, str. 58, v. 1-4. 
tendres et charmantes, étaient blanches et vermeilles, semblables aux roses empourprées au milieu des lis.

Mais dans la romance espagnole de Guiomar, c'est le cristal qui a remplacé les lis ou la neige :

Guiomar, dit le poète anonyme ${ }^{1}$, sort du bain, vermeille comme une rose, son visage brille comme le cristal.

Par leur couleur et leur éclat, la bouche et les lèvres surtout ressemblent à une rose, on ne doit pas ètre surpris aussi de les trouver comparés à cette fleur :

Quand sa voix mélodieuse disait rose, chante Digénis de sa femme, dans un poème grec du $\mathrm{xi}^{\mathrm{e}}$ siecle $^{2}$, je croyais quelle avait une rose sur les levres, tant elles ressemblaient vraiment à une rose nouvellement fleurie.

Les minnesaenger surtout se sont servis de cette gracieuse comparaison.

Sa bouche ressemble à une rose vermeille, s'écrie l'un d'eux, le Durner ${ }^{3}$.

1. Ya se sale Guiomar de los baños de bañar colorada como la rosa su rostro como cristal.

Primavera de romances, no 178, v. 1-4, vol. II, p. 290.

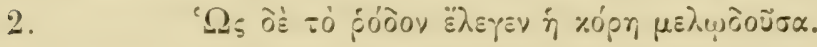

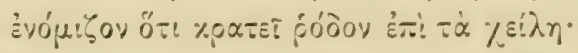

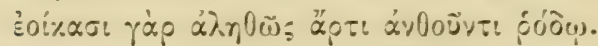

Émile Legrand, Les exploits de Basile Digénis Acritas. Paris, 1892, chap. vi, v. 109-111.

3.

Als ein rôse rôt ist ir der munt.

Bartsch, Deutsche Liederdichter, p. 280, nº XC, v. 30 . 
Vos lèvres, dit un autre ${ }^{1}$, ressemblent à la rose humide de la rosée de mai.

Comme une rose brillait en tout temps sa bouche, chante de la jeune Herrât l'auteur de la Rabenschlacht ${ }^{2}$.

Mon amie, dit un vieux lied $^{3}$, a une bouche si vermeille qu'elle brille comme une rose, quand elle est dans toute sa fleur.

L'éclat du soleil s'éteint à mes yeux, chante non sans afféterie Kraft von Toggenburc ${ }^{4}$, quand je vois les roses qui s'épanouissent sur une bouche vermeille, comme les roses sous la rosée de mai.

Une "bouche qui brille comme une rose » ou qui est " mille fois plus vermeille qu'une rose $)^{*}$, des

1. Als diu rôse in meien touwe ist iuwer lip.

$$
\text { Minnesinger, IV, } 2 .
$$

2. Reht alsam ein rôse brann alle zît ir munt.

Deutsches Heldenbuch, II Teil, éd. E. Martin, str. 121.

3. Mein Lieb, das treit ein Mund so rot

Der prinnet als die Rosen,

Wenn sie in rechter Blüthe staht.

Görres, Volkslieder, p. 14.

'́. diu liehte sunne

erlischet in den ougen min, swanne ich den ròsen schouwe, der bluet uz einem mündel rot, sam die ròsen uz des meien touwe.

Karl Bartsch, Die Schweizer Minnesänger. Frauenfeld, 1887 , in-8, p. $75, x$, v. 28-32.

5 .
Ir ist der munt tủsentstunt

rœter danne ein roselin.

Der Taler, ap. Bartsch, Ibid. p. 67, v. 37-39. 
" joues roses " ou " couleur de rose $)^{1}$ sont des expressions habituelles chez les anciens poètes allemands.

Ha! s'écrie Rost Kilchherre", bouche qui brille comme les roses, quand voudras-tu finir mon long chagrin?

De sa bouche couleur de rose ne sortait jamais une parole qui ne fût bonne,

lit-on dans le Lanzelot allemand ${ }^{3}$.

Pour désigner la bouche et les lèvres de son amie, Wernher von Ilònberc va jusqu’à dire qu'elle « a mangé une rose vermeille $)^{4}$.

Mêmes images chez les poètes italiens. Boccace, dans l'Ameto, dit de la bouche d'une des compagnes de la belle Lia qu'elle "ressemble à une rose vermeille $)^{5}$. Pour Pétrarque, la bouche même est une rose et les dents des perles:

Des perles et des roses vermeilles, où la douleur recueillie formait des sons vifs et charmants ${ }^{6}$.

1. “ Rôsenwengel ». - « Wangen so gar rosenvar ». Drees, Die Naturbetrachtung, p. 31 .

2. Hey, rœselehter munt,

wanne wilt erfrischen mich?

K. Bartsch, Die Schweizer Minnesünger, p. 393, II, v. 1-2.

3. Von ir rosenvarwem munde kom nie wort, ezn waere guot. v. 4026 .

4. Sô hàt si einen rôten rôsen gezzen.

Bartsch, Deutsche Liederdichter, p. 267, v. 7.

5. La bocca... somigliava vermiglia rosa, p. 39.

6. Perle e rose vermiglie, ove l'accolto

Dolor formava ardenti voci e belle.

In vita di Laura. Son. GXXIV, v. 13-14. 
C'est surtout quand elle sourit que la bouche ressemble à une rose épanouie, aussi un minnesaenger, Heinrich von Trostberc, a-t-il comparé le sourire à cette fleur :

Vermeil comme la rose est le sourire de ma bien-aimée dame ${ }^{1}$.

La bruyère est dépouillée de ses fleurs, s'écrie un autre minnesaenger ${ }^{2}$; mais je vois encore des roses lorsque sourit sa bouche vermeille.

Un troisième parle des roses qui tombent des lèvres vermeilles de sa dame ${ }^{3}$.

La bouche de mon amie, chante Nithard ", peut répandre des roses, on le voit à son sourire.

Quand il sourit, dit également un proverbe néerlandais ${ }^{5}$, il neige des roses.

1. Rôsenrôt ist ir daz lachen der vil lieben frouwen mîn.

K. Bartsch, Die Schweizer Minnesünger, p. 273, III, v. 15. 2.

Sô diu heide bar der bluomen lit, dannoch sihe ich rôsen,

swann ir rôtez mündel lachet.

Winli, ap. Ki. Bartsch, Die Schweizer Hinnesänger, p. 156, IV, v. 23-25.

3. Ròsen de vallen an mir frôwen rôder lêr, dàr van wil ic singen.

Wizlàw, ap. K. Bartsch, Deutsche Liederdichter, p. 264, v. 76-78.

4. Der trủten munde künnen rôsen giezen,

Siht mans durch ir lachen lüsteliche ströun.

Minnesinger, vol. III, p. 187, v. 2.

5. "Als hy lacht, dan sneuwt het rosen. " Tuinman, vol. I, p. 306, ap. J. Grimm, Deutsche Wythologie, p. 1054. 
Une chanson calabraise parle d'une "bouche qui jette des roses et des fleurs $)^{1}$.

Quand vous portez la main à votre front, dit aussi une chanson populaire de la Sicile ${ }^{2}$, vous la remplissez de roses et de fleurs.

Dans la version allemande du roman d'Apollonius de Tyr se trouve l'histoire d'un mendiant qui, ayant obtenu l'amour d'une belle reine, sau'e de joie et son sourire répand tant de roses que monts et vaux, arbres et prairies en sont couverts".

Un chant grec parle aussi d'une jeune fille "qui, quand elle sourit, fait pleuroir des roses dans son tablier $)^{4}$. Dans un conte sicilien il est question d'un enchanteur qui confère à une jeune fille vertueuse le don de laisser, chaque fois qu'elle parle, tomber des roses de sa bouche ${ }^{5}$. Cette fiction se retrouve dans le Pentamerone, mais lia aux roses se joignent les

1.

Boccazza che getta rose et fiori.

Nic. Tommaseo e Bellini, Dizionario della lingua italiana.

Boma-Napoli, 1872, in-4, s. v. rosa.

2. Quando mettete la mano alla fronte

Limpiete piena di rose e di fiori.

Nic. Tommaseo e Bellini, Ibid.

$3 . \quad$ Das sach der rosenlachender man,

der lachet, das es voll rosen was,

perg und tal, laub und gras.

Altdeutsche Wülder, vol. I, p. 72, ap. Uhland, vol. III, p. 514, note 183.

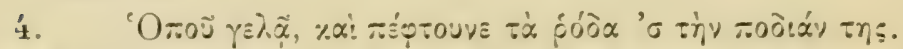

Fauriel, Chants populaires de la Grèce moderne, vol. II, p. $382, n^{\circ}$ III, v. 31 .

5. L. Gonzenbach, Sicilianische Mürchen, vol. I, p. 228, no 34 . 
jasmins ${ }^{1}$. Dans un conte grec il est question d'une " jeune fille qui rit des roses et pleure des perles »². Un conte polonais connaît également la même fiction ${ }^{3}$. Il faut en rapprocher l'expression "parler des roses ", employée, nous l'avons vu", dans l'ancienne poésie grecque, avec le sens de "dire des choses agréables ". On la rencontre dans le Triomphe de l'hiver, pièce de Gil Vicente, du commencement du xvi ${ }^{\circ}$ siècle :

Avec toutes tes querelles, tes paroles sont toutes autant de roses, et tu dis de si douces choses que jai plaisir à t'écouter ${ }^{5}$.

Après avoir comparé à la rose la bouche et son sourire, on fit un pas de plus, on compara à cette fleur gracieuse les baisers que donne ou recoit une bouche aimée. Ln minnesaenger ${ }^{6}$, dans un de ses plus beaux chants, nous présente la rose sous ce

1. Ap. Schleiden, Die Rose, p. 168.

2. J. G. von Hahn, Griechische und albanesische Märchen, gesammelt, übersetzt und erlüutert. Leipzig, 1864, in-12, vol. I, p. $193, \mathrm{n}^{\circ} 28$.

3. Dans un conte suédois du Småland, "Petite Rose » (Lilla Rosa) reçoit le don de laisser tomber de sa bouche un anneau d'or chaque fois qu'elle rit. Schwedische Volkssagen und Märchen. Deutsch von Carl. Oberleitner. Wien, 1848, in-8, p. 151.

4. Voir première partie, chap. III, p. 86 .

5. Con todo tu querellar. y dices tan buenas cosas cuanto hablas todo es rosas, que huelgo de te escuchar.

Tealro español anterior a Lope de Vega. Hamburgo, 1832 , in-8, p. $9^{\prime}$.

6. Minnesinger, vol. I, p. $21 a$, str. 4 . 
triple sens métaphorique et nous montre comment on est passé du premier au troisième.

Fleurs, feuillage, tréfle, montagne et vallée, ainsi que le charme si doux du mois de mai, sont pâles en comparaison des roses que porte ma dame; le brillant soleil s'obscurcit à mes yeux, quand je vois les roses qui s’épanouissent sur sa bouche vermeille, semblables à celles que recouvre la rosée printanière. Qui y cueillit jamais des roses, ajoute-t-il ${ }^{1}$, peut bien se réjouir du fond du cœur; quelques roses que $\mathrm{j}$ aie vues, jamais je $\mathrm{n}$ 'en vis d'aussi plaisantes; quelque belles que soient celles qu'on peut cueillir dans la vallée, sa bouche vermeille en sourit en un instant un millier daussi charmantes.

Le roi Wenceslas de Bohème parle d'une manière non moins poétique des baisers, cueillis sur la bouche de son amie :

Comme une rose qui entrouvre son calice, quand elle boit la douce rosée, elle moffrit ses lèvres tendres et vermeilles $\%$.

Si sa bouche, vermeille comme la rose, voulait me

1. Swer dà ròsen ie gebrach der mac wol im hòchgemüete lôsen;

swaz ich rosen ie gesach dà gesach ich nie sô lòsen ròsen.

swaz man der brichet in dem tal, dà sie die schœenen machet, sà zehant ir ròter munt einen tủsent stunt sò schœenen lachet.

2. Recht als ein rose diu sich ùz ir klòsen làt.

swenn si des süezen touwes gert,

sus bòt si mir ir zuckersüezen ròten munt.

Karl Bartsch, Deutsche Liederdichter, p. 261, v. 21-23. 
donner un baiser, joublierais sur l'heure mes soucis, chante à son tour Kuonrât von Kilchberc ${ }^{1}$.

On rencontre les mèmes comparaisons chez les poètes néerlandais :

Mon amie est mon été, mon amie est ma joie, dit l'un d'eux ${ }^{2}$, et toutes les roses fleurissent, aussi souvent qu'elle me donne un baiser.

Ce ne sont pas là les seules comparaisons que les poètes du moyen âge ont empruntées à la rose, ils s'en sont encore servis comme de symbole et d'emblème pour désigner ce qui était beau et précieux; n'était-elle pas pour eux "la plus noble des fleurs ", digne d'ètre " prisée également de l'empereur et de l'impératrice $)^{3}$, comme s'exprime un minnesaenger? Il était naturel dès lor's qu'on lui comparât ce qu'on estimait ou ce qu'on regardait comme ayant une valeur particulière.

C'est des plus belles la rose, dit un vieux poète ${ }^{4}$.

1. Wolde ir roselehter munt, sorge mich verzihen mir ein küssen lihen, wolt ich så ze stunt.

2. Mijn liefken is mijn somer,

Karl Bartsch, Deutsche Liederdichter, p. 266, v. 62-65.

mijn liefken is mijn lust,

en al de rosen bloejen

so dicmal si mi cust.

Altniederlündische Lieder, p. 73, $\mathrm{n}^{\circ}$ 7, v.

3. Keiser und keiserinne ist diu ròse ein edel werdiu bluome.

Der jüngere Titurel, éd. Boisserée, ch. III, v. 84-85.

4. Man. 6572, fol. 80, ap. Lacurne de Sainte-Palaye, s. v. rose. 
Fleur de chevalerie et vertu esprouvée!

Roze de hardement, car plus qu'achier temprée! s'écrie l'armée acclamant Baudoin qui l'a sauvée'.

Mais li Francois, s'on dire l'ose, Sont de tous cavaliers la rose,

dira Philippe Mouskiés ${ }^{2}$.

De mime Wolfram d'Eschenbach loue Gamuret d'être "pour ses amis une douce fleur de rose " 3 . "Elle brille entre toutes les femmes, comme la rose entre toutes les fleur's s, dit de la fille du roi païen Marchorel l'un des personnages d'Ortnit'. Dietrich, déplorant la perte de Jubart de Latran, l'appelle " une rose de fidélité " ${ }^{5}$, et Wernher n'a pas cru pouvoir donner une plus haute idée de Capharnaüm qu'en disant qu'elle " brille comme une rose au-dessus des autres villes ${ }^{\circ}$.

A cause de la couleur écarlate de certaines espèces de roses, un trouvère n'a pas hésité à leur comparer des murs couverts de sang :

Et li mur sont vermeil comme rose esmerée ${ }^{\text {. }}$

1. Li romans de Baudoin de Sebourc, chant VIII, v. 405406. Valenciennes, 1841, in-8, rol. I, p. 214.

2. Lacurne de Sainte-Palaye, s. v. rose.

$3 . \quad$ Den friunden ein süeziu rosenblüete.

$$
\text { Titurel, éd. Pfeiffer, str. 159, v. } 4 \text {. }
$$

'. Siu lûht uz allen wîben, reht als diu ròse tuot. Str. 15, 3.

5. Dietrichs Flucht, v. 9983, ap. Lüning, p. 157.

6. Siu lúhte sam diu rôse ob anderen steten. Ap. Otto Lüning, Die Natur, p. 157.

7. Gui de Bourgogne, v. 4296. 
Et dans la Divine Comédie, Dante, par une autre association d'idées, compare à une rose immense, éternelle, dont les feuilles s'épanouissent et se superposent

\section{rosa sempiterna \\ Che si dilata, rigrada ${ }^{1}$,}

les bienheureux, - "milice sainte, que le Christ épousa avec son sang ${ }^{2}{ }^{2}$, - contemplant, rangés autour du Créateur, la lumière divine.

Enfin, à l'imitation des poètes de l'antiquité, les poètes des derniers temps du moyen âge - les écrivains modernes suivront cet exemple - ont comparé les feux de l'aurore à l'éclat d'une rose vermeille. "Je vois, dit Pétrarque", descendre du ciel l'Aurore au front de roses et aux cheveux d'or ». "L'Aurore nous apparait arec les couleurs de la rose la plus fraîche "), dit de mème Boccace dans l'Ametó. "Le soleil, chante à son tour Chaucer", se levait vermeil comme une rose. »).

Le contraste entre la beauté et le parfum de la rose et la rudesse des épines qui l'entourent devait

1. Il Paradiso, canto XXX, str. 42, v. 126-27.

2.

la milizia santa,

Che nel suo sangue Cristo fece sposa.

Il Paradiso, canto XXXI, str. 1, v. 2-3.

3. io veggio dal ciel scender l'Aurora Colla fronte di rose e co' crin d'oro.

In morte di Laura. Sonetto XXIII.

4. "Nell' aurora freschissima rosa si manifesta ), p. 37.

5. The sonne, that roos as rede as rose. The leg. of good women. Prol., v. 112. Cf. p. 369. 
donner naissance aux comparaisons les plus diverses et à de nombreux proverbes. Pour les écrivains mystiques la rose fut l'image de la sainteté, les épines, l'emblème du péché, de l'idolâtrie ou de l'incrédulité; c'est ainsi qu'un minnesaenger appelle la Vierge " une vraie rose sans épines, bien qu'issue de la race juive, c'est-ì-dire du milieu des épines " ${ }^{1}$.

Fleur née sans épines du milieu des épines, chante Adam de Saint-Victor ${ }^{2}$; fleur, gloire des épines, nous, épines, nous sommes ensanglantés par l'épine du péché, dont tu ne sais rien.

Hilarion, remarque de même son historien ${ }^{3}$, qui eut des parents idolâtres, fleurit, comme l'on dit, semblable à une rose au milieu des épines.

Cette image devait se développer et s'appliquer aux choses les plus diverses ${ }^{4}$. C'est ainsi que le poète du Dit de la Rose compare les médisants qui

1. Von judischem kunne also von dorne geborn, ein reht rosa ane dorn.

Legende des Pilatus, v. 112-114. (Wilh. Wackernagel, Deutches Lesebuch, Basel, $4^{\mathrm{e}}$ éd. 1871, p. 266.)

2. Flos de spinis spina carens,

Spina sumus cruentati, Flos spineti gloria.

Nos spinetum, nos peccati Sed tu spina nescia.

In Assumptione, str. 2.

3. Girolamo, ap. Nic. Tommaseo, Dizionario della lingua italiana, s. v. rosa.

4. "L'espine, dit Pierius Valerian, a environné la rose comme représentant le miroir de la vie humaine, en laquelle ce qui est doux et gracieux aigrit et devient amer, estant atteint des aguillons de sang et fascherie. "Commentaires hiéroglyphiques ou images des choses. Lyon, 1576, in-fol., p. 495. 
poursuivent l'amie du poète aux épines qui entourent la rose ${ }^{1}$.

Aussi comme la rose nest

Entre poingnanz espines, est

Cele qui de mon cuer est dame

Entre les mesdisanz, qui blasme

Li porchacent a lor pooir.

C'est dans le même sens que Pétrarque appelle Laure une “blanche rose née entre de dures épines $)^{2}$.

Un de nos vieux poètes ${ }^{3}$, voulant montrer que le plaisir et la douleur sont souvent inséparables, s'écrie :

Li rosiers est poingnauz et s'est souef la rose.

Et la locution “ il n'y a pas de roses sans épines » ", qui exprime la même pensée, se rencontre sous des formes diverses dans toutes les langues. Tel est le proverbe italien $^{5}$ :

1. Bartsch, La langue et la littérature françaises, col. 603, v. 15-19.

2.

Candida rosa nata tra dure spine!

In vita di Laura, son. CGVIII, v. 5.

3. Rustebuef, Li dis des Jacobins (Bartsch, La langue et la littérature françaises, col. 450, v.9).

4. “Nulle rose sans espines. ) Gabr. Meurier, Trésor des sentences, ap. Le Roux de Lincy, Le livre des proverbes francais, vol. I, p. 84.

“Non v'è rosa senza spina. " Tommaseo, Dizionario, s. v. rosa.

"Keine Rose ist ohne Dornen. » Karl Simrock, Die deutschen Sprichwörler. Frankfurt a. M., in-12, $4^{\mathrm{e} e ́ d ., ~ p . ~} 461$.

5. "Ne rosa senza spine, nè amore senza impacci. ) Tommaseo, Ibid. 
Point de roses sans épines, ni d’amour sans ennuis.

Et le proverbe allemand ${ }^{1}$ :

Qui veut la rose ne doit pas redouter l'épine.

Qui peut cueillir des roses sans se piquer à leurs épines, remarque le rabbin Dom Sem Tob².

Cueillir les roses et laisser les épines, dit à son tour Boccace ${ }^{3}$.

De l'épine naît la rose, de la rose l'épine ${ }^{4}$;

Entre les épines naissent aussi les roses ${ }^{5}$; proverbes italiens qui signifient que la joie peut succéder ou ètre mèlée à la tristesse et la tristesse à la joie.

Pour naître sur une épine, la rose certes n'en vaut pas moins - ou n'en sent pas moins bon ${ }^{6}$.

Si elle est rose, elle fleurira; si elle est épine, elle piquera ${ }^{7}$.

1. "Wer die Rose will, darf den Dorn nicht scheuen ». K. Simrock, Ibid.

2.

Quien puede cojer rosas

Syn toccar sus espinas.

Proverbios morales, $\mathrm{n}^{\circ} 110$. (Poetas castellanos anteriores al xv siglo. Madrid, 1864, in-8, p. 334.)

3. Cogliete le rose e lasciate le spine stare.

Decamerone, Giornata quinta, novella x, p. 108.

4. Di spina nasce rosa, da rosa spina.

5. Anco tra le spine nascono le rose.

Nic. Tommaseo, Dizionario, s. v. rosa.

6. Por nascer el spino Non val la rosa cierto menos.

Por nacer en spino La rosa no siente menos.

Proverbios morales, no 47.

7. S'ell' è rosa, fiorirà; s'ell' è spina, pungerà.

Nic. Tommaseo, Dizionario, s. v. rosa. 
autres sentences dont il est inutile d'expliquer le sens.

Mais la rose a donné lieu à bien d'autres expressions proverbiales. Ainsi :

Cueille la rose pendant qu'elle est en fleur ${ }^{1}$.

Qui ne cueille point de roses en été, n’en cueille pas non plus en hiver ${ }^{2}$.

Le temps produit les roses, non le rosier ${ }^{3}$.

Et à cause de sa courte durée :

Périssable comme la rose, - Bien vite effeuillée est la rose ${ }^{4}$.

Il n'est point de rose si belle qui ne finisse par se faner ${ }^{5}$.

Toute vermeille qu'est la rose, elle finit par pâlir ${ }^{6}$.

Voulant montrer que le voisinage de ce qui est laid ne diminue en rien la vraie beauté, Ovide avait dit que "souvent l'ortie croìt près de la rose " ${ }^{\top}$; un

1. “Pflück die Rose, wenn sie blüht. » K. Simrock, Ibid. Proverbe tchèque.

2.

Wer sie (rosen) nicht im sommer bricht,

Der brichts'im winter nicht.

Franz M. Böhme. Altdeutsches Liederbuch, no 137, v. 3-4.

3. "Zeit bringt Rosen, nicht der Stock. " Sebastian Franck.

4. "Vergänglich wie die Rose », - " Rosen sind bald verblättert. ) K. Simrock. Ibid.

5. "Keine Rose so schön, dass sie nicht auch endlich welkte. " K. Simrock. Ibid.

6. Nomis, Oukraïski prikazki, etc. S. Petersburg, 1864, in-8, p. 10 '

7. Voir première partie, chap. III, p. 87. 
trouvère s'est emparé de cette comparaison et l'a développée dans les vers suivants ${ }^{1}$ :

Les roses selonc les orties Ne lor flairor ne lor bonté; Ne perdent mie lor biauté, J'ai veu delez l'ortiier Florir et croistre lou rosier.

Un proverbe de l'Ukraine ${ }^{2}$, preuve que cette métaphore a pénétré jusque chez les peuples slaves, dit de même :

La rose reste toujours rose même entre les orties.

Il faut en rapprocher le proverbe de l'Esthonie, d'un sens tout différent ${ }^{3}$ :

Ce n'est pas à cause de toi que les orties porteront des roses.

L'habitude qu'on prit à la fin du moyen âge de répandre des feuilles de roses sur l'eau du bain donna naissance à l'expression proverbiale " se baigner au milieu des roses », synonyme d'être à son aise, éprouver quelque chose de doux et d'agréable.

Le roys Lois ${ }^{4}$, dit Guillaume Chastelain, se baignoit en roses, ce luy sembloit, d'oyr cette bone aventure.

Et la ballade allemande du chef de bandes Bur-

1. La Bible Guyot, ap. Bartsch, Chrestomathie de l'ancien français, col. 212 , v. 11-15.

2. Nomis, Op.laud., p. 104

3. Esthnische Sprichwörter, gesammelt von Dr J. Altmann, ap. Schleiden, Die Rose, p. 23.

4. Louis XI. Chroniques des ducs de Bourgogne, vol. III, p. 189. 
khardt Münch nous le montre parcourant le champ de bataille, où avec le dauphin, le futur Louis XI, il avait battu près de Bâle les Confédérés, et disant à un de ses compagnons grièvement blessé : "Vois, aujourd'hui nous nous baignons au milieu des roses $)^{1}$.

C'est dans un sens analogue que l'auteur de L'amant devenu cordelier emploie le mot rose, au vers 720 :

Car tels douleurs ne sont que roses,

et qu'un proverbe allemand dit qu' " on ne peut toujours marcher sur des roses $)^{2}$.

1.

schau heut zu Tage hiebei,

Da baden wir in Rosen frei.

Des Knaben Wunderhorn, vol. I, p. 498.

2. Man kann nicht immer auf Rosen gehen.

K. Simrock, op. laud., p. 461. 


\section{CHAPITRE V.}

\section{LA ROSE DANS LES USAGES DE LA VIE, DANS LE CUlte ET DANS L'ART.}

L'extension de la culture de la rose, ainsi que la place considérable qu'elle a prise dans la poésie, pendant les derniers siècles du moyen âge, étaient la conséquence de l'emploi de plus en plus grand qu'on fit à cette époque de cette fleur et de ses produits. De même que chez les anciens, la rose devint alors l'ornement habituel des fètes profanes et religieuses, en mème temps qu'elle figurait dans les usages les plus ordinaires de la vie.

\section{I.}

La proscription dont avait été l'objet cette fleur naguère si recherchée prit bien vite fin, quand on n'eut plus le spectacle de l'abus qui en avait été fait ou des excès dont elle avait été l'accompagnement ordinaire, et elle fut associée au culte nouveau, comme elle l'avait été à celui du paganisme. Ornement du Paradis, la récompense, ainsi que le symbole, du martyr, la rose, avant toutes les autres 
fleurs, avait sa place marquée sur les autels et dans les temples chrétiens.

Paulin de Nole recommandait déjà aux fidèles, à l'occasion de la fète de saint Félix, de devancer le printemps, en jonchant le sol de fleurs et en ornant le seuil de l'église de guirlandes ${ }^{1}$. Dans une épître à Héliodore $^{2}$, saint Jérôme loue l'évèque Népotien de parer les basiliques et les chapelles des saints de fleurs, de feuillage et de pampres.

Le prêtre Sévère, nous apprend Grégoire de Tours $^{3}$, cueillait de ses propres mains des fleurs de lis pour en décorer les murs de son église. Et, dans une pièce de vers connue, Fortunat félicite la reine Radégonde et sa compagne Agnès de ne point imiter les femmes qui se parfument de roses et de réserver pour les temples sacrés les prémices du printemps :

Aux jours de fêtes, dit-il't, vous tressez de vos mains

1. Spargite flore solum; praetexite limina sertis :

Purpureum ver spiret hiems : sit floreus annus Ante diem.

De S. Felice natalitium carmen III, v. 110-112.

2. "Hoc idem possumus et de ista dicere, qui basilicas, ecclesias et martyrum conciliabula diversis floribus et arborum foliis vitiumque pampinis obumbrat. " Epist. LXIII.

3. "Solitus erat flores liliorum tempore quo nascuntur, colligere ac per parietes hujus aedis appendere. „ Liber de gloria confessorum, cap. Iv. Ed. Migne, p. 866.

4. Texistis variis altaria festa coronis Pingitur ut filis floribus ara novis.

Aureus ordo crocis, violis hinc blatteus exit.

Coccinus hinc rubicat, laetus et inde nivet. 
des couronnes pour les autels et les parez de guirlandes de fleurs nouvellement écloses, unissant habilement le safran à la corolle dorée et la violette empourprée. Ici éclate le rouge vif, là la blancheur du lait. Toutes ces couleurs luttent entre elles, et l'on croirait ces fleurs en guerre dans le sanctuaire même de la paix.

Nous savons par le témoignage du poète Thiofried ${ }^{1}$ que sainte Amalbergue prenait plaisir à offrir sur les autels les fleurs, les herbes aromatiques et les fruits qu'elle avait cueillis dans le jardin de son père. Les statuts du monastère de Xante prescrivaient aux diacres de parer le chœur et le maîtreautel de fleurs et d'herbes odoriférantes aux fêtes de saint Jean et des saints Pierre et Paul ${ }^{2}$. Dans la vie de saint Martin qu'il nous a laissée, l'abbé Picher de Metz raconte ${ }^{3}$ que le jour de la fête du patron de son couvent, les religieuses offraient sur l'autel des roses et de blanches couronnes de lis.

Stat prasino venetus, pugnant et flore colores.

Inque loco pacis herbida bella pulas.

Lib. VIII, 7. De floribus super altare, v. 9-14. Ed. Ch. Nisard. Paris, 1887, in-8, p. 206.

1. Floribus et pomis vernant pomeria patris,

Hic altare Deo dicat alma puerpera virgo.

Punica mala, nuces offert, thymiamata, flores.

Adam Reiners, Die Pflanze als Symbol und Schmuck im Heiligtume. Regensburg, 1886, in-8, p. 39.

2. Binterim, Denkwürdigkeiten, ap. Adam Reiners, op. laud., p. 39.

3. Chorus sacrarum virginum, sequendo semper dominum,

Offert rosas et lilia serta quoque candentia.

Adam Reiners, op. laud., p. 39. 
Tous les témoignages sont unanimes pour nous montrer quelle place immense les fleurs et en particulier les roses occupaient dans les cérémonies du culte. Dans un document de $1366^{1}$, preuve manifeste du rôle qu'y jouait cette fleur, l'Ascension est appelée la "fète de la rose ». Mais c'est là, il faut le dire, un cas isolé; la Pentecôte, au contraire, dans la plupart des pays, était bien réellement une fète des roses. A Rome, nous apprend une prescription du chanoine Benedetto ${ }^{2}$, on lançait, en ce jour, du haut de l'église de Santa-Maria-Rotonda, des roses, figure des dons du Saint-Esprit.

Cette coutume n'était pas particulière à la ville éternelle; on en trouve de semblables bien ailleurs, entre autres à Rouen et à Lisicux, ainsi qu'à Senlis, à Orléans et à Tours ${ }^{3}$. Mais aux fleurs que l'on jetait arec des feuilles sur le pavé de l'église, on joignait aussi parfois des étoupes allumées, pour figurer les langues de feu du Saint-Esprit, en mème temps qu'on lâchait des passereaux et surtout des colombes $^{4}$. Cette pluie de roses au jour de la Pentecôte

1. "Comme le jour de l'Ascension, icelui Goeron, demourant au bourc la royne, venoit de la Feste de la Rose. „) Lit. remiss., ap. Du Cange, s. v. Festum rosae.

2. Avellino, Opusculi, vol. III, p. 263. "Statio ad Sanctam Mariam Rotundam, ubi Pontifex debet canere missam et in praedicatione dicere de adventu Spiritus Sancti, quia de altitudine templi mittuntur rosae in figura eiusdem Spiritus Sancti. »

3. Du Cange, s. v. nebula 2. - Dom Martène, De antiquis ecclesiae ritibus libri tres. Venetiis, 1783, in-folio, vol. III, p. $195 a$.

4. "Tunc etiam ex alto ignis projicitur quia Spiritus Sanctus 
a fait donner, en certaines contrées, à cette fète le nom de " dimanche des roses ), et, en Italie et en Espagne, de "pâques des roses " - Rosarum pascha $^{1}$, Pascha rosata ${ }^{2}$ ou rosa ${ }^{3}$.

Les roses figuraient aussi parfois à la fète de saint Jean et de saint Pierre. A Duyven, en IIollande, on ornait, le jour de la Saint-Jean, les maisons de rameaux de noyer et de roses ${ }^{*}$. Dans quelques localités de la Belgique, à l'occasion de la Saint-Pierre, on se pare aujourd hui encore de couronnes de roses ${ }^{3}$.

Quand la fète du Saint-Sacrement eut été instituée, avec la procession solennelle qui en est le complément indispensable, la rose, à cette époque dans toute sa floraison, en fut l'ornement naturel. Le Cérémonial des évéques prescrit de garnir de tentures les rues par lesquelles la procession doit passer et de les joncher de fleurs ct de feuillage ${ }^{6}$. Cette

descendit in discipulos igneis linguis, et etiam flores varii ad denotandum gaudium et diversitatem linguarum et virtutum. Colombae etiam per ecclesiam demittuntur, in quo ipsa Spiritus missio designatur. " Durandus, Ratio divinorum officiorum, lib. VI, cap. 107.

1. Cf. II ${ }^{\mathrm{e}}$ partie, chap. IV, p. 359.

2. “Ita Pentecosten appellant Itali, quod eo fere tempore rosae floreant. "Hist. morlis et miracul. Leonis $I X$, ap. Miklosich, Die Rusalien.

3. L. Foresti, Vocabolario piacentino, s. v.

4. Ad. Kuhn, Sagen, Gebräuche und Märchen aus Westphalen, vol. II, p. 482. A rosa, p. 42.

5. Ad. Kuhn, op. laud., vol. II, p. 490.

6. "Viae per quas processio transire debebit, mundentur et ornentur auleis, pannis, picturis, florilus frondibusque virentibus. " Caerimoniale episcoporum, lib. II, cap. xxxir. 
prescription a toujours et partout été soigneusement observée; mais on ne s'en est pas tenu là; des enfants, au moment de l'adoration du Saint-Sacrement, lancent en l'air des fleurs et en particulier des feuilles de roses. Dans certains diocèses les officiants portent aussi des bouquets de roses; autrefois ils en avaient des couronnes, comme les enfants en ont encore aujourd'hui. Un compte de l'église de Saint-Quiriace de Provins, de l'année 1350, parle de " chapeaux de roses pour la fète du Saint-Sacrement ». Il est égalernent question, dans un compte de Yotre-Dame-du-Val, autre église de Provins, en 1436 , de "chapiaux de roses et fleurs " au jour de la même fète. On les avait payés 2 sols 6 deniers ${ }^{1}$. Une miniature d'un missel du $\mathrm{xv}^{\mathrm{e}}$ siècle montre la confrérie des orfèrres assistant en corps à une procession avec la châsse de leur patron, chaque membre ayant sur la tète et quelques-uns mème autour du cou des guirlandes de roses ${ }^{2}$.

Le Cérémonial des évêques, qui ne fait ici que confirmer une ancienne coutume, recommande, les jours de fètes majeures, d'orner les portes extérieures de l'église, ainsi que le maitre-autel, de feuillage et de fleurs. A Béthune, au $\mathrm{xv}^{\mathrm{e}}$ siècle, on jonchait de fleurs le chøur de l'église à toutes les fètes doubles depuis le jour de l'Ascension jusqu'à celui de l'Élé-

1. "Pro cappellis roseis in festo sancti Sacramenti. ) F. Bourqueiot, Histoire de Provins, p. 259, note 3.

2. Paul Lacroix et Ferd. Seré, Le lis're d'or des métiers, Paris, 1858, in-8, p. 71 . 
vation de la Croix. On rencontre des usages analogues en Espagne, en Italie, en Allemagne, en Belgique et en Angleterre ${ }^{1}$.

Les roses et les lis prirent naturellement une grande place dans le culte de la Vierge, dont elles sont l'emblème :

J'offre à votre royale personne, dit un vieux chant espagnol ${ }^{2}$, des roses dignes d'une tête si auguste. L éclat de la rose vermeille, ô reine excellente, ne messied pas entre les diamants et les émeraudes et il rendra plus brillant l'or de votre couronne.

La coutume païenne de faire des offrandes sur la tombe de ceux qui n'étaient plus fut tout d'abord combattue et proscrite chez les chrétiens; Minutius Félix la regardait comme inutile, " parce que s ils sont heureux les morts n'ont que faire de fleurs, et que, s ils sont malheureux, elles ne sauraient les rejouir » ${ }^{3}$. Saint Jérôme, nous l'arons ru, opposait les œurres de charité de Pammachius aux violettes et aux roses que les autres maris répandent sur le tombeau de leurs épouses". Saint Ambroise dit qu'il ne veut

1. Reiners, op. laud., p. 66.

2. Rosas ofrezco a vuestra real persona, dignas de tal cabeza....

Que no parece mal, Reina excellente, entre el diamante $y$ la esmeralda hermosa, sobra el oro mas puro y refulgente el rosicler de la purpurea rosa.

Böhl de Faber, Florešta, vol. I, n 86, v. 7-12.

3. " Cum et beatus non egeat et miser non gaudeat floribus. » Patrologie, vol. LIX, p. 688, note $c$.

4. Première partie, chap. III, p. 69. 
pas couvrir de fleurs le tombeau de Talentin, mais embaumer son esprit du parfum de Jésus-Christ ${ }^{1}$.

Mais ce dédain pour une pratique si naturelle et si réellement pieuse ne l'empêcha pas de se répandre en Occident, comme en Orient. Les saints eux-mêmes qui l'avaient d'abord condamnée finirent par s'y conformer. Saint Jérôme rappelle les larmes qu'il a répandues avec des fleurs sur la tombe de Népotien². Saint Augustin parle d'une femme aveugle, qui, après avoir prié sur le tombeau de saint Étienne, y fit une offrande de roses ${ }^{3}$. A l'entrée du monument de sainte Agnès, dans les Catacombes de Rome, on voit deux génies ailés qui portent chacun une corbeille remplie de fleurs sur le tombeau de la sainte, image des offrandes qu'on y faisait dans la réalité ${ }^{4}$. Les Actes de saint Nicolas parlent d'offrandes de roses - rosalia ${ }^{3}$ ou rhodismos ${ }^{6}$ - que l'on faisait sur sa tombe.

1. "Ton ego floribus tumulum ejus aspergam, sed spiritum ejus Christi odore perfundam. "In consolatione de obitu Valentini imperatoris.

2. "Quotiescunque nitor... super tumulum ejus flores spargere, toties lacrymis implentur oculi. „ Epist. xxxr. Ad Heliodorum.

3. "Hic caeca mulier, ut ad episcopum portantem duceretur et oravit, flores quos ferebat, dedit. " De civitate Dei, lib. XXII.

4. Martigny, Dictionnaire des antiquités chrétiennes, s. v. Paradis.

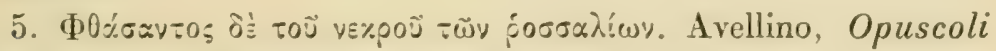
diversi, vol. III. Napoli, 1836, p. 365.

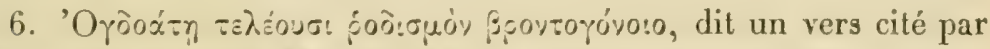
Tomaschek d'après Morelli. Sitzungsberichte der Kais. Aliademie der Wissenschaften, vol. LX, p. 370.) 
Dans son Hymne en l'honneur du martyre de sainte Eulalie, Prudence engage les vierges et les jeunes gens à cueillir les violettes empourprées et le rouge safran, que les champs attićdis et l'hiver près de finir ont produits, et à les offrir avec du vert feuillage sur le tombeau et l'autel de leur patronne ${ }^{1}$. Et dans un autre hymne :

Nous honorerons, dit-il ${ }^{2}$, les restes de nos morts cachés sous terre avec des violettes et du vert feuillage et nous inonderons de parfums liquides leurs vaines inscriptions et leur froide pierre.

Cette pieuse pratique ne devait pas cesser d'ètre en usage chez tous les peuples chrétiens; le rituel romain recommande de parer les enfants après leur mort de couronnes de fleurs ou de plantes odoriférantes et aromatiques ${ }^{3}$. Mais cet hommage n'était pas réservé à leurs seuls restes; des chansons populaires parlent souvent de roses répandues sur le tombeau des êtres qui sont chers. On plantait aussi des rosiers aux lieux où ils reposaient. L'amant

1. Nos tecta fovebimus ossa

Violis et fronde frequenti

Hymnus circa exsequias defuncti, v. 169-172 (Cathemerinon, $\mathrm{X}$ ).

2. Carpite purpureas violas, Sanguineosque crocos metite; Non caret his genialis hyems, Laxat et arva tepens glacies... Ista comantibus e foliis Munera, virgo puerque, date. Sic venerarier ossa libet,

Ossibus altar et impositum.

3. "Imponitur ei corona de floribus, seu de herbis aromaticis et odoriferis, in signum integritatis carnis et virginitatis. » 
malheureux de la chanson serbe citée plus haut demande qu'on en mette un près de sa tête ${ }^{1}$. Dans un lied allemand, une jeune fille en deuil rappelle les rosiers qu'elle a plantés en pleurant sur la tombe de sa mère ${ }^{2}$.

\section{II.}

A partir du $\mathrm{x}^{\circ}{ }^{0}$ siècle surtout, depuis que les mœurs se furent adoucies sous l'influence de la chevalerie et que les progrès du luxe allèrent chaque jour augmentant, l'emploi des fleurs et en particulier des roses prit la plus grande extension dans les usages domestiques; on se croirait, du moins à lire les poètes de l'époque, revenu au temps de l'antiquité, où elles jouaient dans la vie un rôle si considérable. On s'en servait dans les occasions solennelles pour joncher les rues et les places publiques:

De la cité fu li marchies

De fresche herbe trestos joncies ${ }^{3}$.

On en répandait également sur le plancher des appartements les jours de réception, en particulier le jour d'un festin de noces, encore, ce qui peut

1. Deuxième partie, chap. rv, p. 348 .

2. Die Rosen die pflanzt ich ihr weinend aufs Grab.

Mittler, no 310, v. 8 .

3. Durmars, éd. Edm. Stengel, 1873, in-8, v. 945-46.

Totes sont jonchies les rues

Et pardeseure portendues

De cortines, de dras de soie. Guillaume de Palerne, v. 2925. 
surprendre, que ce plancher fut recouvert de tapis ou de riches étoffes, que devaient salir ou gâter les plantes ou les fleurs foulées aux pieds :

Dedans une grande chambre peinte,

Jonchiee de flors et de glai,

Si com drois est el mois de mai ${ }^{1}$,

Et dans Gui de Nanteuil nous voyons que

La sale pourtendue e bien encourtinée

De jonc et de mentastre fu bien englaiolée ${ }^{2}$.

Même spectacle dans le Durmart ${ }^{3}$ :

Totes sont joncies les sales

De roses et de flors de lis

Et de fres jons novel coillis.

Et dans Aiol $^{4}$ :

Li hostes les fis bien servir et honorer;

Sieges orent et coutes et boins tapis ovrés:

De rose et de mentastre font tout joncier l'ostel.

Cet usage n'était pas particulier à la France, on le trouve également en Allemagne. Ainsi dans le Wílleham de Wolfram d'Eschenbach :

De nombreux tapis avaient été étendus dans tout le palais; dessus on jeta une couche épaisse de roses encore

1. Dolopathos, éd. Brunet et Montaiglon, 1856, in-8, p. 364.

2. Éd. Paul Meyer. Paris, 1861, in-8, p. 14 .

3. Ed. Edm. Stengel. Stuttgart, 1873, in-8, v. 940-42.

4. Éd. Jacques Normand et Gaston Raynaud, v. 7083-85. 
humides de rosée; leur brillant éclat fut foulé aux pieds, ce qui n'en répandit pas moins un doux parfum ${ }^{1}$.

De même dans le Tristan de Heinrich von Freiberg: :

De riches tapis de soie le sol fut recouvert et des roses répandues dessus en abondance ${ }^{2}$.

Les murailles étaient parfois également tendues de riches tapis ${ }^{3}$ et ornées de roses ou d'autres fleurs ${ }^{4}$, en même temps que le sol en était jonché. Le poème anglais de Richard Cœur de Lion nous montre aussi, à l'époque joyeuse du mois de mai, quand les oiseaux chantent et que les fleurs s'épanouissent, les dames parant leur's demeures de roses vermeilles et de blanes lis ${ }^{5}$.

Dans les premiers temps du moyen âge, on le

1. Vil teppech übr al den palas Lac, dar ûf geworfen was
Touwic rôsen hende dicke :

Den wurdn ir liehte blicke

Zetreten : daz gap doch süezen wàz. Str. 144, v. 1-5.

2. Mit tiuwern teppichen sidin

Wart der esterich beleit

Und rosen vil daruf gespreit.

V. 2526-28.

3. La chambre ou ele jut ot fait encortiner

De riches dras de soie por son cors honorer.

La chanson de Godefroid de Bouillon, publ. par G. Hippeau.

Paris, 1877, in-8, v. 592-93.

4. Die wende gar bestecket Mit bluomen und das hûs bestreut. Wigalois, p. 265, v. 12.

5. Floures on appyl trees and perye,

Smale foules synge merye,

Ladyes strouwe here boures

With rede roses and lylye flowres.

Th. Wright, op. laud., p. 283.

Joret. La Rose. 
voit par la description de Fortunat, on ornait aussi de roses la table des festins :

Regarde, heureux convive, ces mets délicieux ${ }^{1}$ que l'odeur embellit, avant que le goût lui-même les pare; la foule de ces fleurs vermeilles te sourit; les champs ont à peine autant de roses que cette table en porte.

Toutefois cet emploi des roses emprunté aux Romains ne paraît pas s'ètre conservé longtemps après Fortunat; du moins je n'en ai pas trouvé de trace dans les siècles postérieurs; les trouvères et les minnesaenger n'en parlent pas; tout au plus est-il question dans quelques écrits, par exemple dans la vie de saint Martin, par l'abbé Richer, de roses et de plantes aromatiques répandues sur le sol des salles à manger ${ }^{2}$. Dans les fètes nuptiales, au contraire, les roses ont pris place, comme dans l'antiquité, durant tout le moyen âge. On en répandait en particulier avec des lis dans les salles où se donnaient les festins de noces ${ }^{3}$ :

1. Respice delicias, felix conviva, beatas,

Quas prius ornat odor, quam probet ipse sapor.

Molliter arridet rutulantum copia florum,

Vix tot campus habet quot modo mensa rosas.

Lib. XI, cap. XI. De floribus, v. 1-'́.

2. Rosa, storax et galbanum, cum terebintho platanus

Pavimenti planitiem, per florem pingunt speciem.

3. Ouch was ûf dem esterich

Ein pfellor über all gebreitet

Unde dar ûf gespreitet

Von bluomen ein grôziu kraft, Als ez diu vrouwe tugenthaft Crône, v. 17409-18.
Durch ir selber êre gebot:

Liljen unde rôsen rồt.

Dise edlen bluomen wàren,

Darumbe daz sie bàren

Dem sal einen edlen smac. 
On étendit sur le sol un précieux tapis et dessus on répandit une grande quantité de fleurs, comme la noble dame l'avait ordonné en son propre honneur : des lis et des roses vermeilles, belles fleurs qui devaient remplir la salle d'un doux parfum.

Une chanson populaire ${ }^{1}$ nous apprend qu'en Serbie on répandait des roses sous les pas des nouveaux époux, et, avant le mariage, des jeunes filles, en . Lusace $^{2}$, distribuaient aux invités des roses qu'elles avaient cueillies elles-mêmes.

Les roses figurent aussi dans des espéces de tournois - le siège du château d'Amour - dont l'origine parait remonter aux premières années du xur siècle. Rolandinus Patavinus nous a laissé la description des fètes de ce genre qui se donnèrent, peut-être pour la première fois, à Trérise en 1214, mais ont persisté dans certaines contrées presque jusqu'à nos jours". Si elles ont été rarement décrites, ces réjouissances, véritables batailles de fleurs aux proportions héroïques, ont été souvent représentées, en particulier sur des coffrets ou boîtes en ivoire ${ }^{4}$. On y voit un château flanqué de tours avec sa poterne et leurs créneaux. Sur les créneaux et aux fenêtres du château des dames se défendent avec

1. Ap. Sobotka, Rostlinstvo, etc.

2. Chanson de la Lusace, ap. Solsotka.

3. Annales Veronenses, an. 1214.

'. "Le siège du Chàteau d'amour ». Mémoires de la Société des Antiquaires de France, vol. I, p. 184-87. L'auteur de l'article dit que ces réjouissances se sont données, dans les cantons de Vaud et de Fribourg, jusqu'au commencement de ce siècle. 
des roses qu'elles jettent sur les assaillants; ceux-ci ont recour's aux mèmes projectiles. L'un d'eux est armé d'une arbalète chargée d'une rose; du côté opposé à celui où il se trouve, un chevalier escalade les murs avec une échelle de corde, bien accueilli par deux dames, qui tiennent à récompenser sa valeur. Plus loin deux autres chevaliers sont occupés à charger un trébuchet, afin d'opérer une action décisive sur la forteresse. Sur le devant deux dames à cheval sont sur le point d'en venir aux mains arec deux guerricrs armés de pied en cap. Au-dessus de la porte d'entrée apparaît un ange ou le dieu d'Amour tantôt arec un arc, d'autres fois armé d'une lance et un faucon sur le poing gauche ${ }^{1}$.

Quand les bains furent devenus d'un usage presque journalier et qu'on regarda comme un des premiers devoirs de l'hospitalité d'en offrir aux étrangers qu'on recevait, on chercha ì en relever le prix et l'agrément en répandant sur l'eau des feuilles de

1. Il y a une de ces boîtes au Musée du Louvre. Le Musée de lÉcole des Braux-Arts de Paris en posside le moulage, ainsi que do celles qui se trouvent à Boulogne, à Marbourg, etc. The Gentleman's . Magazine du mois de février 1835 contient la description d'un coffret, conservé à Goodrich Court, dans le comté de Hereford. Alvin Schulz a donné, dans la tie de cour, la reproduction du Siège du chàteau d'Amour, qu'on voit sur une boìte à miroir conservée au monastère de Reun. Le Musée de South-Kensineton possède également une boite à miroir en ivoire, sur le couvercle de larquelle se trouve représenté le Siège du clàtean d Amour. Descriptive catalogue of the fictile ivories in the $S . K$. Museum by Jo. Westwood. London, 1883 , no 58200 . 
$\operatorname{roses}^{1}$. Le lendemain matin du jour où Parcival est reçu chez Gournemanz, celui-ci lui fait apporter près de sa couche un bain sur lequel on répand des roses en quantité ${ }^{2}$. Dans le Frauendienst, après qu'on a préparé un bain au héros du poème, deux valets lui apportent des roses fraîches et vermeilles, et on en répand sur lui une si grande quantité qu'en vérite, dit-il, on ne voyait plus ni lui, ni l'eau du bain ${ }^{3}$. Une des enluminures du manuscrit des Minnesaenger, placée en tète des chansons de Jacob de Warte ${ }^{4}$, nous représente nu dans une baignoire un chevalier dont la poitrine, ainsi que l'eau, est couverte de fleurs. Une damoiselle s'approche et lui présente une couronne, tandis qu'une autre lui offre une coupe à boire.

Cette couronne ainsi offerte était faite de roses". C'était sous cette forme qu'au moyen âge ces fleurs étaient le plus souvent employées dans les usages de

1. Alvin Schulz, op. laud, vol. I, p. 224 .

$2 . \quad$ man warf dà ròsen oben în.

Parzival, éd. Fr. Pfeiffer, liv. III, v. 1522.

3. Dà er zwên ander knehte vant:

Die truogen nàch im rôsen dar,

Gepletert vrisch und wol gevar,

Der streut er dar ûf mich so vil,

Für wàr ich iu daz sagen wil,

Daz mich noch daz bat niemen sach. p. 228, v. 22-27.

4. Fr. Heinrich von der Hagen, Bildersaal altdeutscher Dichter. Berlin, 1856, in-fol., pl. XI.

5. On en portait sourent pendant le bain :

Et se baignent ensemble es cures...

Les chapels de flors en testes. Rom. de la Rose, v. 11133. 
la vie. Les chapeaux de roses servaient d'ailleurs dans les circonstances les plus diverses:

Il n y avait point de cérémonie d'éclat, dit Le Grand d'Aussy ${ }^{1}$, qui généralise peut-être trop, il est vrai, point de noces, point de festins, oú l'on ne portât un chapel ou chapeau de roses. Les religieuses, quand elles faisaient profession, les filles, quand elles se mariaient, portaient aussi une couronne de roses ${ }^{2}$.

Cet usage existait déjà en Allemagne au $\mathrm{x}^{\mathrm{e}}$ siècle ${ }^{3}$. La couronne qu'elle portait était comme l'emblème de la virginité de la mariée; aussi la lui enlevait-on au moment où elle allait entrer dans la chambre nuptiale ou quelquefois pendant la danse qui suivait le repas de noces". En France, d'après d'anciens coutumiers $^{5}$, un père, en mariant sa fille, pourait ne lui donner qu'un chapeau de roses, c'est-à-dire sa parure de noces. Le chapeau de roses du moyen âge a été, depuis la Renaissance, remplacé, dans les pays romans et germaniques, par une couronne d'oranger; mais chez les nations slaves, du moins en Russie, les fiancés ont continné jusqu'au xrrne siècle de porter des couronnes de roses vermeilles ${ }^{6}$.

1. Histoire de la vie privée des Français, vol. II, p. 245.

2. Dans des Lettres de rémission de 1371, il est question d'une " jeune femme à marier (qui) avait un chappellet de fleurs sur sa teste ». Du Cange, s. v. cappelletus.

3. Karl Weinhold, Die deutschen Frauen in dem Mittelalter. Wien, 1882, in-8, vol. I, p. 385.

4. Karl Weinhold, op. laud., vol. I, p. 400.

5. Le Grand d'Aussy, op. laud., vol. II, p. 246.

6. Resue du Ministère de l'Instruction publique. Arril 1889 Je dois ce renseignement à mon ami, M. L. Léger. 
Les femmes, surtout les jeunes filles, qui restaient le plus souvent tête nue, aimaient à se parer de chapeaux de roses ou de fleurs; ces couronnes servaient à retenir leur chevelure :

Et ele ot un capel de flours

En la tieste ki li tenoit

Ses cheveus et li avenoit,

nous dit l'auteur du Chevalier as deus espées ${ }^{1}$.

Une miniature du manuscrit du Sachsenpiegel de Wolfenbuttel, qui représente le partage de la terre, nous montre les jeunes filles qui y figurent, une couronne de roses sur leurs cheveux flottants². Dans deux miniatures du manuscrit des Minnesaenger, où l'on roit des damoiselles qui assistent à un tournoi, l'une d'elles est aussi représentée portant une couronne de roses sur la tête ${ }^{3}$.

Porter ainsi un chapeau de roses était un honneur et un privilìge auxquels ne pouvaient plus prétendre les jeunes filles qui avaient failli; en Allemagne, elles étaient alor's condamnées, en signe de leur déshonneur, à mettre une couronne de paille ${ }^{4}$, ce dont elles se consolaient d'ailleurs, puisfu'ainsi elles conservaient leur liberté ${ }^{j}$. Les femmes mariées portaient aussi, quoique plus rarement, des cou-

1. Éd. W. Förster. Halle, 1877, in-8, v. ‘294.

2. Karl Weinhold, op. laud, vol. II, p. 232.

3. Von der Hagen, Bildersaal, pl. XII et XXIX.

4. Alvin Schultz, op. laud., vol. I. p. 598.

5. Mir ist von strôwe ein schapel und mîn vrier muol

Lieber danne ein rùsenkranz, so bin ich behuot.

Hêr Burkart von Hôhenvels, ap. Bartsch, Liederdichter, p. 149. 
ronnes, mais elles les mettaient sur leur coiffure, leur guimple, all. gebende ${ }^{1}$ - Cette parure même leur était interdite dans certains pays. On rapporte que la duchesse Dubranka de Bohême fut blâmée pour en avoir porté une ${ }^{2}$.

Les hommes portaient aussi des couronnes de roses, de fleurs ou même de feuillage dans les fètes et dans certaines circonstances particulières. Lorsque le connétable servait le roi à table, il avait une verge blanche à la main et une couronne de roses sur la tète $^{8}$. En Angleterre aussi, quand l'écuyer-tranchant se préparait à remplir son office, il se mettait un chapeau de fleur's sur la tète et ceignait un baudrier ${ }^{*}$. Dans le roman de Foulques Fitz-Marin, écrit au xir ${ }^{\ominus}$ siècle, le héros rencontre un jour dans une forêt un messager qui " jolivement chantait » et avait un chapeau de roses rermeilles sur la tète". Arcite, dans Chaucer ${ }^{6}$, pour célébrer le retour du mois de

1. Karl Weinhold, vol. II, p. 317. Alvin Schultz, vol. I, p. 239.

2. Menken, Script. rer. Saxon., vol. I, p. 1997.

3. Le Grand d'Aussy, op. laud., vol. II, p. 246.

4 .

He set a chaplet upon his hed,

A belt about his sydes two.

Romance of the squyer of Lowe Degree, ap. Th. Wright, op. laud., p. 289.

5. Nouvelles françoises en prose du xrve siècle publ. par L. Moland et C. d'Héricault. Paris, 1858, in-18, p. 59.

6. By adventure his way he gan to hold,

To maken him a gerlond of the greves,

Were it of woodbind or hawthorn leves.

Canterbury tales. Ed. Th. Tyrwhitt, London, 1874, in-8.

The knightes tale, v. 1507-9. 
mai, se rend au bocage voisin pour se faire un chapeau de fleurs de chèvre-feuille ou d'aubépine. Et le mème poète nous représente l'appariteur (Sumpnour) des Cunterbury tales ayant lui-mème une couronne ${ }^{1}$, qu'un manuscrit figure comme étant faite de roses ${ }^{2}$.

Mais les couronnes ou chapeaux de roses étaient plus particulièrement la parure des amants. Chaucer nous montre Vénus ayant sur la tète, "parure qui lui sied si bien "), une guirlande de roses parfumées ${ }^{3}$. Dans un lied de Nithard, l'amie du poète parle du brillant " chapeau de roses " — rosenschapel, mot qui nous révèle l'origine de la coutume allemande, qu'il lui a envoyé ${ }^{4}$.

Printemps verdoyant et plein de fleurs, dit une romance espagnole ${ }^{5}$, couronne mes amours de guirlandes faites de blancs lis, de jasmin, de roses vermeilles et musquées, de violettes et de verveine, d'œillets et de mille autres fleurs.

1. A garland had he set upon his head. V. 668 .

2. Man. Ellesmere, ap. Th. Saunders, Canterbury tales. London, 1884 , in-12, p. 82.

on hire hed full semely for to see

A rose gerlond fressh and wel smelling.

The knightes tale, v. 196'.

4. Er sante mir ein rôsenschapel, dàz het liehten schîn ùf das haubet mìn. Éd. Moriz Haupt, 1858, p. 21, v. 14 .

5. Verde primavera llena de flores coronad de guirnaldas a mis amores, de jasmin y rosa mosqueta olorosa violetta y verbena de blanca azucena, de claveles llena y de otros mil flores. Böhl, Floresta, no 259. 
Dans le Lai d'Aristote, l'amie d'Alexandre

Un chapel en son biau chief pose ${ }^{1}$,

pour séduire le maître du puissant monarque.

Une miniature du manuscrit des Minnesinger, placée en tête des lieds d'Henri de Stretlingen, nous montre un jeune homme - le poète sans doute avec sa dame, qui a une couronne de roses sur la tête. Dans une autre miniature du même manuscrit, qui représente le comte Kraft de Toggenburc escaladant la fenètre de son amie, celle-ci lui offre une couronne de roses et de feuillage ${ }^{2}$. Quand les amants se trouvaient réunis, une de leurs occupations était de tresser des chapeaux de roses ou de fleurs. Tels le poète de Beupe de Commarchis dépeint Limbanor et Malatrie dans le verger où ils se sont rencontrés ${ }^{3}$ :

Puis s'assist les la belle, moult va la regardant, Et que plus la regarde, plus li samble plaisant;

Par tres grant amisté va l'uns l'autre araisnant, Un chapel de floretes vont entre aus deux faisant.

Et une miniature du recueil de Manassé nous montre une dame tressant une couronne de roses, tandis qu'elle s'entretient avec un chevalier ${ }^{4}$.

C'était surtout quand ils se rendaient à la danse

1. Henri d'Andeli, Le Lay d'Aristote, v. 368 (Fabliaux et contes publiés par Barbazan. Paris, 1808, in-8, vol. III. p. 108).

2. Fr. Heinrich von der Hagen, op. laud, pl. XLVI ct VII.

3. P. 85, v. 2425-28.

4. Fr. von der Hagen, op. laud, pl. VI. 
que les amants se paraient de chapeaux de roses: "Nombre de jeunes gens, lit-on dans un ancien lied", sont venus à la danse; chacun portait une couronne de roses. "Et dans une de ses chansons, Walther von der Vogelweide offre à sa dame une couronne de ces fleurs aimées, qui " embelliront la danse ", quand elle la mettra ${ }^{2}$. Cette couronne, d'ailleurs, ne servait pas uniquement de parure aux danseurs; elle devenait anssi une marque de distinction enviée, la danseuse donnait la sienne à celui qu'elle préférait, le danseur à celle dont il recherchait l'affection ${ }^{3}$ :

Avec joie je prends part à cette danse, dit le héros d'un vieux lied ${ }^{4}$; j'espère qu'une belle couronne me sera domnée par une jeune fille non moins belle; aussi je veux être tout à elle.

Il n'était pas rare, cela se comprend, qu'on se disputât avec ardeur ces présents recherchés; de là,

1. Dar kam hin durch tanzen junger liute ein michel teil, jetweder truoc ein rôsenkranz. Minnes, III, $193 b, 3$.

2. Nemt, frowe, disen kranz, Alsò sprach ich z'einer wol getànen maget, so zieret ir den tanz mit den schœenen bluomen, als ir's ûfe traget.

Gedichte, p. 19, no 6, v. 1-4.

3. Franz M. Böhme, Geschichte des Tanzes in Deutschland, Leipzig, 1886, in-8, vol I, chap. III, p. 39.

4.

Mit Lust tritt ich an disen tanz,

ich holf", mir werd ein schöner kranz von einem schön jungfrewelein, darumb wil ich ir eigen sein.

Franz, M. Böhme, Altdeutsches Liederbuch, $n^{n} 281$. 
surtout entre paysans, des querelles dont parlent les poètes du temps ${ }^{1}$; non seulement dans la mèlée les couronnes étaient foulées aux pieds, les coiffures et les cheveux arrachés, mais plus d'une fois le sang coula et des danseurs restèrent sur le terrain. "Pour un chapeau de boutons de roses brillantes, dit un minnesaenger ${ }^{2}$, trente-six d'entre eux ont été tués. "

Les couronnes de roses ou de fleurs servaient aussi de récompense dans les jeux populaires ${ }^{3}$, ainsi que dans les concours de chant ou de danse et parfois dans les tournois. A Provins, nous apprend un document du 19 mai $1414^{4}$, des chocurs de jeunes filles se disputaient les jours de chanchis, nom local de ces réjouissances, le chapeau de roses vermeilles que le chapitre de Saint-Quiriace décernait à celle qui dansait avec le plus de grâce. J'ai déjà eu occasion, en parlant des jardins de roses et des maîtres chanteurs, de faire mention des couronnes données aux vainqueurs dans les défis poétiques. Cet usage se conserva en Allemagne pendant les deux derniers siècles du moyen âge et tout le seizième. "Avec lui je veux chanter pour aroir une belle couronne de

1. Ròsenschapel wart dà vil zerströut;

Hàr unt hủben sach man rizen. Minnes, III, $189 a, 5$. Seht, dà wart verhouwen manic ròsenkranz,

dà daz bluot begunde her nàch dringen. Ibid., III, $221 a$.

2. Umb' ein kranz von manger liehten rôsen knopf wart ir sehs und drizeg erslagen. Ibid., III, $260 b, 11$.

3. "Garlands of flowers were the common rewards for success in the popular games. "Thomas Wright, op. laud., p. 290.

4. F. Bourquelot, Histoire de Provins, vol. II, p. 291. 
roses ", lit-on dans un vieux lied ${ }^{1}$. Et dans un autre:

Aux lointains pays étrangers croissent des fleurs vermeilles et blanches, que cueillent arec grand soin les jeunes filles, elles en font des couronnes, les portent à la danse du soir et invitent les jeunes gens à chanter, jusqu'à ce que l'un d'eux gagne ces couronnes".

La miniature placée en tête des lieds du duc Henri de Breslau, dans le recueil des Minnesinger ${ }^{3}$, représente un tournoi dans lequel un chevalier, probablement le poète lui-mème, reçoit une couronne de roses. Et l'on voit sur la couverture d'une boîte à miroir en ivoire, peut-être d'origine anglaise, qui se trouve au Musée de South-Kensington ${ }^{4}$, trois chevaliers qui combattent, tandis que du haut des créneaux du château au pied duquel se donne le tournoi, des dames leur jettent des roses et l'une d'elles mème tient à la main une couronne de ces fleurs destinée au vainqueur.

Au lieu d'être le prix gagné par l'habileté ou la valeur, les chapeaux de roses, et c'est ce qui explique le grand nombre de redevances qu'on en faisait dans les derniers siècles du moyen âge, étaient souvent considérés comme une simple marque de soumission, l'hommage d'un inférieur enver's son supérieur,

1.

Mit im sô wil ich singen

Umb einen hübschen rôsenkranz.

Mone's Anzeiger, an. 1838, p. 376.

2. Franz M. Böhme, Altdeutsches Liederbuch, no 271, str. 2-3 .

3. Fr. Heinrich $\mathrm{v}$. der Hagen, op. laud., pl. IV.

4. Catalogue of the fictile ivories, n’ 58203. 
d'un tenancier envers son donateur ou son suzerain. C'est ainsi que, en $1124^{1}$, Geffroi, chevalier de Graffart, donnant au prieuré de IIeauville une rente d'un quartier de sel, déclare que pour conserver le souvenir de cette donation, les religieux, quand ils viendront en réclamer le paiement annuel, devront faire hommage d'une guirlande de roses. On roit de même, en $1398^{2}$, Pierre Porte s'engager à donner à l'abbesse, "en l'abbaye de Sainte-Trinité de Caen, au jour Saint-Jean-Baptiste, un chappel de roses vermelles, par raison et à cause d'une pièce de terre au terroir de cette ville».

Le 26 octobre 1438, " haut et puissant seigneur Thomas, sire d'Escalles, de Melles, etc., prit en fief et par hommage de Jean d'Argouges, seigneur de Grestot et de Granville, la roque, manoir et circuits de la dite roque de Granville... et fut par ce fait par en faisant par icelui seigneur au dit écuyer et à ses hoirs un chapeau de roses vermeilles pour chacun an de rente, à la fète Saint Jean Baptiste $)^{3}$. Dans une charte de Henri VI, roi d'Angleterre, datée de la $24^{\mathrm{e}}$ année de son règne, il est imposé à un certain comte Richard, en retour des biens reçus de la couronne, de présenter chaque année à la fète de la nativité de Saint Jean-Baptiste une rose, "chaque fois

1. Léopold Delisle, Études sur la condition de la classe agricole en Normandie, p. 91.

2. Cart. de Calix, ap. Léop. Delisle, op. laud., p. 492, n. 11.

3. Guidelou, Notice sur la ville de Granville. Granville, 1846, in -8, p. 116. 
qu'elle lui serait demandée ". Il est question d'une redevance semblable dans deux autres chartes anglaises de la mème époque, avec la mention que la rose ainsi donnée tiendrait lieu de "tous services " ${ }^{1}$.

Le coutumier des forèts nous apprend que Pierre de Poissi, seigneur de Goui, prenait sur le revenu de la forêt de Roumare un chapeau de roses le jour de la Trinité ${ }^{2}$. Au xiv ${ }^{e}$ et au $x^{e}{ }^{e}$ siècle les redevances de couronnes de roses deviennent très fréquentes. M. Léopold Delisle en a relevé de nombreux exemples dans les " aveux » de seigneur's normands de cette époque. Ces couronnes de roses étaient alors estimées, tantôt six deniers, d'autres fois deux sous tournois.

Je suis loin d'avoir énuméré tous les usages qu'on faisait des roses au moyen âge. En voici encore quelques-uns. Des lettres patentes de Charles VI, délivrées en février $1415^{3}$, obligeaient le crieur juré, à son entrée dans la confrérie, à donner " des chapeaux de roses aux maistres qui allaient quérir leur confrérie à la Saint-Martin le Bouillant » (4 juillet). Une ancienne coutume obligeait aussi les ducs et pairs d'offrir des roses au parlement de Paris, en avril, mai et juin ${ }^{4}$. Cet hommage, appelé "Baillée des

1. Du Cange, Glossarium, s. v. Rosa.

2. Léopold Delisle, op. laud., p. 492.

3. Collection des ordonnances des rois de France, vol. X, p. 279 .

4. Sauval, Histoire des antiquités de la ville de Paris, vol. II, liv. virr, p. 446. Le Grand d'Aussy, op. laud., vol. II, p. 248, peut-être par une confusion avec ce qui précède, dit que les pairs 
roses s, était comme la marque de la suzeraineté du parlement, qui représentait le roi dans ses attributions judiciaires. Le pair, qui était appelé à faire les honneurs de cette cérémonie, allait dans chaque chambre, faisant porter devant lui un grand bassin d'argent, lequel contenait autant de bouquets de roses et d'autres fleurs naturelles ou artificielles, qu'il y avait d'officiers, avec un pareil nombre de couronnes composées des mèmes fleurs et rehaussées de ses armes. On donnait le nom de " rosier du parlement » à l'officier de la cour chargé de fournir ces roses. Il les tirait d'ordinaire de Fontenay-aux-Roses.

Des documents, cités par Sauval, font aussi mention à plusieur's reprises ${ }^{1}$ de roses blanches et de violettes, ainsi que de chapeaux et de bouquets de roses vermeilles distribués aux présidents et conseillers de la Cour du Parlement de Paris la veille de la Pentecôte, en particulier à l'occasion de la délivrance des prisonniers. Il existait des usages analogues dans d'autres villes. A Toulouse, on offrait au parlement des boutons de rose; à Rouen, les magistrats municipaux présentaient à l'échiquier un chapeau de roses et de violettes ${ }^{2}$.

laïques offrirent, depuis la fin du xve siècle, des roses aux magistrats, quand ils avaient un procès devant le Parlement.

1. Op. laud, vol. III, p. 517, an. 1497; p. 521, an. 1496; p. 526, an. 1498. Les roses avaient été achetées à "Marguerite la mercière ", les chapeaux 8 sols parisis et les bouquets 6 sols la douzaine.

2. A. Chéruel, Dictionnaire historique des marurs, institutions et coutumes de la France. Art. Redevances féodales. 
L'usage des chapeaux de roses et de fleurs était si général que ce fut une profession particulière d'en faire ou d'en vendre'. Quiconque le voulait avait le droit de l'exercer, à Paris, du moins ${ }^{2}$; mais il lui était interdit de " cueillir ou faire cueillir au jour du dimanche en ses courtils, nulles herbes, nulles fleurs à chappeaulx faire. " Une exception cependant avait lieu en faveur des roses, dont il était permis de faire " chapeau en toute saison ". Toutefois les chapeliers de roses étaient soumis à diverses obligations:

Il appartient, dit Brussel ", au voyer de faire cueillir chacun an de chacun chappelier qui vend chappeaulx de roses, ung chappel de roses environ l'Ascension. Et chacun chappelier, qui a rosier ou rosiers, est ou sont tenus de apporter chez le voyer plain panier de ponpons de roses à faire eau rose.

C'était le matin, à la première heure du jour, ou même, si l'on en croit un minnesaenger ${ }^{4}$, au milieu de la nuit, alors qu'elles étaient toutes couvertes de

1. Le Grand d'Aussy, op. laud., vol. II, p. 248. Le commerce qu'on en faisait au $\mathrm{xrv}^{\mathrm{e}}$ siècle arait une telle importance que, d'après Guillehert de Metz, les droits dont il ćtait frappé avec le cresson rapportaient au roi dix mille francs l'an. Renan, Discours sur l'état des beaux-arts en France au xive siècle, Paris, 1865, in-8. p. 133.

2. Etienne Boileau, Livre des métiers, éd. R. de Lespinasse et F. Bonnardot. Paris, 1879, in-fol., tre p., ch. xc.

3. Nouvel examen de l'usage général des fiefs en France. Paris, 1750 , in-4, vol. II, p. 746.

4. Die Röslein soll man brechen zu halber mitternacht.

Dann seind sich alle bletter Joret. La Rose. mit dem külen taw beladen, so ist es rösleinbrechens zeit. Böhme, n 138, str. 4. 
rosée, qu'on cueillait les roses ou les fleurs dont on faisait les couronnes ou chapeaux.

Bele Aliz matin leva

Sun cors vesti et para,

Enz un verger s'en entra,
Cinq fluretes i truva, Ung chapelet fet en a De rose flurie,

lit-on dans une vieille chanson ${ }^{1}$, qui nous fait en quelque sorte assister à cette occupation. Elle n'était pas seulement celle des " chapeliers " de profession; c'était aussi un des passe-temps auxquels les dames du moyen âge aimaient le plus à se livrer. Les poètes nous montrent souvent leurs héroïnes cueillant des fleurs et s'en tressant des couronnes. Ainsi la jeune sarrasine d'un fableau publié par Adelbert Keller nous est dépeinte descendant le matin de sa chambre dans le jardin, où elle achève sa toilette à la fontaine, après quoi elle se place sur la tête une couronne de fleurs et de feuillage ${ }^{2}$. Quand Jean de Dammartin se met à la recherche de son amie Blonde d'Oxford, il l'aperçoit “ en un prael », où elle aussi ( faisoit un capel $)^{3}$.

1. Citée, fait curieux, et commentée par Étienne de Langton dans un de ses sermons. B. de Roquefort, De l'état de la poésie française dans les $\mathrm{xir}^{\mathrm{e}}$ et $\mathrm{xuI}^{\mathrm{e}}$ siècles. Paris, 1815, in-8, p. 244.

2.

La sarrazine a landemain

Vint a la fonteinne bien matin

Sy se fut lavee et peinie...

En son chef ot un chapelet

De florettes et de fenoilles.

Zwei fablianx aus einer Neuenburger Handschrift. Stuttgart, 1840 , in-8, p. 12.

3. Jehan et Blonde, r. 861. OEuvres poétiques de Philippe de Remi sive de Beaumanoir. vol. II, p. 29, v. 861-62. 
Le lendemain de ses noces, la reine du Chastiement des dames de Francesco Barberino se rend dans le jardin du palais, au milieu des roses et des fleurs, et de sa propre main elle fait une couronne pour elle et une autre pour le Roi ${ }^{1}$. Nous voyons de mème, dans le Conte du Chevalier de Chaucer, Émilie allant se promener au lever du jour dans le jardin et $\mathrm{y}$ cueillant des fleurs blanches et vermeilles, pour s'en tresser une couronne ${ }^{2}$.

Il est aussi, dans les Romances espagnoles, question de dames qui vont cueillir des roses et en font des couronnes. "Je l'ai perdue dans un jardin, dit l'une d'elles ${ }^{3}$, où elle faisait des couronnes. » Et une autre ${ }^{4}$ raconte comment, le matin de la Saint-Jean, Zara, l'épouse du roi Chico, alla cueillir des guirlandes de roses avec ses femmes les plus chères.

L'auteur anglais du Passetemps agréable nous dé-

1.

le donne menan la Reina

In un giardin tra le rose e tra fiori,

Quivi comincia di sua man la Donna,

E fa per se una sua ghirlandetta,

Una ne fa che la presenta al Re. P. 121.

2. In the gardin at the sone uprist

She walketh up and doun wher as hire list;

She gathereth floures, partly white and red,

To make a sotel gerlond for hire hed.

Canterbury tales, v. 1053-56.

3. Perdíla dentro de un huerto cogiendo rosas y flores.

Böhl de Faber, Floresta, vol. I, nº 313.

4. La mañana de San Juan Zara muger del rey Chico
Salen a coger guirnaldas

Con sus mas queridas damas.

Depping, Sammlung span. Romanzen, p. 387. 
peint lui aussi la "Belle pucelle » gaiement assise aupres de la fontaine de son jardin et faisant de " maintes fleurs belles et plaisantes un brillant chapeau $\left.{ }^{1}\right)$.

Une enluminure d'un manuscrit du British Museum, du commencement du xiv ${ }^{\mathrm{e}}$ siècle, nous montre des dames qui cueillent des fleurs dans un jardin et en font des chapeaux ${ }^{2}$. Lne autre enluminure, celle du mois de mai, dans le calendrier qui précède les Heures de la reine Anne ${ }^{3}$, représente également une dame tressant des couronnes avec des fleurs cueillies par ses femmes sur les rosiers qui entourent le jardin au milieu duquel elle est assise.

\section{III}

Une fleur qui occupait une si grande place dans les usages religieux et profanes du moyen àge ne pouvait manquer de figurer sur les monuments de cette époque, comme elle l'avait fait sur ceux de l'antiquité. Dès les premier's temps de notre ère, les artistes chrétiens furent naturellement amenés à orner de fleurs les cryptes et les cimetieres où reposaient

1. Besyde which fountayne, the most fayre lady

La bel Pucel was gayly sitting :

Of many floures fayre and jolly

A goodly chaplet she was in makyng.

The pastime of pleasure, ap. Th. Wright, p. 430.

2. Thomas Wright, op. laud., p. 289.

3. Bibl. nat., lat. 9474 . 
les restes vénérés des martyrs et mème des simples fidèles ${ }^{1}$; c'était comme un symbole de la gloire céleste devenue leur partage, ainsi que des fleurs divines et des ombrages du paradis, au milieu desquels ils reposaient. On voit aussi le plus souvent sur les tombeaux des premiers chrétiens des arbres, des fleurs, des couronnes, parfois de vrais bosquets, image allégorique du lieu de délices, tout verdoyant d'un printemps éternel ${ }^{2}$, tel qu'on se représentait le Paradis. C'est ainsi qu'au cimetière de Calliste se trouve sculptée autour d'une inscription chrétienne une branche de rosier couverte de boutons et de fleurs ${ }^{3}$.

Parfois l'âme du défunt apparaît figurée par une colombe, reposant au milieu des fleurs " dans le bien par excellence ", comme s'exprime l'inscription du tombeau de Sabinianus", dans la crypte de saint Alexandre, sur la voie Nomentane, inseription qui fait songer, avec les deux arbustes qui l'entourent, aux martyrs réunis dans le céleste verger, à l'ombre des rosiers.

Sur une des fresques de la crypte qui sert de

1. Martigny, Dictionnaire des antiquités chrétiennes. Paris, $3^{\circ}$ éd., 1877, in-8, s. v. Fleurs et Paradis.

2. Tempore continuo velinant ubi gramita rivis.

J. B. de Rossi, Inscriptiones chrislianae srlis Romae septimo saecylo antiqviores. Romae, 1861, in-fol., vol. I, p. 1'1, n $n^{\circ} 317$.

3. J. B. de Rossi, Bullettino di archeologia cristiana. Roma, in-1, vol. VI (1868), p. 12.

4.

Sabiniane spiritys trves in bono.

Martigny, op. laud., art. Fleurs et Paradis. 
monument à sainte Cécile, on voit représenté saint Urbain au milieu de rosiers à fleurs doubles ${ }^{1}$. Il semble bien aussi qu'au haut de l'arcosolium principal de la crypte dite des cinq saints ${ }^{2}$, l'artiste chrétien ait peint des branches entrelacées de rosiers à fleurs rouges, et on y voit sainte Dionysade, en particulier, entourée d'arbustes couverts de fleurs ${ }^{3}$ et de fruits.

Une fresque de l'arcosolium d'une chapelle qui se trouve dans le cimetière de Pontien, sur la via Portuensis, représente d'un côté le baptême du Christ, de l'autre la croix se dressant au milieu d'un buisson de rosiers aux fleurs doubles et épanouies ${ }^{4}$. Dans la crypte de Lucine, au cimetière de Calliste, on voit sur une autre fresque des oiseaux, emblème des âmes délivrées des liens du corps, se faisant face, posés chacun sur un tronc, de chaque côté d'un arbre, au milieu d'un champ semé de roses ${ }^{5}$. Une peinture de la tribune gauche d'une chapelle du même cimetière représente sous une figure allégorique le Printemps cueillant des roses ${ }^{6}$. A chacun

1. G. B. de Rossi, La Roma sotterranea cristiana. Roma, in-fol., vol. II, 1867, pl. 6 .

2. G. B. de Rossi, La Roma sotterranea, vol. III, pl. 1-2, p. 49.

3. Ces fleurs, qui, à en juger d'après la chromolithographie 3 de la Roma sotterranea, pourraient bien être des roses, ressemblent plutòt à des oranges dans la reproduction qu'en a donnée L. Perret, Catacombes de Rome. Paris, 1858, in-folio, pl. 49. 4. Bottari, Sculture et pitture sagre estratte dai cimiteri di Roma. Roma. 1737, in-folio, vol. I, pl. 4́, 2.

5. G. B de Rossi, Roma sotterranea, vol. I, p. 323 , pl. 12.

6. Bottari, op. laud., vol. II, pl. 55, 4. 
des quatre angles d'une fresque de la voûte d'un tombeau situé dans la troisième chambre de ce même cimetière, se trouve également, comme motif de décoration, un rosier couvert de fleurs ${ }^{1}$.

Ce sont encore, je le crois, des branches de rosier qu'on aperçoit de chaque côté des vases mystiques peints sur la voûte d'une chapelle située dans le cimetière de la Voie latine ${ }^{2}$. On ne peut pas ne pas reconnaître non plus deux rosiers, assez grossièrement figurés, il est vrai, et deux roses sur un vitrail symbolique, où est peinte une femme étrangère (jeregrina) entre saint Pierre et saint Paul ${ }^{3}$. Sur un autre vitrail, où sont aussi représentés ces deux apôtres, l'artiste a placé une rose entre eux deux ${ }^{4}$. Enfin une des quatre zones horizontales, dans lesquelles est divisée la voùte si remarquable d'une crypte historique du cimetière de Prétextat, est décorée en entier de rosiers couverts de fleurs ${ }^{5}$.

Ainsi partout dans les monuments de la primitive

1. Bottari, op. laud, vol. II, pl. 67. Dans la fresque de la voûte d'un autre tombeau, pl. 65, deux rosiers et deux lis servent aussi de motifs de décoration; dans les arabesques qui séparent les cinq groupes de la coupole d'une chapelle érigée par le pape saint Fabien, pl. 59, il faut reconnaître aussi des rosiers à fleurs simples.

2. Bottari, op. laud., vol. II, pl. 91.

3. Raffaele Garrucci, Vetri ornati di figure in oro trovati nei cimiteri dei cristiani primitivi di Roma. Roma, 1858, in-fol., p. 49, pl. xxi, 6. Dans la fig. 1, qui représente sainte Annes entre saint Pierre et saint Paul, on voit aussi une rose de chaque côté de la sainte.

4. Raffaele Garrucci, op. laud., p. 82, pl. xxxix, 4.

5. G. B. de Rossi, Bullettino, vol. I (1863), p. 3. 
Église apparaît l'image symbolique de la rose. Quand, sorti victorieux des catacombes, le christianisme les remplaça par les basiliques qui, dans leur institution première, n'étaient que les monuments destinés à protéger ou à garder les tombeaux des martyrs $^{1}$, la rose entra aussi dans l'ornementation de ces nouveaux lieux de réunion pour les fidèles. C'est ainsi que dans une mosaïque de l'église SainteAgathe-Majeure à Ravenne, qui représente le Sauveur assis sur son trône entre deux anges, l'artiste a couvert le sol de rosiers couverts de fleurs ${ }^{2}$. Des rosiers couvrent aussi le sol de la partie inférieure de la mosaïque qu'on voit dans l'église Saint-Michel de Ravenne, mosaïque où se trouve également représenté le Sauveur, mais debout entre les archanges Michel et Gabriel et tenant sa croix à la main ${ }^{3}$. Il y a dans l'église Sainte-Suzanne à Rome une mosaïque ancienne, quoique d'une époque plus récente que celles de Ravenne, sur laquelle Charlemagne est représenté recevant à genoux, de la main de saint Picrre, un étendard semé de roses"

Ce motif de décoration se voit aussi sur des étoffes antiques; Anastase le Bibliothécaire mentionne des draperies, des voiles, ainsi qu'un vêtement d'autel et

1. Ossibus altar et impositum.

Prudence, Peristephanon, III, str. 4'́, v. 2.

2. Ciampini, Velera monimenla, in quibus praecipue musiva opera... illustrantur. Romae, 1690-99, in-fol., vol. I, pl. 46.

3. Ciampini, op. laud., vol. II, pl. 17.

4. Marquis d'Orbessan, Mélanges historiques et littéraires, vol. III, p. 333 . 
des tuniques ornés de roses ${ }^{1}$. Toutefois cette ornementation, du moins dans l'Occident, semble avoir cessé d'ètre employée; elle paraît étrangère au style roman proprement dit, et pendant longtemps on n'en retrouve plus trace dans la plus grande partie de l'Europe; mais elle devait reparaitre avec le style gothique, et durant les trois siècles qu'il fleurit, tous les arts y eurent également recours, l'architecture comme la statuaire, la sculpture sur bois et sur ivoire, ainsi que l'orfévrerie qui à cette époque s'y rattachait si étroitement, la ferronnerie, comme l'émaillerie ou la verrerie, l'enluminure enfin et la peinture.

Les artistes laïques, qui donnèrent sa forme définitive au style gothique, en empruntant à la flore indigène la plupart de leurs motifs de décoration, devaient nécessairement faire une place nouvelle à la rose dans l'ornementation; cependant on ne la rencontre d'abord qu'à titre d'exception dans leurs œuvres, et c'est, non pas en France, mais en Syrie, en Espagne et en Italie que cette fleur apparait pour la premiere fois dans l'ornementation architecturale. Des roses sont sculptées au-dessus de plusieurs des

1. "Cortinas albas holosericas rosatas. " - «Tetravela alba holoserica rosata. " - "Vestem albam holosericam rosatam. " "Vestem albam sigillatam cum rosulis. » - " Vela alia alexandrina, ex quibus unum habens rotas et rosas in medio et aliud arbores et rotas. » - «Vestem habentem arbusta et rosas ». Historia de vitis romanorum pontificum, cap. 98 , an. 795 ; cap. 10, an. 817; an. 827; an. 858 (Migne, Patrologie, vol. CXXVIII, p. 1210 , 1242 (417), 1243 (419), 1266 (441), 1282 (462), 1362 (585). 
portes du château de Kalaat el Hossn, situé près du couvent grec de Saint-Georges en Syrie, mais construit par les Croisés ${ }^{1}$; - on voit les armes du comte de 'Toulouse au-dessus de l'entrée principale. Sur les murs de Djébail, lancienne Byblos, construction de la mème époque, se trouve aussi une grande rose en pierre avec deux plus petites de chaque côté ${ }^{2}$. On voit également, aux angles de l'arc en fer à cheval de la tour des deux søurs à l'Alhambra, une rose entourée d'une guirlande de feuillage ${ }^{3}$. Il y a aussi des roses sculptées, avec des perroquets, sur le narbre du tombeau de Gunther, évèque de Bamberg, travail du $\mathrm{xi}^{e}$ siecle et probablement italien ".

Mais la rose finit par prendre place à côté de la flore indigrene dans la décoration architecturale des monuments gothiques. La corbeille du second chapiteau de la tribune de lecture dans le réfectoire du prieuré de Saint-Martin-des-Champs est couverte de feuilles et de fleurs de rosier". Des branches de rosier garnies de fleurs et de feuilles décorent aussi les chapiteaux du porche de la façade de Notre-

1. Schleiden, Die Rose, p. 175.

2. John Lewis Burckhardt, Travels in Syria. London, 1822, in-4, p. 179.

3. Schleiden, Die Rose, p. 175.

4. Ch. Cahier et Arthur Martin, Mélanges d'archéologie, d'histoire et de littérature. Paris, in-'́, vol. II (1851), p. 258 et pl. $x \times x r$.

5. Statistique monumentale de Paris, Paris, 1867, in-1, - vol. I, p. 133, pl. xy, 
Dame, à Reims ${ }^{1}$. Un rosier également tapisse de ses feuilles, de ses boutons et de ses fleurs la console sur laquelle reposent les pieds de la Beauté (Pulchritudo), une des béatitudes célestes que l'on voit au porche nord de la cathédrale de Chartres, et cette femme puissante est représentée la main gauche appuyée sur un bouclier, où sont sculptées quatre roses épanouies? ${ }^{2}$. On a voulu voir aussi un rosier dans la plante qui sort du vase placé près de la statue couronnée d'une niche de la façade principale de la cathédrale d'Amiens ${ }^{3}$.

Dans le palais de la Ragione (Raison), à Padoue, on voit une femme debout sur une roue d'or et tenant trois roses à la main ${ }^{4}$. Sur le huitième chapiteau du palais des doges à Venise est sculptée l'Espérance une rose a la main". Au chapiteau sept, $2^{e}$ place, du mème palais est représentée une femme couronnée de roses et portant une robe semée de ces mèmes fleụrs, avec cette inscription :

\section{Vanitas in me habUndat ${ }^{6}$.}

Comme les roses, qui se rencontrent sur les monuments religieux et même civils du moyen âge,

1. Jules Gailhabaud, L'architecture du ve $a u$ xvir siècle et les arts qui en dépendent. Paris, 1858, in-4, vol. I.

2. Paul Durand, Monographie de Notre-Dame de Chartres. Paris, 1881, in-8, p. 93, pl. xxr.

3. H. Duserel, Notice historique sur l'église cathédrale d'Amiens. Amiens, 1839, in-8, p. 17.

4. Didron, Annales archéoloriques. Paris, in-4, vol. XXVI (1869), p. 196.

5. Didron, op. laud, vol. XVII (1857), p. 80.

6. Didron, op. laud, vol. XVII (1857), p. 81. 
affectent parfois une forme conventionnelle et ont un nombre variable de pétales, il n'est pas toujours facile de les distinguer du fleuron des monuments orientaux et grecs ou romains. Sont-ce des roses véritables, par exemple, que ces fleurs à six ou cing pétales sculptées sur un chapiteau et un pilastre de l'église de Tyr, construite par l'évêque Paulin, chapiteaux transportés par les Vénitiens sur la place Saint-Marc? ${ }^{1}$ Il est difficile de le dire, comme de beaucoup d'autres ornements de ce genre, appelés indifféremment roses, rosettes ou rosaces.

On voit une de ces roses à six pétales sur un chapiteau de l'église Saint-Laurent de Rome²; des rosettes à cinq pétales ont été sculptées sur les fonts baptismaux de l'église de Bercy et sur un autel d'Asti; il y a aussi des roses, mais a six feuilles, sur la facade du palais bâti par les Visconti a Pavie, ainsi qu'aux chapiteaux d'une vieille maison à SaintYrieix ${ }^{3}$. Il semble également qu'il y en.ait deux à l'un des chapiteaux en marbre de la nef de l'ancienne église de Montmartre ${ }^{4}$. Au-dessus de l'entrée de la tour de Ruprecht, dans la grande cour du château de Heidelberg, on voit, supportée par deux anges, une couronne de roses entrelacées de feuillage ${ }^{\sharp}$.

1. Albert Lenoir, Archilecture monastique. Paris, 1852, in-4, vol. I, p. 363 et 371 .

2. Albert Lenoir, op. laud, vol. I, p. 217.

3. Gailhaubaud, op.laud., vol. III.

4. Stalistique monumentale de Paris, vol. II, pl. жx.

5. E. L. Stieglitz, Von altdeutscher Baukunst. Leipzig, 1820 , in-', p. 184. - Baedeker, Süd-Deutschland, p. 18. 
On a dit parfois que les roses ainsi ouvrées sur les monuments étaient un emblème maçonnique, celles que l'on voit dans chacun des quatre angles de la croix placées sur la façade du Catholicon à Athènes ${ }^{1}$, ainsi que les roses sculptées à l'extrémité des deux bras, au sommet et au milieu de quelques vieilles croix, telles qu'était celle de l'ancien cimetière des Innocents ${ }^{2}$, sont bien, elles, le symbole religieux, auquel les écrivains mystiques ont si souvent comparé le sang divin du Sauveur ${ }^{3}$.

On rencontre également, mais comme simple motif de décoration, des roses sur les dallages d'anciens monuments; telles sont, par exemple, les rosaces vert-olive, qu'on voit, au milieu de rosettes rouges ${ }^{4}$, sur celui de la chapelle Saint-Pellegrin à l'abbaye de Saint-Denis. Ce ne sont là que des figures de convention; la dalle tumulaire de Jean Disse, chanoine de Notre-Dame de Noyon, aujourd'hui au Musée de l'École des Beaux-Arts, nous ramène à l'imitation de la véritable nature. A gauche de ce personnage se dresse, sorti de la gueule d'un dragon qu'il foule aux pieds, un rosier avec ses boutons, ses fruits et ses feuilles ${ }^{5}$.

1. Albert Lenoir, op. laud., vol. I, p. 271.

2. Statistique monumentale de Paris, vol. II, pl. r. Telle est aussi la croix que tient à la main, dans le man. Ellesmere, le Pardoner des Canterbury Tales.

3. W. Menzel, Christliche Symbolik, vol. II, p. 283.

4. Viollet-le-Duc, Diction. de l'architecture, art. dallage.

5. Gailhaubaud, op. laud., vol. III. - E. Müntz, Guide de l'École des Beaux-Arts. Paris, s.d., in-8, p. 57. 
Cette vérité dans la reproduction artistique de la rose que nous retrouvons ici se rencontre encore dans les ouvrages d'orfévrerie. Parmi les joyaux si nombreux que possédait le duc Louis d'Anjou se trouvaient plusieurs statuettes en argent de la Vierge, représentée avec l'enfant Jésus sur le bras gauche et tenant à la main droite une branche de rosier à fleurs vermeilles ou dorées ${ }^{1}$. Dans la volute de la belle crosse, dite de l'abbaye d'Estival, est encadrée une image de la Vierge tenant aussi une rose à la main, en même temps que sur la hampe en ivoire de ce joyau précieux court une branche de rosier couverte de feuilles et de fleurs ${ }^{2}$.

Le duc d'Anjou possédait aussi entre autres un gobelet garni à l'extérieur de feuilles de rosier et à l'intérieur duquel il y avait une rose double, avec un bouton "fait en manière de rose " sur le couvercle $^{3}$. Un bouton de rose blanche se trouvait également sur deux hanaps de Charles $\mathrm{V}$, et parmi les joyaux de ce prince, dont les lis étaient l'ornement le plus ordinaire, on voyait une cassette d'argent doré "cizelé a roses ". Des roses ciselées ou repoussées se trouvaient aussi sur de nombreux joyaux de

1. De Laborde, Inventaire des joyaux du duc Louis d'Anjou. Paris, 1853 , in- $8, n^{\text {os }} 18,37,58,67$.

2. Annales archéologiques, vol. IV (1856), p. 2ł9, pl. xvirr. Ainsi que les feuilles, les fleurs de ce rosier sont simples et à cinq pétales.

3. Inventaire du duc d'Anjou, no 213.

4. Jules Labarte, Inventaire du mobilier de Charles $V$, roi de France. Paris, 1879, in-4, nos 2363, 2365 et 2562. 
-Louis d'Anjou, ce grand curieux d'art, en particulier sur divers bassins d'argent ${ }^{1}$. On en voit mème sur des agrafes à cinq et à six pétales²

Ce n'est pas seulement en France et sur des joyaux princiers ou royaux que la rose fut employée comme motif de décuration; on la rencontre de mème sur des bijoux de fabrication étrangère et jusque dans l'extrême nord; dans une ballade danoise ${ }^{3}$, il est question d'anneaux d'or, sur lesquels sont ciselés des lis et des roses.

Un joyau du commencement du $\mathrm{xv}^{0}$ siècle mérite ici une mention spéciale, à cause de sa beauté et de sa richesse, c'est le Rössel d'or qui se trouve dans le trésor de la sainte chapelle d'Alttötting en Bavière, mais qui est de fabrication française ${ }^{4}$. De chaque côté de la Vierge-mère, qui, avec sainte Catherine, saint Jean-Baptiste et saint Jean l'Évangéliste, compose le groupe le plus important de ce magnifique objet d'art, se dressent deux rosiers, couverts de fleurs à cinq pétales; un personnage agenouillé à droite et au-dessous de ce groupe, et dans lequel on a cru reconnaitre le roi Charles VI, porte lui-mème une couronne de roses et la selle de son coursier, que tient en laisse un page, est elle aussi semée de roses.

1. Inventaire du duc d'Anjou, nos $591,592,617,627$.

2. Viollet-le-Duc, Dictionnaire raisonné du mobilier francais. Paris, in-8, vol. II, p. 198 et 226.

3. Es lagen darinnen Goldringe fünf

Aus Rosen und Lilien getrieben.

Wilh. Grimm, Altdänische Heldenlieder, p. 357, no 88.

4. Annales archéologiques, vol. XXVI (1869), p. 119. 
Si ce produit de l'ancienne orfèvrerie est le plus précieux ou figure la rose, le plus célèbre est la " rose d'or ", joyau qui en réalité est un rosier en or garni de feuilles, de boutons et de fleurs. Ce rosier mystique est béni par le pape, avec une solennité toute particulière, le quatrième dimanche de carême ou de Lætare Hierusalem ${ }^{1}$.

De temps immémorial, la rose d'or a été destinée à récompenser quelque prince ou quelque grand, distingué, soit par son mérite, soit par des services particuliers rendus à l'Église. Le plus ancien document où il en soit fait mention remonte au $\mathrm{xi}^{\mathrm{e}}$ siècle. Ce fut sans doute, en effet, une rose que la "fleur d'or ", remise par Urbain II, lors de sa visite à l'église Saint-Martin de Tours, au duc Foulques d'Anjou. Une lettre d'Eugène III à Alphonse de Castille, en 1145 , paraît bien aussi faire allusion à la rose d'or. Il en est question du moins en 1159 d'une manière irréfragable; cette année-là, Alexandre III en donna une à Louis VII, en témoignage de sa reconnaissance pour l'accueil qu'il avait reçu pendant son vorage en France. Douze ans après, le même pontife remit également une rose d'or au doge de Venise, comme une marque de la "faveur spéciale du Saint-Siège ${ }^{2}$ ".

La statue de Raymond-Béranger IV, qui se trouve à $\mathrm{Aix}$, dans l'église Saint-Jean-de-Malte, serre de la

1. Sacrarum caerimoniarum... sanctae romanae ecclesiae libri tres. Coloniae, 1572, in-8, lib. I, p. 81, et lib. II, p. 223.

2. Du Cange, s. v. Rosa aurea. 
main droite sur sa poitrine une fleur, reproduction de la rose d'or qu'en 124'́ le pape Innocent VI envoya à ce prince pour reconnaître son zèle envers l'église'. Quand, durant le carème de l'année 1367 , Jeanne, reine de Naples, vint à Rome arec Pierre, roi de Chypre, Urbain V, le dimanche de Lxtare, lui donna, "comme à la princesse la plus noble et la plus digne, "la rose d'or qu'il venait de consacrer ${ }^{2}$.

Un “rosier d'or » est mentionné dans l'Inventaire de Charles V, ce qui doit faire supposer que ce prince reçut aussi ce présent papal ${ }^{3}$. Le musée de Cluny possède une magnifique "rose d'or ", composée d'une tige garnie de six feuilles et surmontée par une fleur épanouie, ornée à son centre d'un beau saphir. De cette même tige partent cinq branches, qui portent ensemble vingt-cinq feuilles, trois fleurs et deux boutons". Ce précieux joyau, qui fit longtemps partie du trésor de Bâle, fut donné par le pape Clément $\mathrm{V}$ au prince-évêque de cette ville dans les premières années du xiv $^{\mathrm{e}}$ siècle.

Parmi les princes les plus connus qui ont, depuis

1. Aix ancien et moderne. Aix, 1833, in-8, p. 169. Millin, Voyage dans les départements du midi de la France, Paris, in-8, vol. II (1807), p. 287, dit que Raymond déposa cette rose dans l'église Saint-Sauveur.

2. Vita Urbani $V$, ap. du Cange, s. v. rosa aurea.

3. “Un rosier d'or a tenir en sa main ouquel a in pommelles rons et est la rose que le pape donne le jour de la mi caresme au plus noble. » De Laborde, Émaux, p. 487.

4. E. du Sommerard, Catalogue des objets d'art du Musée de Cluny. Paris, 1884, in-8, p. 403, ${ }^{\circ} 5005$.

JoREt. La Rose. 
cette époque, été gratifiés de la rose d'or, il faut citer l'empereur Sigismond, qui la reçut successirement de Jean XXIII et de Martin V. En 1446, elle fut envoyée par Eugène IV à Henri VI d'Angleterre, et Pie II en fit don en 1461 à Thomas Paléologue, qui venait d'ètre dépouillé de ses États par les Turcs. Jules II et Léon X l'envoyèrent tour à tour à Henri VIII, ce qui n'empêcha pas ce prince de rompre avec la cour de Rome. Le présent qui en fut fait à Frédéric le Sage, électeur de Saxe, ne put le détourner non plus de se poser en protecteur de la Réforme ${ }^{1}$.

Comme dans la sculpture sur pierre et l'orférrerie, on rencontre la rose dans la sculpture sur ivoire et sur bois. On la trouve tout naturellement sur les nombreuses réprésentations du Siège du Château d'Amour. Ici, toutefois, comme dans la représentation du Lai d'Aristote, sur un coffret du $\mathrm{xIv}^{\mathrm{e}}$ siècle, où l'on voit Alexandre jeter d'une fenètre de son palais une rose à son ancien maître et à Campaspe qui l'a

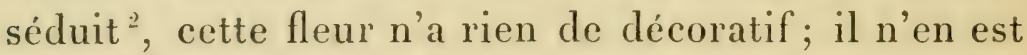
plus de même dans les boiseries où parfois on la trouve sculptée, par exemple sur les vantaux de la porte de l'église à Voulte-Cilhac, décorés de rosettes à six ou sept feuilles, ainsi que sur les boiseries des armoires de la sacristie de Santa Maria in Organo à Vérone, ornées de roses formées d'un double rang de cinq pétales alternés ${ }^{3}$. C'est bien un motif de

1. Schleiden, Die Rose, p. 108.

2. Collection Spitzer.Paris, 1889, in-fol., vol. I, p. 52, pl. xxI.

3. Gailhabaud, op. laud., vol. II. 
décoration qu'on a cherché en sculptant ces roses; c'est dans le mème but aussi qu'ont été ouvrées entre autres les rosettes que l'on voit sur les stalles de l'église Saint-Pierre à Pérouse, et sur celles de Saint-Andoche de Saulieu, ainsi que sur un des panneaux d'une salle de Middle Temple à Londres ${ }^{1}$.

Ce motif de décoration tiré de la rose a été parfois aussi employé dans la ferronnerie; mais là cette fleur affecte les formes les plus fantaisistes; telles sont, par exemple, les rosettes à quatre feuilles quadrangulaires qui terminent les branches des ferrures d'une porte du château de Lahneck, ainsi que celles du grillage d'une maison à Troyes ${ }^{2}$. Sur les vantaux d'une porte en fer, qui se trouve à Rouen, on retrouve, au contraire, des roses aux formes naturelles, encore qu'elles aient six grandes feuilles extérieures, alternant avec six plus petites à l'intérieur. Plus tard la ferronnerie, comme les autres arts industriels, s'appliqua à reproduire les roses avec la plus grande exactitude; c'est ainsi qu'une grille non cataloguée, mais probablement d'une date assez récente, qu'on voit au premier étage du Musée de Cluny, est ornée de roses d'une ressemblance parfaite.

Mais dans les arts dont je viens de parler, la rose ne joue comme ornement et même comme symbole qu'un rôle secondaire; il en est tout autrement dans

1. J. Gailhabaud, op. laud., vol. III et IV. - Viollet-le-Duc, art. Stalles.

2. J. Gailhabaud, op. laud., vol. II et III. Les rosettes du grillage à 'Troyes ont deux fois quatre feuilles. 
l'enluminure et dans la peinture. Dans le premier de ces arts, toutefois, la rose, tant les premier's enlumineurs montrèrent d'indifférence à la reproduire', n'apparait que sous une forme conventionnelle. Ainsi dans la plupart des anciens manuserits du Roman de la Rose, les rosiers que l'amant voit en songe ou qui croissent dans le jardin du dieu d'Amour n'ont pas de feuilles ou n'ont que des feuilles simples, ils n'ont aussi d'ordinaire que des fleurs simples, et presque indifféremment à cinq, six, ou mème quatre pétales ${ }^{2}$; c'est exceptionnellement et relativement assez tard que ces roses sont représentées comme doubles. Le rosier du manuscrit 305 de la Bibliothèque nationale, par exemple, manuscrit $\mathrm{du} \mathrm{xv}^{0}$ siècle, est encore à feuilles simples, mais il a des fleurs doubles et vermeilles; les deux rosiers qu'on roit à la première page du manuscrit 25,526, qui est pourtant seulement du $\mathrm{xiv}^{0}$ siècle, ont non seulement des fleurs doubles, - celles du premier blanches, les roses du second vermeilles, mais les feuilles, quoique assez inexactement dessinées, sont la plupart trilobées ${ }^{3}$; des branches de

1. L'enluminure du man. fr. 22928 de la Bibliothèque nationale qui représente le moine des Miracles de Gautier de Coincy, dans la bouche duquel on trouve après sa mort "cinq roses nouvelles», ne reproduit aucune de ces fleurs.

2. Les fleurs du rosier du man. 1567, fol. $26 b$, xrve siècle, ont l'une six, trois cinq et une quatre pétales. Le man. 1560 n'a que des fleurs à six pétales, etc.

3. Les feuilles des rosiers du man. 803 sont également composées. 
rosier servent de plus d'encadrement au texte de ce premier folio.

La rose devint à cette époque avec quelques autres fleurs, dont le nombre ira bien vite en augmentant, un des motifs de décoration le plus recherchés par les enlumineurs. La passion des princes du temps pour les beaux manuscrits ${ }^{1}$ les avait encouragés à en perfectionner l'ornementation; le développement du sentiment de la nature, dans l'art, dû peutêtre à l'influence des “imagiers » flamands ${ }^{2}$ et des peintres italiens, avec le goût croissant pour les fleurs $^{3}$, en fit multiplier les représentations et porta à y chercher des motifs de décoration; c'est ainsi que la rose, les fraises, l'oillet, plus tard bien d'autres plantes, sont entrés dans l'ornementation des manuscrits de la fin du moyen âge.

Un des plus anciens et des plus beaux où la rose ait été employée à cet usage est le manuscrit 620 de la bibliothèque Méjanes, - les Heures du roi René, - daté de 1458 ; les enluminures de la majuscule du mot Deus ${ }^{4}$ aux pages 418 et 447 , sont un chef-

1. Il suffit de citer Charles V et ses frères, en particulier le duc de Berry, "le plus grand curieux de son temps». Renan, Discours sur l'état des Beaux-Arts au xıve siècle, p. 262. - Louis Gonse, L'art gothique. Paris, 1890, in-8, p. 602.

2. Léon de Laborde, Les ducs de Bourgogne. Études sur les lettres, les arts et l'industrie pendant le xve siècle. Paris, 18'9-52, in-8, vol. I, Introduction, p. 81, note 1.

3. Lecoy de la Marche, Les manuscrits et la miniature. Paris, 1889 , in-8, p. 231.

4. On a prétendu que ces enluminures étaient l'œurre du roi 
d'œuvre d'exécution, surtout celle de la page 447, qui représente une branche de rosier portant trois fleurs vermeilles arec deux feuilles composées et deux entières. Si elles sont moins belles, on rencontre un bien plus grand nombre de roses dans les illustrations du manuscrit 74 de la mème bibliothèque ${ }^{1}$; elles garnissent en partie plusieurs des vignettes qui encadrent le texte.

De magnifiques branches de rosier couvertes de fleurs vermeilles servent aussi d'encadrement aux folios $6 a, b, 18 a, 49 a, b$ du manuserit latin 1159 de la Bibliothèque nationale. Les roses des enluminures du Bresiarium Sarisberiense ${ }^{2}$ sont également remarquables par leur habile reproduction; il en est de même des rosiers en treillis du livre d'Iteures d'Étienne Chevalier, livre antérieur à l'année $1474^{3}$. Mais rien n'égale la perfection de formes et la finesse d'exécution des roses, que Bourdichon a, trente ans plus tard, peintes, arec 338 autres espèces de fleurs",

René lui-mème. Fauris de Saint-Vincens, Mémoires et notices relatifs à la Provence. Paris, 1814, in-8, p. 29.

1. Man. de Mgr. Rey, évêque de Dijon, qui en a fait cadeau à la Méjanes.

2. Bibl. nat., lat. 17294. Par exemple le rosier à fleurs vermeilles qui sert d'encadrement au folio $8 a, b$.

3. Bibl. nat. lat. 1416. C'est la date de sa mort. Quant à la corbeille de roses qu'on voit au-dessous du treillis, c'est l'œurre d'un peintre moderne. Je pourrais encore citer les roses à fleurs doubles et vermeilles du man. $\mathbf{1 1 7 6 .}$

4. Lud. Lalanne, Mém. inédit d'Ant. de Jussieu sur le live d'heures d'Anne de Bretagne, p. 6. (Extr. du Bulletin historique et philologique, an. 1886.) 
dans les Heures d'Anne de Bretagne; les roses vermeilles qui forment l'encadrement du folio 27 a, et les roses blanches et rouges ${ }^{1}$ du folio 31 a sont de la plus exquise beauté.

Dans la peinture proprement dite, qui offre un champ plus libre et plus vaste, la rose devait occuper une place encore plus grande que dans l'enluminure, et elle y apparaît dès les premiers temps de la restauration de ce grand art en Occident. Les artistes qui la firent entrer dans leurs œuvres ne faisaient que se conformer à l'ancienne tradition et continuer ce que l'on n'avait pas cessé de faire en Orient. Le Guide de la peinture de Denys ${ }^{2}$ recommande entre autres de représenter avec une couronne de fleurs - probablement de roses - le moine véritable. Les fresques des premiers monuments chrétiens n'étaient-ils pas, nous l'avons vu, souvent ornés de roses? Les peintres du moyen âge s'inspirèrent de cet exemple.

La Charité de Giotto, que l'on voit sur une fresque de l'église de l'Arena à Padoue, porte une couronne de roses doubles", et dans la corbeille qu'elle tient

1. Ces dernières sont désignées par l'appellation de « roses de la marqque d'Ancone ».

2. 'Traduit par Paul Durand sous le titre de Manuel d'iconographie chrétienne, grecque et latine. Paris, 1845, in-8, p. 403.

3. Didron, Annales archéologiques, vol. XXI, p. 8, a vu dans les fleurs de cette couronne des renoncules, non des roses, les gravures et la pholographie de cette fresque ne permettent pas de douter que Giotto n'ait donné à la Charité une couronne faite de ces dernières fleurs. 
à la main droite se trouvent aussi des roses avec des fleurs de lis et des grenades. Sur une fresque de Benozzo Gozzoli, qui représente l'Adoration des Mages, des buissons de rosiers bordent la route que suivent ses personnages ${ }^{1}$. Dans le tableau du Musée du Lourre, où le Pérugin a peint la Vierge avec l'enfant Jésus, qu'adorent deux anges et deux saintes, l'une de celles-ci, sainte Rose, tient à la main une branche de rosier couverte de boutons et de fleurs². Sandro Botticelli, dans son tableau de "la Vierge, l'enfant Jésus et saint Jean ", a représenté le premier couronné de roses. Un autre tableau du mème peintre, "le couronnement de la Vierge, " nous montre les parvis célestes jonchés de fleurs de roses $^{3}$. Une toile de la galerie de Dresde, attribuée aussi à Gozzoli, représente la Madone avec l'enfant Jésus, qui tient une rose à la main". Dans son tableau de saint Georges et de saint Étienne, Jean Bellini a donné au premier une couronne de roses". Et Léonard de Vinci a représenté la Vanité une fleur à la main et trois roses dans les cheveux ${ }^{6}$.

Dans un tableau de Tan Eyck, "la Vierge et l'enfant Jésus », la Madone tend une branche de rosier au divin enfant et des roses ornent le dossier du

1. E. Müntz, Les précurseur's de la Renaissance, p. 151.

2. Charles Blanc, Histoire des peintres. École ombrienne.

3. Histoire des peintres. École florentine.

4. Le mème sujet a été traité par Carlo Dolce; mais dans son tableau l'enfant Jésus tient à la main une branche de rosier.

5. Histoire des peintres. École vénitienne.

6. Iristoire des peintres, École florentine. 
siège où elle est assise. Un tableau de Van der Goes, à la Pinacothèque de Munich, représente le même sujet, mais c'est un ange qui offre des roses à l'enfant Jésus'. Stéphan Lochner a peint la Madone au milieu d'un bosquet de rosiers². Un tableau de Schœngauer, qui se trouve dans la cathédrale de Colmar, représente aussi la Vierge assise arec l'enfant Jésus sur un gazon verdoyant entouré de rosiers en fleurs, au milieu desquels chantent des oiseaux ${ }^{3}$. Dans un de ses tableaux, qui se trouve à Prague, Albert Durer a peint la Vierge couronnée de roses par deux anges en présence de l'empereur Maximilien, de l'impératrice, de l'artiste lui-même et de son ami Willibald ${ }^{4}$. Lucas de Leyde a représenté aussi la Charité accompagnée de deux enfants, dont l'un lui offre une rose ${ }^{5}$.

Dans toutes ces œuvres, la rose a pris un sens symbolique que l'on ne peut méconnaitre; il apparait d'une manière encore plus manifeste dans une gravure qu'on roit en tìte d'un vieux missel du diocèse de Cologne ${ }^{6}$. La Vierge y est représentée sous les traits d'une jeune fille ayant à sa gauche un rosier portant deux fleurs; plus haut on voit un buisson de rosiers avec la devise Plantatio rosarum.

1. Histoire des peintres. École flamande.

2. Waagen, Geschichte der Malerei, ap. École allemande.

3. W. Menzel, Die christliche Symbolik, vol. II, p. 281.

4. Histoire des peintres. École allemande.

5. Meussel, Miscellanen, ap. Schleiden, p. 188.

6. Rohault de Fleury, La sainte Vierge, études archéologiques et iconographiques. Paris, 1878, in-4, vol. 1, p. 34. 
Les grands peintres de la Renaissance sont restés fidèles à cet emploi symbolique de la rose. Dans une des saintes familles de Raphaël on voit un ange qui répand des roses sur l'enfant Jésus et sa mère ${ }^{1}$. Le Dominiquin a représenté la Madone répandant des roses sur les martyrs. Un ange, dans le tableau de " la Nativité ) de Procaccini, en répand aussi sur la divine crèche ${ }^{2}$. Dans son tableau si gracieux de la "Fuite en Égypte ", Johann Rottenhammer nous montre des anges cueillant des fleurs sur un buisson de rosiers, tandis que le petit Jean-Baptiste en apporte une corbeille à l'enfant Jésus ${ }^{3}$. Claude Lorrain a peint la Madone avec son divin enfant tenant une rose à la main, et, dans son "Assomption ", Vouet a représenté la Vierge qui s'élève au ciel, laissant après elle dans son tombeau des roses et des fleurs ${ }^{4}$. Murillo a perpétué le souvenir du miracle des roses de saint Francois, et Alonso de Tobar peindra mème, allusion au rosaire, la Vierge nourrissant, " divine pastoure $)$, ses brebis avec des roses ${ }^{5}$.

Il va sans dire que, quand ils ont représenté des sujets profanes, les peintres de la fin du moyen àge se sont inspirés de l'emploi que les artistes de l'antiquité ${ }^{6}$ avaient fait de la rose. Dans le tableau où

1. Histoire des peintres. École ombrienne.

2. Histoire des peintres. École bolonaise.

3. Musce de Dresde. Histoire des peintres. École allemande.

4. Histoire des peintres. École francaise.

5. Histoire des peintres. École espagnole, app., p. 26.

6. Sur une plaque de colfret du $r^{\mathbf{e}}$ siècle de notre ère, le tau- 
Botticelli a peint Vénus sortant du sein de la mer, il nous montre une pluie de roses tombant sur la déesse et courrant les flots ${ }^{1}$. Le Titien a représenté aussi la déesse de l'amour étendue sur un coussin de velours rouge couvert de roses blanches². Dans le tableau des Noces de Psyché, par Raphaël, à la Farnésine, des génies ailés répandent des fleurs et des roses sur les deux époux et les serviteurs eux-mêmes sont couronnés de roses ${ }^{3}$. Raphaël a représenté aussi, sur une des fresques de cette galerie célèbre, la "Première heure du jour " un bouquet de roses à la main ${ }^{4}$; de mème, dans un tableau du Guide, on voit l'Aurore répandre, à son apparition, des roses sur la terre $^{5}$, etc.

Mais la rose n'a pas été employée seulement comme symbole par les peintres du moyen âge; ils s'en sont encore servis comme d'ornement; ils ne faisaient en cela qu'imiter leurs précurseurs de l'antiquité, qui ont prodigué les fleurs dans la décoration de leurs édifices privés et publics. Une ancienne fresque du château de Lichtenberg dans le Trrol, qui date du commencement du xive siècle, représente un homme et deux femmes cueillant dans des corbeilles sur un

reau de l'enlèvement d'Europe est représenté portant autour du cou une guirlande de roses. Collection Spitzer, vol. I, p. 29.

1. E. Müntz, Les précurseurs de la Renaissance, p. 203.

2. Histoire des peintres. École vénitienne.

3 Anton Springer, Raffael und Michelangelo. Leipzig, 1878, in-8, p. 345 .

4. Schleiden, Die Rose, p. 187.

5. Histoire des peintres. École bolonaise. 
gigantesque rosier de magnifiques roses doubles, dont les deux femmes portent aussi des couronnes ${ }^{1}$. Les roses de cette fresque, représentation d'une scène de la vie commune, servaient en mème temps à orner les murs de la salle où elles se trouvaient. La peinture finit en effet par employer les roses comme motif de décoration, comme le faisait déjà l'enluminure; c'est ainsi qu'elles servirent, avec les autres fleurs, d'encadrement aux tableaux, en particulier aux portraits. Les artistes y trouvèrent aussi le sujet de quelquesunes de leurs plus gracieuses compositions; mais ce ne fut guère que dans la seconde moitié du xrri siècle que les peintres cherchèrent, dans la reproduction des fleurs et des roses, la matière à de véritables tableaux; elles avaient longtemps auparavant pris place dans la tapisserie et sur les étoffes.

Dès les premiers siècles du moyen âge on avait recherché les "draps de soie » et les "pailes » ornés d'animaux, d'oiseaux ou de fleurs. Si on cessa peutètre pendant plusieurs siècles d'en faire en Occident, ces étoffes continuèrent toujours d'ètre fabriquées en Orient. C'était de là qu'on les faisait venir. Dans un document du $\mathrm{xnI}^{\mathrm{e}}$ siècle $^{2}$, il est question

1. Alvin Schulz, Deutsches Leben im xry. und xr. Jarhundert. Wien u. Prag; 1892, in-8, pl. 9.

2. "Item capa magistri Joh. de S. Claro de quodam panno Tarsico, viridis coloris, cum plurimis piscibus et rosis de aurifilo contextis. " - " Item casula de panno Tarsico, indici coloris, cum pisciculis et rosulis aureis. "Visit. facta in thesauro S. Pauli Lond. ap. Fr. Michel, Recherches sur les étoffes de soie, d'or et d'argent. Paris, 1852, in-4, vol. II, p. 164, note 1. 
d'une chape faite de "drap de Tharse, de couleur verte, orné de poissons et de roses brodées en or ", ainsi que d'une chasuble de mème étoffe, mais de couleur bleue, décorée de petits poissons et de rosettes d'or. Les poètes du Xiri ${ }^{\mathrm{e}}$, du $\mathrm{xIv}^{0}$ et du $\mathrm{xv}^{\mathrm{e}}$ siècle parlent souvent de ces étoffes, dont les grands aimaient à se parer, et qu'on fabrique maintenant en Italie $^{1}$ et bientôt dans les Flandres.

Ils sont d'un drap d'or a oisiax

Vestu, a flors et a lunettes ${ }^{2}$,

lit-on dans le Roman de l'Escoufle. Il est fait mention, dans l'Inventaire du mobilier de Charles $\mathrm{V}$, d'un "chaperon fourré, orné de broderies et à plusieurs roses ", ainsi que d'une pièce de "soudanin sur champ d'azur semé de petites feuilles et de petites roses d'or $^{3}$ ». Le roman de Perceforest parle d'un roi "vestu d'une cotte de samyt blanc, estincelée de rosettes d'or ${ }^{4}$ ). "Il était couvert de broderies, dit Chaucer de son écuyer", et l'on eût dit une

1. "Cinq pieces de drap de soye de Lucques blancs, ourrez a grans osteaulx... et a petites rosettes ou milieu. » Inv. du mobilier de Charles $V, \mathrm{n}^{\circ} 3355$.

2. Cité par Fr. Michel, op. laud., vol. I, p. 360.

3. Nos 3902 et 3377. Le soudanin était une étoffe de soie.

4. Hystoire du roy Perceforest, vol. I, chap. 44. Le samit était une espèce de brocart de soie très fort. Alvin Schulz, Das höfische Leben, vol. I, p. 259.

5. Embroidered was he, as it were a mede All' ful of freshe floures white and rede.

The Canterbury tales. Prol. v. 89. 
prairie toute pleine de fraiches fleurs blanches et vermeilles $)$.

Ces fleurs servaient en particulier d'ornement aux tapis. L'Inventaire de Charles V mentionne entre autres une "chambre blanche a rozes vermeilles", et une autre " chambre de camocas vert tout d'une soye a rozes et à lettres de Damas ${ }^{1}$ ». Dans l'hôtel de Bourgogne qu'habitait a Paris le duc Louis d'Orléans, il y avait " une pièce tendue de drap d'or à roses, bordé de velours vermeil $)^{2}$.

Les "Comptes de Bourgogne " font mention d'une " tapisserie de haute lice... garnie de ciel, dossier et couverture de lit... (dont) les dits dossier et couverture sont au bout d'en hault faiz de trailles de rosiers sur champ vermeil $n^{3}$. Il s'y trouve anssi six tapis blancs " ourrez de roses ». La collection Spitzer renferme plusieurs tapisseries historiques du $\mathrm{xx}^{0}$ siècle dans l'encadrement desquelles on voit des roses de la plus grande beauté. Une autre tapisserie qui représente l'adoration des Nages, mais qui est du $\mathrm{xvi}^{\mathrm{e}}$ siècle et de fabrication italienne, est également encadrée de deux magnifiques rosiers, celui de droite à fleurs blanches, celui de gauche à fleurs

1. Nos 3330 et 3356 . Le mot " chambre " est synonyme de "tenture de chambre ". On donnait le nom de camocas à une espèce de satin.

2. Fr. Michel, op. laud., vol. II, p. 393.

3. Inventaire des joyaux d'or et d'argent de Philippe le Bon, no 4262. (Léon de Laborde, Les ducs de Bourgogne, etc., vol. II, p. 268). 
rouges ${ }^{1}$. Des roses avec des raisins forment aussi l'encadrement des belles tapisscries qui ornent le chøur de l'église métropolitaine d'Aix².

Comme dans l'enluminure, la peinture et la tapisserie, la rose fut employée dans la décoration des émaux, mais elle y a le plus souvent ce caractère conventionnel que nous avons rencontré parfois dans la sculpture; simple d'ordinaire et avec un nombre variable de pétales, elle n'y est aussi bien souvent que de petites dimensions: c'est une "rosette ». C'est ainsi qu'on la rencontre sur un grand nombre de joyaux du duc d'Anjou ${ }^{3}$ et de Charles V ${ }^{4}$. L'aiguière d'argent qui porte le $n^{\circ} 430$ dans l'Inventaire de Louis d'Anjou, par exemple, avait, au dedans émaillé du couvercle, une " rose de trois feuilles vermeilles et trois vertes avec le milieu jaune». Sur le pied d'une croix d'or "à trois marches », que possédait Charles $V^{\prime}$, on voyait des « rozettes esmaillées de rouge cler ». Le couteau du $n^{\circ} 2732$ avait aussi un manche d'émail ( à roses vermeilles et blanches ».

On rencontre également la rose dans l'ornemen-

1. Nos 1, 3, 5 et pl. I et III. Cf. Müntz, La tapisserie, Paris, s. d., in-8.

2. Ces tapisseries, faites en 1511, représentent les principaux épisodes de la vie du Christ; elles passent pour avoir appartenu à l'église Saint-Paul de Londres. F(auris d(c) S(aint) V(incens), Mémoire sur la tapisserie du chour de l'église cathédrale d'Aix, Aix, 1816, in-8.

3. Inventaire du duc d'Anjou, nos 137, 140,350, 391, 431, 432,506 , etc.

4. Inventaire de Charles $V$, n $\mathrm{n}^{\text {os }} 291,397,1303,1387$, etc.

5. No 2574 de l'Inventaire. 
tation des vitraux du $\mathrm{xm}^{0}$ et des $\mathrm{xr}^{\mathrm{e}}$ et $\mathbf{x} \mathrm{v}^{\mathrm{e}}$ siècles; dans le vitrail de sainte Anne a la cathédrale de Chartres, la mère de la Vierge est représentée tenant de la main droite un sceptre qui s'épanouit au sommet en trois roses blanches ${ }^{1}$. Sur une verrière de la cathédrale de Fribourg en Brisgau est peinte la Madone avec l'enfant Jésus, tenant à la main une rose vermeille et à qui elle offre une pomme ${ }^{2}$. Dans le médaillon du vitrail d'une des fenètres de Middle Temple, à Londres, on voit, faisant pendant à une fleur de lis, une rose, mais à quatre pétales et à feuilles simples ${ }^{3}$.

Enfin, la rose a servi dans la décoration des faïences, en Occident, du moins. Il est vrai qu'elle n'y apparaît guère que dans des ouvres postérieures au moỳen âge et la plupart mème modernes; mais on la trouve, dès le $\mathrm{x}^{0}{ }^{0}$ siècle, avec un caractère d'originalité toute particuliere, dans les produits d'une fabrique de l'Europe orientale, ceux de Lindos en Chypre; les nombreux spécimens qu'en possède le Musée de Cluny ${ }^{4}$ nous montrent des branches de rosier garnies de feuilles composées, de fleurs épanouies et de boutons dont la forme trahit l'influence persane qu'avait subie cette fabrication.

1. Paul Durand, op. laud., p. 146.

2. Art. Martin et Cahier, Vitraux peints de Saint-Etienne de Bourges. Paris, 1841, in-fol., pl. XII. On roit aussi des rosaces à six pétales sur la verrière de la planche $\mathrm{V}$ et à huit pétales sur celle de la planche XIII.

3. J. Gailhabaud, op. laud., vol. III.

4. Du Sommerard, Catalogue du Musée de Cluny, p. 180. 
Ce n'est plus comme motif de décoration, mais comme emblème allégorique que la rose apparaît dans les armoiries, ainsi que sur les monnaies et divers insignes. L'Armorial général de la France en dix volumes ${ }^{1}$ ne contient pas moins de soixante écussons, où se trouve la rose, et il est loin de les donner tous; la plupart ne remontent guère au delà du $\mathrm{xv}^{\mathrm{e}}$ siècle, c'est-à-dire à l'époque où la rose est devenue l'objet d'une culture toute particulière; le plus grand nombre sont du $\mathrm{xvi}^{\mathrm{e}}$ siècle, et on les rencontre également dans toutes les provinces.

Parmi les familles les plus anciennes et les plus connues, dans les armoiries desquelles se trouve lo rose, on peut citer les Riquetty de Provence ${ }^{2}$, les Chappuis du Lyonnais ${ }^{3}$, les Lamirault de l'Orléanais $^{4}$, xu ${ }^{e}$ siècle, les Chavagnac d'Auvergne ${ }^{5}$, les Crugi de Marcillac łAuvergne et Quercy), xıv ${ }^{e}$ siècle, les Caquerai (sénéchaussée de Vitré ${ }^{6}$, les Chastenai de Lanti, les Courtoux de Noyan, les Cussi de la Basse-Normandie ${ }^{7}$, les Fournier d'Isamberteville (vicomté d'Arques), les Gervais de Roquepiquet (Agénois, les Longueil de Paris, les Macé de Gastines et les Le Roy de Macé (Normandie), les Malherbe, qui

1. Paris, 1738-68, in-folio.

2. “ D'azur à une bande d'or accompagnée en chef d'une demifleur de lis et en pointe de trois roses d'argent. ")

3. " D'azur à une fasce d'or, accompagnée de trois roses d'argent. »

4. "D'or à une rose de gueules et un chef de même. "

5. "De sable à trois fasces d'argent et trois roses d'or en chef. »

6. " D'or à trois roses de gueules, deux en chef et une en pointe. "

7. “ D'azur à une fasce d'argent, accompagnée en chef de deux roses de mềme. ") 
" portaient d'hermines à six roses de gueules posées trois en chef, deux en face et deux en pointe ${ }^{1}$, etc.

Des roses se trouvaient en particulier dans les armoiries des familles qui portaient le nom de cette fleur ou d'un de ses dérivés rrais ou supposés; c'est ainsi qu'on en voit dans celles des Rose (Paris) et Roze (Berri) ${ }^{2}$, des Rosier (Dauphiné) ${ }^{3}$, et Rosié (Guyenne), des Rozey (Roumois), comme des Rosendal (Flandre). Des villes elles-mêmes en avaient; Grenoble " porte d'or à trois roses de gueules».

La rose ne se rencontre pas moins souvent qu'en France dans les armoiries des autres pays. Florence a. une rose dans son écu; on voit cing roses à cinq feuilles chacune, au milieu d'une rosace à huit pointes, sur le sceau du chapitre de Christ-Church, a Cantorbéry ${ }^{5}$; la rose blanche et la rose rouge, qui se trouvaient dans les armes des maisons d'Yorck et de Lancastre, sont restées célèbres, comme la rivalité de ces deux familles ${ }^{6}$. Il y avait une églantine dans les armes du margrave de Misnie et d'une prin-

1. Armorial général. Bibl, nat. Normandie-Caen, n 277.

2. “Porte d'argent à trois roses de gueules, tigées, feuillées et pointées de sinople. »

3. "Porte d'argent à un rosier de sinople, fleuri de trois roses de gueules. »

4. "Porte de gueules à un chevron d'or, accompagné de trois roses de cinq feuilles chacune. »

5. Revue archéologique, $1^{\text {re }}$ série, vol. X, p. 231.

6. Renowned Yorck the white Rose gave,

Brave Lancaster the red.

R. Johnson, A crown garland of goulden Roses (Percy Sociely, voi. VI, p. 3). 
cesse de Lippe enterrée à Saint-Goar ${ }^{1}$. Le chapitre d'Altenbourg portait une rose rouge dans son écu. On en voit une également dans les armes de Magdebourg, ainsi que dans celles des seigneurs de Gutzkow en Poméranie, des princes-évêques de Schleinitz, des comtes de Stolberg et de Lowenstein. Les barons de Trautmannsdorf, les chevaliers de Schwartzenberg, etc., les seigneurs de Grumbach, Neuendorf, Friesen, de Rosenberg, de Rosenbusch, de Roseneck, etc., les burgraves de Strasbourg, Alversleben, etc., des patriciens de Francfort, de Nurenberg, d'Augsbourg, les villes de Hagenau, de Rosenfeld, Rosenheim, Wertheim, etc., avaient des roses dans leur écusson ${ }^{2}$.

On retrouve encore la rose sur diverses monnaies et plusieurs insignes; cette fleur a donné son nom aux " rosenobles » établis par Édouard III, elle figure sur les "Georgenobles » de Henri VIII; elle sert d'ornement à l'ordre de la Jarretière et à l'ordre du Bain. Une rose était empreinte sur les Oselli de Venise, les Pistole et les Livornini della rosa, de mème que sur les Duetti de Toscane, les Barbone de Lucques et les "pfennigs à la rose » du comté de Lippe. Sur une médaille frappée en 1541 en l'honneur de Luther, on voit, allusion à sa devise, audessous de son effigie, une rose épanouie, au milieu de laquelle repose un cœur à côté d'une croix; à l'exergue on lit la sentence: "Le cœur du chrétien

1. Schleiden, Die Rose, p. 172.

2. Rosenberg, Rhodologia, p. 32. 
est au milieu des roses, quand il est au pied de la croix $^{1}$.)

\section{IV.}

La rose est entrée dans l'onomastique des nations modernes comme dans celles de l'antiquité,; on la rencontre dans la dénomination d'un grand nombre de localités ou de personnes. Les noms des colonies grecques de Gaule et d'Espagne, Rhodê, Rhodai, latinisés en Rhoda, Rhodae, se sont conservés, après la chute de l'Empire, en se transformant, suivant les lois de la dérivation romane; mais ces vocables, rares encore dans la toponomastique latine et propres seulement à quelques localités du litttoral méditerranéen, se répandirent dans tout l'Occident avec la culture de la fleur d'où ils sont tirés; on les rencontre avec leurs dérivés en Italie, en France, en Portugal, surtout en Espagne. Minsi en Italie ${ }^{2}$, Rosa, Rosali, Rosario, Rosata, Rose, Roselle, Roserio, Roseto, etc. En Portugal ${ }^{3}$, Rosa, Rosario, Rosas et Prozas, Rosal et Rosaes, Roseiro. En Espagne ${ }^{4}$, Rosa, Rosal, Rosalejo, Rosales, Rosallo, Rosario, Rosas et las Rosas, Roseiro, Rosel, Rosell (Catalogne),

1. Schleiden, Die Rose, p. 174.

2. Dizionario statistico áei comuni del regno d'ltalia. Savona, 1879 , in-fol. s. v.

3. A. Soares de Azvedo, Portugal antigo e moderno. Diccionario geografico, etc. Lisboa, 1878 , in-8, s. v.

4. Don Pascual Madoz, Diccionario geografico-estadisticohistorico de España. Madrid, 1849, in-4, vol. XIIl, s. v. 
Rosello, Rosera, etc. En France ${ }^{1}$, Rozier et Le Rosier, Rosiers et Les Rosiers, auxquels il faut joindre sans doute la plupart des Roset, Rozet et Rozey, Rosay ${ }^{2}$ et Rozay ou Rozoy, dérivés de rosetum, que Du Cange traduit par "roseraie ", lieu planté de roseaux, mais dont le sens primitif est " lieu planté de rosiers ". Quant au mot Rosières, dérivé de rosaria, et qui sert à désigner un grand nombre de localités, il parait bien plutôt ètre un dérivé du germanique ros (roseau) ${ }^{3}$, que du latin rosa, encore qu'il ait dû prendre parfois ou qu'on ait fini par lui attribuer le sens de "plantation de rosiers ".

Les noms de lieu germaniques dérivés du vocable rose paraissent aussi, du moins à première vue, ètre nombreux; mais beaucoup d'entre cux ne viennent pas de ce radical, comme on l'a souvent répété. Fœrstemann ne regarde aucun des composés vieuxallemand du thème ros-, tel que Rosenburg, Ros(en)heim, comme venant du nom de la rose, il les rattache, soit au radical hrod (rouge), soit à hros (cheval) ${ }^{4}$. Il est évident néanmoins que plus tard le nom de la rose dut entrer dans un certain nombre de noms géographiques allemands, anglais ou scan-

1. Joanne, Dict. des communes de la France. Paris, 1864, s. v.

2. Rosetum. Dict. topographique de l'Eure, s. v.

3. "Rosaria, si bene interpretor, arundinetum. " - "Roseria, arundinetum. » Du Cange, s. v. - " Rosière, lieu planté de roseaux. ») Lacurne, s. v. - Néanmoins les Rosières de Metz avaient trois roses d'or dans leurs armes.

'. Altdeu'sches Yamenbuch. Ortsnamen. Leipzig, 1872, in-8. 
dinaves; tels sont tout d'abord les Rosengarten, - Rosengaard, Rosengard, Rosengarth, - qu'on rencontre dans tant de régions différentes; les Rosen, Rosengrund, les Rosenhagen et Rosenhain, Rosenkran-, Rosenschön, peut-ètre aussi quelques-uns des nombreux Rosenau et Rosenow', Rosenberg, Rosenfeld, Rosenthal, Rosendaal et Rosendael ${ }^{1}$, ou l'idée de rose, comme on le voit par les armoiries, a pénétré, si elle ne s'y trouvait pas à l'origine.

Le nom de la rose et ses dérivés ont servi également à désigner les personnes; on trouve déjà le nom Rosa et son dérivé Prosula dans des Actes fort anciens. Une sainte Rose, originaire de Sardaigne et mère d'un martyr, si elle ne le fut pas elle-mème, vécut au $\mathrm{III}^{\mathrm{e}}$ siècle ${ }^{2}$; une autre sainte du mème nom souffrit, dit-on ${ }^{3}$, le martyre en Perse, peut-ètre au siecle suivant. Sainte Rosule de Filasa (Dacie) fut martyrisée en Afrique sous Dioclétien, avec le prètre Simplice et saint Florence ${ }^{4}$. Au $\mathrm{xi}^{0}$ siècle, sainte Rosalic de Palerme brille par sa piété; au siècle suivant vécurent sainte Rose de Viterbe et sainte Roseline de Villeneuve.

Le nom de Rose, on le voit, prédestinait à la sain. teté; il prédestinait encore plus aux légendes; celles de la rose occupent une place considérable dans la

1. Ritter's Geographisch-Statistisches Lexicon über die Erdtheile, etc. Leipzig, 1883, in-4, s. v.

2. Acta Sanctorum, vol. XLI, p. 107, $1^{\text {er }}$ septembre.

3. Petits Bollandistes, 21 février.

4. Acta Sanctorum, vol. XVI, p. 455,15 mai. 
vie de presque toutes ces saintes; Roseline, en particulier, recut ce nom, parce que sa mère, avant sa naissance, avait rèvé qu'elle portait dans son sein une rose sans épines ${ }^{1}$. Mais c'est surtout dans la vie et dans l'iconographie de sainte Rosalie que la rose, emblème de ses vertus, a pris place. Elle était fille de Sinibaldo, seigneur de Rosas; au lieu de rester dans la maison paternelle, poussée par l'amour de Jésus-Christ, elle se retira, toute jeune encore, dans une grotte ${ }^{2}$. Là elle vit dans la société des anges et des roses; elle offre à Dieu des corbeilles de ces douces fleurs, symbole de sa pièté et de ses prières; c'est aussi couronnée de roses qu'un ange, lui-mème le front ceint de roses, la conduira, divine épouse, ( aux célestes noces ».

Porté d'abord uniquement par des femmes, le nom de Prose le fut aussi plus tard par des hommes: les Rosa d'Italie, les Rose de France et d'Angleterre, les Rosen d'Allemagne et de Suède sont connus. Les noms d'hommes toutefois ont plutôt été empruntés au mot rosier ou aux noms de localités, dans la désignation desquelles entre ou paraît entrer le vocable rose ou l'un de ses composés; tels sont Rosier, Rosiers, Rosenau, Rosenberg, Rosendal ou Rozendael, Rosenthal, Rosenhain, Rosenkranz, Rosenkreuz, Rosenmüller, Rozenzweig', etc., dérivations que la

1. Acta Sanctorum, vol. XXII, p. 486, F, 11 juin.

2. «Ego Rosalia Sinibaldi Rosarum domini filia, amore domini mei Jesu Christi in hoc antro habitari decrevi. " Acta Sanctorum, vol. XLI, 4 septembre, pl: III, vil, xiv. 
légende a cherché parfois à justifier, comme l'ont fait aussi les armoiries ${ }^{1}$. Dans une bataille, le chef qui commandait les troupes vit revenir près de lui un de ses pages tout couvert du sang qui coulait de ses blessures. "Mon pauvre ami, lui dit-il, que t'est-il donc arrivé ? " - "Seigneur, répondit vaillamment le page, j'ai cueilli trois roses que je veux porter à ma mère. ") Le général, frappé du courage de ce jeune homme, le fit chevalier sur-le-champ, en lui disant: "Désormais tu t'appelleras le seigneur de Roses (Rosen) '. "II fut la souche de la famille illustre de ce nom; elle porte trois roses dans ses armes.

Par une métaphore facile à comprendre, le nom de la rose est entré au moyen âge, comme cela arait déjà eu lieu dans l'antiquité, dans la désignation d'un certain nombre d'ètres ou d'objets, qui offraient avec cette fleur quelque analogie de forme ou d'aspect. J'ai parlé des roses, rosaces et rosettes, employées comme motif de décoration dans les arts, et qui offrent plus souvent une ressemblance lointaine avec la fleur dont elles tirent le nom qu'elles n'en sont une reproduction véritable. Telles étaient aussi les roses, espèce d'ornement que les femmes portaient à leur soulier. Dans l'orfèrerie on donnait le nom de

1. On en cite parfois d'autres, en particulier, d'origine germa- nique, comme Rosamunde. Förstemann, Altdeutsches Namenbuch. Personennamen, ne fait dériver aucun de ces noms de rose (rosa), mais de hros (cheval).

2. Schleiden, Die Rose, p. 176. 
rose à une espèce de médaillon " : “Une roze d'or ou est esmaillé le Roy a genoulx devant monseigneur saint Denis et l'évangéliste saint Jean²).

Les ( roses ), une des créations les plus belles de l'architecture gothique, méritent une mention toute spéciale. On donne ce nom aux fenêtres circulaires et à compartiments qu'on voit à la façade et aux deux ailes d'un grand nombre des cathédrales et des églises du xur et du $\mathrm{xIv}^{\mathrm{e}}$ siecle. On les rencontre d'abord dans les monuments de l'Ile-de-France et de la Champagne. La plus ancienne peut-être est la rose qui s'ouvre sur la façade occidentale de l'église de Mantes; elle remonte à la fin du xiı siècle. Les roses de Notre-Dame de Paris, celle de la facade occidentale, qui est de 1220, et surtout les deux roses énormes, postérieures de près de quarante ans, des pignons sud et nord du transept de cet antique édifice, à la fois puissantes et solides, sont les plus beaux spécimens que les architectes de l'lle-de-France aient laissés de ce genre de construction. Les roses de la Champagne les surpassent encore sous le rapport de l'élégance et de la hardiesse. La rose occidentale de la cathédrale de Reims est admirable par la proportion et l'harmonie de ses diverses parties. Il faut en rapprocher les roses de la cathédrale de Chartres, surtout celle de la facade occidentale. On ne trouve rien de compa-

1. L. de Laborde, op. laud., Glossaire, s. v. Rose, p. 486.

2. Cif. Invenţaire du mobilier de Charles $V, n^{\circ} 3138$. "Une roze d'argent, blanche, neellée, à ung escuçon de Bourgogne. » 
rable en Bourgogne; les architectes de cette province ont préféré à ces baies immenses des roses de petite dimension ${ }^{1}$.

Au xrvo siècle, la hardiesse et l'élégance que les architectes de l'âge précédent avaient portées dans la construction des roses, parut diminuer; on le voit bien à la rose de la cathédrale d'Amiens, tout admirable qu'elle est, d'ailleurs ${ }^{2}$. Au delà des limites de la France centrale les roses eurent de la peine à être acceptées. Les architectes normands et anglais ne les employèrent qu'avec hésitation; l'architecture rhénane leur préféra les fenêtres ordinaires; dans la France méridionale on y substitua des ails de un à deux mètres de vide. Cependant on troure aussi de fort belles roses dans ces contrées; par leur exécution et leur style achevé, les roses de la cathédrale de Rouen sont de rrais chefs-d'ourre; celle qui s'ouvre sur la facade de la cathédrale de Clermont n'est pas moins remarquable. On cite aussi comme un modèle d'élégance la rose de l'église de la Batalha à Lisbonne.

On a appelé également du nom de rose diverses plantes qui ressemblent à cette fleur, telle que la rose trémiere, d'outre-mer ou passerose (Althaea rosea L.), la rose de Toël (Helleborus nigrer L.), en all. Weihnachtrose; angl. Christmasrose; la Rose de NotreDame, Rosa del monte esp. (Preonia officinalis L. ;

1. Viollet-le-Duc, Dictionnaire de l'architecture, s. v. Rose.

2. Elle représente les vicissitudes de la vie humaine. Didron, .Hanuel d'iconographie chrétienne, p. 115. 
la Seerose ou Wasserrose en all., Waterrose en angl., nom des Nymphéacées; la Rouselio prov., Rosella cat., Kornrose all., Cankerrose ang. (Papaver rhaeas L.); la Roso de bos pr., rosella pg., nom de divers cistes; la Rose des Alpes, all. Alpenrose (Rhododendron ferrugineum $\mathrm{L}_{\text {.. }}$ ), etc. ${ }^{1}$

Ces noms toutefois ne paraissent pas anciens; aussi je n'en citerai pas d'autres et je me borne à y ajouter la rose de Jéricho (Anastasia Hierochuntica L.), espèce de crucifère, qui, après la dessiccation, s'étale, sous l'influence de l'humidité, en forme de rose. Une légende veut qu'elle fleurisse seulement dans la nuit de Noël; on lui attribue aussi la propriété merveilleuse de protéger contre la foudre la maison où elle se trouve ${ }^{2}$. La "rose du saule ", nom donné aux excroissances foliacées, déterminées sur les rameaux de cet arbre par la piqûre d'un insecte, est, elle, le présage d'un événement important; la fin de la guerre de Trente ans aurait été annoncée par les nombreuses roses que l'on vit sur les saules en $1648^{3}$.

Quelques polypiers, des minéraux même ont pris, surtout en allemand, un nom tiré de la rose, mais à

1. Remberti Dodonaei, Stirpium historia. Antv., 1616, in-fol. - J. Britten and R. Holland, A dictionary of english plantnames. London, 3 v . in-8, 1877-81. - G. Pritzel, und C. Jessen, Die deutschen Volksnamen der Pflanzen. Hannover, 1882, in-8. - Mistral, s. v.

2. Schleiden, Die Rose, p. 108.

3. Abbé de Vallemont, Curiositez de la nature et de l'art. Paris, 1784, in-12, vol. 1, p. $23 \%$. 
une époque postérieure, il semble, au moyen âge. On a dès lors, au contraire, ce qui se faisait déjà dans l'antiquité, donné le nom de "rose » à l'érysipèle; un chroniqueur du $\mathrm{xv}^{\mathrm{e}}$ siècle l'appelle le " mal des roses Nostre-Dame ${ }^{1}$ ), en allemand rosem, rosamo; cette dénomination se rencontre aussi dans les langues slaves.

Enfin on a, à la fin du moyen âge, appelé du nom de " rose », " jardin des roses », ou même de " rosier " divers recueils historiques ou scientifiques. Tels sont les Rosa anglica, Rosa gallicr, livres de médecine célèbres, le "Jardin des roses (Rosengarten) des sages-femmes », la Rosa Ursina, traité d'astronomie, la Rosa ponitentialis, le Rosarium logices, le "Rosaire (Rosenkranz; hermétique ), ouvrage d'alchimie, etc. 11 y a aussi un "Rosier des guerres ), attribué parfois à Louis XI.

1. Lacurne, ร. v. Rose. 


\section{CHAPITRE VI}

LA ROSE DANS LA PIARMACOPÉE, LA CUISINE ET LA TOILETTE

Rose est de grande medecine,

dit un poète anonyme du commencement du xıv siècle ${ }^{1}$, qui n'est ici que l'écho de la croyance générale de son temps aux vertus de cette fleur aimée. Tous les auteurs, chrétiens ou arabes, qui, depuis les premiers siècles du moyen âge, ont écrit sur la thérapeutique ou l'histoire naturelle, ont parlé des vertus médicinales de la rose et des ingrédients fournis par cette fleur, pour eux non moins salutaire que belle.

Bien qu'il se soit surtout attaché à célébrer les beautés de la rose et à en faire connaitre la signification symbolique, Walahfrid mentionne néanmoins l'huile de roses, en ajoutant — on ne pouvait en faire un plus grand éloge - que " personne ne saurait dire combien de fois elle a guéri les maux des mor-

1. "Poème moralisé ". Romania, vol. XIV, p. 458, xxxI, v. 29 . 
tels $)^{1}$. IIildegarde est déjà bien mieux informée ${ }^{2}$; d'après elle, les pétales écrasés de la rose, appliqués sur des yeux chassieux, les guérissent; ils font également disparaître les ulcères qui n'ont pas un caractère malin. Mélangés aux potions, aux onguents, aux divers remèdes, ils les rendent d'autant plus efficaces, qu'ils y ont été ajoutés en plus grande quantité.

Après Hildegarde, il faut placer le faux Emilius Macer, l'auteur d'un traité sur les vertus des plantes ${ }^{3}$, célèbre pendant les derniers siècles du moyen âge. Plus instruit et mieux renseigné que l'abbesse de Saint-Rupert, le pseudo-Macer connait nombre de propriétés médicinales de la rose ignorées de la docte religieuse. Ainsi les pétales écrasés de cette fleur guérissent, d'après lui, le feu sacré ; mélangés à du vin, ils arrêtent le flux du ventre; le suc qu'on en retire, ajoute-t-il, entre dans la composition de divers collyres; réduits en poudre, ils font disparaitre, seuls ou mêlés à du miel, les ulcères de la bouche; appliqués en cataplasme ou bien écrasés dans du mout, ils calment la fièrre. Enfin Macer rappelle, d'après Palladius, et comme Walahfrid, de quelle utilité médicale peut être l'huile de roses

1. Inficit hic oleum proprio de nomine dictum,

Quod quam saepe fiat mortalibus utile curis,

Nec meminisse potest hominum nec dicere quisquam.

Hortulus, XXVI, v. 402-404.

2. Liber de plantis, cap. xxir, éd. Migne, p. 1139.

3. Macer Floridus, De viribus herbarum. S. 1. n. d., in-4, cap. $\mathrm{xxr}$. 
convenablement préparée. Il y a progrès, on le voit, sur Hildegarde; néanmoins le poète-médecin en est encore, en somme, à la pharmacopée grecque et romaine; mais la rose allait servir à bien d'autres usages thérapeutiques ignorés des anciens et entrer dans des produits que ni Macer, ni les écrivains qu'il a suivis n'avaient connus.

La pharmacopée a, depuis le $x_{1}{ }^{e}$ siècle, fait de grands progrès, grâce aux travaux des savants arabes et aux découvertes de l'École de Salerne. Les Arabes avaient inventé les sirops, qui d'Orient et d'Espagne devaient passer dans toutes les officines de l'Occident; la distillation perfectionnée par eux permit également de fabriquer des produits nouveaux; il n'est pas surprenant aussi que, depuis lors, l'emploi médical de la rose se soit étendu. Eissa ibn Massa reconnaissait à cette fleur, surtout à la variété à pétales rouges, une vertu fortifiante, en mème temps qu'elle rafraîchit, suivant lui, les inflammations de la tête ${ }^{1}$. Pour Ishac ibn Amrâm, la rose convient à l'estomac et au foie; elle réduit en particulier les obstructions de ce dernier viscere, occasionnées par la chaleur. Razès, de son côté, dit que la rose calme la fièvre, mais il affirme en même temps qu'elle provoque le coryza. D’après lui encore, le miel rosat est bon à l'estomac, " qui contient des humeurs ». Razès vante également le sirop de roses et le sucre rosat ${ }^{2}$.

1. Ibn el Beithar, Traité des Simples, $\mathrm{n}^{\circ} 2274$ (Notices et extraits des manuscrits, vol. XXVI, p. 406).

2. Opera. Venise, 1500 , p. 12 et 15. 
Avicenne, et après lui Vincent de Beauvais ${ }^{1}$, regardent la rose à la fois comme acide, styptique et amère; pour eux encore elle est apéritive, ainsi que détersive, et calme l'effervescence de la bile; en infusion, elle est bonne contre les nausées et les palpitations fébriles et salutaire à tous les viscères; elle fortifie les membres inférieurs. En cataplasme, elle attire les piquants et les échardes, résout les apostèmes, guérit les excoriations de la peau et régénère les chairs rongées par les ulcères. Grâce à sa froideur, dit-il encore, la rose calme les douleurs des yeux, et celles de la matrice. Une infusion de pétales séchés est bonne contre la chassie des yeux et les ulcères des intestins. Le suc de la rose est excellent pour entretenir l'humidité de l'estomac; dans une infusion il est efficace pour les maux d'oreilles et de gencives. 11 est souverain aussi dans les syncopes. Les jeunes pousses et les tètes de la rose, à l'en croire, sont excellentes pour les crachements de sang et ses graines raffermissent les gencives. Enfin, suivant lui, l'eau et l'huile de roses font éternuer, quand on les respire.

D'après le Livre des Expériences, Ibn el Beithar" enseigne que les pétales de roses desséchés et réduits en poudre cicatrisent et assouplissent la peau des malades atteints de la variole, et il vante également, d’après le mème ourrage, les propriétés salu-

1. Liber canonis. Basiliae, 1556, in-fol. Tract. II, p. 291. Speculum naturae, p. 761 et 762.

2. Op. laud., p. 407. 
taires du sirop de roses, qu'il recommande entre autres dans la fièvre bilieuse. Nous retrouverons à l'instant cette préparation d'origine arabe, dans la pharmacopée de l'Occident, où la rose n'occupe pas moins de place que dans celle de l'Orient. Son emploi y a été généralisé surtout par l'École de Salerne. Nicolas Praepositus, qui contribua à en fonder la renommée au $\mathrm{xu}^{\mathrm{e}}$ siècle $^{1}$, recommande l'emploi de la rose dans les maladies d'estomac et de foie; elle fait cesser, dit-il ${ }^{2}$, les embarras gastriques, dégage la tête des "fumées" de la bile et arrête les hémorrhagies nasales. Cuite dans du vin elle apaise les douleurs d'oreilles et en gargarismes guérit les ulcérations des gencives. Ses graines pilées produisent le même effet. Enfin, les pétales en cataplasme, ainsi que l'écorce en décoction, guérissent, d'après lui, les hémorrhoïdes.

Mais c'est dans Platearius qu'on trouve l'énumération la plus complete des usages de la rose au moyen îge et des propriétés qu'on lui supposait; le célèbre médecin nous a laissé dans son Livre de simple médecine ou Circa instans, une véritable pharmacopée de cette fleur; il n'a omis aucune des préparations dans lesquelles elle peut entrer, ni aucun de ses emplois ${ }^{3}$. Aussi a-t-il servi de modèle et de guide à tous les auteurs de thérapeutique qui

1. Ernst Meyer, Geschichte der Botanik, vol. III, p. 506.

2. Magrstri Nrcolai Prepositi Dispensarium ad aromalorios nuper diligentissime recognitum. Lugduni, 1512, in-4, fol. 4 .

3. Liber de simplici medicina secundum Platearium, dictus Circa instans. Basileae, 1528, in-4, fol. 121 b.

Joret. La Rose. 
sont venus après lui. Vincent de Beauvais le cite en tète des autorités qu'il invoque; si Albert le Grand ne parait rien lui devoir, Thomas de Cantimpré, Barthélemy l'Anglais et Pierre de Crescence, dans son traité d'agriculture, l'ont suivi presque textuellement; il en est de même, à plus forte raison, des Herbiers ou Arboristes français du $\mathrm{xv}^{\mathrm{e}}$ siècle, qui ne sont que des adaptations ou même des traductions plus ou moins complètes du Livre de simple médecine du savant Salernitain, ainsi que des Arbolayres et Grants Herbiers, simples éditions de ces curieux manuscrits. Inutile de parler de Conrad de Megenberg, qui n'a le plus souvent fait que reproduire Thomas de Cantimpré. Quant à l'Herbarius de Mayence et au Ghart de Gesundheit, s'ils invoquent d'autres autorités, ils citent eux aussi Platearius au premier rang et lui ont emprunté le meilleur de ce qu'ils disent.

D'après le plus illustre représentant de l'École de Salerne, les principaux ingrédients faits avec la rose sont le miel et le sucre rosat, le sirop et l'huile de roses. Quant à l'eau de roses qu'il ne décrit pas, comme s'il la supposait trop comme, il ne s'agit point d'un liquide auquel des pétales de roses auraient donné, par un contact plus ou moins prolongé, quelque chose de leur parfum, mais d'une eau distillée avec des roses; Ibn el-Awam déjà en avait indiqué la préparation; elle était analogue à ce qu' elle est aujourd'hui. Ainsi que le Ménagier de Paris', les Herbiers français du $\mathrm{xv}^{\mathrm{e}}$ siècle, donnent un moyen plus simple de l'obtenir:

1. Vol. II, p. 252. 
La manière comme ${ }^{1}$ l'en fait l'eaue rose ne pouvons pas exprimer se l'en ne la voit faire. Aucuns toutefois la font ainsi. Ilz mettent roses avec eaue en une fiole de voire, en cette fiole mettent dedens ung pot ${ }^{2}$ plain d'eaue bouillant, et ainsi cuisent les roses de la fiole avec l'eaue qui y est et devient rouge ${ }^{3}$ et met on la fiole au soleil et se l'en met moult d'eaue avec ung peu de roses elle n'en est pas si bonne. Aucuns cueillent les roses avec la rosée qu'elles ont et les mettent en la fiole comme dit est, sans y mettre autre eaue et celle eaue rose faicte ainsi est tres bonne.

L'eau de roses distillée, dont on a parfois attribué la découverte à Razès ${ }^{4}$, ou même à l'alchimiste Claber, qui vivait en Mésopotamie un siècle avant lui", fut connue de bonne heure en Occident; Constantin Porphyrogenète en fait déjà mention dans la description d'une fète donnée à la cour de Constantinople en $946^{6}$; elle avait donc pénétré en Europe dès le $\mathrm{x}^{0}$ siècle; elle ne tarda pas non plus à pénétrer avec la fleur qui la produit dans l'Hindoustan, à la suite des Gaznévides.

Si l'eau de roses du moyen âge était tout autre que celle des anciens, le miel rosat décrit par Platearius n'était pas moins différent que celui dont parlent

1. Bibl. nationale, fr. 1230 , fol. $164 b$. Le man. 9136 , f. $243 a$, donne "comment », le 9163 " dont ».

2. Fir. 9136 et 12319 , « vaissel ».

3 Fr. 9136, "vermeille », " bouter ».

4. Loiseleur-Deslongchamps, La rose, p. 92.

5. Gmelin, Geschichte der Chemie, vol. I, p. 20.

6. Schleiden, Die Rose, p. 269. 
Pline et les autres écrivains latins. Pour le préparer, on commençait par faire bouillir le miel qu'on voulait aromatiser; on l'écumait, puis on le coulait; ensuite on ajoutait des pétales frais de rose, dont on avait coupé l'onglet et on faisait bouillir le tout quelque temps.

Pour préparer le sucre rosat, on pilait dans un mortier avec du sucre des pétales encore frais de rose; puis on mettait le mélange dans un vase de terre ou de verre, qu'on laissait pendant trente jours exposé au soleil, en ayant soin de le remuer chaque jour. Le Ménagier de Paris donne la recette plus simple de faire bouillir du sucre réduit en poudre dans de l'(c eaue rose » jusqu'à consistance sirupeuse ${ }^{1}$. Le sirop de roses s'obtenait en faisant bouillir des pétales de ces fleurs dans de l'eau, à laquelle on ajoutait ensuite du sucre. On faisait l'huile de roses en mettant bouillir des roses dans de l'huile commune, puis on coulait le tout; ou bien on plaçait des roses et de l'huile dans un vase de verre que l'on faisait chauffer au bain-marie. Nous voila loin des recettes primitives de Pline. Enfin Platearius parle encore d'une décoction de roses dans du vinaigre, sans rien dire toutefois de la manière de la préparer. Elle était la même, sans doute, que pour l'huile de roses.

Je n'ai point l'intention d'énumérer toutes les propriétés médicales que le Livre de simple médecine

1. Vol. II, p. 274. Il convenait de plus, d'après lui, de mettre par chaque livre de sucre un blanc d'œuf bien battu. 
attribue à la rose ${ }^{1}$ ou à ses produits; je me bornerai à en indiquer quelques-unes ${ }^{2}$. Le miel rosat, d'après lui, fortifie et mondifie, c'est un dissolvant puissant; avec de l'eau chaude il relâche, avec de la froide il resserre. Le sucre rosat passait aussi, et cela ne doit pas surprendre, pour avoir des propriétés astringentes et fortifiantes; il en était de mème du sirop et de l'eau de roses. Platearius conseille le premier contre la dyssenteric et la lienterie. Il était réputé bon encore contre les vomissements de bile, contre les syncopes et les troubles du cour. Le sirop de roses était donné avec de l'eau de pluie contre le flux de ventre et les vomissements, et dans de l'eau de rose aux malades atteints de fievre. On l'administrait dans de l'eau froide contre les syncopes. L'huile de roses servait à oindre la région du foie dans les inflammations de cet organe; on en frottait également le front et les tempes pour combattre les migraines produites par le chaud ou le froid.

L'eau de roses était employée contre la dyssenterie accompagnée de vomissements; on y ajoutait avec succès une décoction de mastix et de girofle. Aujour-

1. Voici ce qu'en dit, v. 19-22, le poète anonyme cité au commencement de ce chapitre :

Humeur maise dedens le cors

Ele degaste et boute hors.

Le cervel, c'est desconforté,

Par la rose est reconforté.

2. Ces propriétés ont été résumées en quelques mots dans le man. fr. 12308, fol. 165 b, de la Bibl. nationale : "Pour le saintfeu, pour la matrice et le ventre, pour la bouche, pour restreindre chaleur, pour l'estomac et le ventre, pour le chief, pour plaies, pour douleurs de dos, pour les yeulx. » 
d'hui encore, dans l'Hindoustan, c'est, avec quelques grains d'un médicament mystérieux, le zaharmora (contrepoison), qu'on y dissout, presque le seul remède qu'on emploie contre le choléra ${ }^{1}$. Mèlée avec de la poudre et du sucre de roses, et concentrée au soleil, elle servait à oindre les gencives. On la prenait en boisson dans les syncopes et les affections du cœur; en collyre elle était excellente pour les yeux; elle servait à composer des espèces de pommades pour la figure, à cause de la propriété qu'elle avait d'assouplir la peau et de faire disparaitre les éphélides de la face.

Elle passait en Orient pour avoir une vertu purificative. Quand Saladin se fut emparé de Jérusalem sur les Croisés en 1187, il fit laver avec de l'eau de roses les murailles et le parvis de la mosquée d'Omar, qui avait été transformée en église par les Chrétiens. Sanuto rapporte, par une exagération manifeste, que cinq cents chameaux furent nécessaires pour porter l'eau de roses employée en cette occasion $^{2}$. Bibars, quatrième sultan de la dynastie des Mamelouks-Baharites, fit aussi laver, quand il la visita, la Kasbah de la Mekke avec de l'eau de roses ${ }^{3}$.

1. Schleiden, Die Rose, p. 271.

2. Les écrivains orientaux ne sont pas tombés dans cette exagération; l'un d'eux, en rapportant ce fait, se borne à dire que TakiEddin, neveu de Malek-Adel, après avoir "lavé avec de l'eau les murs et les lambris à plusieurs reprises, y passa ensuite de l'eau de rose." Reinaud, Extraits des historiens arabes relatifs aux guerres des Croisades. Paris, 1829, in-8, p. 214.

3. La Grande Encyclopédie, s. v. Bibars. 
Et Mahomet II, après la prise de Constantinople, n'entra, dit-on ${ }^{1}$, dans l'église de Sainte-Sophie, convertie en mosquée, qu'après l'avoir fait purifier avec cette même eau. Les Chrétiens d'Orient ont adopté cet usage, et dans l'église du Saint-Sépulcre c'est encore la coutume de laver avec ce précieux liquide la pierre sur laquelle, d'après la tradition, aurait été déposé le corps du Christ après la descente de la croix ${ }^{2}$.

Quant au sucre rosat ou conserve de roses, il était recommandé aux estomacs débilités; on en prenait, ainsi que du diarrhodon à la rhubarbe, pour se donner du ton, surtout au printemps et en été $e^{3}$. Celui que l'on préparait à Provins était si renommé, que c'était un des présents qu'on offrait aux princes et aux rois, quand ils traversaient cette ville ${ }^{4}$.

Le Livre de simple médecine, on doit s'y attendre, connaissait encore d'autres produits dans lesquels entrait la rose. Il donne entre autres comme un spécifique souverain contre les douleurs de reins ou de poitrine un emplâtre fait de pétales de roses (pilés) avec du blanc d'œuf et du vinaigre faible. Il préconise de même contre les vomissements une

1. Lebeau, Histoire du Bas-Empire, vol. XXI, p. 282.

2. T. R. Joliffs, Reise in Palästina, etc., ap. Schleiden, p. 270.

3. "Pour conforter l'estomac », dit le Ménagier de Paris, vol. II, p. 27 '

En estyeu o vas lo pascor,

(e) tu prent de sucre rosat, dyarrodon reubarbizat.

Diätetik. v. 81-83 (Herm. Suchier, Denkmäler provenzalischer Literatur und Sprache, Halle, 1883, in-8, p. 203).

'. I, oiseleur-Deslongchamps, La rose, p. 100. 
décoction de roses dans du vinaigre, dont on imbibe une éponge qu'on place sur "l'orifice de l'estomac».

La rose était aussi employée seule; ses pétales desséchés, dit Platearius ${ }^{1}$, mis sous les narines, fortifient le cerveau; contre la diarrhée cholériforme, il conseille aussi une décoction de roses dans de l'eau de pluie. Un cataplasme de pétales de roses préalablement bouillis était excellent, d'après lui encore, contre la rougeur des yeux. Les étamines, que Platearius appelle anthères, déjà utilisées dans l'antiquité, ont été également employées par l'École de Salerne. Elles passaient pour bonnes contre le flux de ventre et les vomissements. En décoction dans du vinaigre pur ou rosat, on s'en serrait pour se gargariser, après l'extraction d'une dent. Réduites en poudre elles calmaient l'inflammation de la luette.

Si le moyen âge s'en tint aux propriétés attribuées à la rose par le Livre de simple médecine, les pharmacologues de la Renaissance devaient renchérir encore sur l'importance médicale attribuée à la rose par le maitre de l'École de Salerne. "Il n'est point de simple, remarque Rosenberg ${ }^{2}$, indigène ou exotique, qui soit plus utile, plus nécessaire et plus agréable. " "Il n'y en a point, ajoute-t-il, qui fournisse autant de remèdes, soit internes, soit externes.» " Elle est, dit un autre auteur ${ }^{3}$, la lumière et la richesse des pharmacies. ”

1. Circa instans, p. 122.

2. Rhodologia, “Praeludium», p. 1 et pars II, cap. xvin, p. 225.

3. "Non esset vel lux vel luxus apothecis, si Rosa pharmacopaeis 
A cette époque de faveur extrême, la rose eut le privilege d'entrer dans la composition de quelquesuns des spécifiques les plus célèbres: tel que le sirop d'or du duc de Mantoue, l'or potable de Roderic a Fonseca, le secret royal, envoyé en présent par Élisabeth d'Angleterre à l'empereur Rodolphe II ${ }^{1}$, et, plus anciennement, l'aquasit de Frédéric III. Le hasard heureux qui fit découvrir, au commencement du $\mathrm{xviI}^{\mathrm{e}}$ siècle $^{\mathrm{Z}}$, l'essence de roses, - attargul, - vint encore augmenter, avec le nombre des produits, le renom pharmaceutique de la rose.

La rose sauvage n'était guère d'un moindre emploi que la rose cultivée. D’après Albert le Grand ${ }^{3}$, une infusion de ses pétales est bonne pour les enfants atteints de rachitisme; en gargarisme ils calment les maux de dents; sa racine passait aussi pour salutaire contre les crachements de sang et pour les estomacs débilités; on la disait encore bonne contre la constipation, ainsi que pour les fractures anciennes. Elle passait également pour souveraine

desideraretur. ") Minderer, Aloedarium. Vindeb., 1616, cap. xI, p. 153. "On en retire tant de choses, dira encore Pomet à la fin du $\mathrm{Xvil}^{\mathrm{e}}$ siècle, en ne parlant toutefois que de la rose de Provins, que sans elle la médecine ne serait pas si florissante. " Histoire générale des drogrues. Paris, 169', in-fol., p. 174.

1. J. G. Benemann, Die Rose zum Lobe ihres Schöpfers. Leipzig, 1742, p. 43, ap. Schleiden, p. 186.

2. En 1612, à l'occasion d'une fête donnée par la sultane favorite Nur-Djihan au grand-mogol Djihan-Guir. L. Langlès, Recherches sur la découverte de l'essence de rose. Paris, 180', in-8.

3. De segretabilibus, lib. VI, tract. I, cap. Ix (44), p. 359. Ce que dit Albert s'applique surtout à l'églantier rouillé. 
contre les fièvres de longue durée. Mâchée et mise sur une piqûre de scorpion, elle en attirait le venin. Ses graines aussi étaient excellentes contre la morsure des reptiles et dans beaucoup d'autres cas.

Loin de diminuer, l'efficacité supposée de l'églantier ne fit que croître à la fin du moyen âge et au commencement des temps modernes. Une décoction de ses graines, dit Conrad de Megenberg ${ }^{1}$, guérit les enfants dont les membres ont souffert de mouvements trop brusques. D'après lui encore, la sève en est bonne en gargarismes contre les maux de dents. Et il ajoute avec Albert que sa racine est excellente contre les crachements de sang, ainsi que dans les maladies d'estomac et les fièrres paludéennes. Hagendorn ${ }^{2}$, un siecle et demi plus tard, reconnaitra à l'églantier encore bien d'autres vertus; il n'énumère pas moins de trente-trois maladies, la plupart même dangereuses, telles que l'épilepsie, l'hydropisie, le croup, l'hémoptysie, le goître, les hémorrhoïdes et la podagre, contre lesquelles cet arbuste offrait un remède assuré:

Mais on n'attribua pas seulement des propriétés curatives ordinaires à la rose cultivée ou sauvage, on lui en reconnut aussi de merveilleuses et de surnaturelles. Telle était la vertu soporifique attribuée par les anciens Germains à l'églantier. Odin, irrité contre la walkyrie Sigurdrifa, qui avait tué Hialmgunnar son protégé, la plongea dans le sommeil, en la tou-

1. Das Buch der Natur, p. 316, 8. G. von Negenberg parle plus particulièrement des fruits de la $R$. rubiginosa.

2. Cynosbatologia, ap. Schleiden, p. 186. 
chant avec une branche de cet arbuste - le svefinthorn ${ }^{1}$. L'excroissance moussue produite sur l'églantier par la piqûre du Cynips rosae - le bédégar - passait surtout pour posséder cette propriété d'endormir ${ }^{2}$; on croyait qu'un homme plongé dans le sommeil, sous la tête duquel on place cette excroissance, ne se réveille point qu'on ne l'ait enlevée.

Près d'une image de Notre-Dame, non loin de Lucques, croissent des roses dont le parfum suffit un jour pour faire parler un petit berger muet de naissance, qui cueillit l'une d'elles ${ }^{3}$. Il y a dans ce fait merveilleux quelque chose d'analogue à l'action miraculeuse que la rose exerce sur les possédés. Mais c'est dans les incantations surtout que cette fleur a pris place; en Westphalie, on arrête le sang au nom des trois roses symboliques qui sont supposées se trouver dans le jardin du ciel ${ }^{4}$ :

Dans le jardin de Dieu, il y a trois roses; l'une sappelle bonté de Dieu; la seconde, sang de Dieu; la troisième, volonté de Dieu; sang, arrête-toi, je te l'ordonne.

1. Die Edda, die ältere und jüngere übersetzt von Karl Simrock. Stuttgart, 1864, in-8. "Sigrdrifumàl», p. 204.

2. J. J. Grimm, Deutsche Mythologie, vol. II, p. 1007.

3. Wolfg. Menzel, op. laud., vol. II, p. 282.

4 .

In Gottes Garten stehn drei Rosen,

Die eine heisst Gottes Güte,

Die andere Gottes Geblüte,

Die dritte Gottes Wille,

Blut, ich gebiete dir, stehe stille.

1. Kuhn, Sagen, Gebräuche und Märchen aus Westphalen, vol. II, p. 199, 
Certains idiomes, nous l'avons vu, ont donné à l'érysipèle le nom de rose; ce mot est ainsi entré dans plusicurs formules magiques destinées à gruérir la maladie qu'il désigne. En voici une qui est usitée dans la région du Minho ${ }^{1}$ :

Que faire à la rose vermeille qui ici mord, brûle et point? Donne-lui du sel de la mer et de l'herbe de la montagne... et Notre-Dame permettra que ce mal diminue.. Que Dieu te rende à ton premier état, tel que tu naquis et fus créé.

La suivante est employée dans la Lithuanie ${ }^{2}$ :

Neuf roses flottent sur la mer, trois noires, trois blanches et trois grises. Les noires s'enfoncent sous l'eau, les grises aussi, seules les blanches surnagent et fleurissent. Qu ainsi, semblables aux roses abîmées dans les flots, disparaisse cette maladie; quainsi, semblable aux roses blanches et fleuries, fleurisse de nouveau ta santé.

II.

Comme dans l'antiquité, la rose et ses produits ne furent pas, au moyen âge, employés seulement dans la médecine, ils le furent encore comme cosmétiques et dans la cuisine. L'eau de roses jouait un rôle considérable dans les usages de l'Orient; on en offrait

1. Que faria a rosa vermelha Que aqui come, arde e doe?

Dá-lhe com sal do mar

E herva do monte...
Que a Senhora permittirá

Que este mal abrandará...

Deus te torne a teu estado,

Coino foste nado e creado.

Ad. Coelho, Romances sacros, oracöes e ensalmos populares do Minho (Romania, vol. III, p. 276.)

2. Potebnia, Obiasnenia, etc., p. 110. 
aux hôtes à leur arrivée; dans les festins on en répandait sur les convives. Sucrée et rafraichie avec de la neige, c’était aussi, dès le vin ${ }^{\mathrm{e}}$ siècle, un breuvage recherché des femmes musulmanes ${ }^{1}$. Dans les fètes princières de l'Inde, on offre aujourd'hui encore de l'eau de roses aux invités pour se parfumer la barbe et les cheveux. Cet emploi de l'eau de roses passa de l'Orient en Occident, peut-ètre à la suite des Croisades; c'est depuis lor's du moins qu'on rencontre l'usage de se laver la figure avec ce liquide?. Avant de se mettre à table, les dames se lavaient les mains avec de l'eau de roses ${ }^{3}$. Après le repas, on se nettoyait aussi la bouche, en France du moins, avec une espèce de poudre et cette mème eau ${ }^{4}$. Dans le Chátiment des clames de Francesco Barberino, on voit les femmes de la jeune reine lui laver les mains et le visage avec de l'eau de roses, avant de la conduire à la couche nuptiale ${ }^{5}$.

1. Kremer, Kulturgeschichte des Orients. Wien, 1875, vol. I, p. 149. Ibn-Batuta dit qu'on en offrait aux invités avec du sirop. Il parle aussi de sorbets que les Persans faisaient avec du jus d'orange et de citron, des roses et des violettes. Voyages, I, 247 et 366, II, 78.

2. D'eve rose (ont) lor vis lavés. Parten., v. 10660.

3. Prisent l'aigue en dorés bacins

Aigue rose tot a fuison,

Onques d'autre n'i lava on. Parten., v. 10846.

Et quant ce vint a la parclose

Letuaires et eve rose

Por laver sa bouche et son vis.

Cortois d'Arras, v. 79 (Méon, Fabliaux, vol. I, p. 382).

5. Lavano il viso elle mani alla donna

D'acqua rosata. Parte V, p. 128, v. 58. 
Comme aujourd'hui, on recherchait les roses et les violettes, à cause de leur parfum, regardé comme fortifiant; le traité provençal de Diététique, publié par M. Suchier, recommande de s'en faire apporter en été, "pour sentir bon " ${ }^{1}$. On faisait aussi usage de pétales desséchés de roses, surtout de roses de Provins, pour parfumer les vêtements, et peut-être pour les préserver des mites ${ }^{2}$.

L'eau de roses et la rose elle-mème n'occupaient pas moins de place, au moyen âge, dans les usages culinaires que dans la toilette. Les habitants des lacustres de la Suisse paraissent déjà aroir mangé les fruits de l'églantier ${ }^{3}$. Au moyen âge on en faisait une espèce de compote. Peut-ètre aussi faisaiton déjà frire alors dans une pâte légère des pétales de rose, comme on le fait aujourd'hui dans l'Allemagne méridionale.

En Orient, nous apprend Abdellatif, ils entraient dans la composition de divers mets et pâtisseries. Le sirop de roses, lui, servait, avec du gingembre, de la cannelle, du jus de citron et plusieurs autres ingrédients, à faire une sauce qu'on versait sur une espèce de pâté rempli de poulets et de petits oiseaux ${ }^{\natural}$. Dans l'Allemagne méridionale les pétales de roses

1. En aprop ti fai aportar en estieu entorn lo pascor... d'espetias per bon flairar...

de rozas o de violetas. V. 93-99.

2. Ménagier de Paris, vol. II, p. 252.

3. Heer, Pflanzen der Pfahlbauten. Zurich, 1866, in-4, p. 29 .

4. Relation de l'Égypte, trad. de S. de Sacy, p. 311 et 317. 
servaient aussi à assaisonner les " poulets à la grecque s, plat qui paraît avoir été prisé au $\mathrm{xv}^{e}$ siècle".

Mais c'est l'eau de roses surtout qui, depuis le xm siècle, a joué dans la cuisine un rôle considérable; on l'employait comme assaisonnement dans les sauces, dans les ragoùts et mème dans diverses soupes². Arnaud de Villeneuve, qui blâmait l'usage immodéré des épices, permettait, dit-on, d'apprèter la volaille avec du vin, du sel et de l'eau de roses. D'après le Ménagier de Paris ${ }^{3}$, les perdrix rôties se mangeaient " au sel menu ou à l'eaue rose et un petit de vin ». Elle entrait aussi dans la préparation de ce "blancmanger », que le cuisinier des Canterbury Tales excellait à faire*. Suivant Le Grand d'Aussy, l'eau

1. On les préparait en mettant ensemble des poulets rôtis et de la chair de porc bouillie; puis on y ajoutait un quarteron de roses. du gringembre et du poivre, du vin et du vinaigre, avec du sucre et du miel; après quoi on faisait revenir le tout sur le feu. Edm. O. ron Lippmann, Geschichte des Zucker's. Leipzigr, 1890, in-8, p. 2'10.

2. Le Grand d'Aussy, op. laud., vol. II, p. 244. Le Grand cite en particulier, p. 230 et 232 , la " soupe dorée » et la "soupe au chènevis » où entrait de l'eau de roses.

3. Vol. II, p. 183.

4. For blanc manger he madè with the best.

John Saunders, Chaucer's Canterbury tales annotated, p. 162, donne la recette de ce mets, d'après $A$ proper new Booke of Cookery de 15:5; on le faisait avec le blanc d'un chapon bouilli, puis séché et ensuite cardé, qu'on mèlait avec du lait, de la crème et de la farine de seigle; on mettait le tout dans une poèle sur le feu, on l'agitait et quand il commençait à bouillir, on ajoutait une demi-livre de sucre en poudre et un gobelet deau de rose. Taillevant, cité par Le Grand d'Aussy, II, 252, avait, un siècle auparavant, donné de ce mets une recette analogue. 
de roses servait également dans la confection de certains desserts; on l'employait en particulier, dit-il, pour assaisonner les cerneaux. Il parle aussi de tartes aux prunes et à l'eau de roses ${ }^{1}$.

Chose surprenante, la rose, dont le parfum est si suave et si recherché, a été parfois un objet d'antipathie. D’après Michel Glykas ${ }^{2}$, qui se fait ici l'écho d'une croyance de l'antiquité, les vautours et les coléoptères fuient le parfum des roses. Il suffit aussi pour tuer les escargots, suivant le persan Enweri ${ }^{3}$. Des hommes eux-mêmes, dit-on, l'ont redouté; le cardinal Olivier Caraffa quittait Rome dans la saison des roses et allait s'enfermer dans son parc, voisin du Quirinal, afin d'éviter toute visite qui pût lui apporter l'odeur de ces fleurs. Quand le doge de Venise, Francesco Venerio, allait à l'église les jours de grandes fètes, il avait soin d'en faire enlever les guirlandes de roses, dont l'odeur l'eùt fait tomber sans connaissance ${ }^{4}$. Il arrivait au cardinal Henri de Cordoue, et à un dominicain de la famille des Barberigi de Venise, de tomber aussi sans connaissance lorsqu'ils sentaient et le dernier mème quand il voyait de loin une rose $e^{5}$.

1. Op. laud., vol. III, p. 275.

2. M. Glykas, Annales. Venetiae, 1729, in-fol., p. 39, a.

3. Schleiden, Die Rose, p. 266.

4. Rosenberg, Rhodologia, p. 216.

5. Schleiden, Die Rose, p. 182. 


\section{BIBLIOGRAPHIE 1}

BeL (J.), La rose. Histoire et culture. Paris, 1892, in-8.

*Benemanx (J.-C.), Die Rose zum Ruhme ihres Schöpfers. Leipzig, 1742.

Buc'hoz (J.-P.), Monographie de la rose et de la violette, considérées sous leur aspect d'utilité et d'agrément, etc. Paris, 1804 , in-8.

Caetani-Lovatelli (E.), La festa delle rose. (Nuova antologia, 1er nov. 1888, vol. XVIII, 3e série).

Ghesnel (A. DE), La rose chez les différents peuples anciens et modernes. Description, culture et propriétés des roses. Paris, 2e éd., 1838, in-8.

Guerrapatn (T.), Almanach des Roses. Troyes, 1811, in-18. Gurlemeau, Histoire naturelle de la rose. Paris, 1800, in-12.

*Hagendorn (Ehr.-Fried.), Cynosbatologia. Ienae, 1681, in-8.

* Hermax (Joh.), Dissertatio inauguralis de rosa. Argentorati, 1672, in-4.

Joret (Charles), La légende de la rose au moyen âge chez les nations romanes et germaniques. Macon, 1890, in-8.

Linder (E.), Monographie du genre rosier, trad. par M. de Pronville. Paris, 182' in-8.

Loiseleur-Deslongchaips (J.-L.-A.), La rose, son histoire, sa culture, sa poésie. Paris, 184', in-12.

Moxardus (Nic.), De rosa et partibus eius. Antverpiae, 1564, in-8.

Orbessax (Mis D'), Essai sur les roses (Mélanges hisloriques et critiques, etc. Paris, 1768, in-8, vol. IJ, p. 297-338).

Redouté et 'T'Honx, Les roses. Paris, 1815-24, in-fol.

Rosexberg (Joh.-Car.), Rhodologire, seu philosophico-medica generosae rosae descriptio. Francof., 1624, in-12.

Schleiden (M. J.), Die Rose. Geschichte und Symbolik in ethnographischer und kulturhistorischer Beziehung. Leipzig, 1873, in-8.

Schmot-Braxco (Cecilia), A rosa na vida dos povos. Madrid, 1886. in-8 (Bibl. de las tradiciones populares españolas, vol. VIII).

*Vrbert, Essai sur les roses. Paris, 1824-30, in-8.

*Walroth, Rosae plantarum generis historia succincta. Nordh. 1828, in-8.

1. Cette bibliographie ne comprend que les ourrages qui traitent exclusivement de la rose; jai marqué d'un astérisque ceux que je n'ai pu consulter moi-même.

Joret. La Rose. 


\section{ADDITIONS ET CORRECTIONS}

Page 3 , note 5 , der alten lire : des alten

- 48, note $1, \omega_{5} \quad-\omega_{5}$

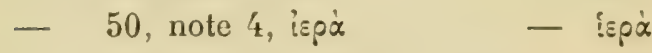

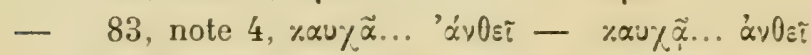

- 140. note 1. scabaei - scarabaei

- 169, lig. 5, épines _ aiguillons

- 193, note 3. Miklosisch _ Miklosich

- 207, lig. 3, gaement - gaiement

- 235, note 4, vividario - viridario

- 219. Un renseignement, qui me paraissait digne de toute confiance, m'a fait croire que la poésie religieuse de la Grèce ignorait la comparaison de la Vierge avec la rose; le fragment suivant d'un poème du mont Ithos, communiqué par le cardinal Pitra à Rohault de Fleury (La sainte Vierge, etc., I, 382), nous offre néanmoins celte comparaison :

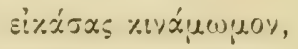

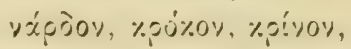

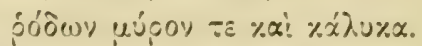

Page 285, note 1, 1. 4, effacer pg.

- 293, note 1, v. 2, geitchte lire: getichte

- 315, note 1, 1. 3, Antwerpener Liederbueklire: Antwerper Liederboek.

- 315, note 3, v. 2, eel lire: edel

- 319, note 2, v. 3. krant - kraut.

- 356, note 4, 1.6, Stuckins - Stuckius. 


\section{TA B L E}

Pages

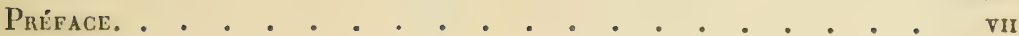

\section{PREMIERE PARTIE.}

\section{LA ROSE DANS L'ANTIQUiTÉ.}

Ginapitre $I^{e r}$. - Des espèces de roses connues dans l'antiquité. . 1

Cirapitre II. - Culture de la rose dans l'antiquité. . . . . . 30

Cinapitre III. - La rose dans les légendes et dans la poésie des Grecs et des Romains. . . . . . . 45

Cincitre IV. - Usages de la rose chez les Grecs et les Romains. . 88

Cirapite V. - La rose dans l'ancien Orient. . . . . . . 122

Cirapitre VI. - La rose dans la pharmacopée grecque et romaine. 129

\section{SEGONDE PARTIE.}

\section{LA ROSE AU MOYEN AGE.}

Cunpitre $I^{\text {er }}$. - Gulture de la rose dans l'Orient et dans l'Occident. . . . . . . . . . 1't 1

Chiprtre II. - La rose dans les légendes et dans la poésie de l'Orient. La rose et le rossignol. . . . . 196

Ginpitre III. - La rose dans les légendes chrétiennes. . . . 231

Chaprtre IV. - La rosedans les légendes profanes et dans la poésie de l'Occident. . . . . . . . 28.5

Cirapitre V. - La rose dans les usages de la vie, dans le culte et dans l'art . . . . . . . . . . . :3!0

Cinpitre VI. - La rose dans la pharmacopée et dans l'art culinaire. . . . . . . . . . 461

Bibliograpine . . . . . . . . . . . . . . 481

Adpitions et corrections. . . . . . . . . . . . 482 


